\title{
TOXICOLOGICAL PROFILE FOR NICKEL
}

U.S. DEPARTMENT OF HEALTH AND HUMAN SERVICES

Public Health Service

Agency for Toxic Substances and Disease Registry

August 2005 


\section{DISCLAIMER}

The use of company or product name(s) is for identification only and does not imply endorsement by the Agency for Toxic Substances and Disease Registry. 


\section{UPDATE STATEMENT}

A Toxicological Profile for Nickel, Draft for Public Comment was released in September 2004. This edition supersedes any previously released draft or final profile.

Toxicological profiles are revised and republished as necessary. For information regarding the update status of previously released profiles, contact ATSDR at:

Agency for Toxic Substances and Disease Registry Division of Toxicology/Toxicology Information Branch 1600 Clifton Road NE

Mailstop F-32

Atlanta, Georgia 30333 



\section{FOREWORD}

This toxicological profile is prepared in accordance with guidelines* developed by the Agency for Toxic Substances and Disease Registry (ATSDR) and the Environmental Protection Agency (EPA). The original guidelines were published in the Federal Register on April 17, 1987. Each profile will be revised and republished as necessary.

The ATSDR toxicological profile succinctly characterizes the toxicologic and adverse health effects information for the hazardous substance described therein. Each peer-reviewed profile identifies and reviews the key literature that describes a hazardous substance's toxicologic properties. Other pertinent literature is also presented, but is described in less detail than the key studies. The profile is not intended to be an exhaustive document; however, more comprehensive sources of specialty information are referenced.

The focus of the profiles is on health and toxicologic information; therefore, each toxicological profile begins with a public health statement that describes, in nontechnical language, a substance's relevant toxicological properties. Following the public health statement is information concerning levels of significant human exposure and, where known, significant health effects. The adequacy of information to determine a substance's health effects is described in a health effects summary. Data needs that are of significance to protection of public health are identified by ATSDR and EPA.

Each profile includes the following:

(A) The examination, summary, and interpretation of available toxicologic information and epidemiologic evaluations on a hazardous substance to ascertain the levels of significant human exposure for the substance and the associated acute, subacute, and chronic health effects;

(B) A determination of whether adequate information on the health effects of each substance is available or in the process of development to determine levels of exposure that present a significant risk to human health of acute, subacute, and chronic health effects; and

(C) Where appropriate, identification of toxicologic testing needed to identify the types or levels of exposure that may present significant risk of adverse health effects in humans.

The principal audiences for the toxicological profiles are health professionals at the Federal, State, and local levels; interested private sector organizations and groups; and members of the public.

This profile reflects ATSDR's assessment of all relevant toxicologic testing and information that has been peer-reviewed. Staff of the Centers for Disease Control and Prevention and other Federal scientists have also reviewed the profile. In addition, this profile has been peer-reviewed by a nongovernmental panel and was made available for public review. Final responsibility for the contents and views expressed in this toxicological profile resides with ATSDR.

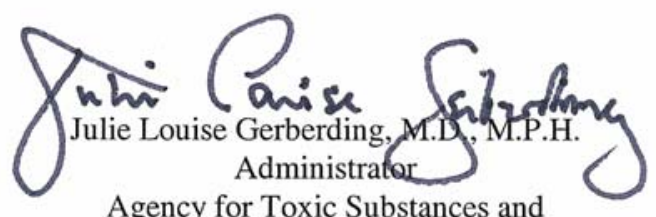

Agency for Toxic Substances and Disease Registry 


\section{* Legislative Background}

The toxicological profiles are developed in response to the Superfund Amendments and Reauthorization Act (SARA) of 1986 (Public law 99-499) which amended the Comprehensive Environmental Response, Compensation, and Liability Act of 1980 (CERCLA or Superfund). This public law directed ATSDR to prepare toxicological profiles for hazardous substances most commonly found at facilities on the CERCLA National Priorities List and that pose the most significant potential threat to human health, as determined by ATSDR and the EPA. The availability of the revised priority list of 275 hazardous substances was announced in the Federal Register on November 17, 1997 (62 FR 61332). For prior versions of the list of substances, see Federal Register notices dated April 29, 1996 (61 FR 18744); April 17, 1987 (52 FR 12866); October 20, 1988 (53 FR 41280); October 26, 1989 (54 FR 43619); October 17, 1990 (55 FR 42067); October 17, 1991 (56 FR 52166); October 28, 1992 (57 FR 48801); and February 28, 1994 (59 FR 9486). Section 104(i)(3) of CERCLA, as amended, directs the Administrator of ATSDR to prepare a toxicological profile for each substance on the list. 


\section{QUICK REFERENCE FOR HEALTH CARE PROVIDERS}

Toxicological Profiles are a unique compilation of toxicological information on a given hazardous substance. Each profile reflects a comprehensive and extensive evaluation, summary, and interpretation of available toxicologic and epidemiologic information on a substance. Health care providers treating patients potentially exposed to hazardous substances will find the following information helpful for fast answers to often-asked questions.

\section{Primary Chapters/Sections of Interest}

Chapter 1: Public Health Statement: The Public Health Statement can be a useful tool for educating patients about possible exposure to a hazardous substance. It explains a substance's relevant toxicologic properties in a nontechnical, question-and-answer format, and it includes a review of the general health effects observed following exposure.

Chapter 2: Relevance to Public Health: The Relevance to Public Health Section evaluates, interprets, and assesses the significance of toxicity data to human health.

Chapter 3: Health Effects: Specific health effects of a given hazardous compound are reported by type of health effect (death, systemic, immunologic, reproductive), by route of exposure, and by length of exposure (acute, intermediate, and chronic). In addition, both human and animal studies are reported in this section.

NOTE: Not all health effects reported in this section are necessarily observed in the clinical setting. Please refer to the Public Health Statement to identify general health effects observed following exposure.

Pediatrics: Four new sections have been added to each Toxicological Profile to address child health issues:

Section 1.6 How Can (Chemical X) Affect Children?

Section 1.7 How Can Families Reduce the Risk of Exposure to (Chemical X)?

Section 3.7 Children's Susceptibility

Section 6.6 Exposures of Children

Other Sections of Interest:

Section 3.8 Biomarkers of Exposure and Effect

Section 3.11 Methods for Reducing Toxic Effects

\section{ATSDR Information Center}

Phone: 1-888-42-ATSDR or (404) 498-0110 Fax: $\quad$ (770) 488-4178

E-mail: atsdric@cdc.gov Internet: http://www.atsdr.cdc.gov

The following additional material can be ordered through the ATSDR Information Center:

Case Studies in Environmental Medicine: Taking an Exposure History-The importance of taking an exposure history and how to conduct one are described, and an example of a thorough exposure history is provided. Other case studies of interest include Reproductive and Developmental 
Hazards; Skin Lesions and Environmental Exposures; Cholinesterase-Inhibiting Pesticide Toxicity; and numerous chemical-specific case studies.

Managing Hazardous Materials Incidents is a three-volume set of recommendations for on-scene (prehospital) and hospital medical management of patients exposed during a hazardous materials incident. Volumes I and II are planning guides to assist first responders and hospital emergency department personnel in planning for incidents that involve hazardous materials. Volume IIIMedical Management Guidelines for Acute Chemical Exposures-is a guide for health care professionals treating patients exposed to hazardous materials.

Fact Sheets (ToxFAQs) provide answers to frequently asked questions about toxic substances.

\section{Other Agencies and Organizations}

The National Center for Environmental Health (NCEH) focuses on preventing or controlling disease, injury, and disability related to the interactions between people and their environment outside the workplace. Contact: NCEH, Mailstop F-29, 4770 Buford Highway, NE, Atlanta, GA 30341-3724 • Phone: 770-488-7000 • FAX: 770-488-7015.

The National Institute for Occupational Safety and Health (NIOSH) conducts research on occupational diseases and injuries, responds to requests for assistance by investigating problems of health and safety in the workplace, recommends standards to the Occupational Safety and Health Administration (OSHA) and the Mine Safety and Health Administration (MSHA), and trains professionals in occupational safety and health. Contact: NIOSH, 200 Independence Avenue, SW, Washington, DC 20201 • Phone: 800-356-4674 or NIOSH Technical Information Branch, Robert A. Taft Laboratory, Mailstop C-19, 4676 Columbia Parkway, Cincinnati, OH 45226-1998 - Phone: 800-35-NIOSH.

The National Institute of Environmental Health Sciences (NIEHS) is the principal federal agency for biomedical research on the effects of chemical, physical, and biologic environmental agents on human health and well-being. Contact: NIEHS, PO Box 12233, 104 T.W. Alexander Drive, Research Triangle Park, NC 27709 • Phone: 919-541-3212.

\section{Referrals}

The Association of Occupational and Environmental Clinics (AOEC) has developed a network of clinics in the United States to provide expertise in occupational and environmental issues. Contact: AOEC, 1010 Vermont Avenue, NW, \#513, Washington, DC 20005 • Phone: 202-347-4976 •FAX: 202-347-4950•e-mail: AOEC@AOEC.ORG•Web Page: http://www.aoec.org/.

The American College of Occupational and Environmental Medicine (ACOEM) is an association of physicians and other health care providers specializing in the field of occupational and environmental medicine. Contact: ACOEM, 55 West Seegers Road, Arlington Heights, IL 60005• Phone: 847-818-1800• FAX: 847-818-9266. 


\section{CONTRIBUTORS}

\section{CHEMICAL MANAGER(S)/AUTHOR(S):}

Mike Fay, Ph.D.

Sharon Wilbur, M.A.

Henry Abadin, M.S.P.H.

ATSDR, Division of Toxicology and Environmental Medicine, Atlanta, GA

Lisa Ingerman, Ph.D.

Steven G. Swarts, Ph.D.

Syracuse Research Corporation, North Syracuse, NY

THE PROFILE HAS UNDERGONE THE FOLLOWING ATSDR INTERNAL REVIEWS:

1. Health Effects Review. The Health Effects Review Committee examines the health effects chapter of each profile for consistency and accuracy in interpreting health effects and classifying end points.

2. Minimal Risk Level Review. The Minimal Risk Level Workgroup considers issues relevant to substance-specific Minimal Risk Levels (MRLs), reviews the health effects database of each profile, and makes recommendations for derivation of MRLs.

3. Data Needs Review. The Research Implementation Branch reviews data needs sections to assure consistency across profiles and adherence to instructions in the Guidance.

4. Green Border Review. Green Border review assures the consistency with ATSDR policy. 



\section{PEER REVIEW}

A peer review panel was assembled for nickel. The panel consisted of the following members:

1. George Daston, Ph.D., Research Fellow, Miami Valley Laboratories, The Procter \& Gamble Company, Cincinnati, $\mathrm{OH}$;

2. A. Phillip Leber, Ph.D., DABT, Consultant in Toxicology, Akron, OH; and

3. Sam Kacew, Ph.D., ATS, Professor, Department of Cellular and Molecular Medicine, University of Ottawa, Ottawa, ON, Canada.

These experts collectively have knowledge of nickel's physical and chemical properties, toxicokinetics, key health end points, mechanisms of action, human and animal exposure, and quantification of risk to humans. All reviewers were selected in conformity with the conditions for peer review specified in Section 104(I)(13) of the Comprehensive Environmental Response, Compensation, and Liability Act, as amended.

Scientists from the Agency for Toxic Substances and Disease Registry (ATSDR) have reviewed the peer reviewers' comments and determined which comments will be included in the profile. A listing of the peer reviewers' comments not incorporated in the profile, with a brief explanation of the rationale for their exclusion, exists as part of the administrative record for this compound.

The citation of the peer review panel should not be understood to imply its approval of the profile's final content. The responsibility for the content of this profile lies with the ATSDR. 



\section{CONTENTS}

DISCLAIMER
UPDATE STATEMENT
FOREWORD
QUICK REFERENCE FOR HEALTH CARE PROVIDERS
CONTRIBUTORS
PEER REVIEW
CONTENTS
LIST OF FIGURES
LIST OF TABLES

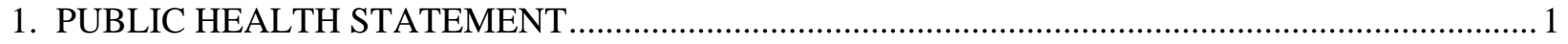

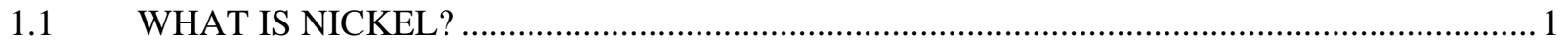

1.2 WHAT HAPPENS TO NICKEL WHEN IT ENTERS THE ENVIRONMENT? ..................... 2

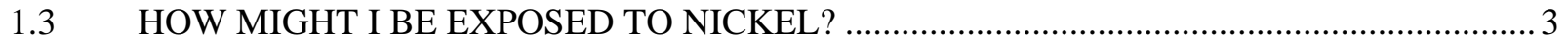

$1.4 \quad$ HOW CAN NICKEL ENTER AND LEAVE MY BODY? ............................................ 5

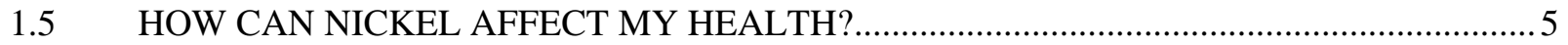

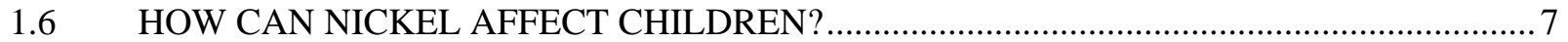

1.7 HOW CAN FAMILIES REDUCE THE RISK OF EXPOSURE TO NICKEL?...................... 8

1.8 IS THERE A MEDICAL TEST TO DETERMINE WHETHER I HAVE BEEN

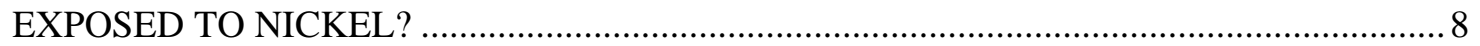

1.9 WHAT RECOMMENDATIONS HAS THE FEDERAL GOVERNMENT MADE

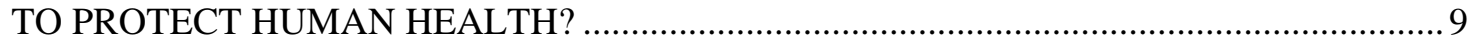

1.10 WHERE CAN I GET MORE INFORMATION? ................................................................ 10

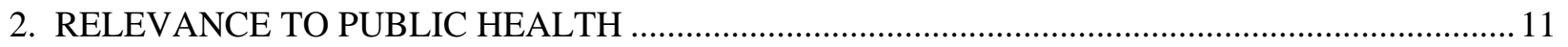

2.1 BACKGROUND AND ENVIRONMENTAL EXPOSURES TO NICKEL IN THE

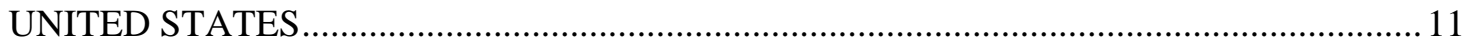

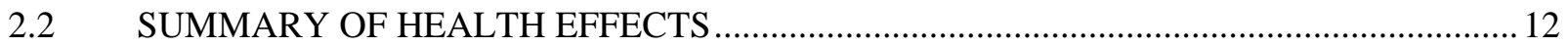

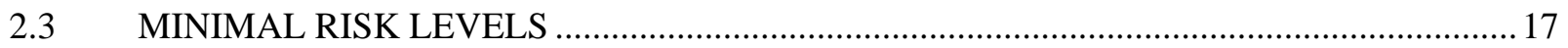

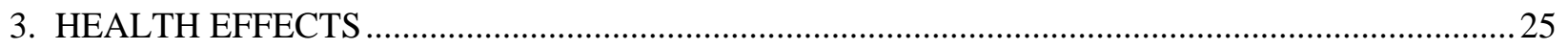

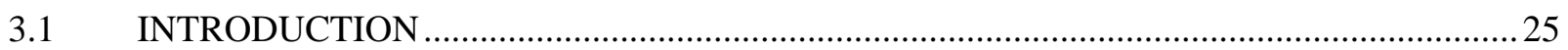

3.2 DISCUSSION OF HEALTH EFFECTS BY ROUTE OF EXPOSURE ................................. 25

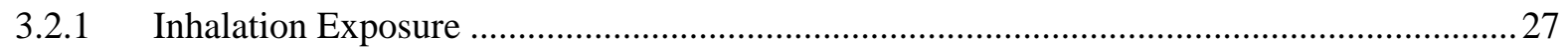

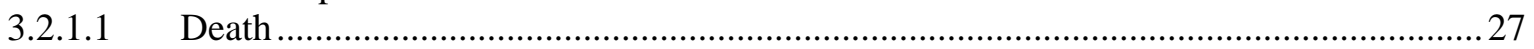

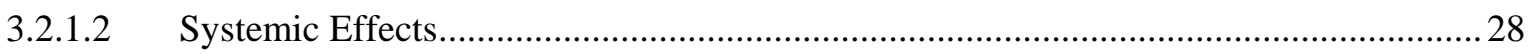

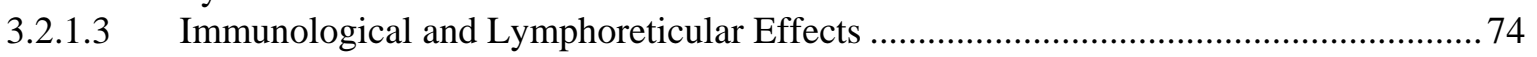

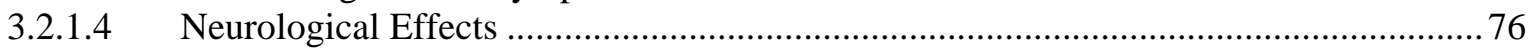

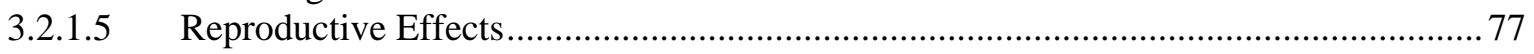

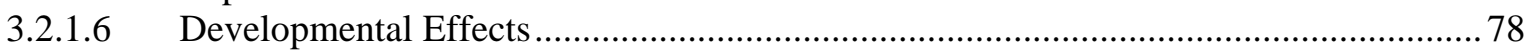

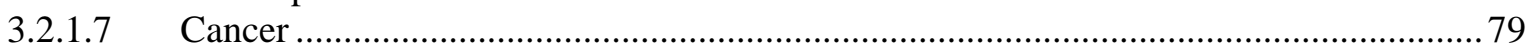

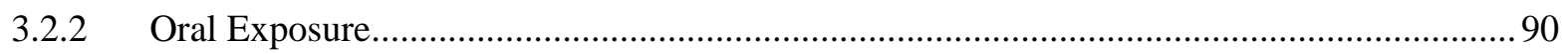

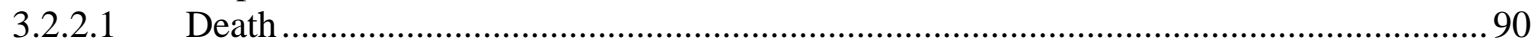

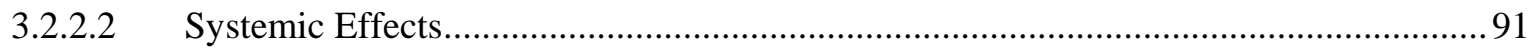

3.2.2.3 Immunological and Lymphoreticular Effects ....................................................... 116

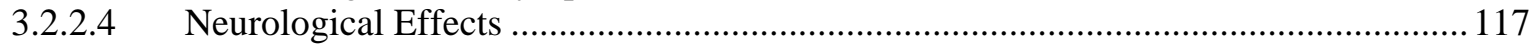




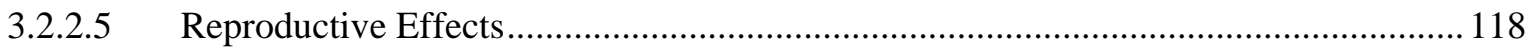

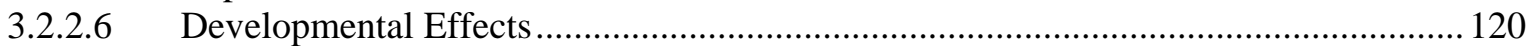

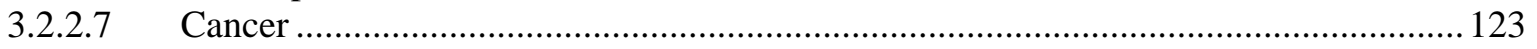

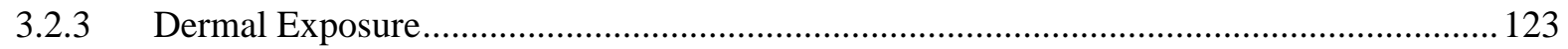

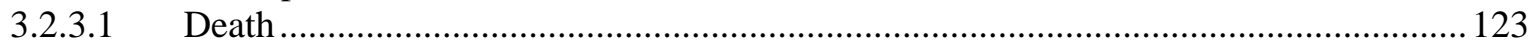

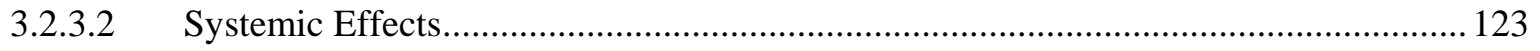

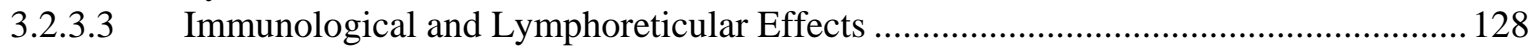

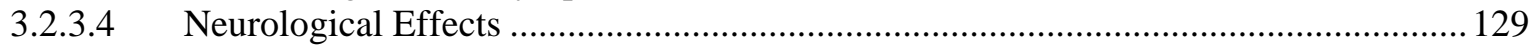

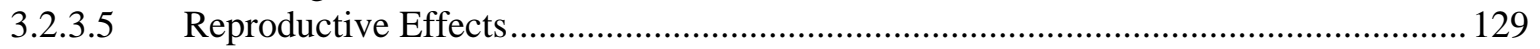

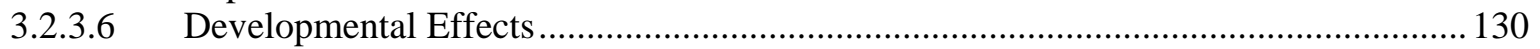

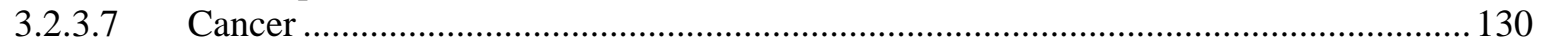

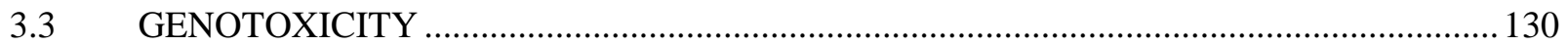

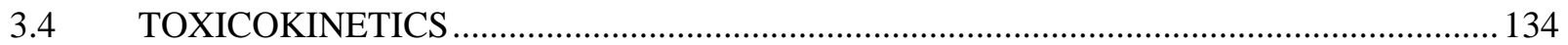

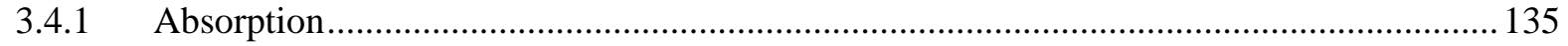

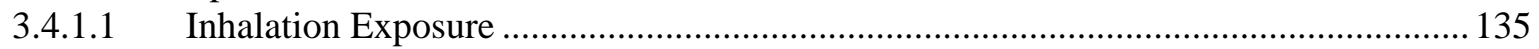

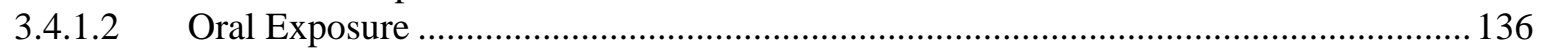

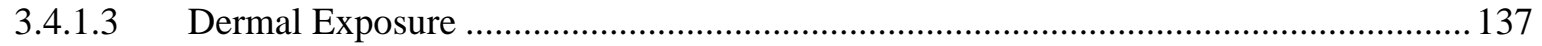

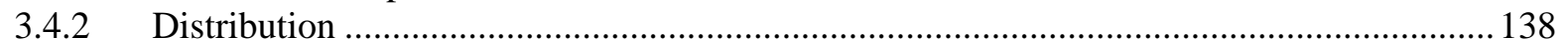

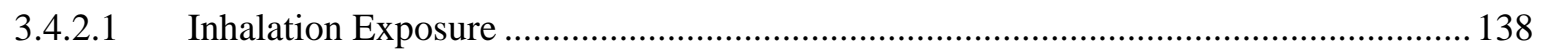

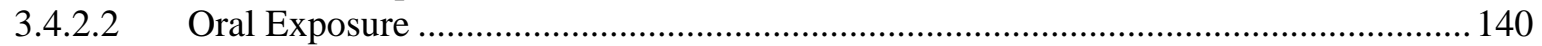

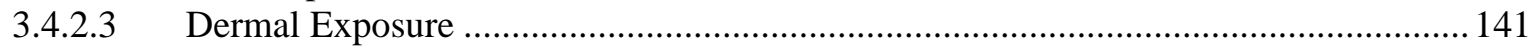

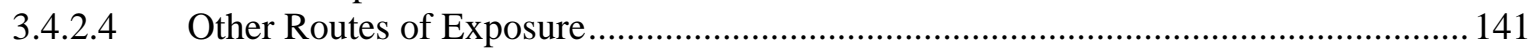

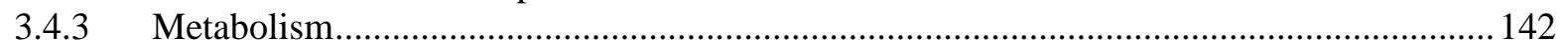

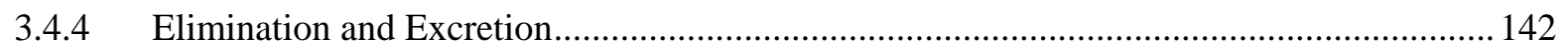

3.4.4.1 Inhalation Exposure ……....................................................................... 142

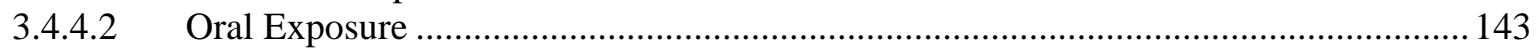

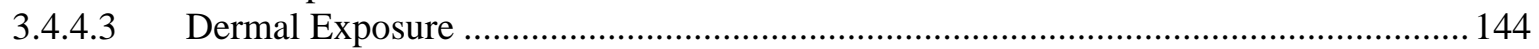

3.4.5 Physiologically Based Pharmacokinetic (PBPK)/Pharmacodynamic (PD) Models ........... 144

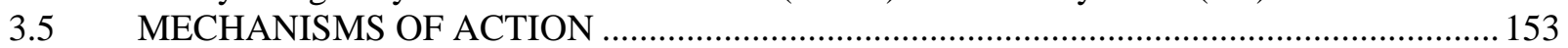

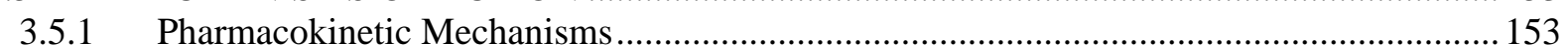

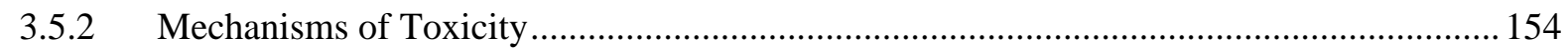

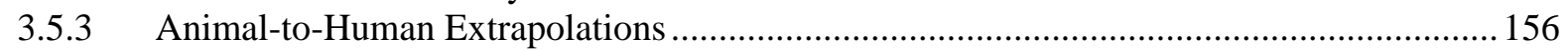

3.6 TOXICITIES MEDIATED THROUGH THE NEUROENDOCRINE AXIS ....................... 157

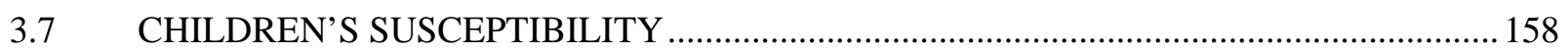

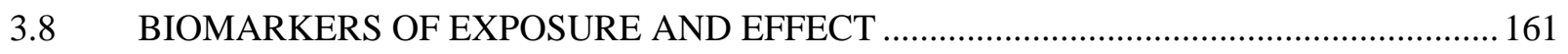

3.8.1 Biomarkers Used to Identify or Quantify Exposure to Nickel ........................................ 162

3.8.2 Biomarkers Used to Characterize Effects Caused by Nickel ............................................ 164

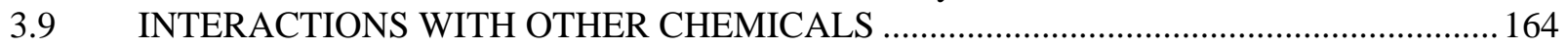

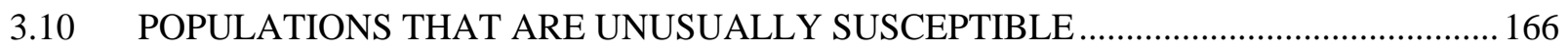

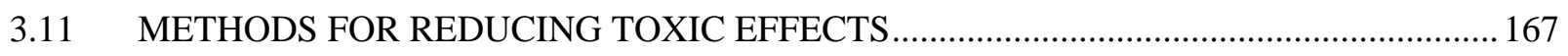

3.11.1 Reducing Peak Absorption Following Exposure ....................................................... 167

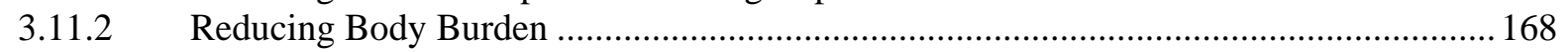

3.11.3 Interfering with the Mechanism of Action for Toxic Effects ........................................ 169

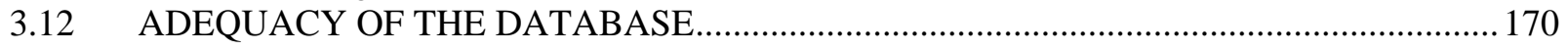

3.12.1 Existing Information on Health Effects of Nickel ......................................................... 170

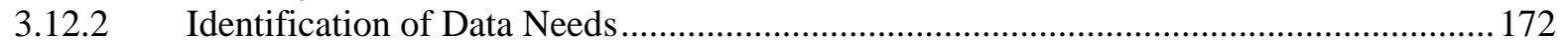

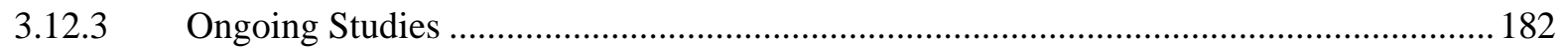

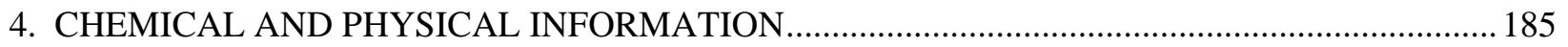

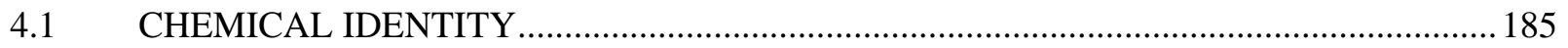

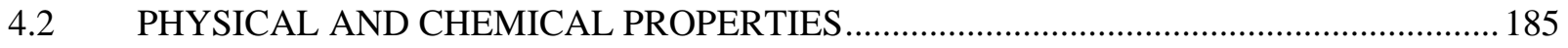




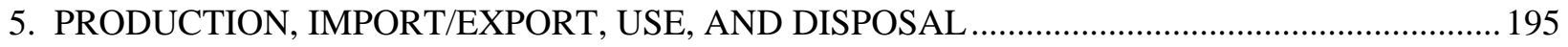

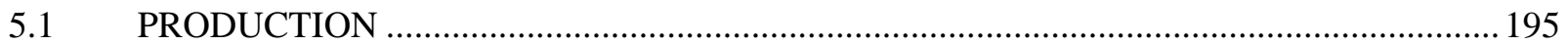

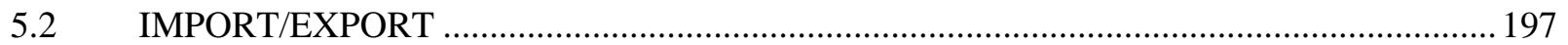

$5.3 \quad$ USE

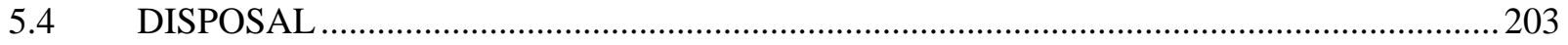

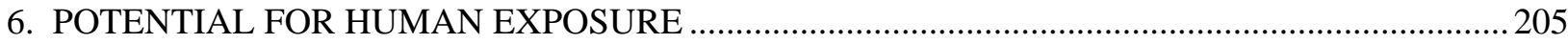

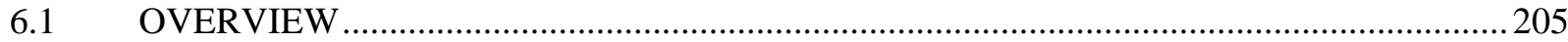

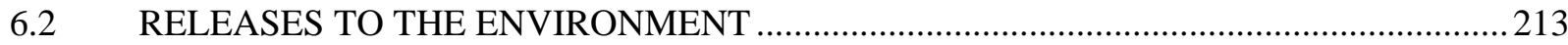

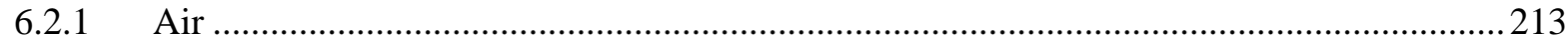

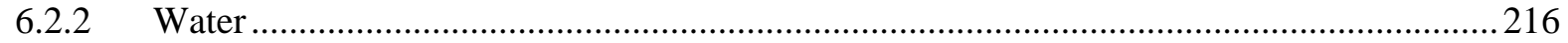

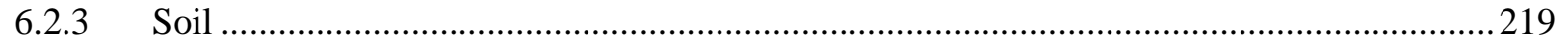

6.3 ENVIRONMENTAL FATE

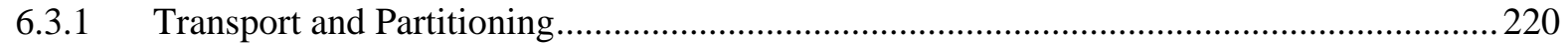

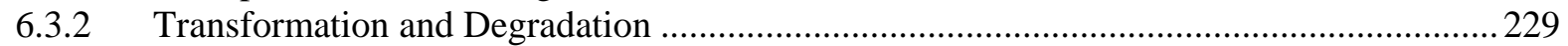

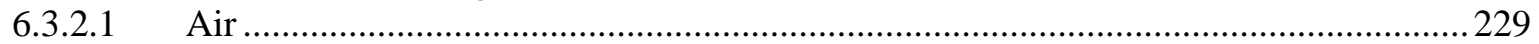

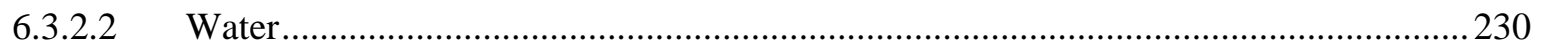

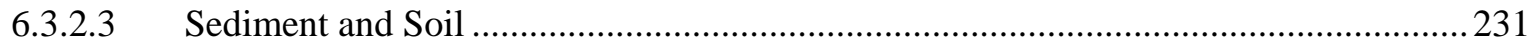

6.4 LEVELS MONITORED OR ESTIMATED IN THE ENVIRONMENT …..........................231

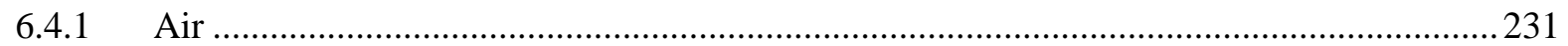

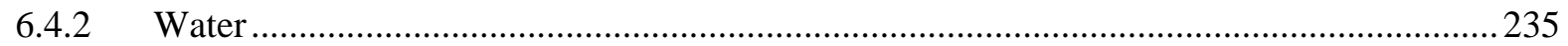

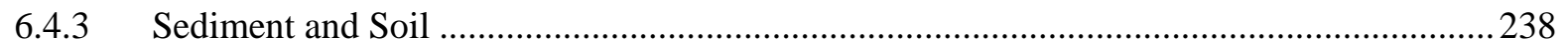

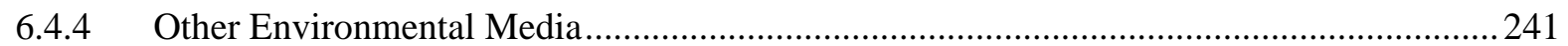

6.5 GENERAL POPULATION AND OCCUPATIONAL EXPOSURE ....................................243

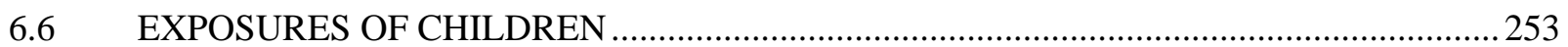

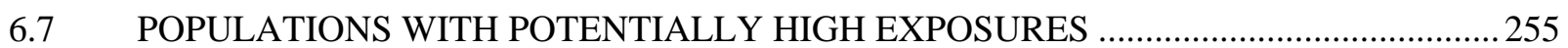

6.8 ADEQUACY OF THE DATABASE

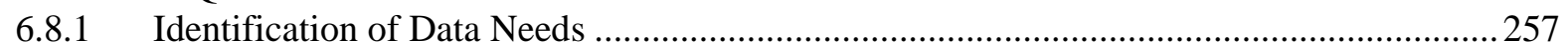

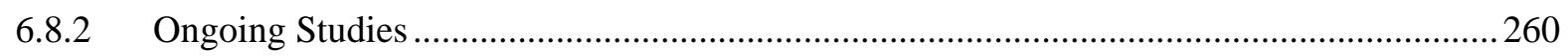

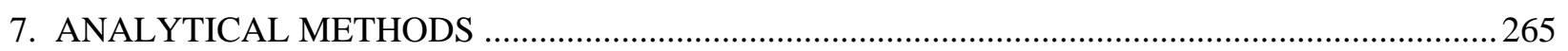

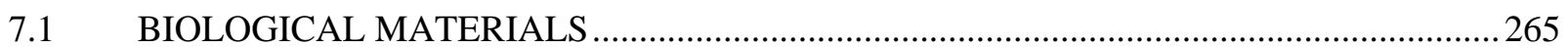

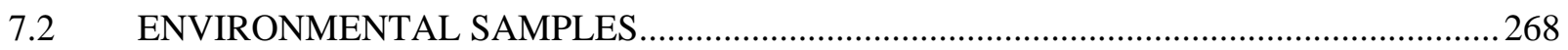

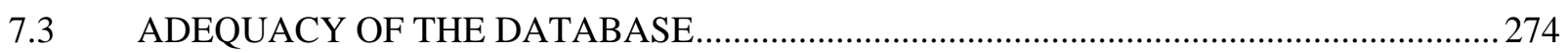

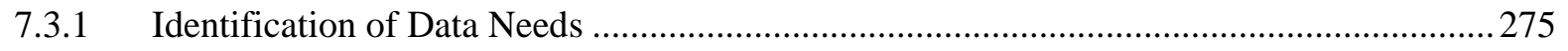

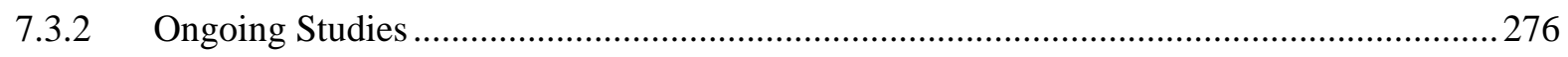

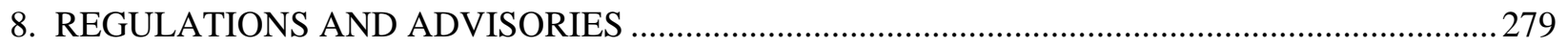

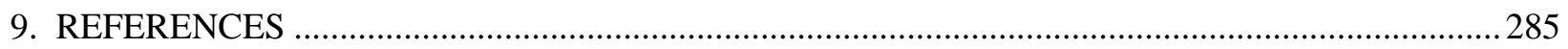

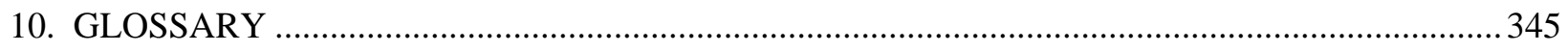




\section{APPENDICES}

A. ATSDR MINIMAL RISK LEVELS AND WORKSHEETS …..................................................... A-1

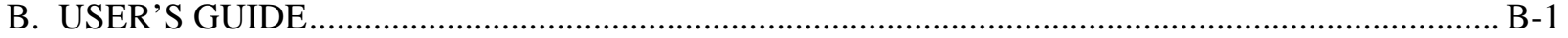

C. ACRONYMS, ABBREVIATIONS, AND SYMBOLS..................................................................

D. INDEX 


\section{LIST OF FIGURES}

3-1. Levels of Significant Exposure to Nickel - Inhalation ....................................................................59

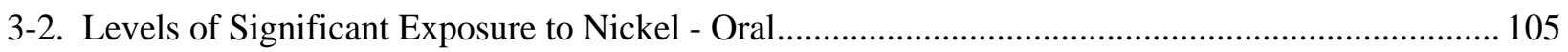

3-3. Conceptual Representation of a Physiologically Based Pharmacokinetic (PBPK) Model for a

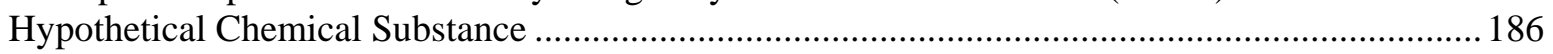

3-4. Diagram of the Compartmental Model of Nickel Metabolism ..................................................... 188

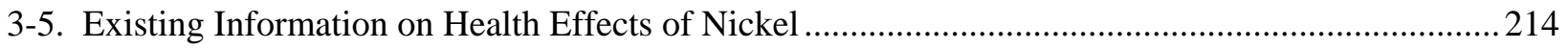

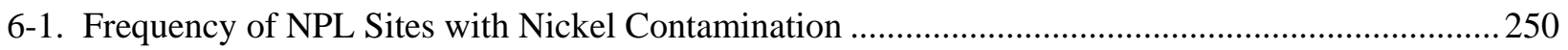





\section{LIST OF TABLES}

3-1. Levels of Significant Exposure to Nickel - Inhalation 29

3-2. Comparison of Risk of Dying of Lung Cancer at Different Levels of Cumulative Exposure to Sulfidic Nickel by Different Levels of Combined Cumulative Exposure to Oxidic and Soluble Nickel in the Mond/INCO (Clydach) Nickel Refinery

3-3. Comparison of Risk of Dying of Lung Cancer at Different Levels of Cumulative Exposure to Oxidic Nickel by Different Levels of Combined Cumulative Exposure to Sulfidic and Soluble Nickel in the Mond/INCO (Clydach) Nickel Refinery.

3-4. Comparison of Risk of Dying of Lung Cancer at Different Levels of Cumulative Exposure to Soluble Nickel by Different Levels of Combined Cumulative Exposure to Sulfidic and Oxidic Nickel in the Mond/INCO (Clydach) Nickel Refinery...

3-5. Comparison of Risk of Dying of Lung Cancer at Different Levels of Cumulative Exposure to Metallic Nickel by Different Levels of Combined Cumulative Exposure to Sulfidic, Oxidic, and Soluble Nickel in the Mond/INCO (Clydach) and Falconbridge (Kristainsand)

Nickel Refineries

3-6. Alveolar/Bronchiolar Neoplasms and Adrenal Medulla Proliferative Lesions in Rats 88

3-7. Alveolar/Bronchiolar Neoplasms in Mice.

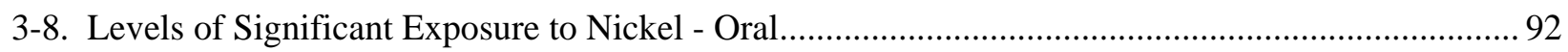

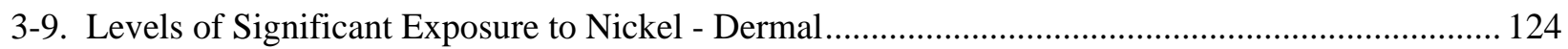

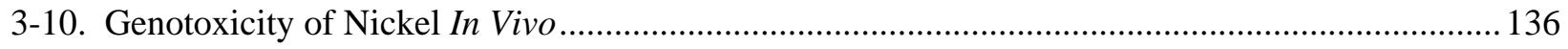

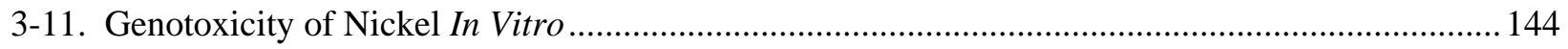

3-12. Kinetic Parameters of Nickel Sulfate Absorption, Distribution, and Elimination in Humans ....... 189

3-13. Clearance Rate Coefficient Constants of Nickel Compounds .................................................... 193

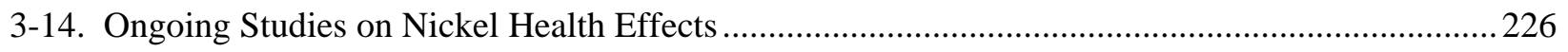

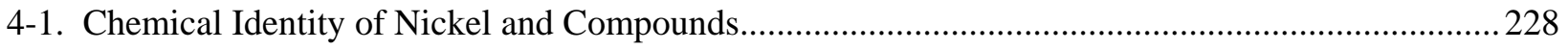

4-2. Physical and Chemical Properties of Nickel and Compounds ......................................................2234

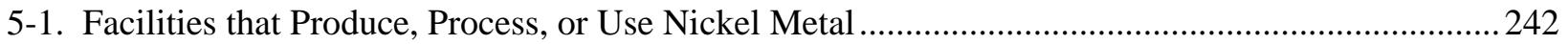

5-2. Facilities that Produce, Process, or Use Nickel Compounds ......................................................... 244

6-1. Releases to the Environment from Facilities that Produce, Process, or Use Nickel.......................251

6-2. Releases to the Environment from Facilities that Produce, Process, or Use Nickel Compounds .... 253 
6-3. Total Dietary Exposure Estimates of Study Participants to Nickel Based on the Dietary Information Obtained from the NHEXAS Arizona Study

6-4. Dietary Exposure Estimates of U.S. Populations to Nickel Based on the Dietary Exposure Potential Model (DEPM).

6-5. Nickel Levels in Air and Distribution of Different Forms of Nickel as a Proportion (by Weight) of Total Nickel in Selected Departments and Time Periods at a Nickel Refinery in Norway.....

6-6. Ongoing Studies on Environmental Fate and the Potential for Human Exposure to Nickel

7-1. Analytical Methods for Determining Nickel in Biological Materials 331

7-2. Analytical Methods for Determining Nickel in Environmental Samples 334

7-3. Ongoing Studies Involving Sample Collection and the Characterization and Quantification of Nickel

8-1. Regulations and Guidelines Applicable to Nickel and Nickel Compounds 345 


\section{PUBLIC HEALTH STATEMENT}

This public health statement tells you about nickel and the effects of exposure to it.

The Environmental Protection Agency (EPA) identifies the most serious hazardous waste sites in the nation. These sites are then placed on the National Priorities List (NPL) and are targeted for long-term federal clean-up activities. Nickel has been found in at least 882 of the 1,662 current or former NPL sites. Although the total number of NPL sites evaluated for this substance is not known, the possibility exists that the number of sites at which nickel is found may increase in the future as more sites are evaluated. This information is important because these sites may be sources of exposure and exposure to nickel may harm you.

When a substance is released either from a large area, such as an industrial plant, or from a container, such as a drum or bottle, it enters the environment. Such a release does not always lead to exposure. You can be exposed to a substance only when you come in contact with it. You may be exposed by breathing, eating, or drinking the substance, or by skin contact.

If you are exposed to nickel, many factors will determine whether you will be harmed. These factors include the dose (how much), the duration (how long), and how you come in contact with it. You must also consider any other chemicals you are exposed to and your age, gender, diet, family traits, lifestyle, and state of health.

\subsection{WHAT IS NICKEL?}

Pure nickel is a hard, silvery-white metal, which has properties that make it very desirable for combining with other metals to form mixtures called alloys. Some of the metals that nickel can be alloyed with are iron, copper, chromium, and zinc. These alloys are used in making metal coins and jewelry and in industry for making items such as valves and heat exchangers. Most nickel is used to make stainless steel. There are also compounds consisting of nickel combined with many other elements, including chlorine, sulfur, and oxygen. Many of these nickel 
compounds are water soluble (dissolve fairly easily in water) and have a characteristic green color. Nickel and its compounds have no characteristic odor or taste. Nickel compounds are used for nickel plating, to color ceramics, to make some batteries, and as substances known as catalysts that increase the rate of chemical reactions.

Nickel combined with other elements occurs naturally in the earth's crust. It is found in all soil, and is also emitted from volcanoes. Nickel is the 24th most abundant element. In the environment, it is primarily found combined with oxygen or sulfur as oxides or sulfides. Nickel is also found in meteorites and on the ocean floor in lumps of minerals called sea floor nodules. The earth's core is composed of $6 \%$ nickel. Nickel is released into the atmosphere during nickel mining and by industries that make or use nickel, nickel alloys, or nickel compounds. These industries also might discharge nickel in waste water. Nickel is also released into the atmosphere by oil-burning power plants, coal-burning power plants, and trash incinerators.

There are no nickel mining operations in the United States. Much of our nickel used in industries comes from recycling nickel-containing alloys or is imported mainly from Canada and Russia.

See Chapters 4 and 5 of this profile for more information on the properties, sources, and uses of nickel and its compounds.

\subsection{WHAT HAPPENS TO NICKEL WHEN IT ENTERS THE ENVIRONMENT?}

Nickel may be released to the environment from the stacks of large furnaces used to make alloys or from power plants and trash incinerators. The nickel that comes out of the stacks of power plants attaches to small particles of dust that settle to the ground or are taken out of the air in rain or snow. It usually takes many days for nickel to be removed from the air. If the nickel is attached to very small particles, it can take more than a month to settle out of the air. Nickel can also be released in industrial waste water. A lot of nickel released into the environment ends up in soil or sediment where it strongly attaches to particles containing iron or manganese. Under acidic conditions, nickel is more mobile in soil and might seep into groundwater. Nickel does 
not appear to concentrate in fish. Studies show that some plants can take up and accumulate nickel. However, it has been shown that nickel does not accumulate in small animals living on land that has been treated with nickel-containing sludge.

See Chapter 6 for more information on the fate of nickel in the environment.

\subsection{HOW MIGHT I BE EXPOSED TO NICKEL?}

Nickel normally occurs at very low levels in the environment, so very sensitive methods are needed to detect nickel in most environmental samples. Food is the major source of exposure to nickel. You may also be exposed to nickel by breathing air, drinking water, or smoking tobacco containing nickel. Skin contact with soil, bath or shower water, or metals containing nickel, as well as, metals plated with nickel can also result in exposure. Stainless steel and coins contain nickel. Some jewelry is plated with nickel or made from nickel alloys. Patients may be exposed to nickel in artificial body parts made from nickel-containing alloys. Exposure of an unborn child to nickel is through the transfer of nickel from the mother's blood to fetal blood. Likewise, nursing infants are exposed to nickel through the transfer of nickel from the mother to breast milk. However, the concentration of nickel in breast milk is either similar or less than the concentration of nickel in infant formulas and cow's milk.

We often do not know the exact form of nickel we are exposed to, including at most hazardous waste sites. Much of the nickel found in air, soil, sediment, and rock is so strongly attached to dust and soil particles or embedded in minerals that it is not readily taken up by plants and animals and, therefore, cannot easily affect your health. In water and waste water, nickel can exist either dissolved in water or attached to material suspended in water.

Nickel in air is attached to small particles. Over a 6-year period (1977-1982) in the United States, average nickel concentrations in cities and in the country ranged from 7 to 12 nanograms per cubic meter (ng/ $\mathrm{m}^{3} ; 1 \mathrm{ng} / \mathrm{m}^{3}$ is equivalent to 1 billionth of a gram in a cubic meter of air). More recently, EPA estimates that the average nickel concentration in air in the United States has decreased to $2.2 \mathrm{ng} / \mathrm{m}^{3}$, based on air quality information obtained from 1996. 
The concentration of nickel in the water of rivers and lakes is very low, with the average concentration usually less than 10 parts of nickel in a billion parts of water (ppb). The level of nickel in water is often so low that we cannot measure it unless we use very sensitive instruments. The average concentration of nickel in drinking water in the United States is between 2 and 4.3 ppb. However, you may be exposed to higher-than-average levels of nickel in drinking water if you live near industries that process or use nickel. The highest levels of nickel in drinking water, about $72 \mathrm{ppb}$, were found near areas of a large natural nickel deposit where nickel is mined and refined.

Soil usually contains between 4 and 80 parts of nickel in a million parts of soil (ppm; $1 \mathrm{ppm}=1,000 \mathrm{ppb}$ ). The highest soil concentrations (up to 9,000 ppm) are found near industries that extract nickel from ore. High concentrations of nickel occur as dust that is released into air from stacks during processing and settles on the ground. You may be exposed to nickel in soil by skin contact. Children may also be exposed to nickel by eating soil.

Food contains nickel and is the major source of nickel exposure for the general population. You eat about 170 micrograms ( $\mu \mathrm{g} ; 1 \mu \mathrm{g}=1$ millionth of a gram) of nickel in your food every day. Foods naturally high in nickel include chocolate, soybeans, nuts, and oatmeal. Our daily intake of nickel from drinking water is only about $2 \mu \mathrm{g}$. We breathe in between 0.1 and $1 \mu \mathrm{g}$ nickel/day, excluding nickel in tobacco smoke. We are exposed to nickel when we handle coins and touch other metals containing nickel.

You may be exposed to higher levels of nickel if you work in industries that process or use nickel. You also may be exposed to nickel by breathing dust or fumes (as from welding) or by skin contact with nickel-containing metal and dust or solutions containing dissolved nickel compounds. A national survey conducted from 1980 to 1983 estimated that 727,240 workers are potentially exposed to nickel metal, nickel alloys, or nickel compounds.

For more information on the potential for exposure to nickel, please see Chapter 6 . 


\subsection{HOW CAN NICKEL ENTER AND LEAVE MY BODY?}

Nickel can enter your body when you breathe air containing nickel, when you drink water or eat food that contains nickel, and when your skin comes into contact with nickel. If you breathe air that contains nickel, the amount of nickel you inhale that reaches your lungs and enters your blood depends on the size of the nickel particles. If the particles are large, they stay in your nose. If the particles are small, they can enter deep into your lungs. More nickel is absorbed from your lungs into your body when the nickel particles can dissolve easily in water. When the particles do not dissolve easily in water, the nickel may remain in your lungs for a long time. Some of these nickel particles can leave the lungs with mucus that you spit out or swallow. More nickel will pass into your body through your stomach and intestines if you drink water containing nickel than if you eat food containing the same amount of nickel. A small amount of nickel can enter your bloodstream from skin contact. After nickel gets into your body, it can go to all organs, but it mainly goes to the kidneys. The nickel that gets into your bloodstream leaves in the urine. After nickel is eaten, most of it leaves quickly in the feces, and the small amount that gets into your blood leaves in the urine. For more information on how nickel can enter and leave your body, see Chapter 3.

\subsection{HOW CAN NICKEL AFFECT MY HEALTH?}

Scientists use many tests to protect the public from harmful effects of toxic chemicals and to find ways for treating persons who have been harmed.

One way to learn whether a chemical will harm people is to determine how the body absorbs, uses, and releases the chemical. For some chemicals, animal testing may be necessary. Animal testing may also help identify health effects such as cancer or birth defects. Without laboratory animals, scientists would lose a basic method for getting information needed to make wise decisions that protect public health. Scientists have the responsibility to treat research animals with care and compassion. Scientists must comply with strict animal care guidelines because laws today protect the welfare of research animals. 
The most common harmful health effect of nickel in humans is an allergic reaction. Approximately $10-20 \%$ of the population is sensitive to nickel. A person can become sensitive to nickel when jewelry or other items containing nickel are in direct contact and prolonged contact with the skin. Wearing jewelry containing nickel in ears or other body parts that have been newly pierced may also sensitize a person to nickel. However, not all jewelry containing nickel releases enough of the nickel ion to sensitize a person. Once a person is sensitized to nickel, further contact with the metal may produce a reaction. The most common reaction is a skin rash at the site of contact. In some sensitized people, dermatitis (a type of skin rash) may develop in an area of the skin that is away from the site of contact. For example, hand eczema (another type of skin rash) is fairly common among people sensitized to nickel. Some workers exposed to nickel by inhalation can become sensitized and have asthma attacks, but this is rare. People who are sensitive to nickel have reactions when nickel comes into prolonged contact with the skin. Some sensitized individuals react when they eat nickel in food or water or breathe dust containing nickel. More women are sensitive to nickel than men. This difference between men and women is thought to be a result of greater exposure of women to nickel through jewelry and other metal items.

People who are not sensitive to nickel must eat very large amounts of nickel to suffer harmful health effects. Workers who accidentally drank light-green water containing $250 \mathrm{ppm}$ of nickel from a contaminated drinking fountain had stomach aches and suffered adverse effects in their blood (increased red blood cells) and kidneys (increased protein in the urine). This concentration of nickel is more than 100,000 times greater than the amount usually found in drinking water.

The most serious harmful health effects from exposure to nickel, such as chronic bronchitis, reduced lung function, and cancer of the lung and nasal sinus, have occurred in people who have breathed dust containing certain nickel compounds while working in nickel refineries or nickelprocessing plants. The levels of nickel in these workplaces were much higher than usual (background) levels in the environment. Lung and nasal sinus cancers occurred in workers who were exposed to more than $10 \mathrm{mg}$ nickel $/ \mathrm{m}^{3}$ as nickel compounds that were hard to dissolve (such as nickel subsulfide). Exposure to high levels of nickel compounds that dissolve easily in water (soluble) may also result in cancer when nickel compounds that are hard to dissolve (less 
soluble) are present, or when other chemicals that can produce cancer are present. The concentrations of soluble and less-soluble nickel compounds that were found to have produced cancers were 100,000 to 1 million times greater than the usual level of nickel in the air in the United States. The U.S. Department of Health and Human Services (DHHS) has determined that nickel metal may reasonably be anticipated to be a carcinogen and nickel compounds are known human carcinogens. The International Agency for Research on Cancer (IARC) has determined that some nickel compounds are carcinogenic to humans and that metallic nickel may possibly be carcinogenic to humans. The EPA has determined that nickel refinery dust and nickel subsulfide are human carcinogens. These cancer classifications were based on studies of nickel workers and laboratory animals.

Lung inflammation and damage to the nasal cavity have been observed in animals exposed to nickel compounds. At high concentrations, the lung damage is severe enough to affect lung function. Long-term exposure to lower levels of a nickel compound that dissolves easily in water did not produce cancer in animals. Lung cancer developed in rats exposed for a long time to nickel compounds that do not dissolve easily in water.

Oral exposure of humans to high levels of soluble nickel compounds through the environment is extremely unlikely. Because humans have only rarely been exposed to high levels of nickel in water or food, much of our knowledge of the harmful effects of nickel is based on animal studies. Eating or drinking levels of nickel much greater than the levels normally found in food and water have been reported to produce lung disease in dogs and rats and to affect the stomach, blood, liver, kidneys, and immune system in rats and mice, as well as their reproduction and development.

See Chapter 3 for more information on the health effects of nickel exposure.

\subsection{HOW CAN NICKEL AFFECT CHILDREN?}

This section discusses potential health effects in humans from exposures during the period from conception to maturity at 18 years of age. 
It is likely that the health effects seen in children exposed to nickel will be similar to the effects seen in adults. We do not know whether children differ from adults in their susceptibility to nickel. Human studies that examined whether nickel can harm the developing fetus are inconclusive. Animal studies have found increases in newborn deaths and decreases in newborn weight after ingesting nickel. These doses are 1,000 times higher than levels typically found in drinking water. It is likely that nickel can be transferred from the mother to an infant in breast milk and can cross the placenta. The nickel levels in breast milk are likely to be similar to the levels in cow’s milk-based or soy-milk-based infant formula.

\subsection{HOW CAN FAMILIES REDUCE THE RISK OF EXPOSURE TO NICKEL?}

If your doctor finds that you have been exposed to substantial amounts of nickel, ask whether your children might also have been exposed. Your doctor might need to ask your state health department to investigate.

People may be exposed to nickel by wearing jewelry that contains nickel. In some people, wearing jewelry that contains nickel produces skin irritation. Avoiding jewelry containing nickel will eliminate risks of exposure to this source of this metal.

Other sources of nickel exposure are through foods that you eat and drinking water. However, the amount of nickel in foods and drinking water are too low to be of concern.

\subsection{IS THERE A MEDICAL TEST TO DETERMINE WHETHER I HAVE BEEN EXPOSED TO NICKEL?}

Measurements of the amount of nickel in your blood, feces, and urine can be used to estimate your exposure to nickel. More nickel was found in the urine of workers who were exposed to nickel compounds that dissolve easily in water (soluble) than in the urine of workers exposed to compounds that are hard to dissolve (less soluble). This means that it is easier to tell if you have been exposed to soluble nickel compounds than less-soluble compounds. The nickel 
measurements do not accurately predict potential health effects from exposure to nickel. More information on medical tests can be found in Chapters 3 and 7.

\subsection{WHAT RECOMMENDATIONS HAS THE FEDERAL GOVERNMENT MADE TO PROTECT HUMAN HEALTH?}

The federal government develops regulations and recommendations to protect public health. Regulations can be enforced by law. The EPA, the Occupational Safety and Health Administration (OSHA), and the Food and Drug Administration (FDA) are some federal agencies that develop regulations for toxic substances. Recommendations provide valuable guidelines to protect public health, but cannot be enforced by law. The Agency for Toxic Substances and Disease Registry (ATSDR) and the National Institute for Occupational Safety and Health (NIOSH) are two federal organizations that develop recommendations for toxic substances.

Regulations and recommendations can be expressed as "not-to-exceed” levels, that is, levels of a toxic substance in air, water, soil, or food that do not exceed a critical value that is usually based on levels that affect animals; they are then adjusted to levels that will help protect humans. Sometimes these not-to-exceed levels differ among federal organizations because they used different exposure times (an 8-hour workday or a 24-hour day), different animal studies, or other factors.

Recommendations and regulations are also updated periodically as more information becomes available. For the most current information, check with the federal agency or organization that provides it. Some regulations and recommendations for nickel include the following:

OSHA has set an enforceable limit of $1.0 \mathrm{mg}$ nickel $/ \mathrm{m}^{3}$ for metallic nickel and nickel compounds in workroom air to protect workers during an 8-hour shift over a 40-hour work week. EPA recommends that drinking water levels for nickel should not be more than $0.1 \mathrm{mg}$ per liter. 


\subsection{WHERE CAN I GET MORE INFORMATION?}

If you have any more questions or concerns, please contact your community or state health or environmental quality department, or contact ATSDR at the address and phone number below.

ATSDR can also tell you the location of occupational and environmental health clinics. These clinics specialize in recognizing, evaluating, and treating illnesses that result from exposure to hazardous substances.

Toxicological profiles are also available on-line at www.atsdr.cdc.gov and on CD-ROM. You may request a copy of the ATSDR ToxProfiles ${ }^{\mathrm{TM}} \mathrm{CD}$-ROM by calling the toll-free information and technical assistance number at 1-888-42ATSDR (1-888-422-8737), by e-mail at atsdric@cdc.gov, or by writing to:

Agency for Toxic Substances and Disease Registry Division of Toxicology 1600 Clifton Road NE Mailstop F-32

Atlanta, GA 30333

Fax: 1-770-488-4178

Organizations for-profit may request copies of final Toxicological Profiles from the following:

National Technical Information Service (NTIS)

5285 Port Royal Road

Springfield, VA 22161

Phone: $1-800-553-6847$ or $1-703-605-6000$

Web site: http://www.ntis.gov/ 


\section{RELEVANCE TO PUBLIC HEALTH}

\subsection{BACKGROUND AND ENVIRONMENTAL EXPOSURES TO NICKEL IN THE UNITED STATES}

Nickel is a very hard metal that occurs naturally in soils and volcanic dust. Nickel is used in combination with other metals to form alloys used for coins, jewelry, and stainless steel. Nickel compounds are used for electroplating, to color ceramics, and in battery production.

Nickel is released to the atmosphere by windblown dust, volcanoes, combustion of fuel oil, municipal incineration, and industries involved in nickel refining, steel production, and other nickel alloy production. The form of nickel emitted to the atmosphere is dependent upon the source. Complex nickel oxides, nickel sulfate, and metallic nickel are associated with combustion, incineration, and smelting and refining processes. Ambient air concentrations of nickel range between 7 and $12 \mathrm{ng} / \mathrm{m}^{3}$, mainly in the form of aerosols and can be as high as $150 \mathrm{ng} / \mathrm{m}^{3}$ near point sources. Based on 1996 air quality data, EPA has reported average U.S. ambient air levels of $2.2 \mathrm{ng} / \mathrm{m}^{3}$. Ambient air levels of nickel are expected to be higher in urban air than in rural air. Concentrations of nickel in indoor air are generally $10 \mathrm{ng} / \mathrm{m}^{3}$.

Background levels of nickel in soils vary widely depending on local geology and anthropogenic inputs, but concentrations typically range between 4 and 80 ppm. Some areas of the United States may contain natural levels as high as 5,000 ppm. Concentrations of nickel in household dust can be high and therefore pose an increased risk to young children who have greater contact with floors. Nickel concentrations in surface water and groundwater range between 3 and $10 \mu \mathrm{g} / \mathrm{L}$. Nickel levels in drinking water in the United States generally range from 0.55 to $25 \mu \mathrm{g} / \mathrm{L}$ and average between 2 and $4.3 \mu \mathrm{g} / \mathrm{L}$. Based on these average nickel concentrations and a reference water intake of $2 \mathrm{~L} /$ day, the estimated average intake of nickel from drinking water ranges from 4 to $8.6 \mu \mathrm{g} /$ day. Elevated levels of nickel may exist as a result of the corrosion and leaching of nickel alloys used in valves and faucets. For the general population, the predominant route of exposure to nickel is through food intake. Nickel intake in the United States ranges between 69 and $162 \mu \mathrm{g} /$ day for adults (>18 years of age). Based on these average water and food nickel levels, a daily dose of $0.001-0.0024 \mathrm{mg} / \mathrm{kg} /$ day can be estimated using a reference body weight of $70 \mathrm{~kg}$. In children, mean daily nickel intakes of 9, 39, 82, and $99 \mu \mathrm{g} /$ day have been determined for children aged 0-6 months, 7-12 months, 1-3 years, and 4-8 years, respectively. The mean daily dietary intakes of 
nickel in children aged 9-18 years (128-137 $\mu \mathrm{g} /$ day in males and 101-109 $\mu \mathrm{g} / \mathrm{day}$ for females) are similar to the mean intakes determined in adults ( $>18$ years of age).

A $70 \mathrm{~kg}$ reference man contains $10 \mathrm{mg}$ of nickel, giving an average body concentration of $0.1 \mathrm{ppm}$. Reference values for nickel in healthy adults is $0.2 \mu \mathrm{g} / \mathrm{L}$ in serum and $1-3 \mu \mathrm{g} / \mathrm{L}$ in urine. A National Health and Nutritional Examination Survey II of hair found mean nickel levels of 0.39 ppm, with 10\% of the population having levels $>1.50 \mathrm{ppm}$.

About $20-35 \%$ of the inhaled nickel that is retained in the lungs is absorbed into the blood. Absorption of nickel following oral exposure has been shown to vary (3-40\%) depending on whether the nickel was in drinking water or food, with greater absorption occurring with drinking water. Fasting individuals have also been shown to absorb more nickel from the gastrointestinal tract. Most of the absorbed nickel is excreted in the urine, regardless of the route of exposure.

Nickel does not bioaccumulate to a great extent in animals. There is evidence of uptake and accumulation in certain plants.

Nickel is an essential trace element in animals, although the functional importance of nickel has not been clearly demonstrated. It is considered essential based on reports of nickel deficiency in several animal species (e.g., rats, chicks, cows, goats). Nickel deficiency is manifested primarily in the liver; effects include abnormal cellular morphology, oxidative metabolism, and increases and decreases in lipid levels. Decreases in growth and hemoglobin concentration and impaired glucose metabolism have also been observed. The essentiality of nickel in humans has not been established, and nickel dietary recommendations have not been established for humans.

\subsection{SUMMARY OF HEALTH EFFECTS}

The general population can be exposed to nickel via inhalation, oral, and dermal routes of exposure. Based on occupational exposure studies, reports of allergic contact dermatitis, and animal exposure studies, the primary targets of toxicity appear to be the respiratory tract following inhalation exposure, the immune system following inhalation, oral, or dermal exposure, and possibly the reproductive system and the developing organism following oral exposure. 
The most commonly reported adverse health effect associated with nickel exposure is contact dermatitis. Contact dermatitis is the result of an allergic reaction to nickel that has been reported in the general population and workers exposed via dermal contact with airborne nickel, liquid nickel solution, or prolonged contact with metal items such as jewelry and prosthetic devices that contain nickel. After an individual becomes sensitized to nickel, dermal contact with a small amount of nickel or oral exposure to fairly low doses of nickel can result in dermatitis. Approximately $10-20 \%$ of the general population is sensitized to nickel.

Adverse respiratory effects have been reported in humans and animals exposed to nickel compounds at concentrations much higher than typically found in the environment. The available data on noncancerous respiratory effects in humans are limited. In nickel workers, exposure to nickel did not result in increases in the risk of death from nonmalignant respiratory system disease. Studies examining potential nonlethal respiratory effects have not found consistent results. Animal data provide strong evidence that nickel is a respiratory toxicant; lung inflammation is the predominant effect. Evidence of lung inflammation has been observed following acute-, intermediate-, and chronic-duration exposure of rats to nickel sulfate, nickel subsulfide, or nickel oxide. Nickel sulfate was the most toxic of the three compounds and nickel oxide was the least toxic. For all three compounds, the threshold for lung effects decreased as the duration of exposure increased. Exposure to nickel sulfate or nickel subsulfide also produced damage to the nasal olfactory epithelium. Human and animal data provide strong evidence that inhalation exposure to some nickel compounds can induce lung cancer. As described in greater detail later in this section, carcinogenic responses have been observed following inhalation exposure to nickel subsulfide and nickel oxide; in the absence of exposure to other carcinogenic agents, nickel sulfate does not appear to be carcinogenic following inhalation exposure.

The potential for nickel compounds to induce reproductive effects has not been firmly established. Several animal studies have reported adverse effects in the male reproductive system following oral exposure to nickel sulfate, nickel chloride, or nickel nitrate. The observed effects included histological alterations in the epididymis and seminal vesicles, decreases in sperm concentration, motility, and abnormalities, and decreases in fertility following male exposure, but not female only exposure. However, the poor reporting of study results, particularly incidence data and statistical analysis, limits the interpretation of these studies. Additionally, other studies have not found histological alterations in the male reproductive system following long-term oral exposure or impaired fertility following oral exposure. A number of studies have reported decreases in survival of the offspring of animals exposed prior to mating and during the gestation and lactation periods. Interpretation of these data are complicated by 
maternal toxicity, particularly decreases in body weight gain, which frequently occurred at the same dose levels.

The most consistently reported adverse effects resulting from exposure to nickel are contact dermatitis and respiratory effects, including cancer; a more detailed discussion of these effects follows. The reader is referred to Section 3.2, Discussion of Health Effects by Route of Exposure, for additional information on other health effects.

Contact Dermatits. Nickel sensitivity is a form of delayed hypersensitivity that is found in $10-20 \%$ of the general population. The prevalence of nickel sensitivity is higher among young women than any other segment of the population, which is probably the result of higher rates of ear and other types of body piercing rather than increased susceptibility to sensitization. There is some evidence of a genetic susceptibility factor that may predispose certain individuals to the development of nickel sensitivity. A significant increase in human leukocyte antigen (HLA)-DRw6 antigens were found among individuals with nickel contact dermatitis compared to individuals with no history of atopy or contact dermatitis. The relative risk of individuals with the HLA-DRw6 allele developing nickel sensitivity was estimated to be 3.3 .

Nickel sensitization typically involves initial prolonged contact with nickel or exposure to a very large nickel dose. In the general population, the initial nickel contact often comes from body piercing with jewelry that releases large amount of nickel ions. The resulting dermatitis, which is an inflammatory reaction mediated by type IV hypersensitivity, typically occurs beneath the metal object. With repeated exposure, the area of sensitization can spread to other locations, particularly the hands. Shorter contact with nickel items, such as nickel-plated coins or door handles, does not result in nickel sensitization. After an individual becomes sensitized to nickel, much lower concentrations are needed to elicit a response. There is limited information on nickel levels resulting in sensitization. One study found that the sensitizing nickel level was 100-1,000 times higher than the level eliciting dermatitis in a previously sensitized individual. Among sensitized individuals, a direct relationship between nickel exposure level and severity of the dermatitis has been found. A weak reaction has been reported in individuals exposed to nickel alloys that release nickel ions at a rate of $<0.5 \mu \mathrm{g} / \mathrm{cm}^{2} /$ week; a strong reaction was observed for nickel alloys that release $>1 \mu \mathrm{g} / \mathrm{cm}^{2} /$ week. No reaction was seen in nickel-sensitized subjects undergoing patch testing with $0.01 \%$ nickel as nickel sulfate in petrolatum; however, exposure to $0.03 \%$ nickel resulted in dermatitis. Similarly, an oral challenge dose of $0.02 \mathrm{mg} \mathrm{Ni} / \mathrm{kg}$ can induce dermatitis in a small percentage of nickel-sensitized individuals, whereas exposure to higher doses (0.06 mg Ni/kg) will often 
result in dermatitis in most nickel-sensitized individuals. Exposure to these nickel concentrations will not result in dermatitis in nonsensitized individuals.

Respiratory Effects. Both noncancerous and cancerous respiratory effects have been observed in humans and animals exposed to airborne nickel compounds. Chronic bronchitis, emphysema, pulmonary fibrosis, and impaired lung function have been observed in nickel welders and foundry workers. These effects were not consistently seen across studies, and co-exposure to other toxic metals such as uranium, iron, lead, and chromium confounds the interpretation of the results. Studies examining the risk of death from nonmalignant respiratory disease among nickel workers have not found significant increases; however, many studies found that the number of observed deaths were significantly lower than expected, suggesting a healthy worker effect.

In animals, the predominant noncancerous effect is lung inflammation following exposure to nickel sulfate, nickel subsulfide, and nickel oxide. The toxicity of nickel in the respiratory tract appears to be related to the solubility of the individual nickel compounds, with soluble nickel sulfate being the most toxic and insoluble nickel oxide being the least toxic. The pulmonary toxicity appears to be related to exposure concentration rather than nickel lung burden. It has been postulated that the higher toxicity of soluble nickel is due to the higher concentrations of free nickel ions, which can diffuse across the cell membrane and interact with cytoplasmic proteins. In contrast, insoluble nickel compounds are phagocytized and a smaller amount of nickel ions interact with cytoplasmic proteins. Following an intermediate-duration exposure, the respective no-observed-adverse effect level (NOAEL) and lowestobserved-adverse effect level (LOAEL) values for lung inflammation were 0.06 and $0.11 \mathrm{mg} \mathrm{Ni} / \mathrm{m}^{3}$ for nickel sulfate, 0.11 and $0.22 \mathrm{mg} \mathrm{Ni} / \mathrm{m}^{3}$ for nickel subsulfide, and 2 and $3.9 \mathrm{mg} \mathrm{Ni} / \mathrm{m}^{3}$ for nickel oxide. At approximately $0.4 \mathrm{mg} \mathrm{Ni} / \mathrm{m}^{3}$ as nickel sulfate, nickel subsulfide, and nickel oxide, the lung burdens following a 13-week exposure were 6, 7, and $80 \mu \mathrm{g} \mathrm{Ni/g} \mathrm{lung,} \mathrm{respectively.} \mathrm{For} \mathrm{all} \mathrm{durations} \mathrm{and} \mathrm{nickel}$ compounds tested, rats appear to be more sensitive to the lung effects than mice; significant increases in the incidence of lung inflammation were observed at lower concentrations in the rats than mice. However, mice were more susceptible to the lethal effects (presumably from impaired lung function) than rats. In addition to the pulmonary effects, atrophy of the nasal olfactory epithelium was observed in rats exposed to nickel sulfate or nickel subsulfide for acute, intermediate, and chronic durations; nasal effects were not observed following exposure to nickel oxide.

The carcinogenicity of nickel has been well documented in occupationally-exposed individuals. Significant increases in the risk of mortality from lung or nasal cancers were observed in several cohorts 
of nickel refinery workers. Studies of workers in other nickel industries, including nickel mining and smelting, nickel alloy production, stainless steel production, or stainless steel welding, which typically involve exposure to lower concentrations of nickel, have not found significant increases in cancer risks. In most of the occupational exposure studies, the workers were exposed to several nickel species, thus making it difficult to compare carcinogenic potential across nickel species. An extensive re-evaluation of the studies published prior to 1990 found the strongest evidence of carcinogenicity for sulfidic nickel; exposure to high concentrations ( $>10 \mathrm{mg} \mathrm{Ni} / \mathrm{m}^{3}$ ) resulted in increased lung cancer risks. There is weaker evidence that high concentrations $\left(>10 \mathrm{mg} \mathrm{Ni} / \mathrm{m}^{3}\right)$ of oxidic nickel, particularly when there is co-exposure to soluble nickel, is also carcinogenic. Soluble nickel does not appear to be carcinogenic in the absence of exposure to other carcinogenic agents. There is no evidence that exposure to low levels of nickel is carcinogenic in humans. The conclusions drawn from the occupational exposure studies are supported by animal inhalation studies. Significant increases in the incidence of lung tumors were observed in rats chronically exposed to nickel subsulfide or nickel oxide. The carcinogenic response was stronger for nickel subsulfide compared to nickel oxide. In contrast, no increases in lung tumor incidences were observed in rats exposed to nickel sulfate; however, the highest concentration tested $\left(0.11 \mathrm{mg} \mathrm{Ni} / \mathrm{m}^{3}\right)$ was lower than the cancer effect levels for nickel subsulfide $\left(0.73 \mathrm{mg} \mathrm{Ni} / \mathrm{m}^{3}\right)$ or nickel oxide $\left(1 \mathrm{mg} \mathrm{Ni} / \mathrm{m}^{3}\right)$.

Although the evidence is sufficient to consider less-soluble nickel compounds as carcinogens following inhalation exposure, how environmental exposure to nickel affects cancer risk is not clear. Nickel levels in the environment are much lower than those that were associated with cancer in workers. In the environment, nickel is also more likely to be in the form of a mineral lattice rather than the more active nickel refinery dust that contains nickel subsulfide, the form of nickel most consistently associated with cancer. Although soluble nickel compounds may not be directly carcinogenic, as indicated by the negative results in the nickel sulfate bioassay, inhalation of nickel sulfate did result in an inflammatory response in the lungs of animals. Because sustained tissue damage can serve to promote carcinogenesis, epidemiology studies of humans who are exposed to many substances may not be able to distinguish between the carcinogenic activity of less-soluble nickel compounds and the promoting activity of toxic concentrations of soluble nickel compounds.

The Department of Health and Human Services has determined that metallic nickel may reasonably be anticipated to be a human carcinogen and nickel compounds are known to be human carcinogens. Similarly, IARC classified metallic nickel in group 2B (possibly carcinogenic to humans) and nickel compounds in group 1 (carcinogenic to humans). EPA has classified nickel refinery dust and nickel subsulfide in Group A (human carcinogen). Other nickel compounds have not been classified by the 
EPA. Based on the occupational data, inhalation unit risk levels of $2.4 \times 10^{-4}\left(\mu \mathrm{g} / \mathrm{m}^{3}\right)^{-1}$ and $4.8 \times 10^{-4}\left(\mu \mathrm{g} / \mathrm{m}^{3}\right)^{-1}$ were derived by EPA for nickel refinery dust and nickel subsulfide, respectively.

\subsection{MINIMAL RISK LEVELS}

Estimates of exposure levels posing minimal risk to humans (MRLs) have been made for nickel. An MRL is defined as an estimate of daily human exposure to a substance that is likely to be without an appreciable risk of adverse effects (noncarcinogenic) over a specified duration of exposure. MRLs are derived when reliable and sufficient data exist to identify the target organ(s) of effect or the most sensitive health effect(s) for a specific duration within a given route of exposure. MRLs are based on noncancerous health effects only and do not consider carcinogenic effects. MRLs can be derived for acute, intermediate, and chronic duration exposures for inhalation and oral routes. Appropriate methodology does not exist to develop MRLs for dermal exposure.

Although methods have been established to derive these levels (Barnes and Dourson 1988; EPA 1990), uncertainties are associated with these techniques. Furthermore, ATSDR acknowledges additional uncertainties inherent in the application of the procedures to derive less than lifetime MRLs. As an example, acute inhalation MRLs may not be protective for health effects that are delayed in development or are acquired following repeated acute insults, such as hypersensitivity reactions, asthma, or chronic bronchitis. As these kinds of health effects data become available and methods to assess levels of significant human exposure improve, these MRLs may be revised.

\section{Inhalation MRLs}

The acute toxicity of nickel has been assessed in several animal studies involving exposure to nickel sulfate (Evans et al. 1995; NTP 1996c), nickel chloride (Adkins et al. 1979; Graham et al. 1978), nickel subsulfide (Benson et al. 1995b; NTP 1996b), and nickel oxide (NTP 1996a). The observed effects include inflammatory changes in the lungs (Benson et al. 1995a; NTP 1996a, 1996b, 1996c), atrophy of the nasal olfactory epithelium (Evans et al. 1995; NTP 1996b, 1996c), hyperplasia in the bronchial and mediastinal lymph nodes (NTP 1996b, 1996c), impaired immune function (Adkins et al. 1979; Graham et al. 1978), and decreases in body weight gain (NTP 1996b, 1996c), which are probably secondary to the

lung damage. NOAEL values for respiratory tract effects were not established for nickel sulfate or nickel subsulfide. In studies by the National Toxicology Program (NTP 1996b, 1996c) (6 hours/day for 12 days in a 16-day period), chronic lung inflammation and atrophy of the nasal olfactory epithelium were 
observed at the lowest tested nickel sulfate $\left(0.7 \mathrm{mg} \mathrm{Ni} / \mathrm{m}^{3}\right)$ and nickel subsulfide $\left(0.44 \mathrm{mg} \mathrm{Ni} / \mathrm{m}^{3}\right)$ concentrations. At 0.7 and $3.65 \mathrm{mg} \mathrm{Ni} / \mathrm{m}^{3}$ as nickel sulfate and nickel subsulfide, respectively, the inflammation was accompanied by labored breathing, suggestive of impaired lung function. Alveolitis was also observed in rats exposed to $0.22 \mathrm{mg} \mathrm{Ni} / \mathrm{m}^{3}$ as nickel subsulfide 6 hours/day for 7 days (Benson et al. 1995b). In mice, the LOAELs for chronic lung inflammation were 0.7 and $1.83 \mathrm{mg} \mathrm{Ni} / \mathrm{m}^{3}$ for nickel sulfate and nickel subsulfide, respectively. Nickel oxide was less toxic than the other two nickel compounds. The NOAEL and LOAEL values for acute lung inflammation were 3.9 and $7.9 \mathrm{mg} \mathrm{Ni} / \mathrm{m}^{3}$ in rats, respectively; in mice, the highest concentration tested $\left(23.6 \mathrm{mg} \mathrm{Ni} / \mathrm{m}^{3}\right)$ was a NOAEL for respiratory effects. Based on these data and data from longer-term studies (NTP 1996a, 1996b, 1996c), nickel sulfate appears to be the most toxic to the respiratory tract of the three nickel compounds tested by NTP. Although the acute-duration nickel subsulfide study used lower concentrations than the nickel sulfate study, there is some evidence to suggest that the nickel sulfate effects were more severe. At $0.7 \mathrm{mg}$ $\mathrm{Ni} / \mathrm{m}^{3}$ as nickel sulfate, the chronic lung inflammation was given a severity score of 1.2-1.8 (minimal to mild) and was accompanied by labored breathing and a 28\% decrease in body weight. The lung inflammation in rats exposed to 0.44 or $0.88 \mathrm{mg} \mathrm{Ni} / \mathrm{m}^{3}$ as nickel subsulfide was scored as minimal (1.0) and was not accompanied by altered respiration or body weight effects.

These acute-duration studies provide strong evidence that the respiratory tract is the most sensitive target following inhalation exposures. The three NTP (1996a, 1996b, 1996c) studies demonstrate that nickel sulfate is more toxic to the lungs than nickel subsulfide or nickel oxide. Because the lowest concentration tested in the nickel sulfate study $\left(0.7 \mathrm{mg} \mathrm{Ni} / \mathrm{m}^{3}\right)$ was a serious LOAEL for respiratory and body weight effects, this study cannot be used for MRL derivation. An immunotoxicity study by Graham et al. (1978) established a lower LOAEL $\left(0.25 \mathrm{mg} \mathrm{Ni} / \mathrm{m}^{3}\right)$ for a soluble nickel compound, nickel chloride; the NOAEL was $0.1 \mathrm{mg} \mathrm{Ni} / \mathrm{m}^{3}$. This study was not selected as the basis for MRL because the respiratory tract was not examined and it is not known if the NOAEL for immunotoxicity would also be a NOAEL for respiratory effects.

- An MRL of $0.0002 \mathrm{mg} \mathrm{Ni} / \mathrm{m}^{3}$ has been derived for intermediate-duration exposure to nickel.

The intermediate-duration toxicity of nickel has been assessed in several animal studies involving exposure to metallic nickel, nickel sulfate, nickel chloride, nickel subsulfide, and nickel oxide. The observed effects include inflammatory changes in the lungs (Benson et al. 1995b; Horie et al. 1985; NTP 1996a, 1996b, 1996c), alveolar macrophage hyperplasia (Benson et al. 1995b; Johansson and Camner 1986; NTP 1996a, 1996b, 1996c), atrophy of the nasal olfactory epithelium (NTP 1996b, 1996c), 
hyperplasia in the bronchial and mediastinal lymph nodes (NTP 1996b, 1996c), impaired immune function (Adkins et al. 1979; Graham et al. 1978; Haley et al. 1990; Johansson et al. 1980, 1987, 1988a, 1989; Johansson and Camner 1986; Morimoto et al. 1995; Spiegelberg et al. 1984), decreases in body weight gain which are probably secondary to the lung damage (NTP 1996b, 1996c; Weischer et al. 1980), decreased sperm concentration (NTP 1996a), and developmental toxicity (Weischer et al. 1980).

As with the acute-duration studies, the most sensitive target of nickel toxicity is the lungs. Chronic lung inflammation was observed at the lowest-adverse-effect levels following 13-week (6 hours/day, 5 days/week) exposures to nickel sulfate, nickel subsulfide, or nickel oxide (NTP 1996a, 1996b, 1996c). Intermediate-duration studies clearly demonstrate that nickel sulfate is more toxic than nickel subsulfide and nickel oxide. In rats, the respective NOAEL and LOAEL values for chronic lung inflammation were 0.06 and $0.11 \mathrm{mg} \mathrm{Ni} / \mathrm{m}^{3}$ for nickel sulfate (NTP 1996c), 0.11 and $0.22 \mathrm{mg} \mathrm{Ni} / \mathrm{m}^{3}$ for nickel subsulfide (NTP 1996b), and 2.0 and $3.9 \mathrm{mg} \mathrm{Ni} / \mathrm{m}^{3}$ for nickel oxide (NTP 1996a). Atrophy of the nasal olfactory epithelium was observed at 0.22 and $0.44 \mathrm{mg} \mathrm{Ni} / \mathrm{m}^{3}$ as nickel sulfate (NTP 1996c) and nickel subsulfide (NTP 1996b), respectively. Similar effects were observed in mice. For nickel sulfate and nickel subsulfide, the LOAEL values for mice were higher than the LOAELs identified in rats; the LOAEL for chronic inflammation following exposure to nickel oxide was the same in rats and mice. The LOAEL values for immunotoxicity, reproductive toxicity, and developmental toxicity were higher than the LOAEL values for respiratory effects in rats exposed to nickel sulfate.

Derivation of an intermediate-duration MRL based on the NTP study of nickel sulfate (NTP 1996c) would be protective against the toxicity of other nickel compounds. In the nickel sulfate study, alveolar macrophage hyperplasia was observed in rats exposed at the two lowest concentrations $(0.03$ and $0.06 \mathrm{mg}$ $\mathrm{Ni} / \mathrm{m}^{3}$ ). NTP noted that when lung effects only consisted of alveolar macrophage hyperplasia, there was only a slight increase in the number of alveolar macrophages and the differences between controls and nickel-exposed animals were subtle; the severity score for the alveolar macrophage hyperplasia was 1.0 (minimal). The minimal alveolar macrophage hyperplasia was not considered adverse because it is considered to be part of the normal physiologic response to inhaled particles and it is not believed to compromise the lung's ability to clear foreign matter. This is supported by the Benson et al. (1995a) study, which found no effect on the clearance of a nickel sulfate tracer in animals exposed to 0.03 or $0.11 \mathrm{mg} \mathrm{Ni} / \mathrm{m}^{3}$ as nickel sulfate for 6 months. Thus, the $0.06 \mathrm{mg} \mathrm{Ni} / \mathrm{m}^{3}$ concentration was identified as a NOAEL and adjusted for intermittent exposure (NOAEL $L_{A D J}$ ). 
The intermediate-duration inhalation MRL of $0.0002 \mathrm{mg} \mathrm{Ni} / \mathrm{m}^{3}$ was derived by dividing the NOAEL $\mathrm{HEC}$ of $0.0052 \mathrm{mg} \mathrm{Ni} / \mathrm{m}^{3}$ by an uncertainty factor of 30 (3 for species to species extrapolation with dosimetric adjustments and 10 for human variability). The NOAEL $\mathrm{HEC}_{\text {was }}$ calculated using the following equations:

$$
\begin{aligned}
& \text { NOAEL }_{\text {ADJ }}=0.06 \mathrm{mg} \mathrm{Ni} / \mathrm{m}^{3} \times 6 \text { hours } / 24 \text { hours } \times 5 \text { days } / 7 \text { days }=0.011 \mathrm{mg} \mathrm{Ni} / \mathrm{m}^{3} \\
& \text { NOAEL }_{\text {HEC }}=\mathrm{NOAEL}_{\text {ADJ }} \times \mathrm{RDDR}=0.011 \mathrm{mg} \mathrm{Ni} / \mathrm{m}^{3} \times 0.474=0.0052 \mathrm{mg} \mathrm{Ni} / \mathrm{m}^{3}
\end{aligned}
$$

The regional deposited dose ratio (RDDR) for the pulmonary region was used to extrapolate deposited doses in rats to deposited doses in humans. The RDDR was calculated using EPA software and the following parameters: particle size (mass median aerodynamic diameter, MMAD) of $2.11 \mu \mathrm{m}$ with a geometric standard deviation (sigma g) of 2.7 (as reported in Table K1 of NTP 1996c); default human body weight (70 kg), minute volume (13 L), and pulmonary surface area (54 $\left.\mathrm{m}^{2}\right)$; and default female F344 rat body weight $(0.124 \mathrm{~kg})$, minute volume $(101.3 \mathrm{~mL})$, and pulmonary surface area $\left(0.34 \mathrm{~m}^{2}\right)$.

No intermediate-duration human inhalation exposure studies were identified; a number of chronic exposure studies have examined the potential of nickel and nickel compounds to induce respiratory effects in workers. Most of these studies are cohort mortality studies that did not find significant increases in the number of deaths from nonmalignant respiratory system disease (Arena et al. 1998; Cox et al. 1981; Cragle et al. 1984; Egedahl et al. 2001; Enterline and Marsh 1982; Redmond 1984; Roberts et al. 1989b; Shannon et al. 1984b, 1991). A few studies have examined workers for possible nonlethal respiratory effects. Two studies examined chest x-rays of workers: one found an increased risk of moderate pulmonary fibrosis (Berge and Skyberg 2003) and the other did not find any significant alterations (Muir et al. 1993). Although most of occupational exposure studies did not report exposure levels, workers were typically exposed to nickel levels that far exceed levels found in ambient air.

- An MRL of $9 \times 10^{-5} \mathrm{mg} \mathrm{Ni} / \mathrm{m}^{3}$ has been derived for chronic-duration exposure to nickel.

One human study (Vyskocil et al. 1994a) and several animal studies (NTP 1996a, 1996b, 1996c; Ottolenghi et al. 1974; Takenaka et al. 1985; Tananka et al. 1988) assessed the noncarcinogenic toxicity of nickel sulfate, nickel chloride, nickel subsulfide, and nickel oxide. These studies found inflammatory changes in the lungs (NTP 1996a, 1996b, 1996c; Ottolenghi et al. 1974; Tanaka et al. 1988), atrophy of the nasal olfactory epithelium (NTP 1996b, 1996c), evidence of renal damage (Vyskocil et al. 1994a), adverse adrenal effects (NTP 1996a), decreased body weight gain, which was probably associated with 
impaired lung function (NTP 1996b, 1996c; Takenaka et al. 1985), and damage to the bronchial lymph nodes (NTP 1996a, 1996b, 1996c).

As with the acute- and intermediate-duration exposures, chronic exposure to nickel sulfate, nickel subsulfide, or nickel oxide resulted in chronic active lung inflammation. A 2-year exposure (6 hours/day, 5 days/week) to nickel sulfate (NTP 1996c) resulted in chronic lung inflammation and bronchialization at $0.06 \mathrm{mg} \mathrm{Ni} / \mathrm{m}^{3}$ and atrophy of the olfactory epithelium at $0.11 \mathrm{mg} \mathrm{Ni} / \mathrm{m}^{3}$; no adverse respiratory effects were observed at $0.03 \mathrm{mg} \mathrm{Ni} / \mathrm{m}^{3}$. A similar exposure to nickel subsulfide (NTP 1996b) resulted in chronic inflammation, alveolar epithelium hyperplasia, fibrosis, and rapid and shallow breathing at $0.11 \mathrm{mg} \mathrm{Ni} / \mathrm{m}^{3}$, and atrophy of the nasal olfactory epithelium at $0.73 \mathrm{mg} \mathrm{Ni} / \mathrm{m}^{3}$. Chronic lung inflammation and alveolar epithelial hyperplasia were observed at the lowest nickel oxide concentration tested (0.5 mg Ni/m³) (NTP 1996a). Similar effects were observed in mice exposed to nickel sulfate, nickel subsulfide, or nickel oxide for 2 years; however, the LOAEL values were higher than for rats. The NTP (1996c) study of nickel sulfate identified the lowest LOAEL for respiratory effects $\left(0.06 \mathrm{mg} \mathrm{Ni} / \mathrm{m}^{3}\right)$; the NOAEL of $0.03 \mathrm{mg} \mathrm{Ni} / \mathrm{m}^{3}$ associated with this LOAEL was used to derive a chronic-duration inhalation MRL for nickel.

The chronic-duration inhalation MRL of $9 \times 10^{-5} \mathrm{mg} \mathrm{Ni} / \mathrm{m}^{3}$ was derived by dividing the NOAEL $\mathrm{HEC}_{\text {of }}$ $0.0027 \mathrm{mg} \mathrm{Ni} / \mathrm{m}^{3}$ by an uncertainty factor of 30 (3 for species to species extrapolation with dosimetric adjustments and 10 for human variability). The NOAEL ${ }_{\mathrm{HEC}}$ was calculated using the following equations:

$$
\begin{aligned}
& \text { NOAEL }_{\text {ADJ }}=0.03 \mathrm{mg} \mathrm{Ni} / \mathrm{m}^{3} \times 6 \text { hours } / 24 \text { hours } \times 5 \text { days } / 7 \text { days }=0.0054 \mathrm{mg} \mathrm{Ni} / \mathrm{m}^{3} \\
& \text { NOAEL }_{\text {HEC }}=\mathrm{NOAEL}_{\text {ADJ }} \times \mathrm{RDDR}=0.0054 \mathrm{mg} \mathrm{Ni} / \mathrm{m}^{3} \times 0.506=0.0027 \mathrm{mg} \mathrm{Ni} / \mathrm{m}^{3}
\end{aligned}
$$

The RDDR for the pulmonary region was used to extrapolate deposited doses in rats to deposited doses in humans. The following parameters were used to calculated the RDDR: mean particle size (MMAD) of $2.5 \mu \mathrm{m}$ with a geometric standard deviation (sigma g) of 2.38 (as reported in Table K1 of NTP 1996c); default human body weight (70 kg), minute volume (13 L), and pulmonary surface area $\left(54 \mathrm{~m}^{2}\right)$; and default female F344 rat body weight $(0.229 \mathrm{~kg})$, minute volume $(167.3 \mathrm{~mL})$, and pulmonary surface area $\left(0.34 \mathrm{~m}^{2}\right)$.

As discussed for the intermediate-duration inhalation MRL, the potential of nickel to induce nonmalignant respiratory tract effects has been examined in a number of cohort mortality studies. In general, these studies did not find significant increases in the risk of dying from nonmalignant respiratory 
system disease (Arena et al. 1998; Cox et al. 1981; Cragle et al. 1984; Egedahl et al. 2001; Enterline and Marsh 1982; Redmond 1984; Roberts et al. 1989b; Shannon et al. 1984b, 1991). Mixed results have been found in the few studies examining nonlethal respiratory tract effects. Two studies examined chest x-rays of nickel workers: one found an increased risk of moderate pulmonary fibrosis (Berge and Skyberg 2003) and the other did not find any significant alterations (Muir et al. 1993). Although most of occupational exposure studies did not report exposure levels, workers were typically exposed to nickel levels that far exceed levels found in ambient air.

\section{Oral MRLs}

Information on the acute oral toxicity of nickel in humans comes from reports of accidental exposures and studies of nickel-sensitized individuals. Gastrointestinal upset (vomiting, cramps, diarrhea) and neurological symptoms (giddiness, headache, weariness) were observed in workers accidentally ingesting water containing approximately 7.1-35.7 mg Ni/kg as nickel sulfate and nickel chloride; boric acid was also present in the water (Sunderman et al. 1988). Allergic dermatitis was observed in previously nickelsensitized individuals ingesting a single challenge dose of greater than $0.01 \mathrm{mg} \mathrm{Ni} / \mathrm{kg}$ as nickel sulfate (Hindsén et al. 2001; Jensen et al. 2003; Menne and Maibach 1987). Reliable data on the acute oral toxicity of nickel in animals is limited to two studies that examined a limited number of end points. A reproductive toxicity study in mice found significant increases in sperm head abnormalities in mice exposed to a single gavage dose of $23 \mathrm{mg} \mathrm{Ni} / \mathrm{kg}$ as nickel nitrate (Sobti and Gill 1989). No developmental effects were observed in the offspring of mice exposed via gavage to $90.6 \mathrm{mg} \mathrm{Ni} / \mathrm{kg} / \mathrm{day}$ as nickel chloride on gestational days 8-12 (Seidenberg et al. 1986). Intermediate-duration studies suggest that the developing organism may be a sensitive target of nickel toxicity; however, this end point has not been adequately examined following acute-duration exposure; thus, an acute-duration oral MRL for nickel has not been derived.

A number of animal studies have assessed the toxicity of nickel following intermediate-duration oral exposure. Significant decreases in body weight and organ weight (liver, kidney, pituitary) were consistently observed in rats exposed to $8.6 \mathrm{mg} \mathrm{Ni} / \mathrm{kg} /$ day and higher as nickel chloride (American Biogenics Corporation 1988; RTI 1988a, 1988b), nickel acetate (Hanger 1973), or nickel sulfate (Dieter et al. 1988). Other systemic effects included kidney damage (minimal convoluted tubular damage) at $108 \mathrm{mg} \mathrm{Ni} / \mathrm{kg} /$ day as nickel sulfate (Dieter et al. 1988) and adverse lung effects at 8.6 and $20 \mathrm{mg}$ $\mathrm{Ni} / \mathrm{kg}$ /day as nickel chloride (American Biogenic Corporation 1988; RTI 1988b). Inconsistent results have been reported for the reproductive toxicity of nickel. Decreased sperm motility and count and sperm 
abnormalities were observed at $1.9 \mathrm{mg} \mathrm{Ni} / \mathrm{kg} /$ day and higher as nickel sulfate (Pandey and Srivastava 2000; Pandey et al. 1999) and decreased fertility was observed in studies in which males and females were exposed to $3.6 \mathrm{mg} \mathrm{Ni/kg/day} \mathrm{as} \mathrm{nickel} \mathrm{chloride} \mathrm{(Käkelä} \mathrm{et} \mathrm{al.} \mathrm{1999).} \mathrm{However,} \mathrm{impaired}$ reproduction has not been observed in multigeneration studies of rats orally exposed to nickel sulfate or nickel chloride (RTI 1988a, 1988b; Springborn Laboratories 2000a). There is stronger evidence that prenatal exposure to nickel results in decreased survival, as measured by live litter size and neonatal mortality, in pups of rat dams exposed to nickel chloride in drinking water prior to mating and during gestation and lactation (Ambrose et al. 1976; Käkelä et al. 1999; RTI 1988a, 1988b; Smith et al. 1993; Springborn Laboratories 2000b). Interpretation and comparison of the studies is complicated by differences in study design and maternal toxicity, which often occurs at the same dose levels as the developmental effects. The available data are not sufficient to establish a threshold for developmental effects to nickel chloride in rats; the lowest LOAEL values identified in the studies range from 1.3 to $90 \mathrm{mg} \mathrm{Ni} / \mathrm{kg} /$ day and the highest NOAEL values range from 2.2 to $45 \mathrm{mg} \mathrm{Ni} / \mathrm{kg} /$ day. Because decreased pup survival is considered a serious LOAEL and a NOAEL for developmental effects has not been clearly identified, an intermediate-duration oral MRL was not derived for nickel.

Data on the chronic toxicity of ingested nickel are limited to one animal study that found significant decreases in body weight and liver weights in rats exposed to $75 \mathrm{mg} \mathrm{Ni} / \mathrm{kg} /$ day as nickel sulfate in the diet and decreases in body weight, increases in liver weight, and adverse renal and lung effects in dogs $62.5 \mathrm{mg} \mathrm{Ni} / \mathrm{kg} /$ day (Ambrose et al. 1976). The available chronic-duration database was considered inadequate for MRL derivation because intermediate-duration studies found significant decreases in survival of the offspring of rats exposed to $\geq 1.3 \mathrm{mg} \mathrm{Ni} / \mathrm{kg} /$ day (Ambrose et al. 1976; Käkelä et al. 1999; RTI 1988a, 1988b; Smith et al. 1993; Springborn Laboratories 2000b). 



\section{HEALTH EFFECTS}

\subsection{INTRODUCTION}

The primary purpose of this chapter is to provide public health officials, physicians, toxicologists, and other interested individuals and groups with an overall perspective on the toxicology of nickel. It contains descriptions and evaluations of toxicological studies and epidemiological investigations and provides conclusions, where possible, on the relevance of toxicity and toxicokinetic data to public health.

A glossary and list of acronyms, abbreviations, and symbols can be found at the end of this profile.

Several different nickel compounds are discussed in this profile. These compounds can be grouped according to their solubility in water: soluble compounds include nickel chloride, nickel sulfate, and nickel nitrate, and less-soluble compounds include nickel oxide and nickel subsulfide. Both the soluble and less-soluble nickel compounds are important with regard to all relevant routes of exposure.

Generally, the soluble compounds are considered more toxic than the less-soluble compounds, although the less-soluble compounds are more likely to be carcinogenic at the site of deposition. Metallic nickel is also considered in this profile. All doses are presented as the amount or concentration of nickel to which subjects were exposed. Nickel carbonyl, a highly toxic nickel compound, is not considered in this profile. The data regarding the toxicity of nickel carbonyl are substantial; however, the likelihood of exposure at hazardous waste sites is very low. In ambient air, nickel carbonyl is relatively unstable with a half-life of $\approx 100$ seconds (Stedman and Hiked 1980). Because nickel carbonyl is highly reactive, it is not likely to be found at hazardous waste sites. Also, nickel carbonyl is not very soluble in water; therefore, it will not be found in drinking water.

\subsection{DISCUSSION OF HEALTH EFFECTS BY ROUTE OF EXPOSURE}

To help public health professionals and others address the needs of persons living or working near hazardous waste sites, the information in this section is organized first by route of exposure (inhalation, oral, and dermal) and then by end point (death, systemic, immunological, neurological, reproductive, developmental, and carcinogenic effects). These data are discussed in terms of three exposure periods: acute (14 days or less), intermediate (15-364 days), and chronic (365 days or more). 
Levels of significant exposure for each route and duration are presented in tables and illustrated in figures. The points in the figures showing no-observed-adverse-effect levels (NOAELs) or lowestobserved-adverse-effect levels (LOAELs) reflect the actual doses (levels of exposure) used in the studies. LOAELs have been classified into "less serious" or "serious" effects. "Serious" effects are those that evoke failure in a biological system and can lead to morbidity or mortality (e.g., acute respiratory distress or death). "Less serious" effects are those that are not expected to produce significant dysfunction or death, or those whose significance to the organism is not entirely clear. ATSDR acknowledges that a considerable amount of judgment may be required in establishing whether an end point should be classified as a NOAEL, "less serious" LOAEL, or "serious" LOAEL, and that in some cases, there will be insufficient data to decide whether the effect is indicative of significant dysfunction. However, the Agency has established guidelines and policies that are used to classify these end points. ATSDR believes that there is sufficient merit in this approach to warrant an attempt at distinguishing between "less serious" and "serious" effects. The distinction between "less serious" effects and "serious" effects is considered to be important because it helps the users of the profiles to identify levels of exposure at which major health effects start to appear. LOAELs or NOAELs should also help in determining whether or not the effects vary with dose and/or duration, and place into perspective the possible significance of these effects to human health.

The significance of the exposure levels shown in the Levels of Significant Exposure (LSE) tables and figures may differ depending on the user's perspective. Public health officials and others concerned with appropriate actions to take at hazardous waste sites may want information on levels of exposure associated with more subtle effects in humans or animals (LOAELs) or exposure levels below which no adverse effects (NOAELs) have been observed. Estimates of levels posing minimal risk to humans (Minimal Risk Levels or MRLs) may be of interest to health professionals and citizens alike.

Levels of exposure associated with carcinogenic effects (Cancer Effect Levels, CELs) of nickel are indicated in Table 3-1 and Figure 3-1. Because cancer effects could occur at lower exposure levels, Figure 3-1 also shows a range for the upper bound of estimated excess risks, ranging from a risk of 1 in 10,000 to 1 in $10,000,000\left(10^{-4}\right.$ to $\left.10^{-7}\right)$, as developed by EPA.

A User's Guide has been provided at the end of this profile (see Appendix B). This guide should aid in the interpretation of the tables and figures for Levels of Significant Exposure and the MRLs. 


\subsubsection{Inhalation Exposure}

\subsubsection{Death}

Death from adult respiratory distress syndrome was reported in one person who sprayed nickel with a metal arc process without wearing personal protective equipment (Rendell et al. 1994). Several days after the exposure, urinary concentrations of nickel were $700 \mu \mathrm{g} / \mathrm{L}$, in comparison to levels of $<0.1-13.3 \mu \mathrm{g} / \mathrm{L}$ in persons not occupationally exposed to nickel (Sunderman 1993). The death occurred 13 days after the 90-minute exposure to an estimated concentration of $382 \mathrm{mg} \mathrm{Ni} / \mathrm{m}^{3}$ of principally metallic nickel with the majority of particle sizes of $<1.4 \mu \mathrm{m}$. Histological examination of the lungs revealed alveolar wall damage and edema in alveolar spaces, and marked tubular necrosis was noted in the kidneys.

Human data regarding chronic inhalation exposure to nickel are limited to occupational exposure studies. The majority of these studies analyzed the toxicity of nickel, usually in the form of nickel oxide, metallic nickel, or nickel refinery dust, by calculating Standard Mortality Ratios (SMR) for all causes of death. Generally, the studies report a higher incidence of cancer deaths from lung and nasal cancers in the exposed workers (see Section 3.2.1.8). Two studies have also reported a higher incidence of deaths resulting from nonmalignant respiratory disease (Cornell and Landis 1984; Polednak 1981). However, all of the workers were exposed to other metals (arsenic, uranium, iron, lead, chromium), so it cannot be concluded that nickel was the sole causative agent. Other studies of humans occupationally exposed to nickel compounds have not reported increased mortality resulting from respiratory diseases (Cox et al. 1981; Cragle et al. 1984; Enterline and Marsh 1982; Redmond 1984; Shannon et al. 1984b, 1991).

During the first 2 days after a single 2-hour exposure, 4 of 28 rats died after exposure to nickel sulfate at $36.5 \mathrm{mg} \mathrm{Ni} / \mathrm{m}^{3}$ (Hirano et al. 1994b). Severe hemorrhage of the lungs was observed in the lungs of the rats that died. During inhalation exposure of 6 hours/day, 5 days/week, for up to 12 exposures, rats and mice exposed to 12.2 or $1.4 \mathrm{mg} \mathrm{Ni} / \mathrm{m}^{3}$, respectively, as nickel sulfate and mice exposed to $7.33 \mathrm{mg} \mathrm{Ni} / \mathrm{m}^{3}$ as nickel subsulfide died, but those exposed to nickel oxide did not (NTP 1996a, 1996b, 1996c). Mice were more sensitive to lethality than rats; at $1.4 \mathrm{mg} \mathrm{Ni} / \mathrm{m}^{3}$ as nickel sulfate, all mice and no rats died, and at $7.33 \mathrm{mg} \mathrm{Ni} / \mathrm{m}^{3}$ as nickel subsulfide, all mice and 2 of 10 rats died. No rats or mice died following exposure to $23.6 \mathrm{mg} \mathrm{Ni} / \mathrm{m}^{3}$ as nickel oxide. No deaths were reported in rats or mice following 13 weeks of exposure (6 hours/day, 5 days/week) to nickel at 7.9, 1.83, or $0.44 \mathrm{mg} \mathrm{Ni} / \mathrm{m}^{3}$ as nickel oxide, nickel subsulfide, or nickel sulfate, respectively (NTP 1996a, 1996b, 1996c). Hamsters survived exposure to $\leq 48.4 \mathrm{mg} \mathrm{Ni} / \mathrm{m}^{3}$ as nickel oxide for 15 or 61 days (Werner and Craig 1972). 
Significant mortality was observed during the last 26 weeks of a 78-week inhalation study of rats exposed to $0.7 \mathrm{mg} \mathrm{Ni} / \mathrm{m}^{3}$ as nickel subsulfide (Ottolenghi et al. 1974). Less than $5 \%$ of the treated rats survived the study (78 weeks of exposure plus 30 weeks of observation) compared to $31 \%$ of the controls (Ottolenghi et al. 1974). All rats, guinea pigs, and mice exposed to $15 \mathrm{mg} \mathrm{Ni} / \mathrm{m}^{3}$ as metallic nickel for $\leq 21$ months died before the end of the study, with most of the guinea pigs and mice dying by 15 months (Hue per 1958). Lung lesions including edema, hyperemia, and hemorrhage were the principal effects noted. However, no controls were used in this study. A significant decrease in mean survival time was observed in rats exposed 23 hours/day for life to $0.06 \mathrm{mg} \mathrm{Ni} / \mathrm{m}^{3}$ as nickel oxide (Takenaka et al. 1985). The average survival times for rats exposed to 0 or $0.06 \mathrm{mg} \mathrm{Ni} / \mathrm{m}^{3}$ were 125.2 and 87.7 weeks, respectively. Survival was not affected in rats exposed to nickel oxide, nickel subsulfide, or nickel sulfate at concentrations up to 2, 0.73, or $0.11 \mathrm{mg} \mathrm{Ni} / \mathrm{m}^{3}$, respectively, for 104 weeks (NTP 1996a, 1996b, 1996c). Survival of mice was also not affected by exposure to nickel oxide, nickel subsulfide, or nickel sulfate at concentrations up to $3.9,0.88$, or $0.22 \mathrm{mg} \mathrm{Ni} / \mathrm{m}^{3}$, respectively, for 104 weeks (NTP 1996a, 1996b, 1996c).

LOAEL values from each reliable study for death in each species, duration category, and nickel compound are recorded in Table 3-1 and plotted in Figure 3-1.

\subsubsection{Systemic Effects}

No studies were located regarding ocular effects in humans or animals after inhalation exposure to nickel. Other systemic effects are discussed below. The highest NOAEL values and all LOAEL values from each reliable study for systemic effects in each species, duration category, and nickel compound are recorded in Table 3-1 and plotted in Figure 3-1.

Respiratory Effects. A number of human studies have examined the potential of nickel and nickel compounds to induce respiratory effects. Most of these studies were cohort mortality studies in nickelexposed workers. A significant excess of deaths from nonmalignant respiratory system disease was found among foundry workers that was associated with the duration of foundry employment, regardless of exposure to nickel (Cornell and Landis 1984). Other studies of refinery workers or workers exposed to nickel alloys have not found increases in deaths from respiratory disease (Arena et al. 1998; Cox et al. 1981; Cragle et al. 1984; Egedahl et al. 2001; Enterline and Marsh 1982; Redmond 1984; Roberts et al. 1989b; Shannon et al. 1984b, 1991). Two studies of welders also did not find significant increases in the 
Table 3-1 Levels of Significant Exposure to Nickel - Inhalation

\begin{tabular}{|c|c|c|c|c|c|c|c|}
\hline \multirow[b]{2}{*}{$\begin{array}{l}\text { Key to } \\
\text { Figure }\end{array}$} & \multirow[b]{2}{*}{$\begin{array}{l}\text { Species } \\
\text { (Strain) }\end{array}$} & \multirow{2}{*}{$\begin{array}{l}\text { Exposure/ } \\
\text { Duration/ } \\
\text { Frequency } \\
\text { (Route) }\end{array}$} & \multirow[b]{2}{*}{ System } & \multirow[b]{2}{*}{$\begin{array}{l}\text { NOAEL } \\
\left(\mathrm{mg} / \mathrm{m}^{3}\right)\end{array}$} & \multicolumn{2}{|c|}{ LOAEL } & \multirow[b]{2}{*}{$\begin{array}{l}\text { Reference } \\
\text { Chemical Form }\end{array}$} \\
\hline & & & & & $\begin{array}{l}\text { Less Serious } \\
\qquad\left(\mathrm{mg} / \mathrm{m}^{3}\right)\end{array}$ & $\begin{array}{l}\text { Serious } \\
\left(\mathrm{mg} / \mathrm{m}^{3}\right)\end{array}$ & \\
\hline
\end{tabular}

ACUTE EXPOSURE

Death

1 Human $90 \mathrm{~min}$

2 Rat

$2 \mathrm{hr}$

(Wistar)

$36.5 \mathrm{M}(4 / 28$ died $)$

$12.2 \mathrm{~F}(5 / 5$ died $)$

$\begin{array}{lll}3 & \text { Rat } & 12 \text { days in } 16 \\ \text { (Fischer- 344) day period }\end{array}$

$6 \mathrm{hr} / \mathrm{day}$

382 M (death of one man) $\quad \begin{aligned} & \text { Rendall et al. } 1994 \\ & \text { metal }\end{aligned}$

$4 \quad$ Mouse 12 days in 16

(B6C3F1) day period

5 Mouse $\quad \begin{aligned} & 12 \text { days in } 16 \\ & \text { day period }\end{aligned}$

(B6C3F1) $\quad$ day perio

Systemic

$6 \quad \begin{aligned} & \text { Rat } 1,2,4,7,12 \mathrm{~d} \\ & \text { (Fischer- 344) } 6 \mathrm{hr} / \mathrm{d}\end{aligned}$ Resp

0.22 (alveolitis)

7 Rat 4, 8, 12 or $16 \mathrm{~d}$ Resp (Long- Evans) 6 hr/d

$0.635 \mathrm{M}$ (atrophy of olfactory epithelium) metal

Hirano et al. 1994b

sulfate

NTP 1996c

sulfate

NTP 1996b

subsulfide

NTP 1996c

sulfate

Benson et al. 1995b

subsulfide

Evans et al. 1995

sulfate 
Table 3-1 Levels of Significant Exposure to Nickel - Inhalation

\begin{tabular}{|c|c|c|c|c|c|c|c|}
\hline \multirow[b]{2}{*}{$\begin{array}{l}\text { Key to } \\
\text { Figure }\end{array}$} & \multirow[b]{2}{*}{$\begin{array}{l}\text { Species } \\
\text { (Strain) }\end{array}$} & \multirow{2}{*}{$\begin{array}{c}\text { Exposure/ } \\
\text { Duration/ } \\
\text { Frequency } \\
\text { (Route) }\end{array}$} & \multirow[b]{2}{*}{ System } & \multirow[b]{2}{*}{$\begin{array}{l}\text { NOAEL } \\
\left(\mathrm{mg} / \mathrm{m}^{3}\right)\end{array}$} & \multicolumn{2}{|c|}{ LOAEL } & \multirow[b]{2}{*}{$\begin{array}{l}\text { Reference } \\
\text { Chemical Form }\end{array}$} \\
\hline & & & & & $\begin{array}{l}\text { Less Serious } \\
\left(\mathrm{mg} / \mathrm{m}^{3}\right)\end{array}$ & $\begin{array}{l}\text { Serious } \\
\qquad\left(\mathrm{mg} / \mathrm{m}^{3}\right)\end{array}$ & \\
\hline
\end{tabular}

$8 \quad$ Rat 12 days in 16

\begin{tabular}{|c|c|c|c|}
\hline \multirow[t]{9}{*}{$\begin{array}{ll}\text { Rat } & 12 \text { days in } 16 \\
\text { (Fischer- } 344) \text { day period } & 6 \text { hours/day }\end{array}$} & Resp & $3.9 \mathrm{~F}$ & $7.9 \mathrm{~F}$ (acute lung inflammation) \\
\hline & Cardio & 23.6 & \\
\hline & Gastro & 23.6 & \\
\hline & Musc/skel & 23.6 & \\
\hline & Hepatic & 23.6 & \\
\hline & Renal & 23.6 & \\
\hline & Endocr & 23.6 & \\
\hline & Dermal & 23.6 & \\
\hline & $\mathrm{Bd} \mathrm{Wt}$ & 23.6 & \\
\hline
\end{tabular}

NTP 1996a

oxide 
Table 3-1 Levels of Significant Exposure to Nickel - Inhalation

\begin{tabular}{|c|c|c|c|c|c|c|c|}
\hline \multirow[b]{2}{*}{$\begin{array}{l}\text { Key to } \\
\text { Figure }\end{array}$} & \multirow[b]{2}{*}{$\begin{array}{l}\text { Species } \\
\text { (Strain) }\end{array}$} & \multirow{2}{*}{$\begin{array}{l}\text { Exposure/ } \\
\text { Duration/ } \\
\text { Frequency } \\
\text { (Route) }\end{array}$} & \multirow[b]{2}{*}{ System } & \multirow[b]{2}{*}{$\begin{array}{l}\text { NOAEL } \\
\left(\mathrm{mg} / \mathrm{m}^{3}\right)\end{array}$} & \multicolumn{2}{|c|}{ LOAEL } & \multirow[b]{2}{*}{$\begin{array}{l}\text { Reference } \\
\text { Chemical Form }\end{array}$} \\
\hline & & & & & $\begin{array}{l}\text { Less Serious } \\
\qquad\left(\mathrm{mg} / \mathrm{m}^{3}\right)\end{array}$ & $\begin{array}{l}\text { Serious } \\
\left(\mathrm{mg} / \mathrm{m}^{3}\right)\end{array}$ & \\
\hline
\end{tabular}

9 Rat 12 days in 16

$\begin{array}{ll}\text { Rat } & 12 \text { days in } 16 \\ \text { (Fischer- 344) } & \begin{array}{l}\text { day period } \\ 6 \text { hours/day }\end{array}\end{array} \quad$ Resp

inflammation, atrophy of

olfactory epithelium)

3.65 F (chronic lung

inflammation with

necrosis and labored

NTP 1996b

breathing)

subsulfide

$\begin{array}{ll}\text { Cardio } & 7.33 \\ \text { Gastro } & 7.33 \\ \text { Hepatic } & 7.33 \\ \text { Renal } & 7.33 \\ \text { Endocr } & 7.33 \\ \text { Dermal } & 7.33 \\ \text { Bd Wt } & 1.83\end{array}$

$3.65 \quad(22-28 \%$ decrease in

body weight gain) 
Table 3-1 Levels of Significant Exposure to Nickel - Inhalation

\begin{tabular}{llll}
\hline Key to & & $\begin{array}{c}\text { Exposure/ } \\
\text { Duration/ }\end{array}$ & \\
Figure & $\begin{array}{c}\text { Species } \\
\text { (Strain) }\end{array}$ & $\begin{array}{c}\text { (Route) } \\
\text { (Routency }\end{array}$ & System \\
\hline & & & \\
10 & $\begin{array}{l}\text { Rat } \\
\text { (Fischer- 344) } \begin{array}{l}12 \text { day period } \\
6 \text { hr/day }\end{array}\end{array}$ & Resp \\
& &
\end{tabular}

LOAEL

Reference

NOAEL

\section{Less Serious} $\left(\mathrm{mg} / \mathrm{m}^{3}\right)$

Chemical Form

Serious
$(\mathrm{mg} / \mathrm{m})$

0.7 (chronic lung NTP 1996c

inflammation; sulfate

degeneration of

bronchiolar epithelium;

labored breathing;

atrophy of olfactory

epithelium)

$\begin{array}{ll}\text { Cardio } & 12.2 \\ \text { Gastro } & 12.2 \\ \text { Musc/skel } & 12.2 \\ \text { Hepatic } & 12.2 \\ \text { Renal } & 12.2 \\ \text { Endocr } & 12.2 \\ \text { Dermal } & 12.2 \\ \text { Bd Wt } & \end{array}$

$0.7 \mathrm{M}$ (final body weights $28 \%$

lower than controls) 
Table 3-1 Levels of Significant Exposure to Nickel - Inhalation

\begin{tabular}{|c|c|c|c|c|c|c|c|}
\hline \multirow[b]{2}{*}{$\begin{array}{l}\text { Key to } \\
\text { Figure }\end{array}$} & \multirow[b]{2}{*}{$\begin{array}{l}\text { Species } \\
\text { (Strain) }\end{array}$} & \multirow{2}{*}{$\begin{array}{l}\text { Exposure/ } \\
\text { Duration/ } \\
\text { Frequency } \\
\text { (Route) }\end{array}$} & \multirow[b]{2}{*}{ System } & \multirow[b]{2}{*}{$\begin{array}{l}\text { NOAEL } \\
\left(\mathrm{mg} / \mathrm{m}^{3}\right)\end{array}$} & \multicolumn{2}{|c|}{ LOAEL } & \multirow[b]{2}{*}{$\begin{array}{l}\text { Reference } \\
\text { Chemical Form }\end{array}$} \\
\hline & & & & & $\begin{array}{l}\text { Less Serious } \\
\qquad\left(\mathrm{mg} / \mathrm{m}^{3}\right)\end{array}$ & $\begin{array}{l}\text { Serious } \\
\qquad\left(\mathrm{mg} / \mathrm{m}^{3}\right)\end{array}$ & \\
\hline
\end{tabular}

11 Mouse 12 days in 16

12 days in $16 \quad$ Resp 23.6

(B6C3F1) 6 hours/day

$\begin{array}{ll}\text { Cardio } & 23.6 \\ \text { Gastro } & 23.6 \\ \text { Hepatic } & 23.6 \\ \text { Renal } & 23.6 \\ \text { Endocr } & 23.6 \\ \text { Dermal } & 23.6 \\ \text { Bd Wt } & 23.6\end{array}$

$\begin{array}{lll}12 \text { days in } 16 & \text { Resp } & 0.44 \\ \text { day period }\end{array}$

day period

Resp

0.44

1.83 (chronic lung

inflammation)

NTP 1996b

subsulfide
NTP 1996a

oxide

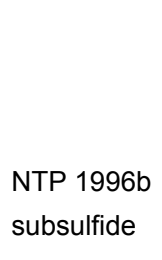

0.88 (atrophy of olfactory

epithelium)

$\begin{array}{ll}\text { Gastro } & 7.33 \\ \text { Hemato } & 7.33 \\ \text { Musc/skel } & 7.33 \\ \text { Hepatic } & 7.33 \\ \text { Renal } & 7.33 \\ \text { Endocr } & 7.33 \\ \text { Dermal } & 7.33 \\ \text { Bd Wt } & 1.83 \mathrm{M}\end{array}$


Table 3-1 Levels of Significant Exposure to Nickel - Inhalation

\begin{tabular}{|c|c|c|c|c|c|c|c|}
\hline \multirow[b]{2}{*}{$\begin{array}{l}\text { Key to } \\
\text { Figure }\end{array}$} & \multirow[b]{2}{*}{$\begin{array}{l}\text { Species } \\
\text { (Strain) }\end{array}$} & \multirow{2}{*}{$\begin{array}{l}\text { Exposure/ } \\
\text { Duration/ } \\
\text { Frequency } \\
\text { (Route) }\end{array}$} & \multirow[b]{2}{*}{ System } & \multirow[b]{2}{*}{$\begin{array}{l}\text { NOAEL } \\
\left(\mathrm{mg} / \mathrm{m}^{3}\right)\end{array}$} & \multicolumn{2}{|c|}{ LOAEL } & \multirow[b]{2}{*}{$\begin{array}{l}\text { Reference } \\
\text { Chemical Form }\end{array}$} \\
\hline & & & & & $\begin{array}{l}\text { Less Serious } \\
\qquad\left(\mathrm{mg} / \mathrm{m}^{3}\right)\end{array}$ & $\begin{array}{l}\text { Serious } \\
\left(\mathrm{mg} / \mathrm{m}^{3}\right)\end{array}$ & \\
\hline
\end{tabular}

13 Mouse 12 days in $16 \quad$ Resp

(B6C3F1) $\quad$ day period

$\begin{array}{ll}\text { Cardio } & 1.4 \\ \text { Gastro } & 1.4 \\ \text { Musc/skel } & 1.4 \\ \text { Hepatic } & 1.4 \\ \text { Renal } & 1.4 \\ \text { Endocr } & 1.4 \\ \text { Dermal } & 1.4 \\ \text { Bd Wt } & 0.7\end{array}$

Immuno/ Lymphoret

14 Rat 12 days in 16

(Fischer- 344) day period

6 hours/day

15

$\begin{array}{ll}\text { Rat } & 12 \text { days in } 16 \\ \text { (Fischer- 344) } & \text { day period } \\ 6 \text { hours/day }\end{array}$

16 Rat

$\begin{array}{ll}\text { Rat } & 12 \text { days in } 16 \\ \text { (Fischer- 344) day period }\end{array}$

$6 \mathrm{hr} / \mathrm{day}$ $\quad 0.7 \mathrm{~F} \quad 1.4 \mathrm{~F}$ (hyperplasia in bronchial and mediastinal lymph nodes)
$1.4 \quad$ (necrotizing lung

inflammation)

NTP 1996c

sulfate

NTP 1996a

oxide

NTP 1996b

subsulfide

NTP 1996c

sulfate 
Table 3-1 Levels of Significant Exposure to Nickel - Inhalation

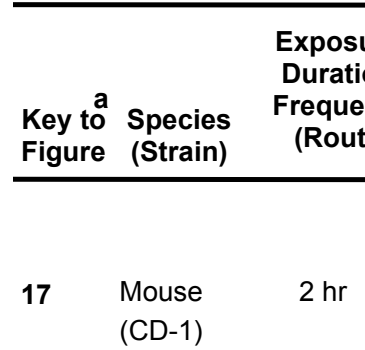

(CD-1)

$\mathrm{hr}$

quency

(Route)

LOAEL

OAEL

System

\section{NOAEL}

$\left(\mathrm{mg} / \mathrm{m}^{3}\right)$

\section{Less Serious} $\left(\mathrm{mg} / \mathrm{m}^{3}\right)$
Reference

Chemical Form
$0.369 \mathrm{~F}$

$18 \quad$ Mouse $2 \mathrm{hr}$

(CD-1)

19 Mouse

$2 \mathrm{hr}$

(CD-1)

20 Mouse

(Swiss)

$2 \mathrm{hr}$

\begin{tabular}{|c|c|c|}
\hline 21 & $\begin{array}{l}\text { Mouse } \\
\text { (B6C3F1) }\end{array}$ & $\begin{array}{l}12 \text { days in } 16 \\
\text { day period } \\
6 \text { hours/day }\end{array}$ \\
\hline 22 & $\begin{array}{l}\text { Mouse } \\
\text { (B6C3F1) }\end{array}$ & $\begin{array}{l}12 \text { days in } 16 \\
\text { day period } \\
6 \text { hours/day }\end{array}$ \\
\hline
\end{tabular}

$0.499 \mathrm{~F}$ (increased susceptibility
to Streptococcal

infection)

Serious

$\left(\mathrm{mg} / \mathrm{m}^{3}\right)$
Adkins et al. 1979

chloride

Adkins et al. 1979

chloride

Adkins et al. 1979

sulfate

to Streptococcal

infection)

$0.1 \mathrm{~F} \quad 0.25 \mathrm{~F}$ (impaired humoral

immunity)

Graham et al. 1978

chloride

NTP 1996a

oxide

NTP 1996b

subsulfide 
Table 3-1 Levels of Significant Exposure to Nickel - Inhalation

\begin{tabular}{lll}
\hline Key to & $\begin{array}{c}\text { Exposure/ } \\
\text { Duration/ } \\
\text { Figure }\end{array}$ \\
\hline (Strain) & $\begin{array}{c}\text { Frequency } \\
\text { (Route) }\end{array}$ \\
$23 \quad \begin{array}{l}\text { Mouse } \\
\text { (B6C3F1) }\end{array}$ & $\begin{array}{l}12 \text { days in } 16 \\
\text { day period } \\
6 \text { hr/day }\end{array}$
\end{tabular}

Neurological

24 Rat 4, 8, 12, $16 \mathrm{~d}$

(Long- Evans) $6 \mathrm{hr} / \mathrm{d}$

\section{Reproductive}

25 Rat

12 days in 16

(Fischer- 344) day period

6 hours/day

$0.635 \mathrm{M}$ (decrease in number of bipolar receptor cells in

nasal olfactory

epithelium)

(Fischer-344) day period

6 hours/day

27 Rat 12 days in 16

(Fischer- 344) day period 6 hr/day

28 Mouse 12 days in 16

$\begin{array}{ll}\text { (B6C3F1) } & \text { day period } \\ 6 \text { hours/day }\end{array}$

29 Mouse 12 days in 16

(B6C3F1) day period

6 hours/day
LOAEL

Serious

Reference

Chemical Form

NTP 1996c

sulfate

Evans et al. 1995

sulfate

NTP 1996a

oxide

NTP 1996b

subsulfide

NTP 1996c

sulfate

NTP 1996a

oxide

NTP 1996b

subsulfide 
Table 3-1 Levels of Significant Exposure to Nickel - Inhalation

\begin{tabular}{|c|c|c|c|c|c|c|c|}
\hline \multirow[b]{2}{*}{$\begin{array}{l}\text { Key to } \\
\text { Figure }\end{array}$} & \multirow[b]{2}{*}{$\begin{array}{l}\text { Species } \\
\text { (Strain) }\end{array}$} & \multirow{2}{*}{$\begin{array}{c}\text { Exposure/ } \\
\text { Duration/ } \\
\text { Frequency } \\
\text { (Route) }\end{array}$} & \multirow[b]{2}{*}{ System } & \multirow[b]{2}{*}{$\begin{array}{l}\text { NOAEL } \\
\left(\mathrm{mg} / \mathrm{m}^{3}\right)\end{array}$} & \multicolumn{2}{|c|}{ LOAEL } & \multirow[b]{2}{*}{$\begin{array}{l}\text { Reference } \\
\text { Chemical Form }\end{array}$} \\
\hline & & & & & $\begin{array}{l}\text { Less Serious } \\
\qquad\left(\mathrm{mg} / \mathrm{m}^{3}\right)\end{array}$ & $\begin{array}{l}\text { Serious } \\
\left(\mathrm{mg} / \mathrm{m}^{3}\right)\end{array}$ & \\
\hline
\end{tabular}

$30 \quad$ Mouse 12 days in 16 $\begin{array}{ll}\text { (B6C3F1) } & \text { day period } \\ 6 \mathrm{hr} / \mathrm{day}\end{array}$

\section{INTERMEDIATE EXPOSURE}

Systemic
Rat up to $6 \mathrm{mo}$
(Fischer- 344) $\frac{5 \mathrm{~d} / \mathrm{wk}}{6 \mathrm{hr} / \mathrm{d}}$

Resp

$0.49 \mathrm{M}$

$1.96 \mathrm{M}$ (moderate alveolitis that persisted at least 4 months after the exposure

Bd Wt $\quad 1.96 \mathrm{M}$

32

$\begin{array}{ll}\text { Rat } & \text { up to } 6 \text { mo } \\ \text { (Fischer- 344) } & \text { Resp } \\ 6 \mathrm{hr} / \mathrm{d}\end{array}$

33

$\begin{array}{ll}\text { Rat } & >2 \mathrm{wk} \\ \text { (Wistar) } & 6 \mathrm{~d} / \mathrm{wk}\end{array}$

$12 \mathrm{hr} / \mathrm{d}$

$34 \quad$ Rat

$\begin{array}{ll}\text { Rat } & >2 \mathrm{wk} \\ \text { (Wistar) } & 6 \mathrm{~d} / \mathrm{wk}\end{array}$

$12 \mathrm{hr} / \mathrm{d}$

$35 \quad$ Rat

$\begin{array}{ll}\text { (Wistar) } & 1 \mathrm{mo} \\ & 5 \mathrm{~d} / \mathrm{wk}\end{array}$

Resp

$0.11 \mathrm{M}$ (alveolitis that persisted exposure)

$0.12 \mathrm{M}$ (alveolar wall thickening)

$0.109 \mathrm{M}$ (hyperplasia of the

bronchial epithelium and

peribronchial lymphocytic infiltration)

Resp

Resp for 4 months after
NTP 1996c

sulfate

Benson et al. 1995a

oxide

Benson et al. 1995a

sulfate

Bingham et al. 1972

oxide

Bingham et al. 1972

chloride

Horie et al. 1985

oxide 
Table 3-1 Levels of Significant Exposure to Nickel - Inhalation

\begin{tabular}{|c|c|c|c|c|c|c|c|}
\hline \multirow[b]{2}{*}{$\begin{array}{l}\text { Key to } \\
\text { Figure }\end{array}$} & \multirow[b]{2}{*}{$\begin{array}{l}\text { Species } \\
\text { (Strain) }\end{array}$} & \multirow{2}{*}{$\begin{array}{l}\text { Exposure/ } \\
\text { Duration/ } \\
\text { Frequency } \\
\text { (Route) }\end{array}$} & \multirow[b]{2}{*}{ System } & \multirow[b]{2}{*}{$\begin{array}{l}\text { NOAEL } \\
\left(\mathrm{mg} / \mathrm{m}^{3}\right)\end{array}$} & \multicolumn{2}{|c|}{ LOAEL } & \multirow[b]{2}{*}{$\begin{array}{l}\text { Reference } \\
\text { Chemical Form }\end{array}$} \\
\hline & & & & & $\begin{array}{l}\text { Less Serious } \\
\left(\mathrm{mg} / \mathrm{m}^{3}\right)\end{array}$ & $\begin{array}{l}\text { Serious } \\
\qquad\left(\mathrm{mg} / \mathrm{m}^{3}\right)\end{array}$ & \\
\hline
\end{tabular}

$36 \quad$ Rat 13 weeks

(Fischer- 344) 5d/wk

Resp

$\left(\mathrm{mg} / \mathrm{m}^{3}\right)$ $\left(\mathrm{mg} / \mathrm{m}^{3}\right)$

Chemical Form

(Fischer- 344) 6hr/d

$6 \mathrm{hr} / \mathrm{d}$
(chronic active lung
inflammation and
granulmatous
granulmatous

NTP 1996a

oxide

$\begin{array}{ll}\text { Cardio } & 7.9 \\ \text { Gastro } & 7.9 \\ \text { Musc/skel } & 7.9 \\ \text { Hepatic } & 7.9 \\ \text { Renal } & 7.9 \\ \text { Endocr } & 7.9 \\ \text { Dermal } & 7.9 \\ \text { Bd Wt } & 7.9\end{array}$


Table 3-1 Levels of Significant Exposure to Nickel - Inhalation

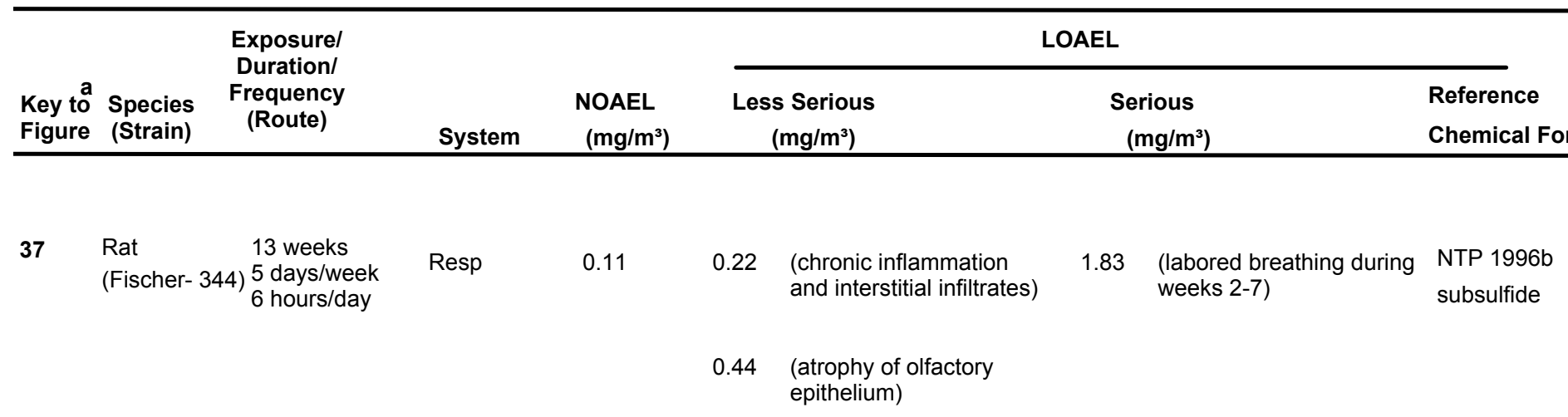

$\begin{array}{ll}\text { Cardio } & 1.83 \\ \text { Gastro } & 1.83 \\ \text { Musc/skel } & 1.83 \\ \text { Hepatic } & 1.83 \\ \text { Renal } & 1.83 \\ \text { Endocr } & 1.83 \\ \text { Dermal } & 1.83 \\ \text { Bd Wt } & 1.83\end{array}$


Table 3-1 Levels of Significant Exposure to Nickel - Inhalation

\begin{tabular}{|c|c|c|c|c|c|c|c|}
\hline \multirow[b]{2}{*}{$\begin{array}{l}\text { Key to } \\
\text { Figure }\end{array}$} & \multirow[b]{2}{*}{$\begin{array}{l}\text { Species } \\
\text { (Strain) }\end{array}$} & \multirow{2}{*}{$\begin{array}{l}\text { Exposure/ } \\
\text { Duration/ } \\
\text { Frequency } \\
\text { (Route) }\end{array}$} & \multirow[b]{2}{*}{ System } & \multirow[b]{2}{*}{$\begin{array}{l}\text { NOAEL } \\
\left(\mathrm{mg} / \mathrm{m}^{3}\right)\end{array}$} & \multicolumn{2}{|c|}{ LOAEL } & \multirow[b]{2}{*}{$\begin{array}{l}\text { Reference } \\
\text { Chemical Form }\end{array}$} \\
\hline & & & & & $\begin{array}{l}\text { Less Serious } \\
\qquad\left(\mathrm{mg} / \mathrm{m}^{3}\right)\end{array}$ & $\begin{array}{l}\text { Serious } \\
\left(\mathrm{mg} / \mathrm{m}^{3}\right)\end{array}$ & \\
\hline
\end{tabular}

$38 \quad$ Rat 13 weeks (Fischer- 344) 5 days/week
6 hours/day
Resp

$0.06 \mathrm{~F}$

$0.11 \mathrm{~F}$ (chronic lung

inflammation, interstitial

infiltrates)

0.22 (atrophy of olfactory epithelium)
NTP 1996c

sulfate

39
$28 \mathrm{~d}$
$23.6 \mathrm{hr} / \mathrm{d}$
Hepatic
$0.784 \mathrm{M}$
Renal
$0.784 \mathrm{M}$
Bd Wt $\quad 0.178 \mathrm{M}$
$0.385 \mathrm{M}(30 \%$ decrease in body weight gain)
Metab
$0.178 \mathrm{M}$
$0.385 \mathrm{M}$ (increased serum glucose)

Weischer et al. 1980

oxide 
Table 3-1 Levels of Significant Exposure to Nickel - Inhalation

\begin{tabular}{|c|c|c|c|c|c|c|c|}
\hline \multirow[b]{2}{*}{$\begin{array}{l}\text { Key to } \\
\text { Figure }\end{array}$} & \multirow[b]{2}{*}{$\begin{array}{l}\text { Species } \\
\text { (Strain) }\end{array}$} & \multirow{2}{*}{$\begin{array}{c}\text { Exposure/ } \\
\text { Duration/ } \\
\text { Frequency } \\
\text { (Route) }\end{array}$} & \multirow[b]{2}{*}{ System } & \multirow[b]{2}{*}{$\begin{array}{l}\text { NOAEL } \\
\left(\mathrm{mg} / \mathrm{m}^{3}\right)\end{array}$} & \multicolumn{2}{|c|}{ LOAEL } & \multirow[b]{2}{*}{$\begin{array}{l}\text { Reference } \\
\text { Chemical Form }\end{array}$} \\
\hline & & & & & $\begin{array}{l}\text { Less Serious } \\
\qquad\left(\mathrm{mg} / \mathrm{m}^{3}\right)\end{array}$ & $\begin{array}{l}\text { Serious } \\
\left(\mathrm{mg} / \mathrm{m}^{3}\right)\end{array}$ & \\
\hline 40 & $\begin{array}{l}\text { Rat } \\
\text { (Wistar) }\end{array}$ & $\begin{array}{l}21 \mathrm{~d} \\
23.6 \mathrm{hr} / \mathrm{d}\end{array}$ & $\mathrm{Bd} \mathrm{Wt}$ & & & $\begin{array}{c}0.8 \mathrm{~F} \text { ( } 36 \% \text { decrease in body } \\
\text { weight gain) }\end{array}$ & $\begin{array}{l}\text { Weischer et al. } 1980 \\
\text { oxide }\end{array}$ \\
\hline \multirow{3}{*}{41} & & & Metab & & $\begin{array}{c}0.8 \mathrm{~F} \text { (decreased serum } \\
\text { glucose level) }\end{array}$ & & \\
\hline & $\begin{array}{l}\text { Mouse } \\
(\mathrm{B} 6 \mathrm{C} 3 \mathrm{~F} 1)\end{array}$ & $\begin{array}{l}\text { up to } 6 \mathrm{mo} \\
5 \mathrm{~d} / \mathrm{wk} \\
6 \mathrm{hr} / \mathrm{d}\end{array}$ & Resp & & $0.98 \mathrm{M}$ (interstitial pneumonia) & & $\begin{array}{l}\text { Benson et al. 1995a } \\
\text { oxide }\end{array}$ \\
\hline & & & $\mathrm{Bd} \mathrm{Wt}$ & $3.93 \mathrm{M}$ & & & \\
\hline 42 & $\begin{array}{l}\text { Mouse } \\
\text { (B6C3F1) }\end{array}$ & $\begin{array}{l}\text { up to } 6 \mathrm{mo} \\
5 \mathrm{~d} / \mathrm{wk} \\
6 \mathrm{hr} / \mathrm{d}\end{array}$ & Resp & $0.06 \mathrm{M}$ & $0.22 \mathrm{M}$ (interstitial pneumonia) & & $\begin{array}{l}\text { Benson et al. } 1995 a \\
\text { sulfate }\end{array}$ \\
\hline
\end{tabular}


Table 3-1 Levels of Significant Exposure to Nickel - Inhalation

\begin{tabular}{|c|c|c|c|c|c|c|c|}
\hline \multirow[b]{2}{*}{$\begin{array}{l}\text { Key to } \\
\text { Figure }\end{array}$} & \multirow[b]{2}{*}{$\begin{array}{l}\text { Species } \\
\text { (Strain) }\end{array}$} & \multirow{2}{*}{$\begin{array}{c}\text { Exposure/ } \\
\text { Duration/ } \\
\text { Frequency } \\
\text { (Route) }\end{array}$} & \multirow[b]{2}{*}{ System } & \multirow[b]{2}{*}{$\begin{array}{l}\text { NOAEL } \\
\left(\mathrm{mg} / \mathrm{m}^{3}\right)\end{array}$} & \multicolumn{2}{|c|}{ LOAEL } & \multirow[b]{2}{*}{$\begin{array}{l}\text { Reference } \\
\text { Chemical Form }\end{array}$} \\
\hline & & & & & $\begin{array}{l}\text { Less Serious } \\
\left(\mathrm{mg} / \mathrm{m}^{3}\right)\end{array}$ & $\begin{array}{l}\text { Serious } \\
\qquad\left(\mathrm{mg} / \mathrm{m}^{3}\right)\end{array}$ & \\
\hline
\end{tabular}

$43 \quad$ Mouse 13 weeks

$\begin{array}{ll}13 \text { weeks } \\ \text { (B6C3F1) } & 5 \mathrm{~d} / \mathrm{wk}\end{array}$

Resp

$2 \mathrm{~F}$

$3.9 \mathrm{~F}$ (perivascular lymphocytic

infiltrates)

NTP 1996a

$6 \mathrm{hr} / \mathrm{d}$

oxide

$\begin{array}{ll}\text { Cardio } & 7.9 \\ \text { Gastro } & 7.9 \\ \text { Musc/skel } & 7.9 \\ \text { Hepatic } & 7.9 \\ \text { Renal } & 7.9 \\ \text { Endocr } & 7.9 \\ \text { Dermal } & 7.9 \\ \text { Bd Wt } & 7.9\end{array}$


Table 3-1 Levels of Significant Exposure to Nickel - Inhalation

\begin{tabular}{|c|c|c|c|c|c|c|c|}
\hline \multirow[b]{2}{*}{$\begin{array}{l}\text { Key to } \\
\text { Figure }\end{array}$} & \multirow[b]{2}{*}{$\begin{array}{l}\text { Species } \\
\text { (Strain) }\end{array}$} & \multirow{2}{*}{$\begin{array}{l}\text { Exposure/ } \\
\text { Duration/ } \\
\text { Frequency } \\
\text { (Route) }\end{array}$} & \multirow[b]{2}{*}{ System } & \multirow[b]{2}{*}{$\begin{array}{l}\text { NOAEL } \\
\left(\mathrm{mg} / \mathrm{m}^{3}\right)\end{array}$} & \multicolumn{2}{|c|}{ LOAEL } & \multirow[b]{2}{*}{$\begin{array}{l}\text { Reference } \\
\text { Chemical Form }\end{array}$} \\
\hline & & & & & $\begin{array}{l}\text { Less Serious } \\
\qquad\left(\mathrm{mg} / \mathrm{m}^{3}\right)\end{array}$ & $\begin{array}{l}\text { Serious } \\
\qquad\left(\mathrm{mg} / \mathrm{m}^{3}\right)\end{array}$ & \\
\hline
\end{tabular}

$44 \quad$ Mouse 13 weeks

13 we

$\begin{array}{ll}\text { (B6C3F1) } & 5 \text { days/week } \\ & 6 \text { hours/day }\end{array}$

Resp

$0.22 \mathrm{M}$

$0.88 \mathrm{M}$ (chronic lung

inflammation and

fibrosis)

NTP 1996b

subsulfide

$0.44 \mathrm{M}$ (atrophy of olfactory

epithelium)

$\begin{array}{ll}\text { Cardio } & 1.83 \\ \text { Gastro } & 1.83 \\ \text { Hemato } & 1.83 \\ \text { Musc/skel } & 1.83 \\ \text { Renal } & 1.83 \\ \text { Endocr } & 1.83 \\ \text { Dermal } & 1.83 \\ \text { Bd Wt } & 1.83\end{array}$


Table 3-1 Levels of Significant Exposure to Nickel - Inhalation

\begin{tabular}{|c|c|c|c|c|c|c|c|}
\hline \multirow[b]{2}{*}{$\begin{array}{l}\text { Key to } \\
\text { Figure }\end{array}$} & \multirow[b]{2}{*}{$\begin{array}{l}\text { Species } \\
\text { (Strain) }\end{array}$} & \multirow{2}{*}{$\begin{array}{l}\text { Exposure/ } \\
\text { Duration/ } \\
\text { Frequency } \\
\text { (Route) }\end{array}$} & \multirow[b]{2}{*}{ System } & \multirow[b]{2}{*}{$\begin{array}{l}\text { NOAEL } \\
\left(\mathrm{mg} / \mathrm{m}^{3}\right)\end{array}$} & \multicolumn{2}{|c|}{ LOAEL } & \multirow[b]{2}{*}{$\begin{array}{l}\text { Reference } \\
\text { Chemical Form }\end{array}$} \\
\hline & & & & & $\begin{array}{l}\text { Less Serious } \\
\qquad\left(\mathrm{mg} / \mathrm{m}^{3}\right)\end{array}$ & $\begin{array}{l}\text { Serious } \\
\left(\mathrm{mg} / \mathrm{m}^{3}\right)\end{array}$ & \\
\hline
\end{tabular}

$45 \quad$ Mouse 13 weeks

13 weeks

$\begin{array}{ll}\text { (B6C3F1) } & 5 \text { days/week } \\ & 6 \text { hours/day }\end{array}$

Resp

$0.22 \mathrm{~F}$

$0.44 \mathrm{~F}$ (chronic lung

inflammation and

fibrosis)

$\begin{array}{ll}\text { Cardio } & 0.44 \\ \text { Gastro } & 0.44 \\ \text { Musc/skel } & 0.44 \\ \text { Hepatic } & 0.44 \\ \text { Renal } & 0.44 \\ \text { Endocr } & 0.44 \\ \text { Dermal } & 0.44 \\ \text { Bd Wt } & 0.44\end{array}$

46

$\begin{array}{lll}\text { Rabbit } & 1-8 \mathrm{mo} & \text { Resp } \\ \text { (NS) } & 5 \mathrm{~d} / \mathrm{wk} \\ & 6 \mathrm{hr} / \mathrm{d} & \end{array}$

Immuno/ Lymphoret

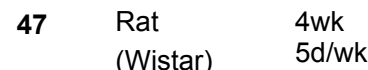

(Wistar) $\quad 5 \mathrm{~d} / \mathrm{wk}$
$8 \mathrm{hr} / \mathrm{d}$

48 Rat

13 weeks

(Fischer- 344) $5 \mathrm{~d} / \mathrm{wk}$
$0.2 \mathrm{M}$ (increased volume density of alveolar type II cells)

9.2 M (increased production of tumor necrosis factor by alveolar macrophages)

0.9 bronchial lymph nodes)

NTP 1996c

sulfate

Johansson and Camner 1986

chloride or metallic

Morimoto et al. 1995

oxide

NTP 1996a

oxide 
Table 3-1 Levels of Significant Exposure to Nickel - Inhalation

\begin{tabular}{|c|c|c|c|c|c|c|c|}
\hline \multirow[b]{2}{*}{$\begin{array}{l}\text { Key to } \\
\text { Figure }\end{array}$} & \multirow[b]{2}{*}{$\begin{array}{l}\text { Species } \\
\text { (Strain) }\end{array}$} & \multirow{2}{*}{$\begin{array}{l}\text { Exposure/ } \\
\text { Duration/ } \\
\text { Frequency } \\
\text { (Route) }\end{array}$} & \multirow[b]{2}{*}{ System } & \multirow[b]{2}{*}{$\begin{array}{l}\text { NOAEL } \\
\left(\mathrm{mg} / \mathrm{m}^{3}\right)\end{array}$} & \multicolumn{2}{|c|}{ LOAEL } & \multirow[b]{2}{*}{$\begin{array}{l}\text { Reference } \\
\text { Chemical Form }\end{array}$} \\
\hline & & & & & $\begin{array}{l}\text { Less Serious } \\
\qquad\left(\mathrm{mg} / \mathrm{m}^{3}\right)\end{array}$ & $\begin{array}{l}\text { Serious } \\
\left(\mathrm{mg} / \mathrm{m}^{3}\right)\end{array}$ & \\
\hline
\end{tabular}

$49 \quad$ Rat 13 weeks

(Fischer- 344) 5 days/week

6 hours/day

$50 \quad$ Rat 13 weeks

(Fischer- 344) 5 days/week

6 hours/day

$51 \quad$ Rat 4 wk

(Wistar) continuous

52

$\begin{array}{ll}\text { Rat } & 4 \text { mo } \\ \text { (Wistar) } & \text { continuous }\end{array}$

53 Mouse $65 d$

(B6C3F1) 5d/wk

$6 \mathrm{hr} / \mathrm{d}$

$54 \quad$ Mouse $65 d$

(B6C3F1) 5d/wk

$6 \mathrm{hr} / \mathrm{d}$

$55 \quad$ Mouse $65 d$

(B6C3F1) $\quad 5 \mathrm{~d} / \mathrm{wk}$
0.11

0.22 (lymphoid hyperplasia in

bronchial lymph nodes)

0.11

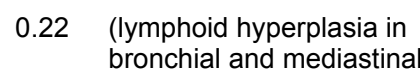
lymph nodes)

0.1

0.2 (impaired humoral immunity)

0.025

0.15 (impaired humoral immunity)

$0.47 \mathrm{~F}$ (decreased alveolar

macrophage activity)

$0.11 \mathrm{~F}$

$0.45 \mathrm{~F}$ (decreased resistance to tumor challenge)

$0.11 \mathrm{~F} \quad 0.45 \mathrm{~F}$ (decreased alveolar macrophage phagocytic activity)
NTP 1996b

subsulfide

NTP 1996c

sulfate

Spiegelberg et al. 1984 oxide

Spiegelberg et al. 1984

oxide

Haley et al. 1990

oxide

Haley et al. 1990

sulfate

Haley et al. 1990

subsulfide 
Table 3-1 Levels of Significant Exposure to Nickel - Inhalation

\begin{tabular}{|c|c|c|}
\hline $\begin{array}{l}\text { Key to } \\
\text { Figure }\end{array}$ & $\begin{array}{l}\text { Species } \\
\text { (Strain) }\end{array}$ & $\begin{array}{c}\text { Exposure/ } \\
\text { Duration/ } \\
\text { Frequency } \\
\text { (Route) }\end{array}$ \\
\hline 56 & $\begin{array}{l}\text { Mouse } \\
\text { (B6C3F1) }\end{array}$ & $\begin{array}{l}13 \text { weeks } \\
5 \mathrm{~d} / \mathrm{wk} \\
6 \mathrm{hr} / \mathrm{d}\end{array}$ \\
\hline
\end{tabular}

57 $\begin{array}{ll}\text { Mouse } & 13 \text { weeks } \\ \text { (B6C3F1) } & 5 \text { days/week } \\ & 6 \text { hours/day }\end{array}$

58
Mouse
5 days/week

6 hours/day

59

$\begin{array}{ll}\text { Rabbit } & 3 \text { or } 6 \text { mo } \\ \text { (NS) } & 5 \mathrm{~d} / \mathrm{wk} \\ & 6 \mathrm{hr} / \mathrm{d}\end{array}$

60

$\begin{array}{ll}\text { Rabbit } & 4-6 \text { wk } \\ \text { (NS) } & 5 \mathrm{~d} / \mathrm{wk} \\ & 6 \mathrm{hr} / \mathrm{d}\end{array}$

61

Rabbit $\quad 4 \mathrm{mo}$

(NS) $\quad 5 \mathrm{~d} / \mathrm{wk}$

\section{Reproductive}
62 Rat
(Fischer- 344) $5 \mathrm{~d} / \mathrm{wk}$
$5 \mathrm{~d} / \mathrm{wk}$

Table

(continued)

LOAEL

$\begin{array}{lcccc} & \text { NOAEL } & \text { Less Serious } & \text { Serious } & \text { Reference } \\ \text { System } & \left(\mathrm{mg} / \mathrm{m}^{3}\right) & \left(\mathrm{mg} / \mathrm{m}^{3}\right) & \left(\mathrm{mg} / \mathrm{m}^{3}\right) & \text { Chemical Form }\end{array}$

0.9

$2 \begin{aligned} & \text { (lymphoid hyperplasia in } \\ & \text { bronchial lymph nodes) }\end{aligned}$

$0.44 \mathrm{~F} \quad 0.88 \mathrm{~F} \begin{aligned} & \text { (lymphoid hyperplasia in } \\ & \text { bronchial lymph nodes) }\end{aligned}$

$0.22 \mathrm{~F} \quad 0.44 \mathrm{~F}$ (hyperplasia of bronchial lymph nodes)

$1 \mathrm{M}$ (inactive macrophage surfaces)

0.6 M (decrease lysozyme

activity in alveola

macrophages)

$0.6 \mathrm{M}$ (decreased macrophage

lysosomal activity)

$3.9 \mathrm{M}$

7.9 (decreased sperm concentration)
NTP 1996a

oxide

NTP 1996b

subsulfide

NTP 1996c

sulfate

Johansson et al. 1987

chloride

Johansson et al. 1988a, 1989

chloride

NTP 1996a

oxide 
Table 3-1 Levels of Significant Exposure to Nickel - Inhalation

\begin{tabular}{|c|c|c|c|c|c|c|c|}
\hline \multirow[b]{2}{*}{$\begin{array}{l}\text { Key to } \\
\text { Figure }\end{array}$} & \multirow[b]{2}{*}{$\begin{array}{l}\text { Species } \\
\text { (Strain) }\end{array}$} & \multirow{2}{*}{$\begin{array}{l}\text { Exposure/ } \\
\text { Duration/ } \\
\text { Frequency } \\
\text { (Route) }\end{array}$} & \multirow[b]{2}{*}{ System } & \multirow[b]{2}{*}{$\begin{array}{l}\text { NOAEL } \\
\left(\mathrm{mg} / \mathrm{m}^{3}\right)\end{array}$} & \multicolumn{2}{|c|}{ LOAEL } & \multirow[b]{2}{*}{$\begin{array}{l}\text { Reference } \\
\text { Chemical Form }\end{array}$} \\
\hline & & & & & $\begin{array}{l}\text { Less Serious } \\
\qquad\left(\mathrm{mg} / \mathrm{m}^{3}\right)\end{array}$ & $\begin{array}{l}\text { Serious } \\
\left(\mathrm{mg} / \mathrm{m}^{3}\right)\end{array}$ & \\
\hline
\end{tabular}

63 Rat 13 weeks

(Fischer- 344) 5 days/week

6 hours/day

$64 \quad$ Rat 13 weeks

(Fischer- 344) 5 days/week

6 hours/day

$65 \quad$ Mouse 13 weeks

(B6C3F1) 5d/wk

$6 \mathrm{hr} / \mathrm{d}$

$66 \quad$ Mouse 13 weeks

(B6C3F1) 5 days/week

6 hours/day

67 Mouse 13 weeks

$\begin{array}{ll}\text { (B6C3F1) } & 5 \text { days/week } \\ 6 \text { hours/day }\end{array}$

Developmental

68 Rat Gd 1-21

(Wistar) $23.6 \mathrm{hr} /$ day

\section{CHRONIC EXPOSURE}

Death

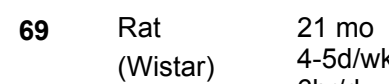

$6 \mathrm{hr} / \mathrm{d}$

70 Rat 78 wk

(Fischer- 344) $\begin{aligned} & 5 \mathrm{~d} / \mathrm{wk} \\ & 6 \mathrm{hr} / \mathrm{d}\end{aligned}$
1.83

1.6 (decreased fetal body weights)
NTP 1996b

subsulfide

NTP 1996c

sulfate

NTP 1996a

oxide

NTP 1996b

subsulfide

NTP 1996c

sulfate

Weischer et al. 1980

oxide

Hueper 1958

metallic

$0.7 \quad(<11 / 226$ survived $)$

Ottolenghi et al. 1974

subsulfide 
Table 3-1 Levels of Significant Exposure to Nickel - Inhalation

\begin{tabular}{|c|c|}
\hline $\begin{array}{l}\text { Key to } \\
\text { Figure }\end{array}$ & $\begin{array}{l}\text { Species } \\
\text { (Strain) }\end{array}$ \\
\hline 71 & $\begin{array}{l}\text { Rat } \\
\text { (Wistar) }\end{array}$ \\
\hline
\end{tabular}

72 Mouse $21 \mathrm{mo}$

(C57) $\quad 4-5 \mathrm{~d} / \mathrm{wk}$

$6 \mathrm{hr} / \mathrm{d}$

$73 \quad$ Gn Pig $21 \mathrm{mo}$

(strain 13) $\quad 4-5 \mathrm{~d} / \mathrm{wk}$

$6 \mathrm{hr} / \mathrm{d}$

Systemic

74 Human occupa-

tional Renal

LOAEL

$\begin{array}{cccc}\text { NOAEL } & \text { Less Serious } & \text { Serious } & \text { Reference } \\ \left(\mathrm{mg} / \mathrm{m}^{3}\right) & \left(\mathrm{mg} / \mathrm{m}^{3}\right) & \left(\mathrm{mg} / \mathrm{m}^{3}\right) & \text { Chemical Form }\end{array}$

$0.06 \mathrm{M}$ (decreased survival time) Takenaka et al. 1985 oxide

$\begin{array}{ll}15 \mathrm{~F}(20 / 20 \text { died }) & \begin{array}{l}\text { Hueper } 1958 \\ \text { metallic }\end{array} \\ 15 \quad(42 / 42 \text { died }) & \begin{array}{l}\text { Hueper } 1958 \\ \text { metallic }\end{array}\end{array}$

$0.75 \mathrm{~F}$ (increased urinary

excretion of

$\mathrm{N}$-acetyl-b-D-

glucosamidase, total

proteins, b2

-microglobulin, and

retinol binding protein)
Vyskocil et al. $1994 a$

sulfate, chloride 
Table 3-1 Levels of Significant Exposure to Nickel - Inhalation

\begin{tabular}{|c|c|c|c|c|c|c|c|}
\hline \multirow[b]{2}{*}{$\begin{array}{l}\text { Key to } \\
\text { Figure }\end{array}$} & \multirow[b]{2}{*}{$\begin{array}{l}\text { Species } \\
\text { (Strain) }\end{array}$} & \multirow{2}{*}{$\begin{array}{l}\text { Exposure/ } \\
\text { Duration/ } \\
\text { Frequency } \\
\text { (Route) }\end{array}$} & \multirow[b]{2}{*}{ System } & \multirow[b]{2}{*}{$\begin{array}{l}\text { NOAEL } \\
\left(\mathrm{mg} / \mathrm{m}^{3}\right)\end{array}$} & \multicolumn{2}{|c|}{ LOAEL } & \multirow[b]{2}{*}{$\begin{array}{l}\text { Reference } \\
\text { Chemical Form }\end{array}$} \\
\hline & & & & & $\begin{array}{l}\text { Less Serious } \\
\left(\mathrm{mg} / \mathrm{m}^{3}\right)\end{array}$ & $\begin{array}{l}\text { Serious } \\
\qquad\left(\mathrm{mg} / \mathrm{m}^{3}\right)\end{array}$ & \\
\hline
\end{tabular}

Rat $2 \mathrm{yr}$

(Fischer- 344) $5 \mathrm{~d} / \mathrm{wk}$

Resp

$6 \mathrm{hrs} / \mathrm{d}$

0.5 (chronic lung

inflammation)

NTP 1996a

oxide

$\begin{array}{ll}\text { Cardio } & 2 \\ \text { Gastro } & 2 \\ \text { Hemato } & 2 \\ \text { Musc/skel } & 2 \\ \text { Hepatic } & 2 \\ \text { Renal } & 2 \\ \text { Endocr } & 1\end{array}$

$2 \mathrm{~F}$ (benign

pheochromocytoma and

adrenal medulla

hyperplasia)

$\begin{array}{ll}\text { Dermal } & 2 \\ \text { Bd Wt } & 2\end{array}$


Table 3-1 Levels of Significant Exposure to Nickel - Inhalation

\begin{tabular}{|c|c|c|c|c|c|c|c|}
\hline \multirow[b]{2}{*}{$\begin{array}{l}\text { Key to } \\
\text { Figure }\end{array}$} & \multirow[b]{2}{*}{$\begin{array}{l}\text { Species } \\
\text { (Strain) }\end{array}$} & \multirow{2}{*}{$\begin{array}{l}\text { Exposure/ } \\
\text { Duration/ } \\
\text { Frequency } \\
\text { (Route) }\end{array}$} & \multirow[b]{2}{*}{ System } & \multirow[b]{2}{*}{$\begin{array}{l}\text { NOAEL } \\
\left(\mathrm{mg} / \mathrm{m}^{3}\right)\end{array}$} & \multicolumn{2}{|c|}{ LOAEL } & \multirow[b]{2}{*}{$\begin{array}{l}\text { Reference } \\
\text { Chemical Form }\end{array}$} \\
\hline & & & & & $\begin{array}{l}\text { Less Serious } \\
\left(\mathrm{mg} / \mathrm{m}^{3}\right)\end{array}$ & $\begin{array}{l}\text { Serious } \\
\qquad\left(\mathrm{mg} / \mathrm{m}^{3}\right)\end{array}$ & \\
\hline
\end{tabular}

$76 \quad$ Rat 2 years

$\begin{array}{lll}\text { Rat } & 2 \text { years } & \text { Resp }\end{array}$

(Fischer- 344) 6 hours/day
5 days/week
0.73 (atrophy of nasal

olfactory epithelium)
0.11 (chronic inflammation, NTP 1996b
alveolar epithelium
hyperplasia, fibrosis
subsulfide
rapid and shallow
breathing)

$\begin{array}{lll}\text { Cardio } & 0.73 & \\ \text { Gastro } & 0.73 & \\ \text { Musc/skel } & 0.73 & \\ \text { Renal } & 0.73 & \\ \text { Endocr } & & 0.11 \mathrm{M} \text { (pheochromocytoma) } \\ \text { Bd Wt } & 0.11 & 0.73 \quad \begin{array}{l}(11-12 \% \text { decrease in } \\ \text { body weight gain) }\end{array}\end{array}$


Table 3-1 Levels of Significant Exposure to Nickel - Inhalation

\begin{tabular}{|c|c|c|c|c|c|c|c|}
\hline \multirow[b]{2}{*}{$\begin{array}{l}\text { Key to } \\
\text { Figure }\end{array}$} & \multirow[b]{2}{*}{$\begin{array}{l}\text { Species } \\
\text { (Strain) }\end{array}$} & \multirow{2}{*}{$\begin{array}{l}\text { Exposure/ } \\
\text { Duration/ } \\
\text { Frequency } \\
\text { (Route) }\end{array}$} & \multirow[b]{2}{*}{ System } & \multirow[b]{2}{*}{$\begin{array}{l}\text { NOAEL } \\
\left(\mathrm{mg} / \mathrm{m}^{3}\right)\end{array}$} & \multicolumn{2}{|c|}{ LOAEL } & \multirow[b]{2}{*}{$\begin{array}{l}\text { Reference } \\
\text { Chemical Form }\end{array}$} \\
\hline & & & & & $\begin{array}{l}\text { Less Serious } \\
\qquad\left(\mathrm{mg} / \mathrm{m}^{3}\right)\end{array}$ & $\begin{array}{l}\text { Serious } \\
\qquad\left(\mathrm{mg} / \mathrm{m}^{3}\right)\end{array}$ & \\
\hline
\end{tabular}

$77 \quad$ Rat $2 \mathrm{yr}$ (Fischer- 344) $6 \mathrm{~d} / \mathrm{wk}$

Resp
$0.03^{c}$

0.11 (atrophy of olfactory

epithelium)

NTP 1996c

$6 \mathrm{hr} / \mathrm{d}$

0.06 (chronic inflammation,

bronchialization)

sulfate

$\begin{array}{ll}\text { Cardio } & 0.11 \\ \text { Gastro } & 0.11 \\ \text { Hemato } & 0.11 \\ \text { Hepatic } & 0.11 \\ \text { Renal } & 0.11 \\ \text { Endocr } & 0.11 \\ \text { Dermal } & 0.11 \\ \text { Bd Wt } & 0.11\end{array}$


Table 3-1 Levels of Significant Exposure to Nickel - Inhalation

\begin{tabular}{|c|c|c|c|c|c|c|c|}
\hline \multirow[b]{2}{*}{$\begin{array}{l}\text { Key to } \\
\text { Figure }\end{array}$} & \multirow[b]{2}{*}{$\begin{array}{l}\text { Species } \\
\text { (Strain) }\end{array}$} & \multirow{2}{*}{$\begin{array}{l}\text { Exposure/ } \\
\text { Duration/ } \\
\text { Frequency } \\
\text { (Route) }\end{array}$} & \multirow[b]{2}{*}{ System } & \multirow[b]{2}{*}{$\begin{array}{l}\text { NOAEL } \\
\left(\mathrm{mg} / \mathrm{m}^{3}\right)\end{array}$} & \multicolumn{2}{|c|}{ LOAEL } & \multirow[b]{2}{*}{$\begin{array}{l}\text { Reference } \\
\text { Chemical Form }\end{array}$} \\
\hline & & & & & $\begin{array}{l}\text { Less Serious } \\
\qquad\left(\mathrm{mg} / \mathrm{m}^{3}\right)\end{array}$ & $\begin{array}{l}\text { Serious } \\
\qquad\left(\mathrm{mg} / \mathrm{m}^{3}\right)\end{array}$ & \\
\hline
\end{tabular}

$78 \quad$ Rat $78 w k$

$\begin{array}{lll}\text { Rat } & 78 w k \\ \text { (Fischer- 344) } & 5 \mathrm{~d} / \mathrm{wk} & \text { Resp }\end{array}$

$\begin{array}{ll}\text { Cardio } & 0.7 \\ \text { Gastro } & 0.7 \\ \text { Hepatic } & 0.7 \\ \text { Renal } & 0.7 \\ \text { Endocr } & 0.7 \\ \text { Bd Wt } & \end{array}$

$\mathrm{Bd} \mathrm{Wt}$
$0.06 \mathrm{M}$ (increased lung weight congestion; alveolar proteinosis)

$\mathrm{Bd} \mathrm{Wt}$

$80 \quad$ Rat

$\begin{array}{lll} & 12 \mathrm{mo} & \text { Resp } \\ \text { (Wistar) } & 5 \mathrm{~d} / \mathrm{wk} \\ 7 \mathrm{hr} / \mathrm{d} & \end{array}$

$\begin{array}{ll}\text { Hepatic } & 0.9 \\ \text { Renal } & 0.9 \\ \text { Bd Wt } & 0.9\end{array}$

0.7 (body weight 20-30\% less than controls)

0.7 (pneumonitis; bronchitis; emphysema)

\section{Ottolenghi et al. 1974} subsulfide

Takenaka et al. 1985 oxide

$0.06 \mathrm{M}$ (weight loss amount not stated)

0.2 (pneumonia)
Tanaka et al. 1988 oxide 
Table 3-1 Levels of Significant Exposure to Nickel - Inhalation

\begin{tabular}{|c|c|c|c|c|c|c|c|}
\hline \multirow[b]{2}{*}{$\begin{array}{l}\text { Key to } \\
\text { Figure }\end{array}$} & \multirow[b]{2}{*}{$\begin{array}{l}\text { Species } \\
\text { (Strain) }\end{array}$} & \multirow{2}{*}{$\begin{array}{l}\text { Exposure/ } \\
\text { Duration/ } \\
\text { Frequency } \\
\text { (Route) }\end{array}$} & \multirow[b]{2}{*}{ System } & \multirow[b]{2}{*}{$\begin{array}{l}\text { NOAEL } \\
\left(\mathrm{mg} / \mathrm{m}^{3}\right)\end{array}$} & \multicolumn{2}{|c|}{ LOAEL } & \multirow[b]{2}{*}{$\begin{array}{l}\text { Reference } \\
\text { Chemical Form }\end{array}$} \\
\hline & & & & & $\begin{array}{l}\text { Less Serious } \\
\qquad\left(\mathrm{mg} / \mathrm{m}^{3}\right)\end{array}$ & $\begin{array}{l}\text { Serious } \\
\qquad\left(\mathrm{mg} / \mathrm{m}^{3}\right)\end{array}$ & \\
\hline
\end{tabular}

\section{$81 \quad$ Mouse $2 \mathrm{yr}$}

$\begin{array}{ll}\text { (B6C3F1) } & 5 \mathrm{~d} / \mathrm{wk} \\ 6 \mathrm{hrs} / \mathrm{d}\end{array}$

Resp

$\begin{array}{ll}\text { Cardio } & 3.9 \\ \text { Gastro } & 3.9 \\ \text { Hemato } & 3.9 \\ \text { Musc/skel } & 3.9 \\ \text { Hepatic } & 3.9 \\ \text { Renal } & 3.9 \\ \text { Endocr } & 3.9 \\ \text { Dermal } & 3.9 \\ \text { Bd Wt } & 3.9\end{array}$

1 (chronic lung

inflammation,

bronchialization, alveolar

proteinosis)
NTP 1996a

oxide 
Table 3-1 Levels of Significant Exposure to Nickel - Inhalation

\begin{tabular}{|c|c|c|c|c|c|c|c|}
\hline \multirow[b]{2}{*}{$\begin{array}{l}\text { Key to } \\
\text { Figure }\end{array}$} & \multirow[b]{2}{*}{$\begin{array}{l}\text { Species } \\
\text { (Strain) }\end{array}$} & \multirow{2}{*}{$\begin{array}{l}\text { Exposure/ } \\
\text { Duration/ } \\
\text { Frequency } \\
\text { (Route) }\end{array}$} & \multirow[b]{2}{*}{ System } & \multirow[b]{2}{*}{$\begin{array}{l}\text { NOAEL } \\
\left(\mathrm{mg} / \mathrm{m}^{3}\right)\end{array}$} & \multicolumn{2}{|c|}{ LOAEL } & \multirow[b]{2}{*}{$\begin{array}{l}\text { Reference } \\
\text { Chemical Form }\end{array}$} \\
\hline & & & & & $\begin{array}{l}\text { Less Serious } \\
\qquad\left(\mathrm{mg} / \mathrm{m}^{3}\right)\end{array}$ & $\begin{array}{l}\text { Serious } \\
\qquad\left(\mathrm{mg} / \mathrm{m}^{3}\right)\end{array}$ & \\
\hline
\end{tabular}

82 Mouse 2 years

$\begin{array}{lll}\text { Mouse } & 2 \text { years } & \text { Resp }\end{array}$

$\begin{array}{ll}\text { (B6C3F1) } & 6 \text { hours/day } \\ & 5 \text { days/week }\end{array}$
0.44 (chronic active lung

inflammation,

bronchialization, alveolar

proteinosis, fibrosis)
NTP 1996b

subsulfide

$\begin{array}{ll}\text { Cardio } & 0.88 \\ \text { Gastro } & 0.88 \\ \text { Hepatic } & 0.88 \\ \text { Renal } & 0.88 \\ \text { Endocr } & 0.88 \\ \text { Dermal } & 0.88 \\ \text { Bd Wt } & 0.88\end{array}$


Table 3-1 Levels of Significant Exposure to Nickel - Inhalation

\begin{tabular}{|c|c|c|c|c|c|c|c|}
\hline \multirow[b]{2}{*}{$\begin{array}{l}\text { Key to } \\
\text { Figure }\end{array}$} & \multirow[b]{2}{*}{$\begin{array}{l}\text { Species } \\
\text { (Strain) }\end{array}$} & \multirow{2}{*}{$\begin{array}{l}\text { Exposure/ } \\
\text { Duration/ } \\
\text { Frequency } \\
\text { (Route) }\end{array}$} & \multirow[b]{2}{*}{ System } & \multirow[b]{2}{*}{$\begin{array}{l}\text { NOAEL } \\
\left(\mathrm{mg} / \mathrm{m}^{3}\right)\end{array}$} & \multicolumn{3}{|c|}{ LOAEL } \\
\hline & & & & & $\begin{array}{l}\text { Less Serious } \\
\qquad\left(\mathrm{mg} / \mathrm{m}^{3}\right)\end{array}$ & $\begin{array}{l}\text { Serious } \\
\left(\mathrm{mg} / \mathrm{m}^{3}\right)\end{array}$ & $\begin{array}{l}\text { Reference } \\
\text { Chemical Form }\end{array}$ \\
\hline
\end{tabular}

$83 \quad$ Mouse $2 \mathrm{yr}$

(B6C3F1) $\quad 5 \mathrm{~d} / \mathrm{wk}$
$6 \mathrm{hr} / \mathrm{d}$

Resp

epithelium)

$0.06 \mathrm{~F}$ (chronic active lung

inflammation, alveola

proteinosis)

$\begin{array}{ll}\text { Cardio } & 0.22 \\ \text { Gastro } & 0.22 \\ \text { Hemato } & 0.22 \\ \text { Hepatic } & 0.22 \\ \text { Renal } & 0.22 \\ \text { Endocr } & 0.22 \\ \text { Dermal } & 0.22 \\ \text { Bd Wt } & 0.22\end{array}$

Immuno/ Lymphoret

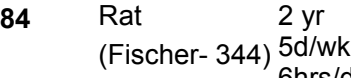

$6 \mathrm{hrs} / \mathrm{d}$

85 Rat 2 years

(Fischer- 344) 6 hours/day 5 days/week

86
Rat
$2 \mathrm{yr}$
$5 \mathrm{~d} / \mathrm{wk}$
(Fischer- 344) $5 \mathrm{~d} / \mathrm{wk}$

$0.5 \mathrm{M}$ (lymphoid hyperplasia in

bronchial lymph node)

0.11 (lymphoid hyperplasia in bronchial lymph nodes)

0.06
NTP 1996c

sulfate
NTP 1996a

oxide

NTP 1996b

subsulfide

NTP 1996c

sulfate 
Table 3-1 Levels of Significant Exposure to Nickel - Inhalation

\begin{tabular}{|c|c|c|}
\hline $\begin{array}{l}\text { Key to } \\
\text { Figure }\end{array}$ & $\begin{array}{l}\text { Species } \\
\text { (Strain) }\end{array}$ & $\begin{array}{r}\text { Exposur } \\
\text { Duratio } \\
\text { Frequen } \\
\text { (Route }\end{array}$ \\
\hline 87 & $\begin{array}{l}\text { Mouse } \\
\text { (B6C3F1) }\end{array}$ & $\begin{array}{l}2 \mathrm{yr} \\
5 \mathrm{~d} / \mathrm{wk}\end{array}$ \\
\hline
\end{tabular}
$6 \mathrm{hrs} / \mathrm{d}$

88
Mouse
(B6C3F1) 6 hours/day
6 hours/day
5 days/week

$89 \quad$ Mouse

(B6C3F1) 5d/wk

$6 \mathrm{hr} / \mathrm{d}$

0.22 (bronchial lymph node
hyperplasia)

2
LOAEL

NOAEL Less Serious Serious

$\left(\mathrm{mg} / \mathrm{m}^{3}\right)$

Reference

Chemical Form

NTP 1996a

oxide

NTP 1996b

subsulfide

NTP 1996c

sulfate

NTP 1996b

subsulfide

NTP 1996c

sulfate

NTP 1996a

oxide

NTP 1996b

subsulfide 
Table 3-1 Levels of Significant Exposure to Nickel - Inhalation

\begin{tabular}{|c|c|c|c|c|c|c|c|}
\hline \multirow[b]{2}{*}{$\begin{array}{l}\text { Key to } \\
\text { Figure }\end{array}$} & \multirow[b]{2}{*}{$\begin{array}{l}\text { Species } \\
\text { (Strain) }\end{array}$} & \multirow{2}{*}{$\begin{array}{l}\text { Exposure/ } \\
\text { Duration/ } \\
\text { Frequency } \\
\text { (Route) }\end{array}$} & \multirow[b]{2}{*}{ System } & \multirow[b]{2}{*}{$\begin{array}{l}\text { NOAEL } \\
\left(\mathrm{mg} / \mathrm{m}^{3}\right)\end{array}$} & \multicolumn{2}{|c|}{ LOAEL } & \multirow[b]{2}{*}{$\begin{array}{l}\text { Reference } \\
\text { Chemical Form }\end{array}$} \\
\hline & & & & & $\begin{array}{l}\text { Less Serious } \\
\qquad\left(\mathrm{mg} / \mathrm{m}^{3}\right)\end{array}$ & $\begin{array}{l}\text { Serious } \\
\left(\mathrm{mg} / \mathrm{m}^{3}\right)\end{array}$ & \\
\hline
\end{tabular}

\section{$95 \quad$ Mouse $2 \mathrm{yr}$}

(B6C3F1) $\quad 5 \mathrm{~d} / \mathrm{wk}$

Cancer

96 Human occupa-

tional

97 Human occupa-

tional

$98 \quad$ Rat

(Fischer- 344) $5 \mathrm{~d} / \mathrm{wk}$

99 Rat 2 years

(Fischer- 344) 6 hours/day 5 days/week

0.22

\section{NTP 1996c}

sulfate

$10 \mathrm{M}$ (CEL: lung and nasal cancers)

1 (CEL: lung and nasal cancers)

$1 \mathrm{M}$ (CEL:

alveolar/bronchiola

adenoma or carcinoma)

0.73 (CEL:alveolar/bronchiolar NTP 1996b

adenoma or carcinoma) subsulfide
Int Committee on $\mathrm{N}$

Carcinogenesis in Man 1990

soluble and less soluble forms combined

Int Committee on $\mathrm{N}$

Carcinogenesis in Man 1990

soluble

NTP 1996a

oxide 
Table 3-1 Levels of Significant Exposure to Nickel - Inhalation

(continued)

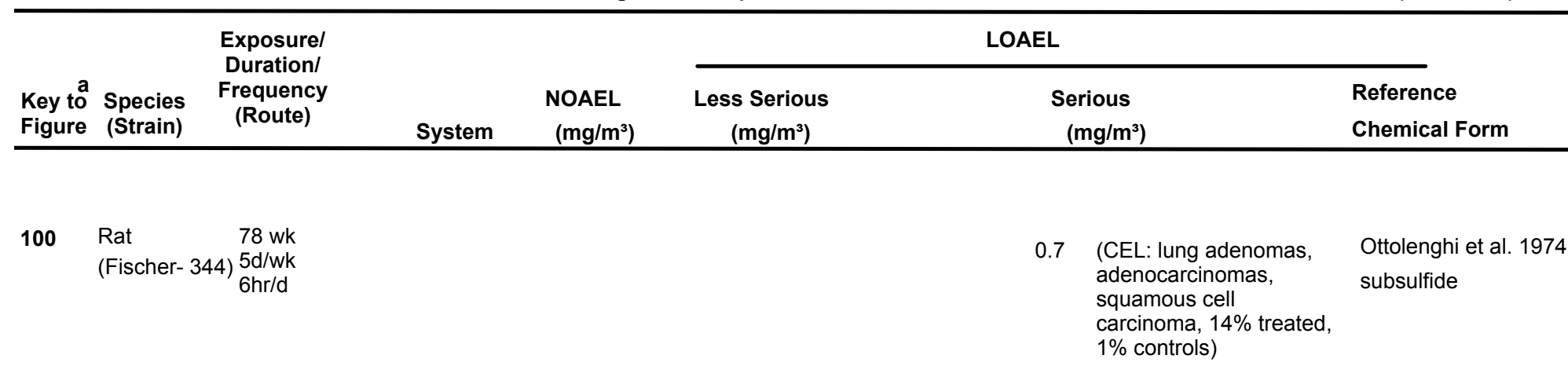

a The number corresponds to entries in Figure 3-1.

b Used to derive an intermediate-duration inhalation minimal risk level (MRL) of $0.0002 \mathrm{mg} \mathrm{Ni} / \mathrm{m} 3$; concentration adjusted for intermittent exposure ( 6 hours/24 hours, 5 days $/ 7$ days) multiplied by the Regional Deposited Dose Ratio (RDDR) of 0.474 for the pulmonary region, and divided by an uncertainty factor of 30 ( 3 for extrapolation from animals to human with dosimetric adjustment, and 10 for human variability).

c Used to derive a chronic-duration inhalation minimal risk level (MRL) of $0.00009 \mathrm{mg} \mathrm{Ni} / \mathrm{m} 3$; concentration adjusted for intermittent exposure (6 hours/24 hours, 5 days/7 days), multiplied by the Regional Deposited Dose Ratio (RDDR) of 0.506 for the pulmonary region, and divided by an uncertainty factor of 30 ( 3 for extrapolation from animals to human with dosimetric adjustment, and 10 for human variability).

d Differences in levels of health effects between male and females are not indicated in Figure 3-1. Where such differences exist, only the levels of effect for the most sensitive gender are presented.

$\mathrm{Bd} \mathrm{Wt}=$ body weight; Cardio = cardiovascular; $\mathrm{CEL}=$ cancer effect level; $\mathrm{d}=$ day $(\mathrm{s})$; Endocr = endocrine; F = Female; Gastro = gastrointestinal; Gd = gestational day; Gn pig =

guinea pig; hemato = hematological; $\mathrm{hr}=$ hour(s); Immuno = immunological; LOAEL = lowest-observed-adverse-effect level; $\mathrm{M}=\mathrm{male} ; \mathrm{mo}=\mathrm{month}(\mathrm{s}) ; \mathrm{Musc} / \mathrm{skel}=\mathrm{musculoskeletal}$; $\mathrm{Ni}=$ nickel; NOAEL = no-observed-adverse-effect level; NS = not specified; Resp = respiratory; wk = week(s) 
Figure 3-1 Levels of Significant Exposure to Nickel - Inhalation

Acute ( $\leq 14$ days)

Systemic

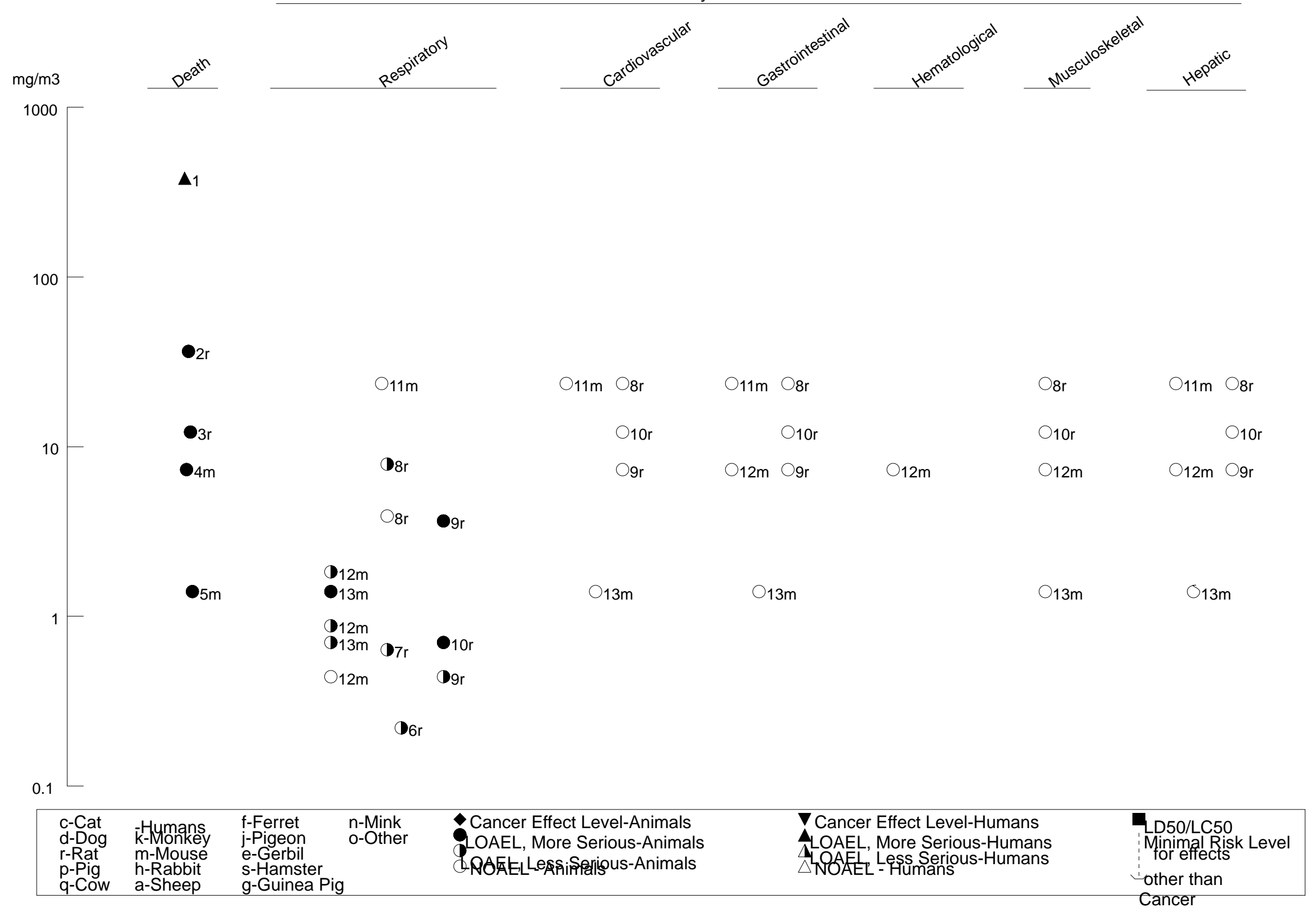

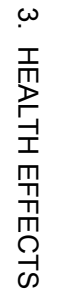


Figure 3-1 Levels of Significant Exposure to Nickel - Inhalation (Continued)

Acute ( $\leq 14$ days)

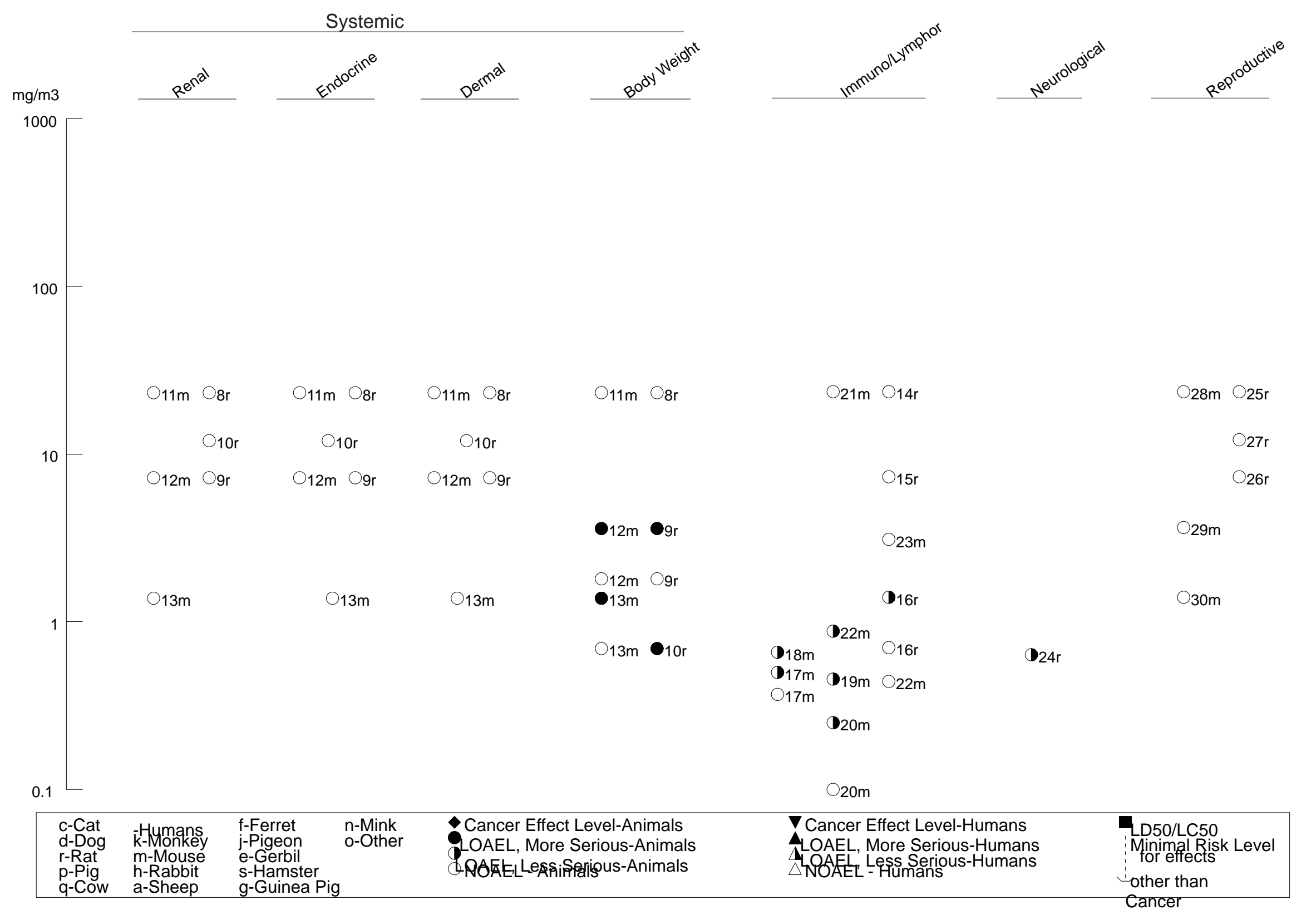


Figure 3-1 Levels of Significant Exposure to Nickel - Inhalation (Continued)

Intermediate (15-364 days)

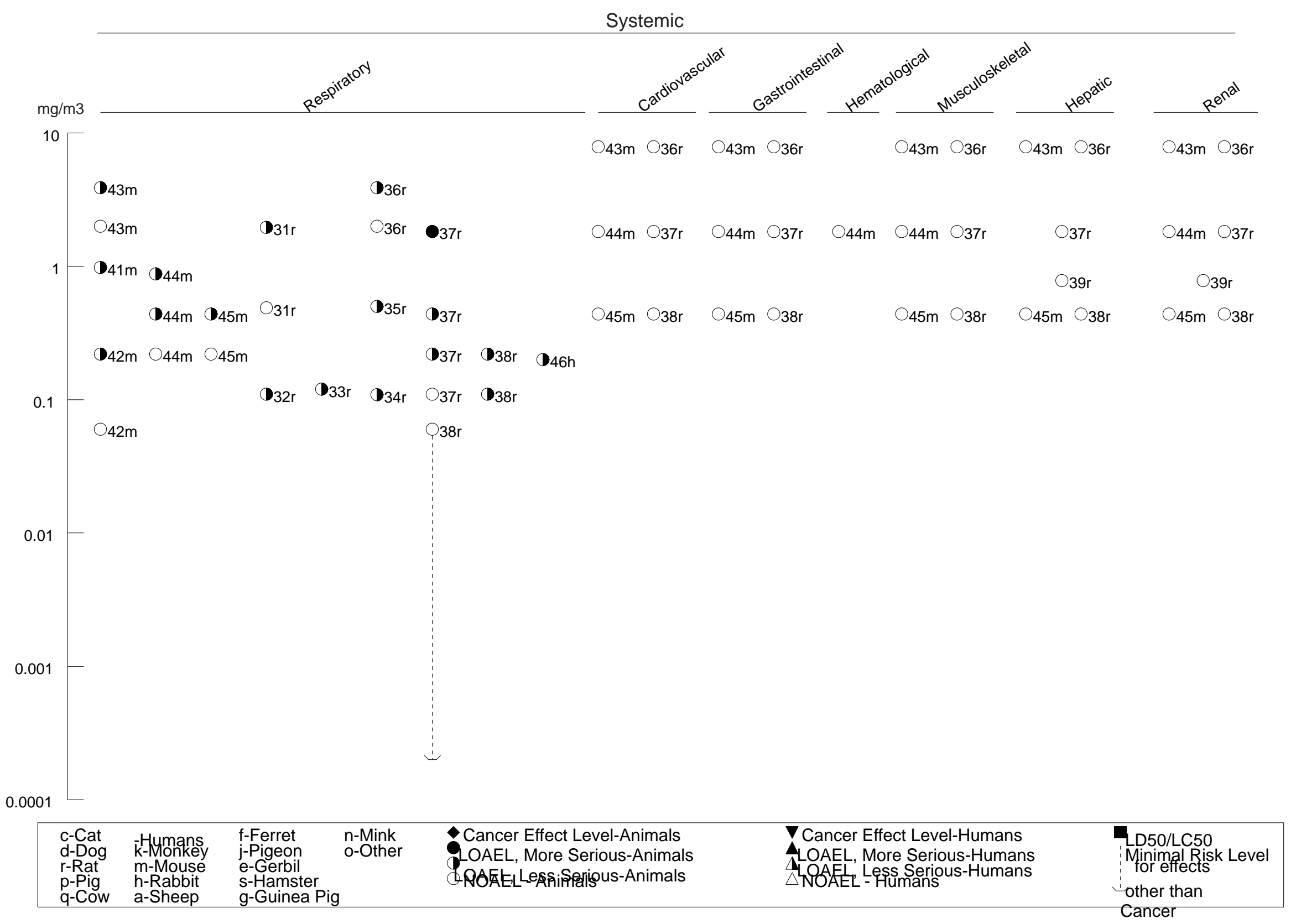


Figure 3-1 Levels of Significant Exposure to Nickel - Inhalation (Continued)

Intermediate (15-364 days)

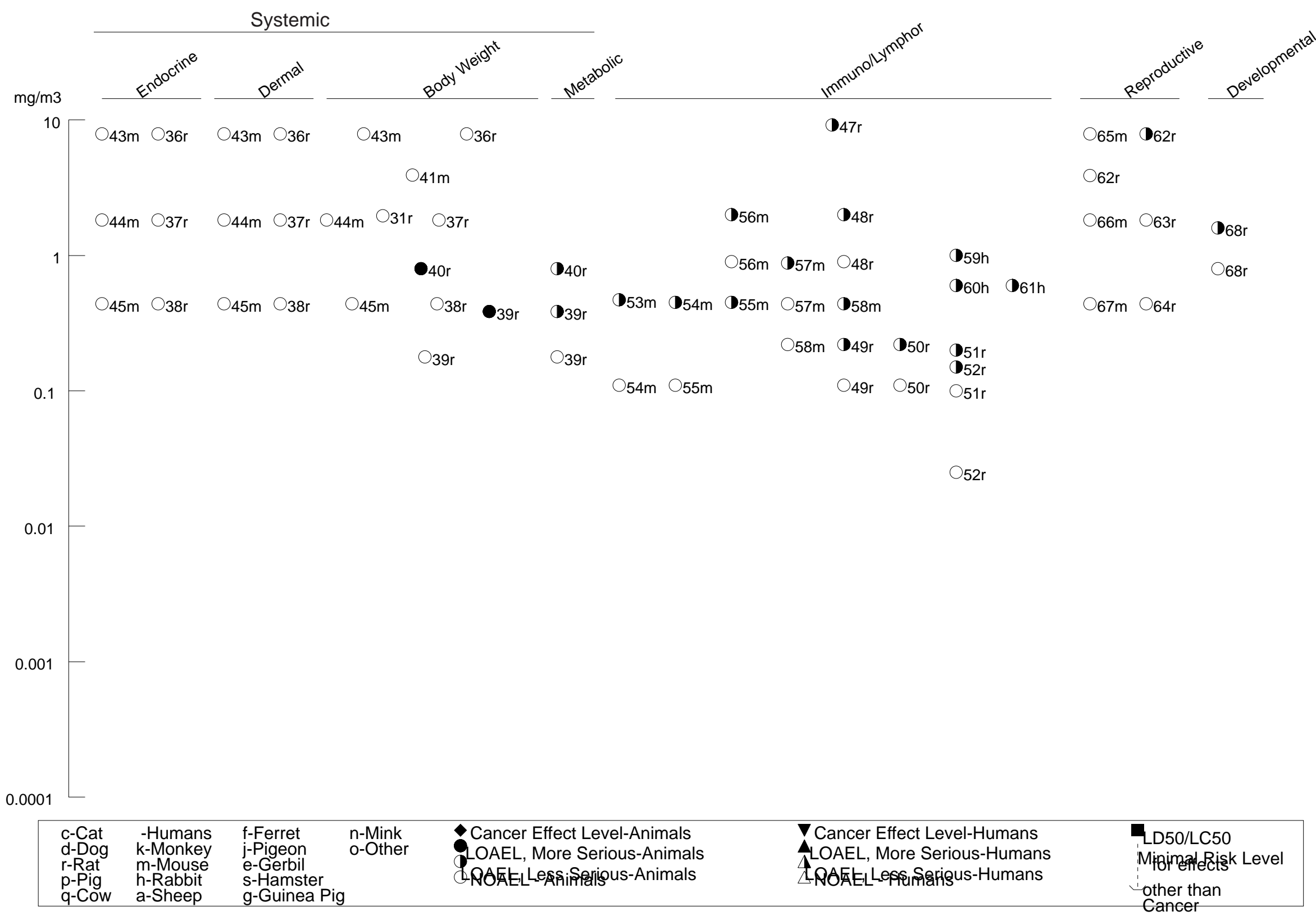


Figure 3-1 Levels of Significant Exposure to Nickel - Inhalation (Continued)

Chronic ( $\geq 365$ days)

Systemic

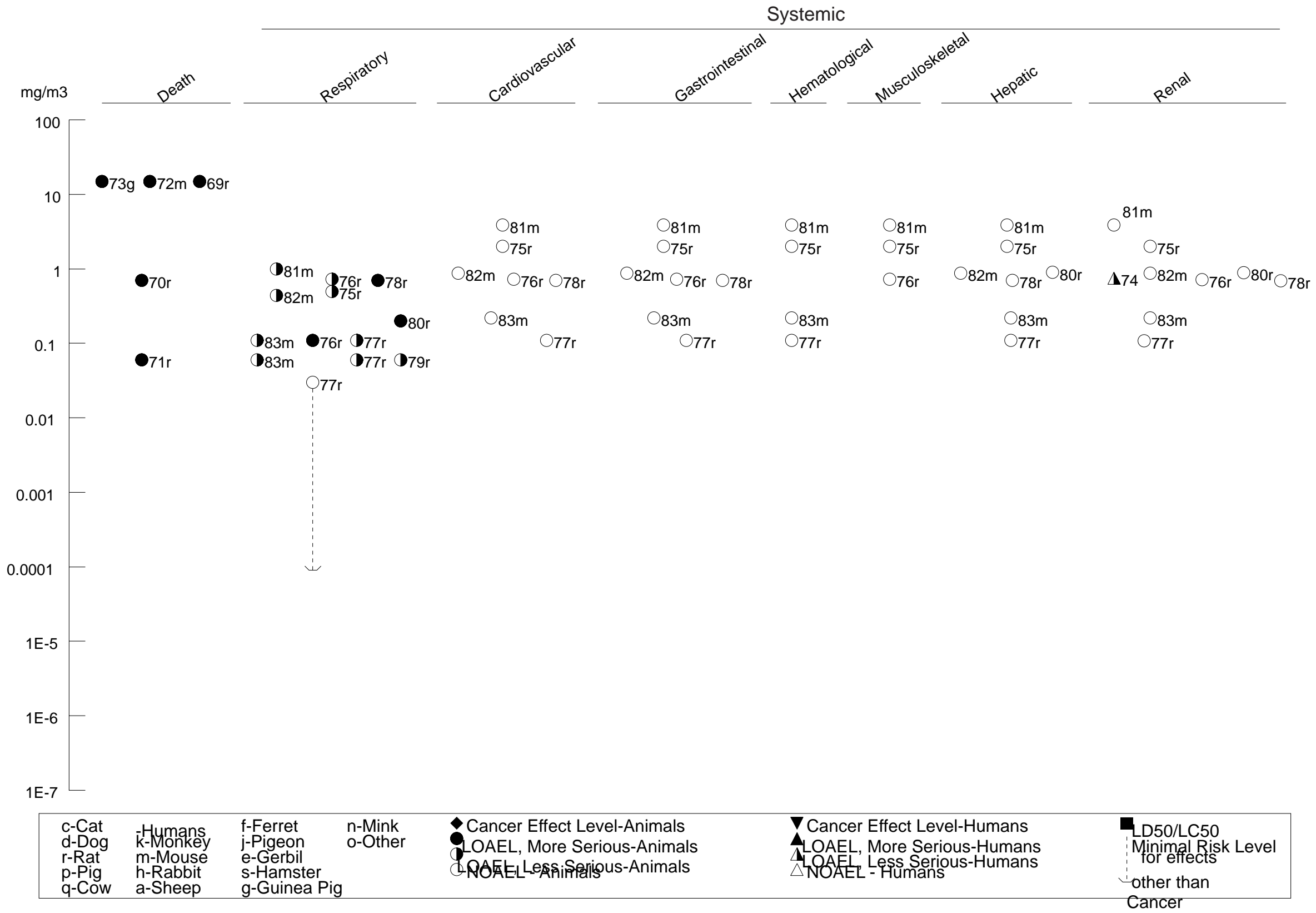


Figure 3-1 Levels of Significant Exposure to Nickel - Inhalation (Continued)

Chronic ( $\geq 365$ days)

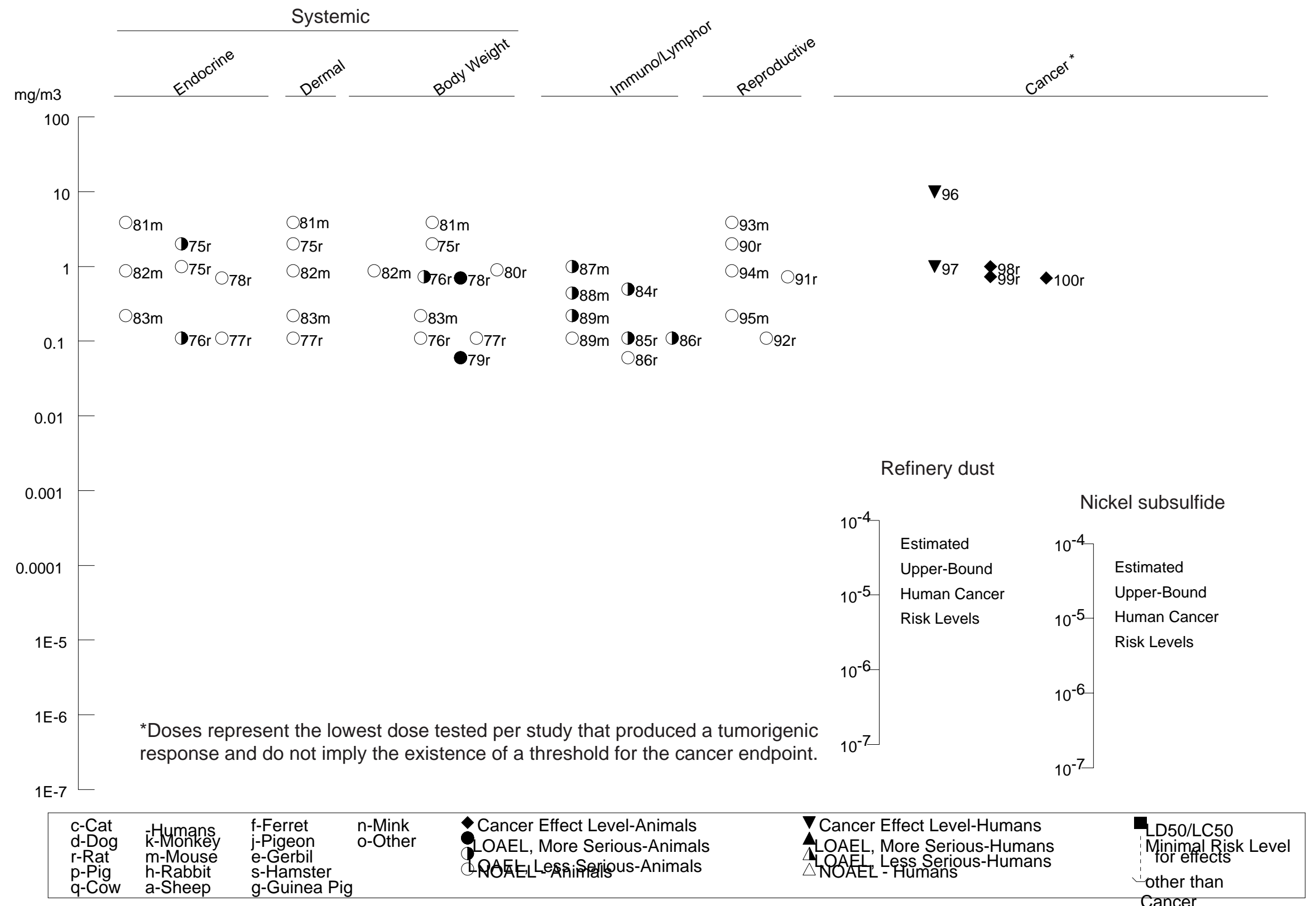


risk of nonmalignant respiratory disease deaths (Moulin et al. 2000; Polednak 1981). A common limitation of the cohort mortality studies is that the number of observed deaths from all causes were lower (in many cases significantly lower) than the number expected deaths, suggesting a healthy worker effect. Additionally, the workers were exposed to other respiratory toxicants; this is particularly true for welders exposed to elevated levels of chromium. A single case of death from adult respiratory distress syndrome has been reported following a 90-minute exposure to a very high concentration $\left(382 \mathrm{mg} / \mathrm{m}^{3}\right)$ of metallic nickel of small particle size $(<1.4 \mu \mathrm{m})$ (Rendell et al. 1994). Histological changes noted in the lungs of this case included alveolar wall damage, with fibrotic changes, and edema in the alveolar space.

A small number of studies have examined potential respiratory tract effects, not associated with lethality. Reduced vital capacity and expiratory flows were observed in stainless steel welders exposed to elevated levels of nickel and chromium (Kilburn et al. 1990). When the welders were divided into two groups based on smoking status, only the forced expiratory volume $\left(\mathrm{FEV}_{75-85}\right)$ was significantly different from the referent population, suggesting that current smoking status may have contributed to the observed effects. The study also found that the prevalence of chronic bronchitis was higher in both the current smoker and non-smoker groups, as compared to the referent population. Although this study provides suggestive evidence of respiratory effects in welders, establishing a causal relationship between nickel and the observed effects is limited by co-exposure to chromium and the lack of a comparison group of non-nickel-exposed welders. Examination of chest radiographs of nickel sinter plant workers exposed to nickel at concentrations as high as $100 \mathrm{mg} / \mathrm{m}^{3}$ did not reveal an increase in small irregular opacities, which would be indicative of inflammatory or fibrogenic response in the lungs (Muir et al. 1993). Another study found an increased risk of moderate pulmonary fibrosis, after controlling for age and smoking, among nickel refinery workers with cumulative exposure to soluble nickel or sulfidic nickel (Berge and Skyberg 2003). A dose-response trend was also found for soluble nickel among cases in the three highest cumulative exposure categories $\left(0.04-\leq 0.15,0.15-\leq 0.6\right.$, and $>0.6 \mathrm{mg} / \mathrm{m}^{3} \mathrm{x}$ years $)$, after adjusting for age, smoking, and exposure to asbestos. Asthma induced by occupational exposure to nickel has been documented in a small number of individuals (Dolovich et al. 1984; Novey et al. 1983; Shirakawa et al. 1990). The asthma can result from either primary irritation or an allergic response. Interpretation of these data is limited by the small number of cases, as well as by possible exposure to other sensitizing metals.

Studies in rats and mice demonstrate that chronic active inflammation in the lungs is the most prominent effect following inhalation exposure to nickel sulfate, nickel subsulfide, or nickel oxide. In acutelyexposed rats, chronic lung inflammation was observed at the lowest nickel sulfate $\left(0.7 \mathrm{mg} \mathrm{Ni} / \mathrm{m}^{3}\right)$ and 
nickel subsulfide $\left(0.44 \mathrm{mg} \mathrm{Ni} / \mathrm{m}^{3}\right)$ concentrations tested in 12-day exposure studies (6 hours/day, 12 days in a 16-day period) (NTP 1996b, 1996c). At higher concentrations of nickel sulfate and nickel subsulfide (1.4 and $3.65 \mathrm{mg} \mathrm{Ni} / \mathrm{m}^{3}$, respectively), the inflammation was accompanied by labored breathing. The chronic active lung inflammation was characterized by focal accumulation of alveolar macrophages and interstitial (nickel subsulfide) or inflammatory cell (nickel sulfate) infiltrates. At the higher concentrations, necrotic cellular debris was also present. Bronchiolar epithelium degeneration was also observed in rats exposed to $0.7 \mathrm{mg} \mathrm{Ni} / \mathrm{m}^{3}$ as nickel sulfate (NTP 1996c). Consistent with these findings, is the observation of alveolitis in rats exposed to $0.22 \mathrm{mg} \mathrm{Ni} / \mathrm{m}^{3}$ as nickel subsulfide 6 hours/day for 7 days (Benson et al. 1995b). Additionally, exposure to $0.95 \mathrm{mg} \mathrm{Ni} / \mathrm{m}^{3}$ as nickel subsulfide resulted in alveolitis and alveolar proteinosis after 4 days of exposure, but not after 1 or 2 days of exposure (Benson et al. 1995b). In contrast, acute lung inflammation, consisting of neutrophilic infiltrates, was first observed in rats exposed to nickel oxide at $7.9 \mathrm{mg} \mathrm{Ni} / \mathrm{m}^{3}$ (NTP 1996a); chronic lung inflammation was not observed at doses as high as $23.6 \mathrm{mg} \mathrm{Ni} / \mathrm{m}^{3}$. Mice appear to be less sensitive than rats to the acute toxicity of nickel with LOAELs for chronic inflammation of $0.7,1.83$, and $>23.6 \mathrm{mg} \mathrm{Ni} / \mathrm{m}^{3}$ as nickel sulfate, nickel subsulfide, and nickel oxide, respectively (NTP 1996a, 1996b, 1996c).

As with acute exposure, chronic lung inflammation was typically observed at the lowest adverse effect level following intermediate-duration exposure. Thirteen-week (6 hours/day, 5 days/week) studies of rats exposed to nickel sulfate, nickel subsulfide, or nickel oxide (NTP 1996a, 1996b, 1996c) identified LOAELs for chronic active lung inflammation of $0.11,0.22$, and $3.9 \mathrm{mg} \mathrm{Ni} / \mathrm{m}^{3}$, respectively; NOAEL values of $0.06,0.11$, and $2 \mathrm{mg} \mathrm{Ni} / \mathrm{m}^{3}$, respectively, were also identified for chronic inflammation. Alveolitis was reported in rats exposed to $0.11 \mathrm{mg} \mathrm{Ni} / \mathrm{m}^{3}$ as nickel sulfate and $1.96 \mathrm{mg} \mathrm{Ni} / \mathrm{m}^{3}$ as nickel oxide for 6 months (6 hours/day, 5 days/week) (Benson et al. 1995a) and interstitial pneumonia was observed at $0.5 \mathrm{mg} \mathrm{Ni} / \mathrm{m}^{3}$ as nickel oxide for 1 month (6 hours/day, 5 days/week) (Horie et al. 1985). A number of other lung effects have also been observed in rats exposed to nickel for intermediate durations. Minimal alveolar macrophage hyperplasia was observed at the lowest nickel sulfate, nickel subsulfide, and nickel oxide concentrations tested (0.03, 0.11, and $0.4 \mathrm{mg} \mathrm{Ni} / \mathrm{m}^{3}$, respectively) (NTP 1996a, 1996b, 1996c). These slight changes in the number of macrophages were not considered adverse because it is considered to be part of the normal physiologic response to inhaled particles and it is not believed to compromise the lung's ability to clear foreign matter. At higher nickel concentrations, mild to moderate changes in alveolar macrophage hyperplasia were found. The effect of nickel on alveolar macrophages is also discussed in Section 3.2.1.3, Immunological and Lymphoreticular Effects. Interstitial infiltrates were observed in rats exposed to $\geq 0.11$ or $0.22 \mathrm{mg} \mathrm{Ni} / \mathrm{m}^{3}$ as nickel sulfate or nickel subsulfide (NTP 1996b, 1996c) or $0.109 \mathrm{mg} \mathrm{Ni} / \mathrm{m}^{3}$ as nickel chloride (Bingham et al. 1972), granulomatous inflammation was 
observed in rats exposed to $3.9 \mathrm{mg} \mathrm{Ni} / \mathrm{m}^{3}$ as nickel oxide (NTP 1996a), alveolar wall thickening was observed in rats exposed to $0.12 \mathrm{mg} \mathrm{Ni} / \mathrm{m}^{3}$ as nickel oxide (Bingham et al. 1972), and hyperplasia of the bronchial epithelium was observed in rats exposed to $0.109 \mathrm{mg} \mathrm{Ni} / \mathrm{m}^{3}$ as nickel chloride (Bingham et al. 1972). The highest NOAEL values for respiratory effects in rats exposed to nickel sulfate, nickel subsulfide, or nickel oxide for intermediate durations were $0.06 \mathrm{mg} \mathrm{Ni} / \mathrm{m}^{3}$ (NTP 1996c), $0.11 \mathrm{mg} \mathrm{Ni} / \mathrm{m}^{3}$ (NTP 1996b), and $0.49 \mathrm{mg} \mathrm{Ni} / \mathrm{m}^{3}$ (Benson et al. 1995a). An intermediate-duration inhalation MRL was derived from the NOAEL $\left(0.06 \mathrm{mg} \mathrm{Ni} / \mathrm{m}^{3}\right)$ and LOAEL $\left(0.11 \mathrm{mg} \mathrm{Ni} / \mathrm{m}^{3}\right)$ identified from the NTP $(1996 \mathrm{c})$ study of nickel sulfate, as described in the footnote to Table 3-1 and Appendix A.

Similar effects have been observed in mice exposed to nickel for intermediate durations, although the LOAELs for the lung effects tend to be higher suggesting a lower sensitivity compared to rats. Chronic active lung inflammation was observed in mice exposed to $\geq 0.44$ and $0.88 \mathrm{mg} \mathrm{Ni} / \mathrm{m}^{3}$ as nickel sulfate or nickel subsulfide, respectively (NTP 1996b, 1996c). Lung inflammation was not found in mice exposed to nickel oxide at concentrations as high as $7.9 \mathrm{mg} \mathrm{Ni} / \mathrm{m}^{3}$ (NTP 1996a); however, perivascular lymphocyte infiltrates were observed at 3.9 and $7.9 \mathrm{mg} \mathrm{Ni} / \mathrm{m}^{3}$ (NTP 1996a). Interstitial pneumonia has also been observed in mice exposed to 0.22 or $0.98 \mathrm{mg} \mathrm{Ni} / \mathrm{m}^{3}$ as nickel sulfate or nickel oxide (Benson et al. 1995a). Other lung effects in mice include minimal alveolar macrophage hyperplasia at $0.11,0.22$, or $0.4 \mathrm{mg} \mathrm{Ni} / \mathrm{m}^{3}$ as nickel sulfate, nickel subsulfide, or nickel oxide, respectively (NTP 1996a, 1996b, 1996c); interstitial infiltrates at $\geq 0.44$ or $0.44 \mathrm{mg} \mathrm{Ni} / \mathrm{m}^{3}$ as nickel subsulfide or nickel sulfate, respectively (NTP 1996b, 1996c), and fibrosis at 0.44 and $0.88 \mathrm{mg} \mathrm{Ni} / \mathrm{m}^{3}$ as nickel sulfate or nickel subsulfide, respectively (NTP 1996b, 1996c). As with the rats, minimal alveolar macrophage hyperplasia was not considered adverse. The highest NOAEL values for respiratory effects in mice exposed to nickel sulfate, nickel subsulfide, and nickel oxide for intermediate durations were $0.22,0.22$, and $2.0 \mathrm{mg} \mathrm{Ni} / \mathrm{m}^{3}$, respectively (NTP 1996a, 1996b, 1996c).

Chronic exposure to nickel (6 hours/day, 5 days/week for 2 years) resulted in chronic active lung inflammation (or pneumonia) in rats and mice at $0.06 \mathrm{mg} \mathrm{Ni} / \mathrm{m}^{3}$ as nickel sulfate, in rats at $0.11 \mathrm{mg} \mathrm{Ni} / \mathrm{m}^{3}$ and higher as nickel subsulfide (NTP 1996b; Ottolenghi et al. 1990), in mice at $0.44 \mathrm{mg} \mathrm{Ni} / \mathrm{m}^{3}$ and higher as nickel subsulfide (NTP 1996b), in rats at $0.2 \mathrm{mg} \mathrm{Ni} / \mathrm{m}^{3}$ and higher as nickel oxide (NTP 1996a; Tanaka et al. 1988), and in mice at $1 \mathrm{mg} \mathrm{Ni} / \mathrm{m}^{3}$ as nickel oxide (NTP 1996a). Additional lung effects that were found at the same dose levels as inflammation included alveolar epithelium hyperplasia (or bronchialization), fibrosis in rats and mice exposed to nickel subsulfide (NTP 1996b), and bronchialization and/or alveolar proteinosis in mice exposed to nickel oxide (NTP 1996a; Takenaka et al. 1985). With the exception of the NTP (1996c) study of nickel sulfate in rats, NOAEL values for 
respiratory effects following chronic duration exposure were not identified. The NOAEL of $0.03 \mathrm{mg}$ $\mathrm{Ni} / \mathrm{m}^{3}$ and LOAEL of $0.06 \mathrm{mg} \mathrm{Ni} / \mathrm{m}^{3}$ identified in rats exposed to nickel sulfate (NTP 1996c) were used to derive a chronic-duration inhalation MRL for nickel, as described in the footnote to Table 3-1 and Appendix A.

The NTP (1996a, 1996b, 1996c) studies allow for the comparison of the toxicity of nickel sulfate, nickel subsulfide, and nickel oxide in rats and mice. Following acute- or intermediate-duration exposure, the toxicity of the different nickel compounds is related to its solubility, with soluble nickel sulfate being the most toxic and insoluble nickel oxide being the least toxic. The difference in the toxicity across compounds is probably due to the ability of water-soluble nickel compounds to cross the cell membrane and interact with cytoplasmic proteins. In contrast, the severity of inflammatory and proliferative lesions following chronic exposure was greater in rats exposed to nickel subsulfide or nickel oxide, as compared to nickel sulfate. Additionally, parenchymal damage secondary to inflammation was evident in the rats exposed to nickel subsulfide and nickel oxide, but not nickel sulfate. For all durations and nickel compounds tested, rats appear to be more sensitive to the lung effects than mice; significant increases in the incidence of chronic lung inflammation were observed at lower concentrations in the rats than mice. Intermediate-duration studies (Benson et al. 1995a; Horie et al. 1985) that monitored animals for months after exposure termination suggest that nickel-induced lung damage is not readily reversible after exposure termination. In the Benson et al. (1995a) studies, alveolitis was observed in rats exposed to $0.11 \mathrm{mg} \mathrm{Ni} / \mathrm{m}^{3}$ as nickel sulfate and $1.96 \mathrm{mg} \mathrm{Ni} / \mathrm{m}^{3}$ as nickel oxide at the end of the 6-month exposure period and 4 months after exposure termination. Horie et al. (1985) reported interstitial pneumonia in rats exposed 6 hours/day, 5 days/week to $0.5 \mathrm{mg} \mathrm{Ni} / \mathrm{m}^{3}$ as nickel oxide for $1 \mathrm{month}$. Twelve and 20 months after termination of exposure to $6.3 \mathrm{mg} \mathrm{Ni} / \mathrm{m}^{3}$, squamous metaplasia of the bronchial epithelium, hyperplasia of the bronchial gland, and chronic bronchitis were observed.

In addition to the lung effects, several studies have demonstrated that exposure to nickel sulfate or nickel subsulfide can induce atrophy of the nasal olfactory epithelium (Evans et al. 1995; NTP 1996b, 1996c). The nasal lesions are typically observed at higher concentrations than the lung effects. In a study designed specifically to examine the effects of nickel on the olfactory system, rats were exposed to nickel sulfate at 0 or $0.635 \mathrm{mg} \mathrm{Ni} / \mathrm{m}^{3} 6$ hours/day for 16 days (Evans et al. 1995). Histological changes in the olfactory epithelium of exposed rats included a slight reduction in the number of bipolar sensory receptor cells, a decrease in the thickness of the olfactory epithelium resulting from a loss of sustentacular cells, a thinning of apical cytoplasm, and a reduction in the number of sensory cilia on the surface of the cells. 
After a recovery period of 22 days, fewer sensory cilia was the only change that remained, indicating that the effects of an intermediate-duration exposure to nickel were reversible.

Cardiovascular Effects. No increases in the number of deaths from cardiovascular diseases were reported in workers exposed to nickel (Cornell and Landis 1984; Cox et al. 1981; Cragle et al. 1984).

Microscopic examinations of the hearts of rats and mice exposed to nickel sulfate, nickel subsulfide, or nickel oxide for 12 6-hour exposures over 16 days did not reveal any changes at concentrations as high as $12.2,7.33$, or $23.6 \mathrm{mg} \mathrm{Ni} / \mathrm{m}^{3}$, respectively, in rats and $1.4,7.33$, or $23.6 \mathrm{mg} \mathrm{Ni} / \mathrm{m}^{3}$, respectively, in mice (NTP 1996a, 1996b, 1996c). No cardiovascular effects were observed in rats or mice exposed to 0.44 , 1.83 , or $7.9 \mathrm{mg} \mathrm{Ni} / \mathrm{m}^{3}$ as nickel sulfate, nickel subsulfide, or nickel oxide, respectively, 6 hours/day, 5 days/week for 13 weeks (NTP 1996a, 1996b, 1996c). Similarly, chronic exposure (6 hours/day, 5 days/week) of rats to nickel sulfate, nickel subsulfide, or nickel oxide at concentrations up to $0.11,0.73$, or $2 \mathrm{mg} \mathrm{Ni} / \mathrm{m}^{3}$, respectively, or exposure of mice to, $0.22,0.88$, or $3.9 \mathrm{mg} \mathrm{Ni} / \mathrm{m}^{3}$, respectively, did not result in microscopic changes in the heart (NTP 1996a, 1996b, 1996c). Intermittent exposure ( 6 hours/day, 5 days/week) of rats to $0.7 \mathrm{mg} \mathrm{Ni} / \mathrm{m}^{3}$ as nickel subsulfide for 78 weeks also did not affect the microscopic appearance of the heart (Ottolenghi et al. 1974).

Gastrointestinal Effects. No studies were located regarding gastrointestinal effects in humans after inhalation exposure to nickel.

Microscopic examinations of the gastrointestinal tract of mice and rats exposed to nickel sulfate, nickel subsulfide, or nickel oxide for 12 6-hour exposures did not reveal any changes at concentrations as high as $12.2,7.33$, or $23.6 \mathrm{mg} \mathrm{Ni} / \mathrm{m}^{3}$, respectively, in rats and $1.4,7.33$, or $23.6 \mathrm{mg} \mathrm{Ni} / \mathrm{m}^{3}$, respectively, in mice (NTP 1996a, 1996b, 1996c). Likewise, no histological alterations were observed in the gastrointestinal tracts of rats and mice exposed to $0.44,1.83$, or $7.9 \mathrm{mg} \mathrm{Ni} / \mathrm{m}^{3}$ as nickel sulfate, nickel subsulfide, or nickel oxide, respectively, 6 hours/day, 5 days/week for 13 weeks (NTP 1996a, 1996b, 1996c). Chronic exposure of rats to nickel sulfate, nickel subsulfide, or nickel oxide at concentrations up to $0.11,0.73$, or $2 \mathrm{mg} \mathrm{Ni} / \mathrm{m}^{3}$, respectively, or exposure of mice to $0.22,0.88$, or $3.9 \mathrm{mg} \mathrm{Ni} / \mathrm{m}^{3}$ as nickel sulfate, nickel subsulfide, or nickel oxide, respectively, did not result in microscopic changes in the gastrointestinal tract (NTP 1996a, 1996b, 1996c). Intermittent exposure (6 hours/day, 5 days/week) of rats to $0.7 \mathrm{mg} \mathrm{Ni} / \mathrm{m}^{3}$ as nickel subsulfide for 78 weeks also did not affect the microscopic appearance of the intestines (Ottolenghi et al. 1974). 
Hematological Effects. No studies were located regarding hematological effects in humans after inhalation exposure to nickel.

A number of hematological alterations were observed in studies by Weischer et al. (1980) and NTP (1996a, 1996b, 1996c). A decrease in hematocrit level was observed in male rats continuously exposed to 0.178 or $0.385 \mathrm{mg} \mathrm{Ni} / \mathrm{m}^{3}$ as nickel oxide for 28 days (Weischer et al. 1980); no significant alterations were observed at $0.785 \mathrm{mg} \mathrm{Ni} / \mathrm{m}^{3}$. The biological significance of a decrease in hematocrit level in the absence of hemoglobin or erythrocyte alterations is not known. In non-pregnant females continuously exposed to nickel oxide for 21 days, increases in hematocrit and hemoglobin levels were observed at $0.8 \mathrm{mg} \mathrm{Ni} / \mathrm{m}^{3}$ and higher; an increase in mean cell volume and a decrease in erythrocyte levels were observed at $1.6 \mathrm{mg} \mathrm{Ni} / \mathrm{m}^{3}$ and higher (Weischer et al. 1980). Similarly, increases in hematocrit, hemoglobin, and erythrocyte levels were observed in rats exposed to nickel subsulfide at $0.73 \mathrm{mg} \mathrm{Ni} / \mathrm{m}^{3}$ 6 hours/day, 5 days/week for 2 years (NTP 1996b). As noted by NTP (1996b), increases in hematocrit, hemoglobin, and erythrocytes are consistent with erythropoietin production in response to tissue hypoxia, possibly as a result of the nickel-induced lung damage. Chronic exposure of rats to nickel oxide or nickel sulfate at concentrations up to 2 or $0.11 \mathrm{mg} \mathrm{Ni} / \mathrm{m}^{3}$, respectively, and chronic exposure of mice to nickel oxide, nickel subsulfide, or nickel sulfate at concentrations up to $3.9,0.88$, or $0.22 \mathrm{mg} \mathrm{Ni} / \mathrm{m}^{3}$, respectively, did not result in significant hematological effects (NTP 1996a, 1996b, 1996c).

Musculoskeletal Effects. No studies were located regarding musculoskeletal effects in humans after inhalation exposure to nickel.

No histological alterations were observed in bone of rats and mice exposed to nickel sulfate 6 hours/day for 12 days/16 days (highest NOAEL is $\left.12.2 \mathrm{mg} \mathrm{Ni} / \mathrm{m}^{3}\right), 5$ days/week for 13 weeks $\left(0.44 \mathrm{mg} \mathrm{Ni} / \mathrm{m}^{3}\right)$, or 5 days/week for 2 years ( 0.11 and $0.22 \mathrm{mg} \mathrm{Ni} / \mathrm{m}^{3}$ for rats and mice) (NTP 1996c); the muscles were not examined histologically in these studies. No alterations were observed in bone or muscle of rats and mice exposed to nickel oxide ( 6 hours/day, 5 days/week) at $23.6 \mathrm{mg} \mathrm{Ni} / \mathrm{m}^{3}$ for 16 days (12 days $/ 16$ days), $7.9 \mathrm{mg} \mathrm{Ni} / \mathrm{m}^{3}$ for 13 weeks, or 2 (rats) or $3.9 \mathrm{mg} \mathrm{Ni} / \mathrm{m}^{3}$ (mice) for 2 years (NTP 1996a). Similarly, exposure to nickel subsulfide 6 hours/day, 5 days/week did not result in alterations in bone or muscle in rats at $7.33 \mathrm{mg} \mathrm{Ni} / \mathrm{m}^{3}$ for 13 weeks or $0.73 \mathrm{mg} \mathrm{Ni} / \mathrm{m}^{3}$ for 2 years or mice at $7.33 \mathrm{mg} \mathrm{Ni} / \mathrm{m}^{3}$ for 16 days, $1.83 \mathrm{mg} \mathrm{Ni} / \mathrm{m}^{3}$ for 13 weeks, or $0.88 \mathrm{mg} \mathrm{Ni} / \mathrm{m}^{3}$ (mice) for 2 years (NTP 1996b).

Hepatic Effects. No studies were located regarding hepatic effects in humans after inhalation exposure to nickel. 
No histological alterations were observed in the livers of rats or mice exposed to nickel subsulfide, nickel sulfate, or nickel oxide at concentrations of $7.33,12.2$, or $23.6 \mathrm{mg} \mathrm{Ni} / \mathrm{m}^{3}$, respectively, in rats and 1.4 , 12.2 , or $23.6 \mathrm{mg} \mathrm{Ni} / \mathrm{m}^{3}$, respectively, in mice exposed 6 hours/day, 12 days in a 16-day period (NTP 1996a, 1996b, 1996c) or 1.83, 0.44, or $7.9 \mathrm{mg} \mathrm{Ni} / \mathrm{m}^{3} 6$ hours/day, 5 days/week, for 13 weeks (NTP 1996a, 1996b, 1996c). Following chronic exposure, no histological changes were observed in the livers of rats exposed to nickel subsulfide at $0.7 \mathrm{mg} \mathrm{Ni} / \mathrm{m}^{3}$ (Ottolenghi et al. 1974) or $0.73 \mathrm{mg} \mathrm{Ni} / \mathrm{m}^{3}$ (NTP 1996b), to nickel oxide at $0.9 \mathrm{mg} \mathrm{Ni} / \mathrm{m}^{3}$ (Tanaka et al. 1988) or $2 \mathrm{mg} \mathrm{Ni} / \mathrm{m}^{3}$ (NTP 1996a), or to nickel sulfate at $0.11 \mathrm{mg} \mathrm{Ni} / \mathrm{m}^{3}$ (NTP 1996c). Chronic exposure of mice to nickel oxide, nickel subsulfide, or nickel sulfate at concentrations up to $3.9,0.88$, or $0.22 \mathrm{mg} \mathrm{Ni} / \mathrm{m}^{3}$, respectively, did not result in microscopic changes in the liver (NTP 1996a, 1996b, 1996c).

Renal Effects. Marked tubular necrosis was observed in the kidneys of a man who died of adult respiratory distress syndrome 13 days after a 90-minute exposure to a very high concentration $\left(382 \mathrm{mg} / \mathrm{m}^{3}\right)$ of metallic nickel of small particle size $(<1.4 \mu \mathrm{m})$ (Rendall et al. 1994). Several days after the exposure, urinary concentrations of nickel were $700 \mu \mathrm{g} / \mathrm{L}$, in comparison to levels of $<0.1-13.3 \mu \mathrm{g} / \mathrm{L}$ in persons not occupationally exposed to nickel (Sunderman 1993).

In nickel refinery workers, a significant association was found between increased levels of nickel in urine and increased urinary $\beta_{2}$-microglobulin levels (Sunderman and Horak 1981). A significant increase in urinary $\beta_{2}$-microglobulin levels was observed in a group of workers with urinary nickel levels exceeding $100 \mu \mathrm{g} / \mathrm{L}$; urinary $\beta_{2}$-microglobulin levels were not significantly altered in workers with urine nickel levels of less than $100 \mu \mathrm{g} / \mathrm{L}$. Urinary levels of total proteins, $\beta_{2}$-microglobulin, retinol binding protein, and $N$-acetyl- $\beta$-D-glucosaminidase (NAG) were increased in 12 women, and urinary lysozyme and NAG were increased in 14 men occupationally exposed to soluble nickel (sulfate, chloride) compounds at an average concentration of $0.75 \mathrm{mg} \mathrm{Ni} / \mathrm{m}^{3}$ (Vyskocil et al. 1994a). Although the average exposure concentration was the same for women and men, women were more highly exposed as indicated by urine concentrations of $10.3 \mu \mathrm{g} \mathrm{Ni} / \mathrm{g}$ creatinine in women compared to $5 \mu \mathrm{g} \mathrm{Ni} / \mathrm{g}$ creatinine in men. The markers that were changed reflected tubular dysfunction. No effects on markers of glomerular function, urinary albumin levels, or transferrin levels were noted. Sanford and Nieboer (1992) did not find significant alterations in urinary $\beta_{2}$-microglobulin levels in nickel refinery workers with urine nickel levels of less than $60 \mu \mathrm{g} / \mathrm{L}$. Sanford and Nieboer (1992) noted that elevated urinary $\beta_{2}$-microglobulin levels were found in spot urine samples of three workers; however, when the levels were averaged over 
three or more voids, the average levels were within the normal range. A study of 17 electroforming workers did not find evidence of proteinuria (Wall and Calnan 1980).

No histological alterations were observed in the kidneys of rats or mice exposed to nickel sulfate, nickel subsulfide, or nickel oxide 6 hours/day, 5 days/week, at concentrations of $\leq 12.2,7.33$, or $23.6 \mathrm{mg} \mathrm{Ni} / \mathrm{m}^{3}$, respectively, for 16 days (12 days in a 16-day period) (NTP 1996a, 1996b, 1996c), or $\leq 0.44,1.83$, or $7.9 \mathrm{mg} \mathrm{Ni} / \mathrm{m}^{3}$, respectively, for 13 weeks (NTP 1996a, 1996b, 1996c), or $0.9 \mathrm{mg} \mathrm{Ni} / \mathrm{m}^{3}$ as nickel oxide for 12 months (Tanaka et al. 1988). Chronic exposure of rats to nickel oxide (NTP 1996a; Tanaka et al. 1988), nickel subsulfide (NTP 1996b), or nickel sulfate (NTP 1996c) at concentrations up to 2, 0.73, or $0.11 \mathrm{mg} \mathrm{Ni} / \mathrm{m}^{3}$, respectively, did not result in histological alterations in the kidneys. Additionally, no alterations were observed in mice exposed to nickel oxide, nickel subsulfide, or nickel sulfate at concentrations up to $3.9,0.88$, or $0.22 \mathrm{mg} \mathrm{Ni} / \mathrm{m}^{3}$, respectively (NTP 1996a, 1996b, 1996c).

Endocrine Effects. No studies were located regarding endocrine effects in humans following inhalation exposure to nickel.

Histological examinations did not reveal any changes in the adrenal glands, pancreas, parathyroid, pituitary, or thyroid glands in rats or mice exposed to nickel as nickel sulfate, nickel oxide, or nickel subsulfide for 12 6-hour exposures over 16 days or for 6 hours/day, 5 days/week for 13 weeks (NTP 1996a, 1996b, 1996c). The NOAEL values for endocrine effects were 12.2, 23.6, and $7.33 \mathrm{mg} \mathrm{Ni} / \mathrm{m}^{3}$ in rats and mice exposed to nickel sulfate, nickel oxide, and nickel subsulfide, respectively, for the shorter duration study and $0.44,7.9$, and $1.83 \mathrm{mg} \mathrm{Ni} / \mathrm{m}^{3}$, respectively, for the 13-week study. In rats exposed intermittently to nickel subsulfide at $0.7 \mathrm{mg} \mathrm{Ni} / \mathrm{m}^{3}$ for 78 weeks, no histological changes were observed in the thyroid or adrenal glands (Ottolenghi et al. 1974). Adrenal medulla hyperplasia and increased incidences of benign pheochromocytoma were observed in female rats exposed to $2 \mathrm{mg} \mathrm{Ni} / \mathrm{m}^{3}$ as nickel oxide (NTP 1996a) and male and female rats exposed to $0.73 \mathrm{mg} \mathrm{Ni} / \mathrm{m}^{3}$ as nickel subsulfide for 2 years (NTP 1996b); an increased incidence of benign pheochromocytoma was also observed in male rats exposed to $0.11 \mathrm{mg} \mathrm{Ni} / \mathrm{m}^{3}$ as nickel subsulfide. These effects were not observed in rats exposed chronically to nickel sulfate at concentrations up to $0.11 \mathrm{mg} \mathrm{Ni} / \mathrm{m}^{3}$, or in mice exposed to nickel oxide, nickel subsulfide, or nickel sulfate at concentrations of $3.9,0.88$, or $0.22 \mathrm{mg} \mathrm{Ni} / \mathrm{m}^{3}$, respectively (NTP 1996a, 1996b, 1996c).

Dermal Effects. No studies were located regarding dermal effects in humans following inhalation exposure. However, contact dermatitis in persons exposed to nickel compounds is one of the most 
common effects of nickel exposure (see Section 3.2.3.2). In addition, immunological studies indicate that the dermatitis is an allergic response to nickel, and significant effects on the immune system have been noted in workers exposed to nickel (see Section 3.2.1.3).

Microscopic changes in the skin were not observed in rats or mice exposed to nickel as nickel sulfate, nickel subsulfide, or nickel oxide at concentrations up to $12.2,7.33$, or $23.6 \mathrm{mg} \mathrm{Ni} / \mathrm{m}^{3}$, respectively, for 6 hours/day for 12 days in a 16-day period (NTP 1996a, 1996b, 1996c) or $0.44,1.83$, or $7.9 \mathrm{mg} \mathrm{Ni} / \mathrm{m}^{3}$ 6 hours/day, 5 days/week for 13 weeks (NTP 1996a, 1996b, 1996c). Chronic exposure of rats to nickel sulfate, nickel subsulfide, or nickel oxide at concentrations up to $0.11,0.73$, or $2 \mathrm{mg} \mathrm{Ni} / \mathrm{m}^{3}$, respectively, or exposure of mice at concentrations up to $0.22,0.88$, or $3.9 \mathrm{mg} \mathrm{Ni} / \mathrm{m}^{3}$, respectively, did not result in microscopic changes in the skin (NTP 1996a, 1996b, 1996c).

Body Weight Effects. No studies were located regarding body weight effects in humans after inhalation exposure to nickel. Significant decreases in body weight gain have been observed in rats and mice exposed to nickel sulfate, nickel subsulfide, and nickel oxide for acute, intermediate, and chronic exposure durations. In many of the studies, the decreases in body weight gain were associated with lung inflammation, impaired lung function (as evidenced by labored breathing), and lethality. Exposure to nickel sulfate resulted in serious decreases in body weight gain (terminal body weights $>25 \%$ lower than controls) in rats exposed to $0.7 \mathrm{mg} \mathrm{Ni} / \mathrm{m}^{3}$ and higher and in mice exposed to $1.4 \mathrm{mg} \mathrm{Ni} / \mathrm{m}^{3} 6$ hours/day for 12 days in a 16-day period (NTP 1996c); no significant alterations in body weight gain were observed in mice exposed to $0.7 \mathrm{mg} \mathrm{Ni} / \mathrm{m}^{3}$. No significant alterations in body weight gain were observed in rats or mice exposed to $0.44 \mathrm{mg} \mathrm{Ni} / \mathrm{m}^{3}$ for 13 weeks (NTP 1996c), rats exposed to $0.11 \mathrm{mg} \mathrm{Ni} / \mathrm{m}^{3}$ for 2 years (NTP 1996c), or mice exposed to $0.22 \mathrm{mg} \mathrm{Ni} / \mathrm{m}^{3}$ for 2 years (NTP 1996c).

For nickel subsulfide, serious decreases in body weight gain (22-28\%) and emaciation were observed in rats and mice, respectively, exposed to $3.65 \mathrm{mg} \mathrm{Ni} / \mathrm{m}^{3}$ for 6 hours/day for 12 days in a 16-day period (NTP 1996b); a NOAEL of $1.85 \mathrm{mg} \mathrm{Ni} / \mathrm{m}^{3}$ was also identified. No alterations in body weight were observed at $1.83 \mathrm{mg} \mathrm{Ni} / \mathrm{m}^{3} 6$ hours/day, 5 days/week for 13 weeks. Exposure to approximately $0.7 \mathrm{mg}$ $\mathrm{Ni} / \mathrm{m}^{3}$ for 6 hours/day, 5 days/week for a chronic-duration resulted in 11-30\% decreases in body weight gains in rats (NTP 1996b; Ottolenghi et al. 1974). No alterations were observed in mice exposed to $0.88 \mathrm{mg} \mathrm{Ni} / \mathrm{m}^{3}$ for 6 hours/day, 5 days/week for 2 years (NTP 1996b).

Most studies did not find significant alterations in rats and mice exposed to nickel oxide. A NOAEL of $23.6 \mathrm{mg} \mathrm{Ni} / \mathrm{m}^{3}$ was identified in rats and mice exposed to $23.6 \mathrm{mg} \mathrm{Ni} / \mathrm{m}^{3} 6$ hours/day for 12 days in a 
16-day period (NTP 1996a). For intermediate exposure, NOAELs of 1.9-7.9 mg Ni/m were identified in rats and mice (Benson et al. 1995a; NTP 1996a). However, Weischer et al. (1980) reported 30-36\% decreases in body weight gain in male and female rats exposed to 0.385 or $0.8 \mathrm{mg} \mathrm{Ni} / \mathrm{m}^{3}$, respectively, continuously for 21-28 days. In pregnant rats, an 11\% decrease in body weight gain was observed at $0.8 \mathrm{mg} \mathrm{Ni} / \mathrm{m}^{3}$ compared to the $36 \%$ decrease observed in similarly exposed non-pregnant rats. NTP (1996a) did not find significant alterations in body weight gain in rats and mice exposed to 2 or $3.9 \mathrm{mg}$ $\mathrm{Ni} / \mathrm{m}^{3}$, respectively, 6 hours/day, 5 days/week for 2 years; a NOAEL of $0.9 \mathrm{mg} \mathrm{Ni} / \mathrm{m}^{3}$ was also identified in rats exposed 7 hours/day, 5 days/week for 12 months (Tanaka et al. 1988). In contrast, Takenaka et al. (1985) reported weight loss in rats continuously exposed to $0.06 \mathrm{mg} \mathrm{Ni} / \mathrm{m}^{3}$ for 31 months; the weight loss began after 13 months of exposure. These data suggest that continuous exposure is more toxic than intermittent exposure (duration adjusted NOAEL from the rat NTP study is $0.36 \mathrm{mg} \mathrm{Ni} / \mathrm{m}^{3}$ ). Continuous exposure would result in higher lung burdens than intermittent exposure, which would lead to increased lung damage.

Metabolic Effects. No studies were located regarding metabolic effects in humans after inhalation exposure to nickel.

Significant increases in serum glucose levels were observed in male rats continuously exposed to 0.385 or $0.784 \mathrm{mg} \mathrm{Ni} / \mathrm{m}^{3}$ as nickel oxide for 28 days (Weischer et al. 1980). In females rats continuously exposed to nickel oxide, decreases in serum glucose levels were observed at 0.8 and $1.6 \mathrm{mg} \mathrm{Ni} / \mathrm{m}^{3}$; at $3.2 \mathrm{mg}$ $\mathrm{Ni} / \mathrm{m}^{3}$, serum glucose levels did not significantly differ from controls (Weischer et al. 1980). These data suggest that there may be a gender difference. Although no adverse pancreatic effects have been noted in inhalation studies, a single-dose intravenous injection study has reported increases in serum glucose levels and effects on pancreatic cells in rabbits at doses of 4.5-9 mg Ni/kg as nickel chloride (Kadota and Kurita 1955); Weischer et al. (1980) also found increases in serum glucose levels in male rats exposed to nickel chloride in water for 28 days. It is possible that changes in serum glucose levels reflect an effect on the pancreas or may be secondary to the marked decrease in body weight gain also observed in the Weischer et al. (1980) study.

\subsubsection{Immunological and Lymphoreticular Effects}

A number of immunological and lymphoreticular effects have been reported in humans and animals exposed to nickel. In 38 production workers exposed to nickel (compound not specified), significant increases in levels of immunoglobulin $\mathrm{G}$ (IgG), $\operatorname{IgA}$, and $\operatorname{IgM}$ and a significant decrease in IgE levels 
were observed (Bencko et al. 1983, 1986). Significant increases in other serum proteins, which may be involved in cell-mediated immunity (including $\alpha_{1}$-antitrypsin, $\alpha_{2}$-macroglobulin, ceruloplasmin), were also observed. The increase in immunoglobulins and serum proteins suggests that the immune system was stimulated by nickel exposure. Similar but less-pronounced effects were observed in workers exposed to cobalt. A relationship between nickel and cobalt sensitization is further supported by the finding that nickel-reactive IgE antibodies were observed in eight patients with hard-metal asthma induced by cobalt exposure (Shirakawa et al. 1990). Exposure levels were not reported.

Alterations in innate (or non-specific) and acquired immunity have been observed in animals. Several studies examined alveolar macrophage functions. A significant reduction in macrophage phagocytic activity was observed in rats exposed to an unspecified concentration of nickel chloride for 2 hours (Adkins et al. 1979) or in mice exposed to $0.47 \mathrm{mg} \mathrm{Ni} / \mathrm{m}^{3}$ as nickel oxide or $0.45 \mathrm{mg} \mathrm{Ni} / \mathrm{m}^{3}$ as nickel subsulfide 6 hours/day, 5 days/week for 65 days (Haley et al. 1990). No alteration of macrophage phagocytic activity was observed in mice exposed to $\leq 0.45 \mathrm{mg} \mathrm{Ni} / \mathrm{m}^{3}$ as nickel sulfate 6 hours/day, 5 days/week for 65 days (Haley et al. 1990). Other alveolar macrophage alterations include decreased lysozyme activity in rabbits exposed to $0.6 \mathrm{mg} \mathrm{Ni} / \mathrm{m}^{3}$ as nickel chloride 6 hours/day, 5 days/week for 46 weeks (Bingham et al. 1987; Johansson et al. 1987, 1988a, 1989), alterations in macrophage production of tumor necrosis factor (Goutet et al. 2000; Morimoto et al. 1995), and morphological alterations. Morimoto et al. (1995) found increased production of tumor necrosis factor in rats exposed to $9.2 \mathrm{mg}$ $\mathrm{Ni} / \mathrm{m}^{3}$ as nickel oxide 8 hours/day, 5 days/week for 4 weeks. In contrast, Goutet et al. (2000) found a decrease in tumor necrosis factor production in rats following a single intratracheal instillation of nickel sulfate. The conflicting results may be due to exposure route, duration, or concentration differences between the studies. Alveolar macrophages from rabbits exposed to $1 \mathrm{mg} \mathrm{Ni} / \mathrm{m}^{3}$ as metallic nickel 6 hours/day, 5 days/week for 3-6 months (Johansson et al. 1980) or $0.6 \mathrm{mg} \mathrm{Ni} / \mathrm{m}^{3}$ as nickel chloride 6 hours/days, 5 days/week for 4-6 weeks (Johansson et al. 1987) or 4 months (Johansson et al. 1988a, 1989) had increases in membrane-bound lamellar bodies. Exposure to metallic nickel also resulted in macrophages with smooth surfaces; the frequency of occurrence was duration-related (Johansson et al. 1980).

Several studies have examined the relationship between nickel exposures and acquired immune function. An increase in susceptibility to Streptococci infection was observed in mice exposed to $0.499 \mathrm{mg} \mathrm{Ni} / \mathrm{m}^{3}$ as nickel chloride or $0.455 \mathrm{mg} \mathrm{Ni} / \mathrm{m}^{3}$ as nickel sulfate for 2 hours (Adkins et al. 1979); mice exposed to $0.657 \mathrm{mg} \mathrm{Ni} / \mathrm{m}^{3}$ as nickel chloride also developed septicemia from the Streptococci infection and had a reduced ability to clear the inhaled bacteria (Adkins et al. 1979). Other studies have found an impaired 
response to sheep red blood cells (decrease in the number of antibody production spleen cells) in mice exposed to $0.25 \mathrm{mg} \mathrm{Ni} / \mathrm{m}^{3}$ as nickel chloride for 2 hours (Graham et al. 1978) or rats continuously exposed to $0.2 \mathrm{mg} \mathrm{Ni} / \mathrm{m}^{3}$ as nickel oxide for 4 weeks or $0.15 \mathrm{mg} \mathrm{Ni} / \mathrm{m}^{3}$ for 4 months (Spiegelberg et al. 1984). A decreased resistance to a tumor challenge was also observed in mice exposed to $0.45 \mathrm{mg} \mathrm{Ni} / \mathrm{m}^{3}$ as nickel sulfate 6 hours/day, 5 days/week for 65 days (Haley et al. 1990).

A significant portion of nickel that is removed from the lung enters the lymphatic system, often inducing damage to the lymph nodes. Lymphoid hyperplasia in the bronchial and mediastinal lymph nodes was observed in rats exposed to $1.4 \mathrm{mg} \mathrm{Ni} / \mathrm{m}^{3}$ as nickel sulfate (NTP 1996c) or mice exposed to $0.88 \mathrm{mg}$ $\mathrm{Ni} / \mathrm{m}^{3}$ as nickel subsulfide (NTP 1996b) 6 hours/day for 12 days in a 16-day period; no effects were observed in rats exposed to $7.33 \mathrm{mg} \mathrm{Ni} / \mathrm{m}^{3}$ as nickel subsulfide (NTP 1996b), rats and mice exposed to $23.5 \mathrm{mg} \mathrm{Ni} / \mathrm{m}^{3}$ as nickel oxide (NTP 1996a), and mice exposed to $3.1 \mathrm{mg} \mathrm{Ni} / \mathrm{m}^{2}$ as nickel sulfate (NTP 1996c). In intermediate-duration studies, a 6 hour/day, 5 day/week exposure resulted in lymphoid hyperplasia in bronchial lymph nodes of rats exposed to $0.22,0.22$, or $2 \mathrm{mg} \mathrm{Ni} / \mathrm{m}^{3}$ as nickel sulfate, nickel subsulfide, or nickel oxide, respectively, and in mice exposed to $0.44,0.88$, or $2 \mathrm{mg} \mathrm{Ni} / \mathrm{m}^{3}$ as nickel sulfate, nickel subsulfide, or nickel oxide, respectively (NTP 1996a, 1996b, 1996c). Similarly, lymphoid hyperplasia was observed in the bronchial lymph nodes of rats exposed to $0.11,0.11$, or $0.5 \mathrm{mg} \mathrm{Ni} / \mathrm{m}^{3}$ as nickel sulfate, nickel subsulfide, or nickel oxide, respectively, and in mice exposed to $0.22,0.44$, or $1 \mathrm{mg}$ $\mathrm{Ni} / \mathrm{m}^{3}$ as nickel sulfate, nickel subsulfide, or nickel oxide, respectively (NTP 1996a, 1996b, 1996c).

The highest NOAEL values and all LOAEL values from each reliable study for immunological and lymphoreticular effects for each species, duration category, and nickel compound are recorded in Table 3-1 and plotted Figure 3-1.

\subsubsection{Neurological Effects}

No studies were located regarding neurological effects in humans after inhalation exposure to nickel.

Microscopic examinations did not reveal any changes in the whole brains of rats or mice exposed to nickel as nickel sulfate, nickel oxide, or nickel subsulfide for 12 6-hour exposures over 16 days (NTP 1996a, 1996b, 1996c). The maximum concentrations that did not result in deaths or changes in brain histology were $3.1,23.6$, and $7.33 \mathrm{mg} \mathrm{Ni} / \mathrm{m}^{3}$ in rats for nickel sulfate, nickel oxide, and nickel subsulfide, respectively, and $0.7,23.6$, and $3.65 \mathrm{mg} / \mathrm{m}^{3}$ in mice for nickel sulfate, nickel oxide, and nickel subsulfide, respectively. In intermediate-duration studies, no histological alterations were observed in the whole 
brains of rats and mice exposed to $0.44,7.9$, or $1.83 \mathrm{mg} \mathrm{Ni} / \mathrm{m}^{3}$ as nickel sulfate, nickel oxide, or nickel subsulfide, respectively, 6 hours/day, 5 days/week for 13 weeks (NTP 1996a, 1996b, 1996c). In rats exposed intermittently (6 hours/day, 5 days/week) to nickel subsulfide at $0.7 \mathrm{mg} \mathrm{Ni} / \mathrm{m}^{3}$ for 78 weeks, histological changes were not observed in the brain (Ottolenghi et al. 1974). Chronic exposure of rats to nickel oxide, nickel subsulfide, or nickel sulfate at concentrations up to $2,0.73$, or $0.11 \mathrm{mg} \mathrm{Ni} / \mathrm{m}^{3}$, respectively, or exposure of mice to nickel oxide, nickel subsulfide, or nickel sulfate at concentrations up to $3.9,0.88$, or $0.22 \mathrm{mg} \mathrm{Ni} / \mathrm{m}^{3}$, respectively, did not result in microscopic changes in the whole brain (NTP 1996a, 1996b, 1996c).

As noted in Section 3.2.1.2, atrophy of the olfactory epithelium has been observed in rats exposed to nickel sulfate and nickel subsulfide (Evans et al. 1995; NTP 1996a, 1996b, 1996c). To determine if changes in the olfactory epithelium result in any functional changes, Evans et al. (1995) completed behavioral studies of olfactory absolute threshold and olfactory discrimination in rats exposed to nickel sulfate at $0.635 \mathrm{mg} / \mathrm{m}^{3} 6$ hours/day for 16 days. Although histological changes were observed in the olfactory epithelium, including atrophy and a decrease in the number of bipolar receptor cells, no functional changes were noted. Carnosine, a neurochemical marker, was reduced in the olfactory epithelium following 12 days of exposure but was back to control levels by exposure day 16, suggesting adaptation to nickel exposure.

The LOAEL value from the Evans et al. (1995) study is recorded in Table 3-1 and plotted in Figure 3-1; the NOAELs for histological alterations in the brain were not recorded in the LSE table because this is not a sensitive indicator of functional neurotoxicity.

\subsubsection{Reproductive Effects}

An increase in the rate of spontaneous abortions (15.9\%) was reported among a group of 356 women who worked in a nickel hydrometallurgy refining plant in the Arctic region of Russia as compared to the rate (8.5\%) in 342 local female construction workers (Chashschin et al. 1994). Exposure concentrations were $0.08-0.196 \mathrm{mg} \mathrm{Ni} / \mathrm{m}^{3}$, primarily as nickel sulfate, and nickel concentrations in the urine were 3.2$22.6 \mu \mathrm{g} / \mathrm{L}$. Nickel levels in the urine of persons not occupationally exposed are generally $<0.1-13.3 \mu \mathrm{g} / \mathrm{L}$ (Sunderman 1993). The investigators noted that the nickel-exposed women manually lifted heavy nickel anodes and that they may have experienced heat stress. These confounders, plus the lack of information on the selection of control group subjects, possible acute exposure to high concentrations of chlorine, and the lack of adequate control of possible confounding variables such as smoking habits, use of alcohol, and 
intercurrent disease, preclude establishing a causative relationship between nickel exposure and reproductive toxicity from this study.

Testicular degeneration was observed in rats and mice exposed to nickel sulfate $\left(\geq 1.4 \mathrm{mg} \mathrm{Ni} / \mathrm{m}^{3}\right)$ and nickel subsulfide ( $\geq 1.83 \mathrm{mg} \mathrm{Ni} / \mathrm{m}^{3}$ for rats and $\geq 3.65 \mathrm{mg} \mathrm{Ni} / \mathrm{m}^{3}$ for mice) 6 hours/day for 12 days over a 16-day period (NTP 1996a, 1996b, 1996c). The study authors indicated that testicular lesions were probably the result of emaciation rather than a direct effect of nickel. In intermediate-duration studies, sperm concentration was decreased by $21 \%$ in rats exposed to nickel oxide at $7.9 \mathrm{mg} \mathrm{Ni} / \mathrm{m}^{3}$, with no effects at $3.9 \mathrm{mg} / \mathrm{m}^{3}$ (NTP 1996a). No effects on sperm motility, morphology, or concentration were observed in rats exposed to nickel subsulfide or nickel sulfate at concentrations up to 1.83 and $0.44 \mathrm{mg}$ $\mathrm{Ni} / \mathrm{m}^{3}$, respectively, or in mice exposed to nickel oxide, nickel subsulfide, or nickel sulfate at concentrations up to $7.9,1.83$, or $0.44 \mathrm{mg} \mathrm{Ni} / \mathrm{m}^{3}$, respectively (NTP 1996a, 1996b, 1996c). Histological changes in the testes were not observed. No effect on the length of the estrous cycle was noted in mice or rats exposed to nickel sulfate at $\leq 0.44 \mathrm{mg} \mathrm{Ni} / \mathrm{m}^{3}$, nickel oxide at $\leq 7.9 \mathrm{mg} \mathrm{Ni} / \mathrm{m}^{3}$, or nickel subsulfide at $\leq 1.83 \mathrm{mg} \mathrm{Ni} / \mathrm{m}^{3} 6$ hours/day, 5 days/week, for 13 weeks (NTP 1996a, 1996b, 1996c). Chronic exposure of rats to nickel oxide, nickel subsulfide, or nickel sulfate at concentrations up to $2,0.73$, or $0.11 \mathrm{mg}$ $\mathrm{Ni} / \mathrm{m}^{3}$, respectively, and exposure of mice to nickel oxide, nickel subsulfide, or nickel sulfate at concentrations up to $3.9,0.88$, or $0.22 \mathrm{mg} \mathrm{Ni} / \mathrm{m}^{3}$, respectively, did not result in microscopic changes in the reproductive organs (NTP 1996a, 1996b, 1996c).

The highest NOAEL values from each reliable study for reproductive effects in each species, duration category, and nickel compound and the LOAEL for decreased sperm concentration in rats exposed to nickel oxide are recorded in Table 3-1 and plotted in Figure 3-1.

\subsubsection{Developmental Effects}

In addition to the reproductive effects, Chashschin et al. (1994) also reported an increase in the incidence of structural malformations $(16.9 \%)$ in the offspring of female nickel hydrometallurgy refining plant workers as compared to the incidence $(5.8 \%)$ in the female construction workers. Although the specific structural malformations found were not stated, the investigators note that relative risks were 2.9 for all kinds of defects, 6.1 for cardiovascular system defects, and 1.9 for musculoskeletal defects. Exposure concentrations were $0.08-0.196 \mathrm{mg} \mathrm{Ni} / \mathrm{m}^{3}$, primarily as nickel sulfate, and nickel concentrations in the urine were 3.2-22.6 $\mu \mathrm{g} / \mathrm{L}$. Nickel levels in the urine of persons not occupationally exposed are generally $<0.1-13.3 \mu \mathrm{g} / \mathrm{L}$ (Sunderman 1993). As discussed under Reproductive Effects, a number of possible 
confounders including heavy lifting, possible heat stress, lack of information on the selection of control group subjects, possible acute exposure to high concentrations of chlorine, and the lack of adequate control of possible confounding variables such as smoking habits, use of alcohol, and intercurrent disease, preclude establishing a causative relationship between nickel exposure and developmental toxicity from this study.

A decrease in fetal body weight was observed in the offspring of rats exposed to $1.6 \mathrm{mg} \mathrm{Ni} / \mathrm{m}^{3}$ as nickel oxide 23.6 hours/day on gestation days 1-21 (Weischer et al. 1980). No effect on fetal body weight was observed at $0.8 \mathrm{mg} \mathrm{Ni} / \mathrm{m}^{3}$, although decreased maternal body weight gain was observed at this concentration. No effects on the number of fetuses or on the weight of placenta were observed.

The NOAEL value and the LOAEL value from the Weischer et al. (1980) study are recorded in Table 3-1 and plotted Figure 3-1.

\subsubsection{Cancer}

A large number of epidemiology studies have assessed the carcinogenic potential of nickel; it has been estimated that over 100,000 nickel workers have been examined in epidemiology studies (Seilkop and Oller 2003). These workers have been employed in nickel refinery facilities, nickel mining and smelting facilities, nickel alloy production facilities, stainless steel production facilities, nickel-cadmium battery production facilities, or as stainless steel welders. In the mid 1980s, a committee of epidemiologists was formed to investigate the human health risks associated with nickel exposure and to determine the specific forms of nickel that are associated with an increased risk of respiratory cancer (International Committee on Nickel Carcinogenesis in Man 1990). The investigators updated the existing data from 10 previously examined cohorts and estimated levels of exposure to various nickel species. Since no measurements of nickel concentrations were available for workers employed prior to 1950, the investigators estimated total nickel exposure levels using recent monitoring data and historical data on the industrial processes. Based on information on the chemistry of the industrial process, total nickel exposure levels were divided into exposure to four nickel species: soluble nickel (including nickel sulfate and nickel chloride), sulfidic nickel (including nickel subsulfide), oxidic nickel, and metallic nickel. It is noted that interpretation of the results of many of the epidemiology studies of nickel workers is confounded by poor nickel exposure characterization, exposure to relatively high concentrations of other metals, including arsenic, and in some cases, exposure to irritant gases including hydrogen sulfide, ammonia, chlorine, and sulfur dioxide (IARC 1990). 
Statistically significant increases in the risk of nasal and/or lung cancer were found among nickel refinery workers (Andersen et al. 1996; Anttila et al. 1998; Chovil et al. 1981; Doll et al. 1977; Enterline and Marsh 1982; Grimsrud et al. 2003; International Committee on Nickel Carcinogenesis in Man 1990; Karjalainen et al. 1992; Magnus et al. 1982; Muir et al. 1994; Pedersen et al. 1973; Peto et al. 1984; Roberts et al. 1989a). In general, the nickel refinery workers were exposed to high levels of sulfidic and oxidic nickel and low levels of soluble and metallic nickel (International Committee on Nickel Carcinogenesis in Man 1990). At one nickel refinery facility (New Caledonia), the risk of respiratory tract cancers was not significantly elevated in the nickel-exposed workers (Goldberg et al. 1987, 1994; International Committee on Nickel Carcinogenesis in Man 1990). This refinery facility differs from other refineries in that the workers were primarily exposed to silicate oxide ore and oxidic nickel with very little exposure to sulfidic or soluble nickel. Sunderman and associates (Sunderman et al. 1989a) examined the histopathological diagnosis of 100 cases of sinonasal cancer and 259 cases of lung cancer among workers at three nickel refinery facilities. The primary sinonasal cancers were squamous cell carcinomas (48\%), anaplastic and undifferentiated carcinomas (39\%), and adenocarcinomas (6\%). In an analysis of lung cancer, the cancers were primarily squamous cell carcinomas (67\%), anaplastic, small cell, and oat cell carcinomas (15\%), and adenocarcinomas (8\%). The types of sinonasal and lung cancers were similar to those found in the general population, suggesting a lack of nickel-specific tumor types.

In contrast to the findings of nickel refinery workers, most studies in other groups of nickel workers have not found significant increases in the risk of lung cancer among workers employed in nickel mining and smelting facilities (International Committee on Nickel Carcinogenesis in Man 1990; Shannon et al. 1984b, 1991), workers employed at a hydrometallurgical refinery (Egedahl and Rice 1984, Egedahl et al. 1991, 2001), workers employed at nickel alloy and stainless steel production facilities (Cornell 1984; Cornell and Landis 1984; Cox et al. 1981; Enterline and March 1982; International Committee on Nickel Carcinogenesis in Man 1990; Jakobsson et al. 1997; Moulin et al. 1993; Sorahan 2004), workers employed as stainless steel welders (Danielsen et al. 1996; Gerin et al. 1993; Hansen et al. 1996; Simonato et al. 1991), workers involved in nickel-chromium electroplating (Pang et al. 1996), or workers employed at a barrier production facility (Cragle et al. 1984; Godbold and Tompkins 1979; International Committee on Nickel Carcinogenesis in Man 1990). Although some studies of these workers did find significant increases in respiratory tract cancers (Becker 1999; Moulin et al. 1990), the increased risk was attributed to exposure to other carcinogenic agents, such as polycyclic aromatic hydrocarbons or asbestos. Redmond (1984) and Arena et al. (1998) reported significant increases in lung cancer risks among high nickel alloy production workers as compared to the U.S. population. However, when the local population 
was used as the comparison group, the increase in lung cancer risk was no longer statistically significant (Arena et al. 1998). In general, workers employed in these industries were exposed to lower levels of sulfidic or oxidic nickel than the nickel refinery workers who were primarily exposed to metallic nickel (Cragle et al. 1984; Godbold and Tompkins 1979) or soluble nickel (Pang et al. 1996).

Because nickel workers are exposed to several nickel species, it is difficult to assess the carcinogenic potential of a particular nickel species. The International Committee on Nickel Carcinogenesis in Man (1990) investigators used cross-classification analyses to examine the dose-response to a specific nickel species independent of variations in other species. The most comprehensive cross-classification analyses were performed for cohorts of workers in different departments at the Mond/INCO (Clydach) nickel refinery and at the Falconbridge (Kristiansand) nickel refinery (only analyzed for metallic nickel). Summaries of some of these cross-characterizations showing the different risks for low and high exposure to a particular nickel species stratified by the degree of exposure to other nickel species are presented in Tables 3-2 through 3-5. The strongest evidence of carcinogenicity of a particular nickel species is for sulfidic nickel. The highest cancer risk levels were found in cohorts with the highest sulfidic nickel exposure levels, although high oxidic and soluble nickel levels were also found at these same facilities. The increased cancer risks in workers with high sulfidic nickel exposure and low oxidic and soluble nickel exposure (Table 3-2) suggests that sulfidic nickel is the causative agent. The evidence for oxidic nickel is weaker. As presented in Table 3-3, no differences in cancer risks were seen among groups of workers with low sulfidic and soluble nickel exposures when the levels of oxidic nickel were varied. However, when high soluble nickel levels are present, oxidic nickel appears to be carcinogenic. The available weight of evidence does not suggest that exposure to soluble nickel, in the absence of carcinogenic compounds, will increase the risk of cancer. At low sulfidic and oxidic nickel levels, increasing soluble nickel levels do not increase the cancer risk in the Clydach cohort (Table 3-4). However, at high oxidic nickel levels, increasing the soluble nickel levels resulted in at least a 2-fold increase in the cancer risk. There is no evidence that metallic nickel is associated with increased lung or nasal cancer risks in nickel workers based on the results of the cross-classification analyses for two cohorts of nickel refinery workers (Table 3-5) and the lack of increased cancer risk in the workers exposed to metallic nickel alone at the barrier production facility (Cragle et al. 1984; Godbold and Tompkins 1979). The International Committee on Nickel Carcinogenesis in Man (1990) concluded that lung and nasal cancers were related primarily to exposure to less soluble nickel compounds at concentrations of $\geq 10 \mathrm{mg} \mathrm{Ni} / \mathrm{m}^{3}$ (primarily oxidic and sulfidic compounds). Exposure to soluble nickel compounds at concentrations of $>1 \mathrm{mg} \mathrm{Ni} / \mathrm{m}^{3}$ appeared to enhance the carcinogenicity of insoluble nickel 


\section{Table 3-2. Comparison of Risk of Dying of Lung Cancer at Different Levels of Cumulative Exposure to Sulfidic Nickel by Different Levels of Combined Cumulative Exposure to Oxidic and Soluble Nickel in the Mond/INCO (Clydach) Nickel Refinery ${ }^{a}$}

\begin{tabular}{|c|c|c|c|c|c|c|c|}
\hline \multirow{2}{*}{$\begin{array}{l}\text { Degree of exposure to } \\
\text { oxidic and soluble nickel, } \\
\text { respectively }\end{array}$} & \multicolumn{3}{|c|}{$\begin{array}{l}\text { Low exposure to } \\
\text { sulfidic nickel }^{\mathrm{c}}\end{array}$} & \multicolumn{3}{|c|}{$\begin{array}{l}\text { High exposure to } \\
\text { sulfidic nickel }^{\mathrm{d}}\end{array}$} & \multirow{2}{*}{$\begin{array}{l}\text { Difference in SMR } \\
\text { value ( } p \text {-value) }\end{array}$} \\
\hline & $\mathrm{O}$ & $E$ & SMR & $\mathrm{O}$ & $E$ & SMR & \\
\hline Low, low & 51 & 26.01 & 196 & 8 & 1.25 & 638 & 0.004 \\
\hline Low, high & 7 & 4.16 & 168 & 1 & 0.15 & 657 & 0.388 \\
\hline High, low & 18 & 5.14 & 350 & 32 & 6.34 & 505 & 0.455 \\
\hline High, high & 30 & 3.87 & 776 & 28 & 2.36 & 1,187 & 0.265 \\
\hline
\end{tabular}

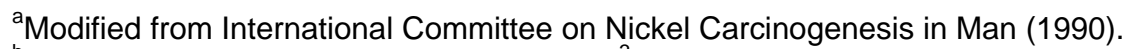

${ }^{b}$ Low oxidic nickel exposure $=<50\left(\mathrm{mg} \mathrm{Ni} / \mathrm{m}^{3}\right) \cdot y e a r s$ and high oxidic nickel exposure $=\geq 50\left(\mathrm{mg} \mathrm{Ni}^{3} \mathrm{~m}^{3}\right) \cdot y e a r s ;$ low soluble nickel exposure $=<10\left(\mathrm{mg} \mathrm{Ni} / \mathrm{m}^{3}\right) \cdot y$ ears and high soluble nickel exposure $=\geq 10\left(\mathrm{mg} \mathrm{Ni} / \mathrm{m}^{3}\right) \cdot$ years.

${ }^{\mathrm{C}}$ Low sulfidic nickel exposure $=<15\left(\mathrm{mg} \mathrm{Ni} / \mathrm{m}^{3}\right)$.years.

${ }^{\mathrm{d}}$ High sulfidic nickel exposure $=\geq 15\left(\mathrm{mg} \mathrm{Ni} / \mathrm{m}^{3}\right) \cdot$ years.

$\mathrm{O}=$ observed; $\mathrm{E}=$ expected; SMR = standardized mortality ratio 


\section{Table 3-3. Comparison of Risk of Dying of Lung Cancer at Different Levels of Cumulative Exposure to Oxidic Nickel by Different Levels of Combined Cumulative Exposure to Sulfidic and Soluble Nickel in the Mond/INCO (Clydach) Nickel Refinery ${ }^{a}$}

\begin{tabular}{|c|c|c|c|c|c|c|c|}
\hline \multirow{2}{*}{$\begin{array}{l}\text { Degree of exposure to sulfidic } \\
\text { and soluble nickel, respectively }\end{array}$} & \multicolumn{3}{|c|}{$\begin{array}{l}\text { Low exposure to } \\
\text { oxidic nickel }^{c}\end{array}$} & \multicolumn{3}{|c|}{$\begin{array}{l}\text { High exposure to } \\
\text { oxidic nickel }^{d}\end{array}$} & \multirow{2}{*}{$\begin{array}{l}\text { Difference in } \\
\text { SMR value } \\
\text { (p-value) }\end{array}$} \\
\hline & $\mathrm{O}$ & $E$ & SMR & $\mathrm{O}$ & $E$ & SMR & \\
\hline Low, low & 51 & 26.01 & 196 & 18 & 5.14 & 350 & 0.100 \\
\hline Low, high & 7 & 4.16 & 168 & 30 & 3.87 & 776 & $<0.001$ \\
\hline High, low & 8 & 1.25 & 638 & 32 & 6.34 & 505 & 0.839 \\
\hline High, high & 1 & 0.15 & 658 & 28 & 2.36 & 1,187 & 0.841 \\
\hline
\end{tabular}

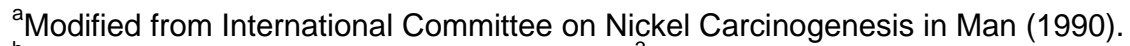

${ }^{b}$ Low sulfidic nickel exposure $=<15\left(\mathrm{mg} \mathrm{Ni}^{3} \mathrm{~m}^{3}\right) \cdot y e a r s$ and high sulfidic nickel exposure $=\geq 15\left(\mathrm{mg} \mathrm{Ni}^{3} \mathrm{~m}^{3}\right) \cdot y e a r s ;$ low soluble nickel exposure $=<10\left(\mathrm{mg} \mathrm{Ni} / \mathrm{m}^{3}\right) \cdot y e a r s$ and high soluble nickel exposure $=\geq 10\left(\mathrm{mg} \mathrm{Ni} / \mathrm{m}^{3}\right) \cdot$ years.

${ }^{\mathrm{C}}$ Low oxidic nickel exposure $=<50\left(\mathrm{mg} \mathrm{Ni} / \mathrm{m}^{3}\right) \cdot$ years.

${ }^{\mathrm{d}}$ High oxidic nickel exposure $=\geq 50\left(\mathrm{mg} \mathrm{Ni} / \mathrm{m}^{3}\right) \cdot$ years.

$\mathrm{O}=$ observed; $\mathrm{E}=$ expected; SMR = standardized mortality ratio 
Table 3-4. Comparison of Risk of Dying of Lung Cancer at Different Levels of Cumulative Exposure to Soluble Nickel by Different Levels of Combined Cumulative Exposure to Sulfidic and Oxidic Nickel in the Mond/INCO (Clydach) Nickel Refinery ${ }^{\mathrm{a}}$

\begin{tabular}{|c|c|c|c|c|c|c|c|}
\hline \multirow{2}{*}{$\begin{array}{l}\text { Degree of exposure to sulfidic } \\
\text { and oxidic nickel, respectively }\end{array}$} & \multicolumn{3}{|c|}{$\begin{array}{l}\text { Low exposure to } \\
\text { soluble nickel }\end{array}$} & \multicolumn{3}{|c|}{$\begin{array}{l}\text { High exposure to } \\
\text { soluble nickel }^{\mathrm{d}}\end{array}$} & \multirow{2}{*}{$\begin{array}{l}\text { Difference in } \\
\text { SMR value } \\
\text { (p-value) }\end{array}$} \\
\hline & $\mathrm{O}$ & $E$ & SMR & $\mathrm{O}$ & $E$ & SMR & \\
\hline Low, low & 51 & 26.01 & 196 & 7 & 4.16 & 168 & 0.931 \\
\hline Low, high & 18 & 5.14 & 350 & 30 & 3.87 & 776 & 0.024 \\
\hline High, low & 8 & 1.25 & 638 & 1 & 0.15 & 658 & 0.999 \\
\hline High, high & 32 & 6.34 & 505 & 28 & 2.36 & 1,187 & 0.003 \\
\hline
\end{tabular}

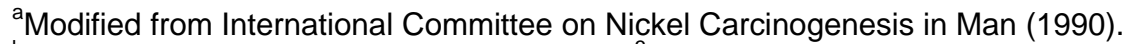

${ }^{b}$ Low sulfidic nickel exposure $=<15\left(\mathrm{mg} \mathrm{Ni} / \mathrm{m}^{3}\right)$.years and high sulfidic nickel exposure $=\geq 15\left(\mathrm{mg} \mathrm{Ni}^{3} \mathrm{~m}^{3}\right) \cdot y e a r s ;$ low oxidic nickel exposure $=<50\left(\mathrm{mg} \mathrm{Ni} / \mathrm{m}^{3}\right) \cdot$ years and high oxidic nickel exposure $=\geq 50\left(\mathrm{mg} \mathrm{Ni} / \mathrm{m}^{3}\right) \cdot y e a r s$.

${ }^{c}$ Low soluble nickel exposure $=<10\left(\mathrm{mg} \mathrm{Ni} / \mathrm{m}^{3}\right) \cdot$ years.

${ }^{\mathrm{d}}$ High soluble nickel exposure $=\geq 10\left(\mathrm{mg} \mathrm{Ni} / \mathrm{m}^{3}\right) \cdot$ years.

$\mathrm{O}=$ observed; $\mathrm{E}=$ expected; SMR = standardized mortality ratio 


\section{Table 3-5. Comparison of Risk of Dying of Lung Cancer at Different Levels of Cumulative Exposure to Metallic Nickel by Different Levels of Combined Cumulative Exposure to Sulfidic, Oxidic, and Soluble Nickel in the Mond/INCO (Clydach) and Falconbridge (Kristainsand) Nickel Refineries ${ }^{a}$}

\begin{tabular}{|c|c|c|c|c|c|c|c|}
\hline \multirow{2}{*}{$\begin{array}{l}\text { Degree of exposure to sulfidic, } \\
\text { oxidic, and soluble nickel, } \\
\text { respectively }\end{array}$} & \multicolumn{3}{|c|}{$\begin{array}{l}\text { Low exposure to metallic } \\
\text { nickel }^{c}\end{array}$} & \multicolumn{3}{|c|}{$\begin{array}{l}\text { High exposure to } \\
\text { metallic nickel }^{d}\end{array}$} & \multirow{2}{*}{$\begin{array}{l}\text { Difference in } \\
\text { SMR value } \\
\text { (p-value) }\end{array}$} \\
\hline & $\mathrm{O}$ & $E$ & SMR & $\mathrm{O}$ & $E$ & SMR & \\
\hline \multicolumn{8}{|l|}{ Mond/INCO (Clydach) Nickel Refinery } \\
\hline None, none, none & 19 & 11.5 & 166 & - & - & - & - \\
\hline Low, low, low & 44 & 23.2 & $190^{\mathrm{e}}$ & 7 & 2.8 & $249^{f}$ & 0.80 \\
\hline Low, low, high & 5 & 3.8 & 133 & 2 & 0.4 & 499 & 0.23 \\
\hline Low, high, low & 17 & 4.7 & $363^{e}$ & 1 & 0.5 & $220^{f}$ & 0.87 \\
\hline Low, high, high & 26 & 3.3 & $783^{\mathrm{e}}$ & 4 & 0.1 & $732^{9}$ & 0.99 \\
\hline High, low, low & 2 & 0.3 & $589^{f}$ & 5 & 0.9 & $656^{\mathrm{e}}$ & 0.99 \\
\hline High, low, high & - & - & - & 1 & 0.2 & 658 & - \\
\hline High, high, low & 2 & 0.9 & 235 & 30 & 5.5 & 547 & 0.49 \\
\hline High, high, high & 3 & 0.3 & $865^{9}$ & 25 & 2.0 & $1,140^{\mathrm{e}}$ & 0.83 \\
\hline \multicolumn{8}{|c|}{ Falconbridge (Kristiansand) Nickel Refinery } \\
\hline None, none, none & 6 & 3.28 & 186 & - & - & - & \\
\hline Low, low, low & 19 & 7.17 & $265^{\mathrm{e}}$ & - & - & - & \\
\hline Low, low, high & 10 & 1.75 & $570^{\mathrm{e}}$ & - & 0.18 & - & \\
\hline Low, high, low & 11 & 4.66 & $236^{\mathrm{e}}$ & 1 & 0.25 & 396 & \\
\hline Low, high, high & 6 & 0.78 & $770^{\mathrm{e}}$ & 2 & 0.26 & $1,112^{\mathrm{g}}$ & \\
\hline High, low, high & 1 & 0.13 & 769 & - & - & - & \\
\hline High, high, low & 2 & 0.58 & 344 & 3 & 0.64 & $471^{f}$ & \\
\hline High, high, high & 4 & 0.76 & $524^{g}$ & 1 & 0.58 & 171 & \\
\hline
\end{tabular}

\footnotetext{
${ }^{a}$ Modified from International Committee on Nickel Carcinogenesis in Man (1990).

${ }^{\mathrm{b}}$ Clydach: low sulfidic nickel exposure $=<15\left(\mathrm{mg} \mathrm{Ni} / \mathrm{m}^{3}\right) \cdot y e a r s$ and high sulfidic nickel exposure $=\geq 15(\mathrm{mg}$ $\left.\mathrm{Ni} / \mathrm{m}^{3}\right) \cdot$ years; low oxidic nickel exposure $=<50\left(\mathrm{mg} \mathrm{Ni}^{3} \mathrm{~m}^{3}\right) \cdot$ years and high oxidic nickel exposure $=\geq 50(\mathrm{mg}$ $\left.\mathrm{Ni} / \mathrm{m}^{3}\right)$.years; low soluble nickel exposure $=<10\left(\mathrm{mg} \mathrm{Ni} / \mathrm{m}^{3}\right)$ years and high soluble nickel exposure $=\geq 10(\mathrm{mg}$ $\left.\mathrm{Ni} / \mathrm{m}^{3}\right) \cdot$ years.

Kristiansand: low sulfidic nickel exposure $=<5\left(\mathrm{mg} \mathrm{Ni}^{3} \mathrm{~m}^{3}\right) \cdot$ years and high sulfidic nickel exposure $=\geq 5(\mathrm{mg}$ $\left.\mathrm{Ni} / \mathrm{m}^{3}\right) \cdot$ years; low oxidic nickel exposure $=<15\left(\mathrm{mg} \mathrm{Ni} / \mathrm{m}^{3}\right) \cdot$ years and high oxidic nickel exposure $=\geq 15(\mathrm{mg}$ $\left.\mathrm{Ni} / \mathrm{m}^{3}\right)$.years; low soluble nickel exposure $=<5\left(\mathrm{mg} \mathrm{Ni}^{3} \mathrm{~m}^{3}\right)$.years and high soluble nickel exposure $=\geq 5(\mathrm{mg}$ $\left.\mathrm{Ni} / \mathrm{m}^{3}\right) \cdot$ years.

${ }^{c}$ Clydach: low exposure $=<15\left(\mathrm{mg} \mathrm{Ni}^{3} \mathrm{~m}^{3}\right.$ ).years; Kristiansand: low exposure $=<5\left(\mathrm{mg} \mathrm{Ni} / \mathrm{m}^{3}\right) \cdot$ years.

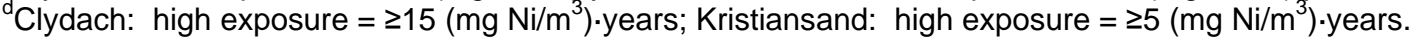
${ }_{\mathrm{p}}^{\mathrm{e}}<0.001$

if $p<0.05$

${ }^{9} \mathrm{p}<0.01$

$\mathrm{O}=$ observed; $\mathrm{E}=$ expected; SMR = standardized mortality ratio
} 
compounds. These concentrations are presented as human cancer effect levels for lung and nasal cancers in Table 3-1 and Figure 3-1.

Significant increases in cancer risks at sites other than the respiratory tract have been found in some cohorts of nickel workers. The International Committee on Nickel Carcinogenesis in Man (1990) noted that if nickel exposure was associated with nonrespiratory tract cancer, increased risks would be seen among the workers with the highest nickel exposures (cohorts that also had increased levels of respiratory tract cancer). Among the three cohorts with the highest nickel exposures (Clydach, INCO Ontario sinter plants, and Kristiansand), no consistent patterns of increased nonrespiratory tract cancer risks were found. When the three cohorts were combined, significant increases in pharynx (SMR 201; 95\% confidence interval 117-322) and bone (SMR 206; 95\% confidence interval 111-353) cancers were found. The investigators noted that cancers of the ethmoid and maxillary sinuses are sometimes classified as bone cancer and that bone cancer is sometimes listed on death certificates if the primary lung cancers are occasionally unrecognized and death is attributed to the site of metastasis. Among workers with low-level nickel exposures without significant increases in respiratory tract cancer, no significant increases in cancer risks were found. Thus, the investigators concluded that there was insufficient evidence that nickel exposure results in tumors outside of the respiratory tract (International Committee on Nickel Carcinogenesis in Man 1990). Two studies published after this analysis found significant increases in the incidence of stomach cancer among nickel refinery workers (Antilla et al. 1998) and nickel platers (Pang et al. 1996). These data are insufficient to conclude whether the increases in stomach cancer risks are due to exposure to nickel, other agents, or chance. A meta-analysis of occupational exposure studies on pancreatic cancer (Ojajärvi et al. 2000) found a significant association between exposure to nickel and pancreatic cancer risk. However, the Ojajärvi et al. (2000) meta-analysis has been criticized (Sielkop 2001) for excluding a study of nickel mining and smelting workers (Shannon et al. 1991) and a study of nickel alloy production workers (Arena et al. 1998). The addition of these studies lowered the meta-analysis ratio from 1.9 (95\% confidence interval 1.2-3.2) to 1.3 (95\% confidence interval 0.9-1.9); Ojajärvi accepted Sielkop's comments. Overall, there does not appear to be sufficient evidence that exposure to airborne nickel is associated with increased cancer risks outside of the respiratory tract.

A number of animal studies have examined the carcinogenic potential of nickel subsulfide, nickel oxide, and nickel sulfate. Chronic exposure to nickel subsulfide resulted in significant increases in lung tumors in two rat studies. Adenomas, adenocarcinomas, squamous cell carcinomas, and fibrosarcoma were observed in rats exposed to $0.7 \mathrm{mg} \mathrm{Ni} / \mathrm{m}^{3}$ as nickel subsulfide 6 hours/day, 5 days/week, for 78 weeks 
(Ottolenghi et al. 1974). Similarly, significant increases in the combined incidences of alveolar/ bronchiolar adenoma or carcinoma were observed in male and female rats exposed to 0.11 or $0.73 \mathrm{mg}$ $\mathrm{Ni} / \mathrm{m}^{3}$ as nickel subsulfide, 6 hours/day, 5 days/week for 2 years (NTP 1996b); incidence data for this study are presented in Table 3-6. Significant increases in the incidence of benign or malignant pheochromocytoma in the adrenal medulla were also observed in males at 0.11 or $0.73 \mathrm{mg} \mathrm{Ni} / \mathrm{m}^{3}$ and females at $0.73 \mathrm{mg} \mathrm{Ni} / \mathrm{m}^{3}$. In contrast to the findings in rats, no significant alterations in tumor incidences were observed in mice exposed to 0.44 or $0.88 \mathrm{mg} \mathrm{Ni} / \mathrm{m}^{3}$ as nickel subsulfide 6 hours/day, 5 days/week for 2 years (NTP 1996b) (see Table 3-7 for incidence data) or in mice following weekly intratracheal injections of $\leq 0.8 \mathrm{mg} \mathrm{Ni} / \mathrm{m}^{3}$ as nickel subsulfide for $\leq 15$ weeks, followed by observation for $\leq 27$ months (Fisher et al. 1986; McNeill et al. 1990). Acute (6 hours/day, 5 days/week, for 1 month) inhalation exposure to $\leq 6.3 \mathrm{mg} \mathrm{Ni} / \mathrm{m}^{3}$ as nickel oxide resulted in no significant increase in lung cancer in rats $\leq 20$ months after exposure (Horie et al. 1985). However, significant increases in the incidence of alveolar/bronchiolar adenoma or carcinoma were observed in male and female rats exposed to 1 or $2 \mathrm{mg}$ $\mathrm{Ni} / \mathrm{m}^{3}$ as nickel oxide 6 hours/day, 5 days/week for 2 years (NTP 1996c) (see Table 3-6 for incidence data), but not in rats exposed to $0.5 \mathrm{mg} \mathrm{Ni} / \mathrm{m}^{3}$ or in mice exposed to 1,2 , or $3.9 \mathrm{mg} \mathrm{Ni} / \mathrm{m}^{3}$. Significant increases in the incidence of benign or malignant pheochromocytoma in the adrenal medulla were also observed in rats exposed to $3.9 \mathrm{mg} \mathrm{Ni} / \mathrm{m}^{3}$ (NTP 1996c). In contrast to the less soluble nickel compounds, chronic (6 hours/day, 5 days/week for 2 years) exposure to nickel sulfate did not result in significant increases in neoplasms in rats or mice (NTP 1996a); the highest concentrations tested were 0.11 and $0.22 \mathrm{mg} \mathrm{Ni} / \mathrm{m}^{3}$, respectively; incidence data reported in Tables 3-6 and 3-7. The nickel concentrations as nickel subsulfide and nickel oxide resulting in cancer in rats are presented as Cancer Effect Levels in Table 3-1 and Figure 3-1.

The Department of Health and Human Services (NTP 2002) has determined that metallic nickel may reasonably be anticipated to be a human carcinogen and that nickel compounds are known to be human carcinogens. Similarly, IARC (1990) classified metallic nickel in group 2B (possibly carcinogenic to humans) and nickel compounds in group 1 (carcinogenic to humans). EPA has classified nickel refinery dust and nickel subsulfide in Group A (human carcinogen) (IRIS 2005). Other nickel compounds have not been classified by the EPA. Based on the occupational data, inhalation unit risk levels of $2.4 \times 10^{4}\left(\mu \mathrm{g} / \mathrm{m}^{3}\right)^{-1}$ and $4.8 \times 10^{-4}\left(\mu \mathrm{g} / \mathrm{m}^{3}\right)^{-1}$ were derived for nickel refinery dust and nickel subsulfide, respectively (IRIS 2003). The risk levels for these compounds are presented in Figure 3-1. The risk levels range from $4 \times 10^{-1}$ to $4 \times 10^{-4} \mu \mathrm{g} / \mathrm{m}^{3}$ for a risk ranging from $1 \times 10^{-4}$ to $1 \times 10^{-7}$, respectively, for nickel refinery dust (IRIS 2005) and from $2 \times 10^{-1}$ to $2 \times 10^{-4} \mu \mathrm{g} / \mathrm{m}^{3}$ for a risk ranging from $1 \times 10^{-4}$ to $1 \times 10^{-7}$, respectively, for nickel subsulfide (IRIS 2005). These risk levels are presented in Figure 3-1. 


\section{Table 3-6. Alveolar/Bronchiolar Neoplasms and Adrenal Medulla Proliferative Lesions in Rats ${ }^{\mathrm{a}}$}

\begin{tabular}{|c|c|c|c|c|c|c|c|c|c|c|c|}
\hline \multirow{3}{*}{ Effect } & \multicolumn{11}{|c|}{ Number of rats with neoplasms or proliferative lesions/number of rats examined } \\
\hline & \multicolumn{4}{|c|}{$\begin{array}{l}\text { Exposure to nickel sulfate } \\
\text { hexahydrate }\left(\mathrm{mg} \text { nickel/ } / \mathrm{m}^{3}\right)\end{array}$} & \multicolumn{3}{|c|}{$\begin{array}{l}\text { Exposure to nickel } \\
\text { subsulfide }\left(\mathrm{mg} \text { nickel } / \mathrm{m}^{3}\right)\end{array}$} & \multicolumn{4}{|c|}{$\begin{array}{l}\text { Exposure to nickel } \\
\text { oxide (mg nickel/m³) }\end{array}$} \\
\hline & 0 & 0.03 & 0.06 & 0.11 & 0 & 0.11 & 0.73 & 0 & 0.5 & 1 & 2 \\
\hline \multicolumn{12}{|l|}{ Male } \\
\hline $\begin{array}{l}\text { Alveolar/ } \\
\text { brochiolar } \\
\text { adenoma/ } \\
\text { carcinoma }\end{array}$ & $2 / 54$ & $0 / 53$ & $1 / 53$ & $3 / 53$ & $0 / 53$ & $6 / 53^{b}$ & $11 / 53^{c}$ & $1 / 54$ & $1 / 53$ & $6 / 53^{b}$ & $4 / 52^{\mathrm{b}}$ \\
\hline $\begin{array}{l}\text { Adrenal medulla } \\
\text { benign, } \\
\text { malignant, or } \\
\text { complex } \\
\text { pheochromo- } \\
\text { cytoma }\end{array}$ & $16 / 54$ & $19 / 53$ & $13 / 53$ & $12 / 53$ & $14 / 53$ & $30 / 52^{c}$ & $42 / 53^{c}$ & $27 / 54$ & $24 / 53$ & $27 / 53$ & $35 / 54^{c}$ \\
\hline \multicolumn{12}{|l|}{ Female } \\
\hline $\begin{array}{l}\text { Alveolar/ } \\
\text { brochiolar } \\
\text { adenoma/ } \\
\text { carcinoma }\end{array}$ & $0 / 52$ & $0 / 53$ & $0 / 53$ & $1 / 54$ & $2 / 53$ & $6 / 53^{d}$ & $9 / 53^{b}$ & $1 / 53$ & $0 / 53$ & $6 / 53^{d}$ & $5 / 54^{d}$ \\
\hline $\begin{array}{l}\text { Adrenal medulla } \\
\text { benign, } \\
\text { malignant, or } \\
\text { complex } \\
\text { pheochromo- } \\
\text { cytoma }\end{array}$ & $2 / 51$ & $4 / 53$ & $3 / 53$ & $3 / 54$ & $3 / 53$ & $7 / 53$ & $36 / 53^{c}$ & $4 / 53$ & $7 / 53$ & $6 / 53$ & $18 / 54^{c}$ \\
\hline
\end{tabular}

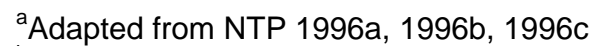

${ }^{b} \mathrm{p} \leq 0.05$

${ }^{c} p \leq 0.01$

${ }^{d} p \leq 0.05$ versus historical data (1.4\%, $3 / 210$ males; $1.4 \%, 4 / 208$ females) (Dunnick et al. 1995) 
Table 3-7. Alveolar/Bronchiolar Neoplasms in Mice ${ }^{a}$

\begin{tabular}{|c|c|c|c|c|c|c|c|c|c|c|c|}
\hline & \multicolumn{11}{|c|}{ Number of mice with tumors/number of mice examined } \\
\hline & \multicolumn{4}{|c|}{$\begin{array}{l}\text { Exposure to nickel sulfate } \\
\text { hexahydrate }\left(\mathrm{mg} \text { nickel } / \mathrm{m}^{3}\right)\end{array}$} & \multicolumn{3}{|c|}{$\begin{array}{l}\text { Exposure to nickel } \\
\text { subsulfide }\left(\mathrm{mg} \text { nickel } / \mathrm{m}^{3}\right)\end{array}$} & \multicolumn{4}{|c|}{$\begin{array}{l}\text { Exposure to nickel oxide } \\
\left(\mathrm{mg} \text { nickel } / \mathrm{m}^{3}\right)\end{array}$} \\
\hline & 0 & 0.06 & 0.11 & 0.22 & 0 & 0.44 & 0.88 & 0 & 1 & 2 & 3.9 \\
\hline Male & $13 / 61$ & $18 / 61$ & $7 / 62$ & $8 / 61$ & $13 / 61$ & $5 / 59$ & $6 / 58$ & $9 / 57$ & $14 / 67$ & $15 / 66$ & $14 / 69$ \\
\hline Female & $7 / 61$ & $6 / 60$ & $10 / 60$ & $2 / 60$ & $9 / 58$ & $2 / 59$ & $3 / 60$ & $6 / 64$ & $15 / 66^{\mathrm{b}}$ & $12 / 63$ & $8 / 64$ \\
\hline
\end{tabular}

${ }^{a}$ Adapted from NTP 1996a, 1996b, 1996c

${ }^{b} \mathrm{p} \leq 0.05$ 


\subsubsection{Oral Exposure}

\subsubsection{Death}

One human death following oral exposure to nickel was reported (Daldrup et al. 1983). Nickel sulfate crystals (rough estimate of $570 \mathrm{mg} \mathrm{Ni} / \mathrm{kg}$ ) were accidentally ingested by a 2-year-old child. Four hours after ingestion, cardiac arrest occurred, and the child died 8 hours after exposure.

Single-dose oral lethality studies indicate that soluble nickel compounds are more toxic than less-soluble nickel compounds. Oral $\mathrm{LD}_{50}$ values of 46 or $39 \mathrm{mg} \mathrm{Ni} / \mathrm{kg}$ as nickel sulfate in male and female rats (Mastromatteo 1986) and 116 and $136 \mathrm{mg} \mathrm{Ni} / \mathrm{kg}$ as nickel acetate in female rats and male mice, respectively (Haro et al. 1968) have been reported for soluble nickel compounds. In contrast, the oral $\mathrm{LD}_{50}$ values in rats for less-soluble nickel oxide and subsulfide were $>3,930$ and $>3,665 \mathrm{mg} \mathrm{Ni} / \mathrm{kg}$, respectively (Mastromatteo 1986).

Increases in mortality $(6 / 52,60 / 60)$ were observed in rats administered via gavage 8.6 or $25 \mathrm{mg} \mathrm{Ni} / \mathrm{kg} /$ day as nickel chloride hexahydrate for 91 days (American Biogenics Corporation 1988). Clinical signs observed included lethargy, ataxia, irregular breathing, hypothermia, salivation, squinting, and loose stools. As part of a longer-term study, rats were provided with drinking water containing 1,000 ppm nickel as nickel chloride (approximately $140 \mathrm{mg} / \mathrm{kg} /$ day) (RTI 1988a). Within 2 weeks, 7/62 died and the dose was eliminated from the study. In other studies, no deaths were observed in rats given doses up to $92 \mathrm{mg} \mathrm{Ni} / \mathrm{kg}$ as nickel chloride in drinking water for 15 days (RTI 1985), $28.8 \mathrm{mg} \mathrm{Ni} / \mathrm{kg} /$ day as nickel sulfate in drinking water for 13 weeks (Obone et al. 1999), or $22 \mathrm{mg} \mathrm{Ni} / \mathrm{kg} /$ day (males) or $33 \mathrm{mg}$ $\mathrm{Ni} / \mathrm{kg} /$ day (females) as nickel sulfide administered via gavage for 90 days (Springborn Laboratories 2002); no deaths were observed in mice provided with nickel sulfate in the drinking water at doses up to $150 \mathrm{mg} \mathrm{Ni} / \mathrm{kg} /$ day for 180 days (Dieter et al. 1988).

In a multigeneration study (RTI 1988a, 1988b) in which rats were treated with nickel chloride in the drinking water, the death of female rats from pregnancy complications at the time of delivery suggests that females are more susceptible to nickel toxicity during parturition. Although the number of deaths was not significantly above controls and not clearly dose related $\left(\mathrm{P}_{0}: 0 / 31\right.$ in controls, $1 / 31$ at $7 \mathrm{mg} / \mathrm{kg} / \mathrm{day}, 3 / 30$ at $30 \mathrm{mg} / \mathrm{kg} / \mathrm{day}$, and $3 / 31$ at $55 \mathrm{mg} / \mathrm{kg} /$ day; $\mathrm{F}_{1}: 0 / 30$ at 0 and $7 \mathrm{mg} / \mathrm{kg} / \mathrm{day}, 3 / 30$ at $30 \mathrm{mg} / \mathrm{kg} / \mathrm{day}$, and $1 / 30$ at $55 \mathrm{mg} / \mathrm{kg} /$ day), death in dams during delivery is a relatively rare event. The results of this study (RTI 1988a, 1988b) are confounded by a decrease in food and water intake observed 
in the exposed animals. Deaths in offspring before weaning have also been reported in multigeneration, multilitter studies (RTI 1988a, 1988b; Schroeder and Mitchener 1971; Smith et al. 1993). Because crossfostering studies have not been completed, it is not possible to know if the pre-weaning deaths are a result of an inherent defect in the pups, nickel exposure through the milk, or a change in the quality or quantity of the milk produced by the dam (Smith et al. 1993).

An increase in mortality was not observed in chronic studies in rats or dogs fed nickel sulfate in the diet at doses up to $188 \mathrm{mg} / \mathrm{kg} / \mathrm{day}$ for rats and $62.5 \mathrm{mg} / \mathrm{kg} / \mathrm{day}$ for dogs (Ambrose et al. 1976). In mice provided with $0.95 \mathrm{mg} / \mathrm{Ni} / \mathrm{kg}$ as nickel acetate in drinking water until death (last death at 991 days for males and 904 days for females), an increase in life expectancy was observed (Schroeder and Mitchener 1975).

Oral $\mathrm{LD}_{50}$ values and all LOAEL values from each reliable study for death in each species and duration category are recorded in Table 3-8 and plotted in Figure 3-2.

\subsubsection{Systemic Effects}

The highest NOAEL values and all LOAEL values from each reliable study for systemic effects for each species, duration category, and nickel compound are recorded in Table 3-8 and plotted in Figure 3-2.

Respiratory Effects. No studies were located regarding respiratory effects in humans after oral exposure to nickel.

Pneumonitis was observed in 6/19 male rats and 9/17 female rats treated for 91 days by gavage with $8.6 \mathrm{mg} \mathrm{Ni} / \mathrm{kg} / \mathrm{day}$ as nickel chloride (American Biogenics Corporation 1988). Significant increases in absolute and relative lung weights were observed in rats exposed to $28.8 \mathrm{mg} \mathrm{Ni} / \mathrm{kg} /$ day as nickel sulfate in drinking water for 13 weeks (Obone et al. 1999). This study also found alterations in enzyme activity in bronchoalveolar lavage (BAL) fluid and lung tissues, including increases in protein levels in BAL fluid at $14.4 \mathrm{mg} \mathrm{Ni} / \mathrm{kg} /$ day and higher, decreases in alkaline phosphatase activity in BAL fluid at $5.75 \mathrm{mg}$ $\mathrm{Ni} / \mathrm{kg} /$ day and higher, and decrease in alkaline phosphatase activity in lung tissue at $28.8 \mathrm{mg} \mathrm{Ni} / \mathrm{kg} / \mathrm{day}$. No histological alterations were observed in the lungs. The study authors suggested that the decrease in alkaline phosphatase activity was indicative of decreased activity of type II alveolar cells and the increased total protein was indicative of increased air-blood barrier permeability. In a multigeneration study (RTI 1988a, 1988b), increased lung weights were observed in rats provided with nickel chloride in 
Table 3-8 Levels of Significant Exposure to Nickel - Oral

\begin{tabular}{|c|c|c|c|c|c|c|c|}
\hline \multirow[b]{2}{*}{$\begin{array}{l}\text { Key to } \\
\text { Figure }\end{array}$} & \multirow[b]{2}{*}{$\begin{array}{l}\text { Species } \\
\text { (Strain) }\end{array}$} & \multirow{2}{*}{$\begin{array}{l}\text { Exposure/ } \\
\text { Duration/ } \\
\text { Frequency } \\
\text { (Route) }\end{array}$} & \multirow[b]{2}{*}{ System } & \multirow[b]{2}{*}{$\begin{array}{l}\text { NOAEL } \\
\text { (mg/kg/day) }\end{array}$} & \multicolumn{2}{|c|}{ LOAEL } & \multirow[b]{2}{*}{$\begin{array}{l}\text { Reference } \\
\text { Chemical Form }\end{array}$} \\
\hline & & & & & $\begin{array}{l}\text { Less Serious } \\
\text { (mg/kg/day) }\end{array}$ & $\begin{array}{l}\text { Serious } \\
\text { (mg/kg/day) }\end{array}$ & \\
\hline
\end{tabular}

ACUTE EXPOSURE

Death

1 Rat once

(Fischer- 344) (G)

$120 \mathrm{M}$ (LD50) $\quad \begin{aligned} & \text { Haro et al. } 1968 \\ & \text { acetate }\end{aligned}$

2 Rat once

(Sprague- (G)

Dawley)

$46 \mathrm{M}$ (LD50)

Mastromatteo 1986

$39 \mathrm{~F}$ (LD50)

sulfate

$3 \quad$ Rat 14

(CD) $\quad(\mathrm{W})$

$140 \quad(7 / 64$ died $)$

RTI 1988a, 1988b

chloride

Haro et al. 1968

$136 \mathrm{M}(\mathrm{LD} 50)$

acetate

(Swiss- (G)

Webster)

\section{Systemic}

5 Human $2 \mathrm{~d}$

$2 \mathrm{~d} / \mathrm{d} \quad$ Dermal 0.03

(C)

$6 \quad$ Human

$\begin{aligned} & \text { once } \\ & \text { or } 1 \text { dose for } 2 \text { Dermal } \\ & \text { d }\end{aligned}$ 0.043 F $\quad 0.097 \mathrm{~F}$ (allergic dermatitis in
sensitized individuals)

(C)

7 Human once
(C)
Dermal
$0.014 \mathrm{~F}$
$0.057 \mathrm{~F}$ (dermatitis in nickel sensitive subjects)

Burrows et al. 1981

sulfate

Gawkrodger et al. 1986

sulfate

Hindsen et al. 2001

sulfate 
Table 3-8 Levels of Significant Exposure to Nickel - Oral

\begin{tabular}{|c|c|c|c|c|c|c|c|}
\hline \multirow[b]{2}{*}{$\begin{array}{l}\text { Key to } \\
\text { Figure }\end{array}$} & \multirow[b]{2}{*}{$\begin{array}{l}\text { Species } \\
\text { (Strain) }\end{array}$} & \multirow{2}{*}{$\begin{array}{l}\text { Exposure/ } \\
\text { Duration/ } \\
\text { Frequency } \\
\text { (Route) }\end{array}$} & \multirow[b]{2}{*}{ System } & \multirow[b]{2}{*}{$\begin{array}{l}\text { NOAEL } \\
\text { (mg/kg/day) }\end{array}$} & \multicolumn{2}{|c|}{ LOAEL } & \multirow[b]{2}{*}{$\begin{array}{l}\text { Reference } \\
\text { Chemical Form }\end{array}$} \\
\hline & & & & & $\begin{array}{l}\text { Less Serious } \\
\text { (mg/kg/day) }\end{array}$ & $\begin{array}{l}\text { Serious } \\
\text { (mg/kg/day) }\end{array}$ & \\
\hline
\end{tabular}

8 Human

once

(C)

$0.014 \mathrm{~F}$

$0.057 \mathrm{~F}$ (dermatitis in inickel

sensitive subjects)

$9 \quad$ Human $1 \mathrm{~d}$

(W)

Gastro

7.1 M (vomiting, cramps,
diarrhea)

10 Dog

3 days

Gastro

25

62.5 (vomiting)

Neurological

11 Human $1 \mathrm{~d}$

Reproductive

12 Mouse once

(lacca) (GW)

Developmental

13 Mouse

(CD-1)

Gd 8-12

$1 \mathrm{x} / \mathrm{da}$

(G)

14 Mouse Gd 8-12

(GW)

\section{INTERMEDIATE EXPOSURE}

Death

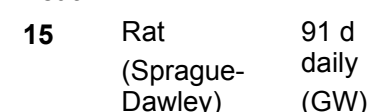

$91 \mathrm{~d}$
daily

GW)
7.1 M (giddiness, headache weariness)
$23 \mathrm{M}$ (3.7-fold increase in
sperm head

abnormalities)

45.3

90.6
Jensen et al. 2003

sulfate

Sunderman et al. 1988

sulfide/chloride

Ambrose et al. 1976

sulfate

Sunderman et al. 1988

sulfate/chloride

Sobti and Gill 1989

nitrate

Gray et al. 1986

chloride

Seidenberg et al. 1986

chloride

American Biogenics Corp 1988

chloride 
Table 3-8 Levels of Significant Exposure to Nickel - Oral

\begin{tabular}{|c|c|c|c|c|c|c|c|}
\hline \multirow[b]{2}{*}{$\begin{array}{l}\text { Key to } \\
\text { Figure }\end{array}$} & \multirow[b]{2}{*}{$\begin{array}{l}\text { Species } \\
\text { (Strain) }\end{array}$} & \multirow{2}{*}{$\begin{array}{c}\text { Exposure/ } \\
\text { Duration/ } \\
\text { Frequency } \\
\text { (Route) }\end{array}$} & \multirow[b]{2}{*}{ System } & \multirow[b]{2}{*}{$\begin{array}{l}\text { NOAEL } \\
(\mathrm{mg} / \mathrm{kg} / \text { day })\end{array}$} & \multicolumn{2}{|c|}{ LOAEL } & \multirow[b]{2}{*}{$\begin{array}{l}\text { Reference } \\
\text { Chemical Form }\end{array}$} \\
\hline & & & & & $\begin{array}{r}\text { Less Serious } \\
\text { (mg/kg/day) }\end{array}$ & $\begin{array}{l}\text { Serious } \\
\text { (mg/kg/day) }\end{array}$ & \\
\hline
\end{tabular}

\section{Systemic}

16 Human

$\begin{array}{lll}91-178 \mathrm{~d} & \text { Dermal } & 0.02 \mathrm{~F}\end{array}$

$02 \mathrm{~F}$

17 Rat $91 \mathrm{~d}$

(Sprague- daily

Dawley)

(GW)

Resp
Gastro

8.6

Hemato $\quad 1.2 \mathrm{~F}$

Hepatic $\quad 8.6$

Renal $\quad 8.6$

Dermal $\quad 8.6$

Ocular $\quad 8.6$

$\mathrm{Bd} \mathrm{Wt} \quad 1.2$

Metab

$1.2 \mathrm{~F}$

8.6 F ( $12 \%$ decrease in body weight gain)

$1.2 \mathrm{~F}$$$
\text { weight gain) }
$$

8.6 F (decreased blood
8.6 (pneumonitis)

8.6 F (increased platelet count) glucose level)
Santucci et al. 1994

sulfate

American Biogenics Corp 1988

chloride
25 (ulcerative gastritis and enteritis) 
Table 3-8 Levels of Significant Exposure to Nickel - Oral

\begin{tabular}{|c|c|c|c|c|c|c|c|}
\hline \multirow[b]{2}{*}{$\begin{array}{l}\text { Key to } \\
\text { Figure }\end{array}$} & \multirow[b]{2}{*}{$\begin{array}{l}\text { Species } \\
\text { (Strain) }\end{array}$} & \multirow{2}{*}{$\begin{array}{l}\text { Exposure/ } \\
\text { Duration/ } \\
\text { Frequency } \\
\text { (Route) }\end{array}$} & \multirow[b]{2}{*}{ System } & \multirow[b]{2}{*}{$\begin{array}{l}\text { NOAEL } \\
\text { (mg/kg/day) }\end{array}$} & \multicolumn{2}{|c|}{ LOAEL } & \multirow[b]{2}{*}{$\begin{array}{l}\text { Reference } \\
\text { Chemical Form }\end{array}$} \\
\hline & & & & & $\begin{array}{r}\text { Less Serious } \\
(\mathrm{mg} / \mathrm{kg} / \mathrm{day})\end{array}$ & $\begin{array}{l}\text { Serious } \\
\text { (mg/kg/day) }\end{array}$ & \\
\hline
\end{tabular}

18 Rat

$\begin{array}{ll}\text { Rat } & \text { daily } \\ \text { (Sprague- } & 13 \text { weeks } \\ \text { Dawley) } & \text { (W) }\end{array}$

Dawley) (W)

$\begin{array}{lll}\text { Rat } & F: 27-30 w k & \\ \text { (CD) } & M: 21-24 w k & \text { Resp } \\ & \text { (W) }\end{array}$

20
Resp

(mg/kg/day)

Chemical Form

Obone et al. 1999

sulfate

phosphatase activity in

bronchioalveolar lavage

fluid)

$\begin{array}{ll}\text { Cardio } & 28.8 \mathrm{M} \\ \text { Gastro } & 28.8 \mathrm{M} \\ \text { Hepatic } & 28.8 \mathrm{M} \\ \text { Renal } & 5.75 \mathrm{M}\end{array}$

14.4 M (increased realtive kidney

weight, decreased urine

volume and urine

glucose)

RTI 1988a, 1988b

chloride

infiltration in lungs in $\mathrm{F}$ 1

generation)

Smith et al. 1993

chloride 
Table 3-8 Levels of Significant Exposure to Nickel - Oral

\begin{tabular}{|c|c|c|c|c|}
\hline $\begin{array}{l}\text { Key to } \\
\text { Figure }\end{array}$ & $\begin{array}{l}\text { Species } \\
\text { (Strain) }\end{array}$ & $\begin{array}{l}\text { Exposure/ } \\
\text { Duration/ } \\
\text { Frequency } \\
\text { (Route) }\end{array}$ & System & $\begin{array}{r}\text { NOAE } \\
(\mathrm{mg} / \mathrm{kg} /\end{array}$ \\
\hline \multirow[t]{5}{*}{21} & $\begin{array}{l}\text { Rat } \\
\text { (Sprague- } \\
\text { Dawley) }\end{array}$ & $\begin{array}{l}\text { daily } \\
18 \text { weeks } \\
\text { (GW) }\end{array}$ & Cardio & 2.2 \\
\hline & & & Gastro & 2.2 \\
\hline & & & Hepatic & 2.2 \\
\hline & & & Renal & 2.2 \\
\hline & & & $\mathrm{Bd} \mathrm{Wt}$ & 2.2 \\
\hline
\end{tabular}

22 Rat Daily

LOAEL

Reference

Chemical Form

Daily Resp

(GW)

Cardio $\quad 22 \mathrm{M}$

Gastro $\quad 22 \mathrm{M}$

Hepatic $22 \mathrm{M}$

Renal $22 \mathrm{M}$

Endocr $22 \mathrm{M}$

Bd Wt $\quad 11 \mathrm{M} \quad 17 \mathrm{M}(12.2 \%$ decrease in final

body weight)

23 Rat

3 or 6 mo

(Wistar)

(W)

Renal

7.6 $\mathrm{F}$ (increased urinary

albumin)

Springborn Laboratories 2000a

sulfate

Springborn Laboratories 2002

sulfate

Vyskocil et al. 1994b

sulfate 
Table 3-8 Levels of Significant Exposure to Nickel - Oral

\begin{tabular}{|c|c|c|c|c|c|c|c|}
\hline \multirow[b]{2}{*}{$\begin{array}{l}\text { Key to } \\
\text { Figure }\end{array}$} & \multirow[b]{2}{*}{$\begin{array}{l}\text { Species } \\
\text { (Strain) }\end{array}$} & \multirow{2}{*}{$\begin{array}{l}\text { Exposure/ } \\
\text { Duration/ } \\
\text { Frequency } \\
\text { (Route) }\end{array}$} & \multirow[b]{2}{*}{ System } & \multirow[b]{2}{*}{$\begin{array}{l}\text { NOAEL } \\
(\mathrm{mg} / \mathrm{kg} / \text { day })\end{array}$} & \multicolumn{2}{|c|}{ LOAEL } & \multirow[b]{2}{*}{$\begin{array}{l}\text { Reference } \\
\text { Chemical Form }\end{array}$} \\
\hline & & & & & $\begin{array}{r}\text { Less Serious } \\
\text { (mg/kg/day) }\end{array}$ & $\begin{array}{l}\text { Serious } \\
\text { (mg/kg/day) }\end{array}$ & \\
\hline
\end{tabular}

24 Rat (OSU brown) (F)

6 wk (OSU brown)

$\mathrm{Bd} W \mathrm{t}$

$5 \mathrm{M}$

25

$\begin{array}{ll}\text { Mouse } & 180 \\ \text { (B6C3F1) } & \text { daily }\end{array}$
$180 \mathrm{~d}$
daily

Hepatic

$150 \mathrm{~F}$

(W)

Renal

$44 \mathrm{~F}$

$108 \mathrm{~F}$ (hyaline casts, loss of tubular epithelial cells)

Bd Wt

$44 \mathrm{~F}$

$108 \mathrm{~F}$ (body weight $10 \%$ lower than controls)

$5.75 \mathrm{M}$

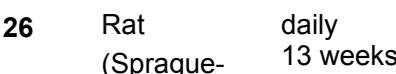

Dawley) (W)

27 Mouse

(B6C3F1) daily

(W)
14.4 M (alterations in spleen and thymus lymphocyte T-cell and B-cell
subpopulations)

$44 \mathrm{~F}$ (mild thymic atrophy, impaired B-cell immune function, decreased

granulocyte macrophage

progenitor cell levels)
Whanger 1973

acetate

$25 \mathrm{M}(88 \%$ decrease in body weight gain)

Dieter et al. 1988

sulfate

Obone et al. 1999

sulfate

Dieter et al. 1988

sulfate 
Table 3-8 Levels of Significant Exposure to Nickel - Oral

\begin{tabular}{lll}
\hline Key to & & $\begin{array}{c}\text { Exposure/ } \\
\text { Duration/ } \\
\text { Figure } \\
\text { Frequency } \\
\text { (Route) }\end{array}$ \\
\hline (Strain) & & \\
28 & $\begin{array}{l}\text { Mouse } \\
\text { (BALB/c) }\end{array}$ & $\begin{array}{l}10-11 \mathrm{wk} \\
\text { (W) }\end{array}$
\end{tabular}

Neurological

29 Rat

$\begin{array}{ll}\text { Rat } & 91 \mathrm{~d} \\ \text { (Sprague- } & \text { daily } \\ \text { Dawley) } & \text { (GW) }\end{array}$

\section{Reproductive}

30 Rat

(Wistar)

about 24 wk

$(\mathrm{F})$

90

31 Rat

(Wistar) 62 days

(W)

$32 \quad$ Rat

(Wistar) 28 or 42 days

(W)

$33 \quad$ Rat

daily

(Wistar) 28--76 days

(W)

$34 \quad$ Rat

daily

(Sprague- 13 wee

Dawley) (W)
20.3 F (enhanced inflammatory

response in the hearts of

mice challenged with

coxsackie virus B3)

8.6 (ataxia, prostation, hypothermia)

American Biogenics Corp 1988

Ambrose et al. 1976

3.6 M (decreased fertility)

3.6 (decreased fertility)

$28.8 \mathrm{M}$ chloride

sulfate

Kakela et al. 1999

chloride

llback et al. 1994

chloride

Kakela et al. 1999

chloride

Kakela et al. 1999

chloride

Obone et al. 1999

sulfate 
Table 3-8 Levels of Significant Exposure to Nickel - Oral

\begin{tabular}{|c|c|c|c|c|c|c|c|}
\hline \multirow[b]{2}{*}{$\begin{array}{l}\text { Key to } \\
\text { Figure }\end{array}$} & \multirow[b]{2}{*}{$\begin{array}{l}\text { Species } \\
\text { (Strain) }\end{array}$} & \multirow{2}{*}{$\begin{array}{l}\text { Exposure/ } \\
\text { Duration/ } \\
\text { Frequency } \\
\text { (Route) }\end{array}$} & \multirow[b]{2}{*}{ System } & \multirow[b]{2}{*}{$\begin{array}{l}\text { NOAEL } \\
(\mathrm{mg} / \mathrm{kg} / \text { day })\end{array}$} & \multicolumn{2}{|c|}{ LOAEL } & \multirow[b]{2}{*}{$\begin{array}{l}\text { Reference } \\
\text { Chemical Form }\end{array}$} \\
\hline & & & & & $\begin{array}{r}\text { Less Serious } \\
\text { (mg/kg/day) }\end{array}$ & $\begin{array}{l}\text { Serious } \\
\text { (mg/kg/day) }\end{array}$ & \\
\hline
\end{tabular}

$35 \quad$ Rat $\quad F: 27-30 w k$

(CD) M:21-24 wk

(W)

$36 \quad$ Rat

(Long- Evans)
lactation

2 litters

(W)

$37 \quad$ Rat

(Sprague- 18 weeks

Dawley) (GW)

$38 \quad$ Rat

$\begin{array}{ll}\text { Rat } & \text { daily } \\ \text { (Sprague- } & 35-56 \text { days } \\ \text { Dawley) } & (\mathrm{GW})\end{array}$

39 Mouse 5 days/week

(NS) $\quad 35$ days

(GW)

$40 \quad$ Mouse 5 days/week

(NS) $\quad 35$ days

(GW)

\section{F $\quad 30 \mathrm{~F}$ (increased gestation \\ length in first PO \\ pregnancy)}

31.6

$1.1 \mathrm{M}$

2.2 M (decreased sperm

motility; increased sperm

abnormalities)

$1.2 \mathrm{M}$

$2.5 \mathrm{M}$ (decreased sperm

motility and count;

increased sperm

abnormalities)
RTI 1988a, 1988b

chloride

Smith et al. 1993

chloride

Springborn Laboratories 2000a

sulfate

Springborn Laboratories 2000b

sulfate

Pandey and Srivastava 2000

sulfate

Pandey and Srivastava 2000

chloride 
Table 3-8 Levels of Significant Exposure to Nickel - Oral

\begin{tabular}{lll}
\hline Key to & & $\begin{array}{c}\text { Exposure/ } \\
\text { Duration/ } \\
\text { Figure } \\
\text { Frequency } \\
\text { (Route) }\end{array}$ \\
\hline & & \\
41 & Mouse & \\
& (Swiss) & $\begin{array}{l}5 \text { days/week } \\
35 \text { days } \\
\text { (GW) }\end{array}$ \\
& & \\
42 & $\begin{array}{l}\text { Mouse } \\
\text { (Swiss) }\end{array}$ & $\begin{array}{l}5 \text { days/week } \\
35 \text { days } \\
\text { (GW) }\end{array}$ \\
& &
\end{tabular}

LOAEL

Reference

Chemical Form

\section{Developmental}

43 Rat

about 24 wk

(Wistar)

(F)

44 Rat

(Wistar) 62 days

$4 \mathrm{~F}$

(W)

45 Rat daily

(Wistar) 28 or 42 days

(W)

$46 \quad$ Rat

daily

(Wistar) 28--76 days

(W)

NOAEL
System $\quad(\mathrm{mg} / \mathrm{kg})$

Less Serious
(mg/kg)

(mg/kg)

Pandey et al. 1999

sulfate

(increased total number

2.2 M (decreased fertility)

Pandey et al. 1999

sulfate

22.5 (increased number of stillborns)

Ambrose et al. 1976

sulfate

13 F (decreased litter size and Kakela et al. 1999 pup survival)

chloride

3.6 M (decreased pup viability and survival)

Kakela et al. 1999

chloride

3.6 (increased fetal mortality and decreased pup

Kakela et al. 1999

survival) 
Table 3-8 Levels of Significant Exposure to Nickel - Oral

\begin{tabular}{lll}
\hline Key to & Species & $\begin{array}{c}\text { Exposure/ } \\
\text { Duration/ } \\
\text { Figure } \\
\text { Frequency } \\
\text { (Route) }\end{array}$ \\
\hline & & \\
47 & Rat & F: $27-30 \mathrm{wk}$ \\
& (CD) & $\begin{array}{l}\text { M:21-24 wk } \\
\text { (W) }\end{array}$
\end{tabular}

(W)

$48 \quad$ Rat

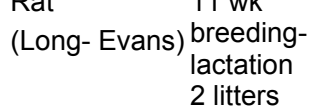

(W)

$49 \quad$ Rat

(Sprague- 18 weeks

Dawley) (GW)

50 Rat

$\begin{array}{ll}\text { Rat } & \text { daily } \\ \text { (Sprague- } & 49-70 \text { days }\end{array}$

Dawley) (GW)

$51 \quad$ Mouse $\quad$ Gd 2-17

(CD-1) (W)
LOAEL

NOAEL

Less Serious

(mg/kg/day)

System

(mg/kg/day)

80
$30 \mathrm{M}$ (increased mortality in

$\mathrm{F} 1 \mathrm{~b}$ rats on pnd 22-42;

decreased pup body

weight in $\mathrm{F} 1 \mathrm{~b}$ rats)

1.3 (decreased pup survival)

Smith et al. 1993

chloride

Reference

Chemical Form

RTI 1988a, 1988b

chloride

Springborn Laboratories 2000a

sulfate

Springborn Laboratories 2000b

$$
\text { post-implantation loss) sulfate }
$$

160 (increased spontaneous

Berman and Rehnberg 1983

chloride 
Table 3-8 Levels of Significant Exposure to Nickel - Oral

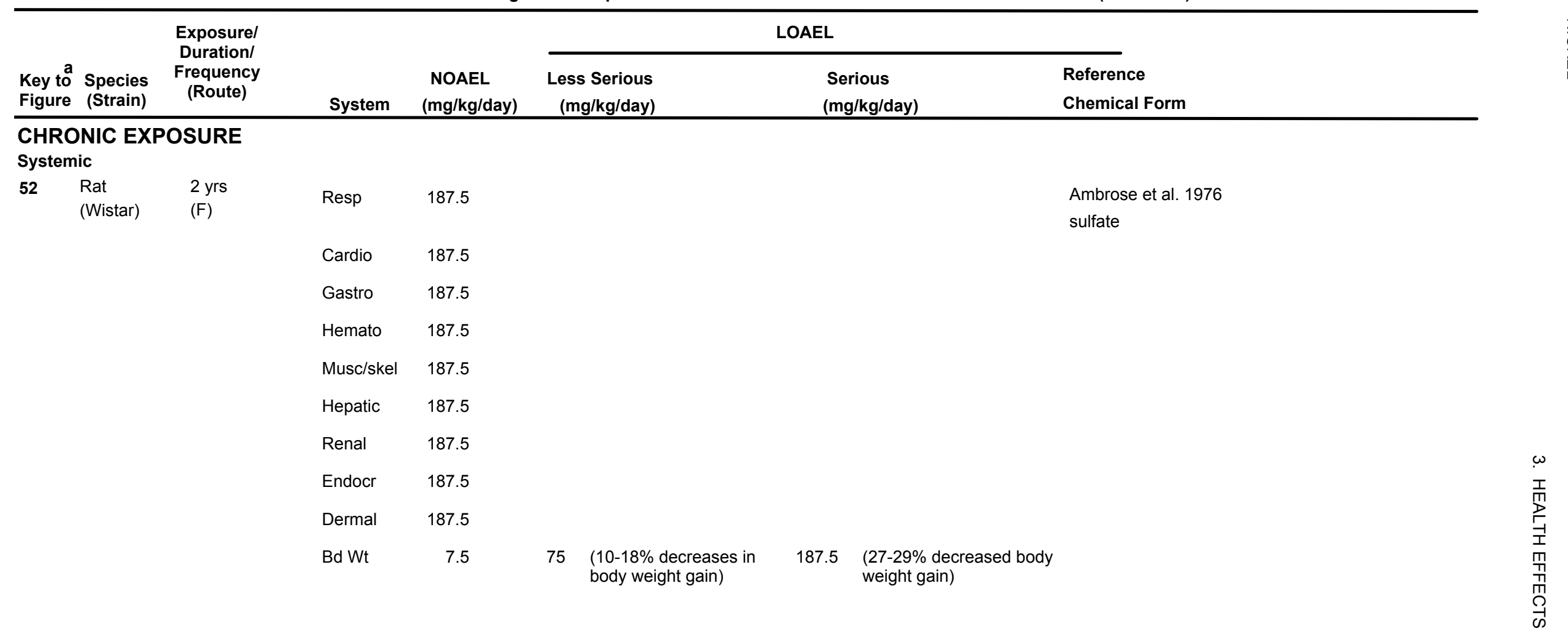


Table 3-8 Levels of Significant Exposure to Nickel - Oral

\begin{tabular}{|c|c|c|c|c|c|c|c|}
\hline \multirow[b]{2}{*}{$\begin{array}{l}\text { Key to } \\
\text { Figure }\end{array}$} & \multirow[b]{2}{*}{$\begin{array}{l}\text { Species } \\
\text { (Strain) }\end{array}$} & \multirow{2}{*}{$\begin{array}{l}\text { Exposure/ } \\
\text { Duration/ } \\
\text { Frequency } \\
\text { (Route) }\end{array}$} & \multirow[b]{2}{*}{ System } & \multirow[b]{2}{*}{$\begin{array}{l}\text { NOAEL } \\
\text { (mg/kg/day) }\end{array}$} & \multicolumn{2}{|c|}{ LOAEL } & \multirow[b]{2}{*}{$\begin{array}{l}\text { Reference } \\
\text { Chemical Form }\end{array}$} \\
\hline & & & & & $\begin{array}{l}\text { Less Serious } \\
\text { (mg/kg/day) }\end{array}$ & $\begin{array}{l}\text { Serious } \\
\text { (mg/kg/day) }\end{array}$ & \\
\hline
\end{tabular}

53 Dog 2 yrs

(Beagle) (F)

Resp

25

Cardio $\quad 625$

Gastro $\quad 62.5$

Hemato

25

Musc/skel $\quad 62.5$

Hepatic $\quad 62.5$

Renal 25

Endocr $\quad 62.5$

Dermal $\quad 62.5$

Bd Wt $\quad 25$
62.5 (decreased hematocrit and hemoglobin levels) $62.5 \begin{array}{ll}\text { (cholesterol granulomas, } \\ \text { emphysema, } \\ \text { bronchiolectasis) }\end{array}$ Ambrose et al. 1976

Immuno/ Lymphoret

$\begin{array}{lllll}\mathbf{5 4} & \begin{array}{l}\text { Rat } \\ \text { (Wistar) }\end{array} & \begin{array}{l}2 \text { yrs } \\ \text { (F) }\end{array} & 187.5 & \text { Ambrose et al. } 1976 \\ \text { sulfate }\end{array}$

$2.5 \quad(10 \%$ decrease in body weight gain)

62.5 (polyuria in 2/6 dogs,

increased kidney weight)

sulfate 
Table 3-8 Levels of Significant Exposure to Nickel - Oral

\begin{tabular}{|c|c|c|c|c|c|c|c|}
\hline \multirow[b]{2}{*}{$\begin{array}{l}\text { Key to } \\
\text { Figure }\end{array}$} & \multirow[b]{2}{*}{$\begin{array}{l}\text { Species } \\
\text { (Strain) }\end{array}$} & \multirow{2}{*}{$\begin{array}{c}\text { Exposure/ } \\
\text { Duration/ } \\
\text { Frequency } \\
\text { (Route) }\end{array}$} & \multirow[b]{2}{*}{ System } & \multirow[b]{2}{*}{$\begin{array}{l}\text { NOAEL } \\
(\mathrm{mg} / \mathrm{kg} / \text { day })\end{array}$} & \multicolumn{2}{|c|}{ LOAEL } & \multirow[b]{2}{*}{$\begin{array}{l}\text { Reference } \\
\text { Chemical Form }\end{array}$} \\
\hline & & & & & $\begin{array}{r}\text { Less Serious } \\
\text { (mg/kg/day) }\end{array}$ & $\begin{array}{l}\text { Serious } \\
\text { (mg/kg/day) }\end{array}$ & \\
\hline 57 & $\begin{array}{l}\text { Dog } \\
\text { (Beagle) }\end{array}$ & $\begin{array}{l}2 \mathrm{yrs} \\
(\mathrm{F})\end{array}$ & & 62.5 & & & $\begin{array}{l}\text { Ambrose et al. } 1976 \\
\text { sulfate }\end{array}$ \\
\hline
\end{tabular}

The number corresponds to entries in Figure 3-2.

Bd Wt = body weight; $(C)=$ capsule; Cardio = cardiovascular; $d=$ day $(\mathrm{s}) ;$ Endocr $=$ endocrine; $(F)=$ feed; $F=$ Female; $(G)=$ gavage; Gastro = gastrointestinal; gd = gestational day; $(\mathrm{GW})=$ gavage in water; hemato = hematological; Immuno = immunological; LD50 = lethal dose, $50 \%$ kill; LOAEL = lowest-observed-adverse-effect level; $\mathrm{M}=$ male; $\mathrm{mo}=$ month $(\mathrm{s}) ;$

Musc/skel $=$ musculoskeletal; $\mathrm{Ni}=$ nickel; $\mathrm{NOAEL}=$ no-observed-adverse-effect level; Resp = respiratory;

$\mathrm{x}=$ time $(\mathrm{s}) ;(\mathrm{W})=$ drinking water; $\mathrm{wk}=$ week $(\mathrm{s}) ; \mathrm{yr}=$ year(s) 
Figure 3-2 Levels of Significant Exposure to Nickel - Oral

Acute ( $\leq 14$ days)

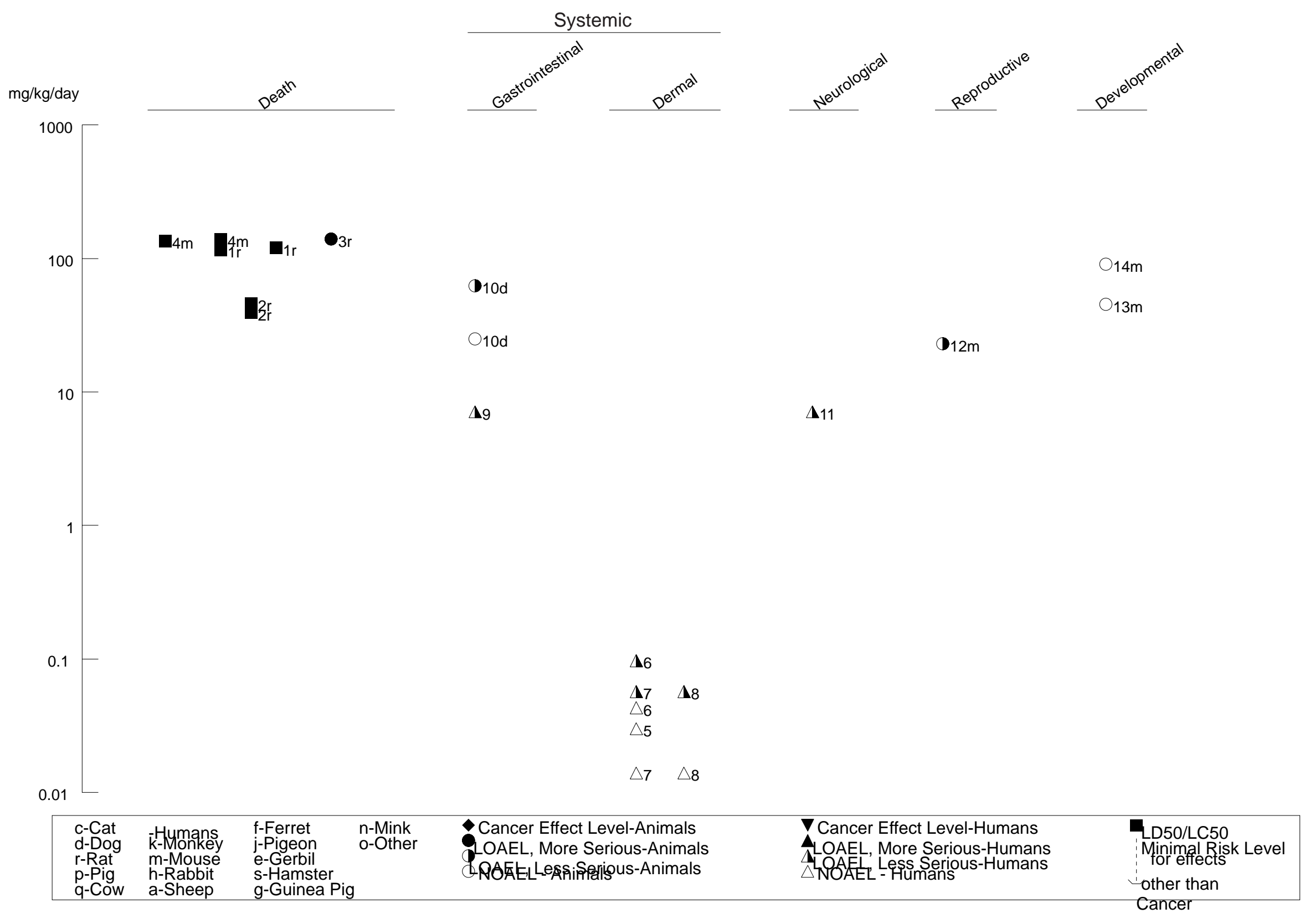


Figure 3-2 Levels of Significant Exposure to Nickel - Oral Intermediate (15-364 days)

Systemic

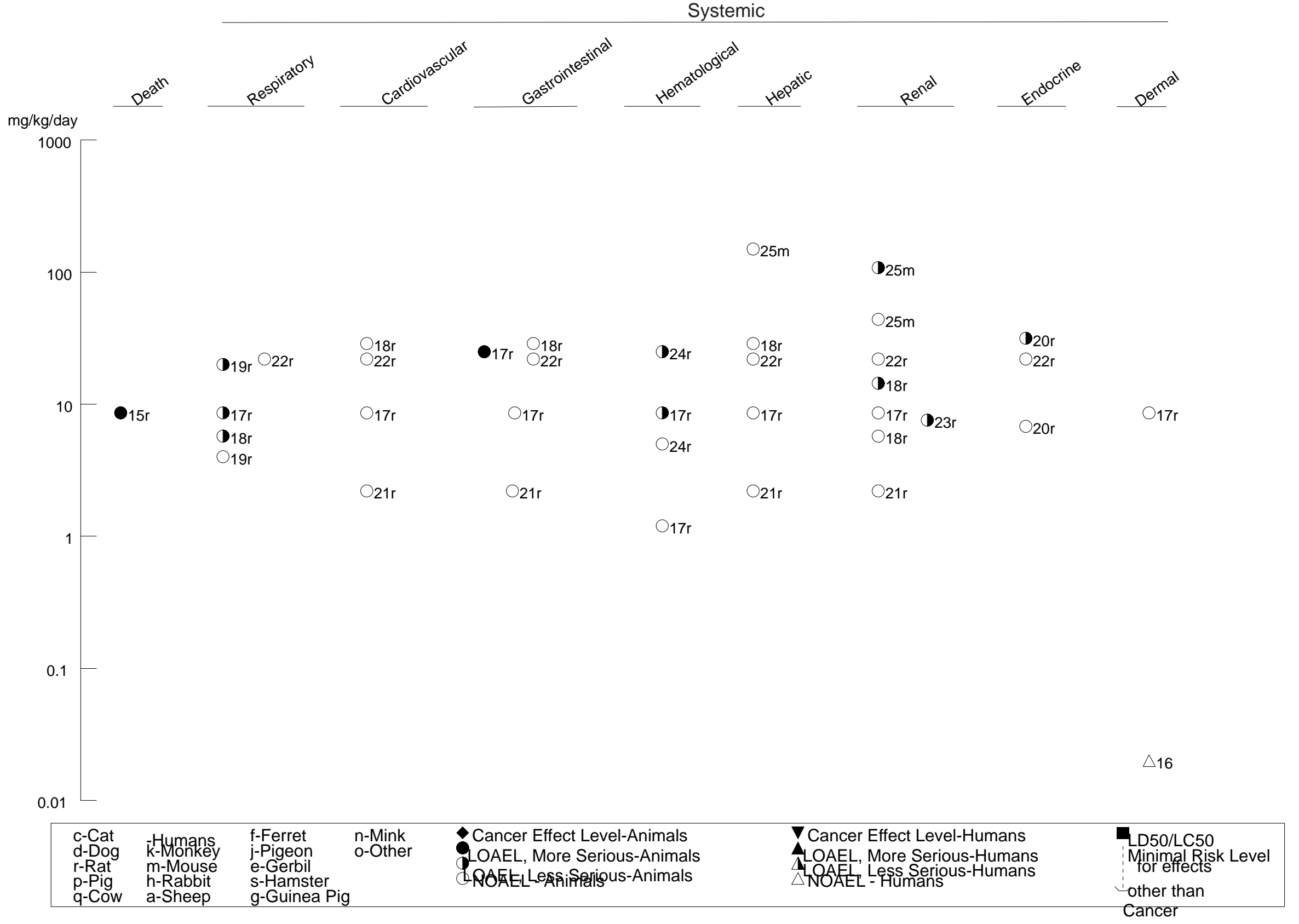


Figure 3-2 Levels of Significant Exposure to Nickel - Oral Intermediate (15-364 days)

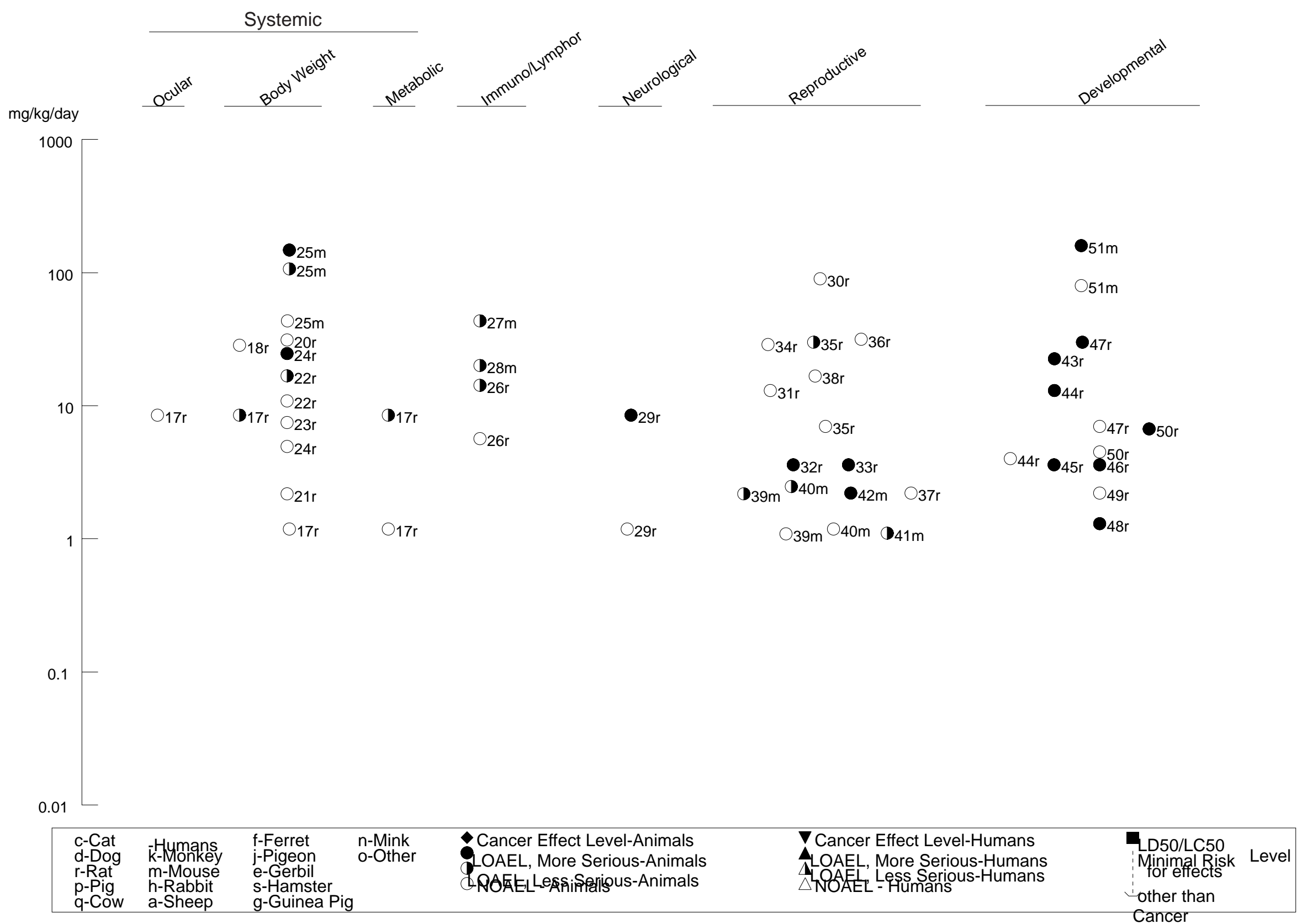


Figure 3-2 Levels of Significant Exposure to Nickel - Oral

Chronic ( $\geq 365$ days)

\section{Systemic}

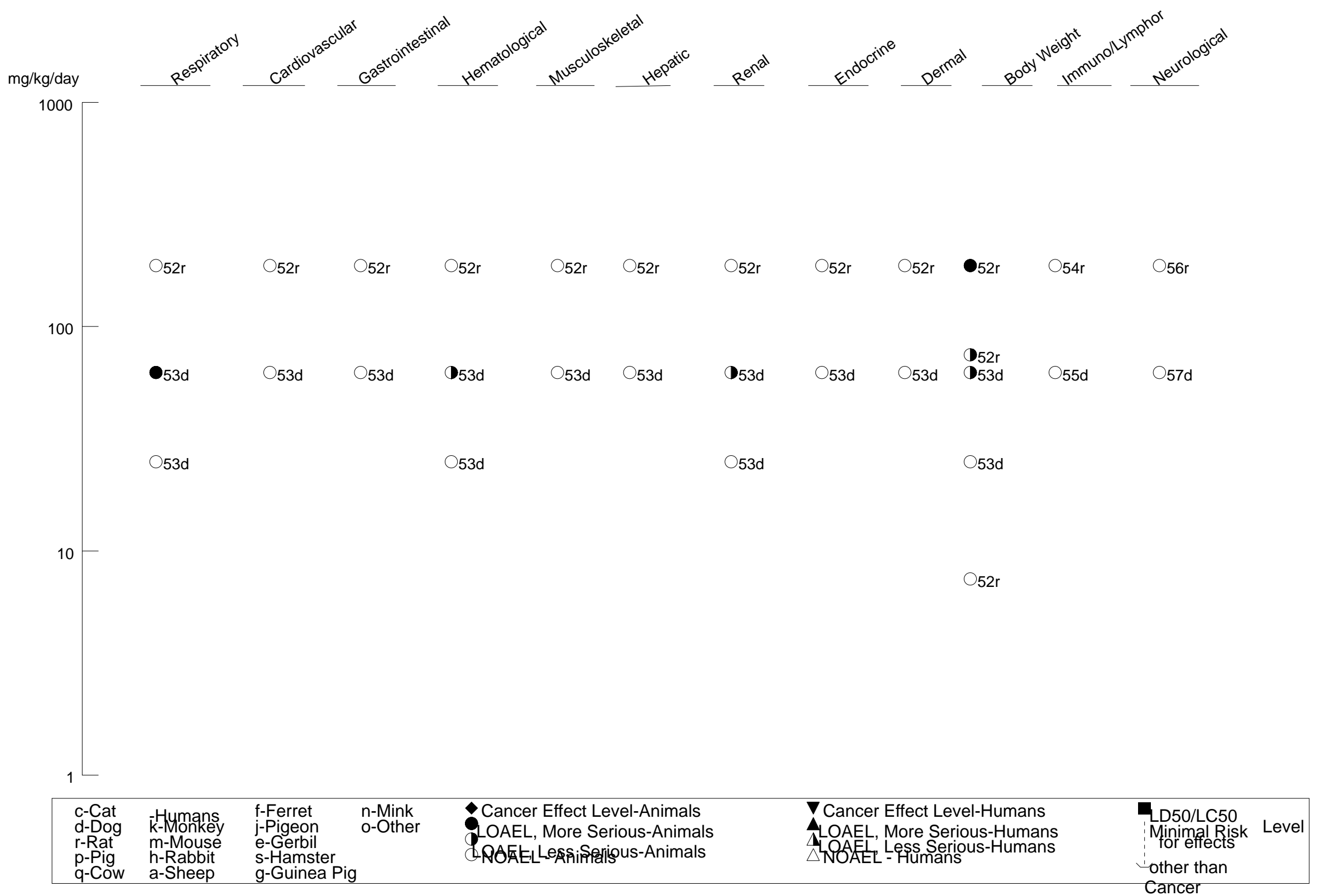


the drinking water at $55 \mathrm{mg} \mathrm{Ni} / \mathrm{kg} / \mathrm{day}$, and an increase in cellular infiltration of the lungs was observed at $20 \mathrm{mg} \mathrm{Ni} / \mathrm{kg} / \mathrm{day}$. This study is confounded by decreased food and water intake observed in exposed animals. Emphysema, bronchiolectasis, and cholesterol granulomas were also observed in dogs exposed to $62.5 \mathrm{mg} \mathrm{Ni} / \mathrm{kg} /$ day as nickel sulfate in the diet for 2 years, but not in rats exposed at up to $187.5 \mathrm{mg} / \mathrm{kg} /$ day for 2 years (Ambrose et al. 1976).

Cardiovascular Effects. Nickel sulfate crystals (rough estimate of $570 \mathrm{mg} \mathrm{Ni} / \mathrm{kg}$ ) were accidentally ingested by a 2-year-old child (Daldrup et al. 1983). Four hours after ingestion, cardiac arrest occurred, and the child died 8 hours after exposure.

Rats exposed to $8.6 \mathrm{mg} \mathrm{Ni} / \mathrm{kg} /$ day as nickel chloride for 91 days had decreased heart weight (American Biogenics Corporation 1988), whereas rats exposed to $75 \mathrm{mg} \mathrm{Ni} / \mathrm{kg} /$ day as nickel sulfate for 2 years had increased heart weight (Ambrose et al. 1976). Because the changes in heart weight were not accompanied by histological changes and decreases in body weight gain were also observed, the significance of these changes is not known. Histological changes in the heart were not observed in rats treated with nickel chloride in the drinking water at $40 \mathrm{mg} / \mathrm{kg} / \mathrm{day}$ for up to 30 weeks (RTI 1988a), rats exposed to $28.8 \mathrm{mg}$ $\mathrm{Ni} / \mathrm{kg} /$ day as nickel sulfate in drinking water (Obone et al. 1999), rats exposed to $187.5 \mathrm{mg} \mathrm{Ni} / \mathrm{kg} / \mathrm{day}$ as nickel sulfate in the diet for 2 years (Ambrose et al. 1976), rats administered via gavage $22 \mathrm{mg} \mathrm{Ni} / \mathrm{kg} / \mathrm{day}$ (males) or $33 \mathrm{mg} \mathrm{Ni} / \mathrm{kg} /$ day (females) as nickel sulfate for 90 days (Springborn Laboratories 2002), or dogs provided with nickel sulfate in the diet at a dose of $62.5 \mathrm{mg} \mathrm{Ni} / \mathrm{kg} / \mathrm{day}$ for 2 years (Ambrose et al. 1976).

Gastrointestinal Effects. Symptoms of gastrointestinal distress were reported by workers who drank water during one work shift from a water fountain contaminated with nickel sulfate, nickel chloride, and boric acid (Sunderman et al. 1988). Thirty-five workers were exposed, 20 reported symptoms, and 10 were hospitalized. The workers who reported symptoms were exposed to an estimated dose of 7.1$35.7 \mathrm{mg} \mathrm{Ni} / \mathrm{kg}$. The symptoms included nausea (15 workers), abdominal cramps ( 14 workers), diarrhea (4 workers), and vomiting ( 3 workers). Although the actual contribution of boric acid to these effects is not known, the investigators (Sunderman et al. 1988) indicate that the intake of 20-200 $\mathrm{mg}$ boric acid probably did not contribute to the observed effects because the effects of boric acid are generally observed only following ingestion of $\geq 4 \mathrm{~g}$ by adults.

Gastrointestinal effects were observed in rats that died following treatment by gavage with $25 \mathrm{mg}$ $\mathrm{Ni} / \mathrm{kg} / \mathrm{day}$ as nickel chloride hexahydrate for up to 91 days (American Biogenics Corporation 1988). The 
effects included discolored gastrointestinal contents, ulcerative gastritis, and enteritis. Discolored (green) gastrointestinal contents were also observed at 1.2 and $8.6 \mathrm{mg} / \mathrm{kg} / \mathrm{day}$. The discoloration may have been due to the presence of nickel chloride in the gastrointestinal tract and is not considered an adverse effect. Adverse gastrointestinal effects were not observed in rats exposed to $28.8 \mathrm{mg} \mathrm{Ni} / \mathrm{kg} / \mathrm{day}$ as nickel sulfate in drinking water for 13 weeks (Obone et al. 1999), rats treated with nickel sulfate in the diet at $187.5 \mathrm{mg}$ $\mathrm{Ni} / \mathrm{kg} /$ day for 2 years (Ambrose et al. 1976), or rats receiving gavage doses of 22 (males) or 33 (females) $\mathrm{mg} \mathrm{Ni} / \mathrm{kg} /$ day as nickel sulfate (Springborn Laboratories 2002). During the first 3 days of a 2-year study, dogs vomited following treatment with nickel sulfate in the diet at $62.5 \mathrm{mg} \mathrm{Ni} / \mathrm{kg} /$ day (Ambrose et al. 1976). The dose was lowered to $37.5 \mathrm{mg} \mathrm{Ni} / \mathrm{kg} / \mathrm{day}$ for 2 weeks, and then incrementally raised at 2-week intervals back to $62.5 \mathrm{mg} / \mathrm{kg} / \mathrm{day}$, at which time, no further gastrointestinal distress was noted. These studies indicate that high doses of nickel can be irritating to the gastrointestinal tract, although acclimation to high levels of dietary nickel can occur. The toxicological significance of the results of the American Biogenics Corporation (1988) is not known, particularly since studies in rats (Ambrose et al. 1976; Obone et al. 1999; Springborn Laboratories 2002) have not reported gastrointestinal effects.

Hematological Effects. A transient increase in blood reticulocytes was observed in workers who were hospitalized after drinking water during one work shift from a water fountain contaminated with nickel sulfate, nickel chloride, and boric acid (Sunderman et al. 1988). Thirty-five workers were exposed, 20 reported symptoms, and 10 were hospitalized. The workers who reported symptoms were exposed to an estimated dose of 7.1-35.7 mg Ni/kg. The contribution of boric acid to these effects is not known.

Rat studies have indicated that intermediate-duration exposure to $\geq 0.7 \mathrm{mg} \mathrm{Ni} / \mathrm{kg} / \mathrm{day}$ as various nickel salts produce hematological effects. Effects included a decrease in hemoglobin level in rats exposed to $25 \mathrm{mg} \mathrm{Ni} / \mathrm{kg} /$ day as nickel acetate in the diet for 6 weeks (Whanger 1973), an increase in leukocyte levels in rats exposed to $0.49 \mathrm{mg} \mathrm{Ni} / \mathrm{kg} /$ day as nickel chloride in drinking water for 28 days, but not at $0.97 \mathrm{mg}$ $\mathrm{Ni} / \mathrm{kg} /$ day (Weischer et al. 1980), and an increase in platelet counts in rats administered via gavage $8.6 \mathrm{mg} \mathrm{Ni} / \mathrm{kg} /$ day as nickel chloride for 91 days (American Biogenics Corporation 1988). No hematological effects were observed in rats treated with nickel sulfate in the diet at a dose of $187.5 \mathrm{mg}$ $\mathrm{Ni} / \mathrm{kg} /$ day for 2 years (Ambrose et al. 1976). Low hematocrit levels were observed in dogs after chronic dietary exposure to $62.5 \mathrm{mg} \mathrm{Ni} / \mathrm{kg} /$ day as nickel sulfate (Ambrose et al. 1976).

Musculoskeletal Effects. Muscular pain was reported by one worker who drank water contaminated with nickel sulfate, nickel chloride, and boric acid during one work shift (Sunderman et al. 1988). Thirtyfive workers were exposed, 20 reported symptoms, and 10 were hospitalized. The workers who reported 
symptoms were exposed to an estimated dose of 7.1-35.7 $\mathrm{mg} \mathrm{Ni} / \mathrm{kg}$. The contribution of boric acid to these effects is not known.

Microscopic changes in skeletal muscle were not observed in rats or dogs fed nickel sulfate in the diet at doses up to $187.5 \mathrm{mg} \mathrm{Ni} / \mathrm{kg}$ /day for rats (Ambrose et al. 1976; Springborn Laboratories 2002) and $62.5 \mathrm{mg} \mathrm{Ni} / \mathrm{kg} /$ day for dogs (Ambrose et al. 1976).

Hepatic Effects. A transient increase in serum bilirubin levels was observed in 3 of 10 workers who were hospitalized after drinking water during one work shift from a water fountain contaminated with nickel sulfate, nickel chloride, and boric acid (Sunderman et al. 1988). The workers who reported symptoms ( 20 of 35 ) or were hospitalized (10 of 35) were exposed to an estimated dose of 7.1-35.7 mg $\mathrm{Ni} / \mathrm{kg}$. The contribution of boric acid to these effects is not known.

Decreased liver weight was observed in rats exposed to $0.97-75 \mathrm{mg} \mathrm{Ni} / \mathrm{kg} /$ day as nickel chloride or nickel sulfate for 28 days to 2 years (Ambrose et al. 1976; American Biogenics Corporation 1988; Obone et al. 1999; Weischer et al. 1980) and mice exposed to $150 \mathrm{mg} \mathrm{Ni} / \mathrm{kg} /$ day as nickel sulfate in drinking water for 180 days (Dieter et al. 1988). No alterations in absolute liver weights were observed in male and female rats administered via gavage 22 or $33 \mathrm{mg} \mathrm{Ni} / \mathrm{kg} /$ day as nickel sulfate, respectively, for 90 days (Springborn Laboratories 2002); no histological alterations were reported in this study. A significant increase in relative liver weight, however, was observed in dogs exposed to $62.5 \mathrm{mg} \mathrm{Ni} / \mathrm{kg} / \mathrm{day}$ as nickel sulfate for 2 years (Ambrose et al. 1976). Because histological changes in the liver were not observed in these studies and decreases in body weight gain were often observed at the same dose levels, the significance of the liver weight changes is unclear.

Renal Effects. A transient increase in urine albumin levels was observed in 3 of 10 workers who were hospitalized after drinking water during one work shift from a water fountain contaminated with nickel sulfate, nickel chloride, and boric acid (Sunderman et al. 1988). Thirty-five workers were exposed, 20 reported symptoms, and 10 were hospitalized. The workers who reported symptoms were exposed to an estimated dose of 7.1-35.7 $\mathrm{mg} \mathrm{Ni} / \mathrm{kg}$. The contribution of boric acid to these effects is not known.

Renal tubular damage at the corticomedullary junction described as minor was observed in mice exposed to $\geq 108 \mathrm{mg} \mathrm{Ni} / \mathrm{kg} / \mathrm{day}$ as nickel sulfate in the drinking water for 180 days (Dieter et al. 1988). The renal effects included the loss of renal tubular epithelial cells and the presence of hyaline casts in the tubule (suggesting protein loss). No changes in markers of renal tubular function (urinary lactate dehydrogenase 
and NAG levels and $\beta_{2}$-microglobulin levels) were observed in rats exposed to nickel sulfate in the drinking water for 6 months at a concentration that supplied doses of $6.9 \mathrm{mg} / \mathrm{kg} / \mathrm{day}$ for males and $7.6 \mathrm{mg} / \mathrm{kg} /$ day for females (Vyskocil et al. 1994b). Urinary albumin levels, a marker of glomerular barrier dysfunction, was significantly increased in nickel-exposed female rats. Albumin excretion also tended to be higher in male rats, but did not reach statistical significance because of two control rats with very high values. The investigators noted that male rats develop a spontaneous nephrosis as they age and that this may have obscured the effect of nickel. Significant decreases in urine volume and urine glucose levels and increases in relative kidney weight at 14.4 or $28.8 \mathrm{mg} \mathrm{Ni} / \mathrm{kg} /$ day and increases in blood urea nitrogen (BUN) at $28.8 \mathrm{mg} \mathrm{Ni} / \mathrm{kg} / \mathrm{day}$ were observed in rats exposed to nickel sulfate in drinking water for 13 weeks (Obone et al. 1999); no changes in $\gamma$-glutamyl transpeptidase activity, NAG activities, or histological alterations were observed.

In dogs, polyuria and increased kidney weight were observed after exposure to $62.5 \mathrm{mg} \mathrm{Ni} / \mathrm{kg} / \mathrm{day}$ as nickel sulfate for 2 years; however, renal effects were not observed in similarly treated rats (Ambrose et al. 1976). Several studies in rats have reported significant changes in kidney weights following exposure to $0.97-55 \mathrm{mg} \mathrm{Ni} / \mathrm{kg} /$ day as nickel salts for 28 days to 9 months (American Biogenics Corporation 1988; RTI 1988b; Weischer et al. 1980). However, there was no consistency in direction of the change; some studies reported increases in kidney weights while others reported decreases. The toxicological significance of these data is not known. Additionally, no histological alterations were observed in the kidneys of male and female rats exposed to 22 or $33 \mathrm{mg} \mathrm{Ni} / \mathrm{kg} /$ day, respectively, as nickel sulfate administered via gavage for 90 days (Springborn Laboratories 2002).

Endocrine Effects. No studies were located regarding endocrine effects in humans after oral exposure to nickel.

Although histological changes were not observed, increases in pituitary weights were observed in male rats, but not female rats, treated with nickel chloride at doses $\geq 20 \mathrm{mg} \mathrm{Ni} / \mathrm{kg} /$ day for up to 30 weeks (RTI 1986, 1988a, 1988b). The multigeneration study (RTI 1988a, 1988b) is confounded by a decrease in both food and water intake. Decreased prolactin levels were observed in female rats treated with $31 \mathrm{mg}$ $\mathrm{Ni} / \mathrm{kg} /$ day as nickel chloride in the drinking water throughout the breeding and lactation of two litters (11 weeks before breeding, 2-week rest period after weaning of the first litter, followed by a second breeding), but not at a $6.8-\mathrm{mg} / \mathrm{kg} /$ day dose (Smith et al. 1993). Histological examinations did not reveal any adverse effects in the pituitary, thyroid, and adrenal glands or in the pancreas of rats and dogs treated 
with nickel sulfate in the diet for 2 years at $187.5 \mathrm{mg} \mathrm{Ni} / \mathrm{kg} / \mathrm{day}$ for rats and $62.5 \mathrm{mg} \mathrm{Ni} / \mathrm{kg} / \mathrm{day}$ for dogs (Ambrose et al. 1976).

Dermal Effects. Contact dermatitis, which results from dermal exposure to nickel, is the most prevalent effect of nickel in the general population (see Section 3.2.3.2). Several studies indicate that a single oral dose of nickel given as nickel sulfate can result in a flare-up in the dermatitis in nickelsensitive individuals (Burrows et al. 1981; Christensen and Moller 1975; Cronin et al. 1980; Gawkrodger et al. 1986; Hindsén et al. 2001; Jensen et al. 2003; Kaaber et al. 1978; Veien et al. 1987). Observed effects included erythema on the body, worsening of hand eczema, and a flare-up at the patch test site. Although some of the older studies reported fairly low LOAEL values (e.g., $0.009 \mathrm{mg} \mathrm{Ni} / \mathrm{kg}$ ), these studies have several design limitations including small sample size, the observation of placebo effects, and non-double-blind study designs (possibly introducing investigator bias). Two more recent studies have addressed a number of these concerns by using a large number of test subjects and a double-blind study design. One month after patch testing, an oral challenge dose of $1.0 \mathrm{mg}$ nickel as nickel sulfate $(0.014 \mathrm{mg} / \mathrm{kg})$ resulted in dermatitis in two of nine nickel-sensitive subjects (not significantly different than placebo incidence of 0/9); exposure to $4.0 \mathrm{mg}$ nickel $(0.057 \mathrm{mg} / \mathrm{kg})$ resulted in dermatitis in nine of nine subjects (Hindsén et al. 2001). Similarly, an oral challenge of 0, 0.3, 1.0, or $4.0 \mathrm{mg}$ nickel as nickel sulfate $(0,0.0043,0.014$, or $0.057 \mathrm{mg} / \mathrm{kg})$ administered 1 month after patch testing resulted in dermatitis in $1 / 10,4 / 10,4 / 10$, and $7 / 10$ nickel-sensitized individuals, respectively; no cutaneous reactions were observed in healthy controls receiving an oral challenge dose of 0 or $4.0 \mathrm{mg}$ nickel (Jensen et al. 2003). Although some sensitive individuals may react to very low oral doses of nickel, the threshold for dermatitis in nickel-sensitized individuals appears to be around $0.01 \mathrm{mg} \mathrm{Ni} / \mathrm{kg}$; a dose of approximately $0.06 \mathrm{mg} \mathrm{Ni} / \mathrm{kg}$ will result in a response in the most sensitized individuals.

Nielsen et al. (1990) fed 12 women with hand eczema and known allergy to nickel a diet (oatmeal, soy beans, cocoa) with 5 times the normal level of nickel (about $0.007 \mathrm{mg} / \mathrm{kg} /$ day) for 4 days. An aggravation of hand eczema was found in $6 / 12$ by day 4 after the start of the challenge, and although excess nickel was excreted by 2 days after the last treatment, further exacerbation of hand eczema was observed in $10 / 12$ by day 11 . It is not clear how well the diets were controlled after the challenge period, and the subjects may have eaten foods that contained vasoactive substances that could exacerbate an allergic reaction. This study also suggests that withdrawal of nickel rather than the peak nickel levels may contribute to the dermatitis observed in some sensitive individuals. 
Intermediate-duration studies suggest that longer-term oral exposure can be tolerated by some nickelsensitive individuals and may even serve to desensitize some individuals. Jordan and King (1979) found flaring of dermatitis in only $1 / 10$ nickel-sensitive women given nickel sulfate at $0.007 \mathrm{mg} / \mathrm{kg} /$ day for 2 weeks. Patch test responses to nickel were reduced in nickel-sensitive women given one weekly dose of 0.05 or 0.07 (but not 0.007 ) $\mathrm{mg} \mathrm{Ni} / \mathrm{kg}$ as nickel sulfate for 6 weeks (Sjovall et al. 1987). Santucci et al. (1994) gave increasing daily doses of nickel (0.01-0.03 mg/kg/day) as nickel sulfate to eight nickelsensitive women for up to 178 days. A significant clinical improvement in hand eczema was observed in all subjects after 1 month of treatment, and continued treatment resulted in healing of all dermal lesions except for those on the hands. Measurement of urine and serum nickel suggested a decrease in the absorption of nickel and an increase in the excretion of nickel with longer exposure. The Santucci et al. (1994) study indicates that a daily dose of $0.01-0.03 \mathrm{mg} \mathrm{Ni} / \mathrm{kg}$ can be tolerated by some nickel-sensitive people and may also serve to reduce their sensitivity. Among 44 sensitive subjects treated with a regimen of 1-2 ng nickel sulfate every other day, or daily for up to 2-3 years, 7 stopped the treatment for unspecified reasons, 7 had reactivation of symptoms, and complete (29) or partial (1) disappearance of symptoms for 2-4 years was observed in 30 subjects. In guinea pigs sensitized before oral treatment with nickel, only a transient desensitization was observed (van Hoogstraten et al. 1991).

Oral exposure before the sensitizing exposure may also help prevent nickel sensitization in some individuals. A study of 2,159 subjects examining the relationship between ear piercing and orthodontic treatment found that nickel sensitivity was reduced significantly when orthodontic treatment preceded ear piercing (23.3 versus 38.1\%) (van Hoogstraten et al. 1991). The investigators hypothesized that the oral nickel exposure that occurred during orthodontic treatment helped prevent the sensitization that occurred following ear piercing with earrings containing nickel. Orthodontic treatment after ear piercing did not affect the risk of nickel sensitization. Further evidence that oral exposure to nickel before a sensitizing exposure can prevent hypersensitivity is provided by the observation that nickel sensitivity in mice could be consistently produced only when metal frames to cover the cages and metal water nipples that released nickel were replaced with glass covers and nipples free of nickel (van Hoogstraten et al. 1991). Oral treatment of guinea pigs with nickel sulfate (30 mg/week for 6 weeks) has also been shown to prevent dermal sensitization (van Hoogstraten et al. 1991). Skin exposure of guinea pigs to nickel (nonsensitizing contacts) before oral exposure was also shown to interfere with oral tolerance induction.

Histological changes in the skin have not been observed in rats treated by gavage with nickel chloride at a dose of $8.6 \mathrm{mg} \mathrm{Ni} / \mathrm{kg} / \mathrm{day}$ for 91 days (American Biogenics Corporation 1988), or in rats and dogs exposed to nickel sulfate in the diet for 2 years at doses of 187.5 and $62.5 \mathrm{mg} \mathrm{Ni} / \mathrm{kg} /$ day, respectively 
(Ambrose et al. 1976). These studies suggest that the skin is not affected by orally administered nickel in animals that have not been previously sensitized to nickel.

Ocular Effects. In a pharmacokinetic study in humans, transient left homonymous hemianopsia (loss of sight in the corresponding lateral half of the eyes) occurred in one male subject following ingestion of $0.05 \mathrm{mg} \mathrm{Ni} / \mathrm{kg}$ as nickel sulfate in the drinking water (Sunderman et al. 1989b). No adverse effects were found in other subjects $(\mathrm{n}=9)$ when lower doses of 0.018 and $0.012 \mathrm{mg} \mathrm{Ni} / \mathrm{kg}$ were used.

No treatment-related ophthalmological changes were observed in rats treated by gavage with $8.6 \mathrm{mg}$ $\mathrm{Ni} / \mathrm{kg} /$ day as nickel chloride for 91 days (American Biogenics Corporation 1988).

Body Weight Effects. No studies were located regarding body weight effects in humans after oral exposure to nickel.

Decreased body weight gain of $10 \%$ or more, associated with reduced food and/or water intake, has been observed in rats treated by gavage with nickel chloride at $8.6 \mathrm{mg} \mathrm{Ni} / \mathrm{kg} /$ day for 91 days (American Biogenics Corporation 1988) or $55 \mathrm{mg} \mathrm{Ni} / \mathrm{kg}$ /day for 30 weeks (RTI 1988a), in rats exposed to 0.23 $0.97 \mathrm{mg} \mathrm{Ni} / \mathrm{kg} /$ day as nickel chloride in the drinking water for 28 days (Weischer et al. 1980), and in rats treated with nickel sulfate in the diet at $75 \mathrm{mg} \mathrm{Ni} / \mathrm{kg} / \mathrm{day}$ for 2 years (Ambrose et al. 1976). The concomitant decreases in food and/or water consumption limit the interpretation of these results. Decreases in body weight gain were also observed in male and female rats administered via gavage 17 or $28 \mathrm{mg} \mathrm{Ni} / \mathrm{kg} /$ day, respectively, as nickel sulfate (Springborn Laboratories 2002); however, the 10-13\% decreases in body weight gain were not associated with consistent alterations in food intake (water consumption data were not reported). Decreased body weight gain has also been reported in mice treated with nickel sulfate in drinking water at a dose of $108 \mathrm{mg} \mathrm{Ni} / \mathrm{kg} /$ day for 180 days (Dieter et al. 1988), and in dogs treated with nickel sulfate in the diet at a dose of $62.4 \mathrm{mg} / \mathrm{kg} / \mathrm{day}$ for 2 years (Ambrose et al. 1976). Decreases in body weight gain of $10 \%$ or more were not observed in rats treated with nickel chloride in the drinking water at $31.6 \mathrm{mg} \mathrm{Ni} / \mathrm{kg} /$ day for 11 weeks (Smith et al. 1993), with nickel sulfate in drinking water at $28.8 \mathrm{mg} \mathrm{Ni} / \mathrm{kg}$ /day for 13 weeks (Obone et al. 1999), or with nickel chloride at a dose of $7.6 \mathrm{mg} \mathrm{Ni} / \mathrm{kg} /$ day for 3 or 6 months (Vyskocil et al. 1994b).

Metabolic Effects. No studies were located regarding metabolic effects in humans after oral exposure to nickel. 
Two studies reported significant alterations in serum glucose levels in rats exposed to nickel chloride. A significant decrease in blood glucose levels was observed in rats administered $8.6 \mathrm{mg} \mathrm{Ni} / \mathrm{kg} /$ day via gavage for 91 days (American Biogenics Corporation 1988). In contrast, Weischer et al. (1980) reported a significant increase in blood glucose levels in rats administered $0.23 \mathrm{mg} \mathrm{Ni} / \mathrm{kg} / \mathrm{day}$ via drinking water for 28 days. In both studies, significant decreases in body weight gain (20\% and higher) were also observed at the same dose effect levels. Thus, it is difficult to assess whether this is a direct effect of nickel or secondary to the effect on body weight.

\subsubsection{Immunological and Lymphoreticular Effects}

Dermatitis resulting from nickel allergy is well reported in the literature (see Section 3.2.2.2 for further discussion of allergic dermatitis following oral exposure).

Effects on the immunological system following exposure to $44 \mathrm{mg} \mathrm{Ni} / \mathrm{kg} /$ day and higher as nickel sulfate in the drinking water for 180 days were assessed in mice (Dieter et al. 1988). Mild thymic atrophy was observed at $44 \mathrm{mg} \mathrm{Ni} / \mathrm{kg} /$ day and higher and mild splenic atrophy was observed at $108 \mathrm{mg} \mathrm{Ni} / \mathrm{kg} / \mathrm{day}$ and higher. Although several tests of immune function were performed, only two alterations were founddecreased spleen cellularity at $150 \mathrm{mg} \mathrm{Ni} / \mathrm{kg} /$ day and impaired lymphoproliferative response to the B-cell mitogen, Escherichia coli lipopolysaccharide (LPS), at $44 \mathrm{mg} \mathrm{Ni} / \mathrm{kg} /$ day and higher; a marginal response to sheep red blood cells was also observed at $150 \mathrm{mg} \mathrm{Ni} / \mathrm{kg} /$ day. No response to concanavalin A (con A), natural killer cell activity, or resistance to Listeria monocytogenes challenge were observed. In addition to the immune function responses, exposure to nickel sulfate resulted in alterations in bone marrow: decreases in bone marrow cellularity at $108 \mathrm{mg} \mathrm{Ni} / \mathrm{kg} /$ day and higher, decreases in granulocytemacrophage progenitor cells (CFU-GM) at $44 \mathrm{mg} \mathrm{Ni} / \mathrm{kg} /$ day and higher, and multipotential stem cells (CFU-S) at $108 \mathrm{mg} \mathrm{Ni} / \mathrm{kg} / \mathrm{day}$ and higher. The stem cell alterations were associated with alterations in glucose-6-phosphate dehydrogenase activity—increased at $44 \mathrm{mg} \mathrm{Ni} / \mathrm{kg} /$ day and decreased at 108 and $150 \mathrm{mg} \mathrm{Ni} / \mathrm{kg} /$ day. Obone et al. (1999) reported alterations in T-cell and B-cell subpopulations in the thymus and splenic lymphocytes in rats exposed to nickel sulfate in drinking water for 13 weeks. In the spleen, the changes consisted of an increase in the total number of cells at $14.4 \mathrm{mg} \mathrm{Ni} / \mathrm{kg} / \mathrm{day}$ and a decrease at $28.8 \mathrm{mg} \mathrm{Ni} / \mathrm{kg} /$ day; an increase in $\mathrm{CD}^{4+} \mathrm{T}$ cells at $14.4 \mathrm{mg} \mathrm{Ni} / \mathrm{kg} /$ day and a decrease at $28.8 \mathrm{mg} \mathrm{Ni} / \mathrm{kg} /$ day; increases in $\mathrm{CD}^{8+} \mathrm{T}$ cells at 14.4 and $28.8 \mathrm{mg} \mathrm{Ni} / \mathrm{kg} /$ day; an increase in the number of B cells at $14.4 \mathrm{mg} \mathrm{Ni} / \mathrm{kg} /$ day; and a decrease in the ratio of B cells to total cells at $14.4 \mathrm{mg} \mathrm{Ni} / \mathrm{kg} / \mathrm{day}$. In the thymus, the changes consisted of an increase in the total number of cells at $14.4 \mathrm{mg} \mathrm{Ni} / \mathrm{kg} / \mathrm{day}$ and a decrease at $28.8 \mathrm{mg} \mathrm{Ni} / \mathrm{kg} / \mathrm{day}$; an increase in $\mathrm{CD}^{4+} \mathrm{T}$ cells at $14.4 \mathrm{mg} \mathrm{Ni} / \mathrm{kg} / \mathrm{day}$ and a decrease at 
$28.8 \mathrm{mg} \mathrm{Ni} / \mathrm{kg} /$ day; a decrease in the ratio of $\mathrm{CD}^{4+} \mathrm{T}$ cells to total cells at $28.8 \mathrm{mg} \mathrm{Ni} / \mathrm{kg} / \mathrm{day}$; increases in $\mathrm{CD}^{8+} \mathrm{T}$ cells at 5.75 and $14.4 \mathrm{mg} \mathrm{Ni} / \mathrm{kg} /$ day and a decrease at $28.8 \mathrm{mg} \mathrm{Ni} / \mathrm{kg} /$ day; increases in the ratio of $\mathrm{CD}^{8+} \mathrm{T}$ cells to total cells at $5.75 \mathrm{mg} \mathrm{Ni} / \mathrm{kg} /$ day and higher; and an increase in the number of B cells at $14.4 \mathrm{mg} \mathrm{Ni} / \mathrm{kg} /$ day and a decrease at $28.8 \mathrm{mg} \mathrm{Ni} / \mathrm{kg} / \mathrm{day}$. When challenged with Coxsackie virus B3, an enhanced inflammatory response was observed in the hearts of mice treated with nickel chloride in drinking water at $20.3 \mathrm{mg} \mathrm{Ni} / \mathrm{kg} /$ day for 10-11 weeks (Ilback et al. 1994). Nickel treatment had no adverse effect on virus-induced lethality, spleen or thymus weights, or the number of cells in the spleen or thymus. Gross and microscopic examinations of the spleen did not reveal any adverse effects in rats or dogs fed nickel sulfate in the diet for 2 years at doses of $187.5 \mathrm{mg} / \mathrm{kg} /$ day for rats and $62.5 \mathrm{mg} / \mathrm{kg} / \mathrm{day}$ for dogs (Ambrose et al. 1976).

The highest NOAEL values and all LOAEL values from each reliable study for immunological effects in each species, duration category, and nickel compound are recorded in Table 3-8 and plotted in Figure 3-2.

\subsubsection{Neurological Effects}

Neurological effects were observed in workers who drank water during one work shift from a water fountain contaminated with nickel sulfate, nickel chloride, and boric acid (Sunderman et al. 1988). Thirty-five workers were exposed, 20 reported symptoms, and 10 were hospitalized. The dose to which the workers with symptoms were exposed was estimated to be $7.1-35.7 \mathrm{mg} \mathrm{Ni} / \mathrm{kg}$. The neurological effects included giddiness (seven workers), weariness (six workers), and headache (five workers). The contribution of boric acid to these effects is not known.

In a study designed to determine the absorption and elimination of nickel in humans, one male who ingested a single dose of $0.05 \mathrm{mg} \mathrm{Ni} / \mathrm{kg}$ as nickel sulfate in drinking water developed left homonymous hemianopsia (loss of sight in the corresponding lateral half of the eyes) 7 hours later; the condition lasted for 2 hours (Sunderman et al. 1989b). The loss of sight occurred soon after the peak serum concentration of nickel was reached, leading the investigators to suspect a causal relationship between nickel exposure and the loss of sight. The doses given to other subjects were lowered to 0.018 and $0.012 \mathrm{mg} \mathrm{Ni} / \mathrm{kg}$ with no adverse effects.

In a 90-day study, lethargy, ataxia, prostration, irregular breathing, and cool body temperature were observed in rats treated by gavage with nickel chloride (American Biogenics Corporation 1988). These effects were observed frequently at $25 \mathrm{mg} \mathrm{Ni} / \mathrm{kg} / \mathrm{day}$, a dose at which all rats died, and at lower 
incidences at $8.6 \mathrm{mg} \mathrm{Ni} / \mathrm{kg} / \mathrm{day}$, a dose at which 6/52 rats died. At the lower dose, it is not clear if the adverse neurological effects were observed only in the animals that died. No signs of neurological dysfunction were observed at $1.2 \mathrm{mg} / \mathrm{kg} / \mathrm{day}$. Microscopic examinations of whole brains did not reveal any changes in the brains of rats or dogs treated with nickel salts at doses of $8.6 \mathrm{mg} \mathrm{Ni} / \mathrm{kg} / \mathrm{day}$ for up to 2 years (Ambrose et al. 1976; American Biogenics Corporation 1988).

The highest NOAEL values and all LOAEL values from each reliable study for neurological effects in each species, duration category, and nickel compound are recorded in Table 3-8 and plotted in Figure 3-2.

\subsubsection{Reproductive Effects}

No studies were located regarding reproductive effects in humans after oral exposure to nickel.

A number of studies have examined the reproductive toxicity of nickel following oral exposure to rats, mice, or dogs. The studies have found conflicting results, with some studies identifying LOAELs for serious health effects and others identifying NOAELs at very similar dose levels. Pandey et al. (1999) reported an accumulation of nickel (in descending order of concentration) in the epididymis, testes, seminal vesicles, and prostate gland in mice orally exposed to nickel sulfate for 35 days. The accumulation of nickel in male reproductive tissues resulted in histological damage in the epididymis and seminal vesicles and sperm damage. Regressed epithelium and vacuolated cells were observed in the epididymis of mice administered $1.1 \mathrm{mg} \mathrm{Ni} / \mathrm{kg}$ as nickel sulfate via gavage 5 days/week for 35 days (Pandey et al. 1999). In the seminiferous tubules, the damage consisted of atrophy of centrally located tubules and disturbed spermatogenesis in mice administered $1.1 \mathrm{mg} \mathrm{Ni} / \mathrm{kg}$ as nickel sulfate (5 days/week) (Pandey et al. 1999). The significance of these findings is not known because the incidence data and statistical analysis were not reported. Käkelä et al. (1999) reported a statistically significant decrease in seminiferous tubule diameter in rats exposed to $3.6 \mathrm{mg} \mathrm{Ni} / \mathrm{kg} /$ day as nickel chloride in drinking water for 28 or 42 days. A significant decrease in basal spermatogonia was also observed in the rats exposed for 28 days, but not in the rats exposed for 42 days. Although it was not discussed in the report, the final body weights of males exposed for 28 days appear to be lower than control body weights; this may have contributed to the histological findings. Other studies have not found histological alterations in male or female reproductive tissues in rats administered up to $25 \mathrm{mg} \mathrm{Ni} / \mathrm{kg} / \mathrm{day}$ as nickel chloride for 91 days (American Biogenic Corp 1988), rats exposed to $28.8 \mathrm{mg} \mathrm{Ni} / \mathrm{kg} /$ day as nickel sulfate in drinking water for 90 days (Obone et al. 1999), rats exposed to $2.2 \mathrm{mg} \mathrm{Ni} / \mathrm{kg} /$ day as nickel sulfate administered via gavage for 18 weeks (Springborn Laboratories 2000a), rats exposed to $187.5 \mathrm{mg} \mathrm{Ni} / \mathrm{kg} /$ day as nickel sulfate in the 
diet for 2 years (Ambrose et al. 1976), or dogs exposed to $62.5 \mathrm{mg} \mathrm{Ni} / \mathrm{kg} /$ day as nickel sulfate in the diet for 2 years (Ambrose et al. 1976).

Significant decreases in sperm count and sperm motility and sperm abnormalities (banana and detached head; acrosome up, down, or missing; curved neck and curved, bent, round, loop, and folded tail) were observed in mice administered $\geq 2.2 \mathrm{mg} \mathrm{Ni} / \mathrm{kg}$ as nickel sulfate (decreased sperm count significant at $4.5 \mathrm{mg} \mathrm{Ni} / \mathrm{kg}$ ) or $2.5 \mathrm{mg} \mathrm{Ni} / \mathrm{kg}$ as nickel chloride 5 days/week for 35 days (Pandey and Srivastava 2000); no sperm effects were observed at 1.1 or $1.2 \mathrm{mg} \mathrm{Ni} / \mathrm{kg}$ as nickel sulfate or nickel chloride, respectively. Although the route of administration was not reported, it is assumed that the nickel chloride and nickel sulfate were administered via gavage. The investigators reported a dose-related decrease in body weight gain and decreases in absolute and relative testes, epididymis, seminal vesicle, and prostate gland weights at the two highest dose levels (2.2 and $4.5 \mathrm{mg} \mathrm{Ni} / \mathrm{kg}$ as nickel sulfate and 2.5 and $4.9 \mathrm{mg} \mathrm{Ni} / \mathrm{kg}$ as nickel chloride). Similarly, Pandey et al. (1999) reported decreases in sperm count and motility in mice administered $2.2 \mathrm{mg} \mathrm{Ni} / \mathrm{kg}$ as nickel sulfate, 5 days/week for 35 days; an increase in sperm abnormalities was also observed at $1.1 \mathrm{mg} \mathrm{Ni} / \mathrm{kg}$. Although Pandey et al. (1999) did not report alterations in body weight gain, significant decreases in testes, epididymis, seminal vesicle, and prostate gland weights were observed. In both studies by Pandey and associates, there were no significant alterations in the occurrence of a particular sperm abnormality; the total number of abnormalities was increased. Sobti and Gill (1989) reported increases in sperm head abnormalities in mice receiving a single gavage dose of 23, 28 , or $43 \mathrm{mg} / \mathrm{kg}$ as nickel nitrate, nickel sulfate, or nickel chloride, respectively; it should be noted that this study was poorly reported and no information on number of animals tested was given. No alterations in sperm count, concentration, motility, or morphology were observed in the F0 or F1 rats administered $2.2 \mathrm{mg} \mathrm{Ni} / \mathrm{kg} /$ day as nickel sulfate via gavage for 18 weeks (Springborn Laboratories 2000a).

In addition to the histological alterations and sperm alterations, alterations in fertility were observed in some studies, but not in all studies. Male-only exposure or male and female exposure to $3.6 \mathrm{mg}$ $\mathrm{Ni} / \mathrm{kg} /$ day as nickel chloride in drinking water resulted in decreased fertility in rats exposed for 28 days prior to mating (Käkelä et al. 1999). However, male rats exposed to $3.6 \mathrm{mg} \mathrm{Ni} / \mathrm{kg} /$ day for 42 days prior to mating with unexposed females resulted in a small decrease in fertility (83 versus 100\%) (Käkelä et al. 1999); suggesting regeneration of damaged tissues. Female-only exposure to concentrations as high as $13 \mathrm{mg} / \mathrm{kg} / \mathrm{day}$ as nickel chloride in drinking water did not adversely affect fertility in rats (Käkelä et al. 1999). Interpretation of this study is limited by the small number of animals tested (six gender/group) and the limited reporting of the results. No adverse effects on fertility were observed in a multigeneration study in which male and female rats exposed to doses as high as $55 \mathrm{mg} \mathrm{Ni} / \mathrm{kg} /$ day as nickel chloride in 
drinking water for 11 weeks prior to mating (RTI 1988a, 1988b), in a single generation study in which rats were administered $16.7 \mathrm{mg} \mathrm{Ni} / \mathrm{kg} /$ day as nickel sulfate via gavage for 2-weeks prior to mating, during mating, and during gestation (Springborn Laboratories 2000b), in a two-generation study involving gavage administration of up to $2.2 \mathrm{mg} \mathrm{Ni} / \mathrm{kg} / \mathrm{day}$ for 10 weeks prior to mating, during mating, gestation, and lactation (Springborn Laboratories 2000a), or in a multilitter study in which female rats were exposed to doses as high as $31.6 \mathrm{mg} \mathrm{Ni} / \mathrm{kg} /$ day (Smith et al. 1993).

The highest NOAEL value and all LOAEL values from each reliable study for reproductive effects in each species, duration category, and nickel compound are recorded in Table 3-8 and plotted in Figure 3-2.

\subsubsection{Developmental Effects}

No studies were located regarding developmental effects in humans after oral exposure to nickel.

The available animal data on developmental toxicity provide suggestive evidence that the developing fetus and neonates are sensitive targets of nickel toxicity. The most commonly reported end point is fetal loss and decreased survival observed in the rat and mouse offspring in studies involving male-only exposure, female-only exposure, and combined male and female exposure in single generation, multilitter, and multigeneration studies. The developmental effects were often reported at maternally toxic doses. Other developmental end points that have been examined include body weights, gross necropsy for abnormalities, and neurodevelopmental toxicity.

Male-only exposure to $3.6 \mathrm{mg} \mathrm{Ni} / \mathrm{kg} /$ day as nickel chloride in drinking water for 28 days resulted in decreases in the number of pups born alive (2.7/dam versus 10.2/dam in controls), the number of pups surviving until postnatal day 4 (56\% versus 100\% in controls), and litter size at postnatal day 21 (1.3 pups versus 9.2 pups in controls) (Käkelä et al. 1999). However, when the male rats were exposed to $3.6 \mathrm{mg}$ $\mathrm{Ni} / \mathrm{kg} /$ day for 42 days, no significant alterations in pup viability or survival were observed (Käkelä et al. 1999). A NOAEL was not identified in this study.

Several studies examined female-only exposure to nickel (Berman and Rehnberg 1983; Käkelä et al. 1999; Smith et al. 1993). An increase in spontaneous abortions was observed in female mice exposed to $160 \mathrm{mg} \mathrm{Ni} / \mathrm{kg} /$ day as nickel chloride in drinking water on gestational days 2-17 (Berman and Rehnberg 1983); no effects were observed at $80 \mathrm{mg} \mathrm{Ni} / \mathrm{kg} /$ day. In contrast, no effects on the average number of neonates per litter were observed when mouse dams were treated by gavage on gestation days $8-12$ with 
$90.6 \mathrm{mg} \mathrm{Ni} / \mathrm{kg} /$ day as nickel chloride (a dose that resulted in a significant decrease in maternal body weight) (Seidenberg et al. 1986). Exposure of rats to $13 \mathrm{mg} \mathrm{Ni} / \mathrm{kg} /$ day as nickel chloride in drinking water for 14 days prior to mating, during mating, gestation, and lactation resulted in a decreased pup survival from birth to postnatal day 4 (87 versus 100\% in controls) and from postnatal day 4 to 21 (52 versus 90\% in controls) (Käkelä et al. 1999); no significant alterations were observed at $4.0 \mathrm{mg}$ $\mathrm{Ni} / \mathrm{kg} /$ day. Pup mortality was also observed in a multilitter study in which rats were exposed to $0,1.3$, 6.8 , or $31.6 \mathrm{mg} \mathrm{Ni} / \mathrm{kg} /$ day as nickel chloride in drinking water for 11 weeks prior to breeding and during two successive gestation and lactation periods (Smith et al. 1993). In the first litter, the percentages of dead pups per litter at postnatal day 1 were $1.7,3.1,0$, and $13.2 \%$ (statistically significant at the high dose only); no significant alterations were observed in the number of dead pups at postnatal day 21 . In the second litter, the number of litters with dead pups at birth $(2,7,6$, and $10 \%$; statistically significant at high dose only), the percentages of dead pups per litter at postnatal day 1 (1.0, 4.3, 4.6, and 8.8\%; statistically significant at all three dose levels), and the percentage of dead pups at postnatal day 21 (12.5, $13.4,19.4$, and $29.2 \%$; significant at high dose only) were increased.

Offspring mortality was also observed in four studies involving combined male and female exposure (Ambrose et al. 1976; Käkelä et al. 1999; RTI 1988a, 1988b; Springborn Laboratories 2000b). Exposure of rats to $3.6-4.0 \mathrm{mg} \mathrm{Ni} / \mathrm{kg} /$ day as nickel chloride in drinking water for 28 days prior to mating, during mating, gestation, and lactation adversely affected the litter size at postnatal day 21 (2.7/dam versus 9.2/dam in controls) and pup survival from postnatal day 4 to 21 (44 versus $90 \%$ in controls) (Käkelä et al. 1999); a NOAEL was not identified. Significant increases in post-implantation losses were observed in the offspring of rats administered $6.7 \mathrm{mg} \mathrm{Ni} / \mathrm{kg} /$ day as nickel sulfate via gavage for 14 days prior to mating, during mating, and gestation (Springborn Laboratories 2000b); at $16.7 \mathrm{mg} \mathrm{Ni} / \mathrm{kg} / \mathrm{day}$, an increased number of dead pups at lactation day 0 and a decreased mean litter size were observed. This study identified a NOAEL of $4.5 \mathrm{mg} \mathrm{Ni} / \mathrm{kg} / \mathrm{day}$. In a multigeneration study (Ambrose et al. 1976) involving exposure of rats to $0,22.5,45$, or $90 \mathrm{mg} \mathrm{Ni} / \mathrm{kg} / \mathrm{day}$ as nickel chloride in the diet for 11 weeks prior to mating, during mating, gestation, and lactation, a dose-related increase in the number of stillborn pups was observed. An independent statistical analysis of the data using the Fisher Exact Test found significant increases in the total number pups born dead at $22.5 \mathrm{mg} \mathrm{Ni} / \mathrm{kg} /$ day and higher for the F1a generation, 45 and $90 \mathrm{mg} \mathrm{Ni} / \mathrm{kg} /$ day for the F1b generation, $90 \mathrm{mg} \mathrm{Ni} / \mathrm{kg} /$ day for the F2a generation, $22.5 \mathrm{mg} \mathrm{Ni} / \mathrm{kg} /$ day for the $\mathrm{F} 2 \mathrm{~b}$ generation, and 45 and $90 \mathrm{mg} \mathrm{Ni} / \mathrm{kg} /$ day for the F3b generation. The study authors noted that the number of offspring (dead and alive) was progressively less with increasing nickel levels above $45 \mathrm{mg} / \mathrm{kg} / \mathrm{day}(10.3,10.6,9.8$, and 9.0 for $0,22.5,45$, and $90 \mathrm{mg} / \mathrm{kg} / \mathrm{day}$, respectively); the number of offspring weaned per litter was also decreased with increasing nickel levels $(8.1,7.2,6.8$, and 
6.4 for $0,22.5,45$, and $90 \mathrm{mg} / \mathrm{kg} /$ day, respectively). The third study (RTI 1988a, 1998b) is a twogeneration study in which the P0 generation was exposed to nickel chloride in drinking water for 11 weeks before mating and during gestation and lactation, and the F1b generation animals were mated to produce the F2 generations. A reduction in live litter size was observed in the F1a, F1b, and F2a offspring of rats exposed to $55 \mathrm{mg} \mathrm{Ni} / \mathrm{kg} / \mathrm{day}$. Increases in mortality were also observed in the F1b rats on postnatal days 22 through 42; these increases were statistically significant in males at 30 and $55 \mathrm{mg}$ $\mathrm{Ni} / \mathrm{kg} /$ day and in females at $55 \mathrm{mg} \mathrm{Ni} / \mathrm{kg} /$ day. No adverse developmental effects were observed in the cesarean delivered F2b rats, suggesting that the nickel-induced decrease in live litter size occurred postnatally. No alterations in offspring mortality or survival were observed in a two-generation study in which rats were administered up to $2.2 \mathrm{mg} \mathrm{Ni} / \mathrm{kg} /$ day as nickel sulfate via gavage for approximately 18 weeks (Springborn Laboratories 2000a).

Decreases in pup body weights were reported in the offspring of rats exposed to $90 \mathrm{mg} \mathrm{Ni} / \mathrm{kg} / \mathrm{day}$ (Ambrose et al. 1976), 30, and 55 mg Ni/kg/day (RTI 1988a, 1988b). Neither the Ambrose et al. (1976) nor the RTI (1988a, 1988b) multigeneration studies found significant, nickel-related gross abnormalities in the surviving offspring of rats exposed to nickel. Käkelä et al. (1999) noted that the pups that died during lactation were runts: the heads were disproportionately large and the posteriors of the bodies were underdeveloped. No effects on figure eight maze reactive locomotor activity levels were observed in the offspring of mice treated by gavage at $45.3 \mathrm{mg} \mathrm{Ni} / \mathrm{kg} / \mathrm{day}$ as nickel chloride on gestation days 8-12 (Gray et al. 1986).

In summary, these data provide suggestive evidence that exposure to nickel prior to mating and during gestation and lactation results in decreased survival (Ambrose et al. 1976; Käkelä et al. 1999; RTI 1988a, 1988b; Smith et al. 1993). Decreased survival was also observed in the offspring of male rats exposed prior to mating to unexposed females (Käkelä et al. 1999) and increased spontaneous abortions were observed following gestation-only exposure of mice (Berman and Rehnberg 1983). Interpretation of these data is complicated by the maternal toxicity, in particular, a decrease in maternal body weight gain, which was also observed at these dose levels (Ambrose et al. 1976; Käkelä et al. 1999; RTI 1988a, 1988b; Smith et al. 1993). Decreases in food and water intake have also been observed (RTI 1988a, 1988b; Smith et al. 1993).

The highest NOAEL values and all LOAEL values from each reliable study for developmental effects in each species, duration category, and nickel compound are recorded in Table 3-8 and plotted in Figure 3-2. 


\subsubsection{Cancer}

No studies were located regarding cancer in humans after oral exposure to nickel.

In lifetime drinking water studies in rats and mice, nickel acetate $(0.6 \mathrm{mg} \mathrm{Ni} / \mathrm{kg} /$ day for rats; $0.95 \mathrm{mg}$ $\mathrm{Ni} / \mathrm{kg} /$ day for mice) was found to be noncarcinogenic (Schroeder et al. 1964, 1974). The incidence of tumors was comparable to that observed in controls.

\subsubsection{Dermal Exposure}

\subsubsection{Death}

No studies were located regarding death in humans or animals after dermal exposure to nickel.

\subsubsection{Systemic Effects}

No studies were located regarding adverse cardiovascular, gastrointestinal, musculoskeletal, or ocular effects in humans or animals after dermal exposure to nickel.

The highest NOAEL values and all LOAEL values from each reliable study for systemic effects for each species, duration category, and nickel compound are recorded in Table 3-9.

Respiratory Effects. Scratch tests and intradermal tests were performed on a patient diagnosed with nickel-related asthma (McConnell et al. 1973). Nonasthmatic controls were also tested. Testing resulted in respiratory distress in the patient but not in the controls, with a more severe response resulting from the scratch test.

No studies were located regarding adverse respiratory effects in animals after dermal exposure to nickel.

Hematological Effects. No studies were located regarding adverse hematological effects in humans after dermal exposure to nickel. 
Table 3-9 Levels of Significant Exposure to Nickel - Dermal

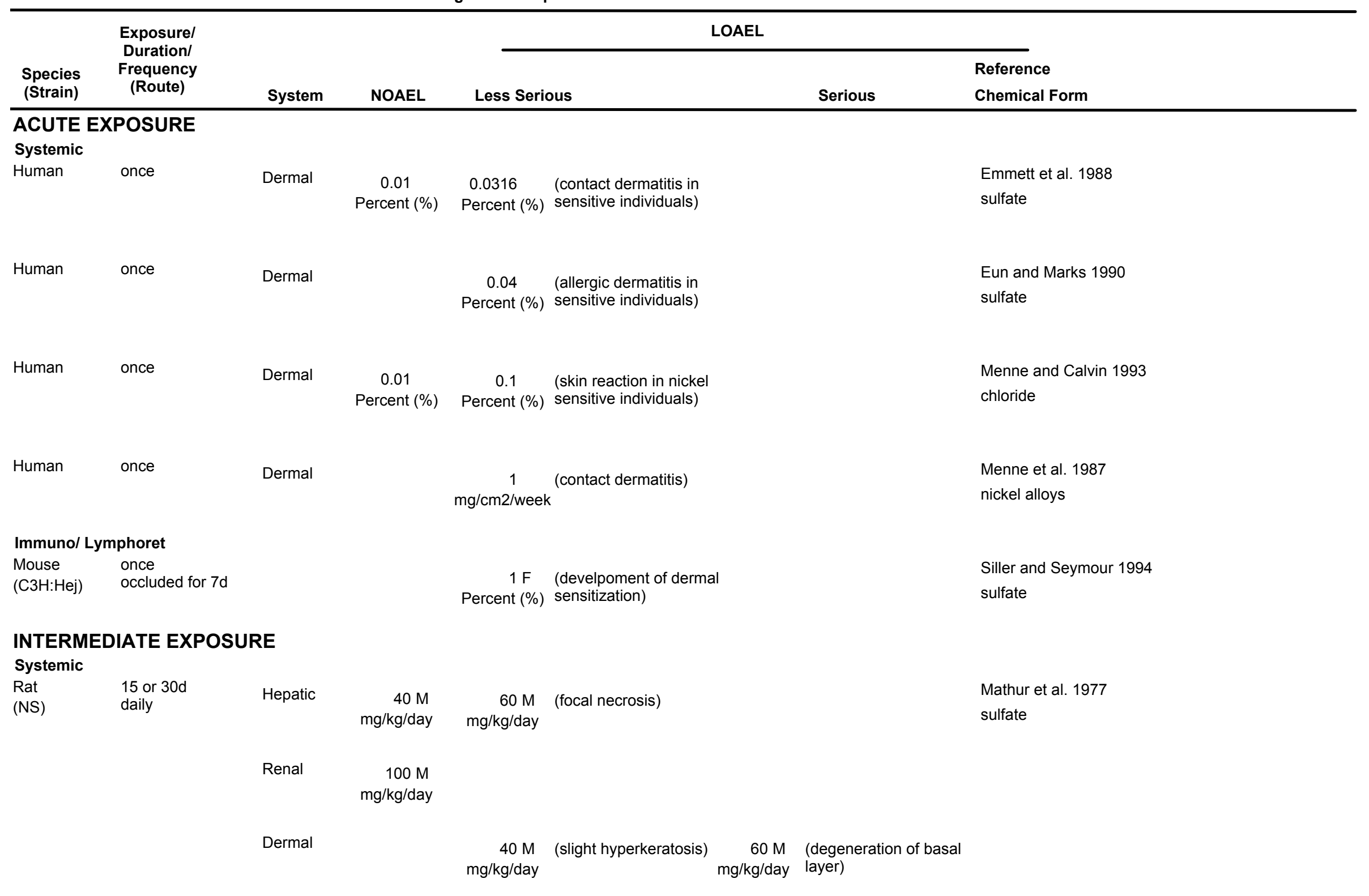


Table 3-9 Levels of Significant Exposure to Nickel - Dermal

(continued)

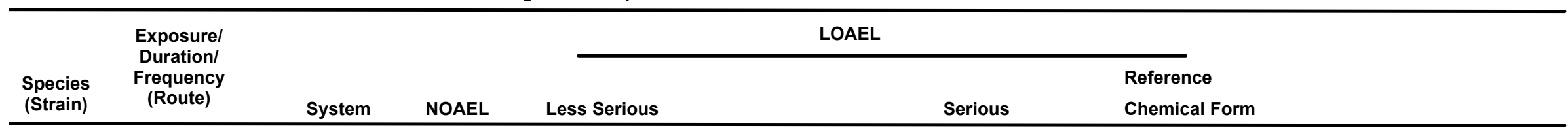

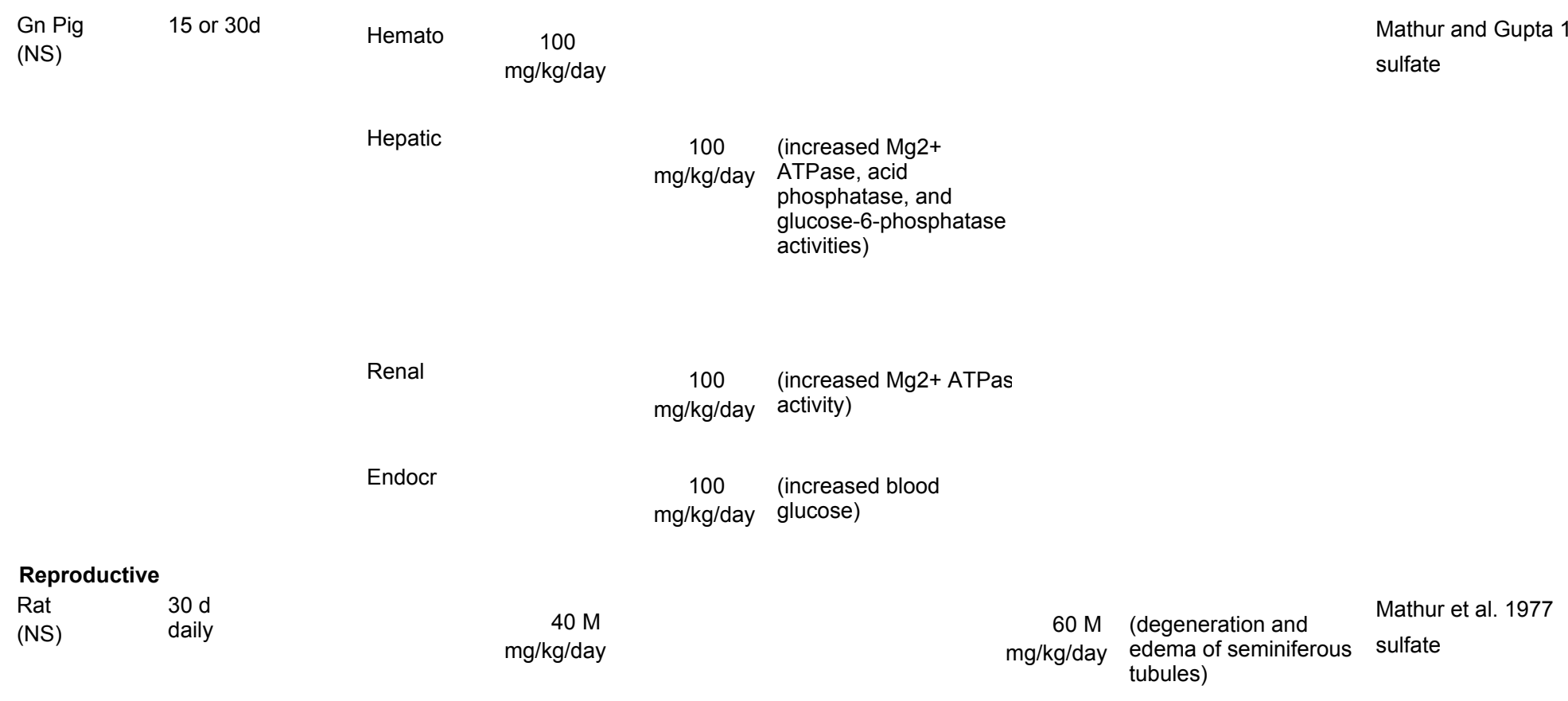


Hematocrit and hemoglobin levels were not affected in guinea pigs treated with $100 \mathrm{mg} \mathrm{Ni} / \mathrm{kg} / \mathrm{day}$ as nickel sulfate placed on skin of the back for 15 or 30 days (Mathur and Gupta 1994). Only one dose level was used in this study.

Hepatic Effects. No studies were located regarding adverse hepatic effects in humans after dermal exposure to nickel.

Effects on the liver were observed in rats treated dermally (lateral abdominal area) with daily doses of $60 \mathrm{mg} \mathrm{Ni} / \mathrm{kg} /$ day as nickel sulfate for 15 or 30 days (Mathur et al. 1977). The effects included swollen hepatocytes and feathery degeneration after 15 days and focal necrosis and vacuolization after 30 days.

In this study, there was no indication that the rats were prevented from licking the nickel from the skin; therefore, these effects could have resulted from oral exposure. Increased $\mathrm{Mg}^{2+}$ ATPase activity was observed in the livers of guinea pigs treated with $100 \mathrm{mg} \mathrm{Ni} / \mathrm{kg} / \mathrm{day}$ as nickel sulfate placed on skin of the back for 15 or 30 days (Mathur and Gupta 1994). Acid phosphatase and glucose-6-phosphatase activities were increased only after 30 days of treatment.

Renal Effects. Proteinuria was not observed in electroforming industry workers exposed to nickel. No information was provided on exposure level or nickel compound (Wall and Calnan 1980).

No gross or microscopic lesions were observed in the kidneys of rats treated dermally with $\leq 100 \mathrm{mg}$ $\mathrm{Ni} / \mathrm{kg} /$ day as nickel sulfate for 15 or 30 days (Mathur et al. 1977). In this study, there was no indication that the rats were prevented from licking the nickel from the skin; therefore, the animals could have been orally exposed. Increased $\mathrm{Mg}^{2+}$ ATPase activity was observed in the kidneys of guinea pigs treated with $100 \mathrm{mg} \mathrm{Ni} / \mathrm{kg} /$ day as nickel sulfate placed on skin of the back for 30 days (Mathur and Gupta 1994). No adverse effect was noted at 15 days, and dermal nickel exposure had no effect on kidney acid phosphatase or glucose-6-phosphatase activities.

Endocrine Effects. No studies were located regarding adverse endocrine effects in humans after dermal exposure to nickel.

Blood glucose levels were significantly increased in guinea pigs treated with $100 \mathrm{mg} \mathrm{Ni} / \mathrm{kg} / \mathrm{day}$ as nickel sulfate placed on skin of the back for 15 or 30 days (Mathur and Gupta 1994). 
Dermal Effects. Allergic contact dermatitis is a commonly reported effect in humans exposed to nickel. Contact dermatitis was found in $15.5 \%$ of approximately 75,000 individuals undergoing patch tests with nickel sulfate (5\% in petrolatum) (Uter et al. 2003). Smaller scale studies reported a similar frequency: $19.1 \%$ of 542 subjects (Akasya-Hillenbrand and Özkaya-Bayazit 2002), 21.2\% of 1,729 subjects (Wantke et al. 1996), and 20.13\% of 3,040 subjects (Simonetti et al. 1998). In the general population (a random sample of 567 people aged 15-69 years responding to a mailed screening questionnaire on respiratory allergy symptoms), $11 \%$ of the subjects had a positive reaction to nickel patch tests (Nielsen et al. 2002). Contact dermatitis in response to nickel exposure is more frequently observed in females, particularly younger females, than in males or older individuals (Uter et al. 2003; Wantke et al. 1996). This increased prevalence appears to be related to previous nickel exposure rather than increased susceptibility. Prolonged exposure to nickel in consumer products, especially jewelry, rather than occupational exposure, is often the sensitizing source. An association has been observed between ear piercing and nickel sensitivity (Akasya-Hillenbrand and Özkaya-Bayazit 2002; Dotterud and Falk 1994; Larsson-Stymne and Widstrom 1985; Meijer et al. 1995; Uter et al. 2003). The prevalence of nickel allergy was $9 \%$ among girls (age 8,11 , and 15; $\mathrm{n}=960$ ) with pierced ears compared to $1 \%$ among girls without pierced ears. Girls with more than one hole in each ear were also more likely to be sensitive to nickel than girls with only one hole in each ear (19 versus 11\%) (Larsson-Stymne and Widstrom 1985). In a study in schoolchildren age 7-12, the frequency of nickel allergy was $30.8 \%$ among girls with pierced ears and 16.3\% among girls who did not have pierced ears (Dotterud and Falk 1994). Similarly, $14 \%$ of females with pierced ears developed nickel allergy compared to $4 \%$ in females without pierced ears (Nielsen et al. 2002). Among a group of Swedish men (age 18-24) completing military service, $4.6 \%$ with pierced ears reacted to nickel, while $0.8 \%$ who did not have pierced ears had a positive reaction to nickel (Meijer et al. 1995). Keczkes et al. (1982) have shown that sensitivity to nickel remains for many years. Fourteen people who tested positively for nickel sensitivity using nickel sulfate also tested positive 10 years later. However, the time interval between exposures can influence the degree of reactivity (Hindsén et al. 1997). A stronger reaction was found in nickel sensitized women when there was a 1-month period between nickel sulfate exposures compared to a 4-month period. This study also found a stronger reaction when nickel sulfate was applied to an area with previous allergic contact dermatitis.

Patch test studies in sensitive individuals using nickel sulfate have shown a dose-response relationship between the amount of nickel and the severity of the test response (Emmett et al. 1988; Eun and Marks 1990). In a study of 12 individuals, a nickel concentration of $0.0316 \%$ (316 ppm) in petrolatum resulted 
in dermatitis, while a concentration of $0.01 \%$ (100 ppm) did not produce adverse effects (Eun and Marks 1990). In aqueous solution, the nickel concentration of $0.0316 \%$ (316 ppm) did not result in dermatitis.

Although most patch testing is done with nickel sulfate because it is less irritating than nickel chloride, nickel alloys on the skin interact with human sweat, resulting in the release of nickel chloride. Therefore, nickel chloride is the more relevant form of nickel for examining threshold concentrations (Menne 1994). Menne and Calvin (1993) examined skin reactions to various concentrations of nickel chloride in 51 sensitive and 16 nonsensitive individuals. Although inflammatory reactions in the sweat ducts and hair follicles were observed at $0.01 \%$ and lower, positive reactions to nickel were not observed. To be scored as a positive reaction, the test area had to have both redness and infiltration, while the appearance of vesicles and/or a bullous reaction were scored as a more severe reaction. At $0.1 \%, 4 / 51$ and 1/51 tested positive with and without $4 \%$ sodium lauryl sulfate. Menne et al. (1987) examined the reactivity to different nickel alloys in 173 nickel-sensitive individuals. With one exception (Inconel 600), alloys that released nickel into synthetic sweat at a rate of $<0.5 \mu \mathrm{g} / \mathrm{cm}^{2} /$ week showed weak reactivity, while alloys that released nickel at a rate of $>1 \mu \mathrm{g} / \mathrm{cm}^{2} /$ week produced strong reactions.

Nickel sensitivity has been induced in guinea pigs following skin painting or intradermal injection with nickel sulfate (Turk and Parker 1977; Wahlberg 1976; Zissu et al. 1987). As discussed in Section 3.2.2.2, nickel sensitivity can also be induced in mice if oral exposure to nickel is reduced (Moller 1984; van Hoogstraten et al. 1991).

Adverse effects on the skin were observed in rats treated dermally with $\geq 40 \mathrm{mg} \mathrm{Ni} / \mathrm{kg} / \mathrm{day}$ as nickel sulfate for 15 or 30 days (Mathur et al. 1977). The effects included distortion of the epidermis and dermis after 15 days and hyperkeratinization, vacuolization, hydropic degeneration of the basal layer, and atrophy of the epidermis at 30 days. Biochemical changes in the skin (enzymatic changes, increased lipid peroxidation, and an increase in the content of sulfhydryl groups and amino nitrogen) were observed in guinea pigs dermally exposed to nickel sulfate for up to 14 days (Mathur et al. 1988, 1992). Additive effects were observed when nickel sulfate was given in combination with sodium lauryl sulfate.

\subsubsection{Immunological and Lymphoreticular Effects}

Contact dermatitis resulting from nickel allergy is well reported in the literature (see Section 3.2.3.2 for further discussion of allergic reactions to nickel following dermal exposure). A relationship between human lymphocyte antigens (HLA) and nickel sensitivity was observed in individuals who had contact 
allergic reactions and positive results in the patch test (Mozzanica et al. 1990). The individuals had not been occupationally exposed to nickel. The HLA typing found a significantly greater prevalence of HLADRw6 antigen in the nickel-sensitive group compared to normal controls. The relative risk for individuals with DRw6 to develop a sensitivity to nickel was approximately 3.3. In individuals with allergic contact dermatitis to nickel, nickel directly bound and activated T-cells (Kapsenberg et al. 1988).

The dose-response relationship for the development of nickel sensitivity has been examined in a mouse model (Siller and Seymour 1994). The sensitization exposure involved placing a 6-mm pad containing $45 \mu \mathrm{L}$ of a $0,1,5,10,15$, or $20 \%$ nickel sulfate solution on the shaved abdominal skin of mice. This pad was left on the skin under occlusion for 7 days. Seven days after the sensitization procedure, the mice were challenged with $10 \mu \mathrm{L}$ of a $0.4 \%$ aqueous nickel sulfate solution injected into the footpad. Saline was injected into the opposite footpad as a control. Contact hypersensitivity, indicated by footpad swelling, was elicited at all doses, although the degree of swelling was minimal at the $1 \%$ concentration. Footpad swelling increased as the sensitizing dose increased and generally peaked between 24 and 48 hours after the challenge. In a comparison of the responses between male and female mice, males showed a weaker and more variable response than females, and the response peaked at 72 hours in males compared to 48 hours in females. The LOAEL for sensitization in mice is recorded in Table 3-9.

\subsubsection{Neurological Effects}

No studies were located regarding adverse neurological effects in humans or animals after dermal exposure to nickel.

\subsubsection{Reproductive Effects}

No studies were located regarding adverse reproductive effects in humans after dermal exposure to nickel.

Tubular degeneration of the testes was observed in rats treated dermally with nickel sulfate at $60 \mathrm{mg}$ $\mathrm{Ni} / \mathrm{kg} /$ day for 30 days (Mathur et al. 1977). No effects were found at $40 \mathrm{mg} \mathrm{Ni} / \mathrm{kg} /$ day after 30 days or at doses of $\leq 100 \mathrm{mg} \mathrm{Ni} / \mathrm{kg} /$ day after 15 days of treatment. In this study, there was no indication that the rats were prevented from licking the nickel sulfate from the skin; therefore, these effects could have resulted from oral exposure. Consequently, these values do not appear in Table 3-9. 


\subsubsection{Developmental Effects}

No studies were located regarding adverse developmental effects in humans or animals after dermal exposure to nickel.

\subsubsection{Cancer}

No studies were located regarding cancer in humans or animals after dermal exposure to nickel.

\subsection{GENOTOXICITY}

A number of studies have examined the genotoxicity of a variety of nickel compounds; the results of these in vivo and in vitro tests are presented in Tables 3-10 and 3-11, respectively. The available weight of evidence suggests that nickel does not alter the frequency of gene mutations in nonmammalian organisms (Arlauskas et al. 1985; Biggart and Costa 1986; Green et al. 1976; Marzin and Phi 1985; Rasmuson 1985; Wong 1988), although some studies have found gene mutations (Ogawa et al. 1994; Pikalek and Necasek 1983; Rodriguez-Arnaiz and Ramos 1986). Mixed results for gene mutations have been found in mammalian test systems. Increases in the frequency of gene mutations have been found at the HGPRT locus in Chinese hamster V79 cells (Hartwig and Beyersmann 1989; Miyaki et al. 1979) but not in Chinese hamster ovary cells (Hsie et al. 1979). An increase in gene mutation frequency has also been found in Chinese hamster ovary AS52 cells (grp locus) (Fletcher et al. 1994), mouse lymphoma cells (Amacher and Paillet 1980; McGregor et al. 1988), and virus-infected mouse sarcoma cells (Biggart and Murphy 1988; Biggart et al. 1987). Gene mutation frequency was not affected in transgenic mouse and rat respiratory tissue following inhalation exposure to nickel subsulfide (Mayer et al. 1998).

There is some evidence to suggest that nickel is clastogenic and can damage DNA. Chromosome gaps or chromosome aberrations have been reported in lymphocytes from nickel refinery workers (Waksvik and Boysen 1982), mouse bone marrow cells following intraperitoneal injection (Dhir et al. 1991), and in in vitro assays using hamster cells (Conway and Costa 1989; Larramendy et al. 1981; Sen and Costa 1986b; Sen et al. 1987), human lymphocytes (Larramendy et al. 1981; Lechner et al. 1984), and human bronchial epithelial cells (Lechner et al. 1984). No alterations in the occurrence of sister chromatid exchange were observed in lymphocytes from nickel refinery workers (Waksvik and Boysen 1982), but increases were found in in vitro assays of human lymphocytes (Andersen 1983; Arrouijal et al. 1992; Larramendy et al. 
Table 3-10. Genotoxicity of Nickel In Vivo

\begin{tabular}{|c|c|c|c|c|}
\hline Species (test system) & End point & Results & Reference & Compound \\
\hline $\begin{array}{l}\text { Drosophilia } \\
\text { melanogaster }\end{array}$ & Gene mutation & - & Rasmuson 1985 & Nickel nitrate or chloride \\
\hline D. melanogaster & Recessive lethal & + & $\begin{array}{l}\text { Rodriguez-Arnaiz } \\
\text { and Ramos } 1986\end{array}$ & Nickel sulfate \\
\hline D. melanogaster & $\begin{array}{l}\text { Gene mutation } \\
\text { (wing spot test) }\end{array}$ & \pm & Ogawa et al. 1994 & Nickel chloride \\
\hline \multicolumn{5}{|l|}{ Mammalian cells: } \\
\hline Human lymphocytes & $\begin{array}{l}\text { Chromosome } \\
\text { gaps }\end{array}$ & + & $\begin{array}{l}\text { Waksvik and Boysen } \\
1982\end{array}$ & $\begin{array}{l}\text { Nickel oxide, nickel } \\
\text { subsulfide }\end{array}$ \\
\hline Human lymphocytes & $\begin{array}{l}\text { Sister chromatid } \\
\text { exchange }\end{array}$ & - & $\begin{array}{l}\text { Waksvik and Boysen } \\
1982\end{array}$ & $\begin{array}{l}\text { Nickel oxide, nickel } \\
\text { subsulfide }\end{array}$ \\
\hline $\begin{array}{l}\text { Rat bone marrow and } \\
\text { spermatogonial cells }\end{array}$ & $\begin{array}{l}\text { Chromosome } \\
\text { aberrations }\end{array}$ & - & Mathur et al. 1978 & Nickel sulfate \\
\hline $\begin{array}{l}\text { Mouse bone marrow } \\
\text { cells }\end{array}$ & $\begin{array}{l}\text { Chromosome } \\
\text { aberrations (ip) }\end{array}$ & + & Dhir et al. 1991 & Nickel chloride \\
\hline $\begin{array}{l}\text { Mouse bone marrow } \\
\text { cells }\end{array}$ & $\begin{array}{l}\text { Micronucleus } \\
\text { test (ip) }\end{array}$ & - & Morita et al. 1997 & $\begin{array}{l}\text { Nickel chloride, nickel } \\
\text { sulfate, nickel oxide }\end{array}$ \\
\hline Rat bone marrow cells & $\begin{array}{l}\text { Micronucleus } \\
\text { test (oral) }\end{array}$ & - & $\begin{array}{l}\text { Covance } \\
\text { Laboratories, Inc. } \\
2003\end{array}$ & Nickel sulfate \\
\hline $\begin{array}{l}\text { Mouse bone marrow } \\
\text { cells }\end{array}$ & $\begin{array}{l}\text { Micronucleus } \\
\text { test (ip) }\end{array}$ & - & $\begin{array}{l}\text { Deknudt and } \\
\text { Leonard } 1982\end{array}$ & Nickel chloride \\
\hline $\begin{array}{l}\text { Mouse lung, mouse } \\
\text { nasal mucosa, rat lung, } \\
\text { rat nasal mucosa }\end{array}$ & $\begin{array}{l}\text { Gene mutation } \\
\text { (inhalation) }\end{array}$ & - & Mayer et al. 1998 & Nickel subsulfide \\
\hline Mouse & $\begin{array}{l}\text { Dominant lethal } \\
\text { (ip) }\end{array}$ & - & $\begin{array}{l}\text { Deknudt and } \\
\text { Leonard } 1982\end{array}$ & Nickel acetate \\
\hline
\end{tabular}

- = negative result; $+=$ positive result; $\pm=$ weakly positive; (ip) $=$ intraperitoneal 
Table 3-11. Genotoxicity of Nickel In Vitro

\begin{tabular}{|c|c|c|c|c|}
\hline Species (test system) & End point & Results & Reference & Compound \\
\hline \multicolumn{5}{|l|}{ Prokaryotic organisms: } \\
\hline Salmonella typhimurium & $\begin{array}{l}\text { Gene mutation } \\
\text { frequency }\end{array}$ & - & $\begin{array}{l}\text { Arlauskas et al. 1985; } \\
\text { Biggart and Costa 1986; } \\
\text { Marzin and Phi 1985; } \\
\text { Wong } 1988\end{array}$ & $\begin{array}{l}\text { Nickel chloride, } \\
\text { nickel nitrate, } \\
\text { nickel sulfate }\end{array}$ \\
\hline Escherichia coli WP2 & $\begin{array}{l}\text { Gene mutation } \\
\text { frequency }\end{array}$ & - & Green et al. 1976 & Nickel chloride \\
\hline Cornebacterium sp. & $\begin{array}{l}\text { Gene mutation } \\
\text { frequency }\end{array}$ & + & $\begin{array}{l}\text { Pikalek and Necasek } \\
1983\end{array}$ & Nickel chloride \\
\hline E. coli & DNA replication rate & + & Chin et al. 1994 & Nickel chloride \\
\hline Bacillus subtilis & $\begin{array}{l}\text { DNA damage } \\
\text { (rec assay) }\end{array}$ & - & Kanematsu et al. 1980 & $\begin{array}{l}\text { Nickel oxide and } \\
\text { trioxide }\end{array}$ \\
\hline \multicolumn{5}{|l|}{ Eukaryotic organisms } \\
\hline \multicolumn{5}{|l|}{ Fungi } \\
\hline $\begin{array}{l}\text { Saccharomyces } \\
\text { cerevesiae }\end{array}$ & Reverse mutation & - & Singh 1984 & Nickel sulfate \\
\hline \multicolumn{5}{|l|}{ Mammalian cells: } \\
\hline $\mathrm{CHO}$ cells & $\begin{array}{l}\text { Gene mutation at } \\
\text { HGPRT locus }\end{array}$ & - & Hsie et al. 1979 & Nickel chloride \\
\hline $\begin{array}{l}\text { Virus-infected mouse } \\
\text { sarcoma cells }\end{array}$ & $\begin{array}{l}\text { Induction of revertant } \\
\text { foci }\end{array}$ & + & $\begin{array}{l}\text { Biggart and Murphy } \\
\text { 1988; Biggart et al. } 1987\end{array}$ & Nickel chloride \\
\hline Mouse lymphoma cells & Forward mutation & + & $\begin{array}{l}\text { Amacher and Paillet } \\
\text { 1980; McGregor et al. } \\
1988\end{array}$ & $\begin{array}{l}\text { Nickel chloride, } \\
\text { nickel sulfate }\end{array}$ \\
\hline $\begin{array}{l}\text { Chinese hamster V79 } \\
\text { cells }\end{array}$ & $\begin{array}{l}\text { Gene mutation at } \\
\text { HGPRT locus }\end{array}$ & + & $\begin{array}{l}\text { Harwig and Beyersmann } \\
\text { 1989; Miyaki et al. } 1979\end{array}$ & Nickel chloride \\
\hline $\begin{array}{l}\text { Chinese hamster ovary } \\
\text { AS52 cells }\end{array}$ & $\begin{array}{l}\text { Gene mutation at grp } \\
\text { locus }\end{array}$ & + & Fletcher et al. 1994 & $\begin{array}{l}\text { Nickel oxide (black } \\
\text { and green); } \\
\text { amorphous nickel } \\
\text { sulfide; nickel } \\
\text { subsulfide nickel } \\
\text { chloride; nickel } \\
\text { sulfate; nickel } \\
\text { acetate }\end{array}$ \\
\hline $\begin{array}{l}\text { CD2F1 mouse lung } \\
\text { and nasal mucosa } \\
\text { cells }\end{array}$ & DNA fragmentation & + & Mayer et al. 1998 & Nickel subsulfide \\
\hline $\begin{array}{l}\text { Chinese hamster ovary } \\
\text { cells }\end{array}$ & $\begin{array}{l}\text { DNA protein } \\
\text { crosslinks/single } \\
\text { strand breaks }\end{array}$ & + & $\begin{array}{l}\text { Hamilton-Koch et al. } \\
\text { 1986; Patierno and } \\
\text { Costa } 1985\end{array}$ & $\begin{array}{l}\text { Crystalline NiS, } \\
\text { nickel chloride }\end{array}$ \\
\hline $\begin{array}{l}\text { Human diploid } \\
\text { fibroblasts }\end{array}$ & $\begin{array}{l}\text { DNA single strand } \\
\text { breaks }\end{array}$ & - & $\begin{array}{l}\text { Hamiltion-Koch et al. } \\
1986\end{array}$ & Nickel chloride \\
\hline
\end{tabular}


Table 3-11. Genotoxicity of Nickel In Vitro

\begin{tabular}{|c|c|c|c|c|}
\hline Species (test system) & End point & Results & Reference & Compound \\
\hline $\begin{array}{l}\text { Human gastric } \\
\text { mucosal cells }\end{array}$ & $\begin{array}{l}\text { DNA damage } \\
\text { (comet analysis) }\end{array}$ & $-{ }^{a}$ & Pool-Zobel et al. 1994 & Nickel sulfate \\
\hline Human HeLa cells & DNA replication & + & Chin et al. 1994 & Nickel chloride \\
\hline Hamster cells & $\begin{array}{l}\text { Sister chromatid } \\
\text { exchange }\end{array}$ & + & $\begin{array}{l}\text { Andersen 1983; } \\
\text { Larramendy et al. 1981; } \\
\text { Ohno et al. 1982; } \\
\text { Saxholm et al. } 1981\end{array}$ & $\begin{array}{l}\text { Nickel sulfate, } \\
\text { nickel chloride; } \\
\text { crystalline NiS }\end{array}$ \\
\hline Human lymphocytes & $\begin{array}{l}\text { Sister chromatid } \\
\text { exchange }\end{array}$ & + & $\begin{array}{l}\text { Andersen 1983; } \\
\text { Larramendy et al. 1981; } \\
\text { Saxholm et al. 1981; } \\
\text { Wulf } 1980\end{array}$ & $\begin{array}{l}\text { Nickel sulfate, } \\
\text { nickel sulfide }\end{array}$ \\
\hline Hamster cells & $\begin{array}{l}\text { Chromosome } \\
\text { aberration }\end{array}$ & + & $\begin{array}{l}\text { Conway and Costa 1989; } \\
\text { Larramendy et al. 1981; } \\
\text { Sen and Costa 1986b; } \\
\text { Sen et al. } 1987\end{array}$ & $\begin{array}{l}\text { Nickel sulfate, } \\
\text { nickel chloride, } \\
\text { nickel mono-sulfide }\end{array}$ \\
\hline Human lymphocytes & $\begin{array}{l}\text { Chromosome } \\
\text { aberration }\end{array}$ & + & Larramendy et al. 1981 & Nickel sulfate \\
\hline \multirow[t]{3}{*}{ Human lymphocytes } & $\begin{array}{l}\text { Sister chromatid } \\
\text { exchange }\end{array}$ & + & Arrouijal et al. 1992 & Nickel subsulfide \\
\hline & Metaphase analysis & + & & \\
\hline & $\begin{array}{l}\text { Micronucleus } \\
\text { formation }\end{array}$ & + & & \\
\hline $\begin{array}{l}\text { Human bronchial } \\
\text { epithelial cells }\end{array}$ & $\begin{array}{l}\text { Chromosome } \\
\text { aberration }\end{array}$ & + & Lechner et al. 1984 & Nickel sulfate \\
\hline $\begin{array}{l}\text { Hamster cell and } \\
\text { C3H/10T1/2 cells }\end{array}$ & Cell transformation & + & $\begin{array}{l}\text { Conway and Costa 1989; } \\
\text { Costa and Heck 1982; } \\
\text { Costa and Mollenhauer } \\
\text { 1980; Costa et al. 1982; } \\
\text { DiPaolo and Casto 1979; } \\
\text { Hansen and Stern 1984; } \\
\text { Saxholm et al. } 1981\end{array}$ & $\begin{array}{l}\text { Nickel monosulfide, } \\
\text { nickel subsulfide, } \\
\text { nickel chloride, } \\
\text { nickel, nickel oxide } \\
\text { or trioxide }\end{array}$ \\
\hline $\begin{array}{l}\text { Mouse embryo } \\
\text { fibroblasts }\end{array}$ & Cell transformation & - & Miura et al. 1989 & $\begin{array}{l}\text { Nickel sulfate, } \\
\text { nickel chloride }\end{array}$ \\
\hline $\begin{array}{l}\text { Mouse embryo } \\
\text { fibroblasts }\end{array}$ & Cell transformation & + & Miura et al. 1989 & $\begin{array}{l}\text { Nickel subsulfide, } \\
\text { nickel monosulfide, } \\
\text { nickel oxide }\end{array}$ \\
\hline Human foreskin cells & Cell transformation & + & $\begin{array}{l}\text { Biedermann and } \\
\text { Landolph } 1987\end{array}$ & $\begin{array}{l}\text { Nickel subsulfide, } \\
\text { nickel oxide, nickel } \\
\text { sulfate, nickel } \\
\text { acetate }\end{array}$ \\
\hline
\end{tabular}

${ }^{a}$ Nickel was genotoxic and cytotoxic at the same concentration $(9.5 \mu \mathrm{mol} / \mathrm{mL})$, so it was not a selective genotoxicant. - = negative result; + = positive result; DNA = dexoyribonucleic acid; NiS = nickel sulfide 
1981; Ohno et al. 1982; Saxholm et al. 1981; Wulf 1980) and hamster cells (Andersen 1983; Larramendy et al. 1981; Saxholm et al. 1981). Micronucleus formation was not affected in rat or mouse bone marrow cells following oral or intraperitoneal exposure (Covance Laboratories 2003; Deknudt and Leonard 1982; Morita et al. 1997). DNA damage consisted of fragmentation in mouse lung and nasal mucosal cells (Mayer et al. 1998) and DNA protein crosslink and/or single strand breaks in Chinese hamster ovary cells (Hamilton-Koch et al. 1986; Patierno and Costa 1985). However, DNA single strand breaks and damage (as assessed using comet analysis) were not found in human diploid fibroblasts (Hamilton-Koch et al. 1986) or human gastric mucosal cells (Pool-Zobel et al. 1994), respectively.

\subsection{TOXICOKINETICS}

Following inhalation exposure, about 20-35\% of nickel deposited in the lungs of humans is absorbed into the bloodstream. Absorption from the respiratory tract is dependent on the solubility of the nickel compound, with higher urinary nickel levels observed in workers exposed to soluble nickel compounds (nickel chloride, nickel sulfate) than in those exposed to less-soluble nickel compounds (nickel oxide, nickel subsulfide). Following oral exposure, about $27 \%$ of the nickel given to humans in drinking water was absorbed, while only about $1 \%$ was absorbed when nickel was given with food. Nickel applied directly to the skin can be absorbed into the skin where it may remain rather than entering the bloodstream.

Autopsy data from nonoccupationally exposed individuals indicate that the highest concentrations of nickel are found in the skin, adrenal glands, and intestines. Following inhalation exposure, nickel also tends to accumulate in the lungs. The pituitary may accumulate nickel if exposure occurs during pregnancy. Nickel has been shown to cross the placenta, and nickel can accumulate in milk, resulting in exposure of the offspring. In human serum, the exchangeable pool of nickel is bound to albumin, L-histidine, and $\alpha_{2}$-macroglobulin. There is also a nonexchangeable pool of nickel in the serum, which is tightly bound to nickeloplasmin. Regardless of the route of exposure, absorbed nickel is excreted in the urine. Nickel that is not absorbed from the gastrointestinal tract is excreted in the feces. 


\subsubsection{Absorption}

\subsubsection{Inhalation Exposure}

Inhaled nickel particles are deposited in the upper and lower respiratory tract and are subsequently absorbed by several mechanisms. The deposition pattern in the respiratory tract is related to particle size, which determines the degree to which particles are affected by inertial impaction, sedimentation, and diffusion. Large particles $(5-30 \mu \mathrm{m})$ deposit in the nasopharyngeal area where higher airstream velocities and airway geometry promote inertial impaction (Gordon and Amdur 1991). Smaller particles (1-5 $\mu$ m) enter the trachea and bronchiolar region where they deposit principally by sedimentation. The smallest particles $(<1 \mu \mathrm{m})$ enter the alveolar region of the lungs where diffusion and electrostatic precipitation of the particles occurs. Fractional deposition can be expected to vary considerably with age and breathing patterns.

In humans, about $20-35 \%$ of the inhaled nickel that is retained in the lungs is absorbed into the blood (Bennett 1984; Grandjean 1984; Sunderman and Oskarsson 1991). The remainder is either swallowed, expectorated, or remains in the respiratory tract. Nickel is detected in the urine of workers exposed to nickel (Angerer and Lehnert 1990; Elias et al. 1989; Ghezzi et al. 1989; Hassler et al. 1983; Torjussen and Andersen 1979). Higher concentrations of urinary nickel were found in workers exposed to soluble nickel compounds (nickel chloride, nickel sulfate) than in those exposed to less-soluble nickel compounds (nickel oxide, nickel subsulfide), indicating that the soluble compounds were more readily absorbed from the respiratory tract (Torjussen and Andersen 1979). A man who died of adult respiratory distress syndrome 13 days after being exposed to a very high concentration of metallic nickel fume (approximately $380 \mathrm{mg} / \mathrm{m}^{3}$ ) had very high concentrations of nickel in his urine (700 $\mu \mathrm{g} / \mathrm{L}$ ) (Rendall et al. 1994). This case report indicates that metallic nickel can be absorbed from the lungs if levels are high enough to result in lung damage.

The half-life of nickel in the lungs of rats exposed by inhalation has been reported to be 32 hours for nickel sulfate (mass median aerodynamic diameter [MMAD] $0.6 \mu \mathrm{m}$ ) (Hirano et al. 1994b), 4.6 days for nickel subsulfide ( ${ }^{63} \mathrm{Ni}_{3} \mathrm{~S}_{2}$ activity median aerodynamic diameter [AMAD] $1.3 \mu \mathrm{m}$ ), and 120 days for green nickel oxide $\left({ }^{63} \mathrm{NiO}\right.$, AMAD $\left.1.3 \mu \mathrm{m}\right)$ (Benson et al. 1994). Elimination half-times from the lung of rats of 7.7, 11.5, and 21 months were calculated for green nickel oxide with MMADs of 0.6, 1.2, and $4.0 \mu \mathrm{m}$, respectively (Tanaka et al. 1985, 1988). 
Following exposure to green nickel oxide, nickel was only excreted in the feces indicating that the dominant mechanism for removing nickel oxide from the lungs is macrophage-mediated rather than dissolution-absorption (Benson et al. 1994). Following exposure to nickel subsulfide, nickel was excreted in both the urine and the feces, with greater amounts in the urine on days 6-14 post-exposure. These results indicate that dissolution-absorption plays an important role in the removal of nickel subsulfide in the lungs, and the study authors concluded that in the lungs, nickel subsulfide acts more like a soluble compound (Benson et al. 1994).

\subsubsection{Oral Exposure}

A human study using a stable nickel isotope estimated that $29-40 \%$ of the ingested label was absorbed (based on fecal excretion data) (Patriarca et al. 1997). Other human absorption studies show that 40 times more nickel was absorbed from the gastrointestinal tract when nickel sulfate was given in the drinking water $(27 \pm 17 \%)$ than when it was given in food $(0.7 \pm 0.4 \%)$ (Sunderman et al. 1989b). The bioavailability of nickel, as measured by serum nickel levels, was elevated in fasted subjects given nickel sulfate in drinking water (peak level of $80 \mu \mathrm{g} / \mathrm{L}$ after 3 hours), but not when nickel was given with food (Solomons et al. 1982). The bioavailability of nickel increased when nickel was administered in a soft drink, but decreased when nickel was given with whole milk, coffee, tea, or orange juice. In another study (Nielsen et al. 1999) examining the relationship between nickel absorption and food intake, the highest nickel absorption (11.07-37.42\% of dose), as evidenced by the amount excreted in urine, was found when the subjects were administered $12 \mu \mathrm{g} \mathrm{Ni} / \mathrm{kg} 4$ hours after ingestion of a scrambled egg meal. The lowest absorption level (2.83-5.27\%) was found when nickel was administered at the same time as the meal. Ethylenediamine tetraacetic acid (EDTA) added to the diet decreased nickel bioavailability to below fasting levels (Solomons et al. 1982). These data indicate that the presence of food profoundly reduced the absorption of nickel. The observation of a decreased serum-nickel to urine-nickel ratio with increasing nickel doses in nickel-sensitive individuals suggests that at least some sensitive people adapt to increasing oral doses of nickel by reducing absorption by the gastrointestinal tract (Santucci et al. 1994). Urinary excretion of nickel following a single oral dose given to women after an overnight fast was found to decrease with increasing age, suggesting that nickel absorption may decrease with age (Hindsen et al. 1994).

Studies in rats and dogs indicate that $1-10 \%$ of nickel, given as nickel, nickel sulfate, or nickel chloride in the diet or by gavage, is rapidly absorbed by the gastrointestinal tract (Ambrose et al. 1976; Ho and Furst 1973; Tedeschi and Sunderman 1957). In a study in which rats were treated with a single gavage dose of 
a nickel compound (10 mg nickel) in a 5\% starch saline solution, the absorption was found to be directly correlated with the solubility of the compound (Ishimatsu et al. 1995). The percentages of the dose absorbed were $0.01 \%$ for green nickel oxide, $0.09 \%$ for metallic nickel, $0.04 \%$ for black nickel oxide, $0.47 \%$ for nickel subsulfide, $11.12 \%$ for nickel sulfate, $9.8 \%$ for nickel chloride, and $33.8 \%$ for nickel nitrate. Absorption was higher for the more-soluble nickel compounds. Unabsorbed nickel is excreted in the feces.

\subsubsection{Dermal Exposure}

Human studies show that nickel can penetrate the skin (Fullerton et al. 1986; Norgaard 1955). In a study in which radioactive nickel sulfate was applied to occluded skin, 55-77\% was absorbed within 24 hours, with most being absorbed in the first few hours (Norgaard 1955). It could not be determined whether the nickel had been absorbed into the deep layers of the skin or into the bloodstream. Compared to normal subjects, nickel absorption did not differ in nickel-sensitive individuals. In a study using excised human skin, only $0.23 \%$ of an applied dose of nickel chloride permeated skin after 144 hours when the skin was not occluded, while 3.5\% permeated occluded skin (Fullerton et al. 1986). Nickel(II) ions from a chloride solution passed through the skin $\approx 50$ times faster than nickel(II) ions from a sulfate solution (Fullerton et al. 1986). Application of nickel chloride in a sodium lauryl sulfate solution (0.25, 2, or 10\%) to excised human skin resulted in a dose-related increase in the penetration of nickel during a 48-hour period (Frankild et al. 1995).

Studies in animals also indicate that nickel can penetrate the skin (Lloyd 1980; Norgaard 1957). Radioactive nickel sulfate was absorbed through the depilated skin of rabbits and guinea pigs after 24 hours and appeared primarily in the urine (Norgaard 1957). A small percentage of radioactive nickel chloride was absorbed through the skin of guinea pigs 4-24 hours after application, as indicated by radioactivity in the blood and urine (0.005-0.51\%) (Lloyd 1980). Most of the nickel remained in the skin, primarily in the highly keratinized areas. Increased levels of nickel in the liver and kidneys in guinea pigs treated dermally with nickel sulfate for 15 or 30 days also indicate that nickel can be absorbed through the skin (Mathur and Gupta 1994). 


\subsubsection{Distribution}

An autopsy study of individuals not occupationally exposed to nickel has shown the highest concentrations of nickel ( $\mu \mathrm{g} / \mathrm{kg}$ dry weight) in the lungs $(174 \pm 94)$, followed by the thyroid $(141 \pm 83)$,

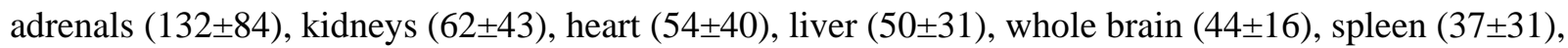
and pancreas (34 \pm 25$)$ (Rezuke et al. 1987). In an autopsy study, median levels of 0.046, 0.084, and $0.33 \mu \mathrm{g} \mathrm{Ni} / \mathrm{g}$ wet weight were found in the adrenal glands, colon, and skin, respectively (Tipton and Cook 1963). The total amount of nickel found in the human body has been estimated as $6 \mathrm{mg}$ or $86 \mu \mathrm{g} / \mathrm{kg}$ for a 70-kg person (Sumino et al. 1975).

\subsubsection{Inhalation Exposure}

Workers occupationally exposed to nickel have higher lung burdens of nickel than the general population. Dry weight nickel content of the lungs at autopsy was $330 \pm 380 \mu \mathrm{g} / \mathrm{g}$ in roasting and smelting workers exposed to less-soluble compounds, $34 \pm 48 \mu \mathrm{g} / \mathrm{g}$ in electrolysis workers exposed to soluble nickel compounds, and $0.76 \pm 0.39 \mu \mathrm{g} / \mathrm{g}$ in unexposed controls (Andersen and Svenes 1989). In an update of this study, Svenes and Andersen (1998) examined 10 lung samples takes from different regions of the lungs of 15 deceased nickel refinery workers; the mean nickel concentration was $50 \mu \mathrm{g} / \mathrm{g}$ dry weight. Nickel levels in the lungs of cancer victims did not differ from those of other nickel workers (Kollmeier et al. 1987; Raithel et al. 1989). Nickel levels in the nasal mucosa are higher in workers exposed to lesssoluble nickel compounds relative to soluble nickel compounds (Torjussen and Andersen 1979). These results indicate that, following inhalation exposure, less-soluble nickel compounds remain deposited in the nasal mucosa.

Higher serum nickel levels have been found in occupationally exposed individuals compared to nonexposed controls (Angerer and Lehnert 1990; Elias et al. 1989; Torjussen and Andersen 1979). Serum nickel levels were found to be higher in workers exposed to soluble nickel compounds compared to workers exposed to less-soluble nickel compounds (Torjussen and Andersen 1979). Concentrations of nickel in the plasma, urine, and hair were similar in nickel-sensitive individuals compared to nonsensitive individuals (Spruit and Bongaarts 1977).

Following a single 70 -minute inhalation exposure of rats to green nickel oxide $\left({ }^{63} \mathrm{NiO} ; 9.9 \mathrm{mg} \mathrm{Ni} / \mathrm{m}^{3}\right.$; AMAD $1.3 \mu \mathrm{m}$ ), the fraction of the inhaled material deposited in the total respiratory tract was 0.13 , with 0.08 deposited in the upper respiratory tract and 0.05 deposited in the lower respiratory tract (Benson et 
al. 1994). During the 180 days postexposure, nickel was not detected in extrarespiratory tract tissues. Following a single 120-minute inhalation exposure of rats to nickel subsulfide $\left({ }^{63} \mathrm{Ni}_{3} \mathrm{~S}_{2} ; 5.7 \mathrm{mg} \mathrm{Ni} / \mathrm{m}^{3}\right.$; AMAD $1.3 \mu \mathrm{m}$ ), the fraction of inhaled material deposited in the upper respiratory tract was similar to that observed for nickel oxide ( 0.14 in the total respiratory tract, 0.09 in the upper respiratory tract, and 0.05 in the lower respiratory tract). In contrast to nickel from nickel oxide, nickel from nickel subsulfide was detected in the blood, kidneys, and carcass between 4 and 24 hours after the exposure.

Data in rats and mice indicate that a higher percentage of less-soluble nickel compounds was retained in the lungs for a longer time than soluble nickel compounds (Benson et al. 1987, 1988; Dunnick et al. 1989; Tanaka et al. 1985) and that the lung burden of nickel decreased with increasing particle size $(\leq 4 \mu \mathrm{m})$ (Kodama et al. 1985a, 1985b). Nickel retention was $\approx 6$ times (mice) to 10 times (rats) greater in animals exposed to less-soluble nickel subsulfide compared to soluble nickel sulfate (Benson et al. 1987, 1988). The lung burdens of nickel generally increased with increasing exposure duration and increasing levels of the various nickel compounds (Dunnick et al. 1988, 1989). From weeks 9 to 13 of exposure, lung levels of nickel sulfate and nickel subsulfide remained constant while levels of nickel oxide continued to increase (Dunnick et al. 1989).

Slow clearance of nickel oxide from the lungs was also observed in hamsters (Wehner and Craig 1972). Approximately $20 \%$ of the inhaled concentration of nickel oxide was retained in the lungs at the end of exposure for 2 days, 3 weeks, or 3 months. The retention was not dependent on the duration of exposure or exposure concentration. By 45 days after the last exposure to nickel oxide (2-day exposure), $45 \%$ of the initial lung burden was still present in the lungs (Wehner and Craig 1972). The nickel oxide used in this study was not further identified.

The clearance of nickel compounds from the lungs was studied following intratracheal injection (Carvalho and Ziemer 1982; Valentine and Fisher 1984). Nickel subsulfide (less soluble) was cleared from the lungs of mice in two phases: $38 \%$ of the dose was cleared with a half-time of 1.2 days, and $42 \%$ was cleared with a half-time of 12.4 days. After 35 days, $10 \%$ of the dose remained in the lungs (Valentine and Fischer 1984). Soluble nickel chloride was cleared from the lungs much faster: $71 \%$ of the dose was cleared from the lungs in 24 hours, and only $0.1 \%$ remained in the lungs by day 21 (Carvalho and Ziemer 1982).

In a study that examined the effect of green nickel oxide and nickel sulfate on the clearance of nickel from the lungs, rats and mice were exposed 6 hours/day, 5 days/week, for up to 6 months and then given a 
single nose-only exposure to a ${ }^{63} \mathrm{Ni}$-labeled compound (Benson et al. 1995a). Nickel sulfate at concentrations up to $0.11 \mathrm{mg} \mathrm{Ni} / \mathrm{m}^{3}$ had no effect on lung clearance of nickel sulfate. Nickel oxide exposure did reduce the lung clearance of nickel oxide. When measured 184 days after the single exposure, a 6-month exposure of rats to nickel oxide at $0,0.49$, and $1.96 \mathrm{mg} \mathrm{Ni} / \mathrm{m}^{3}$ was found to result in the retention of 18,33 , and $96 \%$ of the dose, respectively. In mice exposed to nickel oxide at $0,0.98$, or $3.93 \mathrm{mg} / \mathrm{m}^{3}$ for 6 months, 4,20 , and $62 \%$, respectively, of the dose was retained 214 days after the single exposure to radiolabelled compound.

\subsubsection{Oral Exposure}

Serum nickel levels peaked 1.5 and 3 hours after ingestion of nickel (Christensen and Lagesson 1981; Patriarca et al. 1997; Sunderman et al. 1989b). In workers who accidentally ingested water contaminated with nickel sulfate and nickel chloride, the mean serum half-time of nickel was 60 hours (Sunderman et al. 1988). This half-time decreased substantially (27 hours) when the workers were treated intravenously with fluids.

In animals, nickel was found primarily in the kidneys following both short- and long-term oral exposure to various soluble nickel compounds (Ambrose et al. 1976; Borg and Tjalve 1989; Dieter et al. 1988; Ishimatsu et al. 1995; Jasim and Tjalve 1986a, 1986b; Oskarsson and Tjalve 1979; Whanger 1973). Substantial levels of nickel were also found in the liver, heart, lung, and fat (Ambrose et al. 1976; Dieter et al. 1988; Jasim and Tjalve 1986b; Schroeder et al. 1964; Whanger 1973) as well as in the peripheral nerve tissues and in the brain (Borg and Tjalve 1989; Jasim and Tjalve 1986a). Following a 2-year study in rats in which nickel levels were measured in bone, liver, kidneys, and fat, Ambrose et al. (1976) concluded that there were no important storage sites for nickel. In control rats, bone nickel was $0.53 \mathrm{ppm}$ in female rats and $<0.096 \mathrm{ppm}$ in male rats. An explanation for the difference in bone nickel between male and female rats was not provided. Nickel was found to cross the placenta, as indicated by increases in the levels of nickel in the fetuses of mice given nickel during gestation (Jasim and Tjalve 1986a; Schroeder et al. 1964).

When administered as part of a mixture of nickel, cadmium, arsenic, chromium, and vanadium in drinking water or food, elevated levels of nickel were found in the small intestines, kidneys, pancreas, and femur (only tissues examined) (Radike et al. 2002). The highest levels were found in the small intestine and kidney. When administered in water, significant elevations in nickel levels were found in the small 
intestine after 8 weeks of exposure; maximum levels were reached after 20 weeks. In the kidneys, the nickel levels were only significantly higher than controls at week 20.

In pregnant rats not exposed to nickel, maternal and fetal blood concentrations of nickel were 3.8 and $10.6 \mu \mathrm{g} / \mathrm{L}$, respectively (Szakmary et al. 1995). Twenty-four hours after a single gavage dose of 5.4, 11.3 , or $22.6 \mathrm{mg} \mathrm{Ni} / \mathrm{kg}$ as nickel chloride was given to pregnant rats (gestation day 19), nickel levels in $\mu \mathrm{g} / \mathrm{L}$ were $18.5,90$, and 91.5 , respectively, in maternal blood, $14.5,65.5$, and 70.5 , respectively, in fetal blood, and 16.5, 20, and 17, respectively, in amniotic fluid. This study showed that at higher doses, nickel reached a plateau in maternal and fetal blood, and that nickel concentrations in amniotic fluid were relatively well controlled in that they were similar at all three doses.

\subsubsection{Dermal Exposure}

No data were located regarding the distribution of nickel in humans after dermal exposure.

One hour after application of nickel chloride to the shaved skin of guinea pigs, nickel had accumulated in keratinaceous areas and in hair sacs (Lloyd 1980). After 4 hours, nickel was found in the stratum corneum and stratum spinosum. Twenty-four hours after treatment of depilated skin in rabbits and guinea pigs with nickel-57, radioactivity was detected in the blood, kidneys, and liver with the greatest amounts found in the blood and kidneys (Norgaard 1957). Quantitative data were not provided. Concentrations of nickel in the liver were $2.4 \pm 0.1 \mu \mathrm{g} / \mathrm{g}$ following 15 daily dermal treatments of guinea pigs with nickel sulfate at $100 \mathrm{mg} \mathrm{Ni} / \mathrm{kg} /$ day and $4.4 \pm 0.5 \mu \mathrm{g} / \mathrm{g}$ following 30 days of treatment with the same dose, compared to $0.2 \pm 0.01 \mu \mathrm{g} / \mathrm{g}$ before treatment (Mathur and Gupta 1994). In the kidneys, nickel levels were $0.4 \pm 0.2 \mu \mathrm{g} / \mathrm{g}$ before treatment, $1.5 \pm 0.12 \mu \mathrm{g} / \mathrm{g}$ at 15 days, and $3.52 \pm 0.42 \mu \mathrm{g} / \mathrm{g}$ at 30 days.

\subsubsection{Other Routes of Exposure}

Several researchers have examined the distribution of nickel in pregnant and lactating rats following its injection (Dostal et al. 1989; Mas et al. 1986; Sunderman et al. 1978). Half-lives of nickel in whole blood following intraperitoneal treatment of pregnant and nonpregnant rats were similar (3.6-3.8 hours), while the half-life for nickel in fetal blood was 6.3 hours following treatment on gestation days 12 or 19 (Mas et al. 1986). Intramuscular injection of nickel chloride (12 $\mathrm{mg} \mathrm{Ni} / \mathrm{kg} /$ day) into pregnant and nonpregnant rats resulted in a greater accumulation of nickel in the pituitary of pregnant rats (Sunderman et al. 1978). 
Wet weight nickel concentrations in the pituitary were $0.13 \mu \mathrm{g} / \mathrm{g}$ in nonpregnant rats and 1.1 and $0.91 \mu \mathrm{g} / \mathrm{g}$ in pregnant rats treated on gestation days 8 and 18, respectively. Following subcutaneous exposure of lactating rats to nickel chloride, Dostal et al. (1989) found that peak nickel concentrations in the milk were reached 12 hours after treatment. Relative to treatment with a single dose, four daily subcutaneous doses of nickel resulted in higher nickel concentrations in milk, while serum nickel levels were the same as following a single dose (Dostal et al. 1989). This study suggests that nickel can accumulate in the milk, which would result in exposure of the offspring.

Using whole-body autoradiography, Ilback et al. $(1992,1994)$ examined the distribution of an intravenous dose of nickel given to mice with and without Coxsackie virus B3 infection. Virus infection changed nickel distribution, resulting in accumulation in the pancreas and the wall of the ventricular myocardium. The investigators suggested that the change in distribution may result from repair and immune mechanisms activated in response to the virus.

\subsubsection{Metabolism}

The extracellular metabolism of nickel consists of ligand exchange reactions (Sarkar 1984). In human serum, nickel binds to albumin, L-histidine, and $\alpha_{2}$-macroglobulin. Binding in animals is similar. The principal binding locus of nickel to serum albumins is the histidine residue at the third position from the amino terminus in humans, rats, and bovines (Hendel and Sunderman 1972). Dogs do not have this binding locus, and most of the nickel (>85\%) in dog serum was not bound to protein. A proposed transport model involves the removal of nickel from albumin to histidine via a ternary complex composed of albumin, nickel, and L-histidine. The low molecular weight L-histidine nickel complex can then cross biological membranes (Sarkar 1984). In the serum, there is also a nonexchangeable pool of nickel tightly bound to nickeloplasmin, which is an $\alpha$-macroglobulin (Sunderman 1986).

\subsubsection{Elimination and Excretion}

\subsubsection{Inhalation Exposure}

Absorbed nickel is excreted in the urine, regardless of the route of exposure (Angerer and Lehnert 1990; Elias et al. 1989; Ghezzi et al. 1989; Hassler et al. 1983; Torjussen and Andersen 1979). In nickel workers, an increase in urinary excretion was found from the beginning to the end of the shift, indicating a fraction that was rapidly eliminated. An increase in urinary excretion was also found as the workweek 
progressed, indicating a fraction that was excreted more slowly (Ghezzi et al. 1989; Tola et al. 1979). Nickel was also excreted in the feces of nickel workers, but this probably resulted from mucociliary clearance of nickel from the respiratory system to the gastrointestinal tract (Hassler et al. 1983). Among electrolysis and refinery workers exposed to soluble nickel compounds (nickel sulfate aerosols), nickel concentrations in the urine were 5.2-22.6 $\mu \mathrm{g} / \mathrm{L}$ for those exposed to concentrations of $0.11-0.31 \mathrm{mg}$ $\mathrm{Ni} / \mathrm{m}^{3}$, and 3.2-18 $\mu \mathrm{g} / \mathrm{L}$ for those exposed to $0.08-0.2 \mathrm{mg} \mathrm{Ni} / \mathrm{m}^{3}$ (Chashschin et al. 1994). Higher nickel levels were found in the urine of workers exposed to soluble nickel compounds, indicating that the soluble compounds are more readily absorbed than the less-soluble compounds (Bernacki et al. 1978; Torjussen and Andersen 1979). Although high levels of nickel were found in the urine of a man who died of adult respiratory distress syndrome 13 days after being exposed to a very high concentration of metallic nickel (Rendall et al. 1994), it is not clear if metallic nickel would be absorbed from healthy lungs.

In animals, the route of excretion following intratracheal administration of nickel depends on the solubility of the nickel compound. In rats given soluble nickel chloride or nickel sulfate, $\approx 70 \%$ of the given dose was excreted in the urine within 3 days (Carvalho and Zeimer 1982; Clary 1975; English et al. 1981; Medinsky et al. 1987). By day 21, $96.5 \%$ of the given dose of nickel chloride had been excreted in the urine (Carvalho and Zeimer 1982). Following intratracheal administration of less-soluble compounds (nickel oxide, nickel subsulfide), a greater fraction of the dose was excreted in the feces as a result of mucociliary clearance. Following administration of black nickel oxide to rats or nickel subsulfide to mice, approximately equal amounts of the initial dose were excreted in the urine and the feces (English et al. 1981; Valentine and Fischer 1984). A total of $90 \%$ of the initial dose of nickel subsulfide was excreted within 35 days (Valentine and Fischer 1984), and 60\% of the initial dose of black nickel oxide was excreted within 90 days (English et al. 1981). This is consistent with nickel oxide being less soluble and not as rapidly absorbed as nickel subsulfide (English et al. 1981; Valentine and Fischer 1984).

\subsubsection{Oral Exposure}

In humans, most ingested nickel is excreted in the feces; however, this represents unabsorbed nickel (Patriarca et al. 1997; Sunderman et al. 1989b). However, the nickel that is absorbed from the gastrointestinal tract is excreted in the urine. Nickel administered in the drinking water was absorbed much more readily than when administered in the food $(27 \%$ absorption in water versus $0.7 \%$ absorption in food, respectively) (Sunderman et al. 1989b). By 4 days post-treatment, 26\% of the dose given in water was excreted in the urine and $76 \%$ in the feces, and $2 \%$ of the dose given in food was excreted in the urine and $102 \%$ in the feces (Sunderman et al. 1989b). The elimination half-time for absorbed nickel 
averaged $28 \pm 9$ hours (Sunderman et al. 1989b). These data are consistent with a nickel tracer study that found that $51-82 \%$ of the administered label was excreted in the urine over the 5 days (Patriarca et al. 1997).

In animals, the majority of the ingested dose of nickel is excreted in the feces. One day after administration of nickel chloride in rats, $94-97 \%$ had been excreted in the feces and 3-6\% had been excreted in the urine (Ho and Furst 1973). In dogs fed nickel sulfate in the diet for 2 years, only 1-3\% of the ingested nickel was excreted in the urine (Ambrose et al. 1976). Because dogs lack a major binding site in serum albumin that is found in humans (Hendel and Sunderman 1972), the relevance of dog data to humans is unclear.

\subsubsection{Dermal Exposure}

No studies were located regarding excretion of nickel in humans or animals after dermal exposure to nickel.

\subsubsection{Physiologically Based Pharmacokinetic (PBPK)/Pharmacodynamic (PD) Models}

Physiologically based pharmacokinetic (PBPK) models use mathematical descriptions of the uptake and disposition of chemical substances to quantitatively describe the relationships among critical biological processes (Krishnan et al. 1994). PBPK models are also called biologically based tissue dosimetry models. PBPK models are increasingly used in risk assessments, primarily to predict the concentration of potentially toxic moieties of a chemical that will be delivered to any given target tissue following various combinations of route, dose level, and test species (Clewell and Andersen 1985). Physiologically based pharmacodynamic (PBPD) models use mathematical descriptions of the dose-response function to quantitatively describe the relationship between target tissue dose and toxic end points.

PBPK/PD models refine our understanding of complex quantitative dose behaviors by helping to delineate and characterize the relationships between: (1) the external/exposure concentration and target tissue dose of the toxic moiety, and (2) the target tissue dose and observed responses (Andersen and Krishnan 1994; Andersen et al. 1987). These models are biologically and mechanistically based and can be used to extrapolate the pharmacokinetic behavior of chemical substances from high to low dose, from route to route, between species, and between subpopulations within a species. The biological basis of 
PBPK models results in more meaningful extrapolations than those generated with the more conventional use of uncertainty factors.

The PBPK model for a chemical substance is developed in four interconnected steps: (1) model representation, (2) model parametrization, (3) model simulation, and (4) model validation (Krishnan and Andersen 1994). In the early 1990s, validated PBPK models were developed for a number of toxicologically important chemical substances, both volatile and nonvolatile (Krishnan and Andersen 1994; Leung 1993). PBPK models for a particular substance require estimates of the chemical substancespecific physicochemical parameters, and species-specific physiological and biological parameters. The numerical estimates of these model parameters are incorporated within a set of differential and algebraic equations that describe the pharmacokinetic processes. Solving these differential and algebraic equations provides the predictions of tissue dose. Computers then provide process simulations based on these solutions.

The structure and mathematical expressions used in PBPK models significantly simplify the true complexities of biological systems. If the uptake and disposition of the chemical substance(s) are adequately described, however, this simplification is desirable because data are often unavailable for many biological processes. A simplified scheme reduces the magnitude of cumulative uncertainty. The adequacy of the model is, therefore, of great importance, and model validation is essential to the use of PBPK models in risk assessment.

PBPK models improve the pharmacokinetic extrapolations used in risk assessments that identify the maximal (i.e., the safe) levels for human exposure to chemical substances (Andersen and Krishnan 1994). PBPK models provide a scientifically sound means to predict the target tissue dose of chemicals in humans who are exposed to environmental levels (for example, levels that might occur at hazardous waste sites) based on the results of studies where doses were higher or were administered in different species. Figure 3-3 shows a conceptualized representation of a PBPK model.

If PBPK models for nickel exist, the overall results and individual models are discussed in this section in terms of their use in risk assessment, tissue dosimetry, and dose, route, and species extrapolations. 


\section{Figure 3-3. Conceptual Representation of a Physiologically Based Pharmacokinetic (PBPK) Model for a Hypothetical Chemical Substance}

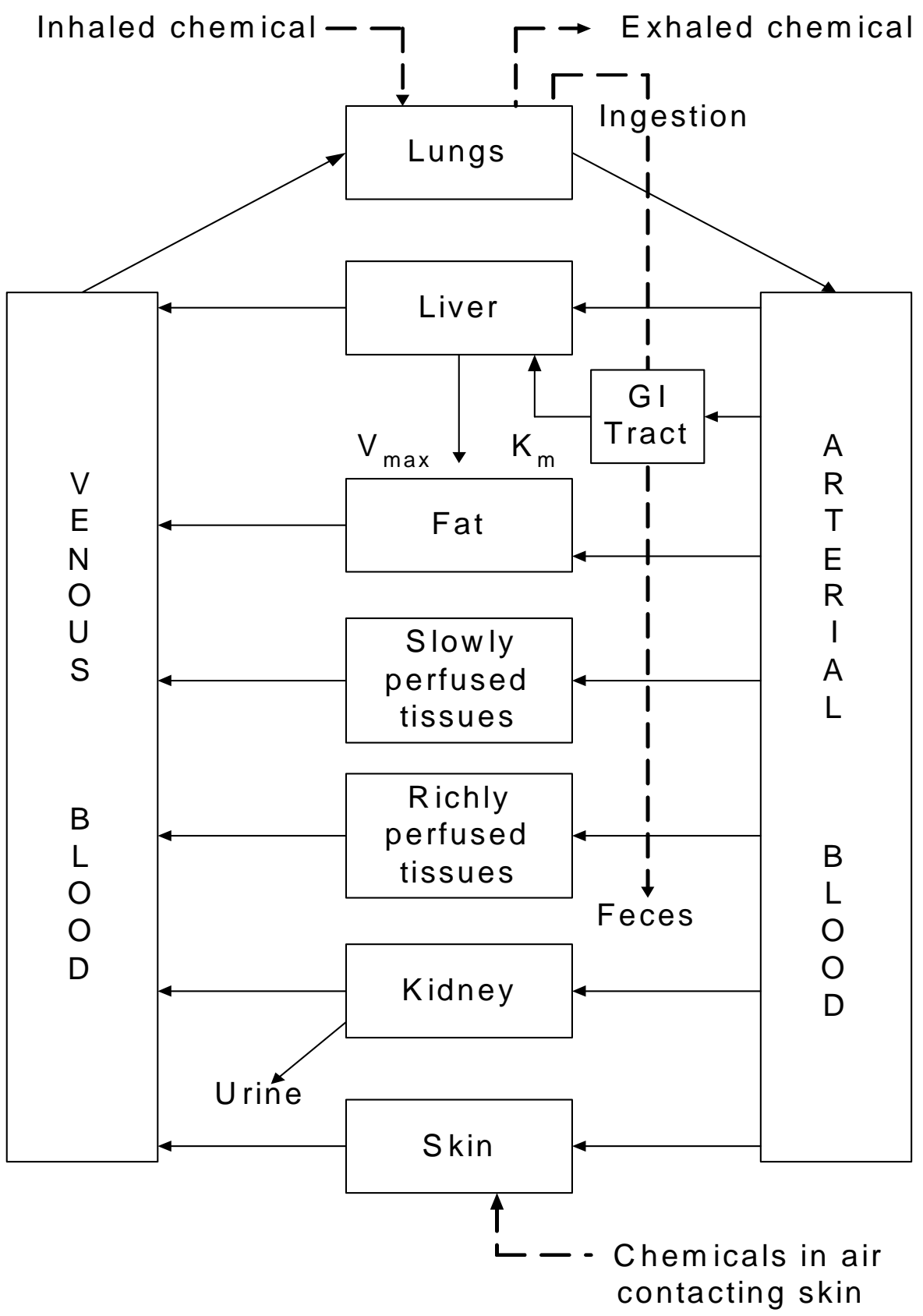

Source: adapted from Krishnan et al. 1994

Note: This is a conceptual representation of a physiologically based pharmacokinetic (PBPK) model for a hypothetical chemical substance. The chemical substance is shown to be absorbed via the skin, by inhalation, or by ingestion, metabolized in the liver, and excreted in the urine or by exhalation. 


\section{Sunderman et al. (1989b) Model}

Description of the Model. Sunderman et al. (1989b) developed a model to predict nickel absorption, serum levels, and excretion following oral exposure to nickel in water and food. The model was developed based on two experiments in humans in which serum nickel levels and urinary and fecal excretion of nickel were monitored for 2 days before and 4 days after eight subjects were given an oral dose of nickel as nickel sulfate $(12,18$, or $50 \mu \mathrm{g} \mathrm{Ni} / \mathrm{kg}$ ) in water (experiment 1) or in food (experiment 2). The data were then analyzed using a linear, compartmental, toxicokinetic model (Figure 3-4). Two inputs of nickel, the single oral dose, in which uptake was considered to be a first-order process, and the baseline dietary ingestion of nickel, in which uptake was considered to be a pseudo-zero order process, were included in the model. Parameters determined for the model from the two experiments are shown in Table 3-12. The only parameter that was significantly different between exposure in water and exposure in food was the fraction of nickel absorbed from the gastrointestinal tract. The absorption rate constant was not different at the different doses, but the investigators indicated that the observations do not exclude the possibility that nickel absorption from the gastrointestinal tract could be saturated at higher doses. At doses low enough to be in the deficiency range, the absorption rate and percentage absorbed are probably larger.

Validation of the Model. The model has been shown to predict serum nickel and cumulative nickel levels in subjects receiving a single dose of nickel in drinking water or food. The study authors (Sunderman et al. 1989b) noted that the model was going to be analyzed using data on individuals accidentally ingesting nickel from a contaminated drinking fountain (toxicity data described in Sunderman et al. 1988); however, it does not appear that this validation of the model has been published.

Risk Assessment. Currently, there are no oral exposure MRLs for nickel. Because the model evaluates the absorption of nickel from different media (food and water), the model can be used in conjunction with MRLs during the assessment of potential health hazards associated with nickel in different environmental media (e.g., soil, water).

Target Tissues. This model was designed to predict nickel absorption. It did not measure nickel in target tissues. 


\section{Figure 3-4. Diagram of the Compartmental Model of Nickel Metabolism*}

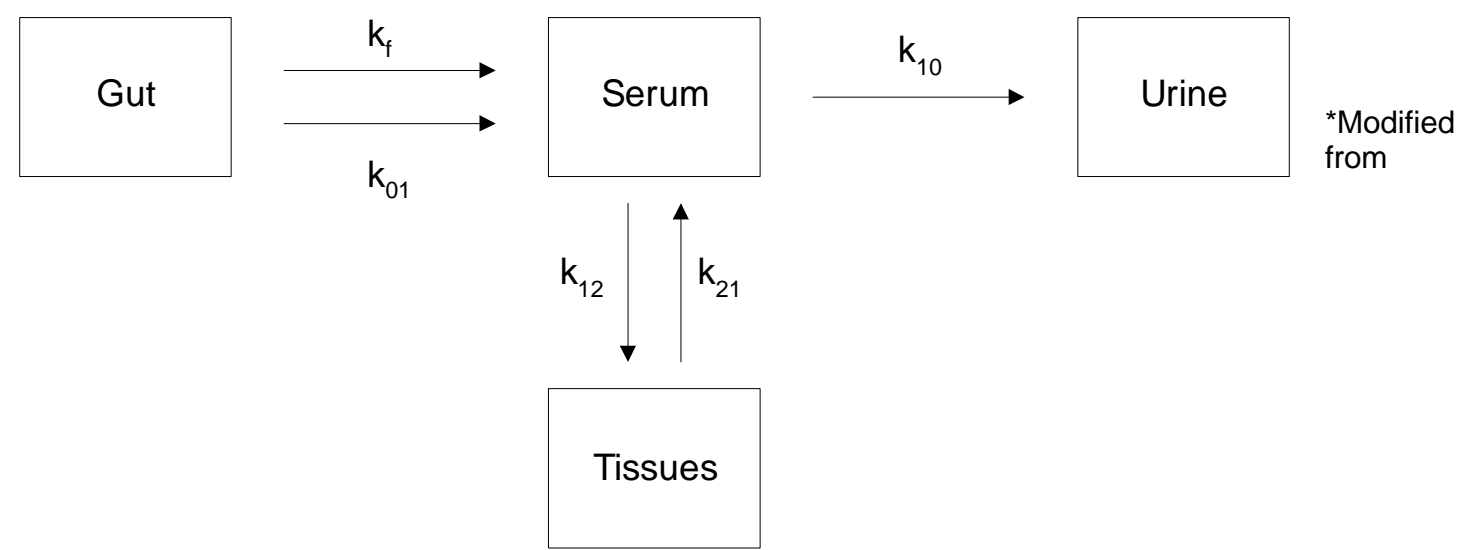

Sunderman et al. $1989 \mathrm{~b}$

$\mathrm{k}_{\mathrm{f}}=$ zero-order rate constant for fractional absorption of dietary nickel

$\mathrm{k}_{01}=$ first-order rate constant for intestinal absorption of nickel from oral $\mathrm{NiSO}_{4}$

$\mathrm{k}_{12}=$ first-order rate constant for nickel transfer from serum to tissues

$\mathrm{k}_{21}=$ first-order rate constant for nickel transfer from tissue to serum

$\mathrm{k}_{10}=$ first-order rate constant for nickel excretion in urine 


\section{Table 3-12. Kinetic Parameters of Nickel Sulfate Absorption, Distribution, and Elimination in Humans ${ }^{a}$}

\begin{tabular}{|c|c|c|}
\hline $\begin{array}{l}\text { Parameters } \\
\text { (symbols and units) }\end{array}$ & $\begin{array}{l}\text { Experiment } 1 \\
\text { (nickel sulfate in water) }\end{array}$ & $\begin{array}{l}\text { Experiment } 2 \\
\text { (nickel sulfate in food) }\end{array}$ \\
\hline $\begin{array}{l}\text { Mass fraction of nickel dose } \\
\text { absorbed from the gastrointestinal } \\
\text { tract (F, percent) }\end{array}$ & $27 \pm 17$ & $0.7 \pm 0.4^{b}$ \\
\hline $\begin{array}{l}\text { Rate constant for alimentary } \\
\text { absorption of nickel from the } \\
\text { nickel dose }\left(k_{01}, \text { hour }{ }^{-1}\right)\end{array}$ & $0.28 \pm 0.11$ & $0.33 \pm 0.24$ \\
\hline $\begin{array}{l}\text { Rate constant for alimentary } \\
\text { absorption of dietary nickel intake } \\
\left(\mathrm{k}_{\mathrm{f}}, \mu \mathrm{g} / \mathrm{hour}\right)\end{array}$ & $0.092 \pm 0.051$ & $0.105 \pm 0.036$ \\
\hline $\begin{array}{l}\text { Rate constant for nickel transfer } \\
\text { from serum to tissues }\left(k_{12}, \text { hour }^{-1}\right)\end{array}$ & $0.38 \pm 0.17$ & $0.37 \pm 0.34$ \\
\hline $\begin{array}{l}\text { Rate constant for nickel transfer } \\
\text { from tissue to serum }\left(k_{21}, \text { hour }^{-1}\right)\end{array}$ & $0.08 \pm 0.03$ & $-^{c}$ \\
\hline $\begin{array}{l}\text { Rate constant for urinary } \\
\text { elimination of nickel }\left(k_{10}, \text { hour }^{-1}\right)\end{array}$ & $0.21 \pm 0.05$ & $0.15 \pm 0.11$ \\
\hline $\begin{array}{l}\text { Rate clearance of nickel } \\
\left(\mathrm{C}_{\mathrm{Ni}}, \mathrm{mL} / \text { minute } / 1.73 \mathrm{mg} / \mathrm{m}^{2}\right)\end{array}$ & $8.3 \pm 2.0$ & $5.8 \pm 4.3$ \\
\hline $\begin{array}{l}\text { Rate clearance of creatinine } \\
\left(\mathrm{C}_{\text {creatinine, }}, \mathrm{mL} / \text { minute } / 1.73 \mathrm{mg} / \mathrm{m}^{2}\right)\end{array}$ & $97 \pm 9$ & $93 \pm 15$ \\
\hline $\begin{array}{l}\text { Nickel clearance as percent of } \\
\text { creatinine clearance }\left(C_{N i} / C_{\text {creatinine }}\right. \\
\text { x100) }\end{array}$ & $8.5 \pm 1.8$ & $6.3 \pm 4.6$ \\
\hline
\end{tabular}

${ }^{\mathrm{a}}$ Data (mean \pm standard deviation) from Sunderman et al. 1989b

${ }^{b} p<0.001$ relative to exposure in food computed by analysis of variance

${ }^{c}$ No value was determined because of the small mass of nickel absorbed from the gastrointestinal tract and transferred from the serum into the tissues. 
Species Extrapolation. This model was designed for application to humans; the study authors noted that studies to use this model for absorption, distribution, and excretion in laboratory animals are being initiated. No publications of these data were located.

Interroute Extrapolation. This model is designed to simulate oral absorption of nickel and cannot be used for other routes of exposure.

\section{Dosimetric Model for Lung Burden (Hsieh et al. 1999a, 1999b; Yu et al. 2001)}

Description of the Model. Hsieh et al. (1999a) describe a dosimetric model of nickel deposition and clearance from the lung. This model was derived using lung burden data from the rat NTP studies of nickel sulfate (NTP 1996c), nickel subsulfide (NTP 1996b), and nickel oxide (NTP 1996a) and existing models of lung deposition. The model considers the alveolar region of the lung as a single compartment; removal of nickel from the compartment occurs via macrophage phagocytosis and migration (mechanical clearance) and/or via dissolution. For nickel sulfate and nickel oxide, dissolution and mechanical clearance, respectively, are assumed to be the primary clearance mechanisms; clearance of nickel subsulfide occurs via both mechanisms. The accumulation of nickel in the lung over time was described by the following equations:

$$
\begin{aligned}
& \frac{d M}{d t}=\dot{r}-\lambda M \\
& \dot{r}=\text { concentration } \times \eta \times M V \\
& \lambda=a \exp \left[-b\left(\frac{m_{s}}{m_{s 0}}\right)^{c}\right]
\end{aligned}
$$

where $\mathrm{M}$ is the mass burden, $\mathrm{r}$ is the deposition rate, $\lambda$ is the total alveolar clearance rate coefficient; $\eta$ is the alveolar deposition fraction, $\mathrm{MV}$ is the minute ventilation, $a, b, c$ are clearance rate coefficient constants, $\mathrm{m}_{\mathrm{s}}=\mathrm{M} / \mathrm{S}$ in which $\mathrm{M}$ is the lung mass burden and $\mathrm{S}$ is the total alveolar surface area $\left(\mathrm{m}_{\mathrm{s}}=5.38 \times 10^{3} \mathrm{~cm}^{2}\right.$ for rats $)$, and $\mathrm{m}_{\mathrm{s} 0}=1 \mathrm{mg} / \mathrm{cm}^{2}$ is the dimensional constant introduced to normalize $\mathrm{m}_{\mathrm{s}}$.

The clearance rate coefficients constants in rats for the three nickel compounds examined are presented in Table 3-13. 
Hsieh et al. (1999b) modified the rat model to develop a model of deposition and clearance of nickel in humans. Deposition rates were calculated for six scenarios: nose-breathing at rest, nose-breathing at light work, nose breathing at moderate work, mouth breathing at rest, mouth breathing at light work, and mouth breathing at moderate work. The clearance rate coefficient constants for humans were modified from the rat values. For nickel oxide, clearance rate coefficient constant $a$ was estimated to be 1/7.6 times the rat value; constants $b$ and $c$ were assumed to be the same as rats. For nickel subsulfide, clearance is due to mechanical transport and dissolution; the clearance rate coefficient constant $a$ was estimated to be the sum of the clearance rate coefficient constant $a$ for insoluble nickel (nickel oxide) and the difference between the clearance rate coefficient constant $a$ for nickel oxide and for nickel subsulfide for rats. For nickel sulfate, clearance rate coefficient constants in humans were assumed to be the same as in rats. The human coefficient constants are presented in Table 3-13.

Yu et al. (2001) further expanded this human model to incorporate three additional factors: inhalability, mixed breathing mode, and clearance rate coefficient of a mixture of nickel compounds.

Validation of the Model. To validate the Hseih et al. (1999a) model, lung burdens for the nickel concentrations used by NTP were compared with measured lung burdens. In general, there was good agreement between the predicted lung burdens and measured burdens. Some differences were noted, particularly for the shorter term studies (16 days and 13 weeks). Hsieh et al. (1999a) noted that the differences may be due to assumptions used in the model (e.g., average body weight, constant respiratory parameters), using lung geometry data for Long Evans rats rather than for the Fischer rats used by NTP, or shortcomings in the experimental data.

The Hsieh et al. (1999b) model modification was not verified.

The Yu et al. (2001) modification of the model was used to predict lung burdens in nickel refinery workers; the predicted burdens were compared to measured lung burdens in deceased nickel refinery workers (Andersen and Svenes 1989). Good agreement between predicted and measured body burdens was found.

Risk Assessment. Currently, the intermediate- and chronic-duration inhalation MRLs for nickel are based on lung effects in rats. Further development of this model (Hseih et al. 1999a) and the modifications of the model (Hseih et al. 1999b; Yu et al. 2001) would allow for the model to be used to 
Table 3-13. Clearance Rate Coefficient Constants of Nickel Compounds

\begin{tabular}{llccl}
\hline & & \multicolumn{3}{c}{ Clearance rate coefficient constant } \\
\cline { 3 - 4 } Species & Nickel compound & $a$ & $b$ & $c$ \\
\hline Rat $^{\mathrm{a}}$ & & & 17.16 & 0.105 \\
& Nickel sulfate & 10.285 & -20.135 & 0.266 \\
& Nickel subsulfide & 0.00768 & 300 & 0.95 \\
Human $^{\mathrm{b}}$ & Nickel oxide & 0.0075 & & \\
& & & 17.16 & 0.105 \\
& Nickel sulfate & 10.285 & -20.135 & 0.266 \\
& Nickel subsulfide & 0.00117 & 300 & 0.95 \\
\hline
\end{tabular}

${ }^{\mathrm{a}}$ Data from Hsieh et al. 1999a

${ }^{\mathrm{b}}$ Data from Hsieh et al. 1999b 
extrapolate from rats to humans with greater certainty than using the standard dosimetric approach. This model could also be used for estimating human equivalent concentrations following low-dose extrapolation of the animal carcinogenicity data, similar to that conducted by Seilkop and Oller (2003).

Target Tissues. Based on limited human data and extensive animal data, the lung has been identified as the critical target of nickel toxicity. Further development of this model would allow nickel lung burdens to be predicted.

Species Extrapolation. The modifications of the Hsieh et al. (1999a) model allow for estimation of human lung burdens.

Interroute Extrapolation. This model is designed to simulate deposition and clearance of nickel from the lung and cannot be used for other routes of exposure.

\subsection{MECHANISMS OF ACTION}

\subsubsection{Pharmacokinetic Mechanisms}

Nickel is thought to be absorbed from the gastrointestinal tract as a lipophilic, low molecular weight compound (Kenney and McCoy 1992). The absorption of nickel from the gut is dependent on the various ligands and ions that are present. For example, food greatly decreases the absorption of nickel (Sunderman et al. 1989b). The results of an in situ perfusion study in rats (Arnich et al. 2000) suggest that at low concentrations ( $\leq 10 \mathrm{mg} \mathrm{Ni} / \mathrm{L})$, nickel is absorbed via active transport and facilitated diffusion; at higher concentrations, the carriers become saturated and nickel is absorbed via passive diffusion. These results are consistent with in vitro data showing that nickel is actively absorbed in the jejunum, but may cross the ileum by passive diffusion (Tallkvist and Tjalve 1994).

In the plasma, nickel is transported by binding to albumin and ultrafiltrable ligands, which include small polypeptides and amino acids; for example, histidine (Sunderman and Oskarsson 1991). The nickel binding site on albumin consists of the terminal amino group, the first two peptide nitrogen atoms at the $N$-terminus, and the imidazole nitrogen of the histidine at the third position from the $N$-terminus. Nickel competes with copper for this albumin binding site. In the plasma, nickel is also found bound to nickeloplasmin, an $\alpha$-macroglobulin, but the nickel associated with nickeloplasmin is not readily exchangeable, and this protein is not thought to play a role in the transport of nickel (Sunderman and 
Oskarsson 1991). An in vitro study of rat hepatocytes found that the calcium channels are involved in nickel uptake by the liver (Funakoshi et al. 1997). At physiological levels, no tissue significantly accumulates orally administered nickel (Nielsen 1990).

Nickel that is absorbed is excreted primarily in the urine. In the urine, nickel is primarily associated with low molecular weight complexes that have free amino acids as indicated by the ninhydrin reaction (Sunderman and Oskarsson 1991). In humans nickel is also eliminated in hair, skin, milk, and sweat.

The physiological role of nickel in animals and humans has not yet been identified. The most likely roles are as cofactors in metalloenzymes or metalloproteins, or as a cofactor that facilitates the intestinal absorption of iron $\left(\mathrm{Fe}^{3+}\right.$ ion) (Nielsen 1982). Support for a role of nickel in enzymes comes from the identification of nickel-containing enzymes in plants and microorganisms. The types of nickel-containing enzymes that have been identified are urease, hydrogenase, methylcoenzyme M reductase, and carbon monoxide dehydrogenase (Nielsen 1990). Nickel may also have a role in endocrine gland function as suggested by its effect on prolactin levels.

\subsubsection{Mechanisms of Toxicity}

The mechanism of adverse respiratory effects following lung exposure of rabbits to metallic nickel or nickel chloride has been examined (Johansson and Camner 1986; Johansson et al. 1980, 1981, 1983, 1987, 1988a, 1989). In these studies, an accumulation of macrophages and granular material (primarily phospholipids) in the alveoli and an increase in volume density of alveolar type II cells were observed. The type II cells contained large amounts of lamellar bodies. Similar results were found following exposure to metallic nickel and nickel chloride, indicating that nickel ions apparently had a direct effect on type II cells (Johansson and Camner 1986). At the end of 6 months, all of the rabbits had foci of pneumonia, indicating an increased susceptibility to infection (Johansson et al. 1981). This may have been a result of the decreased function of the alveolar macrophages.

The substitution of nickel for other essential elements may also contribute to the adverse effects of nickel. Nickel can replace magnesium in certain steps in the activation of complement (McCoy and Kenney 1992). For example, the replacement of nickel for magnesium can increase the formation of $\mathrm{C} 3 \mathrm{~b}, \mathrm{Bb}$ enzyme by 40 times, which amplifies activation of the complement pathway. Nickel has also been shown to activate calcineurin, a phosphatase that binds zinc and iron, and is usually activated by manganese. 
There is some evidence that nickel may have a role in the release of prolactin from the pituitary. In vitro studies have shown that nickel could directly inhibit the release of prolactin by the pituitary, and it has been suggested that nickel may be part of a prolactin inhibiting factor (LaBella et al. 1973). Intravenous exposure to nickel chloride has been shown to reduce serum levels of prolactin in male rats that were pretreated with chlorpromazine, which itself produces hyperprolactinemia (LaBella et al. 1973). The effect was not observed in rats that had not been pretreated with chlorpromazine. Nickel has also been shown to accumulate more in the pituitaries of pregnant rats than nonpregnant rats (Sunderman et al. 1978), suggesting that a toxicological effect through prolactin may only be manifested during maximum prolactin production. A subcutaneous injection study has also shown that nickel can change the quality of the milk produced, resulting in increased milk solids (42\%) and lipids (110\%), and decreased protein (29\%) and lactose (61\%) (Dostal et al. 1989). Because these changes were noted in comparison to pairfed rats, they were not considered to be a result of changes in food intake.

The mechanism of nickel carcinogenicity has not been firmly established; it is likely that the carcinogenic effects result from a variety of mechanisms. The available evidence suggests that, mechanistically, nickel carcinogenicity is probably the result of genetic factors and/or direct (e.g., conformational changes) or indirect (e.g., generation of oxygen radicals) epigenetic factors. Additionally, certain nickel compounds promote cell proliferation, which would convert repairable DNA lesions into nonrepairable mutations. Nickel is considered to be genotoxic, but has a low mutagenic potential (Kasprzak et al. 2003b). The nickel-induced DNA damage has resulted in the formation of chromosomal aberrations (Conway and Costa 1989; Dhir et al. 1991; Larramendy et al. 1981; Lechner et al. 1984; Sen and Costa 1986b; Sen et al. 1987; Waksvcik and Boysen 1982) that could result in deletion of senescence or tumor suppressor genes. Nickel compounds have also been found to be weak inducers of sister chromatid exchanges (Andersen 1983; Arrouijal et al. 1992; Larramendy et al. 1981; Ohno et al. 1982; Saxholm et al. 1981; Wulf 1980).

Although nickel has a relatively weak affinity for DNA, it has a high affinity for chromatin proteins, particularly histones and protamines (Costa et al. 1994; Kasprzak et al. 2003b; Oller et al. 1997). The complexing of nickel ions with heterochromatin results in a number of alterations including condensation, DNA hypermethylation, gene silencing, and inhibition of histone acetylation. These alterations have been shown to disturb gene expression (Costa et al. 1994; Kasprzak et al. 2003b; Lee et al. 1995; Oller et al. 1997; Zoroddu et al. 2002). Methylation of DNA may result in critical genes becoming incorporated into heterochromatin where they can no longer be expressed (Costa 1995). Some of the alterations in gene expression may be mediated by activated transcription factors. Nickel has been shown to alter several 
transcription factors including hypoxia-inducible transcription factor (HIF-1), activating transcription factor (ATF-1) involved in inactivation of thrombospondin-1, which suppresses angiogenesis, and $\mathrm{NF}_{\mathrm{K}} \mathrm{B}$ transcription factor involved in the inducible expression of adhesion molecules (Kasprzak et al. 2003b). The strongest epigenetic effects on nickel have been associated with HIF-1. The HIF-1 transcription factor is involved in the regulation of hypoxia-inducible genes involved in cell transformation, tumor promotion, and progression, angiogenesis, altered metabolism, and apoptosis. HIF-1 $\alpha$, one of the HIF-1 subunits, is over-expressed in both primary and metastatic tumors. It is induced in response to hypoxia and exposure to nickel (Li et al. 2004; Salnikow et al. 2000b). Both soluble and insoluble nickel compounds have also been shown to induce Cap43 (also called NDRG2) gene expression, which requires HIF-1 $\alpha$ activation (Costa et al. 2003; Li et al. 2004; Salnikow et al. 2000b). There is also evidence that nickel ions inhibit DNA repair (Hartwig et al. 1994). Nickel enhances the genotoxicity of ultraviolet light, x-rays, cis- and trans-platinum, and mitomycin C. In vitro studies in HeLa cells suggest that nickel inhibits the incision step in excision repair (Hartwig et al. 1994), while studies using Chinese hamster ovary cells suggest that nickel inhibits the ligation step of excision repair (Lee-Chen et al. 1994). The underlying mechanism of how nickel affects DNA repair is unclear. Sunderman and Barber (1988), Sunderman (1989b), and Hartwig et al. (1994) suggest that nickel ions may compete with zinc ions for binding to zinc-finger DNA binding proteins, resulting in structural changes in DNA that prevent repair enzymes from binding. Nickel may also directly interact with enzymes required for DNA repair (Hartwig et al. 1994).

The binding of nickel to the histone protein within heterochromatin could result in the generation of oxygen radicals. These oxygen radicals could subsequently induce damage bases, DNA strand breaks, and DNA protein crosslinks (Costa et al. 1994; Oller et al. 1997). The available evidence suggests that this mechanism would play a minor (if any) role in nickel carcinogenicity because the damage would be confined to heterochromatin regions of DNA, which lack active genes (Oller et al. 1997). However, nickel ions can complex with a number of cellular ligands including amino acids, peptides, and proteins resulting in the generation of oxygen radicals. The reactive oxygen species (ROS) generated could nonselectively damage DNA, possibly resulting in genetic changes in active genes (Kasprzak et al. 2003b; Oller et al. 1997).

\subsubsection{Animal-to-Human Extrapolations}

The available data on the toxicity of inhaled nickel provide strong evidence that the respiratory tract, in particular the lung, is the most sensitive target of nickel toxicity in humans and animals. There are 
limited exposure-response data for noncarcinogenic effects in humans; several well-designed animal studies (Benson et al. 1995a, 1995b; NTP 1996a, 1996b, 1996c) provide good exposure-response data that can be used to predict the thresholds of toxicity. One of these studies (NTP 1996c) was used to derive intermediate- and chronic-duration inhalation MRLs for nickel. A PBPK model (Hsieh et al. 1999a, 1999b) of lung deposition and clearance of inhaled nickel found a higher deposition of nickel in the alveolar region of humans compared to rats; however, adjustment for differences in lung weights resulted in a lower alveolar deposition of nickel in humans than in rats. This model, as described in more detail in Section 3.4.5, allows for prediction of human lung burdens. A cancer bioassay in rats and mice conducted by NTP (1996c) did not find significant increases in the occurrence of lung tumors. However, several occupational exposure studies have reported increases in the occurrence of nasal and lung tumors in workers exposed to soluble nickel compounds (primarily nickel sulfate and nickel chloride) in combination with exposures to other nickel compounds and/or carcinogenic agents (Anttila et al. 1998; Grimsrund et al. 2001, 2002; International Committee on Nickel Carcinogenesis in Man 1990). It is not known if the apparent species differences are due to differences in carcinogenic potential, co-exposure to other nickel compounds or other metals, or differences in exposure concentrations.

The available data on the oral toxicity of nickel are insufficient for comparing sensitive targets of toxicity and dose-response relationships between humans and laboratory animals. With the exception of dogs, the toxicokinetic properties of nickel did not differ between species. In dogs, the serum albumin lacks the histidine residue at the third position from the amino terminus (Hendel and Sunderman 1972); thus, dogs would not be a good model for the disposition of nickel in humans. In the absence of data to the contrary, it is assumed that most laboratory animals are a good model for humans.

\subsection{TOXICITIES MEDIATED THROUGH THE NEUROENDOCRINE AXIS}

Recently, attention has focused on the potential hazardous effects of certain chemicals on the endocrine system because of the ability of these chemicals to mimic or block endogenous hormones. Chemicals with this type of activity are most commonly referred to as endocrine disruptors. However, appropriate terminology to describe such effects remains controversial. The terminology endocrine disruptors, initially used by Colborn and Clement (1992), was also used in 1996 when Congress mandated the EPA to develop a screening program for "...certain substances [which] may have an effect produced by a naturally occurring estrogen, or other such endocrine effect[s]...". To meet this mandate, EPA convened a panel called the Endocrine Disruptors Screening and Testing Advisory Committee (EDSTAC), and in 1998, the EDSTAC completed its deliberations and made recommendations to EPA concerning endocrine 
disruptors. In 1999, the National Academy of Sciences released a report that referred to these same types of chemicals as hormonally active agents. The terminology endocrine modulators has also been used to convey the fact that effects caused by such chemicals may not necessarily be adverse. Many scientists agree that chemicals with the ability to disrupt or modulate the endocrine system are a potential threat to the health of humans, aquatic animals, and wildlife. However, others think that endocrine-active chemicals do not pose a significant health risk, particularly in view of the fact that hormone mimics exist in the natural environment. Examples of natural hormone mimics are the isoflavinoid phytoestrogens (Adlercreutz 1995; Livingston 1978; Mayr et al. 1992). These chemicals are derived from plants and are similar in structure and action to endogenous estrogen. Although the public health significance and descriptive terminology of substances capable of affecting the endocrine system remains controversial, scientists agree that these chemicals may affect the synthesis, secretion, transport, binding, action, or elimination of natural hormones in the body responsible for maintaining homeostasis, reproduction, development, and/or behavior (EPA 1997). Stated differently, such compounds may cause toxicities that are mediated through the neuroendocrine axis. As a result, these chemicals may play a role in altering, for example, metabolic, sexual, immune, and neurobehavioral function. Such chemicals are also thought to be involved in inducing breast, testicular, and prostate cancers, as well as endometriosis (Berger 1994; Giwercman et al. 1993; Hoel et al. 1992).

There is no evidence to suggest that nickel disrupts the normal functioning of the neuroendocrine axis. However, nickel-induced endocrine effects have been observed in laboratory animals. Several studies have found decreases in prolactin levels in lactating animals following oral (Smith et al. 1993), subcutaneous (Dostal et al. 1989), or intravenous (LaBella et al. 1973) administration. Additionally, in vivo and in vitro studies (Forgács et al. 1998, 2001) reported an inhibitory effect of nickel on stimulated testosterone production in mouse Leydig cells.

\subsection{CHILDREN'S SUSCEPTIBILITY}

This section discusses potential health effects from exposures during the period from conception to maturity at 18 years of age in humans, when all biological systems will have fully developed. Potential effects on offspring resulting from exposures of parental germ cells are considered, as well as any indirect effects on the fetus and neonate resulting from maternal exposure during gestation and lactation. Relevant animal and in vitro models are also discussed. 
Children are not small adults. They differ from adults in their exposures and may differ in their susceptibility to hazardous chemicals. Children's unique physiology and behavior can influence the extent of their exposure. Exposures of children are discussed in Section 6.6, Exposures of Children.

Children sometimes differ from adults in their susceptibility to hazardous chemicals, but whether there is a difference depends on the chemical (Guzelian et al. 1992; NRC 1993). Children may be more or less susceptible than adults to health effects, and the relationship may change with developmental age (Guzelian et al. 1992; NRC 1993). Vulnerability often depends on developmental stage. There are critical periods of structural and functional development during both prenatal and postnatal life and a particular structure or function will be most sensitive to disruption during its critical period(s). Damage may not be evident until a later stage of development. There are often differences in pharmacokinetics and metabolism between children and adults. For example, absorption may be different in neonates because of the immaturity of their gastrointestinal tract and their larger skin surface area in proportion to body weight (Morselli et al. 1980; NRC 1993); the gastrointestinal absorption of lead is greatest in infants and young children (Ziegler et al. 1978). Distribution of xenobiotics may be different; for example, infants have a larger proportion of their bodies as extracellular water and their brains and livers are proportionately larger (Altman and Dittmer 1974; Fomon 1966; Fomon et al. 1982; Owen and Brozek 1966; Widdowson and Dickerson 1964). The infant also has an immature blood-brain barrier (Adinolfi 1985; Johanson 1980) and probably an immature blood-testis barrier (Setchell and Waites 1975). Many xenobiotic metabolizing enzymes have distinctive developmental patterns. At various stages of growth and development, levels of particular enzymes may be higher or lower than those of adults, and sometimes unique enzymes may exist at particular developmental stages (Komori et al. 1990; Leeder and Kearns 1997; NRC 1993; Vieira et al. 1996). Whether differences in xenobiotic metabolism make the child more or less susceptible also depends on whether the relevant enzymes are involved in activation of the parent compound to its toxic form or in detoxification. There may also be differences in excretion, particularly in newborns who all have a low glomerular filtration rate and have not developed efficient tubular secretion and resorption capacities (Altman and Dittmer 1974; NRC 1993; West et al. 1948). Children and adults may differ in their capacity to repair damage from chemical insults. Children also have a longer remaining lifetime in which to express damage from chemicals; this potential is particularly relevant to cancer.

Certain characteristics of the developing human may increase exposure or susceptibility, whereas others may decrease susceptibility to the same chemical. For example, although infants breathe more air per kilogram of body weight than adults breathe, this difference might be somewhat counterbalanced by their 
alveoli being less developed, which results in a disproportionately smaller surface area for alveolar absorption (NRC 1993).

There are limited data on the toxicity of nickel in children. Several surveys of nickel-induced dermatitis found higher incidences of nickel sensitivity among young girls (Uter et al. 2003; Wantke et al. 1996). This apparent age-related increase in nickel-induced dermatitis is likely the result of increased nickel exposure in this segment of the population rather than an increase in sensitivity. For most of the general population, the sensitizing exposure is through consumer products, particularly jewelry. The higher prevalence of ear piercing in young women probably results in a higher prevalence of nickel sensitivity (Akasya-Hillenbrand and Özkaya-Bayazit 2002; Dotterud and Falk 1994; Larsson-Stymne and Widstrom 1985; Meijer et al. 1995; Uter et al. 2003). With the exception of nickel sensitization, there are limited toxicity data on age-related differences in toxicity in humans or animals. Zhang et al. (2000) found that elderly rats (aged 20 months) were more susceptible to the proinflammatory effects in the lungs of inhaled ultrafine nickel as compared to juvenile rats (aged 2 months).

A number of inhalation and oral exposure studies in rats and mice provide suggestive evidence that the fetus and neonate are targets of nickel toxicity. Increases in spontaneous abortions and stillbirths and decreases in neonatal survival have been observed in rats (Ambrose et al. 1976; Käkelä et al. 1999; RTI 1988a, 1988b; Smith et al. 1993) and mice (Berman and Rehnberg 1983) following oral exposure to nickel. Decreases in pup body weight have also been observed in rats following inhalation (Weischer et al. 1980) or oral (Ambrose et al. 1976; RTI 1988a, 1988b) exposure.

No human or animal data on the toxicokinetic properties of nickel in children or immature animals or studies examining possible age-related differences in the toxicokinetics of nickel were located. Studies with other metals, notably lead and cadmium (Bhattacharyya 1983), have found higher absorption rates in suckling animals, as compared to adults; it is not known if this is also true for nickel. Parenteral administration studies in rats and mice demonstrate that water-soluble nickel compounds are transferred across the placenta (Olsen and Jonsen 1979) and via maternal milk (Dostal et al. 1989). Subsequent sections of this chapter (Sections 3.8, 3.10, and 3.11) discuss the available information on biomarkers, interactions, and methods for reducing toxic effects. The available information is from adults and mature animals; no child-specific information was identified. It is likely that this information will also be applicable to children. 


\subsection{BIOMARKERS OF EXPOSURE AND EFFECT}

Biomarkers are broadly defined as indicators signaling events in biologic systems or samples. They have been classified as markers of exposure, markers of effect, and markers of susceptibility (NAS/NRC 1989).

Due to a nascent understanding of the use and interpretation of biomarkers, implementation of biomarkers as tools of exposure in the general population is very limited. A biomarker of exposure is a xenobiotic substance or its metabolite(s) or the product of an interaction between a xenobiotic agent and some target molecule(s) or cell(s) that is measured within a compartment of an organism (NAS/NRC 1989). The preferred biomarkers of exposure are generally the substance itself or substance-specific metabolites in readily obtainable body fluid(s) or excreta. However, several factors can confound the use and interpretation of biomarkers of exposure. The body burden of a substance may be the result of exposures from more than one source. The substance being measured may be a metabolite of another xenobiotic substance (e.g., high urinary levels of phenol can result from exposure to several different aromatic compounds). Depending on the properties of the substance (e.g., biologic half-life) and environmental conditions (e.g., duration and route of exposure), the substance and all of its metabolites may have left the body by the time samples can be taken. It may be difficult to identify individuals exposed to hazardous substances that are commonly found in body tissues and fluids (e.g., essential mineral nutrients such as copper, zinc, and selenium). Biomarkers of exposure to nickel are discussed in Section 3.8.1.

Biomarkers of effect are defined as any measurable biochemical, physiologic, or other alteration within an organism that, depending on magnitude, can be recognized as an established or potential health impairment or disease (NAS/NRC 1989). This definition encompasses biochemical or cellular signals of tissue dysfunction (e.g., increased liver enzyme activity or pathologic changes in female genital epithelial cells), as well as physiologic signs of dysfunction such as increased blood pressure or decreased lung capacity. Note that these markers are not often substance specific. They also may not be directly adverse, but can indicate potential health impairment (e.g., DNA adducts). Biomarkers of effects caused by nickel are discussed in Section 3.8.2.

A biomarker of susceptibility is an indicator of an inherent or acquired limitation of an organism's ability to respond to the challenge of exposure to a specific xenobiotic substance. It can be an intrinsic genetic or other characteristic or a preexisting disease that results in an increase in absorbed dose, a decrease in the 
biologically effective dose, or a target tissue response. If biomarkers of susceptibility exist, they are discussed in Section 3.10 "Populations that are Unusually Susceptible."

\subsubsection{Biomarkers Used to Identify or Quantify Exposure to Nickel}

Biological monitoring data are available primarily from occupational settings. Determination of nickel in the urine, feces, serum, hair, and nasal mucosa has been used to demonstrate human exposure to nickel compounds (Angerer and Lehnert 1990; Bencko et al. 1986; Bernacki et al. 1978; Elias et al. 1989; Ghezzi et al. 1989; Hassler et al. 1983; Torjussen and Andersen 1979). Based on an extensive review of biological monitoring data, Sunderman et al. (1993) concluded that serum and urine nickel levels were the most useful biomarkers of nickel exposure. Levels of nickel in urine and serum can provide the most information about levels of nickel exposure if the route, sources, and duration of exposure are known, if the chemical identities and physical-chemical properties of the nickel compounds are known, and if physiological information (e.g., renal function) of the exposed population is known (Sunderman 1993). In the general population, average nickel concentrations in serum and urine are 0.2 and $1-3 \mu \mathrm{g} / \mathrm{L}$, respectively (Templeton et al. 1994).

Significant correlations have been found between occupational exposure to less-soluble nickel compounds (breathing zone samples) and the levels of nickel in the urine and serum in various groups of workers (Morgan and Rouge 1984). Nickel levels in urine and serum of workers inhaling nickel powder, alloys, or slightly soluble compounds reflect the combined influences of long-term accumulation and recent exposures (Sunderman et al. 1986). Correlations between exposure concentration and levels in the urine and serum were found only in groups and not in individual workers. A relationship between exposure concentrations of soluble nickel compounds and levels of nickel in the urine and serum has also been reported (Bernacki et al. 1980). Urine and serum levels of nickel in workers inhaling soluble nickel compounds reflect the amount of nickel absorbed in the previous 1 or 2 days (Sunderman et al. 1986). With respect to monitoring nickel following exposure to soluble compounds, the best correlations between exposure concentration and urine levels were found with "end-of-shift" urine sampling (Bernacki et al. 1980) or "next morning" urine sampling (Tola et al. 1979). A correlation was found between urinary nickel and plasma nickel in workers, with nickel levels in urine being about 8-fold higher than plasma levels (Angerer and Lehnert 1990; Bernacki et al. 1978). Bavazzano et al. (1994) did not find significant correlations between urinary nickel concentrations in nickel electroplating workers and air concentrations of soluble nickel compounds. Among nickel refinery workers, there was a significant correlation between urinary nickel levels (unadjusted or adjusted for creatinine levels) and soluble nickel 
concentrations in air; the correlation coefficients were approximately 0.35 and 0.55 for unadjusted and adjusted urine (Werner et al. 1999). Adding insoluble nickel air concentrations into the regression analysis as a predictor value resulted in a negligible change. Similarly, Oliveira et al. (2000) found significant correlations between postshift urinary nickel levels (adjusted for creatinine excretion) and nickel concentrations in the air among workers at a galvanizing facility exposed to soluble nickel compounds. A lower correlation coefficient was found for the relationship between preshift adjusted urinary levels and airborne nickel concentrations.

Higher concentrations of nickel in the urine and the plasma and lower concentrations of nickel in the nasal mucosa were observed in workers exposed to soluble nickel compounds when compared to workers exposed to less-soluble compounds (Bernacki et al. 1978; Torjussen and Andersen 1979). Less-soluble nickel compounds tended to remain in the nasal mucosa (half-life of $\approx 3.5$ years); therefore, urinary and plasma levels were relatively low (Torjussen and Andersen 1979).

In workers exposed to nickel at a battery factory, a positive correlation was also found between air concentrations of nickel and concentrations of nickel in the feces (Hassler et al. 1983). High concentrations of nickel were found in the feces of workers exposed to nickel dusts containing large particles (as a result of greater mucociliary clearance from the lungs to the gastrointestinal tract) (Hassler et al. 1983).

It has been questioned whether or not levels of nickel in urine or serum are indicators of specific adverse health effects in humans. After reviewing monitoring data in occupationally exposed workers, Sunderman (1993) concluded that with the exception of nickel carbonyl, a relationship between nickel levels in body fluids and a specific health risk could not be established.

Exposure to nickel has also been monitored by assessing the content of nickel in the hair (Bencko et al. 1986). Analysis of the nickel content of hair provides evidence of past exposure and not changes in recent exposure to nickel. Correlations between exposure concentration and the level of nickel in hair were not reported.

Sensitization to nickel produces changes in serum antibodies (an increase in $\operatorname{IgG}, \operatorname{IgA}$, and $\operatorname{IgM}$ and a decrease in IgE) that may be monitored to determine if exposure to nickel has occurred (Bencko et al. 1983, 1986; Novey et al. 1983). These changes were found in both sensitized (Novey et al. 1983) and 
nonsensitized (Bencko et al. 1983, 1986) individuals. Information regarding the exposure concentration of nickel needed to produce serum antibody changes was not reported.

\subsubsection{Biomarkers Used to Characterize Effects Caused by Nickel}

Antibodies to hydroxymethyl uracil, an oxidized DNA base, were determined in workers exposed to nickel and cadmium, and in welders (Frenkel et al. 1994). Compared to controls, a significant increase in these antibodies was noted in the most highly exposed workers. Personal monitoring of 12 workers exposed to nickel and cadmium showed correlation coefficients between exposure concentrations and the antibodies of 0.4699 for cadmium and 0.7225 for nickel. Antibodies to hydroxymethyl uracil were not increased among welders. The levels of antibodies in the control populations for the nickel cadmium workers and for the welders were different, indicating the importance of determining the distribution of a new biomarker in controls for each population that is studied. This preliminary study suggests that antibodies to oxidized DNA products may be useful biomarkers for nickel and other metals that induce oxidative stress.

A preliminary study using imaging cytometry of nasal smears obtained from nickel workers indicates that this method may be useful to detect precancerous and cancerous lesions (Reith et al. 1994). With this method in which the cells were obtained by brushing the inside of the nose, the investigators were able to distinguish between nickel-exposed workers with non-dysplastic normal and suspicious mucosa smears and those with dysplastic lesions.

Although increases in oxidized DNA products and precancerous and cancerous lesions in the nose have been associated with nickel exposure, these effects are not specific to nickel. There are no specific biomarkers for nickel adverse health effects.

\subsection{INTERACTIONS WITH OTHER CHEMICALS}

A number of interactions of nickel with other chemicals are reported in the literature. The toxicity of nickel has been mitigated by treatment with chelating agents (Horak et al. 1976; Misra et al. 1988; Sunderman et al. 1976). Chelation treatment stimulates the excretion of nickel, thereby mitigating its toxicity. Lipophilic chelating agents, such as triethylenetetramine (TETA) and Cyclam (1,4,8,11-tetraazacyclotetradecane), were more effective than hydrophilic chelating agents such as EDTA, 
cyclohexanediamine tetraacetic acid (CDTA), diethylenetriamine pentaacetic acid (DTPA), and hydroxyethylenediamine triacetic acid (HEDTA) (Misra et al. 1988). The higher efficacy of the lipophilic agents may be due to their ability to bind to nickel both intracellularly and extracellularly, while the hydrophilic agents can only bind extracellularly.

A cross-reactivity between nickel and cobalt in sensitive individuals has been noted. For example, eight patients with asthma resulting from cobalt exposure also developed asthma when challenged with nickel sulfate (Shirakawa et al. 1990).

Nickel has also been found to interact with other metals such as iron, chromium, magnesium, manganese, zinc, and cadmium. The toxicity of nickel was mitigated by treatment with zinc (Waalkes et al. 1985) and magnesium (Kasprzak et al. 1986). The data suggest that magnesium, but not zinc, acted by altering the pharmacokinetics of nickel. The mechanism of action for zinc could not be determined from the study (Waalkes et al. 1985). Nickel absorption is increased during iron deficiency (Müller-Fassbender et al. 2003; Talkvist and Tjälve 1997), suggesting that iron deficiency may result in increased nickel toxicity. Coadministration of magnesium and nickel resulted in increased urinary excretion of nickel and decreased deposition of nickel in the lung, liver, and kidneys (Kasprzak et al. 1986). Manganese dust inhibited nickel subsulfide-induced carcinogenesis following simultaneous intramuscular injection of the two compounds (Sunderman and McCully 1983). The inhibition by manganese was a local and not a systemic effect.

Pretreatment of animals with cadmium 1 week before nickel treatment enhanced the nephrotoxicity and hepatotoxicity of nickel (Khandelwal and Tandon 1984). The mechanism of interaction could not be determined from these studies. Pretreatment of mice with cadmium 24 hours before nickel treatment has also been shown to decrease nickel-induced lethality and lipid peroxidation in the liver (Srivastava et al. 1995). The investigators suggested that a cadmium-induced production of ceruloplasmin, which prevented a nickel-induced reduction of ceruloplasmin, provided the protection against nickel toxicity.

More severe respiratory effects (increases in lung weight, in the accumulation of alveolar macrophages, and in the density of type II cell volumes) were observed in rabbits exposed by inhalation to both nickel and trivalent chromium than in rabbits exposed to nickel only (Johansson et al. 1988b).

In iron-deficient rats, nickel enhanced the absorption of iron (Nielsen 1980; Nielsen et al. 1980, 1984). This effect of nickel was only observed when ferric sulfate was given. No interaction was observed when 
iron was given as a $60 \%$ ferric $/ 40 \%$ ferrous sulfate mixture. It has been proposed that nickel facilitates the passive diffusion of ferric ions by stabilizing the transport ligand (Nielsen 1980).

Veien and Menne (1990) have suggested that vasoactive substances found in food can enhance nickel sensitivity reactions. Foods that they suggested that nickel-sensitive people should avoid include beer, wine (especially red wine), herring, mackerel, tuna, tomatoes, onions, carrots, apples, and citrus fruits. The vasoactive substances may increase the amount of nickel that is able to reach the skin.

\subsection{POPULATIONS THAT ARE UNUSUALLY SUSCEPTIBLE}

A susceptible population will exhibit a different or enhanced response to nickel than will most persons exposed to the same level of nickel in the environment. Reasons may include genetic makeup, age, health and nutritional status, and exposure to other toxic substances (e.g., cigarette smoke). These parameters result in reduced detoxification or excretion of nickel, or compromised function of organs affected by nickel. Populations who are at greater risk due to their unusually high exposure to nickel are discussed in Section 6.7, Populations with Potentially High Exposures.

Individuals sensitized to nickel may be unusually susceptible because exposure to nickel by any route may trigger an allergic response. Epidemiology studies indicate that African-Americans have a higher nickel sensitivity than Caucasians and that women of both racial groups have higher reaction rates than men (Nethercott and Holness 1990; North American Contact Dermatitis Group 1973; Prystowsky et al. 1979). The incidence of reactions may be higher in women because they generally wear more metal jewelry than men. Further studies are required to determine if there are true gender and racial differences in nickel sensitivity, or if it is indeed a difference in exposure.

A relationship between HLA and nickel sensitivity was observed in individuals who had a contact allergy and positive results in a patch test for nickel (Mozzanica et al. 1990). The nickel-sensitive group had a significant elevation in HLA-DRw6 antigen, compared to controls with no history of atopy or contact dermatitis. The relative risk for individuals with DRw6 to develop a sensitivity to nickel was approximately 3.3. The presence of DRw6 may be monitored to determine the potential risk of individuals to become sensitized to nickel.

Nickel that has been absorbed into the blood stream is primarily excreted in the urine. Therefore, individuals with kidney dysfunction are likely to be more sensitive to nickel. The increased sensitivity of 
persons with kidney dysfunction is also suggested by increased serum concentrations of nickel in dialysis patients (Hopfer et al. 1989). Because diabetics often have kidney damage, and because of the hyperglycemic effects of nickel observed in animal studies, the sensitivity of diabetics to nickel is also likely to be increased.

\subsection{METHODS FOR REDUCING TOXIC EFFECTS}

This section will describe clinical practice and research concerning methods for reducing toxic effects of exposure to nickel. However, because some of the treatments discussed may be experimental and unproven, this section should not be used as a guide for treatment of exposures to nickel. When specific exposures have occurred, poison control centers and medical toxicologists should be consulted for medical advice. The following texts provide specific information about treatment following exposures to nickel:

Bronstein AC, Currance PL. 1988. Emergency care for hazardous material exposure. Washington, DC: The CV Mosby Company, 147-148.

Gosselin RE, Smith RP, Hodge HC. 1984. Clinical toxicology of commercial products, 5th ed. Baltimore, MD: Williams \& Wilkins, II, 145.

Stutz DR, Janusz SJ. 1988. Hazardous materials injuries--a handbook for pre-hospital care. 2nd ed. Beltsville, MD: Bradford Communications Corporation, 218-219.

\subsubsection{Reducing Peak Absorption Following Exposure}

General recommendations for reducing absorption of nickel following acute inhalation exposure have included moving the patient to fresh air and monitoring for respiratory distress (HSDB 2003). About 20$35 \%$ of less-soluble nickel deposited in the lungs is absorbed into the blood from the respiratory tract (see Section 3.4.1.1). The nickel that is not absorbed into the blood is removed by mucociliary action and is expectorated or swallowed. Since the oral toxicity of metallic nickel is low, treatment with fluid and electrolyte replacement has been considered necessary only in cases with severe vomiting and diarrhea (HSDB 2003), which can occur as a result of nickel-induced gastrointestinal irritation (Sunderman et al. 1988). Thus, further induction of emesis is seldom necessary. EDTA added to the diet of humans decreased the bioavailability of orally administered nickel (Solomons et al. 1982). The presence of food in the stomach also reduced the gastrointestinal absorption of nickel (Christensen and Lagesson 1981). Oral administration of water or milk helps to dilute caustic nickel compounds in the stomach (Bronstein 
and Currance 1988; Stutz and Janusz 1988). In cases of dermal or ocular exposure, the skin or eyes should be thoroughly washed to prevent absorption by the skin or irritation of the eyes (Bronstein and Currance 1988; Stutz and Janusz 1988). Topical application of chelating agents and barrier creams has also been used to reduce dermal absorption in nickel-sensitive subjects (Gawkrodger et al. 1995). The most effective topical ligand for nickel yet described is 5-chloro-7-iodoquinolin-8-ol, but its use may be limited by its toxicity. Propylene glycol, petrolatum, and lanolin have been shown to reduce the dermal absorption of nickel.

\subsubsection{Reducing Body Burden}

Once absorbed into the blood, nickel has been found to distribute to the kidneys, liver, heart, fat, peripheral nervous tissues, and brain of animals (see Section 3.4.2). A mean serum half-time of nickel of 60 hours was measured in humans after oral exposure to nickel sulfate and nickel chloride (Sunderman et al. 1988).

A number of methods to decrease the body burden of nickel have been used or suggested. As discussed in Section 3.9, chelation treatment with a number of agents has been helpful (Horak et al. 1976; Misra et al. 1988; Sunderman et al. 1976). Lipophilic chelating agents such as TETA and Cyclam were more effective than hydrophilic chelating agents such as EDTA, CDTA, DTPA, and HEDTA (Misra et al. 1988). This may reflect differences in the distribution of hydrophilic and lipophilic agents between the intracellular and extracellular compartments. The use of diethyldithiocarbamate (DDC) as a chelating agent has been suggested as the preferred agent (Goldfrank et al. 1990; HSDB 2003). Disulfiram, which is metabolized to two molecules of DDC, might also be effective if DDC is not available. Penicillamine has also been used as a chelating agent for nickel. Intravenous infusion of fluids reduced the half-time for serum clearance of nickel from 60 to 27 hours in humans accidentally exposed to nickel sulfate and nickel chloride in water (Sunderman et al. 1988). The use of chelating agents over the long term to reduce nickel body burden in nickel-sensitive individuals is not recommended because it would also result in the reduction of other essential metals (Veien and Menne 1990). A nickel-restricted diet is useful in some sensitive adults for reducing nickel dermatitis, but this diet must be used with caution in nickel-sensitive children because it may not provide sufficient levels of nutrients for growth (Veien and Menne 1990). 


\subsubsection{Interfering with the Mechanism of Action for Toxic Effects}

Many toxic effects of both soluble nickel and some relatively less-soluble (in water) nickel compounds, which slowly dissolve in serum and cytosol, are due to nickel ions (Sunderman and Oskarsson 1991). In addition to reducing body burden of nickel, chelating agents may effectively mitigate toxicity by binding to nickel ions before toxic effects can be produced. For example, contact dermatitis is a prevalent allergic response to nickel, and disulfiram has been shown to be effective in clearing up cases of nickel dermatitis (Goldfrank et al. 1990; HSDB 2003).

Inhalation exposure to nickel or nickel compounds (along with other metals) in the workplace has resulted in such adverse respiratory effects as chronic bronchitis, emphysema, reduced vital capacity, and asthma (see Section 3.2.1.2). Studies in animals have indicated that the effects of nickel compounds on the respiratory system (chronic inflammation, fibrosis, macrophage hyperplasia) depend on the solubility of the compounds rather than on lung burden. Nickel oxide (low solubility) was less toxic than the soluble nickel sulfate but resulted in a higher lung burden. Nickel compounds have been shown to have effects on lung macrophages of animals, including accumulation of macrophages and granular material in the alveoli and an increase in volume density of alveolar type II cells. A decrease in alveolar macrophage activity was observed in animals after exposure to nickel compounds, and the more-soluble compounds had the greatest effect (Haley et al. 1990). The relationship between the effects on alveolar macrophages and respiratory toxicity is unknown, but since soluble nickel compounds appear to have greater effects, the involvement of the nickel ion is implicated.

The mechanisms of nickel carcinogenicity have not been established. There is a strong evidence that the mechanism involves the binding of nickel to biological macromolecules, including chromatin and proteins (Costa et al. 1994; Kasprzak et al. 2003b; Lee et al. 1995; Oller et al. 1997; Zoroddu et al. 2002). The complexing of nickel ions and chromatin can result in hypermethylation of DNA condensation, which alters gene expression. Additionally, the binding of nickel ions to proteins and peptides can result in the generation of oxygen radicals, which could indirectly damage DNA. Mechanisms that interfere with the binding of nickel ions to macromolecules may interfere with the carcinogenicity of nickel.

In conclusion, it appears that the toxicity of nickel and nickel compounds involves the binding of nickel ions to biological macromolecules. Chelation therapy appears to be effective both in reducing the body burden of nickel and interfering with the mechanism by which nickel exerts toxic effects by competing with the binding sites on biological molecules. 


\subsection{ADEQUACY OF THE DATABASE}

Section 104(i)(5) of CERCLA, as amended, directs the Administrator of ATSDR (in consultation with the Administrator of EPA and agencies and programs of the Public Health Service) to assess whether adequate information on the health effects of nickel is available. Where adequate information is not available, ATSDR, in conjunction with the National Toxicology Program (NTP), is required to assure the initiation of a program of research designed to determine the health effects (and techniques for developing methods to determine such health effects) of nickel.

The following categories of possible data needs have been identified by a joint team of scientists from ATSDR, NTP, and EPA. They are defined as substance-specific informational needs that if met would reduce the uncertainties of human health assessment. This definition should not be interpreted to mean that all data needs discussed in this section must be filled. In the future, the identified data needs will be evaluated and prioritized, and a substance-specific research agenda will be proposed.

\subsubsection{Existing Information on Health Effects of Nickel}

The existing data on health effects of inhalation, oral, and dermal exposure of humans and animals to nickel are summarized in Figure 3-5. The purpose of this figure is to illustrate the existing information concerning the health effects of nickel. Each dot in the figure indicates that one or more studies provide information associated with that particular effect. The dot does not necessarily imply anything about the quality of the study or studies, nor should missing information in this figure be interpreted as a "data need". A data need, as defined in ATSDR's Decision Guide for Identifying Substance-Specific Data Needs Related to Toxicological Profiles (Agency for Toxic Substances and Disease Registry 1989), is substance-specific information necessary to conduct comprehensive public health assessments. Generally, ATSDR defines a data gap more broadly as any substance-specific information missing from the scientific literature.

Humans have been exposed to nickel in nickel mines and processing plants, and numerous epidemiology studies have been performed to assess the cause of death in these workers. Accidental ingestion of nickel also has been documented in a small child and in electroplating workers. Nickel dermatitis is the most prevalent effect of nickel in humans. 
Figure 3-5. Existing Information on Health Effects of Nickel

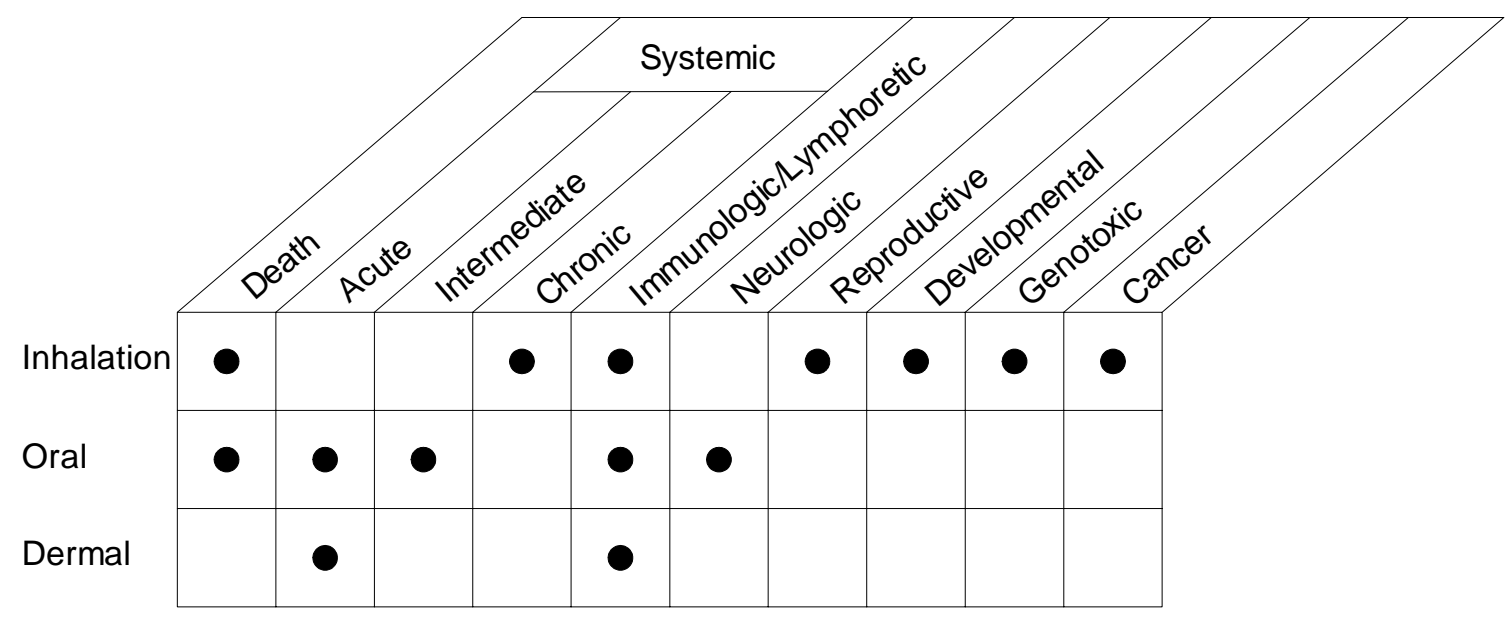

Human

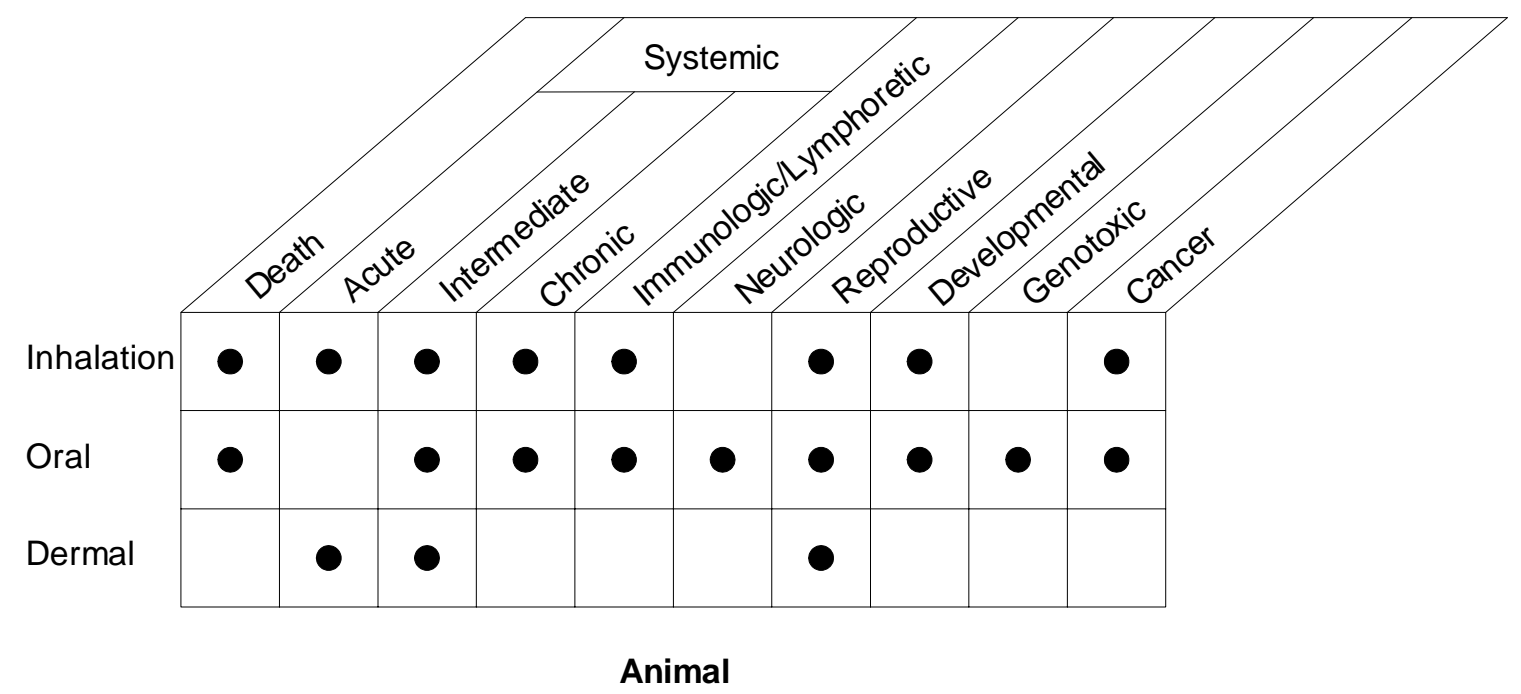

Existing Studies 
Several chronic inhalation and oral studies and acute dermal studies in animals are reported in the literature. These studies exposed several species of animals to both soluble and less-soluble nickel compounds. The target organs were found to be the respiratory system for inhalation exposure and the respiratory system, gastrointestinal tract, hematological system, and kidneys for oral exposure at high levels. Reproductive and developmental effects were observed in animals after inhalation exposure and after oral exposure to nickel. Nickel sensitivity and dermatitis were also observed.

\subsubsection{Identification of Data Needs}

Acute-Duration Exposure. Data on the acute toxicity of nickel come from case reports of individuals exposed to nickel via inhalation, ingestion, or dermal contact, studies of patch testing in humans, and animal inhalation, oral, and dermal exposure studies. Human inhalation data are limited to a study of a worker dying due to respiratory tract injury following a 90-minute exposure to a very high concentration of metallic nickel with a small particle size (Rendall et al. 1994). Adverse gastrointestinal and neurological effects were observed in workers who ingested drinking water contaminated with nickel and boric acid (Sunderman et al. 1988). The contribution of boric acid to these effects is not known. Patch testing and oral nickel challenge testing have been done on individuals with contact dermatitis to determine if an allergy to nickel exists (Christensen and Moller 1975; Cronin et al. 1980; Eun and Marks 1990; Gawkrodger et al. 1986; Jordan and King 1979; Kaaber et al. 1978; Nielsen et al. 1990; Sjovall et al. 1987; Veien et al. 1987). With the exception of nickel sensitivity following dermal contact, the available human data are not sufficient for identifying the most sensitive targets of nickel toxicity.

Acute inhalation studies in animals of nickel sulfate, nickel subsulfide, and nickel oxide indicate that nickel sulfate as the most toxic of the three compounds tested (NTP 1996a, 1996b, 1996c). The most sensitive target of nickel toxicity in animals appears to be the respiratory tract. Alveolitis, chronic lung inflammation, alveolar macrophage hyperplasia, and atrophy of the nasal olfactory epithelium have been observed in rats exposed to nickel sulfate (Evans et al. 1995; NTP 1996c) or nickel subsulfide (Benson et al. 1995b; NTP 1996b), and active lung inflammation has been observed in rats exposed to nickel oxide (NTP 1996a). Chronic lung inflammation was also observed in mice acutely exposed to nickel sulfate (NTP 1996c) or nickel subsulfide (NTP 1996b). In addition to the respiratory effects, adverse immunological effects have been observed in mice exposed to nickel chloride (Adkins et al. 1979; Graham et al. 1978) or nickel sulfate (Adkins et al. 1979). Although the available acute-duration inhalation data are sufficient for identifying the critical target of nickel toxicity, the data were not 
considered adequate for derivation of an inhalation MRL because a serious LOAEL was identified at the lowest concentration tested in a study examining the respiratory tract (NTP 1996c). Although a NOAEL was identified for immunological effects; this study (Graham et al. 1978) was not suitable for MRL derivation due to the uncertainty of whether the NOAEL concentration would also be a no effect level for respiratory effects. A study involving exposure to low concentrations of a soluble nickel compound in which the respiratory tract was examined is might provide data to derive an acute-duration inhalation MRL.

Acute oral studies in animals are limited to $\mathrm{LD}_{50}$ studies (Haro et al. 1968; Mastromatteo 1986), a mouse study reporting increases in the occurrence of sperm head abnormalities (Sobti and Gill 1989), and a developmental toxicity screening study in mice that did not find adverse developmental effects (Seidenberg et al. 1986). Because of the limited number of end points examined, these studies do not provide sufficient information for identifying the most sensitive target of nickel toxicity following acute oral exposure, and are thus inadequate for MRL derivation. Acute oral exposure studies that examine a number of end points, including reproductive and development toxicity, would help to identify the most sensitive target of toxicity. Studies utilizing a number of doses would be useful for establishing the doseresponse relationships for ingested nickel.

The development of nickel sensitivity in mice has been shown to be related to both the concentration of the nickel solution applied to the skin and the duration of exposure (Siller and Seymour 1994). Male mice showed a weaker response than females, and further studies regarding the gender difference in the development of nickel sensitivity would be useful. Additionally, dermal exposure studies examining a number of potential end points would be useful for identifying the most sensitive target of nickel toxicity following dermal exposure.

Intermediate-Duration Exposure. Intermediate-duration inhalation studies in humans were not located. Several studies examining the relationship between nickel ingestion and contact dermatitis were identified (Jordan and King 1979; Santucci et al. 1994; Sjovall et al. 1987). These studies are not useful for identifying the critical target of nickel toxicity or the threshold of toxicity in nonsensitized individuals. No human studies examining the toxicity of nickel following dermal contact for an intermediate duration were located.

A number of adverse health effects have been observed in laboratory animals exposed to airborne nickel; the effects occurred in the respiratory tract (Benson et al. 1995a; Bingham et al. 1972; Horie et al. 1985; 
Johansson and Camner 1986; NTP 1996a, 1996b, 1996c; Tanaka et al. 1988), blood glucose levels (Weischer et al. 1980), immune and lymphoreticular system (Haley et al. 1990; Johansson et al. 1980, 1987, 1988a, 1989; Morimoto et al. 1995; NTP 1996a, 1996b, 1996c; Spiegelberg et al. 1984), reproductive system (NTP 1996a), and the developing organism (Weischer et al. 1980). The available inhalation data provide strong evidence that the respiratory tract is the most sensitive target of nickel toxicity following intermediate-duration exposure. Chronic active lung inflammation was the most sensitive respiratory effect and a NOAEL for this effect (NTP 1996c) was used to derive an intermediateduration inhalation MRL.

A number of animal studies have assessed the toxicity of nickel following intermediate-duration oral exposure. Observed effects include decreases in body weight (American Biogenics Corporation 1988; Dieter et al. 1988; RTI 1988a, 1988b; Springborn Laboratories 2002; Weischer et al. 1980; Whanger 1973), kidney damage (Dieter et al. 1988), adverse lung effects (American Biogenics Corporation 1988; RTI 1988b), adverse reproductive effects (Käkelä et al. 1999; Pandey and Srivastava 2000; Pandey et al. 1999) and decreases in fetal/neonatal survival (Ambrose et al. 1976; Käkelä et al. 1999; RTI 1988a, 1988b; Smith et al. 1993; Springborn Laboratories 2000b). These data provide suggestive evidence that the developing organism may be a sensitive target of nickel toxicity following intermediate-duration exposure. As discussed in the sections on data needs for Reproductive Effects and Developmental Effects, additional studies are needed to confirm the identification of these effects as sensitive targets of nickel toxicity. Additional intermediate-duration studies would be useful for identifying sensitive targets of systemic toxicity and establishing dose-response relationships.

Dose-response data for dermal exposure of humans or animals to nickel were not identified. Dermal exposure studies would be useful for identifying sensitive targets of toxicity and establishing exposureresponse relationships.

Chronic-Duration Exposure and Cancer. A number of epidemiology studies examining the inhaled toxicity of nickel in workers at nickel mines or nickel processing plants have been identified (Bencko et al. 1983, 1986; Berge and Skyberg 2003; Cornell 1984; Cornell and Landis 1984; Enterline and Marsh 1982; Godbold and Tompkins 1979; Kilburn et al. 1990; Muir et al. 1993; Pedersen et al. 1973; Polednak 1981; Redmond et al. 1994; Shannon et al. 1991; Sunderman and Horak 1981). In general, these studies were mortality studies and did not provide nickel monitoring data. Additionally, Chashschin et al. (1994) examined the potential of nickel to induce reproductive and developmental effects in female nickel workers. Chronic oral toxicity data in humans are limited to a study on nickel 
sensitized individuals (Panzani et al. 1995), which examined the occurrence of contact dermatitis. Three studies examined the occurrence of contact dermatitis in individuals chronically exposed to nickel via dermal contact (Lee and Lee 1990; Meijer et al. 1995; Wall and Calnan 1980).

The toxicity of nickel sulfate (NTP 1996c), nickel subsulfide (NTP 1996b; Ottolenghi et al. 1974), and nickel oxide (NTP 1996a; Takenaka et al. 1985, 1988) following chronic inhalation exposure has been investigated in a number of studies in laboratory animals. The results of these studies provide strong evidence that the lung is the most sensitive target of toxicity; inflammatory changes were observed in the lung at the lowest adverse effect levels. Other effects that have been observed include damage to the nasal olfactory epithelium (NTP 1996b, 1996c), decreases in body weight gain (Ottolenghi et al. 1974; Takanaka et al. 1985), and hyperplasia of the bronchial lymph nodes (NTP 1996a, 1996b, 1996c). A chronic-duration inhalation MRL was derived from the NTP (1996c) rat study of nickel sulfate. Data on the chronic toxicity of ingested nickel in laboratory animals are limited to a 2-year study in rats and dogs (Ambrose et al. 1976). The observed effects included decreases in body weight gain, lung damage, and adverse kidney effects. A chronic-duration oral MRL was not derived from this study because intermediate-duration studies provide suggestive evidence that the developing organism and possibly the reproductive system are sensitive targets of toxicity; these end points were not examined in chronicduration studies. Additional oral exposure studies would be useful for identifying the critical targets of toxicity for ingested nickel; studies which examined the systemic toxicity of nickel would be useful in assessing whether the developing organism and/or the reproductive system are most sensitive targets. No chronic-duration dermal studies in laboratory animals were located. Studies by the dermal route of exposure would be useful for identifying the most sensitive targets of toxicity and establishing exposureresponse relationships.

A number of occupational exposure studies have examined the carcinogenic potential of nickel. In general, these studies have found increased risks of lung and/or nasal cancer in workers exposed to lesssoluble nickel compounds (Chovil et al. 1981; Doll et al. 1977; Enterline and Marsh 1982; International Committee on Nickel Carcinogenesis in Man 1990; Magnus et al. 1982; Pedersen et al. 1973; Sunderman et al. 1989a) or soluble nickel compounds (Anttila et al. 1998; Grimsrund et al. 2002, 2003; International Committee on Nickel Carcinogenesis in Man 1990). No studies have examined the carcinogenicity of nickel in humans following oral or dermal exposure. A series of bioassays conducted by NTP (1996a, 1996b, 1996c) and Ottolenghi et al. (1974) examined the carcinogenic risk of inhaled nickel. Significant increases in the occurrence of lung tumors following exposure to nickel oxide (NTP 1996a) and nickel subsulfide (NTP 1996b; Ottolenghi et al. 1974), but not after nickel sulfate (NTP 1996c), were found. No 
additional inhalation studies in laboratory animals are needed at this time. Data on the carcinogenicity of ingested nickel are limited to a rat and mouse study conducted by Schroeder and associates (Schroeder and Mitchener 1975; Schroeder et al. 1974); no increases in the occurrence of malignant tumors were observed. These studies are inadequate for assessing carcinogenic potential because very low doses, below the MTD, were administered. Additional oral exposure carcinogenicity studies are needed to assess whether increased exposure to nickel could lead to an increased risk of developing cancer. Carcinogenicity studies using animals dermally exposed to nickel were not located. Cancer has been observed, however, after parental administration of less-soluble nickel compounds (e.g., nickel oxide, nickel subsulfide), but not soluble nickel compounds (Gilman 1962; Kasprzak et al. 1983; Lumb and Sunderman 1988; Smialowicz et al. 1985; Sunderman and Maenza 1976; Sunderman and McCully 1983).

Genotoxicity. Investigators conducting epidemiology studies have reported a higher incidence of chromosomal aberrations in nickel workers compared to controls (Elias et al. 1989; Waksvik and Boysen 1982). Both in vitro and in vivo studies in mammals indicate that nickel is genotoxic (Andersen 1983; Biedermann and Landolph 1987; Conway and Costa 1989; Costa et al. 1982; DiPaolo and Casto 1979; Hansen and Stern 1984; Larramendy et al. 1981; Miura et al. 1989; Ohno et al. 1982; Saxholm et al. 1981; Sobti and Gill 1989; Wulf 1980), and the mechanism of action of nickel on cellular DNA has been studied (Ciccarelli and Wetterhahn 1982; Patierno and Costa 1985, 1987; Robinson and Costa 1982). Additional studies regarding the genotoxicity of nickel compounds are not needed at this time.

Reproductive Toxicity. Data on the reproductive toxicity of nickel in humans is limited to a study of women working at a nickel hydrometallurgy refining plant (Chashschin et al. 1994). However, interpretation of these study results is limited by the lack of information on the control of potential confounding variables, heavy lifting, and possible heat stress. Several oral exposure studies in animals suggest that nickel can result in testicular and epididymal damage (Käkelä et al. 1999; Pandey et al. 1999) and decreases in sperm motility, count, and sperm abnormalities (Pandy and Srivastava 2000; Pandey et al. 1999; Sobti and Gill 1999), or alterations in fertility (Käkelä et al. 1999; Pandey et al. 1999). Other oral studies have not found histological alterations in male or female reproductive tissues or impaired fertility following intermediate- or chronic- duration exposure (Ambrose et al. 1976; American Biogenics Corporation 1988; Obone et al. 1999; RTI 1988a, 1988b; Springborn Laboratories 2000a). Although testicular effects were also observed following inhalation exposure, the investigators (NTP 1996b, 1996c) considered the testicular effects to be secondary to emaciation. Additionally, fertility was not adversely affected in a single generation study (Springborn Laboratories 2000b) or two multigeneration studies (RTI 1988a, 1988b; Springborn Laboratories 2000a). The poor reporting of the study results, particularly 
incidence data and statistical analysis, limits the interpretation of the Käkelä et al. (1999), Pandey et al. (1999), and Pandey and Srivastava (2000) studies. An expert evaluation of the unpublished results of these studies, along with the other available reproductive toxicity studies (RTI 1988a, 1988b; Springborn Laboratories 2000a, 2000b), may provide insight on the apparent differences between the studies. Nickel treatment of rats during lactation has also been shown to change the quality of the milk (Dostal et al. 1989). Further studies concerning the role of physiological levels, as well as toxic levels, of nickel in the release of prolactin from the pituitary could provide useful information on potential reproductive and developmental effects of nickel.

Developmental Toxicity. There are limited data on the potential developmental toxicity of nickel in humans. An increase in structural malformations was observed in infants of women who worked in a nickel hydrometallurgy refining plant (Chashschin et al. 1994); however, the lack of information on control of potential confounding variables such as smoking and alcohol use and heavy lifting, and possible heat stress limits the interpretation of these results. Decreased fetal body weight was observed in offspring of rats exposed to high levels of nickel via inhalation during gestation (Weischer et al. 1980). Developmental effects such as increased pup mortality, decreased pup survival, and decreased pup body weight were observed in oral exposure single-generation studies involving male-only, female-only, or male and female exposure to nickel (Käkelä et al. 1999), multigeneration studies in rats (Ambrose et al. 1976; RTI 1988a, 1988b; Springborn Laboratories 2000b), and multilitter studies in rats (Smith et al. 1993). Although the available studies have consistently found decreases in pup survival, decreases in maternal body weight, food consumption, and water consumption often occur at the same dose levels. Thus, it is not known if the effects are due to nickel-induced damage to the offspring or are secondary to the maternal toxicity. Studies that controlled for maternal food intake and water consumption would be useful in understanding the mechanism of nickel toxicity. Developmental toxicity studies utilizing a number of dose levels would provide useful information in establishing the dose-response relationships for nickel. Studies assessing the developmental effects following dermal exposure were not located. Developmental effects have also been observed in animals following parental administration of nickel (Chernoff and Kavlock 1982; Lu et al. 1979; Sunderman et al. 1978).

Immunotoxicity. Human exposure to a large dose of nickel can result in sensitization manifested as contact dermatitis. Although there are limited data for the inhalation route, there are extensive data for the oral and dermal routes. Three studies examined immunological end points following inhalation exposure; two of these studies (Bencko et al. 1983, 1986) measured immunoglobulin levels in nickel workers and found significant alterations. The third study (Shirakawa et al. 1990) found positive results 
in patch tests of workers with hard metal lung disease. In nickel-sensitized individuals, oral exposure to fairly low doses of nickel can result in contact dermatitis; this has been tested in several acute-duration studies (Christensen and Moller 1975; Cronin et al. 1980; Gawkrodger et al. 1986; Veien et al. 1987) and two intermediate-duration studies (Jordan and King 1979; Sjovall et al. 1987). There is extensive information on the immunotoxicity of nickel in humans following dermal exposure. In general, the dermal exposure studies fall into two main categories: patch testing in individuals with contact dermatitis (Akasya-Hillenbrand and Özkaya-Bayazit 2002; Cavelier et al. 1988; Emmett et al. 1988; Eun and Marks 1990; Keczkes et al. 1982; Meijer et al. 1995; Menne et al. 1987; Simonetti et al. 1998; Uter et al. 2003; Wantke et al. 1996) and studies designed to assess the occurrence of nickel sensitivity in the general population (Dotterud and Falk 1994; Larsson-Stymme and Widstrom 1985; Menne and Holm 1983; Nielsen et al. 2002).

Animal studies demonstrate that nickel can induce immunological effects in nonsensitized individual. Alterations in nonspecific immunity (e.g., macrophage activity) (Adkins et al. 1979; Haley et al. 1990; Johansson et al. 1980) and humoral and cell mediated immunity (e.g., resistance to bacterial infection, response to foreign substances) (Adkins et al. 1979; Graham et al. 1978; Morimoto et al. 1995; Spiegelberg et al. 1984) has been observed in animals following inhalation exposure. Similarly, oral exposure to nickel has resulted in alterations in natural killer cells (Ilback et al. 1994) and humoral and cell mediated immunity (e.g., resistance to bacterial infection, response to foreign substances) (Dieter et al. 1988; Ilback et al. 1994). One dermal exposure study in mice examined the exposure-response relationship for nickel sensitization in mice (Siller and Seymour 1994). Studies designed to assess the dose-response relationship for contact dermatitis and oral dose are needed; the results of these studies should be considered during the derivation of oral MRLs for nickel. Additionally, studies that examined whether tolerance to nickel can develop and that assess cross sensitization of nickel with other metals would also be useful.

Neurotoxicity. No studies on the neurotoxicity of nickel in humans following inhalation or dermal exposure were located. Neurological effects (giddiness, weariness) were reported in individuals accidentally exposed to nickel and boric acid in drinking water (Sunderman et al. 1988). Temporary blindness in half of each eye occurred shortly after one person took a $0.05-\mathrm{mg} / \mathrm{kg}$ dose of nickel as nickel sulfate in drinking water (Sunderman et al. 1989b). There is limited information on the neurotoxicity of nickel in laboratory animals. No histological alterations were observed in the central nervous system following inhalation (NTP 1996a, 1996b, 1996c) or oral exposure (Ambrose et al. 1976; Obone et al. 1999). Although histological damage to the nasal olfactory epithelium was observed in animals following 
inhalation exposure to nickel sulfate or nickel subsulfide (Evans et al. 1995; NTP 1996b, 1996c), functional changes were not noted (Evans et al. 1995). Neurological signs (lethargy, ataxia, prostration) were observed in dying rats treated with nickel for 3 months; however, these effects were probably associated with overall toxicity (American Biogenics Corporation 1988). No animal dermal exposure studies examined neurological end points. The human data provide suggestive evidence that exposure to nickel may result in neurological effects; additional animal studies examining neurobehavioral performance would provide valuable information on the neurotoxic potential of nickel.

Epidemiological and Human Dosimetry Studies. A number of epidemiology studies regarding nickel toxicity are available in the literature. Most of these studies have focused on the carcinogenicity of inhaled nickel (Anttila et al. 1998; Chovil et al. 1981; Doll et al. 1977; Enterline and Marsh 1982; Grimsrund et al. 2002, 2003; International Committee on Nickel Carcinogenesis in Man 1990; Magnus et al. 1982; Pedersen et al. 1973; Sunderman et al. 1989a) or nickel sensitivity following oral (Christensen and Moller 1975; Cronin et al. 1980; Gawkrodger et al. 1986; Jordan and King 1979; Sjovall et al. 1987; Veien et al. 1987) or dermal (Akasya-Hillenbrand and Özkaya-Bayazit 2002; Cavelier et al. 1988; Dotterud and Falk 1994; Emmett et al. 1988; Eun and Marks 1990; Keczkes et al. 1982; Larsson-Stymme and Widstrom 1985; Meijer et al. 1995; Menne and Holm 1983; Menne et al. 1987; Nielsen et al. 2002; Simonetti et al. 1998; Uter et al. 2003; Wantke et al. 1996) exposure. As nickel exposure levels in the occupational environments have been reduced, continued health monitoring of populations occupationally exposed to nickel would be useful to determine if more subtle adverse health effects occur in humans at lower concentrations. Continued monitoring of nickel sensitization in the general population is needed to assess whether the increased popularity of body piercing will result in increased occurrences of nickel sensitivity. Additional studies on the dose-response relationship of ingested nickel dose and contact dermatitis would be useful. Animal data provide some suggestive evidence that nickel may be a reproductive toxicant and maternal exposure may result in increases in neonatal mortality. Inclusion of these end points in occupational exposure studies may provide valuable information on whether these would also be end points of concern for humans.

\section{Biomarkers of Exposure and Effect.}

Exposure. Nickel is a naturally occurring component of the diet and can be detected in hair, blood, urine, and feces (Angerer and Lehnert 1990; Bencko et al. 1986; Bernacki et al. 1978; Elias et al. 1989; Ghezzi et al. 1989; Hassler et al. 1983; Torjussen and Andersen 1979). In persons exposed to nickel above background levels, positive qualitative correlations have been found between air concentrations of nickel 
and nickel levels in the feces (Hassler et al. 1983) and urine (Angerer and Lehnert 1990; Bavazzano et al. 1994; Bernacki et al. 1978, 1980; Morgan and Rouge 1984; Oliveira et al. 2000; Sunderman et al. 1986; Tola et al. 1979; Torjussen and Andersen 1979; Werner et al. 1999). Additional studies examining the relationship between levels of nickel in the urine and body burden levels and studies associating urinary nickel levels and the manifestation of adverse health effects would be useful in establishing biological exposure indices for nickel.

Effect. A relationship between human lymphocyte antigens and nickel sensitivity exists and predicts that individuals with this antigen have a relative risk of approximately 3.3 for developing nickel sensitivity (Mozzanica et al. 1990). Antibodies to hydroxymethyl uracil, an oxidized DNA base, have also been shown to be increased in some nickel-exposed workers (Frenkel et al. 1994). A preliminary study using imaging cytometry of nasal smears obtained from nickel workers indicates that this method may be useful to detect precancerous and cancerous lesions (Reith et al. 1994). Additional studies that examine markers of early biological effects, such as changes in gene expression measured by microarrays, could be piloted with in vitro cell lines to determine nickel-specific markers, followed by in vivo screening of people living near sites that contain elevated levels of nickel or who have occupational exposures to nickel. Studies that identify nickel-specific biomarkers may be helpful in alerting health professionals to nickel exposure before serious toxicological effects occur.

Absorption, Distribution, Metabolism, and Excretion. Pharmacokinetic studies in humans indicate that nickel is absorbed through the lungs (Bennett 1984; Grandjean 1984; Sunderman and Oskarsson 1991), gastrointestinal tract (Nielsen et al. 1999; Patriarca et al. 1997; Sunderman et al. 1989b), and skin (Fullerton et al. 1986; Norgaard 1955). Food greatly decreases the absorption of nickel from the gastrointestinal tract (Sunderman et al. 1989b). Following absorption from the lungs and the gastrointestinal tract, nickel is excreted in the urine (Angerer and Lehnert 1990; Bernacki et al. 1978; Elias et al. 1989; Ghezzi et al. 1989; Hassler et al. 1983; Sunderman et al. 1989b; Torjussen and Andersen 1979). Increased levels of nickel were found in the lungs, nasal septum, liver, and kidneys of workers inhaling nickel (Andersen and Svenes 1989; Kollmeier et al. 1987; Raithel et al. 1988; Rezuke et al. 1987; Sumino et al. 1975; Svenes and Andersen 1998; Torjussen and Andersen 1979). Animal data indicate that after inhalation, nickel particles can remain in the lungs (nickel oxide) or be absorbed and then excreted in the urine (nickel sulfate). High levels of nickel have been found in the liver, kidneys, and spleen of animals after inhaling high levels of nickel (Benson et al. 1987, 1988, 1994, 1995a; NTP 1996a, 1996b, 1996c; Tanaka et al. 1985). Nickel that has been absorbed after oral exposure is primarily distributed to the kidneys before being excreted in the urine. High levels of nickel were also found in the 
liver, heart, lungs, fat, peripheral nervous tissue, and brain (Ambrose et al. 1976; Borg and Tjalve 1989; Dieter et al. 1988; Jasim and Tjalve 1986a, 1986b; Oskarsson and Tjalve 1979; Whanger 1973). Studies examining the bioavailability of nickel from soil following oral exposure would be useful for determining the absorbed dose from nickel-contaminated soil at a hazardous waste site. Further verification of the toxicokinetic models developed by Hsieh et al. (1999a, 1999b) and Sunderman et al. (1989b) would improve the ability to predict the absorbed dose following inhalation or oral exposure.

Comparative Toxicokinetics. Studies that examine the toxicokinetics of nickel in humans after occupational exposure, ingestion of nickel from food and water, and dermal exposure are available (Bennett 1984; Fullerton et al. 1986; Grandjean 1984; Norgaard 1955; Sunderman and Oskarsson 1991; Sunderman et al. 1989b). The toxicokinetics of both inhaled and ingested nickel have been examined in several species of animals (rats, mice, dogs, hamsters) (Ambrose et al. 1976; Benson et al. 1987, 1988; Borg and Tjalve 1989; Dieter et al. 1988; Jasim and Tjalve 1986a, 1986b; NTP 1996a, 1996b, 1996c; Oskarsson and Tjalve 1979; Tanaka et al. 1985; Whanger 1973). Dermal studies have been performed in guinea pigs and rabbits (Lloyd 1980; Norgaard 1957). The limited human data correlate well with the toxicokinetics observed in animals. Studies that compare the toxicokinetics of humans and animals using the same experimental protocol would be helpful in determining which species of animal is the best model for assessing the effects of nickel in humans.

Methods for Reducing Toxic Effects. Approximately $20-35 \%$ of inhaled less-soluble nickel is absorbed through the lungs (Bennett 1984; Grandjean 1984; Sunderman and Oskarsson 1991). Methods that would enhance the clearance of nickel from the lung, thus preventing or reducing the severity of lung damage (inflammation or fibrosis), would be useful. The administration of EDTA in food (Solomons et al. 1982) and the presence of food in the stomach (Christensen and Lagesson 1981) decrease the amount of nickel that is absorbed through the gastrointestinal tract. Several chelating agents (e.g., TETA, Cyclam, EDTA) have been shown to be effective in reducing the body's nickel burden (Horak et al. 1976; Misra et al. 1988; Sunderman et al. 1976). It is not known if other methods, such as dialysis, would be more effective in reducing the body burden. The mechanism of nickel toxicity involves the binding of nickel ions to macromolecules; chelating agents have been shown to bind to the nickel ions, thus mitigating the toxicity. Studies designed to determine if other methods would be more effective in binding nickel ions would be useful. 
Children's Susceptibility. Data needs relating to both prenatal and childhood exposures, and developmental effects expressed either prenatally or during childhood, are discussed in detail in the Developmental Toxicity subsection above.

There are limited data on the toxicity of nickel in children. Several patch testing studies have included children (Akasya-Hillenbrand and Özkaya-Bayazit 2002; Dotterud and Falk 1994; Larsson-Stymne and Widstrom 1985; Meijer et al. 1995; Uter et al. 2003; Wantke et al. 1996), the results of which suggest that children may be more susceptible than adults. However, the increased susceptibility observed in children may be due to increased prolong exposure to nickel-containing products such as earrings, rather than increased sensitivity; additional studies are needed to verify this assumption. Studies in laboratory animals provide evidence that the fetus and neonates are sensitive targets of nickel toxicity following inhalation or oral exposure (Ambrose et al. 1976; Berman and Rehnberg 1993; Käkelä et al. 1999; RTI 1988a, 1988b; Smith et al. 1993; Weischer et al. 1980). As noted in the Developmental Toxicity section, additional studies are needed to verify this apparent sensitivity. No human or animal data on the toxicokinetic properties of nickel in children or immature animals or studies examining possible agerelated differences in the toxicokinetics of nickel were located. Studies with other metals, notably lead and cadmium (Bhattacharyya 1983), have found higher absorption rates in suckling animals, as compared to adults; it is not known if this is also true for nickel. Additional studies examining potential age-related differences in nickel would provide valuable information on the susceptibility of children to nickel toxicity.

Child health data needs relating to exposure are discussed in Section 6.8.1, Identification of Data Needs: Exposures of Children.

\subsubsection{Ongoing Studies}

Ongoing studies pertaining to nickel have been identified and are shown in Table 3-14. 
Table 3-14. Ongoing Studies on Nickel Health Effects

\begin{tabular}{lll}
\hline Investigator & Affiliation & Research area \\
\hline Costa, M & New York University, & Examination of the epigenetic \\
& School of Medicine & mechanisms of nickel carcinogenesis \\
Rokita, SE & University of Maryland & Mechanisms of nickel carcinogenicity \\
Kasprzak, KS & National Cancer Institute & Mechanisms of nickel carcinogenicity \\
Leikauf, GD & University of Cincinnati & $\begin{array}{l}\text { Genetic determinants on nickel-induced } \\
\text { lung toxicity }\end{array}$ \\
Warshaw, EM & Department of Veterans Affairs, & Treatment of nickel contact dermatitis \\
& Medical Center, Minneapolis & \\
\hline
\end{tabular}

Source: FEDRIP 2004 



\section{CHEMICAL AND PHYSICAL INFORMATION}

\subsection{CHEMICAL IDENTITY}

Nickel is a transition metal in group VIII of the periodic table following iron and cobalt (Cotton and Wilkinson 1980). Its outer shell of electrons has a $4 s^{2} 3 d^{8}$ configuration. While nickel can exist in oxidation states $-1,0,+2,+3$, and +4 , its only important oxidation state is nickel $(+2)$ under normal environmental conditions.

Nickel forms useful alloys with many metals. It is added to metals to increase their hardness, strength, and corrosion resistance. The most familiar are nickeliferous alloys used in stainless steel and coppernickel alloys used in coinage metal.

Nickel oxide also comes in a black crystalline form that has a slightly higher oxygen content than its formula, $\mathrm{NiO}$ (Antonsen 1981). The nickel content of black nickel oxide is 76-77\% compared with $78.5 \%$ for the more stable green nickel oxide. Nickel ammonium sulfate, nickel sulfate, nickel chloride, and nickel nitrate usually exist as hexahydrates, while nickel acetate, nickel cyanide, and nickel sulfamate are in the form of a tetrahydrate.

Information regarding the chemical identity of nickel is located in Table 4-1.

\subsection{PHYSICAL AND CHEMICAL PROPERTIES}

Metallic nickel is a hard, lustrous, silvery white metal, which, in its bulk form, is resistant to attack by air and water at ordinary temperatures. However, powdered nickel is reactive in air and may spontaneously ignite.

Nickel has typical metallic properties; it can be readily rolled, drawn into wire, forged, and polished. It is also ferromagnetic and a good conductor of both heat and electricity. Nickel is positioned after hydrogen in the electrochemical series and slowly displaces hydrogen ions from dilute hydrochloric and sulfuric acids. It reacts more rapidly with nitric acid. Nickel is highly resistant to attack by strong alkalis (Hawley 1981). Black nickel oxide readily yields nickel salts in the presence of acids (WHO 1991). 
Table 4-1. Chemical Identity of Nickel and Compounds ${ }^{a}$

\begin{tabular}{|c|c|c|c|}
\hline Characteristic & Nickel & Nickel acetate & Nickel ammonium sulfate \\
\hline Synonyms & $\begin{array}{l}\text { Cl 77775; Nickel 200; } \\
\text { Nickel 201; Nickel 205; Nickel } \\
\text { 270; Alnicob; NP } 2^{\text {b }}\end{array}$ & $\begin{array}{l}\text { Acetic acid, nickel(2+) salt; } \\
\text { nickel diacetate; nickelous } \\
\text { acetate; nickel(II) acetate }\end{array}$ & $\begin{array}{l}\text { Ammonium nickel sulfate; } \\
\text { sulfuric acid, ammonium } \\
\text { nickel(2+) salt; ammonium } \\
\text { disulfatonickelate(II) }\end{array}$ \\
\hline $\begin{array}{l}\text { Registered } \\
\text { trade name(s) }\end{array}$ & 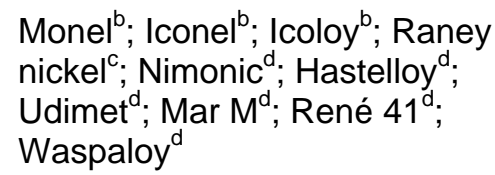 & No data & No data \\
\hline $\begin{array}{l}\text { Chemical } \\
\text { formula }\end{array}$ & $\mathrm{Ni}$ & $\mathrm{Ni}\left(\mathrm{CH}_{3} \mathrm{CO}_{2}\right)_{2}$ & $\mathrm{Ni}\left(\mathrm{NH}_{4}\right)_{2}\left(\mathrm{SO}_{4}\right)_{2}$ \\
\hline $\begin{array}{l}\text { Chemical } \\
\text { structure }\end{array}$ & $\mathrm{Ni}$ & {$\left[\mathrm{Ni}^{2+}\right]\left[\begin{array}{c}\mathrm{O} \\
\mathrm{H}_{3} \mathrm{C}-\mathrm{C} \\
\mathrm{C}-\mathrm{O}^{-}\end{array}\right]_{2}$} & {$\left[\mathrm{Ni}^{2+}\right]\left[\begin{array}{r}+ \\
\mathrm{NH}_{4}\end{array}\right]\left[\begin{array}{rr}\mathrm{O} & 2- \\
\mathrm{O}-\mathrm{S} & -\mathrm{O} \\
\mathrm{O} & \end{array}\right]_{2}$} \\
\hline \multicolumn{4}{|c|}{ Identification numbers: } \\
\hline CAS registry & $7440-02-0$ & $373-02-4$ & $15699-18-0$ \\
\hline $\begin{array}{l}\text { NIOSH } \\
\text { RTECS }\end{array}$ & QR5950000 ${ }^{d}$ & QR6125000 & WS6050000 ${ }^{d}$ \\
\hline $\begin{array}{l}\text { EPA } \\
\text { hazardous } \\
\text { waste }\end{array}$ & No data & No data & No data \\
\hline OHM/TADS & No data & No data & No data \\
\hline $\begin{array}{l}\text { DOT/UN/NA/ } \\
\text { IMCO shipping }\end{array}$ & No data & No data & No data \\
\hline HSDB & 1096 & 1029 & 1241 \\
\hline $\mathrm{NCl}$ & No data & No data & No data \\
\hline
\end{tabular}


Table 4-1. Chemical Identity of Nickel and Compounds ${ }^{a}$

\begin{tabular}{|c|c|c|c|}
\hline Characteristic & Nickel carbonate & Nickel chloride & Nickel cyanide \\
\hline Synonyms & $\begin{array}{l}\text { Cl 77779; Carbonic acid, } \\
\text { nickel(2+) salt; nickel (II) } \\
\text { carbonate; nickelous } \\
\text { carbonate; nickel } \\
\text { monocarbonate }\end{array}$ & $\begin{array}{l}\text { Nickel(II) chloride; nickel } \\
\text { dichloride; nickelous } \\
\text { chloride }\end{array}$ & $\begin{array}{l}\text { Nickel(II) cyanide; nickel } \\
\text { dicyanide; dicyanonickel }\end{array}$ \\
\hline $\begin{array}{l}\text { Registered } \\
\text { trade name(s) }\end{array}$ & No data & No data & No data \\
\hline $\begin{array}{l}\text { Chemical } \\
\text { formula }\end{array}$ & $\mathrm{NiCO}_{3}$ & $\mathrm{NiCl}_{2}$ & $\mathrm{Ni}(\mathrm{CN})_{2}$ \\
\hline $\begin{array}{l}\text { Chemical } \\
\text { structure }\end{array}$ & {$\left[\mathrm{Ni}^{2+}\right]\left[\begin{array}{rr}\mathrm{O}^{\mathrm{II}} & \\
\mathrm{O}^{-\mathrm{C}} & \mathrm{O}\end{array}\right]$} & $\mathrm{Cl}-\mathrm{Ni}-\mathrm{Cl}$ & $\mathrm{NC}-\mathrm{Ni}-\mathrm{CN}$ \\
\hline \multicolumn{4}{|c|}{ Identification numbers: } \\
\hline CAS registry & $3333-67-3$ & $7718-54-9$ & $557-19-7$ \\
\hline $\begin{array}{l}\mathrm{NIOSH} \\
\text { RTECS }\end{array}$ & QR6200000 ${ }^{d}$ & QR6475000 & QR6495000 ${ }^{d}$ \\
\hline $\begin{array}{l}\text { EPA } \\
\text { hazardous } \\
\text { waste }\end{array}$ & No data & No data & No data \\
\hline OHM/TADS & No data & No data & No data \\
\hline $\begin{array}{l}\text { DOT/UN/NA/ } \\
\text { IMCO shipping }\end{array}$ & No data & No data & UN1653 \\
\hline HSDB & 1662 & 860 & 1185 \\
\hline $\mathrm{NCl}$ & No data & No data & No data \\
\hline
\end{tabular}


Table 4-1. Chemical Identity of Nickel and Compounds ${ }^{a}$

\begin{tabular}{|c|c|c|c|}
\hline Characteristic & Nickel oxide & Nickel nitrate & Nickel subsulfide \\
\hline Synonyms & $\begin{array}{l}\text { Bunsenite; Cl 77777; green } \\
\text { nickel oxide; mononickel } \\
\text { oxide; nickel(II) oxide; } \\
\text { nickelous oxide; nickel } \\
\text { monoxide }{ }^{\text {b }} \text { nickel oxide sinter } \\
75^{\text {b; }} \text { nickel protoxide; } \\
\text { mononickel }\end{array}$ & $\begin{array}{l}\text { Nitric acid, nickel(2+) salt, } \\
\text { nickelous nitrate; nickel } \\
\text { dinitrate; nickel(II) nitrate }\end{array}$ & $\begin{array}{l}\text { Trinickel disulfide }{ }^{\text {b; }} \text {, nickel } \\
\text { sulfide; Heazlewoodite; } \\
\text { nickel sesquisulfide }^{\mathrm{b}} \text {; } \\
\text { khislevudite }^{\mathrm{b}} \text {; nickel } \\
\text { tritadisulfide }\end{array}$ \\
\hline $\begin{array}{l}\text { Registered } \\
\text { trade name(s) }\end{array}$ & Nickel oxide & No data & No data \\
\hline $\begin{array}{l}\text { Chemical } \\
\text { formula }\end{array}$ & NO & $\mathrm{Ni}\left(\mathrm{NO}_{3}\right)_{2}$ & $\mathrm{Ni}_{3} \mathrm{~S}_{2}$ \\
\hline $\begin{array}{l}\text { Chemical } \\
\text { structure }\end{array}$ & $\mathrm{Ni}-\mathrm{O}$ & 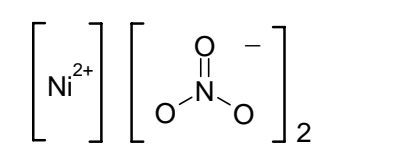 & No data \\
\hline \multicolumn{4}{|c|}{ Identification numbers: } \\
\hline CAS registry & $1313-99-1$ & $13138-45-9$ & $12035-72-2$ \\
\hline $\begin{array}{l}\text { NIOSH } \\
\text { RTECS }\end{array}$ & QR8400000 ${ }^{d}$ & $\mathrm{QR}^{2} 200000^{\mathrm{d}}$ & QR9800000 $^{d}$ \\
\hline $\begin{array}{l}\text { EPA } \\
\text { hazardous } \\
\text { waste }\end{array}$ & No data & No data & No data \\
\hline OHM/TADS & No data & No data & No data \\
\hline $\begin{array}{l}\text { DOT/UN/NA/ } \\
\text { IMCO shipping }\end{array}$ & No data & UN 27525; IMO 5.1 & No data \\
\hline HSDB & 1664 & 1829 & 2965 \\
\hline $\mathrm{NCl}$ & No data & No data & No data \\
\hline
\end{tabular}




\section{Table 4-1. Chemical Identity of Nickel and Compounds ${ }^{a}$}

\begin{tabular}{|c|c|c|}
\hline Characteristic & Nickel sulfamate & Nickel sulfate \\
\hline Synonyms & $\begin{array}{l}\text { Sulfamic acid, nickel(2+) salt } \\
\text { Nickel amidosulfate }{ }^{\mathrm{e}} \text {; Nickel } \\
\text { (II) sulfamate } \\
\text { Aeronikl } 400^{\mathrm{d}} ; \text { Aeronikl } 250^{\mathrm{d}} \text {; } \\
\text { Aeronikl } 575^{\mathrm{d}}\end{array}$ & $\begin{array}{l}\text { Nickel monosulfate; } \\
\text { nickelous sulfate; nickel(II) } \\
\text { sulfate; sulfuric acid nickel } \\
\text { salt }^{b}\end{array}$ \\
\hline $\begin{array}{l}\text { Registered } \\
\text { trade name(s) }\end{array}$ & No data & No data \\
\hline $\begin{array}{l}\text { Chemical } \\
\text { formula }\end{array}$ & $\mathrm{Ni}\left(\mathrm{NH}_{2} \mathrm{SO}_{3}\right)_{2}$ & $\mathrm{NiSO}_{4}$ \\
\hline $\begin{array}{l}\text { Chemical } \\
\text { structure }\end{array}$ & {$\left[\mathrm{Ni}^{2+}\right]\left[\begin{array}{cc}\mathrm{O} & - \\
\mathrm{H}_{2} \mathrm{~N}-\mathrm{S}-\mathrm{O} \\
\mathrm{O}^{-}\end{array}\right]_{2}$} & {$\left[\mathrm{Ni}^{2+}\right]\left[\begin{array}{rr}\mathrm{O}^{2} & 2- \\
0-\mathrm{S} & -\mathrm{O} \\
\mathrm{M} & \end{array}\right]$} \\
\hline \multicolumn{3}{|c|}{ Identification numbers: } \\
\hline CAS registry & $13770-89-3^{d}$ & $7786-81-4$ \\
\hline $\begin{array}{l}\text { NIOSH } \\
\text { RTECS }\end{array}$ & QR9275000 ${ }^{d}$ & QR9350000 ${ }^{d}$ \\
\hline $\begin{array}{l}\text { EPA } \\
\text { hazardous } \\
\text { waste }\end{array}$ & No data & No data \\
\hline OHM/TADS & No data & No data \\
\hline $\begin{array}{l}\text { DOT/UN/NA/ } \\
\text { IMCO shipping }\end{array}$ & No data & ID8027 \\
\hline HSDB & No data & 1114 \\
\hline $\mathrm{NCl}$ & No data & $\mathrm{NCl}-\mathrm{C} 60344^{\mathrm{d}}$ \\
\hline
\end{tabular}

${ }^{a}$ All information obtained from HSDB 2004 except where noted.

${ }^{\mathrm{b}}$ Czerczak and Gromiec 2001

'Tien and Howson 1981; Windholz 1983. Names refer to alloys of nickel. Generally, there is a series of alloys with the same trade name (e.g., Monel alloy K-400, Monel alloy K-500).

${ }^{\mathrm{d}}$ RTECS 2004

e Laschelles and Nicholls 1991

CAS = Chemical Abstracts Service; DOT/UN/NA/IMCO = Department of Transportation/United Nations/North America/International Maritime Dangerous Goods Code; EPA = Environmental Protection Agency; HSDB = Hazardous Substances Data Bank; $\mathrm{NCI}=$ National Cancer Institute; Ni = nickel; NIOSH = National Institute for Occupational Safety and Health; OHM/TADS = Oil and Hazardous Materials/Technical Assistance Data System; RTECS = Registry of Toxic Effects of Chemical Substances 
4. CHEMICAL AND PHYSICAL INFORMATION

Information regarding the physical and chemical properties of nickel and compounds is located in Table 4-2. 
Table 4-2. Physical and Chemical Properties of Nickel and Compounds ${ }^{a}$

\begin{tabular}{|c|c|c|c|c|}
\hline Property & Nickel & Nickel acetate & $\begin{array}{l}\text { Nickel } \\
\text { ammonium } \\
\text { sulfate }\end{array}$ & Nickel carbonate \\
\hline Molecular weight & 58.69 & 176.80 & 286.90 & 118.70 \\
\hline Color & Silvery & Green & Blue-green & Green \\
\hline Physical state & Solid & Solid & Solid & Solid \\
\hline Melting point & $1,455^{\circ} \mathrm{C}$ & Decomposes & No data & Decomposes \\
\hline Boiling point & $2,730^{\circ} \mathrm{C}$ & $\begin{array}{l}16.6^{\circ} \mathrm{C} ; \\
\text { decomposes }^{\mathrm{b}}\end{array}$ & No data & No data \\
\hline Density & $8.91 \mathrm{~g} / \mathrm{cm}^{3}$ & $1.798 \mathrm{~g} / \mathrm{cm}^{3}$ & $1.923 \mathrm{~g} / \mathrm{cm}^{3}$ & $4.39 \mathrm{~g} / \mathrm{cm}^{3}$ \\
\hline Odor & Odorless & Acetic odor & Odorless & No data \\
\hline \multicolumn{5}{|l|}{ Odor threshold: } \\
\hline Water & No data & No data & No data & No data \\
\hline Air & No data & No data & No data & No data \\
\hline \multicolumn{5}{|l|}{ Solubility: } \\
\hline Water & $1.13 \mathrm{mg} / \mathrm{L}$ at $37^{\circ} \mathrm{C}^{\mathrm{c}}$ & $\begin{array}{l}17 \text { weight } \% \text { at } \\
68{ }^{\circ} \mathrm{C}\end{array}$ & $104 \mathrm{~g} / \mathrm{L}$ at $20^{\circ} \mathrm{C}$ & $93 \mathrm{mg} / \mathrm{L}$ at $25^{\circ} \mathrm{C}$ \\
\hline $\begin{array}{l}\text { Organic } \\
\text { solvents }\end{array}$ & No data & $\begin{array}{l}\text { Insoluble in } \\
\text { alcohol }\end{array}$ & \multicolumn{2}{|c|}{ Insoluble in alcohol No data } \\
\hline \multicolumn{5}{|l|}{ Partition coefficients: } \\
\hline $\mathrm{K}_{\mathrm{ow}}$ & No data & No data & No data & No data \\
\hline $\mathrm{K}_{\mathrm{oc}}$ & No data & No data & No data & No data \\
\hline Vapor pressure & $\begin{array}{l}1 \mathrm{mmHg} \text { at } \\
1,810^{\circ} \mathrm{C}\end{array}$ & No data & No data & No data \\
\hline $\begin{array}{l}\text { Henry's law } \\
\text { constant }\end{array}$ & No data & No data & No data & No data \\
\hline $\begin{array}{l}\text { Autoignition } \\
\text { temperature }\end{array}$ & No data & No data & Nonflammable & Nonflammable \\
\hline Flashpoint & No data & No data & Nonflammable & Nonflammable \\
\hline Flammability limits & No data & No data & Nonflammable & Nonflammable \\
\hline Conversion factor & No data & No data & No data & No data \\
\hline Explosive limits & No data & No data & No data & No data \\
\hline
\end{tabular}


Table 4-2. Physical and Chemical Properties of Nickel and Compounds ${ }^{a}$

\begin{tabular}{|c|c|c|c|c|}
\hline Property & Nickel chloride & Nickel cyanide & Nickel oxide & Nickel nitrate \\
\hline Molecular weight & 129.60 & 110.73 & 74.69 & 182.72 \\
\hline Color & Golden yellow & Yellow brown & Green or black & Green \\
\hline Physical state & Solid & Solid & Solid & Solid \\
\hline Melting point & $1,001{ }^{\circ} \mathrm{C}$ & $>200^{\circ} \mathrm{C}$ & $1,955^{\circ} \mathrm{C}$ & $56.7^{\circ} \mathrm{C}^{\mathrm{C}}$ \\
\hline Boiling point & Sublimes at $973^{\circ} \mathrm{C}$ & Decomposes & No data & $136.7^{\circ} \mathrm{C}^{\mathrm{C}}$ \\
\hline Density & $3.55 \mathrm{~g} / \mathrm{cm}^{3}$ & $2.393 \mathrm{~g} / \mathrm{cm}^{3}$ & $6.72 \mathrm{~g} / \mathrm{cm}^{3}$ & $2.05 \mathrm{~g} / \mathrm{cm}^{3 \mathrm{~d}}$ \\
\hline Odor & None & $\begin{array}{l}\text { Weak almond } \\
\text { odor }\end{array}$ & No data & No data \\
\hline \multicolumn{5}{|l|}{ Odor threshold: } \\
\hline Water & No data & No data & No data & No data \\
\hline Air & No data & No data & No data & No data \\
\hline \multicolumn{5}{|l|}{ Solubility: } \\
\hline Water & $642 \mathrm{~g} / \mathrm{L}$ at $20^{\circ} \mathrm{C}$ & Insoluble & $1.1 \mathrm{mg} / \mathrm{L}$ at $20^{\circ} \mathrm{C}$ & $\begin{array}{l}2,385 \mathrm{~g} / \mathrm{L} \text { at } 0{ }^{\circ} \mathrm{C}^{\mathrm{d}} \\
48.5 \text { weight } \% \text { at } 20^{\circ} \mathrm{C}^{\mathrm{d}}\end{array}$ \\
\hline $\begin{array}{l}\text { Organic } \\
\text { solvents }\end{array}$ & $\begin{array}{l}\text { Soluble in ethanol; } \\
180 \mathrm{~g} / \mathrm{L} \text { at } 20^{\circ} \mathrm{C} \text { in } \\
\text { ethylene glycol }\end{array}$ & No data & No data & $\begin{array}{l}\text { Insoluble in alcohol } \\
\text { soluble in alcohol }^{c}\end{array}$ \\
\hline \multicolumn{5}{|l|}{ Partition coefficients: } \\
\hline $\mathrm{K}_{\mathrm{ow}}$ & No data & No data & No data & No data \\
\hline $\mathrm{K}_{\mathrm{oc}}$ & No data & No data & No data & No data \\
\hline Vapor pressure & $1 \mathrm{mmHg}$ at $671^{\circ} \mathrm{C}$ & No data & No data & No data \\
\hline $\begin{array}{l}\text { Henry's law } \\
\text { constant }\end{array}$ & No data & No data & No data & No data \\
\hline $\begin{array}{l}\text { Autoignition } \\
\text { temperature }\end{array}$ & Nonflammable & Nonflammable & No data & No data \\
\hline Flashpoint & Nonflammable & Nonflammable & No data & No data \\
\hline Flammability limits & Nonflammable & Nonflammable & No data & No data \\
\hline Conversion factor & No data & No data & No data & No data \\
\hline Explosive limits & No data & No data & No data & No data \\
\hline
\end{tabular}




\section{Table 4-2. Physical and Chemical Properties of Nickel and Compounds ${ }^{a}$}

\begin{tabular}{|c|c|c|c|}
\hline Property & Nickel subsulfide & $\begin{array}{l}\text { Nickel } \\
\text { sulfamate }\end{array}$ & Nickel sulfate \\
\hline Molecular weight & 240.212 & $322.94^{f, g}$ & 154.75 \\
\hline Color & Pale yellowish ${ }^{\mathrm{e}}$ & No data & Greenish-yellow \\
\hline Physical state & Solid & Solid & Solid \\
\hline Melting point & $787^{\circ} \mathrm{C}$ & No data & $840{ }^{\circ} \mathrm{C}$ \\
\hline Boiling point & No data & No data & Decomposes at $840^{\circ} \mathrm{C}$ \\
\hline Density & $5.87 \mathrm{~g} / \mathrm{cm}^{3}$ & No data & $4.01 \mathrm{~g} / \mathrm{cm}^{3}$ \\
\hline Odor & No data & No data & Odorless \\
\hline \multicolumn{4}{|l|}{ Odor threshold: } \\
\hline Water & No data & No data & No data \\
\hline Air & No data & No data & No data \\
\hline \multicolumn{4}{|l|}{ Solubility: } \\
\hline Water & $517 \mathrm{mg} / \mathrm{L}$ at $37^{\circ} \mathrm{C}^{\mathrm{C}}$ & No data & $293 \mathrm{~g} / \mathrm{L}$ at $0^{\circ} \mathrm{C}$ \\
\hline $\begin{array}{l}\text { Organic } \\
\text { Solvents }\end{array}$ & No data & No data & $\begin{array}{l}\text { Insoluble in ether and acetone; } 0.2 \mathrm{~g} / \mathrm{L} \text { at } \\
35^{\circ} \mathrm{C} \text { in ethanol; } 0.9 \mathrm{~g} / \mathrm{L} \text { at } 35^{\circ} \mathrm{C} \text { in } \\
\text { methanol }\end{array}$ \\
\hline \multicolumn{4}{|l|}{ Partition coefficients: } \\
\hline $\mathrm{K}_{\mathrm{ow}}$ & No data & No data & No data \\
\hline $\mathrm{K}_{\mathrm{oc}}$ & No data & No data & No data \\
\hline Vapor pressure & No data & No data & No data \\
\hline $\begin{array}{l}\text { Henry's law } \\
\text { constant }\end{array}$ & No data & No data & No data \\
\hline $\begin{array}{l}\text { Autoignition } \\
\text { temperature }\end{array}$ & No data & No data & Nonflammable \\
\hline Flashpoint & No data & No data & Nonflammable \\
\hline Flammability limits & No data & No data & Nonflammable \\
\hline Conversion factor & No data & No data & No data \\
\hline Explosive limits & No data & No data & No data \\
\hline
\end{tabular}

${ }^{a}$ All information obtained from HSDB 2004 except where noted.

${ }^{\mathrm{b}}$ Decomposes before melting.

Ishimatsu et al. 1995

${ }^{\mathrm{d}}$ Data are for the hexahydrate.

IARC 1990

'Data are for the tetrahydrate.

${ }^{g}$ Laschelles and Nicholls 1991 



\section{PRODUCTION, IMPORT/EXPORT, USE, AND DISPOSAL}

\subsection{PRODUCTION}

Nickel ranks 24th in order of abundance in the earth's crust, with an average concentration of $0.0086 \%$. Its crustal concentration varies from $>0.0001$ to $>0.3 \%$. Economically exploitable ore deposits typically contain 1-4\% nickel. The concentration of nickel increases towards the center of the earth, and nickel is estimated to comprise $0.22 \%$ of the earth's mantle and $5.8 \%$ of its core (Duke 1980a). Therefore, the nickel concentration is estimated to be $2 \%$ by weight when averaged over all of the Earth, making it the fifth most abundant element after iron, oxygen, magnesium, and silicon. Nickel is found combined with iron in meteorites; the nickel content ranges from 5 to 50\% (Duke 1980a; Mastromatteo 1986). It is also found in sea floor nodules (Mastromatteo 1986).

Nickel ores are of two general types: magmatic sulfide ores, which are mined underground, and lateritic hydrous nickel silicates or garnierites, which are surface mined (Duke 1980a; Warner 1984).

The most important nickel sulfide-arsenide deposits are in hydrothermal veins associated with mafic (i.e., rich in magnesium and iron) and ultramafic igneous rock. These ores typically contain 1-3\% nickel. Pentlandite $(\mathrm{Ni}, \mathrm{Fe})_{9} \mathrm{~S}_{8}$ is the principle ore. Pentlandite often occurs along with the iron mineral pyrrhotite and the copper mineral chalcopyrite, and part of the smelting and refining process separates the copper and iron from the nickel. The ore is concentrated by physical means (i.e., flotation and magnetic separation) after crushing. One of the largest sulfidic nickel deposits is in Sudbury, Ontario, Canada. Nickeliferous sulfide deposits are also found in Thompson, Manitoba, and Voisey's Bay, Labrador, Canada; South Africa; Russia (primarily Siberia); Finland; western Australia; and Minnesota (Ademec and Kihlgren 1967; Duke 1980a; Kuck 2002).

The lateritic hydrous nickel silicate ores are formed by the weathering of rocks rich in iron and magnesium in humid tropical areas. The repeated processes of dissolution and precipitation lead to a uniform dispersal of the nickel that is not amenable to concentration by physical means; therefore, these ores are concentrated by chemical means such as leaching. Lateritic ores are less well defined than sulfide ores. The nickel content of lateritic ores is similar to that of sulfide ore and typically ranges from 1 to 3\% nickel. Important lateritic deposits of nickel are located in Cuba, New Caledonia, Indonesia, Guatemala, the Dominican Republic, the Philippines, Brazil, and Spain. Fossil nickeliferous laterite 
deposits are found in Oregon, Greece, and the former Soviet Union, where humid, tropical climates prevailed in the past. Lateritic deposits constitute the largest nickel reserves (Ademec and Kihlgren 1967; Antonsen and Springer 1967; Duke 1980a). Thirty-five percent of known nickel reserves are in the United States, followed by Russia at 11\% (USGS 2003).

Sulfide ores are processed by a number of pyrometallurgical processes: roasting, smelting, and converting. During these processes, sulfur and iron are removed to yield a sulfur-deficient copper-nickel matte. Especially after roasting and converting, the nickel in the matte may consist primarily of nickel subsulfide. After physical separation of the copper and nickel sulfides, the nickel is refined electrochemically or by the carbonyl process. The treatment of the matte depends on the end use of the nickel. Alternatively, the sulfide can be roasted to form a nickel oxide sinter that is used directly in steel production.

Lateritic ore is processed by pyrometallurgical or hydrometallurgical processes. In the pyrometallurgical process, sulfur is generally added to the oxide ore during smelting, usually as gypsum or elemental sulfur, and an iron-nickel matte is produced. The smelting process that does not include adding sulfur produces a ferronickel alloy, containing $\leq 50 \%$ nickel, which can be used directly in steel production. Hydrometallurgical techniques involve leaching with ammonia or sulfuric acid, after which the nickel is selectively precipitated (Duke 1980b; IARC 1990; Tien and Howson 1981; Warner 1984). Alloys, such as stainless steels, are produced by melting primary metals and scrap in large arc furnaces and adjusting the carbon content and concentration of alloying metals to the desired levels. More information on the mining, smelting, and refining of nickel can be found in Duke (1980b), Tien and Howson (1981), and Warner (1984).

Domestic primary nickel production in the United States ceased in 1986 (Chamberlain 1985; Kirk 1988a) with the closing of the Hanna mine and smelter in Riddle, Oregon, and the AMAX refinery in Braithwaite, Louisiana. However, Glenbrook Nickel Company purchased the Riddle, Oregon, facility in 1989 and had reactivated the mining and smelting operation, but then decommissioned both the mining and smelting operations in 2000. World mine production of nickel in 2002 was estimated at 1,340,000 metric tons, which matched the production level reported for 2001 (Kuck 2002). Secondary nickel production from scrap is a major source of nickel for industrial applications. In 1988, an estimated 59,609 and 3,700 short tons (51,355 and 3,357 metric tons, respectively) of nickel were produced from ferrous and nonferrous scrap, respectively. Nickel recovery from scrap is estimated by using the gross weight of the scrap and a weighted average nickel content (e.g., $7.5 \%$ for stainless steel). The secondary 
recovery from ferrous scrap was considerably higher and the recovery from nonferrous scrap was considerably lower than for the previous 7 years in which the annual recovery of nickel from ferrous and nonferrous scrap ranged from 30,034 to 389,265 short tons (27,246-353,139 metric tons) and from 8,392 to 19,776 short tons $(7,613-17,940$ metric tons), respectively. The production of refined nickel in 1993 has been estimated as 220,700,346,800, 176,200, 52,100, and 96,300 short tons $(200,200,314,600$, 159,800, 47,300, and 87,400 metric tons, respectively) for North America, Europe, Asia, Africa, and Australia, respectively (ABMS 1994). In 2002, 1,210,000 metric tons of refined nickel were produced. Of this total production, there were 259,000 metric tons in the form of ferronickel, 678,000 metric tons as the metal, 97,000 metric tons as the oxide sinter, 17,800 metric tons as chemicals, and 154,000 metric tons as unspecified production (Kuck 2002). The distribution of world plant production of refined nickel was 20.3\%, Russia (Commonwealth of Independent States); 16.4\%, Europe; 12.9\%, Japan; 11.9\%, Canada; $15.8 \%$, Australia/New Caledonia; 4.5\%, China; 4.0\%, Africa; 3.7\%, Colombia; 3.3\%, Cuba, and 7.2\%, Brazil, Dominican Republic, Indonesia, and Venezuela (Kuck 2002). The reported world consumption of refined nickel was 1,150,800 metric tons in 2001, up from 997,800 metric tons in 1997 (ABMS 2002). In 2002, demand for primary nickel in the Western World was 1,032,000 metric tons, up from 968,700 metric tons in 2001 (Kuck 2002).

Tables 5-1 and 5-2 list the facilities that produced, imported, processed, or used nickel and its compounds, respectively, in 2001 according to reports made to the EPA under the requirements of Section 313 of the Emergency Planning and Community Right-to-Know Act of 1986, which were subsequently published in the Toxic Chemical Release Inventory (TRI) (TRI02 2004). Companies were required to report if they produced, imported, or processed $\geq 25,000$ pounds of nickel and its compounds or used $>10,000$ pounds. Also included in Tables 5-1 and 5-2 are the maximum amount of nickel and its compounds, respectively, that these facilities had on site and whether nickel was produced, processed, or used by the facility.

\subsection{IMPORT/EXPORT}

In 2002, the United States imported 130,000 metric tons of nickel, including 121,000 metric tons of unwrought metal (97,200 metric tons of cathodes, pellets, briquets and shot; 12,300 metric tons of ferronickel; 6,970 metric tons of powder and flakes, 1,230 metric tons of metallurgical-grade oxide; 1,280 metric tons of catalysts; and 1,590 metric tons of salts), 6,080 metric tons of stainless steel scrap, and 3,030 metric tons of nickel waste and scrap (Kuck 2002). In 2002, Canada supplied the largest share of primary nickel, 59,400 metric tons (46\%). Russia was the second largest exporter of primary nickel to the United States with 24,200 metric tons (19\%) followed by Australia and Norway with 10,400 and 
Table 5-1. Facilities that Produce, Process, or Use Nickel Metal

\begin{tabular}{|c|c|c|c|c|}
\hline State $^{a}$ & $\begin{array}{l}\text { Number } \\
\text { of } \\
\text { facilities }\end{array}$ & $\begin{array}{l}\text { Minimum } \\
\text { amount on site } \\
\text { in pounds }\end{array}$ & $\begin{array}{l}\text { Maximum } \\
\text { amount on site } \\
\text { in pounds }\end{array}$ & Activities and uses $^{c}$ \\
\hline$\overline{\mathrm{AL}}$ & 73 & 0 & $499,999,999$ & $1,2,3,4,5,6,7,8,9,10,11,12,13,14$ \\
\hline AR & 47 & 0 & $99,999,999$ & $1,2,3,4,5,6,7,8,9,10,11,12,13$ \\
\hline$A Z$ & 41 & 0 & $9,999,999$ & $1,2,3,4,5,6,7,8,9,10,11,12,13$ \\
\hline CA & 146 & 0 & $99,999,999$ & $1,2,3,4,5,6,7,8,9,10,11,12,13,14$ \\
\hline $\mathrm{CO}$ & 39 & 100 & $9,999,999$ & $1,2,3,4,5,6,7,8,10,11,12$ \\
\hline CT & 78 & 0 & $99,999,999$ & $1,2,3,4,5,6,7,8,9,10,11,12,13,14$ \\
\hline DC & 2 & 10,000 & 999,999 & 8 \\
\hline DE & 15 & 100 & 999,999 & $1,2,3,4,5,6,7,8,9,10,11,12$ \\
\hline $\mathrm{FL}$ & 30 & 0 & $499,999,999$ & $1,2,3,4,5,6,7,8,9,10,11,12$ \\
\hline GA & 57 & 0 & $999,999,999$ & $1,2,3,4,5,6,7,8,9,10,11,12,13,14$ \\
\hline $\mathrm{HI}$ & 1 & 10,000 & 99,999 & 10 \\
\hline IA & 71 & 0 & $9,999,999$ & $1,2,3,4,5,6,7,8,9,10,11,12,13,14$ \\
\hline ID & 12 & 0 & 999,999 & $1,3,5,7,8,10,12$ \\
\hline IL & 145 & 0 & $499,999,999$ & $1,2,3,4,5,6,7,8,9,10,11,12,13,14$ \\
\hline IN & 158 & 0 & $499,999,999$ & $1,2,3,4,5,6,7,8,9,10,11,12,13,14$ \\
\hline KS & 41 & 0 & $49,999,999$ & $1,2,3,5,7,8,9,10,11,12,13,14$ \\
\hline KY & 85 & 0 & $999,999,999$ & $1,2,3,4,5,6,7,8,9,10,11,12,13,14$ \\
\hline LA & 42 & 0 & $49,999,999$ & $1,2,3,4,5,6,7,8,9,10,11,12,13,14$ \\
\hline MA & 71 & 0 & $499,999,999$ & $1,2,3,4,5,6,7,8,9,10,11,12$ \\
\hline MD & 44 & 0 & $9,999,999$ & $1,2,3,4,5,6,7,8,9,10,11,12,13$ \\
\hline ME & 13 & 1,000 & $99,999,999$ & $1,3,7,8,12$ \\
\hline MI & 154 & 0 & $49,999,999$ & $1,2,3,4,5,6,7,8,9,10,11,12,13,14$ \\
\hline MN & 69 & 0 & $9,999,999$ & $1,2,3,4,5,6,7,8,9,10,11,12,13,14$ \\
\hline MO & 59 & 0 & $99,999,999$ & $1,2,3,4,5,6,7,8,9,10,11,12,13,14$ \\
\hline MS & 17 & 0 & $9,999,999$ & $1,2,3,6,7,8,10,12$ \\
\hline MT & 8 & 100 & 99,999 & $1,2,3,5,6,8,10,11$ \\
\hline NC & 66 & 0 & $99,999,999$ & $1,2,3,4,5,6,7,8,9,10,11,12,13,14$ \\
\hline ND & 9 & 1,000 & 999,999 & $2,3,5,7,8,9,12$ \\
\hline NE & 39 & 0 & $9,999,999$ & $1,2,3,5,6,7,8,9,10,11,12,13$ \\
\hline $\mathrm{NH}$ & 19 & 0 & $49,999,999$ & $3,7,8,9,11,12$ \\
\hline NJ & 86 & 0 & $49,999,999$ & $1,2,3,4,5,6,7,8,9,10,11,12,13$ \\
\hline NM & 13 & 100 & 999,999 & $2,3,6,7,8,10,11$ \\
\hline NV & 27 & 100 & $9,999,999$ & $1,2,4,5,6,7,8,9,10,11,12,13$ \\
\hline NY & 93 & 0 & $49,999,999$ & $1,2,3,4,5,6,7,8,9,10,11,12,13$ \\
\hline $\mathrm{OH}$ & 204 & 0 & $99,999,999$ & $1,2,3,4,5,6,7,8,9,10,11,12,13,14$ \\
\hline OK & 74 & 0 & $99,999,999$ & $1,2,3,4,5,6,7,8,9,10,11,12,13,14$ \\
\hline OR & 45 & 0 & $499,999,999$ & $1,2,3,4,5,6,7,8,9,10,11,12,13$ \\
\hline PA & 237 & 0 & $499,999,999$ & $1,2,3,4,5,6,7,8,9,10,11,12,13,14$ \\
\hline PR & 6 & 0 & 99,999 & $2,3,7,8,10,11,12$ \\
\hline RI & 36 & 0 & 999,999 & $1,2,3,4,6,7,8,9,10,11,12$ \\
\hline
\end{tabular}


Table 5-1. Facilities that Produce, Process, or Use Nickel Metal

\begin{tabular}{lcrrl}
\hline & $\begin{array}{l}\text { Number } \\
\text { of } \\
\text { facilities }\end{array}$ & $\begin{array}{l}\text { Minimum } \\
\text { amount on site } \\
\text { in pounds }\end{array}$ & $\begin{array}{l}\text { Maximum } \\
\text { amount on site } \\
\text { in pounds }\end{array}$ & $\begin{array}{l}\text { Activities and uses } \\
\text { State }\end{array}$ \\
\hline SC & 77 & 0 & $9,999,999$ & $1,2,3,4,5,6,7,8,9,10,11,12,13,14$ \\
SD & 9 & 1,000 & $\begin{array}{r}999,999 \\
1,5,7,8,9,11\end{array}$ \\
TN & 113 & 0 & $499,999,999$ & $1,2,3,4,5,6,7,8,9,10,11,12,13,14$ \\
TX & 137 & 0 & $9,999,999$ & $1,2,3,4,5,6,7,8,9,10,11,12,13,14$ \\
UT & 40 & 100 & $9,999,999$ & $1,2,3,4,5,6,7,8,10,11,12,13$ \\
VA & 49 & 0 & $49,999,999$ & $1,2,3,4,5,6,7,8,9,10,11,12,13,14$ \\
VT & 15 & 1,000 & 999,999 & $2,4,8,11,12$ \\
WA & 31 & 0 & 999,999 & $1,2,3,4,5,7,8,9,10,11,12,13$ \\
WI & 114 & 0 & $99,999,999$ & $1,2,3,4,5,6,7,8,9,10,11,12,14$ \\
WV & 35 & 0 & $499,999,999$ & $1,2,3,4,5,6,7,8,9,10,11,12,13$ \\
WY & 9 & 100 & 99,999 & $1,4,8,9,10,12,13$ \\
\hline
\end{tabular}

Source: TRI02 2004 (Data are from 2002)

${ }^{\text {a }}$ Post office state abbreviations used

${ }^{\mathrm{b}}$ Amounts on site reported by facilities in each state ${ }^{\mathrm{c}}$ Activities/Uses:
1. Produce
6. Impurity
2. Import
7. Reactant
3. Onsite use/processing
8. Formulation Component
11. Chemical Processing Aid
4. Sale/Distribution
9. Article Component
10. Repackaging
12. Manufacturing Aid
13. Ancillary/Other Uses
14. Process Impurity
5. Byproduct 
Table 5-2. Facilities that Produce, Process, or Use Nickel Compounds

\begin{tabular}{|c|c|c|c|c|}
\hline State $^{a}$ & $\begin{array}{l}\text { Number of } \\
\text { facilities }\end{array}$ & $\begin{array}{l}\text { Minimum amount } \\
\text { on site in pounds }\end{array}$ & $\begin{array}{l}\text { Maximum amount } \\
\text { on site in pounds }\end{array}$ & 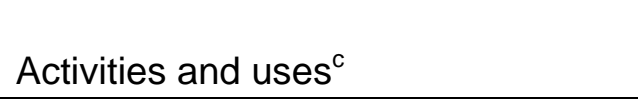 \\
\hline AK & 7 & 10,000 & $9,999,999$ & $1,2,3,5,7,10,11,12,13$ \\
\hline$A L$ & 83 & 0 & $9,999,999$ & $1,2,3,4,5,6,7,8,9,10,11,12,13,14$ \\
\hline AR & 46 & 0 & $99,999,999$ & $1,2,3,4,5,6,7,8,9,10,11,12,13,14$ \\
\hline$A Z$ & 54 & 100 & $99,999,999$ & $1,2,3,4,5,6,7,8,9,10,11,12,13,14$ \\
\hline $\mathrm{CA}$ & 163 & 0 & $499,999,999$ & $1,2,3,4,5,6,7,8,9,10,11,12,13,14$ \\
\hline $\mathrm{CO}$ & 16 & 100 & 999,999 & $1,2,3,4,5,6,7,8,9,11,12$ \\
\hline CT & 81 & 0 & $499,999,999$ & $1,2,3,4,5,6,7,8,9,10,11,12,13$ \\
\hline DC & 4 & 10,000 & 99,999 & $1,3,5,6,8,10,11$ \\
\hline $\mathrm{DE}$ & 19 & 0 & $9,999,999$ & $1,2,3,5,6,7,8,9,10,12,13,14$ \\
\hline $\mathrm{FL}$ & 52 & 0 & 999,999 & $1,2,3,4,5,6,7,8,9,10,11,12,13,14$ \\
\hline GA & 79 & 0 & $9,999,999$ & $1,2,3,4,5,6,7,8,9,10,11,12,13,14$ \\
\hline $\mathrm{HI}$ & 5 & 0 & 999,999 & $1,5,10,12$ \\
\hline IA & 42 & 0 & $49,999,999$ & $1,2,3,4,5,6,7,8,9,10,11,12,13,14$ \\
\hline ID & 20 & 1,000 & $49,999,999$ & $1,2,3,5,6,7,8,9,10,11,12,13,14$ \\
\hline IL & 184 & 0 & $499,999,999$ & $1,2,3,4,5,6,7,8,9,10,11,12,13,14$ \\
\hline IN & 143 & 0 & $9,999,999$ & $1,2,3,4,5,6,7,8,9,10,11,12,13,14$ \\
\hline KS & 35 & 0 & $9,999,999$ & $1,2,3,5,6,7,8,9,10,11,12,13,14$ \\
\hline KY & 82 & 100 & $499,999,999$ & $1,2,3,4,5,6,7,8,9,10,11,12,13,14$ \\
\hline LA & 73 & 0 & $9,999,999$ & $1,2,3,4,5,6,7,8,9,10,11,12,13,14$ \\
\hline MA & 50 & 100 & $9,999,999$ & $1,2,3,4,5,6,7,8,9,10,11,12,13$ \\
\hline MD & 37 & 0 & $49,999,999$ & $1,2,3,4,5,6,7,8,9,12,13$ \\
\hline ME & 12 & 0 & 999,999 & $1,5,8,9,11,12,13$ \\
\hline MI & 149 & 0 & $499,999,999$ & $1,2,3,4,5,6,7,8,9,10,11,12,13,14$ \\
\hline MN & 66 & 100 & 999,999 & $1,2,3,4,5,6,7,8,9,10,11,12,13,14$ \\
\hline MO & 63 & 0 & $499,999,999$ & $1,2,3,4,5,6,7,8,9,10,11,12,13,14$ \\
\hline MS & 37 & 0 & $9,999,999$ & $1,2,3,4,5,6,7,8,9,10,11,12,13,14$ \\
\hline MT & 16 & 0 & $9,999,999$ & $1,2,3,4,5,6,8,9,10,11,12,13,14$ \\
\hline NC & 73 & 0 & $9,999,999$ & $1,2,3,4,5,6,7,8,9,10,11,12,13,14$ \\
\hline ND & 4 & 1,000 & 9,999 & $1,5,12,13,14$ \\
\hline NE & 19 & 100 & 999,999 & $1,3,4,5,6,7,8,9,10,11,12,13,14$ \\
\hline $\mathrm{NH}$ & 12 & 0 & 99,999 & $1,5,7,8,9$ \\
\hline NJ & 91 & 0 & $499,999,999$ & $1,2,3,4,5,6,7,8,9,10,11,12,13,14$ \\
\hline NM & 19 & 100 & $499,999,999$ & $1,3,4,5,8,9,10,11,12,13,14$ \\
\hline NV & 33 & 100 & $10,000,000,000$ & $1,2,3,5,6,7,8,9,10,12,13,14$ \\
\hline NY & 110 & 0 & $499,999,999$ & $1,2,3,4,5,6,7,8,9,10,11,12,13,14$ \\
\hline $\mathrm{OH}$ & 218 & 0 & $99,999,999$ & $1,2,3,4,5,6,7,8,9,10,11,12,13,14$ \\
\hline OK & 42 & 100 & 999,999 & $1,2,3,4,5,6,7,8,9,10,11,12,13,14$ \\
\hline OR & 38 & 100 & $49,999,999$ & $1,2,3,4,5,6,7,8,9,11,12,13$ \\
\hline PA & 229 & 0 & $499,999,999$ & $1,2,3,4,5,6,7,8,9,10,11,12,13,14$ \\
\hline
\end{tabular}


Table 5-2. Facilities that Produce, Process, or Use Nickel Compounds

\begin{tabular}{|c|c|c|c|c|}
\hline State $^{a}$ & $\begin{array}{l}\text { Number of } \\
\text { facilities }\end{array}$ & $\begin{array}{l}\text { Minimum amount } \\
\text { on site in pounds }\end{array}$ & $\begin{array}{l}\text { Maximum amount } \\
\text { on site in pounds }\end{array}$ & Activities and uses ${ }^{c}$ \\
\hline PR & 19 & 100 & 999,999 & $1,2,5,6,7,8,10,11,12,13$ \\
\hline RI & 39 & 0 & $49,999,999$ & $1,2,3,4,5,6,7,8,9,10,11,12$ \\
\hline SC & 61 & 0 & $9,999,999$ & $1,2,3,4,5,6,7,8,9,10,11,12,13,14$ \\
\hline SD & 1 & 10,000 & 99,999 & $1,5,9,13$ \\
\hline TN & 100 & 0 & $499,999,999$ & $1,2,3,4,5,6,7,8,9,10,11,12,13,14$ \\
\hline TX & 194 & 0 & $49,999,999$ & $1,2,3,4,5,6,7,8,9,10,11,12,13,14$ \\
\hline UT & 26 & 100 & $49,999,999$ & $1,2,3,4,5,6,7,8,9,10,11,12,13,14$ \\
\hline VA & 44 & 0 & 999,999 & $1,2,3,4,5,6,7,8,9,10,11,12,13,14$ \\
\hline $\mathrm{VI}$ & 4 & 10,000 & 999,999 & $2,10,11$ \\
\hline WA & 28 & 0 & 999,999 & $1,2,3,4,5,6,7,8,9,10,11,12,13$ \\
\hline WI & 75 & 0 & $9,999,999$ & $1,2,3,4,5,6,7,8,9,10,11,12,13,14$ \\
\hline WV & 45 & 0 & $99,999,999$ & $1,2,3,4,5,6,7,8,9,10,11,12,13,14$ \\
\hline WY & 9 & 100 & 99,999 & $1,2,3,4,5,9,10,12,13$ \\
\hline
\end{tabular}

Source: TRI02 2004 (Data are from 2002)

${ }^{\text {a}}$ Post office state abbreviations used

${ }^{\mathrm{b}}$ Amounts on site reported by facilities in each state

${ }^{\mathrm{c} A c t i v i t i e s / \text { Uses: }}$
1. Produce
6. Impurity
2. Import
7. Reactant
11. Chemical Processing Aid
3. Onsite use/processing
8. Formulation Component
12. Manufacturing Aid
4. Sale/Distribution
9. Article Component
13. Ancillary/Other Uses
5. Byproduct
10. Repackaging
14. Process Impurity 
8,550 metric tons, respectively. The 130,000 metric tons of nickel imported in 2002 was down from the 167,000 and 144,000 metric tons imported in 2000 and 2001, respectively (Kuck 2001, 2002). From 1999 to 2001, nickel imports as a percentage of consumption decreased from 63 to 46\%, with a slight increase to $48 \%$ in 2002 (USGS 2003).

The amount of exported nickel dropped sharply in 1986 to 15,217 short tons (13,805 metric tons) from 35,245 short tons (31,974 metric tons) the previous year (Kirk 1988a), which coincided with the cessation of primary nickel production in the United States. The nickel content of exported primary and secondary nickel in 2002 was 45,900 metric tons, most of which (39,400 metric tons) was in the form of stainless steel scrap and waste scrap (Kuck 2002).

\subsection{USE}

Nickel is primarily used in alloys because it imparts to a product such desirable properties as corrosion resistance, heat resistance, hardness, and strength. Nickel alloys are often divided into categories depending on the primary metal with which they are alloyed and their nickel content. Copper-nickel alloys (e.g., Monel alloys) are used for industrial plumbing, marine equipment, petrochemical equipment, heat exchangers, pumps, and electrodes for welding. Coinage metal contains $75 \%$ copper and $25 \%$ nickel. Nickel-chromium alloys (e.g., Nichrome) are used for heating elements. Nickel-iron-chromium alloys (e.g., Inconel) provide strength and corrosion resistance over a wide temperature range. Hastelloy alloys, which contain nickel, chromium, iron, and molybdenum, provide oxidation and corrosion resistance for use with acids and salts. Nickel-based superalloys have the required high-temperature strength and creep and stress resistance for use in gas-turbine engines. Nickel silvers, and nickel alloys with zinc and copper, have an attractive white color and are used for coatings on tableware and as electrical contacts. Raney nickel, 50\% aluminum and 50\% nickel, is used as a catalyst in hydrogenation reactions. Large amounts of nickel are alloyed with iron to produce alloy steels, stainless steels, and cast irons. Stainless steel may contain as much as $25-30 \%$ nickel, although $8-10 \%$ nickel is more typical. Alloy steels generally contain $0.3-5 \%$ nickel. In addition to imparting characteristics such as strength, toughness, corrosion resistance, and machinability, some applications make use of nickel's magnetic characteristics. Most permanent magnets are made of alloys of iron and nickel (Tien and Howson 1981).

Nickel salts are used in electroplating, ceramics, pigments, and as catalysts. Sinter nickel oxide is used as charge material in the manufacture of alloy steel and stainless steel. Nickel is also used in nickelcadmium (NiCd) and nickel-metal hydride (NiMH) batteries. 
The distribution of nickel consumption by use in 2002 was as follows: stainless and heat-resistant steel, 61\%; nickel-copper and copper-nickel alloys, 4\%; other nickel alloys, 13\%; electroplating, 6\%; superalloys, 9\%; and other, 7\%. Other uses include cast iron; chemicals and chemical use; electric, magnet, expansion alloys; steel alloys, other than stainless steel; batteries; and ceramics. Forty-six percent of primary nickel consumption in 2002 was for the production of stainless steel and low-nickel steels, and 33\% was used for the production of superalloys and related nickel-based alloys (Kuck 2002).

\subsection{DISPOSAL}

Little information concerning the disposal of nickel and its compounds is found in the literature. Much of the nickel used in metal products (e.g., stainless steel, nickel plate, various alloys) is recycled, which is evident from the fact that $53 \%$ of nickel consumption in 2002 was derived from secondary scrap (Kuck 2002). According to the 2002 TRI, $86 \%$ of the $29,698,967$ pounds $(13,483,331 \mathrm{~kg})$ of nickel and nickel compounds released on-site is released to land (see Section 6.1) (TRI02 2004). In addition, >14 million pounds of nickel were transferred to off-site locations that year with about $90 \%$ being recycled. Steel and other nickel-containing items discarded by households and commercial establishments are generally recycled, landfilled, or incinerated along with normal commercial and municipal trash.

Nickel is removed from electroplating wastes by treatment with hydroxide, lime, and/or sulfide to precipitate the metal (HSDB 2004). Adsorption with activated carbon, activated alumina, and iron filings is also used for treating nickel-containing waste water. Ion exchange is also used for nickel removal and recovery.

Nickel and its compounds have been designated as toxic pollutants by EPA pursuant to Section 307(a)(1) of the Federal Water Pollution Control Act (40 CFR 401.15). As such, permits are issued by the states under the National Pollutant Discharge Elimination System (NPDES) for discharges of nickel that meet the applicable requirements (40 CFR 401.12). 



\section{POTENTIAL FOR HUMAN EXPOSURE}

\subsection{OVERVIEW}

Nickel has been identified in at least 872 of the 1,662 hazardous waste sites that have been proposed for inclusion on the EPA National Priorities List (NPL) (HazDat 2005). However, the number of sites evaluated for nickel is not known. The frequency of these sites can be seen in Figure 6-1. Of these sites, 862 are located within the United States, 6 are located in the Commonwealth of Puerto Rico, and 4 are located in the Territory of Guam (the Commonwealth of Puerto Rico and the Territory of Guam are not shown).

Nickel and its compounds are naturally present in the Earth's crust, and releases to the atmosphere occur from natural discharges such as windblown dust and volcanic eruptions, as well as from anthropogenic activities. It is estimated that 8.5 million $\mathrm{kg}$ of nickel are emitted into the atmosphere from natural sources such as windblown dust, volcanoes, and vegetation each year (Bennett 1984; Schmidt and Andren 1980). Five times that quantity is estimated to come from anthropogenic sources (Nriagu and Pacyna 1988). The burning of residual and fuel oil is responsible for $62 \%$ of anthropogenic emissions, followed by nickel metal refining, municipal incineration, steel production, other nickel alloy production, and coal combustion (Bennett 1984; Schmidt and Andren 1980). Table 6-1 lists releases from facilities in the United States that produced, processed, or used nickel metal in 2002, according to TRI (TRI02 2004). These releases, which totaled 6,792,299 pounds (3,081 metric tons), were distributed as follows: $82.2 \%$ to land, $6.0 \%$ to air, $2.2 \%$ to water, and $0.8 \%$ to underground injection. Table 6-2 lists releases from facilities in the United States that produced, processed, or used nickel compounds in 2002, according to TRI (TRI02 2004). These releases, which totaled 37,558,704 pounds (17,037 metric tons), were distributed as follows: $87.1 \%$ to land, $2.5 \%$ to air, $1.4 \%$ to water, and $1.7 \%$ to underground injection. The TRI data should be used with caution because only certain types of facilities are required to report. This is not an exhaustive list.

The general population is exposed to low levels of nickel in ambient air, water, and food. Exposure also occurs from smoking. The general population takes in most nickel through food. The average daily dietary nickel intake for U.S. diets is 69-162 $\mu \mathrm{g}$ (NAS 2002; O’Rourke et al. 1999; Pennington and Jones 1987; Thomas et al. 1999). These values agree with those from European studies. Typical average daily intakes of nickel from drinking water and inhalation of air are approximately 8 and $0.04 \mu \mathrm{g}$, respectively. 
Figure 6-1. Frequency of NPL Sites with Nickel Contamination

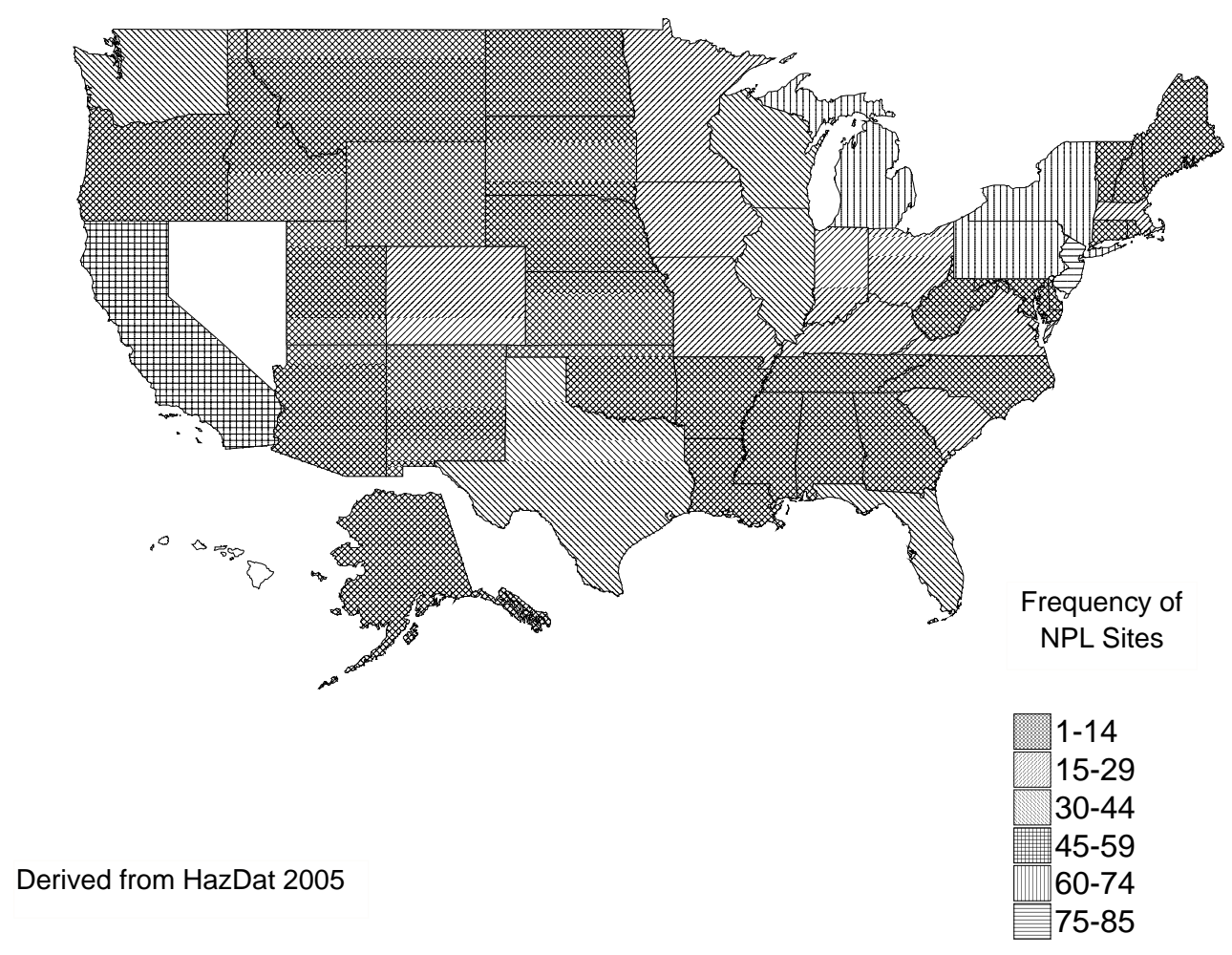


Table 6-1. Releases to the Environment from Facilities that Produce, Process, or Use Nickel ${ }^{\mathrm{a}}$

\begin{tabular}{|c|c|c|c|c|c|c|c|c|c|}
\hline \multirow[b]{3}{*}{ State $^{c}$} & \multirow[b]{3}{*}{$\mathrm{RF}^{\mathrm{d}}$} & \multicolumn{8}{|c|}{ Reported amounts released in pounds per year ${ }^{b}$} \\
\hline & & \multirow[b]{2}{*}{ Air ${ }^{\mathrm{e}}$} & \multirow[b]{2}{*}{ Water ${ }^{f}$} & \multirow[b]{2}{*}{$\mathrm{UI}^{\mathrm{g}}$} & \multirow[b]{2}{*}{ Land $^{\text {h }}$} & \multirow[b]{2}{*}{ Other ${ }^{i}$} & \multicolumn{3}{|c|}{ Total release } \\
\hline & & & & & & & On-site & Off-site ${ }^{k}$ & $\begin{array}{l}\text { On- and off- } \\
\text { site }\end{array}$ \\
\hline$\overline{\mathrm{AL}}$ & 58 & 2,610 & 1,302 & 0 & 81,145 & 256 & 3,786 & 81,526 & 85,312 \\
\hline AR & 38 & 15,621 & 665 & 0 & 11,572 & 13,210 & 16,176 & 24,893 & 41,069 \\
\hline$A Z$ & 19 & 1,421 & 6 & 0 & 674,841 & 5 & 670,775 & 5,498 & 676,273 \\
\hline $\mathrm{CA}$ & 94 & 1,886 & 3,377 & 24,776 & 223,027 & 17,568 & 216,055 & 54,578 & 270,634 \\
\hline $\mathrm{CO}$ & 15 & 97 & 58 & 0 & 1,232 & 19,277 & 357 & 20,308 & 20,665 \\
\hline $\mathrm{CT}$ & 47 & 2,514 & 4,355 & 0 & 58,026 & 18,330 & 3,124 & 80,102 & 83,225 \\
\hline $\mathrm{DE}$ & 2 & 5 & 0 & 0 & 750 & 250 & 5 & 1,000 & 1,005 \\
\hline $\mathrm{FL}$ & 26 & 2,188 & 340 & 3,833 & 8,429 & 29,663 & 9,480 & 34,973 & 44,453 \\
\hline GA & 36 & 2,617 & 1,072 & 0 & 3,459 & 11,575 & 3,222 & 15,502 & 18,724 \\
\hline IA & 62 & 4,459 & 1,623 & 0 & 38,465 & 12,020 & 5,880 & 50,687 & 56,568 \\
\hline ID & 5 & 70 & 5 & 0 & 238,979 & 0 & 239,049 & 5 & 239,054 \\
\hline IL & 129 & 8,057 & 19,525 & 255 & 111,752 & 16,157 & 13,473 & 142,273 & 155,746 \\
\hline IN & 154 & 37,078 & 3,914 & 500 & $2,026,732$ & 43,905 & 42,673 & $2,069,457$ & $2,112,130$ \\
\hline KS & 24 & 2,422 & 94 & 0 & 10,671 & 594 & 11,730 & 2,051 & 13,781 \\
\hline KY & 54 & 2,220 & 2,555 & 0 & 58,811 & 15,529 & 2,701 & 76,414 & 79,115 \\
\hline LA & 33 & 917 & 899 & 0 & 64,942 & 2,940 & 57,072 & 12,626 & 69,698 \\
\hline MA & 41 & 1,866 & 983 & 0 & 33,468 & 27,945 & 2,459 & 61,803 & 64,262 \\
\hline MD & 13 & 72 & 40 & 0 & 728 & 1,418 & 91 & 2,167 & 2,258 \\
\hline ME & 8 & 242 & 69 & 0 & 4,585 & 4,122 & 263 & 8,755 & 9,018 \\
\hline MI & 115 & 17,489 & 6,921 & 0 & 110,495 & 32,153 & 20,683 & 146,374 & 167,057 \\
\hline $\mathrm{MN}$ & 44 & 658 & 312 & 0 & 1,441 & 255 & 910 & 1,756 & 2,666 \\
\hline MO & 51 & 7,304 & 2,103 & 0 & 7,801 & 1,001 & 10,464 & 7,745 & 18,209 \\
\hline MS & 25 & 5,243 & 127 & 0 & 2,326 & 0 & 5,655 & 2,041 & 7,697 \\
\hline MT & 1 & 40 & 0 & 0 & 16,000 & 0 & 16,040 & 0 & 16,040 \\
\hline $\mathrm{NC}$ & 68 & 965 & 2,477 & 0 & 52,612 & 26,814 & 21,099 & 61,769 & 82,868 \\
\hline ND & 5 & 34 & 5 & 0 & 2,554 & 250 & 37 & 2,806 & 2,843 \\
\hline NE & 24 & 2,390 & 586 & 0 & 15,052 & 192 & 2,465 & 15,755 & 18,220 \\
\hline $\mathrm{NH}$ & 16 & 1,000 & 8,364 & 0 & 330 & 38,540 & 1,009 & 47,225 & 48,234 \\
\hline NJ & 22 & 4,408 & 2,287 & 0 & 8,664 & 52,540 & 4,414 & 63,485 & 67,899 \\
\hline NM & 4 & 482 & 7 & 0 & 275 & 7 & 734 & 37 & 771 \\
\hline NV & 10 & 1,040 & 5 & 0 & 64,280 & 0 & 40,214 & 25,111 & 65,325 \\
\hline NY & 67 & 10,149 & 3,763 & 0 & 37,047 & 21,878 & 16,151 & 56,685 & 72,836 \\
\hline $\mathrm{OH}$ & 212 & 20,209 & 13,783 & 5,478 & 708,223 & 11,067 & 196,744 & 562,016 & 758,759 \\
\hline OK & 66 & 16,142 & 190 & 0 & 50,733 & 0 & 45,194 & 21,871 & 67,065 \\
\hline OR & 14 & 2,511 & 919 & 0 & 58,520 & 5 & 2,623 & 59,332 & 61,955 \\
\hline PA & 209 & 203,216 & 6,538 & 0 & 68,001 & 55,213 & 206,110 & 126,858 & 332,967 \\
\hline
\end{tabular}


Table 6-1. Releases to the Environment from Facilities that Produce, Process, or Use Nickel ${ }^{\mathrm{a}}$

\begin{tabular}{|c|c|c|c|c|c|c|c|c|c|}
\hline \multirow[b]{3}{*}{ State $^{c}$} & \multirow[b]{3}{*}{$\mathrm{RF}^{\mathrm{d}}$} & \multicolumn{8}{|c|}{ Reported amounts released in pounds per year ${ }^{b}$} \\
\hline & & \multirow[b]{2}{*}{ Air ${ }^{\mathrm{e}}$} & \multirow[b]{2}{*}{ Water $^{f}$} & \multirow[b]{2}{*}{$\mathrm{UI}^{\mathrm{g}}$} & \multirow[b]{2}{*}{ Land $^{\text {h }}$} & \multirow[b]{2}{*}{ Other $^{\mathrm{i}}$} & \multicolumn{3}{|c|}{ Total release } \\
\hline & & & & & & & On-site ${ }^{j}$ & Off-site ${ }^{k}$ & $\begin{array}{l}\text { On- and off- } \\
\text { site }\end{array}$ \\
\hline$\overline{\mathrm{PR}}$ & 4 & 0 & 0 & 0 & 0 & 0 & 0 & 0 & 0 \\
\hline RI & 7 & 266 & 505 & 0 & 0 & 500 & 271 & 1,000 & 1,271 \\
\hline SC & 52 & 3,633 & 1,226 & 0 & 192,091 & 643 & 12,882 & 184,710 & 197,593 \\
\hline SD & 6 & 324 & 5 & 0 & 47 & 0 & 324 & 52 & 376 \\
\hline TN & 52 & 1,467 & 11,413 & 0 & 54,393 & 1,932 & 9,685 & 59,520 & 69,205 \\
\hline TX & 121 & 6,327 & 28,697 & 20,343 & 211,220 & 82,910 & 42,933 & 306,562 & 349,495 \\
\hline UT & 13 & 297 & 267 & 0 & 760 & 0 & 797 & 528 & 1,325 \\
\hline VA & 25 & 1,082 & 2,490 & 0 & 13,393 & 2,702 & 5,899 & 13,768 & 19,667 \\
\hline VT & 5 & 10 & 10 & 0 & 0 & 16,186 & 10 & 16,196 & 16,206 \\
\hline WA & 20 & 794 & 716 & 0 & 23,067 & 2,809 & 1,482 & 25,904 & 27,386 \\
\hline WI & 147 & 10,440 & 16,873 & 0 & 185,992 & 15,681 & 21,180 & 207,806 & 228,986 \\
\hline WV & 13 & 77 & 252 & 0 & 18,704 & 1,535 & 18,629 & 1,939 & 20,568 \\
\hline WY & 3 & 53 & 1 & 0 & 25,759 & 7 & 25,812 & 8 & 25,820 \\
\hline Total & 2,279 & 404,413 & 151,725 & 55,185 & $5,581,392$ & 599,585 & $2,028,824$ & $4,763,475$ & $6,792,299$ \\
\hline
\end{tabular}

Source: TRI02 2004 (Data are from 2002)

${ }^{a}$ The TRI data should be used with caution since only certain types of facilities are required to report. This is not an exhaustive list. Data are rounded to nearest whole number.

${ }^{\mathrm{b}}$ Data in TRI are maximum amounts released by each facility.

${ }^{c}$ Post office state abbreviations are used.

${ }^{\mathrm{d}}$ Number of reporting facilities.

${ }^{\mathrm{e}}$ The sum of fugitive and point source releases are included in releases to air by a given facility.

'Surface water discharges, wastewater treatment-(metals only), and publicly owned treatment works (POTWs) (metal and metal compounds).

${ }^{\mathrm{g}}$ Class I wells, Class II-V wells, and underground injection.

${ }^{\mathrm{h}}$ Resource Conservation and Recovery Act (RCRA) subtitle C landfills; other on-site landfills, land treatment, surface impoundments, other land disposal, other landfills.

iStorage only, solidification/stabilization (metals only), other off-site management, transfers to waste broker for disposal, unknown

jThe sum of all releases of the chemical to air, land, water, and underground injection wells.

kTotal amount of chemical transferred off-site, including to POTWs.

$\mathrm{RF}=$ reporting facilities; $\mathrm{UI}=$ underground injection 
Table 6-2. Releases to the Environment from Facilities that Produce, Process, or Use Nickel Compounds ${ }^{a}$

\begin{tabular}{|c|c|c|c|c|c|c|c|c|c|}
\hline \multirow[b]{3}{*}{$\begin{array}{l}\text { Stat } \\
e^{c}\end{array}$} & \multirow[b]{3}{*}{$\mathrm{RF}^{\mathrm{d}}$} & \multicolumn{8}{|c|}{ Reported amounts released in pounds per year ${ }^{b}$} \\
\hline & & \multirow[b]{2}{*}{ Air } & \multirow[b]{2}{*}{ Water $^{f}$} & \multirow[b]{2}{*}{$U^{g}$} & \multirow[b]{2}{*}{ Land $^{\mathrm{h}}$} & \multirow[b]{2}{*}{ Other $^{\mathrm{i}}$} & \multicolumn{3}{|c|}{ Total release } \\
\hline & & & & & & & On-site & Off-site ${ }^{k}$ & $\begin{array}{l}\text { On- and off- } \\
\text { site }\end{array}$ \\
\hline$\overline{\mathrm{AK}}$ & 3 & 56 & 221 & 0 & 543,911 & 0 & 544,124 & 64 & 544,188 \\
\hline$A L$ & 31 & 10,093 & 5,431 & 501 & 864,693 & 71,756 & 867,989 & 84,485 & 952,474 \\
\hline AR & 15 & 11,912 & 624 & 0 & 810,593 & 30,419 & 761,710 & 91,838 & 853,548 \\
\hline$A Z$ & 12 & 2,647 & 5 & 100,000 & $5,922,548$ & 505 & $6,011,495$ & 14,210 & $6,025,705$ \\
\hline CA & 73 & 6,129 & 8,742 & 4,981 & $1,102,665$ & 42,528 & $1,038,435$ & 126,611 & $1,165,045$ \\
\hline $\mathrm{CO}$ & 7 & 499 & 92 & 0 & 52,244 & 47 & 33,972 & 18,910 & 52,882 \\
\hline CT & 18 & 1,586 & 2,837 & 0 & 114,292 & 49,426 & 2,503 & 165,638 & 168,141 \\
\hline DC & 1 & 0 & 0 & 0 & 18 & 0 & 0 & 18 & 18 \\
\hline $\mathrm{DE}$ & 7 & 19,291 & 23,985 & 0 & 126,040 & 23,795 & 119,068 & 74,043 & 193,111 \\
\hline $\mathrm{FL}$ & 23 & 144,268 & 4,246 & 0 & $2,534,663$ & 4,052 & $2,629,871$ & 57,358 & $2,687,229$ \\
\hline GA & 24 & 7,599 & 15,428 & 0 & 606,137 & 132 & 610,622 & 18,673 & 629,295 \\
\hline $\mathrm{HI}$ & 2 & 64,800 & 10 & 0 & 4,022 & 0 & 64,810 & 4,022 & 68,832 \\
\hline IA & 18 & 6,529 & 28,253 & 0 & 156,841 & 49,686 & 145,002 & 96,307 & 241,309 \\
\hline ID & 4 & 1,490 & 56 & 0 & 33,000 & 0 & 34,495 & 51 & 34,546 \\
\hline IL & 89 & 18,190 & 13,206 & 0 & 752,162 & 127,262 & 481,610 & 429,210 & 910,820 \\
\hline IN & 76 & 31,891 & 19,303 & 490 & $1,937,458$ & 187,004 & $1,524,230$ & 651,917 & $2,176,146$ \\
\hline KS & 13 & 2,608 & 280 & 0 & 64,708 & 292 & 66,981 & 907 & 67,889 \\
\hline KY & 40 & 23,463 & 12,224 & 0 & $1,784,073$ & 80,688 & $1,255,775$ & 644,673 & $1,900,448$ \\
\hline LA & 35 & 58,235 & 7,085 & 9,841 & 364,170 & 474,747 & 311,507 & 602,571 & 914,078 \\
\hline MA & 16 & 15,582 & 1,270 & 0 & 96,020 & 8,135 & 39,054 & 81,953 & 121,007 \\
\hline MD & 11 & 28,308 & 2,733 & 319 & 10,225 & 148,413 & 40,717 & 149,281 & 189,998 \\
\hline ME & 2 & 50 & 3,336 & 0 & 6 & 80 & 50 & 3,422 & 3,472 \\
\hline MI & 70 & 18,024 & 42,115 & 0 & 580,122 & 90,302 & 282,080 & 448,483 & 730,563 \\
\hline MN & 22 & 14,786 & 5,678 & 0 & 228,322 & 67,133 & 98,965 & 216,954 & 315,919 \\
\hline $\mathrm{MO}$ & 32 & 8,476 & 2,669 & 0 & 661,528 & 21,698 & 597,580 & 96,791 & 694,371 \\
\hline MS & 11 & 3,859 & 1,235 & 72,000 & 87,969 & 907 & 99,004 & 66,966 & 165,970 \\
\hline MT & 7 & 2,468 & 0 & 101,419 & 770,075 & 3,775 & 431,813 & 445,924 & 877,737 \\
\hline NC & 30 & 9,046 & 4,179 & 0 & 551,706 & 8,848 & 562,930 & 10,850 & 573,780 \\
\hline ND & 4 & 4,159 & 10 & 0 & 128,699 & 1,800 & 75,869 & 58,799 & 134,668 \\
\hline NE & 8 & 209 & 1,554 & 0 & 127,490 & 41,645 & 127,720 & 43,178 & 170,898 \\
\hline $\mathrm{NH}$ & 5 & 376 & 126 & 0 & 11,192 & 45 & 1,781 & 9,958 & 11,739 \\
\hline NJ & 15 & 2,415 & 5,661 & 0 & 62,541 & 9,865 & 33,721 & 46,760 & 80,481 \\
\hline NM & 3 & 339 & 1 & 0 & 112,800 & 0 & 73,140 & 40,000 & 113,140 \\
\hline NV & 10 & 8,403 & 930 & 0 & $2,568,163$ & 0 & $2,577,480$ & 16 & $2,577,496$ \\
\hline NY & 33 & 10,228 & 49,939 & 0 & 233,794 & 136,288 & 270,034 & 160,216 & 430,250 \\
\hline $\mathrm{OH}$ & 95 & 21,520 & 122,772 & 271,001 & $2,122,342$ & 243,006 & $1,221,840$ & $1,558,801$ & $2,780,641$ \\
\hline
\end{tabular}




\section{Table 6-2. Releases to the Environment from Facilities that Produce, Process, or Use Nickel Compounds ${ }^{\mathrm{a}}$}

\begin{tabular}{|c|c|c|c|c|c|c|c|c|c|}
\hline \multirow[b]{3}{*}{$\begin{array}{l}\text { Stat } \\
\mathrm{e}^{\mathrm{c}}\end{array}$} & \multirow[b]{3}{*}{$\mathrm{RF}^{\mathrm{d}}$} & \multicolumn{8}{|c|}{ Reported amounts released in pounds per year ${ }^{b}$} \\
\hline & & \multirow[b]{2}{*}{ Air $^{\mathrm{e}}$} & \multirow[b]{2}{*}{ Water $^{f}$} & \multirow[b]{2}{*}{$\mathrm{UI}^{\mathrm{g}}$} & \multirow[b]{2}{*}{ Land $^{\text {h }}$} & \multirow[b]{2}{*}{ Other ${ }^{\mathrm{i}}$} & \multicolumn{3}{|c|}{ Total release } \\
\hline & & & & & & & On-site ${ }^{j}$ & Off-site ${ }^{k}$ & $\begin{array}{l}\text { On- and off- } \\
\text { site }\end{array}$ \\
\hline$\overline{\mathrm{OK}}$ & 17 & 9,129 & 1,800 & 0 & 264,634 & 14,331 & 238,243 & 51,651 & 289,894 \\
\hline OR & 9 & 1,618 & 264 & 0 & 39,697 & 0 & 34,682 & 6,897 & 41,578 \\
\hline PA & 113 & 90,020 & 40,145 & 0 & $2,021,526$ & 298,951 & 553,237 & $1,897,405$ & $2,450,642$ \\
\hline PR & 6 & 85,852 & 2,704 & 0 & 8,914 & 3,300 & 88,556 & 12,214 & 100,770 \\
\hline RI & 7 & 254 & 3,147 & 0 & 447 & 5,099 & 814 & 8,133 & 8,947 \\
\hline SC & 27 & 5,666 & 12,734 & 0 & 208,779 & 86,978 & 178,444 & 135,713 & 314,158 \\
\hline SD & 1 & 208 & 0 & 0 & 19,000 & 0 & 19,208 & 0 & 19,208 \\
\hline $\mathrm{TN}$ & 38 & 14,291 & 14,597 & 0 & 721,360 & 11,733 & 544,837 & 217,144 & 761,981 \\
\hline $\mathrm{TX}$ & 98 & 27,305 & 10,954 & 71,192 & $1,050,243$ & 217,862 & 777,048 & 600,507 & $1,377,555$ \\
\hline UT & 10 & 2,374 & 3,450 & 0 & 891,742 & 80 & 897,517 & 129 & 897,646 \\
\hline VA & 20 & 55,129 & 8,637 & 0 & 314,676 & 37,945 & 371,633 & 44,753 & 416,387 \\
\hline VI & 1 & 227 & 0 & 0 & 3,047 & 0 & 1,703 & 1,571 & 3,274 \\
\hline WA & 7 & 650 & 1,047 & 7 & 143,228 & 234 & 137,877 & 7,289 & 145,166 \\
\hline WI & 46 & 5,615 & 9,474 & 0 & 41,811 & 108,006 & 8,384 & 156,521 & 164,906 \\
\hline WV & 18 & 80,986 & 21,617 & 2,681 & 745,233 & 20,087 & 647,928 & 222,676 & 870,604 \\
\hline WY & 3 & 3,260 & 0 & 0 & 128,797 & 6,100 & 132,057 & 6,100 & 138,157 \\
\hline Total & 286 & 942,117 & 516,804 & 634,432 & $32,730,365$ & $2,734,986$ & $7,670,143$ & 888,561 & $37,558,704$ \\
\hline
\end{tabular}

Source: TRI02 2004 (Data are from 2002)

${ }^{\text {a }}$ The TRI data should be used with caution since only certain types of facilities are required to report. This is not an exhaustive list. Data are rounded to nearest whole number.

${ }^{\mathrm{b}} \mathrm{Data}$ in TRI are maximum amounts released by each facility.

${ }^{\mathrm{C} P}$ ost office state abbreviations are used.

${ }^{\mathrm{d}}$ Number of reporting facilities.

${ }^{\mathrm{e}}$ The sum of fugitive and point source releases are included in releases to air by a given facility.

'Surface water discharges, wastewater treatment-(metals only), and publicly owned treatment works (POTWs) (metal and metal compounds).

${ }^{9}$ Class I wells, Class II-V wells, and underground injection.

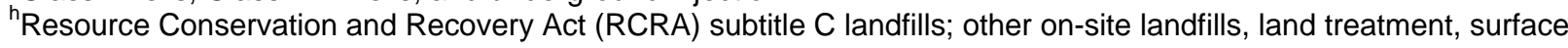
impoundments, other land disposal, other landfills.

iStorage only, solidification/stabilization (metals only), other off-site management, transfers to waste broker for disposal, unknown

'The sum of all releases of the chemical to air, land, water, and underground injection wells.

${ }^{\mathrm{k}}$ Total amount of chemical transferred off-site, including to POTWs.

$\mathrm{RF}=$ reporting facilities; $\mathrm{UI}=$ underground injection 
The highest general population exposures to nickel are typically observed in communities surrounding nickel refineries. This is reflected, for example, in the intakes of nickel from water and air reported in Sudbury, Ontario, Canada, of 140 and $15 \mu \mathrm{g} /$ day, respectively. However, this source of exposure to nickel is not a concern for U.S. populations, due to the absence of refinery operations in the United States. Other potential sources of nickel exposure are from contaminated intravenous fluids, dialysis, and leaching and corrosion of nickel from prostheses.

Occupational exposure to nickel may occur by dermal contact or by inhalation of aerosols, dusts, fumes, or mists containing nickel. Dermal contact may also occur with nickel solutions, such as those used in electroplating, nickel salts, and nickel metal or alloys. Nickel-containing dust may be ingested where poor work practices exist or where poor personal hygiene is practiced. A National Occupational Exposure Survey (NOES) conducted by NIOSH from 1981 to 1983 estimates that 727,240 workers are potentially exposed to some form of nickel metal, alloys, salts, or inorganic nickel compounds in the United States (NIOSH 1990). The forms of nickel that these workers were probably exposed to and the levels of exposure for different industries and operations were reviewed by Warner (1984) and IARC (1990).

Information on nickel exposure from hazardous waste sites is lacking. The most probable route of exposure from hazardous waste sites would be from consumption of contaminated drinking water, inhalation of dust, dermal contact with bath/shower water, soil, or dust, and ingestion of nickelcontaminated soil. Groundwater contamination may occur where the soil has a coarse texture and where acid waste, such as waste from plating industries, is discarded. However, there is no information linking this source of nickel contamination in ground water to levels of nickel in drinking water that would be of concern $(>50 \mu \mathrm{g} / \mathrm{L})$.

Nickel releases to the atmosphere are mainly in the form of aerosols that cover a broad spectrum of sizes. Particulates from power plants tend to be smaller than those from smelters (Cahill 1989; Schroeder et al. 1987). Atmospheric aerosols are removed by gravitational settling and dry and wet deposition. Submicron particles may have atmospheric half-lives as long as 30 days (Schroeder et al. 1987). Monitoring data confirm that nickel can be transported far from its source (Pacyna and Ottar 1985). Average ambient air nickel concentrations in the United States measured during 1977-1982 ranged between 7 and $12 \mathrm{ng} / \mathrm{m}^{3}$ (EPA 1986a). A recent estimate of ambient nickel concentrations in the United States based on data collected in 1996 is $2.22 \mathrm{ng} / \mathrm{m}^{3}$ (EPA 2003u). Nickel concentrations in air particulate matter in remote, rural, and U.S. urban areas have been found in the ranges of 0.01-60, 0.6- 
78, and 1-328 ng/m ${ }^{3}$, respectively (Schroeder et al. 1987). Nickel concentrations in indoor air are typically $<10 \mathrm{ng} / \mathrm{m}^{3}$ (Graney et al. 2004; Kinney et al. 2002; Koutrakis et al. 1992; Van Winkle and Scheff 2001).

The form of nickel emitted to the atmosphere varies according to the type of source. Nickel species associated with combustion, incineration, and metals smelting and refining are often complex nickel oxides, nickel sulfate, and metallic nickel, and in more specialized industries, the species commonly found are nickel silicate, nickel subsulfide, and nickel chloride (EPA 1985a).

Uncontaminated freshwater and seawater generally contain about $0.300 \mu \mathrm{g} / \mathrm{L}$ of nickel (Barceloux 1999). Concentrations of nickel in drinking water commonly range from 0.55 to $25 \mu \mathrm{g} / \mathrm{L}$ and average between 2 and $4.3 \mu \mathrm{g} / \mathrm{L}$. The concentration of nickel in rain has been reported as $\leq 1.5 \mu \mathrm{g} / \mathrm{L}$. Concentrations of nickel in snow in Montreal, Canada, ranged from 2 to $300 \mathrm{ppb}(2,300 \mu \mathrm{g} / \mathrm{L})$ (Landsberger et al. 1983).

Nickel is a natural constituent of soil; levels vary widely depending on local geology and anthropogenic input. The typical concentrations of nickel reported in soil range from 4 to $80 \mathrm{ppm}$. Nickel may be transported into streams and waterways from the natural weathering of soil as well as from anthropogenic discharges and runoff. This nickel accumulates in sediment. Nickel levels in surface water are low. In some studies, nickel could not be detected in a large fraction of analyzed samples. Median nickel concentrations in rivers and lakes range from $\approx 0.5$ to $6 \mu \mathrm{g} / \mathrm{L}$. Levels in groundwater appear to be similar to those in surface water. Levels in seawater are typically $0.1-0.5 \mu \mathrm{g} / \mathrm{L}$.

The speciation and physicochemical state of nickel is important in considering its behavior in the environment and availability to biota. For example, the nickel incorporated in some mineral lattices may be inert and have no ecological significance. Most analytical methods for nickel do not distinguish the form of nickel; the total amount of nickel is reported, but the nature of the nickel compounds and whether they are adsorbed to other material is not known. This information, which is critical in determining nickel's liability and availability, is site specific. Therefore, it is impossible to predict nickel's environmental behavior on a general basis.

Little is known concerning the chemistry of nickel in the atmosphere. The probable species present in the atmosphere include soil minerals, nickel oxide, and nickel sulfate (Schmidt and Andren 1980). In aerobic waters at environmental $\mathrm{pHs}$, the predominant form of nickel is the hexahydrate $\mathrm{Ni}\left(\mathrm{H}_{2} \mathrm{O}\right)_{6}{ }^{2+}$ ion (Richter and Theis 1980). Complexes with naturally occurring anions, such as $\mathrm{OH}^{-}, \mathrm{SO}_{4}{ }^{2-}$, and $\mathrm{Cl}^{-}$, are formed to 
a small degree. Complexes with hydroxyl radicals are more stable than those with sulfate, which in turn are more stable than those with chloride. $\mathrm{Ni}(\mathrm{OH})_{2}{ }^{0}$ becomes the dominant species above $\mathrm{pH} 9.5$. In anaerobic systems, nickel sulfide forms if sulfur is present, and this limits the solubility of nickel. In soil, the most important sinks for nickel, other than soil minerals, are amorphous oxides of iron and manganese. The mobility of nickel in soil is site specific depending mainly on soil type and $\mathrm{pH}$. The mobility of nickel in soil is increased at low $\mathrm{pH}$. At one well-studied site, the sulfate concentration and the surface area of soil iron oxides were also key factors affecting nickel adsorption (Richter and Theis 1980).

\subsection{RELEASES TO THE ENVIRONMENT}

The TRI data should be used with caution because only certain types of facilities are required to report (EPA 1997). This is not an exhaustive list. Manufacturing and processing facilities are required to report information to the Toxics Release Inventory only if they employ 10 or more full-time employees; if their facility is classified under Standard Industrial Classification (SIC) codes 20-39; and if their facility produces, imports, or processes $\geq 25,000$ pounds of any TRI chemical or otherwise uses $>10,000$ pounds of a TRI chemical in a calendar year (EPA 1997).

Most analytical methods for nickel in environmental samples do not distinguish between compounds of nickel or the nature of its binding to soil and particulate matter. It is generally impossible to say with certainty what forms of nickel are released from natural and anthropogenic sources, what forms are deposited or occur in environmental samples, and to what forms of nickel people are exposed. The form of nickel has important consequences as far as its transport, transformations, and bioavailability are concerned.

\subsubsection{Air}

Estimated releases of 404,413 pounds ( 183 metric tons) of nickel and 942,117 pounds ( 427 metric tons) of nickel compounds to the atmosphere from 2,279 and 1,286 domestic manufacturing and processing facilities in 2002, respectively, accounted for about 6.0 and $2.5 \%$ of the estimated total environmental releases, respectively, from facilities required to report to the TRI (TRI02 2004). These releases are summarized in Tables 6-1 and 6-2. 
Nickel and its compounds are naturally present in the Earth's crust, and releases to the atmosphere occur from natural processes such as windblown dust and volcanic eruption, as well as from anthropogenic activities. These latter releases are mainly in the form of aerosols. It is important to consider the background levels that are due to natural sources and distinguish them from levels that may result from anthropogenic activities. It is estimated that 8.5 million $\mathrm{kg}$ of nickel are emitted into the atmosphere from natural sources each year (Bennett 1984; Schmidt and Andren 1980). Based on this value, sources of nickel have been estimated as follows: windblown dust, 56\%; volcanoes, $29 \%$; vegetation, $9 \%$; forest fires, 2\%; and meteoric dust, 2\%. A more recent and higher estimate of 30 million $\mathrm{kg} / \mathrm{year}$ has been given for emission of nickel into the atmosphere from natural sources (Duce et al. 1991; Giusti et al. 1993). Anthropogenic sources of atmospheric nickel include nickel mining, smelting, refining, production of steel and other nickel-containing alloys, fossil fuel combustion, and waste incineration.

Emissions factors (i.e., $\mathrm{kg}$ of nickel emissions per unit consumption or production) have been estimated for various source categories, and these have been used to estimate worldwide emissions (Nriagu and Pacyna 1988). According to Schmidt and Andren (1980), annual anthropogenic emissions are estimated to contain 43 million $\mathrm{kg}$ of nickel (median value), 1.4 times the natural emission rate of 30 million kg/year. Nriagu and Pacyna (1988) estimate annual anthropogenic emissions as 55.6 million $\mathrm{kg}$. The nickel emission factor for burning residual and fuel oil is estimated to be $0.03 \mathrm{~kg} / \mathrm{ton}$, yielding nickel emissions of 26.7 million $\mathrm{kg} / \mathrm{year}$ or $62 \%$ of the total anthropogenic emissions (Schmidt and Andren 1980). The estimated contributions of other anthropogenic sources of nickel are nickel metal and refining, 17\%; municipal incineration, 12\%; steel production, 3\%; other nickel-containing alloy production, 2\%; and coal combustion, 2\% (Bennett 1984; Schmidt and Andren 1980). Wood combustion is also an important source of nickel emissions (Nriagu and Pacyna 1988).

Based on data contained in EPA's 1996 National Toxics Inventory (NTI), which is compilation of emissions data obtained from TRI, state and local databases, and other studies required by the Clean Air Act (CAA), it is estimated that emissions of nickel compounds into air totaled 1,170 tons per year in the United States (EPA 2000). Of this total, 1,196 tons of nickel compounds per year were derived from urban sources, with the major contributors coming from stationary sources that release 10 or more tons of nickel compounds per year. On-road mobile sources, such as cars, motorcycles, trucks, and buses, accounted for only 10 tons per year of nickel released to air, whereas nonroad mobile sources, such as airplanes, boats, and lawn mowers, accounted for a release of 66 tons of nickel compounds per year. 
Deposition of metals around large smelter complexes is a significant local problem. For example, at the Copper Cliff smelter in Sudbury, Ontario, it is estimated that $42 \%$ of nickel particulates emitted from the 381-m stack are deposited within a 60-km radius of the smelter (Taylor and Crowder 1983). The Copper Cliff smelter, one of three large nickel sources in the Sudbury area, emits 592 pounds $(269 \mathrm{~kg})$ of nickel per day.

A typical, modern, coal-fired power plant emits $\approx 25 \mu \mathrm{g}$ nickel per Megajoule (MJ) of power produced, compared with $420 \mu \mathrm{g} / \mathrm{MJ}$ for an oil-fired plant (Hasanen et al. 1986). The nickel concentration in stack emissions from a modern coal-fired power plant with an electrostatic precipitator was $1.3 \mu \mathrm{g} / \mathrm{m}^{3}$ (Lee et al. 1975). In a case study of the emissions of metals from an average sized coal-burning electric power plant (650 MW at a capacity factor of 67\%) equipped with an electrostatic precipitator (ESP), $100 \mathrm{~kg} / \mathrm{year}$ of nickel is emitted into air (Rubin 1999). These nickel emissions are reduced to $16 \mathrm{~kg} / \mathrm{y}$ ear for plants that are fitted with a wet lime/limestone flue gas desulfurization system downstream from the ESP. Highsulfur eastern coal has a higher nickel content than low-sulfur western coal, so power plants using eastern coal emit more nickel than those using western coal (QueHee et al. 1982).

It is estimated that in 1999, 570,000 tons of nickel were released from the combustion of fossil fuels worldwide (Rydh and Svärd 2003). Of this, 326 tons were released from electric utilities (Leikauf 2002).

From a public health point of view, the concentration of nickel associated with small particles that can be inhaled into the lungs is of greatest concern. The nickel content of aerosols from power plant emissions is not strongly correlated with particle size (Hansen and Fisher 1980). In one coal plant, 53 and 32\% of nickel in emissions were associated with particles $<3$ and $<1.5 \mu \mathrm{m}$ in diameter, respectively (Sabbioni et al. 1984). Other studies found that only $17-22 \%$ of nickel emissions from coal-fired power plants were associated with particles of $>2 \mu \mathrm{m}$, and that the mass medium diameter (MMD) of nickel-containing particles from a plant with pollution control devices was $5.4 \mu \mathrm{m}$ (Gladney et al. 1978; Lee et al. 1975). In one study, $40 \%$ of the nickel in coal fly ash was adsorbed on the surface of the particles rather than being embedded in the aluminosilicate matrix (Hansen and Fisher 1980). Surface-adsorbed nickel would be more available than embedded nickel.

Nickel emissions from municipal incinerators depend on the nickel content of the refuse and the design and operation of the incinerator. By comparing the nickel content of particles emitted from two municipal incinerators in Washington, DC, with that of atmospheric particulate matter, Greenberg et al. (1978) concluded that refuse incineration is not a major source of nickel in the Washington area. The 
average nickel concentrations in suspended particles from these incinerators ranged from 170 to $200 \mathrm{ppm}$. Nickel is not primarily associated with very fine or coarse particles. In tests performed under the Canadian National Incinerator Testing and Evaluation Program, $1.0 \mathrm{~g}$ nickel/ton refuse was emitted under normal operating conditions; when the combustion chamber operated at low and high combustion temperatures, nickel emissions increased $\leq 2.2 \mathrm{~g}$ nickel/ton (Hay et al. 1986). These emissions can be compared with a factor of $0.33 \mathrm{~g}$ nickel/ton refuse obtained in a European study (Pacyna 1984). The European study also obtained an emission factor of $1.0 \mathrm{~g}$ nickel/ton for sewage sludge incineration.

An increase in nickel emissions over presettlement levels was assessed by dating and analyzing peat cores from a fen located in northern Indiana, which is downwind from the city of Chicago and the industrial complexes of Gary and East Chicago, areas that contain a large steel mill and a coal-fired power plant. The peak accumulation rate was $7.73 \mathrm{mg}$ nickel $/ \mathrm{m}^{2} /$ year for $1970-1973$, a factor of 21 greater than the accumulation rate in presettlement times (A.D. 1339-1656) (Cole et al. 1990).

Some work has been performed to determine the species of nickel present in air emissions from different source categories (EPA 1985a). This has been determined from analyses of dust by x-ray diffraction, scanning electron microscopy, and energy dispersive $\mathrm{x}$-ray analysis or by an assessment of the reactions and transformations possible for the material present and the process conditions. Nickel resulting from oil combustion is primarily nickel sulfate with lesser amounts of complex metal oxides and nickel oxide. Approximately $90 \%$ of nickel in fly ash from coal combustion consists of complex (primarily iron) oxides. Nickel silicate and iron-nickel oxides would be expected from the mining and smelting of lateritic nickel ore, whereas nickel matte refining would produce nickel subsulfide and metallic nickel. The primary nickel species from secondary nickel smelting and steel and nickel alloys production is ironnickel oxide.

Nickel and nickel compounds have been identified in air samples collected from 20 of the 872 NPL hazardous waste sites where nickel or nickel compounds have been detected in environmental media (HazDat 2005). Nickel or nickel compounds have been detected in air offsite of NPL sites at concentrations ranging from 0.4912 to $4,000 \mathrm{ng} / \mathrm{m}^{3}$.

\subsubsection{Water}

Estimated releases of 151,725 pounds ( $\sim 69$ metric tons) of nickel and 516,804 pounds of nickel compounds ( $\sim 234$ metric tons) to surface water from 2,279 and 1,286 domestic manufacturing and 
processing facilities in 2002, respectively, accounted for about 2.2 and $1.4 \%$ of the estimated total environmental releases, respectively, from facilities required to report to the TRI (TRI02 2004). These releases are summarized in Tables 6-1 and 6-2.

Nickel is a natural constituent of soil and is transported into streams and waterways in runoff either from natural weathering or from disturbed soil. Much of this nickel is associated with particulate matter. Nickel also enters bodies of water through atmospheric deposition.

Emission factors have been estimated for the release of trace metals to water from various source categories and these have been used to estimate inputs of these metals into the aquatic ecosystem. The global anthropogenic input of nickel into the aquatic ecosystem for 1983 is estimated to be between 33 and 194 million kg/year with a median value of 113 million $\mathrm{kg} / \mathrm{year}$ (Nriagu and Pacyna 1988).

A survey of raw and treated waste water from 20 industrial categories indicated that nickel is commonly found in some waste waters. Those industries with mean effluent levels of $>1,000 \mu \mathrm{g} / \mathrm{L}$ in raw waste water were inorganic chemicals manufacturing $(20,000 \mu \mathrm{g} / \mathrm{L})$, iron and steel manufacturing $(1,700 \mu \mathrm{g} / \mathrm{L})$, battery manufacturing $(6,700 \mu \mathrm{g} / \mathrm{L})$, coil coating $(1,400 \mu \mathrm{g} / \mathrm{L})$, metal finishing $(26,000 \mu \mathrm{g} / \mathrm{L})$, porcelain enameling $(19,000 \mu \mathrm{g} / \mathrm{L})$, nonferrous metal manufacturing $(<91,000 \mu \mathrm{g} / \mathrm{L})$, and steam electric power plants $(95,000 \mu \mathrm{g} / \mathrm{L})($ EPA 1981). Those industries with mean effluent levels $>1,000 \mu \mathrm{g} / \mathrm{L}$ in treated waste water were porcelain enameling $(14,000 \mu \mathrm{g} / \mathrm{L})$ and nonferrous metal manufacturing $(14,000 \mu \mathrm{g} / \mathrm{L})$ (EPA 1981). The maximum levels in treated discharges from these industries were 67,000 and $310,000 \mu \mathrm{g} / \mathrm{L}$, respectively. In addition, four other industrial categories had maximum concentrations in treated discharges $>1,000 \mu \mathrm{g} / \mathrm{L}$. These were inorganic chemicals manufacturing $(1,400 \mu \mathrm{g} / \mathrm{L})$, iron and steel manufacturing $(7,800 \mu \mathrm{g} / \mathrm{L})$, aluminum forming $(20,000 \mu \mathrm{g} / \mathrm{L})$, and paint and ink formulation $(80,000 \mu \mathrm{g} / \mathrm{L})$.

Domestic waste water is the major anthropogenic source of nickel in waterways (Nriagu and Pacyna 1988). Concentrations of nickel in influents to 203 municipal waste water treatment plants (9,461 observations) ranged from 2 to $111,400 \mu \mathrm{g} / \mathrm{L}$; the median value was $\approx 300 \mu \mathrm{g} / \mathrm{L}$ (Minear et al. 1981). From a study of influent streams of a waste water treatment plant in Stockholm, Sweden, it was determined that the waste streams from households (e.g., drinking water) and businesses (e.g., drinking water, car washes, chemical uses) account for $29 \%$ of nickel in influent streams (Sörme and Lagerkvist 2002), which is likely to be comparable to what occurs in the United States. Another $31 \%$ of the nickel in influent streams is added at the waste water treatment plant through the addition of water treatment 
chemicals. Storm water accounts for between 1 and 5\% of the nickel in influent streams. Concentrations in treated effluents were not reported. However, nickel may be removed by chemical precipitation or coagulation treatment in publicly owned treatment works, which reduces nickel releases (EPA 1981). For example, improvements in sewage treatment facilities have attributed to a reduction in the flux of nickel in waste water effluents into the Hudson River estuary, decreasing from $518 \mathrm{~kg} /$ day in 1974 to $43 \mathrm{~kg} / \mathrm{day}$ in 1997 (Saňudo-Wilhelmy and Gill 1999).

Effluent water generated from mining and smelting operations comes from seepage, runoff from tailing piles, or from utility water used for mine operations. These discharges consist mostly of less-soluble silicates and sulfides and readily settle out. Tailing effluents from sulfidic ores are acidic due to the bacterial generation of sulfuric acid from the sulfidic minerals in the tailings, and very high concentrations of soluble nickel sulfate may be released. Tailing waters from the Onaping and Sudbury areas of Ontario, Canada, have an average nickel content of $42,500 \mu \mathrm{g} / \mathrm{L}$, a factor of 8,300 greater than that found in river water (Mann et al. 1989). Since there is presently no nickel mining of sulfidic ore in the United States, nickel-containing waste water is not generated by this activity. However, past nickel mining may have contributed to nickel entering our waterways and accumulating in sediment. Old tailing piles may contribute to runoff for decades.

In the EPA-sponsored National Urban Runoff Program, in which 86 samples of runoff from 15 cities throughout the United States were analyzed, nickel was found in $48 \%$ of runoff samples, at concentrations of $1-182 \mu \mathrm{g} / \mathrm{L}$ (Cole et al. 1984). The geometric mean nickel concentration in runoff water from the cities studied was between 5.8 and $19.1 \mu \mathrm{g} / \mathrm{L}$. In a more recent study of nickel concentrations in storm runoff water samples taken from different urban source areas, the arithmetic means of the concentrations for dissolved nickel ranged from $<1$ to $87 \mu \mathrm{g} / \mathrm{L}$, and from 17 to $55 \mu \mathrm{g} / \mathrm{L}$ for nickel that also included the metal associated with particulates (Pitt et al. 1995).

One of the potentially dangerous sources of chemical release at waste sites is landfill leachate. In a study that looked at leachate from three municipal landfills in New Brunswick, Canada, the results were conflicting. Average nickel concentrations in the three leachates (control) were 28 (45) $\mu \mathrm{g} / \mathrm{L}, 33$ (not detectable) $\mu \mathrm{g} / \mathrm{L}$, and 41 (23) $\mu \mathrm{g} / \mathrm{L}$ (Cyr et al. 1987). Sediment at three sites below the leachate outfalls contained 11.9, 37.4, and 71.2 ppm of nickel (dry weight).

Nickel and/or nickel compounds have been identified in surface water samples collected from 292 of the 872 NPL hazardous waste sites where nickel or nickel compounds have been detected in environmental 
media (HazDat 2005). Nickel or nickel compounds have been detected in surface water offsite of NPL sites at concentrations ranging from 2 to $20,000 \mathrm{ppb}$. Nickel and/or nickel compounds have also been identified in groundwater samples collected from 593 of the 872 NPL hazardous waste sites (HazDat 2005). Nickel or nickel compounds have been detected in groundwater offsite of NPL sites at concentrations ranging from 4.2 to $11,400 \mathrm{ppb}$.

\subsubsection{Soil}

Estimated releases of 5.58 million pounds ( 5,530 metric tons) of nickel and 32.7 million pounds ( 14,800 metric tons) of nickel compounds to soils from 2,279 and 1,286 domestic manufacturing and processing facilities in 2002, respectively, accounted for about 82 and $87 \%$ of the estimated total environmental releases, respectively, from facilities required to report to the TRI (TRI02 2004). An additional 55,185 pounds ( $\sim 25$ metric tons) of nickel and 634,432 pounds ( 288 metric tons) of nickel compounds, constituting about $0.8 \%$ and $1.7 \%$ of the total environmental emissions, respectively, were released via underground injection (TRI02 2004). These releases are summarized in Tables 6-1 and 6-2.

Most of the nickel released to the environment is released to land. Emission factors for nickel released to soil have been estimated for various industries (Nriagu and Pacyna 1988). These factors can be used to estimate industrial nickel releases to land. Excluding mining and smelting releases to land, $66 \%$ of estimated anthropogenic environmental releases or 325 million $\mathrm{kg} / \mathrm{year}$ (median) are to soil (Nriagu and Pacyna 1988). Some important sources of nickel released to soil are coal fly ash and bottom ash, waste from metal manufacturing, commercial waste, atmospheric fallout, urban refuse, and sewage sludge.

Based on 1999 production data, the equivalent of 0.6-3.3\% of the nickel that was mined that year was used in the manufacture of portable batteries (Rydh and Svärd 2003). This amounts to approximately 1731 ktons of nickel. Although current battery recycling programs in Europe claim success rates of upwards of 55\%, the global recycling rates are typically lower, ranging between 5 and 50\%. Therefore, on a global level, more than half of the nickel used in battery production will be disposed of in landfills and other waste sites.

Nickel and/or nickel compounds have been identified in soil samples collected from 443 of the 872 NPL hazardous waste sites where nickel or nickel compounds have been detected in environmental media (HazDat 2005). Nickel or nickel compounds have been detected in soils offsite of NPL sites at concentrations ranging from 2 to 10,522 ppb. Nickel and/or nickel compounds have also been identified 
in sediment samples collected from 302 of the 872 NPL hazardous waste sites (HazDat 2005). Nickel or nickel compounds have been detected in sediments offsite of NPL sites at concentrations ranging from 0.009 to $33,100 \mathrm{ppm}$.

\subsection{ENVIRONMENTAL FATE}

It is not always possible to separate the environmental fate processes relating to transport and partitioning from those relating to transformation for a metal and its various compounds and complexes. Because of analytical limitations, investigators rarely identify the form of a metal present in the environment. A change in the transport or partitioning of a metal may result from a transformation. For example, complexation may result in enhanced mobility, while the formation of a less-soluble sulfide would decrease its mobility in water. Adsorption may be the result of strong bonds being formed (transformation) as well as weak ones. Separating data relating to strong and weak adsorption in different sections is awkward and may not always be possible. Section 6.3.1 covers deposition and general adsorption of nickel, and Section 6.3.2 examines areas of environmental fate in which speciation occurs.

\subsubsection{Transport and Partitioning}

Nickel is released to the atmosphere in the form of particulate matter or adsorbed to particulate matter. It is dispersed by wind and removed by gravitational settling (sedimentation), dry deposition (inertial impaction characterized by a deposition velocity), washout by rain (attachment to droplets within clouds), and rainout (scrubbing action below clouds) (Schroeder et al. 1987). The removal rate and distance traveled from the source depends on source characteristics (e.g., stack height), particle size and density, and meteorological conditions.

Gravitational settling governs the removal of large particles $(>5 \mu \mathrm{m})$, whereas smaller particles are removed by other forms of dry and wet deposition. The partitioning between dry and wet deposition depends on the intensity and duration of precipitation, the element in question and its form in the particulate matter, and particle size. The importance of wet deposition relative to dry deposition generally increases with decreasing particle size. Removal of coarse particles may occur in a matter of hours. Small particles within the size range of $0.3-0.5 \mu \mathrm{m}$ may have an atmospheric half-life as long as 30 days and, therefore, have the potential to be transported over long distances (Schroeder et al. 1987). Evidence for the long-range transport of nickel is provided by the fact that emission sources in North America, 
Greenland, and Europe are responsible for elevated atmospheric nickel concentrations in the Norwegian Arctic during both the summer and winter (Pacyna and Ottar 1985).

Available studies indicate that nickel is broadly distributed among aerosol size groups. It has been concluded, based on the chemical and physical properties of atmospheric particles, that the concentrations of nickel in large particles ( $>1 \mu \mathrm{m}$ diameter) that are commonly associated with particulates derived from natural sources are less than concentrations in smaller particles ( $<1 \mu \mathrm{m}$ diameter) that are typically derived from anthropogenic sources (Giusti et al. 1993; Scudlark et al. 1994; Stoessel and Michaelis 1986). However, experiments in Ontario showed that nickel is associated with relatively large particles, 5.6 $\pm 2.4 \mu \mathrm{m}$ (Chan et al. 1986). A 1970 National Air Surveillance Network study of the average nickel size distribution in six American cities indicated that the mass median diameter (MMD) is $\approx 1.0 \mu \mathrm{m}$ in all six cities (Lee et al. 1972). Although the sampling procedure used in this study may have underestimated large particles (Davidson 1980), it represents one of the few studies involving the size distribution of nickel aerosols in U.S. cities.

Nickel concentration and speciation were investigated in particulate matter collected from an urban environment. The sampling sites in Davie, Florida, are within a high population area $(3,837$ persons per square mile) that is influenced by a number of stationary and mobile point source emitters of particulate matter, including residual oil- and natural gas-burning power plants, municipal waste incinerators, automobiles, aircraft, and marine vessel traffic (Galbreath et al. 2003). Nickel concentrations of 86 and $140 \mathrm{ppm}$ were measured in total suspended particulates (TSP) and in particulates with a MMD of $10 \mu \mathrm{m}$ $\left(\mathrm{PM}_{10}\right)$, respectively. The concentration of $140 \mathrm{ppm}$ in the $\mathrm{PM}_{10}$ fraction converts to an airborne nickel concentration of $1.5 \mathrm{ng} / \mathrm{m}^{3}$. Three nickel species were characterized and measured in the particulate fractions, $\mathrm{NiSO}_{4} \bullet \mathrm{xH}_{2} \mathrm{O}, \mathrm{NiFe}_{2} \mathrm{O}_{4}$, and $\mathrm{NiS}$. The proportions of these species in the TSP fraction were 40, 50 , and $10 \%$, respectively; the proportions in the $\mathrm{PM}_{10}$ fraction were 78,22 , and $<5 \%$, respectively.

Metal deposition is characterized by large temporal and spatial variability. Deposition can be associated with precipitation (wet deposition) or result from processes such as gravitational settling of dust (dry deposition). Estimated nickel deposition rates range from 0.01 to $0.5 \mathrm{~kg} /$ hectare/year $\left(1-50 \mathrm{mg} / \mathrm{m}^{2} /\right.$ year) and from 0.1 to $5.95 \mathrm{~kg} /$ hectare/year $\left(10-595 \mathrm{mg} / \mathrm{m}^{2} /\right.$ year) in rural and urban areas, respectively (Schroeder et al. 1987). In the Florida Atmospheric Mercury Study (FAMS) conducted during 19931994, bulk deposition rates for nickel varied between 1.700 and $4.130 \mathrm{mg} / \mathrm{m}^{2} /$ year, depending on local/regional anthropogenic activity (Landing et al. 1995). Nickel deposition from 1980 to 1981 in an industrial area of England where a number of ferrous and nonferrous metal smelting and manufacturing 
works were concentrated had a mean value of $8,800 \mathrm{ng} / \mathrm{cm}^{2} /$ year $\left(88 \mathrm{mg} / \mathrm{m}^{2} /\right.$ year $)$, a factor of $8-25$ above nonurban deposition rates (Pattenden et al. 1982). Wet deposition accounted for half of the deposition. Eighty-one percent of the nickel in rain was dissolved. Schroeder et al. (1987) reported the same percent of dry to wet deposition for nickel, whereas Chan et al. (1986) found that 2.2 times as much wet deposition as dry deposition occurred in Ontario in 1982 with little variability in the ratio across the province. The mean dry deposition rates for southern, central, and northern Ontario in 1982 were 0.25 , 0.28 , and $0.18 \mathrm{mg} / \mathrm{m}^{2} /$ year, respectively. In southern Ontario, Canada, where the average concentration of nickel in rain was $0.557 \mathrm{ppb}$ during $1982,0.5 \mathrm{mg}$ of nickel was deposited annually per square meter as a result of wet deposition (Chan et al. 1986). For central and northern Ontario, the mean concentrations of nickel in rain were 0.613 and $0.606 \mathrm{ppb}$, respectively, and the annual wet depositions averaged 0.5 and $0.4 \mathrm{mg} / \mathrm{m}^{2}$. Wet and dry deposition of particulates emitted from the Claremont Incinerator in Claremont, New Hampshire, were measured within an area between 2 and $15 \mathrm{~km}$ from the incinerator. Wet deposition rates varied between 0.50 and $8.87 \mu \mathrm{g} / \mathrm{m}^{2} /$ day $\left(0.0005-0.00887 \mathrm{mg} / \mathrm{m}^{2} /\right.$ day $)$ with a mean value of $3.0 \mu \mathrm{g} / \mathrm{m}^{2} /$ day $\left(0.003 \mathrm{mg} / \mathrm{m}^{2} /\right.$ day $)$ and depended on distance from the incinerator and wind weight. The mean wet deposition rate of $3.0 \mu \mathrm{g} / \mathrm{m}^{2} /$ day $\left(0.003 \mathrm{mg} / \mathrm{m}^{2} /\right.$ day $)$ was a factor of approximately 19 greater than the mean dry deposition rate of $0.16 \mu \mathrm{g} / \mathrm{m}^{2} /$ day $\left(0.00016 \mathrm{mg} / \mathrm{m}^{2} /\right.$ day $)$, which had been calculated from values ranging from 0.067 to $0.29 \mu \mathrm{g} / \mathrm{m}^{2} /$ day $\left(0.000067-0.00029 \mathrm{mg} / \mathrm{m}^{2} /\right.$ day) (Feng et al. 2000).

Atmospheric deposition of nickel in coastal waters has been reported. Bulk and wet deposition of nickel into Massachusetts Bay was determined to be 7,200 and 3,000 $\mu \mathrm{g} / \mathrm{m}^{2} /$ year (Golomb et al. 1997), respectively, whereas a lower wet deposition rate of $257 \mu \mathrm{g} / \mathrm{m}^{2} /$ year was measured for nickel in Chesapeake Bay (Scudlark et al. 1994). Atmospheric input of nickel into the Great Lakes has been estimated to average $160-590 \mathrm{ng} / \mathrm{m}^{2} /$ year (Nriagu et al. 1996).

Wet and dry deposition of nickel into the world's oceans is estimated to be 8-11 and 14-17 gigagrams $\left(10_{9}\right.$ grams) per year, respectively (Duce et al. 1991). However, atmospheric deposition is only a minor contributor to the flow of nickel into the oceans and coastal waterways as compared to riverine and fluvial input of nickel. The nickel that is carried into oceans in both dissolved and particulate forms through riverine input is rated at 1,411 gigagrams per year, which is a factor of approximately 50 greater than the sum of the wet and dry deposition of nickel of 22-28 gigagrams per year (Duce et al. 1991). In an example of nickel input into Chesapeake Bay, the fluvial input of nickel of 98,700 kg/year (0.0987 gigagrams/year) is 25 times greater than bulk deposition of nickel from the atmosphere (Scudlark et al. 1994). However, for the Great Lakes the atmospheric input of nickel accounts for $60-80 \%$ of the 
total anthropogenic input of nickel into Lake Superior, and 20-70\% of the total inputs into Lakes Erie and Ontario (Nriagu et al. 1996).

The fate of heavy metals in aquatic systems depends on partitioning between soluble and particulate solid phases. Adsorption, precipitation, coprecipitation, and complexation are processes that affect partitioning. These same processes, which are influenced by $\mathrm{pH}$, redox potential, the ionic strength of the water, the concentration of complexing ions, and the species and concentration of the metal, affect the adsorption of heavy metals to soil (Richter and Theis 1980).

Adsorption of nickel onto suspended particles in water is one of the main removal mechanisms of nickel from the water column. The adsorption of nickel on water-borne particulate matter is in competition with adsorption onto dissolved organic matter, which limits the amount of nickel that can be removed from the water column through the settling of suspended particles (Martino et al. 2003). Much of the nickel released into waterways as runoff is associated with particulate matter; it is transported and settles out in areas of active sedimentation such as the mouth of a river. Additionally, when a river feeds into an estuary, the salinity changes may affect absorptivity due to complexation and competition for binding sites (Bowman et al. 1981). During a 4-month study of Lake Onondaga in Syracuse, New York, 36\% of the nickel in the lake was lost to sediment (Young et al. 1982). Seventy-five percent of the nickel load into the lake was soluble and remained in the lake. The soluble nickel is not likely to be as the Ni(II) ion, but is expected to exist as a complex. For example, in an analysis of the speciation of nickel in waste water effluents and runoff discharging into San Francisco Bay, it was found that approximately $20 \%$ of soluble nickel was complexed to moderately strong complexing agents, such as humic acid and biopolymers from activated sludges (Sedlak et al. 1997). However, a larger proportion of the nickel, 75\% in waste water effluent and $25 \%$ in runoff, is found strongly complexed with stability constants that are similar to those found for synthetic chelating agents such as EDTA, DTPA, and phosphonates. Nickel is strongly adsorbed at mineral surfaces such as oxides and hydrous oxides of iron, manganese, and aluminum (Evans 1989; Rai and Zachara 1984). Such adsorption plays an important role in controlling the concentration of nickel in natural waters.

Nickel is strongly adsorbed by soil, although to a lesser degree than lead, copper, and zinc (Rai and Zachara 1984). There are many adsorbing species in soil, and many factors affect the extent to which nickel is adsorbed, so the adsorption of nickel by soil is site specific. Soil properties such as texture, bulk density, $\mathrm{pH}$, organic matter, the type and amount of clay minerals, and certain hydroxides, as well as the extent of groundwater flow, influence the retention and release of metals by soil (Richter and Theis 1980). 
Amorphous oxides of iron and manganese, and to a lesser extent clay minerals, are the most important adsorbents in soil. In alkaline soils, adsorption may be irreversible (Rai and Zachara 1984), which limits nickel's availability and mobility in these soils. For example, in recent studies of nickel speciation in ferromanganese nodules from loess soils of the Mississippi Basin, nickel is found to have a higher partition in the soil nodules than in soil clay matrices (Manceau et al. 2003). This is due to the selective sequesterization of nickel by finely divided iron and manganese oxides in goethite and lithiophorite minerals present in the soils. Cations such as $\mathrm{Ca}^{2+}$ and $\mathrm{Mg}^{2+}$ have been reported to reduce adsorption due to competition for binding sites, whereas anions like sulfate reduce adsorption as a result of complexation. Nickel adsorption depends strongly on metal concentration and $\mathrm{pH}$ (Giusti et al. 1993). For each mole of nickel adsorbed by iron and manganese oxide, $\approx 1-1.5$ moles of hydrogen ions are released (Rai and Zachara 1984). For aluminum oxide, as many as 2.3 moles $\mathrm{H}^{+}$are released. Mustafa and Haq (1988) found that the adsorption of nickel onto iron oxide at $\mathrm{pH} 7.0$ was rapid and increased with increasing temperature. They also found that two hydrogen ions are released into a solution when nickel is adsorbed. These studies indicate that while $\mathrm{Ni}^{2+}$ is the predominant species in solution, $\mathrm{NiOH}^{+}$is preferentially adsorbed, and that both mono- and bidentate complexes may be formed with the iron/manganese/aluminum oxides.

Batch equilibrium studies were performed using seven soils and sediments spiked with varying concentrations of nickel to assess the potential mobility of nickel in contaminated subsoil (LaBauve et al. 1988). The range of Freundlich parameters $\mathrm{K}(1 / \mathrm{n})$, an adsorption constant, ranged from 739 (0.92) to $6,112(0.87)$. One-, two-, and three-parameter models were used to evaluate the relation of soil properties and nickel retention. In the one-parameter model, $\mathrm{pH}$ was the best predictor. Cation exchange capacity (CEC) and iron oxide were the best predictors in the two-parameter models, and CEC, iron oxide, and percent clay were the best predictors in the three-parameter models. Nickel was more mobile in the soils than lead, cadmium, and zinc. The retention of nickel to two of the test subsoils diminished in the presence of synthetic landfill leachate, possibly because of complex formation. In another study in which batch adsorption experiments were conducted with a mixture of cadmium, cobalt, nickel, and zinc, and 38 different agricultural soils, taken from three depths at 13 sites, the adsorption constants ranged from 10 to $1,000 \mathrm{~L} / \mathrm{kg}$ (Anderson and Christensen 1988). Soil $\mathrm{pH}$, and to a lesser extent clay content and the amount of hydrous iron and manganese oxides, most influenced nickel sorption.

In 12 New Mexican soils from agricultural areas and potential chemical waste disposal sites, Freundlich parameters $\mathrm{K}(1 / \mathrm{n})$ ranged from 8.23 to $650(0.87-1.18)$; the median $\mathrm{K}$ was 388 (Bowman et al. 1981). The soil with the K of 8.23 was essentially unweathered rock that was not expected to have good 
adsorbing properties. The study concluded that most soils have an extremely high affinity for nickel and that once sorbed, nickel is difficult to desorb, which may indicate covalent bond formation. Sadiq and Enfield (1984b) observed nickel ferrite formation following adsorption. Bowman et al. (1981) found that when nickel levels were $>10$ ppm, adsorption decreased. High concentrations of chloride decreased adsorption, but not as much as calcium ions, which indicates that calcium competition for sorbing sites is more important than chloride complexation for reducing adsorption. The presence of complexing agents, such as EDTA, dramatically lowers nickel adsorption, which has important implications at waste disposal sites if liquid nickel waste containing chelating agents is released to soil. Chelating agents that are added to soil containing adsorbed nickel appear to have a lesser effect.

The capacity of soil to remove nickel and the nature of the bound nickel were evaluated for 10 mineral and 3 organic soils from the southeastern United States (King 1988). Some soil samples were taken from the subsoil as well as the surface. The amount of adsorbed nickel ranged from 13 to $95 \%$; the low value was found in subsoil, and the high value was found in soil high in organic matter. When extracted with potassium chloride, $5-87 \%$ of the nickel was nonexchangeable. Soil $\mathrm{pH}$ was the most important factor affecting sorbed and nonexchangeable nickel in all soil horizons. Both King (1988) and Tyler and McBride (1982) found much stronger nickel adsorptivity in organic soil than in mineral soils. Adsorption was improved by the quality and quantity of humus in the soil (Hargitai 1989). Nickel was enriched in humic and fulvic acids from Lake Ontario sediment (Nriagu and Coker 1980). It was estimated that 5$10 \%$ of the nickel in this sediment was bound to organic matter.

The leachability of nickel from some soils does not necessarily correlate with the total concentration of nickel in the soil. In an extraction study of soils sampled from the mining and smelting regions of Sudbury, Ontario, the percentage of nickel that is most easily extractable (in acetic acid) varied between 12 and $31 \%$ of the total nickel content $(220-455 \mathrm{mg} / \mathrm{kg}$ ) among the different sampling sites (Adamo et al. 1996). The remaining nickel was found in less extractable forms: 6-11\% was found to be associated with manganese oxides and easily reducible iron oxides, 6-20\% either bound to readily oxidizable organics or sulfides, and the remainder (55-73\%) was associated with sulfides as separate grains or inclusions, iron oxide phases, carbon particles, and silicate spheroids. Similarly, in soils that are naturally enriched in heavy metals sampled from the Port MacQuaire region in Australia, the amount of nickel that can be easily extracted from soil samples is only a small fraction of the total nickel content (Lottermoser 2002). Extraction of these soils with EDTA or acetic acid yielded leachable nickel which amounted to between $<0.1-4.1$ and $<0.01 \%$, respectively, of the total nickel concentrations in the soil samples. Use of stronger extraction methods, for example hydrochloric acid, yielded only leachable nickel in percentages 
(0.1-2.4\%) equivalent to those found for EDTA. The low amount of acetic acid extractable nickel indicates negligible leaching of this metal from these soils into groundwater and surface waters (Lottermoser 2002).

Amendment of soils with exogenous humic acid reduces mobility of dissolved nickel in soil and also increases the bioavailability of this nickel to plants. Halim et al. (2003) showed that humic acid in soils from nickel-humic acid complexes results in the removal of dissolved and exchangeable nickel from soil water. The extractability of nickel increased with the aging time of the organic material. The increased bioavailability of nickel bound to humic acid is temporary and is thought to occur mainly as the result of preventing nickel from undergoing a transformation into insoluble species in soil.

Nickel (II) is poorly removed from waste water in the activated sludge process because of its high solubility (Stephenson et al. 1987). Only 30-40\% of nickel was removed in a pilot activated sludge plant. Nickel removal in activated sludge plants is best correlated with effluent suspended solids (Kempton et al. 1987). Nickel is predominantly soluble in the effluent and is found complexed to humic acid, biopolymers, and other chelating agents (Sedlak et al. 1997).

In order to evaluate the potential of elements to leach from land-spread sewage sludge, Gerritse et al. (1982) studied the adsorption of elements to sandy and sandy loam top soils from water, salt solutions, and sludge solutions. They used metal levels that occurred in the solution phase of sewage sludge, 100$1,000 \mathrm{ppb}$ in the case of nickel. The results indicated that nickel is fairly mobile in these soils; the adsorption constants were $\approx 10-100$ in the sandy soil and a factor of $\approx 10$ higher in the sandy loam soil. The presence of sludge increases the mobility of nickel, particularly in sandy and sandy loam soils, which may be because of complexation with dissolved organic compounds (Kaschl et al. 2002) or increased ionic strength (Gerritse et al. 1982). However, land application of nickel-contaminated sludge did not give rise to increased levels of nickel in groundwater (Demirjian et al. 1984). Higher doses and repeated application of nickel-containing sewage sludge did not result in a proportional increase in nickel mobility (Hargitai 1989).

As part of EPA's National Runoff Program in Fresno, California, the soil water and groundwater at depths $\leq 26 \mathrm{~m}$ beneath five urban runoff retention/recharge basins were monitored during a 2-year study (Nightingale 1987). The results indicated that there were no significant downward movements of nickel with the recharge water. 
Saline sediments from estuaries often contain pyrite and other readily oxidizable sulfur compounds. When these sediments are oxidized, such as when dredged sediment is exposed to oxygen, sulfuric acid may be produced, which may overwhelm the buffering capacity of the sediment, lower the $\mathrm{pH}$ (to $\mathrm{pH}$ 3.1 in a laboratory experiment), and dissolve the ferric oxides and hydroxides that entrap heavy metals (DeLaune and Smith 1985). As a result, significant amounts of nickel may be released from the dredged sediments. An analogous $\mathrm{pH}$ decrease following exposure to oxygen was not observed in freshwater sediment.

The presence of iron-(di)sulfides in wetland sediments has been associated with increased mobilization of nickel into groundwater during periods of drought in Holland (Lucassen et al. 2002). Dessication of sediments leads to oxidation of iron-(di)sulfides and subsequent acidification of the sediments. When the $\mathrm{S} /(\mathrm{Ca}+\mathrm{Mg})$ ratios in these sediments rise above $2 / 3$, mobilization of heavy metals like nickel occurs, leading to groundwater concentrations of nickel that exceeded the Dutch signal level of $50 \mathrm{ppb}$ for nickel in $50 \%$ of the monitoring locations.

It has been reported that nickel is not accumulated in significant amounts by aquatic organisms (Birge and Black 1980; Zaroogian and Johnson 1984). The concentration of nickel in a major carnivorous fish in New York State, the lake trout, was the lowest, and the concentration did not increase appreciably with the age of the fish (Birge and Black 1980). The mean bioconcentration factor (BCF) for three carnivorous fish was 36. The concentration of nickel in mussels and oysters treated with $5 \mu \mathrm{g}$ nickel $/ \mathrm{kg}$ of seawater for 12 weeks averaged 9.62 and $12.96 \mu \mathrm{g}$ nickel/g, respectively, on a dry weight basis (Zaroogian and Johnson 1984). When these data are adjusted for controls and the nickel concentration in tissue is expressed on a wet weight basis, the $\mathrm{BCF}$ for the mussels and oysters is $\approx 100$. After 2 weeks in flowing seawater, 58 and $38 \%$ of the tissue nickel was lost from the mussel and oyster, respectively. No significant loss of nickel occurred during the remainder of the 28-week depuration period. The content of acid volatile sulfide (AVS) in sediment helps determine the bioavailability of metals (Ankley et al. 1991). In studies of nickel and cadmium, the metals were toxic to an amphipod (Hyallela azteca) and an oligocheate (Lumbriculus variegatus) when the extracted metals/AVS ratio was $>1$.

In the work of McGeer et al. (2003), BCFs for nickel in various aquatic organisms (e.g., algae, arthropods, mollusks, and fish) was assessed based on whole-body metal concentrations and exposure concentrations that were obtained from the literature. For exposure concentrations within the range of 5$50 \mu \mathrm{g} / \mathrm{L}$ nickel in water, mean BCF values of 106 \pm 53 (1 standard deviation [SD]) were obtained. When the authors also included data for exposure concentrations outside the range of 5-50 $\mu \mathrm{g} / \mathrm{L}$, a BCF value of 
$157 \pm 135$ was obtained. The authors noted that the BCF values were inversely correlated with the exposure concentrations, where the highest $\mathrm{BCF}$ values were obtained at the lowest exposure concentrations. There was no evidence that nickel biomagnifies in aquatic food webs and, in fact, there is evidence to indicate that the nickel concentrations in organisms decrease with increasing trophic level (McGeer et al. 2003; Suedel et al. 1994).

As part of the U.S. Geological Survey National Water-Quality Assessment (NAWQA) Program, there was no statistically significant correlation between nickel concentrations in bed-sediments collected from streams and rivers in both the Northern Rockies Intermontane Basin study area and the New Jersey study area, and nickel concentrations measured in liver and fillet samples taken from fish collected in the same study areas (USGS 2000a, 2000b). Also, nickel concentrations in fish liver and fillet samples were at or below the detection limits $(<0.1-0.3 \mu \mathrm{g} / \mathrm{g}$, dry weight) for nickel in these studies and are much lower than the concentrations of nickel measured in bed-sediments, which ranged from 12 to $43 \mu \mathrm{g} / \mathrm{g}$ (wet weight).

Uptake and accumulation of nickel into various plant species is known to occur. For example, PeraltaVidea et al. (2002) report the accumulation of nickel in alfalfa grown from soils contaminated with a mixture of four metals (e.g., $\mathrm{Cd}(\mathrm{II}), \mathrm{Cu}(\mathrm{II}), \mathrm{Ni}(\mathrm{II})$, and $\mathrm{Zn}(\mathrm{II})$ ) at a loading of $50 \mathrm{mg} / \mathrm{kg}$ for each metal. Concentration ratios of nickel in plant versus soil (based on dry weights) ranged between 22 and 26 over a $\mathrm{pH}$ range of 4.5-7.1. As with most plant species that hyperaccumulate metals, the alfalfa actively removes and translocates heavy metals, like nickel, from the roots to the shoots. The uptake of nickel into plants is modulated by the acidity $(\mathrm{pH})$ of the soil. Smith (1994) showed that nickel concentrations in rye grass were reduced by a factor of three as the soil $\mathrm{pH}$ was raised from 4 to 7 . This is thought to be due to a decrease in bioavailability of nickel with increasing $\mathrm{pH}$. The bioavailability of nickel to plants is also affected by soil type. Weng et al. (2004) found that the bioavailability of nickel to oat plants grown in soil rich in organic matter is half that of sandy or clay soils in the $\mathrm{pH}$ range of 4.4-7.0. These differences in bioavailability are attributed to a stronger binding of nickel to organic matter than to the silicates and iron hydroxides/oxides in clay and sand under the acidic conditions of the experiment.

Two studies concerning levels in voles and rabbits living on sludge-amended land did not indicate any accumulation of nickel in these herbivores or in the plants they fed upon (Alberici et al. 1989; Dressler et al. 1986). The lack of significant bioaccumulation of nickel in aquatic organisms, voles, and rabbits indicates that nickel is not biomagnified in the food chain. 


\subsubsection{Transformation and Degradation}

Analytical methods do not generally allow identification of the precise form of nickel present in environmental samples or an assessment of the transformations that may occur. Sequential extraction techniques are sometimes employed to determine how tightly nickel is bound to particles or in environmental matrices. Using different and progressively stronger extracting agents, the fractions of a sample that are exchangeable, adsorbed, easily reducible, moderately reducible, or organically bound carbonates, sulfides, and residual can be determined (Rudd et al. 1988; Rybicka 1989).

\subsubsection{Air}

Little is known about the chemical forms and physical and chemical transformations of trace elements in the atmosphere primarily because analytical methods provide information concerning the metal content rather than the specific compounds or species. In the absence of specific information, it is generally assumed that elements of anthropogenic origin, especially those emanating from combustion sources are present as the oxide, and nickel oxide has been identified in industrial emissions (Schroeder et al. 1987). Windblown dust particles may contain nickel in mineral species, which often contain nickel as the sulfide. Increases in the concentration of nickel in Sequoia National Park in California during rain coming from the south correlated with a sharp (7-13 times greater concentration) increase in sulfate (Cahill 1989). Nickel sulfate is a probable atmospheric species resulting from the oxidation of nickel in the presence of sulfur dioxide (Schmidt and Andren 1980).

The form of nickel in particles from different industries varies. The mineralogical composition, chemical content, and form of dusts from nine industries in Krakow, Poland, were examined (Rybicka 1989). The chemical form of a particle-associated heavy metal that was assessed by a five-step extraction scheme classified the metal as exchangeable, easily reducible (manganese oxides, partly amorphous iron oxyhydrates and carbonates), moderately reducible (amorphous and poorly crystallized iron oxyhydrates), organically bound or sulfidic, and residual. Dusts from power plants had a silicate characteristic with quartz and mullite predominant. Approximately $90 \%$ of the nickel from these facilities was in the residual fraction. Only 40-60\% of the nickel from metallurgical, chemical, and cement plants was in the residual fraction. Essentially none of the nickel from any of the industries was in an organic/sulfidic fraction. Dusts from metallurgical, chemical, and cement plants contained between 0 and 10\% (typically $5 \%$ ) of the nickel in the relatively mobile, cation-exchangeable fraction. Thirty percent of the nickel in dust from a slag processing facility was in this form. 


\subsubsection{Water}

In natural waters, nickel primarily exists as the hexahydrate. While nickel forms strong, soluble complexes with $\mathrm{OH}^{-}, \mathrm{SO}_{4}{ }^{2-}$, and $\mathrm{HCO}_{3}{ }^{-}$, these species are minor compared with hydrated $\mathrm{Ni}^{2+}$ in surface water and groundwater with $\mathrm{pH}<9$ (Rai and Zachara 1984). Under anaerobic conditions, such as may exist in deep groundwater, nickel sulfide would reduce free aqueous nickel concentrations to low levels.

Precipitation can remove soluble nickel from water. In aerobic waters, nickel ferrite is the most stable compound (Rai and Zachara 1984). Nickel may also be removed by coprecipitation with hydrous iron and manganese oxides. Nickel removed by precipitation and coprecipitation settles into the sediment.

Nickel in sediment may be strongly bound or present in a removable form. A metal's form in soil or sediment and its availability are determined by measuring the extractability of the metal with different solvents. Sediment samples from western Lake Ontario were analyzed in regard to the compositional associations of nickel by a series of sequential extractions (Poulton et al. 1988). The mean nickel percentages in the various fractions were as follows: exchangeable, $0.7 \pm 1.4$; carbonate, 0.0 ; iron or

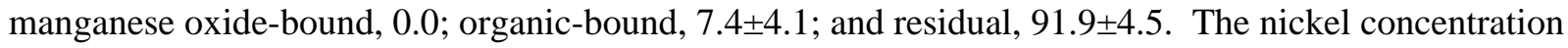
in 450 uncontaminated estuarine and coastal marine sites in the southeastern United States covaried significantly with the aluminum concentration, suggesting that natural aluminosilicates are the dominant natural metal-bearing phase in some aquatic systems (Windom et al. 1989). In 13 random samples of bottom sediment from the highly industrialized Meuse River in The Netherlands, between 0 and $88 \%$ (median 33\%) of the nickel was removable at low $\mathrm{pH}$, showing the great variability of nickel to adsorb to sediments (Mouvet and Bourg 1983).

Nickel removed by coprecipitation can be remobilized by microbial action under anaerobic conditions (Francis and Dodge 1990). Remobilization results from enzymatic reductive dissolution of iron with subsequent release of coprecipitated metals. A lowering of $\mathrm{pH}$ as a result of enzymatic reactions may indirectly enhance the dissolution of nickel. Experiments using mixed precipitates with goethite $(\alpha-\mathrm{FeOOH})$ indicated that a Clostridium species released 55\% of the coprecipitated nickel after 40 hours. Similarly, precipitated nickel sulfides in sediment can be mobilized through sulfur oxidation by Thiobacilli (Wood 1987). In this case, the oxidized sulfur may produce $\mathrm{H}_{2} \mathrm{SO}_{4}$ and decrease the $\mathrm{pH}$. 


\subsubsection{Sediment and Soil}

An analysis of the thermodynamic stability models of various nickel minerals and solution species indicates that nickel ferrite is the solid species that will most likely precipitate in soils (Sadiq and Enfield 1984a). Experiments on 21 mineral soils supported its formation in soil suspensions following nickel adsorption (Sadiq and Enfield 1984b). The formation of nickel aluminate, phosphate, or silicate was not significant. $\mathrm{Ni}^{2+}$ and $\mathrm{Ni}(\mathrm{OH})^{+}$are major components of the soil solution in alkaline soils. In acid soils, the predominant solution species will probably be $\mathrm{Ni}^{2+}, \mathrm{NiSO}_{4}$, and $\mathrm{NiHPO}_{4}($ Sadiq and Enfield 1984a).

A large percentage of nickel in sewage sludges exists in a form that is easily released from the solid matrix (Rudd et al. 1988). Although the availability of nickel to plants grown in sludge-amended soil is correlated with soil-solution nickel, it is only significantly correlated with DTPA-extractable nickel (Adams and Kissel 1989).

\subsection{LEVELS MONITORED OR ESTIMATED IN THE ENVIRONMENT}

Reliable evaluation of the potential for human exposure to nickel depends in part on the reliability of supporting analytical data from environmental samples and biological specimens. Concentrations of nickel in unpolluted atmospheres and in pristine surface waters are often so low as to be near the limits of current analytical methods. In reviewing data on nickel levels monitored or estimated in the environment, it should also be noted that the amount of chemical identified analytically is not necessarily equivalent to the amount that is bioavailable. The analytical methods available for monitoring nickel in a variety of environmental media are detailed in Chapter 7.

\subsubsection{Air}

Mean ambient air concentrations of nickel typically range between 6 and $20 \mathrm{ng} / \mathrm{m}^{3}$ and can be high as $150 \mathrm{ng} / \mathrm{m}^{3}$ near anthropogenic sources of airborne nickel (Barceloux 1999). Schroeder et al. (1987) reported nickel concentrations in particulate matter in the U.S. atmosphere of $0.01-60,0.6-78$, and 1$328 \mathrm{ng} / \mathrm{m}^{3}$ in remote, rural, and urban areas, respectively. Nickel concentrations in particulate matter $\left(\mathrm{PM}_{2.5-8}\right)$, collected in Spokane, Washington, from January 1995 to March 1999, averaged $1.2 \pm 0.9$ (1 SD) $\mathrm{ng} / \mathrm{m}^{3}$ (Claiborn et al. 2002). Based on emission data contained in the EPA $1996 \mathrm{NTI}$ database, an average concentration of nickel in ambient air in the contiguous United States was estimated 
to be $2.22 \mathrm{ng} / \mathrm{m}^{3}$ (median concentration $=0.948 \mathrm{ng} / \mathrm{m}^{3}$ ) (EPA 2003u). The five states with the highest average concentrations of nickel in ambient air were $\left(\mathrm{ng} / \mathrm{m}^{3}\right)$ : West Virginia (6.60), Utah (4.42), Delaware (4.10), New York (3.80), and Pennsylvania (3.69); the five states with the lowest concentrations were: Wyoming (0.127), South Dakota (0.157), North Dakota (0.211), Montana (0.311), and Vermont (0.311). Annual mean nickel concentrations in 11 Canadian cities measured during 1987-1990 ranged from 1 to $20 \mathrm{ng} / \mathrm{m}^{3}$, while at a rural location the mean nickel concentration was $1 \mathrm{ng} / \mathrm{m}^{3}$ (CEPA 1994). In another Canadian study, mean exposure concentrations for nickel in air for residents living near copper smelters and refineries and zinc plants ranged between 0.005 and $0.151 \mu \mathrm{g} / \mathrm{m}^{3}\left(5-151 \mathrm{ng} / \mathrm{m}^{3}\right)$ in comparison to background levels of $0.00069 \mu \mathrm{g} / \mathrm{m}^{3}\left(0.69 \mathrm{ng} / \mathrm{m}^{3}\right)$ (Newhook et al. 2003). Annual average nickel concentrations at three remote sites in the arctic region of Canada ranged from 0.14 to $0.45 \mathrm{ng} / \mathrm{m}^{3}$ (Barrie and Hoff 1985). Levels of nickel and other anthropogenic species peaked during January and February, possibly indicating the significance of combustion sources. Nickel levels in the air at three native villages in northern Alberta, Canada, were $0.779 \pm 0.774,1.1 \pm 0.57$, and $4.97 \pm 9.2 \mathrm{ng} / \mathrm{m}^{3}$, indicating that air concentrations of nickel can be highly variable (Moon et al. 1988).

According to the EPA's National Air Surveillance Network (NASN) report for 1977, 1978, and 1979, median nickel concentrations were below the detection limit for urban and nonurban samples except for 1978; during 1978, the urban median was $6 \mathrm{ng} / \mathrm{m}^{3}$ (Evans et al. 1984). The detection limit for inductively-coupled-plasma atomic emission spectroscopy (ICP-AES), the method used in the EPA study, was $1 \mathrm{ng} / \mathrm{m}^{3}$ (EPA 1986a; Evans et al. 1984). In the EPA study, 10,769 urban samples and 1,402 nonurban 24-hour air samples were analyzed. Five percent of the urban samples were $>33$, 32, and $30 \mathrm{ng}$ nickel $/ \mathrm{m}^{3}$ for 1977,1978 , and 1979 , respectively; $5 \%$ of the nonurban samples were $>10,10$, and $6 \mathrm{ng} / \mathrm{m}^{3}$, respectively, for these 3 years. Ninety-nine percent of the urban and nonurban samples for these 3 years did not exceed 68 and $52 \mathrm{ng} / \mathrm{m}^{3}$, respectively (Evans et al. 1984). Combined urban and nonurban measurements for the 99th percentile from the NASN (1977-1979) and its successor, the National Air Monitoring Filter Sites (NAMFS) (1980-1982), showed a sharp decline from 62 and $67 \mathrm{ng} / \mathrm{m}^{3}$ in 1977 and 1978 to 23 and $30 \mathrm{ng} / \mathrm{m}^{3}$ in 1981 and 1982. Mean levels for the combined urban and nonurban sites over the 6-year period ranged from 7 to $12 \mathrm{ng} / \mathrm{m}^{3}$ (EPA 1986a). According to the NASN data for 1965-1968, the average atmospheric nickel concentration in the air of 28 cities ranged from 3 to $90 \mathrm{ng} / \mathrm{m}^{3}$, with an overall average of $26 \mathrm{ng} / \mathrm{m}^{3}$ (NAS 1975). These data suggest that atmospheric nickel concentrations in the United States have been declining. No reason for this downward trend was suggested (EPA 1986a).

The most intensive study of the nickel concentration in the United States was the result of analyzing air samples collected during 1968-1971 for use in a lead survey (Saltzman et al. 1985). This study is 
significant because numerous sites in four cities were analyzed continuously over 1 year and analyzed by a single, highly experienced laboratory. Samples from 33 sites in Chicago, Houston, New York, and Washington, DC, were analyzed for nickel resulting in respective geometric mean nickel concentrations of $15,18,23$, and $42 \mathrm{ng} / \mathrm{m}^{3}$. The results for Washington, DC, are in basic agreement with the results obtained from Kowalczyk et al. (1982). In this study, 24-hour samples collected at 10 locations yielded average nickel concentrations ranging from 5.7 to $35 \mathrm{ng} / \mathrm{m}^{3}$, with a mean concentration of $17 \mathrm{ng} / \mathrm{m}^{3}$. The two major contributing sources are believed to be oil and coal combustion. The enrichment factor for nickel over crustal levels in 29 cities is 11 (Gladney et al. 1984). An enrichment factor considerably $>1$ indicates that the source of an element is anthropogenic. In Houston, the average concentration of nickel in both the fine $(0.1-2.5 \mu \mathrm{m})$ aerosols and those $>2.5 \mu \mathrm{m}$ was $4 \pm 1 \mathrm{ng} / \mathrm{m}^{3}$ (Johnson et al. 1984).

As part of the Airborne Toxic Element and Organic Substances project for determining patterns of toxic elements in different settings, three urban areas (Camden, Elizabeth, and Newark) and one rural site (Ringwood) in New Jersey were studied during two summers and winters between 1981 and 1983 (Lioy et al. 1987). Each site was sampled every 24 hours for 39 consecutive days. The geometric mean nickel concentrations were 8.0-34.0, 5.0-28.0, 10.0-27.0, and 5.0-13.0 ng/ $\mathrm{m}^{3}$ for Camden, Elizabeth, Newark, and Ringwood, respectively. The nickel levels measured in the industrial urban areas may be compared to the arithmetic mean values reported in the National Air Surveillance survey $\left(9.6-11.0 \mathrm{ng} / \mathrm{m}^{3}\right)$ for $1977-$ 1979 (Evans et al. 1984). Summer and winter maxima in the three urban areas ranged from 24.0 to 39.0 and from 81.0 to $112.0 \mathrm{ng} / \mathrm{m}^{3}$, respectively, and 22.0 and $32.0 \mathrm{ng} / \mathrm{m}^{3}$, respectively, for Ringwood.

The first and second highest annual average nickel concentrations in the air in Texas between 1978 and 1982, according to the Texas Air Control Board, were 49 and $34 \mathrm{ng} / \mathrm{m}^{3}$ at Port Arthur and Beaumont, respectively (Wiersema et al. 1984). The statewide 1978-1982 average was $1 \mathrm{ng} / \mathrm{m}^{3}$. Mean nickel levels showed relatively little geographic variation in Ontario where concentrations in southern, central, and northern Ontario were $0.81,0.91$, and $0.58 \mathrm{ng} / \mathrm{m}^{3}$, respectively (Chan et al. 1986).

Nickel concentrations in particulate matter $\mathrm{PM}_{10}$ was measured at three Midwestern sites, two urban sites with a large industrial component and one rural site, in samples collected from September 1985 to June 1988 (Sweet et al. 1993). Nickel concentrations in the fine $\mathrm{PM}_{10}$ particles $(<1-2.5 \mu \mathrm{m})$ taken from collection sites in East St. Louis and Southeast Chicago averaged 2.1 \pm 1.4 (1 SD) and $2.7 \pm 2.6 \mathrm{ng} / \mathrm{m}^{3}$, respectively, and were similar to those measured in the coarser $\mathrm{PM}_{10}$ particles $(2.5-10 \mu \mathrm{m})$ of $1.8 \pm 1.5$ and $2.1 \pm 1.0 \mathrm{ng} / \mathrm{m}^{3}$, respectively. The concentrations of nickel measured in both the fine and coarse particles collected at the East St. Louis and Southeast Chicago sites were higher than the average concentration of 
nickel of $0.5 \pm 0.3$ and $0.7 \pm 0.5 \mathrm{ng} / \mathrm{m}^{3}$ measured in fine and coarse particles, respectively, collected from a rural site (Bondville, Illinois). The higher concentrations of nickel in the East St. Louis and Southeast Chicago sites are attributed to emissions from zinc smelters and steel mills/oil combustion, respectively.

Nickel concentrations in indoor air are generally $<10 \mathrm{ng} / \mathrm{m}^{3}$. In a study of 10 homes in the southeast Chicago area, indoor and outdoor air samples were regularly sampled between June 1994 and April 1995 (van Winkle and Scheff 2001). Of the 48 samples taken, 35 had nickel concentrations above the detection limit of the assay with a mean $\left( \pm 1 \mathrm{SD}\right.$ ) concentration of $0.002 \pm 0.002 \mu \mathrm{g} / \mathrm{m}^{3}$ and a maximum value of $0.008 \mu \mathrm{g} / \mathrm{m}^{3}$. The median indoor nickel concentration of $0.003 \mu \mathrm{g} / \mathrm{m}^{3}$ was similar to the median outdoor nickel concentration of $0.0034 \mu \mathrm{g} / \mathrm{m}^{3}$. Indoor air samples taken from 394 homes in Suffolk and Onondaga Counties of New York State contained nickel concentrations that were similar to those found in the Chicago study (Koutrakis et al. 1992). A mean indoor nickel concentration of $2 \mathrm{ng} / \mathrm{m}^{3}$ $\left(0.002 \mu \mathrm{g} / \mathrm{m}^{3}\right)$ was derived from a sampling of 28 homes. The New York study also examined nickel concentrations in indoor air as a function of combustion sources within the home (e.g., resident smoker, wood-burning stove, kerosene heater) and found no difference in the mean nickel concentrations between homes containing these combustion sources and homes without. Graney et al. (2004) measured nickel levels in indoor air as part of a 1998 study of metal exposures for residents of a retirement home in Towson, Maryland. The study participants had a mean age of 84 , were all nonsmokers, and did not typically cook their own meals. Median nickel concentrations of 1.02 and $1.71 \mathrm{ng} / \mathrm{m}^{3}$ in air were reported in particulate matter $\left(\mathrm{PM}_{2.5}\right)$ samples collected from indoor air and personal exposure samplers, respectively. In a study of 46 high school students in New York City conducted in the winter and summer of 1999, the concentrations of nickel in collected particulates $\left(\mathrm{PM}_{2.5}\right)$ to which these students were exposed was assessed using personal monitoring devices and stationary measurements of airborne nickel both within and outside the home (Kinney et al. 2002). The mean ( \pm 1 SD) air concentrations of nickel obtained from the outdoor, indoor and personal monitors measured during the winter survey period were similar $\left(32.3 \pm 22.4,31.6 \pm 54.5\right.$, and $49.6 \pm 114 \mathrm{ng} / \mathrm{m}^{3}$, respectively). Likewise, the mean nickel concentrations obtained from all three monitors during the summer survey period were also found to be similar $\left(11.7 \pm 6.3,12.6 \pm 8.4\right.$, and $17.3 \pm 24.7 \mathrm{ng} / \mathrm{m}^{3}$, respectively), although somewhat lower than the winter concentrations. These results suggest that ambient concentrations of nickel are the dominating force in determining both indoor and personal exposures to nickel. 


\subsubsection{Water}

Surface water contains low nickel levels. Uncontaminated freshwater and seawater typically contain about $300 \mathrm{ng} / \mathrm{L}$ of nickel (Barceloux 1999). The concentration in seawater ranges from 100 to 3,000 ng nickel/L. Higher levels are found in deeper waters than in surface water (Mart et al. 1984; Sunderman 1986; van Geen et al. 1988; Yeats 1988). Water from the surface of the Atlantic Ocean, deep within the Atlantic Ocean (400 m), and the Atlantic shelf contained $1.8 \mathrm{nM}(106 \mathrm{ng} / \mathrm{L}), 2.7 \mathrm{nM}(158 \mathrm{ng} / \mathrm{L})$, and $3.5 \mathrm{nM}(205 \mathrm{ng} / \mathrm{L})$ nickel, respectively (van Geen et al. 1988). Nickel concentration in surface water was found to decrease by a factor of approximately 2 with increases in percent salinity from approximately 30 to $36 \%$ and increased with increasing phosphorus concentration (Yeats 1988). Nickel concentrations in South San Francisco Bay were about 3,000 ng/L, with one-third to one-half of the nickel complexed to a class of strong organic ligands (Donat et al. 1994).

The nickel content of fresh surface water has been reported to average between 15 and $20 \mu \mathrm{g}$ nickel/L (Grandjean 1984; NAS 1975). The concentration of dissolved nickel in the lower Mississippi River ranged from 1.2 to $1.5 \mu \mathrm{g} / \mathrm{L}$ in seven samples taken at different flow conditions (Shiller and Boyle 1987). In a 1977-1979 study of representative groundwaters and surface waters throughout New Jersey, in which $>1,000$ wells and 600 surface waters were sampled, the median nickel levels in groundwater and surface water were both $3.0 \mu \mathrm{g} / \mathrm{L}$ (Page 1981). The respective 90 percentile and maximum levels were 11 and $600 \mu \mathrm{g} / \mathrm{L}$ for groundwater and 10 and $45 \mu \mathrm{g} / \mathrm{L}$ for surface water. The nature of the sites with elevated nickel levels was not indicated. However, groundwater polluted with nickel compounds from a nickelplating facility contained as high as $2,500 \mu \mathrm{g} / \mathrm{L}$ (IARC 1990). Nickel concentrations were measured in 30 groundwater samples taken from the South Platte River alluvial aquifer underlying Denver, Colorado (Bruce and McMahon 1996). The samples represented a variety of land-use activities, including commercial, industrial, residential, and agricultural. A median nickel concentration of $3 \mu \mathrm{g} / \mathrm{L}$ was determined, with maximum and minimum concentrations values of 20 and $1 \mu \mathrm{g} / \mathrm{L}$, respectively.

Nickel concentrations from five stations in Lake Huron in 1980 had median and maximum nickel concentrations of 0.54 and $3.8 \mu \mathrm{g} / \mathrm{L}$, respectively (Dolan et al. 1986). In a 1982 survey, nickel concentrations in Hamilton Harbor, Lake Ontario, ranged from $<1$ to $17 \mu \mathrm{g} / \mathrm{L}$, with a median of $6 \mu \mathrm{g} / \mathrm{L}$ (Poulton 1987). The median nickel concentration from an analogous 1980 survey was $4 \mu \mathrm{g} / \mathrm{L}$. Suspended sediment in surface samples (0.2 m) at Hamilton Harbor, Lake Ontario, contained 17-23 ppm nickel; samples from a depth of $20 \mathrm{~m}$ contained $67-87 \mathrm{ppm}$, similar to the $66 \mathrm{ppm}$ of nickel found in bottom sediment samples (Poulton 1987). These findings suggest that resuspension of bottom sediment is 
a major contributor to the suspended sediment at $20 \mathrm{~m}$ depth. In a 1993 survey of heavy metal concentrations in the Great Lakes, average nickel concentrations of 872 and $752 \mathrm{ng} / \mathrm{L}$ were measured in Lakes Erie and Ontario, respectively (Nriagu et al. 1996). Concentrations were highest in near-shore waters due to their proximity to urban centers and polluted river mouths. A decrease in the average concentration of nickel measured in Lake Ontario, from $838 \mathrm{ng} / \mathrm{L}$ measured in May/June to a value of $751 \mathrm{ng} / \mathrm{L}$ obtained in October, indicates that sedimentation of suspended particles results in a fast depletion of nickel during the summer stratification (Nriagu et al. 1996).

Tap water that is used for drinking purposes generally contains nickel at concentrations ranging from 0.55 to $25 \mu \mathrm{g} / \mathrm{L}$ in the United States (FDA 2000; O'Rourke et al. 1999; Thomas et al. 1999). Nickel concentrations in tap water measured in the Total Diet Study 1991-1999 ranged from 0 to $0.025 \mathrm{mg} / \mathrm{kg}$ $(\approx 0-25 \mu \mathrm{g} / \mathrm{L})$ with a mean value of $0.002 \mathrm{mg} / \mathrm{kg}(\approx 2 \mu \mathrm{g} / \mathrm{L})$ (FDA 2000). Analysis of data obtained during 1995-1997 from the National Human Exposure Assessment Study (NHEXAS) yielded median concentrations of nickel in tap water (used as drinking water) of $4.3 \mu \mathrm{g} / \mathrm{L}(10.6 \mu \mathrm{g} / \mathrm{L}, 90 \%$ percentile) in the Arizona study and $4.0 \mu \mathrm{g} / \mathrm{L}(11 \mu \mathrm{g} / \mathrm{L}, 90 \%$ percentile) in the EPA Region 5 (Illinois, Indiana, Michigan, Minnesota, Ohio, and Wisconsin) study (O’Rourke et al. 1999; Thomas et al. 1999). In a 1969-1970 survey of 969 water supplies in the United States representing all water supplies in eight metropolitan areas and one state (2,503 samples), $21.7 \%$ of samples had concentrations $<1 \mu \mathrm{g} / \mathrm{L}, 43.2 \%$ of the samples contained between 1 and $5 \mu \mathrm{g}$ nickel/L, $25.6 \%$ of the samples contained between 6 and $10 \mu \mathrm{g}$ nickel/L, $8.5 \%$ of the samples contained between 11 and $20 \mu \mathrm{g}$ nickel/L, and $1 \%$ had levels $>20 \mu \mathrm{g}$ nickel/L (NAS 1975).

In a national survey of raw, treated, and distributed water from 71 municipalities across Canada, the median nickel concentration in both treated and distributed provincial drinking water were $\leq 0.6-1.3 \mu \mathrm{g} / \mathrm{L}$ for treated water and $1.8 \mu \mathrm{g} / \mathrm{L}$ for distributed water (Meranger et al. 1981). The maximum value was $72.4 \mu \mathrm{g} / \mathrm{L}$ from Sudbury, Ontario. The similarity between median and maximum values for treated and distributed water suggests that nickel is not generally picked up in the distribution system. An exception is in Quebec where the maximum nickel concentration increased from 8.3 to $22.0 \mu \mathrm{g} / \mathrm{L}$ between the treated and distributed water. The median nickel levels in the provincial raw water ranged from $\leq 0.6$ to $2.3 \mu \mathrm{g} / \mathrm{L}$. The maximum levels in tap waters from British Columbia, Prince Edward Island, the Yukon, and Northwest Territories were below the detection limit. The similarity in values between raw and treated water indicates that treatment methods (mainly treatment with lime, alum, or soda ash) did not remove nickel effectively. 
Elevated nickel levels may exist in drinking water as a result of the corrosion of nickel-containing alloys used as valves and other components in the water distribution system as well as from nickel-plated or chromium-nickel-plated faucets. In a Seattle study, mean and maximum nickel levels in standing water were 7.0 and $43 \mu \mathrm{g} / \mathrm{L}$, respectively, compared with 2.0 and $28 \mu \mathrm{g} / \mathrm{L}$ in running water (Ohanian 1986). A similar result was observed in a comparison of the mean ( \pm 1 standard deviation) and $90^{\text {th }}$ percentile concentrations of nickel measured during the NHEXAS EPA Region 5 study in standing tap water of $(9.2[ \pm 21]$ and $16 \mu \mathrm{g} / \mathrm{L})$ and in tap water sampled after the water line had been flushed for 3 minutes (5.3 [ \pm 4.4$]$ and $11 \mu \mathrm{g} / \mathrm{L})$ (Thomas et al. 1999). Even if an individual was to consume only first draw water (containing nickel at the maximum concentration $[48 \mu \mathrm{g} / \mathrm{L}]$ obtained from the Seattle study) as their sole source of drinking water, their daily intake of $96 \mu \mathrm{g} / \mathrm{day}$ is still less than the lifetime daily limit of 1,400 $\mu \mathrm{g} /$ day (see Table 8-1) set by EPA, assuming a drinking water equivalent level (DWEL) of $700 \mu \mathrm{g} / \mathrm{L}$ and a consumption of $2 \mathrm{~L} /$ day (EPA 2000). Although leaching of metals from pipes generally increases with decreasing $\mathrm{pH}$, none of the nickel studies reported the $\mathrm{pH}$ of the tap water. First water drawn from hot water taps plated with nickel may contain concentrations as high as $1-1.3 \mathrm{mg} / \mathrm{L}$ (Barceloux 1999).

Nickel concentrations were measured as part of a study of heavy metal content in streams and creeks, located in the Black Hills of South Dakota that are impacted by abandoned or active mining operations (May et al. 2001). The concentrations of nickel in these surface waters generally ranged between 1.3 and $7.6 \mu \mathrm{g} / \mathrm{L}$ and were typically highest near where they received drainage water from abandoned or active mining operations. At one location, nickel concentrations as high as $20 \mu \mathrm{g} / \mathrm{L}$ were determined and were attributed to effluent and entrained streambed tailings from previous mining activities. The concentrations of nickel in water did not correlate with the concentrations of nickel in the underlying sediments.

Several investigators reported the presence of nickel concentrations in rain. The annual mean nickel concentration in precipitation at Lewes, Delaware, was $0.79 \mu \mathrm{g} / \mathrm{L}$ (Barrie et al. 1987). The mean concentration ( \pm standard deviation) of nickel collected from rain showers in southern Ontario, Canada, in 1982 was $0.56 \pm 0.07 \mu \mathrm{g} / \mathrm{L}$ (Chan et al. 1986). The mean concentrations in northern and central Ontario were both $0.61 \mu \mathrm{g} / \mathrm{L}$, indicating a lack of spatial variability. Sudbury, the site of a large nickel smelter, is located in central Ontario. The nickel concentration in rainwater collected near a large municipal incinerator in Claremont, New Hampshire, was measured at a mean value of $0.69 \mu \mathrm{g} / \mathrm{L}$ (Feng et al. 2000). Nickel concentrations in rain collected between 1985 and 1990 from remote regions of the Atlantic Ocean ranged from 0.63 to $1.42 \mu \mathrm{g} / \mathrm{L}$ (Helmers and Schrems 1995). The concentration of nickel in cloud water 
sampled on the Olympic Peninsula of Washington State in May 1993 was measured at $0.5 \pm 0.4 \mu \mathrm{g} / \mathrm{L}$; the air-equivalent concentration is $0.2 \mathrm{ng} / \mathrm{m}^{3}$ (Vong et al. 1997).

Nickel in snow from Montreal, Canada, was highly enriched compared with ambient air, ranging from 2 to $300 \mathrm{ppb}$ (Landsberger et al. 1983). The nickel content of snow particulate matter was 100-500 ppb. Nickel concentrations were highly correlated with those of vanadium, suggesting that oil combustion was a source. The nickel concentration in snow collected near a large municipal incinerator in Claremont, New Hampshire, was measured at a mean value of $0.62 \mu \mathrm{g} / \mathrm{L}$ (Feng et al. 2000). Snow samples were collected several hundred kilometers from the nearest known nickel emission sources (e.g., smelters and ore processing facilities) in northwestern Russia, near the Finish and Norwegian borders. Mean nickel concentrations of $0.0019 \mathrm{mg} / \mathrm{L}(1.9 \mu \mathrm{g} / \mathrm{L})$ were measured in the snow melt or, based on the volume of accumulated snow, $0.26 \mathrm{mg} / \mathrm{m}^{3}$ (Kashulin et al. 1997).

\subsubsection{Sediment and Soil}

Sediment is an important sink for nickel in water. Mean nickel levels in pristine sediment from five sites off the northern coast of Alaska ranged from 25 to 31 ppm (Sweeney and Naidu 1989). Of this amount, $\approx 10 \%$ was extractable. Nickel was most highly associated with silt and clay. Background nickel concentrations in sediment cores from open water of Lake St. Clair ranged from 8.5 to $21.1 \mathrm{ppm}$, with mean concentrations of 13.6 and 17.6 ppm in sand and silty clay sediment, respectively (Rossmann 1988). The average nickel concentrations in surface sediment of four Rocky Mountain lakes ranged from 9.6 to $18 \mathrm{ppm}$ (dry weight). The nickel concentrations of the five other lakes reported in the literature ranged from 6.4 to 38 ppm (Heit et al. 1984). Nickel concentrations measured in the sediments taken in 1998 from the Clark Fork-Pend Oreille and Spokane River Basins in the region adjoined by the states of Washington, Idaho, and Montana ranged from 12 to $27 \mu \mathrm{g} / \mathrm{g}$, dry weight (USGS 2000a).

The range and mean nickel levels in surface sediment of Penobscot Bay, Maine, were 8.22-35.0 and $26.6 \mathrm{ppm}$ (dry weight), respectively (Larsen et al. 1983). This is higher than the levels found at cleaner sites in Casco Bay in the Gulf of Maine (17.6 ppm) and Eastern Long Island (7.6 ppm) (Larsen et al. 1983). As part of the Long Island-New Jersey National Water-Quality Assessment (LINJ-NAWQA) Program, nickel concentrations were measured in bed-sediments taken from streams and rivers in New Jersey in the fall of 1997 (USGS 2000b). A median nickel concentration of $30 \mu \mathrm{g} / \mathrm{g}$ (wet weight) was determined in bed-sediments, with values ranging from 18 to $43 \mu \mathrm{g} / \mathrm{g}$. In a similar NAWQA study of the Northern Rockies Intermontane Basins study area, a median nickel concentration in bed-sediments of 
$18 \mu \mathrm{g} / \mathrm{g}$ (wet weight) was determined, with a range of values of 12-24 $\mu \mathrm{g} / \mathrm{g}$ (USGS 2000a). Rice (1999) gives a summary of trace metal concentrations in 541 bed-sediment samples taken from throughout the conterminous United States as part of the NAWQA study, reporting a median nickel concentration of $27 \mu \mathrm{g} / \mathrm{g}$, with a larger range of values 6-530 $\mu \mathrm{g} / \mathrm{g}$ than found from the results of the separate NAWQA studies noted above. Nickel is more highly associated with fine-grained sediment with a higher organic carbon content. Levels reflect anthropogenic input as well as mineralization of the regional bedrock.

Nickel content in sediments is expected to be high near sources of nickel emissions. For example, nickel carried into creeks and streams from drainage and runoff originating from active or abandoned mining operations in the Black Hills of South Dakota can lead to increased concentrations of this metal in sediments (May et al. 2001). Nickel concentrations varied between 10 and $64 \mu \mathrm{g} / \mathrm{g}$, dry weight, depending on proximity to nearby mines.

Nickel occurs naturally in the Earth's crust with an average concentration of $0.0086 \%$ (86 ppm) (Duke 1980a). The nickel content of soil may vary depending on local geology. A nickel content of $0.5 \%$ $(5,000 \mathrm{ppm})$ is common in podzol soil in southeastern United States, and nickel concentrations of $>1,000 \mathrm{ppm}$ are not unusual in glacial till in southern Quebec. Both areas are underlaid with ultramafic rock, which is rich in nickel. Typical nickel levels reported in soil range from 4 to $80 \mathrm{ppm}$. In a region north of Sydney, Australia, nickel concentrations as high as 2,030 ppm have been reported in ferrosol topsoils, which are naturally-enriched in nickel through the weathering of underlying haematite, magnetite, quartz, and kaolinite minerals (Lottermoser 2002). A soil survey by the U.S. Geological Survey throughout the United States reported that nickel concentrations ranged from $<5$ to $700 \mathrm{ppm}$, with a geometric mean of $13 \pm 2.31 \mathrm{ppm}$, ranking 15th among the 50 elements surveyed (Shacklette and Boerngen 1984). In this survey, samples were taken at $20 \mathrm{~cm}$ from 1,318 sampling sites. Cultivated soils contained 5-500 ppm of nickel, with a typical concentration of $50 \mathrm{ppm}$ (Bennett 1984). Nickel concentrations in Canadian soils were generally 5-50 ppm (Webber and Shamess 1987). An extensive survey in England and Wales reported that nickel concentrations typically ranged from 4 to $80 \mathrm{ppm}$, with a median value of $26 \mathrm{ppm}$ (Bennett 1984). The average farm soil in the United States contained >30 ppm nickel (NAS 1975). Mean nickel concentrations in the forest floor from samples collected from 78 sites in nine northeastern states averaged $11 \pm 0.8$ ppm (Friedland et al. 1986).

Nickel concentrations in contaminated soils within $\approx 8 \mathrm{~km}$ of the large nickel smelter at Sudbury, Ontario, ranged from 80 to 5,100 ppm (Duke 1980b). A study of wetland soil-sediment in Sudbury found 9,372 and 5,518 ppm of nickel at sites located 2.0 and $3.1 \mathrm{~km}$ from the smelter, respectively (Taylor and 
Crowder 1983). Nickel concentrations declined logarithmically with increasing distance from the smelter. This indicates that nickel accumulations result from atmospheric deposition and soil runoff (Taylor and Crowder 1983). In a more recent survey of nickel content in soils in the Sudbury region, soil samples were taken within $5 \mathrm{~km}$ of each of the three smelters located in the area, Copper Cliff, Coniston, and Falconbridge (Adamo et al. 1996). Mean total concentrations of nickel in soil (based on dry weight) of 580,286 , and $210 \mathrm{mg} / \mathrm{kg}$ were obtained for the three sites, respectively. Concentration ranges were 80-2149, 156-628, and 23-475 mg/kg for the Copper Cliff, Coniston and Falconbridge sites, respectively.

The concentrations of nickel in soils near another smelting operation have been investigated. At the Pechenganikel Smelter in northwestern Russia, near the border with Norway, deposition rates and soil concentrations of nickel were measured in sampling sites either near the facility or up to $41 \mathrm{~km}$ south or southeast of the facility (Koptsik et al. 2003). Nickel deposition rates of $>1.75,0.7,0.26$, and $0.05 \mathrm{mmoles}$ nickel $/ \mathrm{m}^{2} /$ year (>103, 41,15 , and $3 \mathrm{mg} / \mathrm{m}^{2} /$ year, respectively) were determined at distances of $1,8,16$, and $41 \mathrm{~km}$, respectively, from the smelter. Consistent with the decrease in deposition rates, the concentrations of nickel in soils were also found to decrease from 30 mmoles $/ \mathrm{kg}(1,760 \mathrm{mg} / \mathrm{kg}) \mathrm{within}$ $1 \mathrm{~km}$ of the facility to 9.6 mmoles $/ \mathrm{kg}(560 \mathrm{mg} / \mathrm{kg})$ at $8 \mathrm{~km}, 6.5 \mathrm{mmoles} / \mathrm{kg}(380 \mathrm{mg} / \mathrm{kg})$ at $16 \mathrm{~km}$, and $1.0-1.2 \mathrm{mmoles} / \mathrm{kg}(59-7 \mathrm{mg} / \mathrm{kg})$ at $41 \mathrm{~km}$. The nickel concentrations in soils taken from the farthest sampling site $(41 \mathrm{~km})$ are similar to the background concentrations of nickel in soils within the regions that are not influenced by the deposition of nickel from the smelter.

Soils from the Idaho National Engineering Laboratory (INEL) and two background sites in southern Idaho had geometric mean nickel concentrations of 11.8-23.4 ppm dry weight; concentrations are significantly higher near INEL (Rope et al. 1988). The coal-fired steam plant that was constructed at the laboratory in 1982-1983 may be responsible for the higher nickel concentrations.

Nickel concentrations in 57 sludge-treated soils in an agricultural area of Ontario, Canada, ranged from 6.2 to $34 \mathrm{ppm}$ (dry weight), with a mean of $20 \mathrm{ppm}$ (Webber and Shamess 1987). For 252 untreated soils, the range and mean values were 4.0-48 and $16.2 \mathrm{ppm}$, respectively. The mean for sludge-treated soil was significantly elevated. 


\subsubsection{Other Environmental Media}

There have been several studies regarding nickel content in an average diet. Current information on the dietary intake of nickel in the United States is based on data gathered from the NHEXAS study. Nickel concentrations were measured in duplicate diet samples which, in combination with study participant's estimates of food and water intake, were used to determine both the overall concentration of nickel in combined solids and liquids in the total diet and the average nickel intake of study participants. In the EPA Region 5 (Illinois, Indiana, Michigan, Minnesota, Ohio, and Wisconsin) study, the mean and median concentrations of nickel in combined dietary solids and liquids were 47 and $43 \mu \mathrm{g} / \mathrm{kg}$, respectively (Thomas et al. 1999).

In other studies of nickel in the diet, Myron et al. (1978) analyzed nine institutional diets consisting of three meals each. Four of these meals were sampled from the student center at the University of North Dakota. The remaining five diets were special diets from a rehabilitation hospital. The average nickel concentration and nickel content of the student meals ranged from 0.19 to $0.29 \mathrm{ppm}$ and from 140 to $221 \mu \mathrm{g}$, respectively. For the hospital meals, the nickel concentration ranged from $0.21 \mathrm{ppm}(107 \mu \mathrm{g})$ in the puree meals to $0.41 \mathrm{ppm}(176 \mu \mathrm{g})$ in the low-calorie meal. Breakfast had the lowest nickel content. The average daily dietary nickel intake for the nine diets was $168 \pm 11 \mu \mathrm{g}$. The average nickel concentration in the food was $0.27 \mathrm{ppm}$ (dry weight). These results are comparable with estimated daily intakes of nickel of $150 \mu \mathrm{g}$ in Denmark, 73-142 $\mu \mathrm{g}$ in Switzerland, and 140-150 $\mu \mathrm{g}$ in the United Kingdom (IARC 1990; Nielsen and Flyvholm 1984). A 1962 study that used the nickel content of individual foods to estimate average dietary nickel intake reported intakes of 300-600 $\mu \mathrm{g}$, which are much higher than those reported above (Grandjean 1984).

The foods with the highest mean nickel content were oatmeal, spinach, asparagus, and peas (IARC 1990). Nuts and cocoa may have nickel levels as high as 3 and 10 ppm, respectively. In a market basket survey completed in the United States (Pennington and Jones 1987), the highest average levels of nickel in $\mu \mathrm{g} / 100 \mathrm{~g}$ were found in nuts (128.2), legumes (55), sweeteners (31.6), grains and grain products (26.2), and mixed dishes and soups (25.3). From data gathered in the FDA Total Diet Study 1991-1996, the mean and median nickel concentrations in the food items that were surveyed were 0.136 and $0.057 \mathrm{mg} / \mathrm{kg}$, respectively (Capar and Cunningham 2000). The highest concentrations of nickel were found in mixed nuts $(3.04 \mathrm{mg} / \mathrm{kg})$, oat ring cereal $(2.32 \mathrm{mg} / \mathrm{kg})$, chocolate syrup $(1.04 \mathrm{mg} / \mathrm{kg})$, granola cereal $(1.01 \mathrm{mg} / \mathrm{kg})$, and peanuts $(0.956 \mathrm{mg} / \mathrm{kg})$. Other products with notable nickel concentrations are legumes and nuts $(0.368-3.04 \mathrm{mg} / \mathrm{kg})$, cereals containing largely whole wheat, corn, oats, or rice $(0.216-$ 
$2.32 \mathrm{mg} / \mathrm{kg})$, chocolate products $(0.19-1.04 \mathrm{mg} / \mathrm{kg})$, and canned peaches and pineapple juice $(0.408-$ $0.668 \mathrm{mg} / \mathrm{kg}$ ). In an analysis of trace metals in tissue samples taken from livestock and poultry, mean nickel concentrations were $0.23-0.82 \mathrm{ppm}$ in muscle, $0.23-0.29 \mathrm{ppm}$ in liver, and $0.28-0.57 \mathrm{ppm}$ in kidneys (Coleman et al. 1992).

A Canadian survey of nickel in infant formulas gave a median value of $3.53 \mu \mathrm{g} / \mathrm{L}$ for evaporated milk (Dabeka 1989). Different types of milk-based formulas contained from 5.8 to $28.9 \mu \mathrm{g}$ nickel/L (Dabeka 1989). All concentrations are on a ready-to-use basis. Formulas fortified with iron had a higher nickel content. The median nickel content of soy-based formula ranged from 31.2 to $187 \mu \mathrm{g}$ nickel/L. The average daily dietary intake of nickel by infants between the ages of 0 and 12 months could vary from $35.7 \mu \mathrm{g}(4.5 \mu \mathrm{g} / \mathrm{kg})$ (if evaporated milk was fed) to $74.7 \mu \mathrm{g}(10.2 \mu \mathrm{g} / \mathrm{kg}$ ) (if concentrated liquid soy-based formula was used). Infant formula (base not stated) in the United States contained an average of $0.2 \mu \mathrm{g}$ nickel/100 g (Pennington and Jones 1987).

There is limited evidence that stainless steel pots and utensils may release nickel into acid solution (IARC 1990). Six stainless steel pots of different origins were tested to see whether they would release nickel by boiling $350 \mathrm{~mL}$ of $5 \%$ acetic acid in them for 5 minutes (Kuligowski and Halperin 1992). The resulting concentrations of nickel ranged from 0.01 to $0.21 \mathrm{ppm}$. Cooking acidic fruits in new stainless steel pans resulted in an increase of nickel that was about one-fifth the average daily nickel intake (Flint and Packirisamy 1995). Further use of the pans did not result in any release of nickel into the food. The use of nickel-containing catalysts in the hydrogenation of food fats may contribute to elevated nickel levels in food (Mastromatteo 1986). Grain milling may also lead to higher nickel levels (IARC 1990). The results from a recent study that attempted to identify the influence of the container on the trace metal content of preserved pork products showed no clear evidence that the metal container contributed to the metal content of the food (Brito et al. 1990). The nickel concentration was highest in products in china and glass containers, rather than those in metal and plastic containers.

The nickel content of cigarettes is $1-3 \mu \mathrm{g} ; \approx 10-20 \%$ of this nickel is released in mainstream smoke (Sunderman 1986). This indicates that 2-12 $\mu \mathrm{g}$ of nickel are inhaled for each pack of cigarettes smoked. Torjussen et al. (2003) reported a mean nickel concentration of $0.03 \mu \mathrm{g} / \mathrm{g}$ in smoke condensate isolated from the control combustion of five packets of cigarettes representing five U.S. brands. This compares to mean concentrations of nickel of 1.72 and $1.57 \mu \mathrm{g} / \mathrm{g}$ in the tobacco and ash, respectively. Most of the nickel is in the gaseous phase, but the chemical form of the nickel is unknown (IARC 1990). 
In a comprehensive survey of heavy metals in sewage sludge, 31 sludges from 23 American cities were analyzed by electrothermal atomic absorption spectroscopy (AAS) (Mumma et al. 1984). The nickel concentration in the sludges ranged from 29.0 to $800 \mathrm{ppm}$ (dry weight) and had a median value of 195 ppm. The highest concentration of nickel in sludge was in Detroit, Michigan. For comparison, the concentration of nickel in cow manure was $28.0 \mathrm{ppm}$. In another study of heavy metal in sludges generated at waste water treatment plants in 16 large U.S. cities, nickel concentrations (dry weight) were found to range from 18 to $186 \mathrm{ppm}$, with a median value of $66.8 \mathrm{ppm}$ (Gutemann et al. 1994).

Nickel was detected in a large number of the 283 point samples taken from leachate collected from 48 codisposal, hazardous, or municipal solid waste (MSW) sites (Gibbons et al. 1999). Codisposal sites were defined as those facilities accepting municipal wastes and relatively large volumes of industrial sludges, liquids, and solids. Dissolved nickel was detected in 43 of 45 codisposal sites, all 48 old (accepting waste before 1986) hazardous waste sites, all 29 old (accepting waste before 1984) MSW sites, and 1 of 1 new (accepting waste after 1984) MSW site. Solid nickel was detected in 105 of 111 codisposal sites, 108 of 126 old (accepting waste before 1986) hazardous waste sites, 108 of 116 old (accepting waste before 1984) MSW sites, and 36 of 43 new (accepting waste after 1984) MSW sites.

Mosses are used to monitor atmospheric deposition of metals. The metals measured in mosses are obtained only from the passive uptake of metals that are deposited onto the organism from the air. In a study of heavy metal concentrations measured mainly in Pleurozium schreberi taken from 16 regions in West Germany and the former German Democratic Republic (GDR), a decrease in the mean nickel concentration occurred in moss samples taken in 1990-1991 $(2.3 \mu \mathrm{g} / \mathrm{g})$ and 1995-1996 (1.63 $\mu \mathrm{g} / \mathrm{g})$ (Herpin et al. 2004). This decrease follows a decrease in the total airborne emissions of nickel in Germany from 277 to 159 tons/year.

\subsection{GENERAL POPULATION AND OCCUPATIONAL EXPOSURE}

Nickel occurs naturally in the Earth's crust, and the general population will be exposed to low levels of nickel in ambient air, water, and food. Based on several dietary studies, the average daily dietary intake of nickel in food ranges between 69 and $162 \mu \mathrm{g} /$ day (NAS 2002; O'Rourke et al. 1999; Pennington and Jones 1987; Thomas et al. 1999). In a more recent total dietary study (NAS 2002), the mean daily dietary intake of nickel ranged from 101 to $162 \mu \mathrm{g}$ /day for individuals > 18 years of age with males ranging from 136 to $140 \mu \mathrm{g} /$ day and females ranging from 107 to $109 \mu \mathrm{g} /$ day. Pregnant females averaged a daily dietary intake of $121 \mu \mathrm{g} /$ day, whereas lactating females averaged $162 \mu \mathrm{g} / \mathrm{day}$. Also, based on data 
obtained from the National Health and Examination Survey III (NHANES III), it has been estimated that approximately $5 \mu \mathrm{g} /$ day of the daily dietary intake of nickel comes from dietary supplements (NAS 2002). The daily intake from drinking water is $\approx 8 \mu \mathrm{g}$, assuming a median nickel concentration of $\approx 4 \mu \mathrm{g} / \mathrm{L}$ and a consumption rate of $2 \mathrm{~L}$ water/day. For the highest municipal level in drinking water, which is $68 \mu \mathrm{g} / \mathrm{L}$ in Sudbury, Ontario, the average daily intake of nickel would be $140 \mu \mathrm{g}$. Assuming that a person inhales $20 \mathrm{~m}^{3}$ air/day and the average nickel concentration in ambient air in the United States is $2.22 \mathrm{ng} / \mathrm{m}^{3}$, the average nickel intake by inhalation would be $0.0444 \mu \mathrm{g} / \mathrm{day}$. Based on the highest ambient nickel levels reported for the Copper Cliff $\left(6,100 \mathrm{ng} / \mathrm{m}^{3}\right)$ and Sudbury basin region $\left(732 \mathrm{ng} / \mathrm{m}^{3}\right)$ in Ontario (CEPA 1994), the daily inhalation intake for individual living in these areas could have been as high as 122 and $15 \mu \mathrm{g} /$ day, respectively. However, based on the mean ambient nickel concentrations measured in Sudbury area of $21 \mathrm{ng} / \mathrm{m}^{3}$ (CEPA 1994) the daily nickel the average daily nickel intake is estimated to be $0.42 \mu \mathrm{g} / \mathrm{day}$. The nickel intake via inhalation is, therefore, a minor source of nickel to the general, nonsmoking population.

A market basket survey in England completed in 1984 estimated a dietary intake of 154-166 $\mu \mathrm{g} /$ day or 2.2-2.4 $\mu \mathrm{g} / \mathrm{kg} /$ day for a 70-kg person (Smart and Sherlock 1987). Dietary intake of nickel in the United States has been estimated to range from $69 \mu \mathrm{g} / \mathrm{day}$ for 6-11-month-old infants to $162 \mu \mathrm{g} / \mathrm{day}$ for teenage boys, with a level of $146.2 \mu \mathrm{g} / \mathrm{day}$ or $2 \mu \mathrm{g} / \mathrm{kg} /$ day for a $25-30$-year-old male weighing $70 \mathrm{~kg}$ (Pennington and Jones 1987). More recent data on nickel intakes from the U.S. diet come from the results of the NHEXAS studies. Mean and median dietary intakes of nickel for study participants in the EPA Region 5 study were calculated to be 2.2 and $1.4 \mu \mathrm{g} / \mathrm{kg}$ body weight/day, respectively, or 154 and $98 \mu \mathrm{g} /$ day for a 70-kg adult, respectively (Thomas et al. 1999). O'Rourke et al. (1999) have taken the dietary nickel data obtained from the Arizona study and determined the dietary nickel intake for various subpopulations (Table 6-3). The mean daily nickel intake for all subjects was $153 \mu \mathrm{g} / \mathrm{day}$, with the highest mean intake for an adult male $(163 \mu \mathrm{g} /$ day $)$ and lowest intake for children $(125 \mu \mathrm{g} /$ day $)$. Hispanic study participants were found to have a lower mean dietary intake $(141 \mu \mathrm{g} / \mathrm{day})$ than non-Hispanic participants $(155 \mu \mathrm{g} /$ day). Total nickel intake for Canadians in the general population has been estimated to range from 4.4 to $22.1 \mu \mathrm{g} / \mathrm{kg} / \mathrm{day}$, with greater intake estimated for infants than for adults (CEPA 1994). Food, from which nickel is poorly absorbed, accounted for most of the intake (4.4-22 $\mu \mathrm{g} / \mathrm{kg} /$ day). It was estimated that cigarette smoking may increase total daily intake by $0.12-0.15 \mu \mathrm{g} / \mathrm{kg} / \mathrm{day}$. Living in the vicinity of Sudbury, Ontario, where large nickel deposits are found, water intake of nickel for individuals aged 12 years old or older ranged from $0.6-2.5 \mu \mathrm{g} / \mathrm{kg} /$ day. However, the estimated water intake of nickel increased with decreasing age of the study group, for example $0.87-3.6 \mu \mathrm{g} / \mathrm{kg} / \mathrm{day}$ for children ages 511 years old to $2.8-12 \mu \mathrm{g} / \mathrm{kg} /$ day for newborns and infants under the age of 0.5 years. 
Table 6-3. Total Dietary Exposure Estimates of Study Participants to Nickel Based on the Dietary Information Obtained from the NHEXAS Arizona Study ${ }^{\mathrm{a}}$

\begin{tabular}{lcccc}
\hline \multirow{2}{*}{ Exposure population } & \multicolumn{2}{c}{$\begin{array}{c}\text { Number of participants } \\
\text { evaluated }\end{array}$} & \multicolumn{3}{l}{ Daily nickel intake $(\mu \mathrm{g})$} \\
\cline { 3 - 5 } & 176 & 153 & 135 & $27-562$ \\
\hline All subjects & 55 & 163 & 145 & $38-372$ \\
Adult male (>18 years of age) & 86 & 157 & 135 & $23-563$ \\
Adult female (>18 years of age) & 35 & 125 & 107 & $31-343$ \\
Children (<18 years of age) $)^{\mathrm{b}}$ & 54 & 141 & 134 & $27-401$ \\
Hispanic & 119 & 155 & 132 & $42-563$ \\
Non-Hispanic & & & & \\
\hline
\end{tabular}

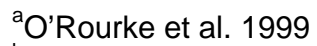

${ }^{\mathrm{b}}$ Either gender 
In another approach to determining daily nickel intake within subpopulations in the United States, Moschandreas et al. (2002) used the Dietary Exposure Potential Model (DEPM) and data obtained from Combined National Residue Database (CNRD) to estimate dietary nickel intake based on food consumption patterns in subpopulations and nickel content in specific food items. The food items used in the model are based on 11 food groups consisting of approximately 800 exposure core foods that represent 6,500 common food items. The results of their model (Table 6-4) yielded an average dietary nickel intake in the U.S. population of $0.374 \mu \mathrm{g} / \mathrm{kg}$ body weight/day, or $26.2 \mu \mathrm{g} / \mathrm{day}$ for a $70-\mathrm{kg}$ adult. Their results also indicate that children under the age of 7 have a nickel intake that is at least 1.8 times higher than the average for the overall population. However, the estimates obtained for dietary nickel intake from the DEPM model are lower than the daily nickel intakes determined from the NHEXAS study (Table 6-3). Moschandreas et al. (2002) attribute these differences in intake values for nickel to differences in study populations, methods for assigning values to measurements that are below the level of detection, and potential errors in recording portion sizes in the NHEXAS study.

The general population is also exposed to nickel in nickel alloys and nickel-plated materials including steel, coins, and jewelry (Barceloux 1999). Jewelry and other items made of silver may either contain, or be coated with, nickel to reduce oxidation. White gold contains 10-15\% nickel and some gold-plated items may have a nickel undercoating. Residual nickel may be present in soaps, fats, and oils hydrogenated with nickel catalysts (Sunderman 1986). In U.S. coinage, the 5-cent coin is composed of a cupronickel alloy containing $25 \%$ nickel and $75 \%$ copper, whereas only the faces of the 10 -cent, quarterdollar, half-dollar, and 1 dollar coins contain the cupronickel alloy (United States Treasury 2004). Studies of European coins, especially the recently introduced Euro coinage, show the transfer of nickel from coins to hands (Fournier and Govers 2003). For example, as part of a 58-coin counting exercise, it was determined that an average of $0.23 \mu \mathrm{g}$ of nickel was transferred to three fingers during a 2-3-second manipulation of an unwashed Euro coin that had been in circulation for 2-5 months.

A NOES survey conducted by NIOSH from 1981 to 1983 estimated that 727,240 workers are potentially exposed to nickel metal, alloys, dust, fumes, salts, or inorganic nickel compounds in the United States (NIOSH 1990). Seventy percent of these estimated exposures are to nickel of an unknown molecular formula. The numbers of workers estimated to be exposed to nickel chloride, nickel oxide, and nickel sulfate are $48,717,18,166$, and 56,844 , respectively. The estimate is provisional because all of the data for trade name products that may contain nickel have not been analyzed. The NOES was based on field surveys of 4,490 businesses employing nearly 1.8 million workers and was designed as a nationwide survey based on a statistical sample of virtually all workplace environments in the United States where 
Table 6-4. Dietary Exposure Estimates of U.S. Populations to Nickel Based on the Dietary Exposure Potential Model (DEPM) ${ }^{a}$

\begin{tabular}{lc}
\hline Subpopulation & Nickel intake $(\mu \mathrm{g} / \mathrm{kg}$ body weight/day $)$ \\
\hline U.S. population & 0.374 \\
Age/gender & $0.870^{\mathrm{b}}$ \\
Nonnursing infants & 0.669 \\
Children 1-6 & 0.425 \\
Children 7-12 & 0.281 \\
Females 13-19 & 0.350 \\
Females 20+ & 0.368 \\
Females 55+ & 0.324 \\
Males 13-19 & 0.342 \\
Males 20+ & 0.369 \\
Males 55+ & \\
Ethnicity & 0.407 \\
Hispanic & $0.424^{\mathrm{b}}$ \\
Non-Hispanic white & 0.295 \\
Non-Hispanic black & 0.258 \\
Non-Hispanic other & \\
Geographic region & \\
North central & 0.238 \\
Northeast & 0.379 \\
Southern & 0.359 \\
Western & $0.423^{\mathrm{b}}$ \\
Family income & \\
Poverty 0-130\% & $0.420^{\mathrm{b}}$ \\
Poverty 131\%+ & 0.362 \\
\hline
\end{tabular}

${ }^{\mathrm{a}}$ Moschandreas et al. 2002

${ }^{b}$ Values indicate the maximum exposure to nickel for each subpopulation group.

${ }^{\mathrm{C}}$ The regional classification is as defined by the U.S. Department of Agriculture, and is based upon U.S. Census Bureau regions.

${ }^{\mathrm{d}}$ Annual household income as a percentage of the Poverty Index. 
eight or more persons are employed in all standard industrial codes except mining or agriculture.

Industries with especially large numbers of potentially exposed workers include the following: plumbing, heating, air conditioning, pressed and blown glass, steel, plating and polishing, aircraft, shipbuilding, railroad, control and measuring instruments, and repair services (NIOSH 1990).

Occupational exposure to nickel will be highest for those involved in production, processing, and use of nickel. There are currently no people in the United States employed in nickel mines, smelters, and refineries at the end of 2001. Primary nickel production in the United States ceased for several years in the late 1980s (Kirk 1988a). The mining and smelting operation in Riddle, Oregon, was reactivated in 1989-1990, and was decommissioned in 2001 (Kuck 2002). The concentration range of airborne nickel that workers were exposed to in laterite mining and smelting in Riddle, Oregon, was 4-420 $\mu \mathrm{g} \mathrm{nickel} / \mathrm{m}^{3}$ (Warner 1984). The ranges of airborne nickel concentrations reported for other industries are as follows: stainless steel production, $<1-189 \mu \mathrm{g} / \mathrm{m}^{3}$; high nickel alloy production, $1-4.4 \mu \mathrm{g} / \mathrm{m}^{3}$; foundry operations, from not detectable to $900 \mu \mathrm{g} / \mathrm{m}^{3}$; electroplating, $<2-<16 \mu \mathrm{g} / \mathrm{m}^{3}$; nickel-cadmium battery manufacture, $20-1,910 \mu \mathrm{g} / \mathrm{m}^{3}$; nickel catalyst production from nickel sulfate, $1-1,240 \mu \mathrm{g} / \mathrm{m}^{3}$; production of nickel salts from nickel or nickel oxide, $9-590 \mu \mathrm{g} / \mathrm{m}^{3}$; and production of wrought nickel and alloys from metal powder, 1-60,000 $\mu \mathrm{g} / \mathrm{m}^{3}$ (Anttila et al. 1998; Haber et al. 2000; Magari et al. 2002; Seilkop and Oller 2003; Warner 1984). The average nickel concentration for selected work areas or operations in these industries, other than producing wrought nickel and alloys from metal powder, is $\left\langle 3-378 \mu \mathrm{g} / \mathrm{m}^{3}\right.$; for wrought nickel and alloy production from metal powder, the average concentration is $1,500 \mu \mathrm{g} / \mathrm{m}^{3}$.

Operations that produce the highest levels of airborne nickel are those that involve grinding, welding, and handling powders. In a survey of 20 individuals involved in the welding or grinding of stainless steel components, nickel concentrations of $0.340-10.129 \mathrm{mg} / \mathrm{m}^{3}$ were measured in the workplace atmosphere (Borská et al. 2003). Not only do occupational exposures vary widely among these operations and industries, but also the form of nickel that workers are exposed to varies as is exemplified in Table 6-5. In another example, Berge and Skyberg (2003) determined the exposure factors and species of nickel for 1,046 nickel refinery workers. Mean exposures factors (range of values) of $5.59 \mathrm{mg} / \mathrm{m}^{3} / \mathrm{year}(0$ $101.38 \mathrm{mg} / \mathrm{m}^{3} /$ year), $1.43 \mathrm{mg} / \mathrm{m}^{3} /$ year (0-24.27 mg/m $/ 3 /$ year), $0.55 \mathrm{mg} / \mathrm{m}^{3} /$ year $\left(0-15.21 \mathrm{mg} / \mathrm{m}^{3} /\right.$ year $)$, $0.52 \mathrm{mg} / \mathrm{m}^{3} /$ year $\left(0-14.61 \mathrm{mg} / \mathrm{m}^{3} /\right.$ year $)$, and $3.09 \mathrm{mg} / \mathrm{m}^{3} /$ year $\left(0-72.99 \mathrm{mg} / \mathrm{m}^{3} /\right.$ year $)$ were determined for total nickel, soluble nickel, sulfidic nickel, metallic nickel and oxidic nickel, respectively. Because sulfur has a deleterious effect on many metals and alloys, nickel sulfate and sulfidic nickel compounds are generally not found in metallurgical workplaces (Warner 1984). Nickel subsulfide is known to exist in only one application in nickel-using industries, namely in certain spent catalysts. Nickel oxide is used as 
Table 6-5. Nickel Levels in Air and Distribution of Different Forms of Nickel as a Proportion (by Weight) of Total Nickel in Selected Departments and Time Periods at a Nickel Refinery in Norway ${ }^{a}$

\begin{tabular}{|c|c|c|c|c|c|}
\hline \multirow{2}{*}{$\begin{array}{l}\text { Department and } \\
\text { period }\end{array}$} & \multirow{2}{*}{$\begin{array}{l}\text { Total nickel in air } \\
\left(\mathrm{mg} / \mathrm{m}^{3}\right)\end{array}$} & \multicolumn{4}{|c|}{ Proportion of total nickel } \\
\hline & & \multicolumn{2}{|c|}{ Soluble nickel Sulfidic nickel } & \multicolumn{2}{|c|}{ Metallic nickel Oxidic nickel } \\
\hline \multicolumn{6}{|c|}{ Crushing and grinding } \\
\hline 1990-1994 & $0.7-1.4$ & 0.12 & 0.72 & 0.11 & 0.04 \\
\hline \multicolumn{6}{|l|}{ Smelter } \\
\hline 1910-1929 & 4.0 & 0.10 & 0.05 & 0.01 & 0.84 \\
\hline 1930-1950 & 4.0 & 0.10 & 0.05 & 0.08 & 0.77 \\
\hline 1951-1977 & $2.6-4.4$ & 0.10 & 0.04 & 0.18 & 0.68 \\
\hline \multicolumn{6}{|l|}{ Calcining, smelting } \\
\hline 1951-1977 & $1.5-3.4$ & 0.10 & 0.05 & 0.01 & 0.84 \\
\hline 1978-1994 & 0.5 & 0.12 & 0.13 & 0.01 & 0.74 \\
\hline \multicolumn{6}{|l|}{ Roasting } \\
\hline 1910-1977 & $1.9-5.3$ & 0.10 & 0.15 & 0.03 & 0.72 \\
\hline 1978-1994 & 0.4 & 0.15 & 0.05 & 0.00 & 0.80 \\
\hline \multicolumn{6}{|l|}{ Copper leaching } \\
\hline 1910-1994 & $0.1-1.5$ & 0.49 & 0.01 & 0.01 & 0.49 \\
\hline \multicolumn{6}{|l|}{ Copper electrolysis } \\
\hline 1910-1994 & $0.03-0.2$ & 0.80 & 0.04 & 0.04 & 0.13 \\
\hline \multicolumn{6}{|l|}{$\begin{array}{l}\text { Copper } \\
\text { cementation }\end{array}$} \\
\hline 1927-1977 & $0.6-1.2$ & 0.45 & 0.05 & 0.45 & 0.05 \\
\hline \multicolumn{6}{|l|}{$\begin{array}{l}\text { Electrolytic } \\
\text { purification }\end{array}$} \\
\hline 1927-1977 & $0.2-0.5$ & 0.80 & 0.03 & 0.15 & 0.02 \\
\hline 1978-1994 & $0.03-0.2$ & 0.98 & 0.01 & 0.00 & 0.01 \\
\hline \multicolumn{6}{|l|}{ Nickel electrolysis } \\
\hline 1910-1977 & $0.1-0.2$ & 0.87 & 0.05 & 0.01 & 0.08 \\
\hline 1978-1994 & $0.03-0.1$ & 0.83 & 0.04 & 0.02 & 0.11 \\
\hline
\end{tabular}


a raw material in steel production, and oxide fumes and dust may occur in melting, casting, and welding operations. There are probably more exposures to metallic nickel in nickel-using industries than in nickel-producing industries. These occur during powder handling, grinding, and polishing operations and in casting operations.

Nickel is an essential trace element for animals (Sunderman 1986), and a 70-kg reference man contains $10 \mathrm{mg}$ of nickel, giving an average body concentration of $0.1 \mathrm{ppm}$ (Iyengar 1986). The highest tissue concentrations of nickel are found in the lungs of nickel smelting and refinery workers. The highest nickel concentration reported in lung tissue of 39 nickel refinery workers autopsied in 1978-1984 was 1,344 ppm (dry weight), compared to $1.7 \mathrm{ppm}$ in unexposed persons (Andersen and Svenes 1989). In another study of nickel content in the lungs of 15 former nickel refinery workers, the arithmetic mean ( $\pm 1 \mathrm{SD}$ ) for nickel concentrations in workers was $50 \pm 150 \mu \mathrm{g} / \mathrm{g}$, dry weight, in comparison to a value of $0.74 \pm 0.44 \mu \mathrm{g} / \mathrm{g}$ in 10 individuals not connected to the refinery industry (Svenes and Anderson 1998). In a multi-element analysis of lung tissue obtained from former nickel refinery workers who were retired for 10-15 years, it was found that one form of nickel remained in the lung following 15-20 years of the occupational exposure (Andersen and Svenes 2003). The remaining nickel is contained within an inhaled mineral, trevorite, which is water-insoluble. The concentration of nickel in the lung tissue within one donor varied from 3.0 to $64.1 \mu \mathrm{g} / \mathrm{g}$ dry weight, depending on the sampling site within the lung.

Ten studies of nickel in human milk gave disparate results. Six median values ranged from 5 to $16 \mu \mathrm{g} / \mathrm{L}$, and 10 mean values ranged from 1.5 to $39 \mu \mathrm{g} / \mathrm{L}$ (Iyengar 1989). Five of the six medians ranged from 11 to $16 \mu \mathrm{g} / \mathrm{L}$. The lowest and highest mean values were from Finland and Germany GDR, respectively. Biego et al. (1998) were not able to detect nickel in breast milk above the detection limit of $2.9 \mu \mathrm{g} / \mathrm{L}$ of the assay. However, mean ( $\pm 1 \mathrm{SD})$ nickel concentrations were determined for formula $(30 \pm 3 \mu \mathrm{g} / \mathrm{L})$ and soya milk $(450 \pm 220 \mu \mathrm{g} / \mathrm{L})$. Individual values ranged from not detectable to $130 \mu \mathrm{g}$ nickel/L. In one study from the United States, the nickel concentrations in human breast milk were measured between 1 and 38 days postpartum (Casey and Neville 1987). The concentrations ranged from 0.52 to $2.04 \mathrm{ng} / \mathrm{mL}$ with a mean value of $1.16 \mathrm{ng} / \mathrm{mL}$. There was no significant change in the values as a function of time postpartum.

Nickel concentrations in human serum taken from 30 individuals not occupationally exposed to nickel ranged from $<0.05$ to $1.05 \mu \mathrm{g} / \mathrm{L}$ with a mean value of $0.34 \mu \mathrm{g} / \mathrm{L}$ (Barceloux 1999). In another example, nickel concentrations in serum obtained from individuals without occupational exposures to nickel ranged from 0.18 to $0.54 \mu \mathrm{g} / \mathrm{L}$ with an average of $0.36 \mu \mathrm{g} / \mathrm{L}$ (Christensen 1995). Serum nickel levels in hospital 
workers averaged 0.6 $\pm 0.3 \mu \mathrm{g} / \mathrm{L}$ in Sudbury, Ontario, versus $0.2 \pm 0.2 \mu \mathrm{g} / \mathrm{L}$ in Hartford, Connecticut (Hopfer et al. 1989). Measurements of nickel content of tap water in these communities were reported as $109 \pm 46$ and $0.4 \pm 0.2 \mu \mathrm{g} / \mathrm{L}$, respectively (Hopfer et al. 1989). A mean nickel concentration of 4.80 $\pm 2.69 \mu \mathrm{g} / \mathrm{L}$ was measured in urine samples collected for the NHEXAS Arizona study (EPA 2003t). Concentrations of nickel in the blood and urine of workers at a rolling mill in Poland were $18.5 \pm 4.0$ and 25.7 $\pm 5.1 \mu \mathrm{g} / \mathrm{L}$, respectively (Baranowska-Dutkiewicz et al. 1992). Mean concentrations of nickel in urine of individuals not occupationally exposed to nickel are generally $<2 \mu \mathrm{g} / \mathrm{L}$ and can range as high as 9 $10 \mu \mathrm{g} / \mathrm{L}$ (95\% upper confidence limit) in healthy adults (Barceloux 1999). Workers at a galvanizing plant in Brazil exposed to airborne nickel sulfate at concentrations of $2.8-116.7 \mu \mathrm{g} / \mathrm{m}^{3}$ had nickel concentrations in their urine ranging between 2.1 and $58.7 \mu \mathrm{g} / \mathrm{g}$ creatinine $(2.3-54.9 \mu \mathrm{g} \mathrm{Ni} / \mathrm{L})$ with mean values of $8.7 \pm 7.8$ and $14.7 \pm 13.5 \mu \mathrm{g} / \mathrm{g}$ creatinine $(10.5 \pm 7.5$ and $20.6 \pm 18.1 \mu \mathrm{g} \mathrm{Ni} / \mathrm{L})$ in preshift and postshift samples, respectively (Oliveira et al. 2000). Mean concentrations of nickel in the urine of the control group (workers in a zinc plating plant) were $3.7 \pm 2.5 \mu \mathrm{g} / \mathrm{g}$ creatinine or $4.9 \pm 2.2 \mu \mathrm{g} / \mathrm{L}$. Danadevi et al. (2003) reported a high mean nickel concentration in the urine obtained from 57 welders of $131.0 \mu \mathrm{g} / \mathrm{L}$ (standard deviation of $\pm 52.6 \mu \mathrm{g} / \mathrm{L}$ ) compared to a mean concentration of $17.4 \mu \mathrm{g} / \mathrm{L}$ (standard deviation of $\pm 8.9 \mu \mathrm{g} / \mathrm{L}$ ) in urine taken from control individuals who were not occupationally exposed to nickel. However, airborne concentrations of nickel were not reported in this study. Nickel concentrations in the urine of preschool children in Poland were 10.6 \pm 4.1 and $9.4 \pm 4.7 \mu \mathrm{g} / \mathrm{L}$ for children from an industrial region and a health resort, respectively (Baranowska-Dutkiewicz et al. 1992). After reviewing studies of nickel concentrations in humans, Templeton et al. (1994) indicated that the most reliable reference values were $0.2 \mu \mathrm{g} / \mathrm{L}$ for nickel in serum of healthy adults and $1-3 \mu \mathrm{g} / \mathrm{L}$ for nickel in urine. These values are dependent on food and fluid intake and environmental factors. Fewer studies of nickel in whole blood were identified, and a reference value was not suggested.

Fingernail samples from 71 Americans contained $0.57 \mathrm{ppm}$ of nickel; samples from residents of Japan, India, Canada, and Poland had nickel concentrations that ranged from 1.1 to $3.9 \mathrm{ppm}$ (Takagi et al. 1988). Nickel levels are higher in males than in females. Higher levels occur in younger people and decrease with age. The mean concentration of nickel in hair samples from 55 men and women from Scranton, Pennsylvania, was 1.01 ppm; populations from cities in Japan, India, Canada, and Poland had mean nickel levels between 0.26 and 2.70 ppm (Takagi et al. 1986). A more recent National Health and Nutritional Examination Survey II of hair from a random sample of 271 adults, ages 20-71, from three communities had geometric mean and median nickel levels of 0.39 and $0.45 \mathrm{ppm}$, respectively. Ten percent of the group had nickel levels $>1.50 \mathrm{ppm}$ (DiPietro et al. 1989). 
The nickel content of most natural vegetation is $0.05-5 \mathrm{ppm}$ on a dry weight basis (NAS 1975). Near source areas, nickel on plants may be elevated because of direct foliar deposition. Some species of plants have the ability to hyperaccumulate nickel (Brooks 1980). The concentration in the leaves of Alyssum bertolonii contained $120 \mathrm{ppm}$ nickel. These plants are thought to produce organic ligands that complex with nickel.

The modal concentration of nickel in 159 species of edible fin fish from the U.S. National Marine Fisheries Survey was 0.2-0.3 ppm (wet weight) (Heit et al. 1989). Jenkins (1980) has compiled literature concentrations of nickel levels in aquatic species. The ranges of nickel concentrations in freshwater fish, marine fish, and mollusks from areas thought to be uncontaminated are from $<0.2$ to 2.0 , from not detectable to 4.0 , and from 0.4 to $2.0 \mathrm{ppm}$ (wet weight), respectively. The highest levels found near sources of pollution, especially near nickel smelters, were $51.6 \mathrm{ppm}$ for freshwater fish and $191.0 \mathrm{ppm}$ for mollusks. The nickel content of muscle tissue of several fish species collected from metal-contaminated lakes near Sudbury, Ontario, was below the detection limit (2.0 ppm dry weight), except for two of four yellow perch, which had levels of 2.8 and 3.4 ppm (Bradley and Morris 1986). A more recent survey of metals in stocked lake trout in five lakes near Sudbury, Ontario, reported that concentrations of metal in trout muscle were not significantly different from those in the hatchery $(0.34-0.83 \mathrm{ppm}$ wet weight versus 0.49 ppm) (Bowlby et al. 1988). Nickel concentrations in the lower Savannah River and Savannah National Wildlife Refuge in Georgia were higher than those reported above for uncontaminated areas. These levels were consistently higher in gar (2.35-6.67 ppm wet weight) than in catfish (0.371.41 ppm) (Winger et al. 1990). Nickel could not be measured above the detection limit ( $0.5 \mu \mathrm{g} / \mathrm{g}$, dry weight) in livers taken from lesser scaup collected along the Mississippi Flyway between Manitoba and Louisiana (Custer et al. 2003). As part of the National Status and Trends Program for Marine Environmental Quality, the concentration of nickel in oysters and mussels was investigated (NOAA 1987). The nickel concentration in bivalve tissue collected in 1986 ranged from 0.55 to $12.57 \mathrm{ppm}$ (dry weight). The highest tissue concentration was found in Matagorda Bay, Texas, and the second highest concentration, $6.57 \mathrm{ppm}$, was found in both Tomales Bay, California, and Chesapeake Bay, Maryland. Oysters around three coastal marinas in South Carolina with sediment nickel levels of 25.8-40.8 ppm (dry weight) had levels of 0.35.2 ppm (Marcus and Thompson 1986). Mean nickel levels in oysters at four sites in the Mississippi Sound varied from <0.5 to $4.7 \mathrm{ppm}$ (wet weight) (Lytle and Lytle 1990). 


\subsection{EXPOSURES OF CHILDREN}

This section focuses on exposures from conception to maturity at 18 years in humans. Differences from adults in susceptibility to hazardous substances are discussed in Section 3.7, Children's Susceptibility.

Children are not small adults. A child's exposure may differ from an adult's exposure in many ways. Children drink more fluids, eat more food, breathe more air per kilogram of body weight, and have a larger skin surface in proportion to their body volume. A child's diet often differs from that of adults. The developing human's source of nutrition changes with age: from placental nourishment to breast milk or formula to the diet of older children who eat more of certain types of foods than adults. A child's behavior and lifestyle also influence exposure. Children crawl on the floor, put things in their mouths, sometimes eat inappropriate things (such as dirt or paint chips), and spend more time outdoors. Children also are closer to the ground, and they do not use the judgment of adults to avoid hazards (NRC 1993).

Exposures of children to airborne nickel are expected to be similar to those found for adults. However, differences in the exposure to nickel contained in deposited particulates (e.g., household dust) are expected to be higher in children, due to greater contact of children with floors and other surfaces, in addition to greater oral and dermal contact with these deposited particulates through the mouthing of toys, hands, feet, etc. Concentrations of nickel in dust collected from homes in Ottawa, Ontario, averaged $62.9 \mathrm{mg} / \mathrm{kg}$ with values as high as $116.4 \mathrm{mg} / \mathrm{kg}$ (Butte and Heinzow 2002). However, it is not known how much nickel a child absorbs through oral or dermal contact with household dust.

Nickel that is dissolved in water is expected to be a minor exposure route for children, due to the generally low concentrations of nickel in drinking water and groundwater (Sections 6.4.2 and 6.5). However, in areas near nickel smelters and refineries where source water used to produce drinking water is contaminated with nickel, intake of nickel through drinking water for individuals in the affected area will be elevated above that for individuals in the surrounding region whose drinking water is unaffected by these sources of nickel contamination, but is expected to be less than nickel intake through food. Exposure to nickel through consumption of human breast milk is expected to be comparable to or lower than milk-based and soy-based formulas, based on the similar concentration ranges of nickel in these fluids (Biego et al. 1998; Dabeka 1989; FDA 2000; Iyengar 1989). Based on the data from Casey and Neville (1987), the intake of nickel through consumption of breast milk averages $0.8 \mu \mathrm{g} / \mathrm{day}$ between the ages of 4 and 38 days postpartum. For 3-month-old children, it is estimated that daily intake of nickel through the consumption of breast, bottled, dried, and evaporated milk is below $2 \mu \mathrm{g} /$ day (Biego et al. 
1998). However, daily intake could be higher when consuming some formulas and soya milk, with estimates as high as 21 and $315 \mu \mathrm{g} /$ day, respectively, being reported (Biego et al. 1998).

Another source of nickel exposure in children is through soil. Children generally receive higher exposure to soil contaminants per unit body weight than adults (Lottermoser 2002). Small children have large surface-to-mass ratios, which provide a greater potential to transdermally absorbed compounds, especially for children crawling in dirt. Also, the skin of newborns and children is more permeable than adult skin. Nickel in an ionized form, such has nickel salts, penetrates intact skin at a slow rate and can also be absorbed at sites of injury to skin (Barceloux 1999). However, nickel exposure through dermal exposure is minimal compared to exposures to nickel through ingestion of soil. The population at greatest risk are children between the ages of 2 and 3 years old as a result of hand-to-mouth activities and those with soileating disorders (Lottermoser 2002). A child's intake of nickel through ingestion of soil could be especially important in areas where soils that are naturally enriched with nickel (for example, some soils in the southeastern United States, southern Ontario, or eastern Australia) or have been contaminated with nickel (for example in the Sudbury, Ontario, region) (Section 6.4.3). However, due to the limited bioavailability of nickel in some soils, the amount of nickel that a child actually absorbs from ingested soils could be rather limited. For example, ingestion of $100 \mathrm{mg}$ of ferrosol soil containing $149 \mathrm{mg}$ nickel per $\mathrm{kg}$ of soil is calculated to contribute an intake of $0.000149 \mathrm{mg}$ nickel/day, assuming a relative bioavailability for nickel of $1 \%$ (Lottermoser 2002).

The primary route of nickel exposure in children is expected to be through the diet. Measurements of nickel in duplicate diet samples obtained from the EPA Region 5 studies indicates that average nickel concentration in combined solids and liquids of $47 \mu \mathrm{g} / \mathrm{kg}$, which is higher than the average nickel concentration in drinking water of approximately $5 \mu \mathrm{g} / \mathrm{kg}(5.3 \mu \mathrm{g} / \mathrm{L})$ (Thomas et al. 1999). Using the portion size information recorded by study participants in the NHEXAS Arizona study, daily dietary intakes of nickel for children ( $<18$ years of age) have been calculated to range from 31 to $343 \mu \mathrm{g}$, with a mean value of $125 \mu \mathrm{g}$ (O'Rourke et al. 1999). These intake levels were lower than the average dietary nickel intake of $153 \mu \mathrm{g} /$ day calculated for the overall study population (Table 6-3). Likewise, in another total dietary study taken between 1991 and 1997 the mean daily dietary intake of nickel in food of 9$99 \mu \mathrm{g} /$ day for children under the age of 9 years was also found to be lower than the mean intake derived for the total study population of $114 \mu \mathrm{g} /$ day (NAS 2002). Mean daily nickel intakes of 9, 39, 82, and $99 \mu \mathrm{g} /$ day were determined for children aged 0-6 months, 7-12 months, 1-3 years, and 4-8 years, respectively. Nevertheless, the ranges in the mean daily dietary intakes of nickel in children aged 918 years (128-137 $\mu \mathrm{g} /$ day in males and $101-109 \mu \mathrm{g} / \mathrm{day}$ for females) are similar to the mean intakes 
determined in adults (>18 years of age) (see Section 6.5). Information on dietary nickel intake for nonnursing children and children ages 1-6 (Table 6-4) obtained from DEPM model (Moschandreas et al. 2002) indicates that these children have a higher intake of nickel than the average intake for the U.S. population. Even so, the daily intake of nickel in these children is estimated (4-13 $\mu \mathrm{g} / \mathrm{day}$ based on $6-$ $20 \mathrm{~kg}$ total body weight) to be much lower than the average dietary nickel intake ( $125 \mu \mathrm{g} / \mathrm{day})$ obtained from the NHEXAS study. Also, results from a study of dietary nickel in 2-year-old children in the United Kingdom, where an average daily intake of $81 \mu \mathrm{g} /$ day (range of 14-260 $\mu \mathrm{g} /$ day) was estimated from the weekly nickel intake of $0.57 \mathrm{mg}$ of nickel (range of $0.1-1.8 \mathrm{mg}$ ) given in the reference (Smart et al. 1987), would suggest a higher daily nickel intake in young children than is indicated based on the results of the DEPM model.

For infants who are exclusively breastfed, the daily intake of nickel is estimated to be approximately $1 \mu \mathrm{g} /$ day (NAS 2002). This estimate is based on a concentration of mean nickel concentration of $1.2 \mathrm{ng} / \mathrm{mL}$ in breast milk and an average secretion of $0.78 \mathrm{~L} /$ day.

\subsection{POPULATIONS WITH POTENTIALLY HIGH EXPOSURES}

In discussing nickel exposure, it is important to consider what form of nickel a person is exposed to and its bioavailability. Such information is not often available. Although high concentrations of nickel may be found in contaminated soil and sediment, it may be embedded in a crystalline matrix or bound to hydrated iron, aluminum, and manganese oxides and, therefore, not bioavailable.

Nickel-containing alloys are used in patients in joint prostheses, sutures, clips, and screws for fractured bones. Corrosion of these implants may lead to elevated nickel levels in the surrounding tissue and to the release of nickel into extracellular fluid (IARC 1990; Ries et al. 2003; Sunderman 1989a; Sunderman et al. 1986, Sunderman et al. 1989c). For example, elevation of nickel blood concentrations (up to 3 times higher than before implantation) has been observed in patients receiving the Amplatzer occluder to repair atrial septal defects up to 1 month following implantation (Ries et al. 2003). However, the nickel concentrations return to preimplantation levels within 12 months. Short-term elevations in nickel concentrations measured in blood and urine of up to a factor of 5 and 10 above preimplant levels, respectively, were seen in patients receiving knee and hip protheses within 1-2 days of implant (Sunderman et al. 1989c). The rapid rise is attributed to the leaching of nickel from nickel-bearing particles from drills, cutting jigs, and drilling jigs. These nickel concentrations were found to decrease to near preimplant concentrations within 2 weeks of implant. Serum albumin solutions used for intravenous 
infusion fluids have been reported to contain as much as $222 \mu \mathrm{g}$ nickel/L, but are very rarely encountered. Dialysis fluid has been reported to contain as much as $0.82 \mu \mathrm{g}$ nickel/L. Studies of nickel in serum preand post-dialysis show between 0 and 33\% increases in nickel concentrations in patients (IARC 1990).

People who live near or work at facilities in the United States that produce stainless steel and other nickelcontaining alloys, oil-fired power plants, coal-fired power plants, refuse incinerators, or waste sites that receive nickel-producing or using industries have the potential to be exposed to levels of nickel in airborne dust, soil, and vegetation that are greater than those for the general population by virtue of their proximity to these sites. Nickel uptake is expected to result from one or more (simultaneous) exposure pathways, such as inhalation, dermal contact, or ingestion of contaminated soil or vegetation. Inhalation and ingestion of particulate matter are likely the main routes of occupational exposures. In the Unites States, these exposures are minimized through the limiting of allowable concentrations of nickel in air in the workplace (see Chapter 8) and the recommended use of protective clothing and equipment (HSDB 2005). For residents living near NPL sites and other facilities that produce, use, handle, or store nickel or nickel-containing material, many of the nickel concentrations measured in air $\left(0.4912-4,000 \mathrm{ng} / \mathrm{m}^{3}\right)$, surface water (2-20,000 ppb), groundwater (4.2-11,400 ppb), and soils (2-10,522 ppb) offsite from NPL sites (HazDat 2005; Newhook et al. 2003) exceed the average concentrations of nickel in air (6$\left.20 \mathrm{ng} / \mathrm{m}^{3}\right)$, surface water (15-20 ppb), groundwater (3 ppb), and soils $(<5,000-700,000 \mathrm{ppb})$ measured in the United States (ATSDR 2003; Barceloux 1999; Grandjean 1984; NAS 1975; Page 1981; Shacklette and Boerngen 1984). It is likely that the greatest exposures to nickel from these facilities are expected to be through inhalation of particulate matter containing nickel or through ingestion of drinking water obtained from private wells. The relationship between these increased nickel concentrations measured in media offsite of these facilities and exposure of nearby residents to nickel will vary between facilities and depend mainly on the location of a resident to the facility.

\subsection{ADEQUACY OF THE DATABASE}

Section 104(i)(5) of CERCLA, as amended, directs the Administrator of ATSDR (in consultation with the Administrator of EPA and agencies and programs of the Public Health Service) to assess whether adequate information on the health effects of nickel is available. Where adequate information is not available, ATSDR, in conjunction with NTP, is required to assure the initiation of a program of research designed to determine the health effects (and techniques for developing methods to determine such health effects) of nickel. 
The following categories of possible data needs have been identified by a joint team of scientists from ATSDR, NTP, and EPA. They are defined as substance-specific informational needs that if met would reduce the uncertainties of human health assessment. This definition should not be interpreted to mean that all data needs discussed in this section must be filled. In the future, the identified data needs will be evaluated and prioritized, and a substance-specific research agenda will be proposed.

\subsubsection{Identification of Data Needs}

Physical and Chemical Properties. Except for differences between black and green nickel oxide, the physical and chemical properties of nickel and its compounds reported in Table 4-2 (HSDB 2004) have been adequately characterized.

\section{Production, Import/Export, Use, Release, and Disposal. According to the Emergency} Planning and Community Right-to-Know Act of 1986, 42 U.S.C. Section 11023, industries are required to submit substance release and off-site transfer information to the EPA. The TRI, which contains this information for 2002, became available in May of 2004. This database is updated yearly and should provide a list of industrial production facilities and emissions.

Information on the production, import, export, and use of nickel metal, nickel alloys, and nickel compounds is readily available (ABMS 1994, 2002; Chamberlain 1985; Kirk 1988a, 1988b; Kuck 2002, 2002; NTD 1996; Tien and Howson 1981; USGS 2003). Except for recycling of metal scrap, little information is available regarding the disposal of nickel and its compounds.

Releases according to the TRI database are reported in Tables 6-1 and 6-2 (TRI02 2004). The TRI data should be used with caution because only certain types of facilities are required to report. This is not an exhaustive list. Much of the nickel released to the environment is transferred off-site for disposal and probably landfilled. Nickel wastes from former mining and smelting operations may have been discarded in large tailing piles. Acid conditions are often created in tailing piles from sulfidic ores that increase the potential for leaching (Wood 1987). This is not the case with lateritic deposits such as those found in Riddle, Oregon. Information regarding nickel leaching from slag heaps is important in assessing releases to the environment. More detailed information regarding disposal methods and the form of nickel disposed of is necessary to assess potential nickel exposure. 
Environmental Fate. Nickel is an element and therefore, is not destroyed in the environment. In assessing human exposure, one must consider the form of nickel and its bioavailability. This information is site specific. Data regarding the forms of nickel in air, soil, and sediment are fragmentary and inadequate (Galbreath et al. 2003; Sadiq and Enfield 1984a; Schroeder et al. 1987). Also lacking is adequate information on the transformations that may occur, the transformation rates, and the conditions that facilitate these transformations. Information relating to the adsorption of nickel by soil and sediment is not adequate. In some situations, adsorption appears to be irreversible. In other situations, however, adsorption is reversible. More data would be helpful in detailing those situations where adsorbed nickel may be released and those where release is unlikely.

Bioavailability from Environmental Media. The absorption and distribution of nickel as a result of inhalation, ingestion, and dermal exposure are discussed in Sections 3.4.1 and 3.4.2. Quantitative data relating the physical/chemical properties of nickel (e.g., particle size, chemical forms of nickel) with its bioavailability are available for inhaled nickel. In aqueous media, nickel is in the form of the hexahydrate ion, which is poorly absorbed by most living organisms (Sunderman and Oskarsson 1991), although uptake of nickel into rye and oats has been reported (Smith 1994; Weng et al. 2004). Additional studies that examine the absorption of nickel from soil would be useful.

Food Chain Bioaccumulation. Data are available on the bioconcentration of nickel in fish and aquatic organisms (Birge and Black 1980; EPA 1979; McGeer et al. 2003; Suedel et al. 1994; Zaroogian and Johnson 1984). Higher levels of nickel have been found in gar compared with catfish from the same environment (Winger et al. 1990). More data on different species of fish at different sites would be useful in explaining these results. Data are limited on the nickel levels in wild birds and mammals (Alberici et al. 1989; Dressler et al. 1986; Jenkins 1980). A larger database including information on both herbivorous and carnivorous species living in both polluted and unpolluted environments is desirable in establishing whether nickel biomagnification in the food chain occurs under some circumstances.

Exposure Levels in Environmental Media. Reliable monitoring data for the levels of nickel in contaminated media at hazardous waste sites are needed so that the information obtained on levels of nickel in the environment can be used in combination with the known body burden of nickel to assess the potential risk of adverse health effects in populations living in the vicinity of hazardous waste sites.

Representative and recent monitoring data for nickel in air, water, and foods are essential for estimating the extent of exposure from each of these sources. Nickel levels in environmental media are often below 
the detection limit of the analytical method employed. When a substantial fraction of determinations of nickel levels in environmental samples are below the detection limit, an arithmetic mean may not adequately represent the data. Data on the levels of nickel in ambient and indoor air are available (Claiborne et al. 2002; EPA 1986a; Evans et al. 1984; Graney et al. 2004; Kinney et al. 2002; Koutrakis et al. 1992; Kowalczyk et al. 1982; Lioy et al. 1987; Galbreath et al. 2003; Salztman et al. 1985; Sweet et al. 1993; van Winkle and Scheff 2001; Wiersema et al. 1984). Data provided by EPA's National Human Exposure Assessment Study (NHEXAS) have contributed to the assessment of current levels of exposure to nickel by the U.S. population via inhalation, drinking water and food. Analyses of data obtained from the Arizona and EPA Region 5 NHEXAS studies (O'Rourke et al. 1999; Thomas et al. 1999) and another total dietary study (NAS 2002) have provided information on daily dietary nickel intake for these study populations. These data have provided the first update of nickel content within the U.S. diet since the last comprehensive survey of nickel in U.S. drinking water in 1969-1970 (NAS 1975) and the information on dietary nickel that had been limited to one study from North Dakota (Myron et al. 1978). While these recent results are in agreement with ones from Europe (Alberti-Fidanza et al. 2003; IARC 1990), they do differ from the estimated dietary nickel intakes obtained by Moschandreas et al. (2002). Therefore, additional data on nickel content within the U.S. diet, especially information covering a larger geographic area in the United States, is desirable. Also, few data are available regarding nickel levels at contaminated or hazardous waste sites (Agency for Toxic Substances and Disease Registry 2003; Bradley and Morris 1986; Duke 1980b; Taylor and Crowder 1983). This information is necessary for exposure assessment analysis at these sites. This should include monitoring of air and drinking water concentrations of nickel surrounding these sites. Since nickel is found in all soil, studies should focus on waste sites where nickel levels are substantially above those found in ordinary soil.

Exposure Levels in Humans. Nickel levels in body fluids, tissue, hair, nails, and breast milk are available (Andersen and Svenes 2003; DiPietro et al. 1989; Hopfer et al. 1989; IARC 1990; Iyengar 1989; Takagi et al. 1986, 1988). Serum and urine levels in some exposed workers have also been published (Angerer and Lehnert 1990; Barceloux 1999; Bencko et al. 1986; Bernacki et al. 1978; Danadevi et al. 2003; Elias et al. 1989; Ghezzi et al. 1989; Hassler et al. 1983; Morgan and Rouge 1984; Oliveira et al. 2000; Torjussen and Andersen 1979). These data do not refer to populations living around hazardous waste sites that contain elevated levels of nickel. It is recommended that additional studies be conducted that examine biomarkers of exposure or markers of early biological effects, such as changes in gene expression measured by microarrays. These studies could be piloted with in vitro cell lines to determine nickel-specific markers, followed by in vivo screening of people living near sites that contain levels of nickel that are elevated above background concentrations or who have occupational exposures to nickel. 
This information is necessary for assessing the need to conduct health studies on these populations.

Exposures of Children. This information is necessary for assessing the need to conduct health studies on children. Some data on daily intake of nickel is available for children under the age of 18 years (Thomas et al. 1999), including data for various age ranges of children (Moschandreas et al. 2002; NAS 2002; O'Rourke et al. 1999). The nickel levels in urine are available (Baranowska-Dutkiewicz et al. 1992), but information on levels in other body fluids, tissue, hair, and nails is not available. These data do not refer to populations living around the hazardous waste sites that contain elevated levels of nickel. Additional studies that examine nickel levels in body fluids and tissues from children living near hazardous waste sites that contain elevated levels of nickel would be useful.

Child health data needs relating to susceptibility are discussed in Section 3.12.2, Identification of Data Needs: Children's Susceptibility.

Exposure Registries. Although there is no U.S. exposure registry for nickel, a Finnish exposure registry for occupational carcinogens exists, and this registry contains information on nickel and inorganic nickel compounds (Grandjean 1984).

This substance is not currently one of the compounds for which a sub-registry has been established in the National Exposure Registry. The substance will be considered in the future when chemical selection is made for sub-registries to be established. The information that is amassed in the National Exposure Registry facilitates the epidemiological research needed to assess adverse health outcomes that may be related to exposure to this substance.

\subsubsection{Ongoing Studies}

A number of ongoing studies concerning the fate/transport of nickel and human exposures to nickel were identified in the FEDRIP (2004) database. These studies are summarized in Table 6-6. 


\section{Table 6-6. Ongoing Studies on Environmental Fate and the Potential for Human Exposure to Nickel ${ }^{\mathrm{a}}$}

\begin{tabular}{|c|c|c|c|}
\hline Investigator & Affiliation & Research description & Sponsor \\
\hline Angle, JS & University of Maryland & $\begin{array}{l}\text { Identify indigenous microorganisms that } \\
\text { affect both metal solubility in soil and } \\
\text { uptake into plants }\end{array}$ & Hatch \\
\hline $\begin{array}{l}\text { Baligar, VC; Clark, } \\
\text { RB; Zelazny, LC; } \\
\text { Persaud, N; } \\
\text { Ritchey, KD; } \\
\text { Martens, DC }\end{array}$ & $\begin{array}{l}\text { Virgina Polytechnical } \\
\text { Institute and State } \\
\text { University }\end{array}$ & $\begin{array}{l}\text { Evaluate the mineralogy and chemistry of } \\
\text { trace elements in soil treated with coal-fired } \\
\text { power plant byproducts }\end{array}$ & USDA \\
\hline Baker, JC & $\begin{array}{l}\text { Virgina Polytechnic } \\
\text { Institute }\end{array}$ & $\begin{array}{l}\text { Determine chemical, physical, and soil } \\
\text { water status in } 1,200 \text { Virgina soil horizons, } \\
\text { including quantifying trace metal } \\
\text { concentrations }\end{array}$ & Hatch \\
\hline Bleam, WF & University of Wisconsin & $\begin{array}{l}\text { Characterizing trace metal complexes with } \\
\text { humic acids containing thiols or amines }\end{array}$ & Hatch \\
\hline $\begin{array}{l}\text { Bleam, WF; } \\
\text { Helmke, PA }\end{array}$ & $\begin{array}{l}\text { University of Wisconsin } \\
\text { at Madison }\end{array}$ & $\begin{array}{l}\text { Improve understanding of how humic } \\
\text { substances in soil bind trace metals by } \\
\text { elucidating specific binding sites and their } \\
\text { affinities for trace metals }\end{array}$ & USDA \\
\hline Chaney, RL & $\begin{array}{l}\text { Beltsville Agricultural } \\
\text { Research Center }\end{array}$ & $\begin{array}{l}\text { Characterize trace element absorbants in } \\
\text { municipal, industrial, and agricultural } \\
\text { byproduct-amended soils }\end{array}$ & USDA \\
\hline $\begin{array}{l}\text { Franklin, RE; } \\
\text { Mylavarapu, R }\end{array}$ & Clemson University & $\begin{array}{l}\text { Establish baselines for metal } \\
\text { concentrations in soils in South Carolina } \\
\text { and vicinity; measure metal concentrations } \\
\text { in plants grown in soils amended with } \\
\text { waste waters and solid wastes }\end{array}$ & Hatch \\
\hline Hopkins, DG & $\begin{array}{l}\text { North Dakota State } \\
\text { University }\end{array}$ & $\begin{array}{l}\text { Evaluate the total and bioavailable trace } \\
\text { metals in North Dakota Drift Prairie }\end{array}$ & Hatch \\
\hline Koenig, RT & Utah State University & $\begin{array}{l}\text { Assess concentrations of metals, } \\
\text { phosphorous, and nitrogen in soils } \\
\text { amended with biosolids }\end{array}$ & Hatch \\
\hline Lave, LB & $\begin{array}{l}\text { Carnegie-Mellon } \\
\text { University }\end{array}$ & $\begin{array}{l}\text { Development of input-output models for } \\
\text { assessing heavy metal flows throughout } \\
\text { some } 500 \text { economic sectors in the United } \\
\text { States }\end{array}$ & NSF \\
\hline Martinez, CE & $\begin{array}{l}\text { Pennsylvania State } \\
\text { University }\end{array}$ & $\begin{array}{l}\text { Characterize the distribution and elemental } \\
\text { associations of nickel, copper, and zinc in } \\
\text { field-collected soils and evaluate the long- } \\
\text { term partitioning and redistribution of these } \\
\text { elements using computer modeling }\end{array}$ & USDA \\
\hline Odom, JW & Auburn University & $\begin{array}{l}\text { Determine the normal occurrence of both } \\
\text { total and extractable trace metals in } \\
\text { selected Alabama soil profiles }\end{array}$ & Hatch \\
\hline
\end{tabular}




\section{Table 6-6. Ongoing Studies on Environmental Fate and the Potential for Human Exposure to Nickel ${ }^{\mathrm{a}}$}

\begin{tabular}{|c|c|c|c|}
\hline Investigator & Affiliation & Research description & Sponsor \\
\hline Parker, DR & University of California & $\begin{array}{l}\text { Determine binding constants between } \\
\text { mugineic acids and trace metals; evaluate } \\
\text { competition between iron and other trace } \\
\text { metals in soils in binding reactions with } \\
\text { mugineic acids; elucidate mechanisms } \\
\text { through which mugineic acids contribute to } \\
\text { the uptake of trace metals into plants }\end{array}$ & USDA \\
\hline Ross, DS & University of Vermont & $\begin{array}{l}\text { Characterize the reactive sites on soil } \\
\text { manganese oxides and elucidating the } \\
\text { oxidation of trace metals at these sites }\end{array}$ & Hatch \\
\hline Sparks, DL & University of Delaware & $\begin{array}{l}\text { Determine the effects of reaction conditions } \\
\text { and residence time on the sorption/release } \\
\text { of important metals/metalloids on soil } \\
\text { components and Delaware soils }\end{array}$ & USDA \\
\hline $\begin{array}{l}\text { Sparks, DL; Ford, } \\
\text { RG }\end{array}$ & University of Delaware & $\begin{array}{l}\text { Examine nickel and zinc sorption- } \\
\text { desorption kinetic behavior on model and } \\
\text { natural soil components, characterize } \\
\text { structure of the sorption complex, and } \\
\text { investigate effect of competition of soil } \\
\text { components with metal-aluminum } \\
\text { precipitates }\end{array}$ & USDA \\
\hline $\begin{array}{l}\text { Sparks, DL; } \\
\text { Scheidegger, AM; } \\
\text { Lamble, GM }\end{array}$ & University of Delaware & $\begin{array}{l}\text { Determine the effects of residence time on } \\
\text { the mechanisms of nickel sorption/release } \\
\text { on soils and soils components; develop } \\
\text { predictions about long-term fate of nickel in } \\
\text { soils }\end{array}$ & USDA \\
\hline $\begin{array}{l}\text { Thompson, ML; } \\
\text { Horton, R; } \\
\text { Tabatabai, MA }\end{array}$ & Iowa State University & $\begin{array}{l}\text { Identify and quantify the fundamental } \\
\text { processes that determine the fate and } \\
\text { transport of metals and pesticides once } \\
\text { they are applied to the soil or where they } \\
\text { occur in contaminated soils }\end{array}$ & USDA \\
\hline Tu, S & $\begin{array}{l}\text { Eastern Regional } \\
\text { Research Center, } \\
\text { Wyndmoor, } \\
\text { Pennsylvania }\end{array}$ & $\begin{array}{l}\text { Elucidate the kinetics and mechanisms of } \\
\text { heavy metal retention/release by soil } \\
\text { mineral colloids as affected by inorganic } \\
\text { anions }\end{array}$ & USDA \\
\hline $\begin{array}{l}\text { Volk, VV; } \\
\text { Roseberg, RJ; } \\
\text { Baham, J }\end{array}$ & Oregon State University & $\begin{array}{l}\text { Assess potential of plants to remove trace } \\
\text { metals from soils and determine impact of } \\
\text { trace metals on plant health }\end{array}$ & USDA \\
\hline
\end{tabular}

${ }^{\mathrm{a}}$ FEDRIP 2004

NSF = National Science Foundation; USDA = U.S. Department of Agriculture 
As part of the Third National Health and Nutrition Evaluation Survey (NHANES III), the Environment Health Laboratory Sciences Division of the National Center for Environment Health, Centers for Disease Control and Prevention, will be analyzing human urine samples for nickel. These data will give an indication of the frequency of occurrence and background levels of these compounds in the general population. 



\section{ANALYTICAL METHODS}

The purpose of this chapter is to describe the analytical methods that are available for detecting, measuring, and/or monitoring nickel, its metabolites, and other biomarkers of exposure and effect to nickel. The intent is not to provide an exhaustive list of analytical methods. Rather, the intention is to identify well-established methods that are used as the standard methods of analysis. Many of the analytical methods used for environmental samples are the methods approved by federal agencies and organizations such as EPA and the National Institute for Occupational Safety and Health (NIOSH). Other methods presented in this chapter are those that are approved by groups such as the Association of Official Analytical Chemists (AOAC) and the American Public Health Association (APHA). Additionally, analytical methods are included that modify previously used methods to obtain lower detection limits and/or to improve accuracy and precision.

\subsection{BIOLOGICAL MATERIALS}

Analytical methods that determine nickel in biological materials are the same as those used for environmental samples. The most common methods determine the total nickel content of the sample instead of the particular nickel compound that may be present. Methodological differences are a function of the nickel level in the sample, digestion procedure required to solubilize the sample, and the level of potentially interfering substances that may be present. Either wet ashing with sulfuric acid or dry ashing through dissolution of the ash with dilute sulfuric or hydrochloric acid is generally a satisfactory method to detect nickel in tissue or food (Boyer and Horowitz 1986; Coleman et al. 1992). Another methodological approach utilizes digestion of biological samples with nitric acid (Custer et al. 2003; Odland et al. 2003) that can also be followed by treatment with hydrogen peroxide to remove residual biological material (USGS 2000). Digestion procedures for biological and environmental samples with particular reference to nickel determinations have been reviewed (Stoeppler 1980; Sunderman 1993; Versieck 1985). As the digestion procedures require the use of strong acids and substances with explosion hazards (e.g., perchloric acid), all safety procedures should be carefully reviewed before the analyses are completed.

Nickel is normally present at very low levels in biological samples. To determine trace nickel levels in these samples accurately, sensitive and selective methods are required. Atomic absorption spectrometry (AAS) and inductively coupled plasma-atomic emission spectroscopy (ICP-AES), with or without 
preconcentration or separation steps, are the most common methods. These methods have been adopted in standard procedures by EPA, NIOSH, IARC, and the International Union of Pure and Applied Chemistry (Brown et al. 1981). Direct aspiration into a flame and atomization in an electrically heated graphite furnace or carbon rod are the two variants of atomic absorption. The latter is sometimes referred to as electrothermal AAS (ETAAS). Typical detection limits for ETAAS are $<0.4 \mu \mathrm{g} / \mathrm{L}$, while the limit for flame AAS and ICP-AES is $3.0 \mu \mathrm{g} / \mathrm{L}$ (Stoeppler 1984; Sunderman 1993; Todorovska et al. 2002). The precision of analytical techniques for elemental determinations in blood, muscles, and various biological materials has been investigated (Iyengar 1989). Good precision was obtained with flame AAS after preconcentration and separation, electrothermal AAS, and ICP-AES. Inductively coupled plasmamass spectrometry (ICP-MS) techniques have been used to quantify nickel in urine with detection sensitivities down to approximately $1 \mu \mathrm{g} / \mathrm{L}$ (Sunderman 1993). The quantification of nickel in biological materials is hampered by the presence of calcium, sodium, and potassium and requires the use of isotope dilution techniques to validate the measurements of nickel in samples.

Voltammetric techniques are becoming increasingly important for nickel determinations since such techniques have extraordinary sensitivity as well as good precision and accuracy. Direct measurement of nickel in urine in the presence of other trace metals (e.g., cadmium, cobalt, and lead) was demonstrated using adsorption differential pulse cathodic stripping voltammetry at a detection limit of $0.027 \mu \mathrm{g} / \mathrm{L}$ (Horng et al. 2003). The addition of dimethylglyoxime, a chelating agent, to the electrolyte significantly enhances the method's sensitivity (IARC 1990; Stoeppler 1984). Detection limits of $<0.001 \mu \mathrm{g} / \mathrm{L}$ have been achieved with differential pulse anodic stripping voltammetry (DPASV) using dimethylglyoxime chelation (Sunderman 1993).

Analytical methods and detection limits for nickel in biological materials are reported in Table 7-1. The presence of nickel in other biological materials such as hair and nails can be determined by the same analytical techniques used for blood and tissue after suitable procedures for dissolving the sample have been utilized (Stoeppler 1980; Takagi et al. 1986, 1988). It should be noted that assays of metals in hair are difficult to interpret because of the likelihood of external contamination on the hair shaft, and due caution is advised.

Detailed reviews regarding the methodology used to determine nickel in environmental and biological samples are available (Stoeppler 1980, 1984; Sunderman 1993). 
Table 7-1. Analytical Methods for Determining Nickel in Biological Materials

\begin{tabular}{|c|c|c|c|c|c|}
\hline $\begin{array}{l}\text { Sample } \\
\text { matrix }\end{array}$ & Preparation method & $\begin{array}{l}\text { Analytical } \\
\text { method }\end{array}$ & $\begin{array}{l}\text { Sample } \\
\text { detection limit }\end{array}$ & $\begin{array}{l}\text { Percent } \\
\text { recovery }\end{array}$ & Reference \\
\hline $\begin{array}{l}\text { Blood fluid, } \\
\text { tissue, and } \\
\text { excreta }^{\mathrm{a}}\end{array}$ & $\begin{array}{l}\text { Acid digestion in mixture of } \\
\text { nitric, sulfuric, and perchloric } \\
\text { acid }\end{array}$ & $\begin{array}{l}\text { Electrothermal } \\
\text { AAS }\end{array}$ & $\begin{array}{l}0.2 \mu \mathrm{g} \mathrm{Ni} / \mathrm{L} \\
\text { fluid; } 0.49 \mu \mathrm{g} \\
\mathrm{Ni} / \mathrm{kg} \text { of tissue }\end{array}$ & $\begin{array}{l}98 \% \text { at } 5 \mu \mathrm{g} \\
\mathrm{Ni} / \mathrm{L} ; 97 \% \text { at } \\
8 \mu \mathrm{g} \mathrm{Ni} / \mathrm{L}\end{array}$ & $\begin{array}{l}\text { IARC } 1986 \\
\text { (Method 11) }\end{array}$ \\
\hline Urine & $\begin{array}{l}\text { Polydithiocarbamate resin } \\
\text { extraction; ash filter and resins } \\
\text { in a low temperature oxygen } \\
\text { plasma asher or digest with } \\
\mathrm{HNO}_{3}: \mathrm{HClO}_{4}\end{array}$ & $\begin{array}{l}\text { ICP-AES; } \\
\text { NIOSH } 8310\end{array}$ & $0.1 \mu \mathrm{g} / \mathrm{sample}$ & $80 \%$ & NIOSH 1994b \\
\hline Urine & Diluted 1:1 in water & STPGFAA & $0.56 \mu \mathrm{g} / \mathrm{L}$ & $100.7 \%$ & $\begin{array}{l}\text { Oliveira et al. } \\
2000\end{array}$ \\
\hline $\begin{array}{l}\text { Blood or } \\
\text { tissue }\end{array}$ & $\begin{array}{l}\text { Acid digestion in 3:1:1 (v/v/v) } \\
\mathrm{HNO}_{3}: \mathrm{HClO}_{4}: \mathrm{H}_{2} \mathrm{SO}_{4}\end{array}$ & $\begin{array}{l}\text { ICP-AES; } \\
\text { NIOSH } 8005\end{array}$ & $\begin{array}{l}1 \mu \mathrm{g} / 100 \mathrm{~g} \\
\text { blood; } 0.2 \mu \mathrm{g} / \mathrm{g} \\
\text { tissue }\end{array}$ & $\begin{array}{l}86 \% \text { in } \\
\text { blood }\end{array}$ & NIOSH 1994b \\
\hline $\begin{array}{l}\text { Serum or } \\
\text { urine }\end{array}$ & $\begin{array}{l}\text { Sample }(10 \mu \mathrm{L}) \text { injected into } \\
\text { graphite furnace with equal } \\
\text { volume of } 30 \% \text { hydrogen } \\
\text { peroxide; pyrolyzed at } \\
1,200^{\circ} \mathrm{C} \text {; atomized at } 2,100- \\
2,200^{\circ} \mathrm{C}\end{array}$ & ETAAS & $0.2 \mu \mathrm{g} / \mathrm{L}$ & $\begin{array}{l}95-97 \% \text { at } \\
1-20 \mu \mathrm{g} / \mathrm{L}\end{array}$ & $\begin{array}{l}\text { Todorovska et } \\
\text { al. } 2002\end{array}$ \\
\hline $\begin{array}{l}\text { Lung } \\
\text { tissue }\end{array}$ & $\begin{array}{l}\text { Acid digestion in 4:2:1 (v/v/v) } \\
\mathrm{HNO}_{3}: \mathrm{HClO}_{4}: \mathrm{H}_{2} \mathrm{SO}_{4}\end{array}$ & $\begin{array}{l}\text { Electrothermal } \\
\text { AAS }\end{array}$ & $5 \mathrm{ng} / \mathrm{g}$ & No data & $\begin{array}{l}\text { Svenes and } \\
\text { Andersen } \\
1998\end{array}$ \\
\hline
\end{tabular}

af substantial quantities of iron are present (e.g., whole blood, tissues), hydrochloric acid is added, and the resulting ferric chloride is extracted with methyl isobutyl ketone.

AAS = atomic absorption spectrometry; ETAAS = electrothermal atomic absorption spectrometry; $\mathrm{HClO}_{4}=$ perchloric acid; $\mathrm{HNO}_{3}=$ nitric acid; $\mathrm{H}_{2} \mathrm{SO}_{4}=$ sulfuric acid; ICP-AES = inductively coupled plasma-atomic emission spectroscopy; Ni = nickel; NIOSH = National Institute for Occupational Safety and Health; STPGFAA = stabilized temperature graphite furnace atomic absorption; $v$ = volume 


\subsection{ENVIRONMENTAL SAMPLES}

Analytical methods that detect nickel in environmental samples generally determine the total nickel content of the sample; determining specific nickel compounds is difficult. Filtering a water sample through a $0.45-\mu \mathrm{m}$ membrane filter can distinguish between total and dissolved nickel (Martin et al. 1992). The most common methods used to detect nickel in environmental samples are AAS, either flame or graphite furnace, ICP-AES, or ICP-MS. Nickel in water and waste water samples can be analyzed using ASTM Test Methods D1976 (ICP-AES) (ASTM 2000) and D5673 (ICP-MS) (ASTM 2000) or EPA Test Methods 249.1 (atomic absorption, direct aspiration) (EPA 1983), 249.2 (atomic absorption, furnace technique) (EPA 1983), 200.7 (ICP-AES) (EPA 1983), 200.8 (ICP-MS) (EPA 1994), 1638 (ICPMS) (EPA 1996e), and 200.12 (atomic absorption, graphite furnace technique) (EPA 1997b), or a direct current plasma atomic emission spectrophotometric method (EPA 1990b). Nickel can also be analyzed in ambient and marine water using stabilized temperature graphite furnace atomic absorption (STGFAA) detection techniques as described in EPA methods 1639 (EPA 1996d) and 200.12 (EPA 1997b), respectively, which give limits of detection for nickel concentrations ranging between 0.65 and $1.8 \mu \mathrm{g} / \mathrm{L}$ and recoveries of $>92 \%$.

Although these methods are suitable for groundwater and surface water samples and domestic and industrial effluents, the nickel concentration in some groundwater, surface water, marine water, and drinking water is often below the method detection limits. Therefore, the sample must be preconcentrated or other test methods must be used. One EPA standardized test method, 1640, uses a chelation preconcentration step to increase the detection sensitivity of the ICP-MS based assay (EPA 1996c). Two other EPA standard test methods, 200.10 and 200.13, also use preconcentration techniques in conjunction with ICP-MS (EPA 1997c) or graphite furnace AAS (EPA 1997d) detection techniques, respectively, for analysis of nickel in marine water. One method uses activated charcoal to preconcentrate nickel in natural waters, followed by elution with $20 \%$ nitric acid and analysis by inductively coupled plasma-optical emission spectrometry (ICP-OES) (Yunes et al. 2003). This method achieved a detection limit of $82 \mathrm{ng} / \mathrm{L}$. Measurement of trace metals, including nickel, in waste water, surface runoff, and seawater can be completed using an in-line system with stripping voltammetry or chronopotentiometry (Sedlak et al. 1997; van den Berg and Achterberg 1994). These methods provide rapid analysis (115 minutes) with little sample preparation. The detection limit of these methods for nickel was not stated. Recommended EPA methods for soil sediment, sludge, and solid waste are Methods 7520 (AAS) and 6010B (ICP-AES). Before the widespread use of AAS, colorimetric methods were employed, and a number of colorimetric reagents have been used (Stoeppler 1980). 
With analytical methods such as x-ray fluorescence (XRF), proton-induced x-ray emission (PIXE), and instrumental neutron activation analysis (INAA), many metals can be simultaneously analyzed without destroying the sample matrix. Of these, XRF and PIXE have good sensitivity and are frequently used to analyze nickel in environmental samples containing low levels of nickel such as air, rain, snow, and soil (Adamo et al. 1996; EPA 1999; Hansson et al. 1988; Landsberger et al. 1983; Nygren 2002; Schroeder et al. 1987; Sweet et al. 1993; Wiersema et al. 1984). The Texas Air Control Board, which uses XRF in its network of air monitors, reported a mean minimum detectable value of $6 \mathrm{ng}$ nickel $/ \mathrm{m}^{3}$ (Wiersema et al. 1984). In the EPA method IO-3.3, detection limits of 0.18 and $1.89 \mathrm{ng} / \mathrm{m}^{3}$ are reported in the analysis of nickel contained in fine (ca. $2.5 \mu \mathrm{m})$ and coarse $(>10 \mu \mathrm{m})$ particulate matter $(\mathrm{PM})$, respectively, collected on Teflon filters (EPA 1999). A detection limit of $30 \mathrm{ng} / \mathrm{L}$ was obtained using PIXE with a nonselective preconcentration step (Hansson et al. 1988). Lower detection limits of $2.37 \mathrm{ng} / \mathrm{m}^{3}$ are reported for the EPA method IO-3.6 based on dichotomous sampling for 24 hours using a Teflon filter at a sampling rate of $0.9 \mathrm{~m}^{3} /$ hour (EPA 1999). Energy dispersive x-ray analysis, in conjunction with a four-step metal extraction technique, has been used to measure the speciation of nickel in soils (Adamo et al. 1996). In these techniques, the sample (e.g., air particulates collected on a filter) is irradiated with a source of x-ray photons or protons. The excited atoms emit their own characteristic energy spectrum, which is detected with an $\mathrm{x}$-ray detector and multichannel analyzer. INAA and neutron activation analysis (NAA) with prior nickel separation and concentration have poor sensitivity and are rarely used (Schroeder et al. 1987; Stoeppler 1984).

There are other standardized analytical methods for quantifying airborne nickel. These techniques utilize an extraction procedure to isolate nickel and other trace metals from PMs collected on air sampler filters. The extraction methods typically involve the use of hot nitric acid or microwave digestion techniques, for example as described in EPA Method IO-3.1 (EPA 1999). The extracted metals are commonly analyzed using instrumental techniques as described in EPA test methods IO-3.2 (atomic absorption, furnace technique), IO-3.4 (ICP-AES), and IO-3.5 (ICP-MS) (EPA 1999), providing limits of detection for concentrations of nickel in air ranging between 0.02 and $0.10 \mathrm{ng} / \mathrm{m}^{3}$ (Table 7-2; Vousta and Samara 2002). Use of trace-metal-free acids and sample extraction methods that are designed to exclude contamination of samples from adventitious metals can yield detection limits for determining airborne nickel concentrations down to $0.013-0.02 \mathrm{ng} / \mathrm{m}^{3}$ when using ICP-MS techniques (EPA 1999; Magari et al. 2002). 
Table 7-2. Analytical Methods for Determining Nickel in Environmental Samples

\begin{tabular}{|c|c|c|c|c|c|}
\hline $\begin{array}{l}\text { Sample } \\
\text { matrix }\end{array}$ & Preparation method & $\begin{array}{l}\text { Analytical } \\
\text { method }\end{array}$ & $\begin{array}{l}\text { Sample } \\
\text { detection limit }\end{array}$ & $\begin{array}{l}\text { Percent } \\
\text { recovery }\end{array}$ & Reference \\
\hline $\begin{array}{l}\text { Air, airborne } \\
\text { particulates }\end{array}$ & $\begin{array}{l}\text { Collection on cellulose acetate } \\
\text { filter; digestion with } \\
\text { concentrated nitrated and } \\
\text { perchloric acids }\end{array}$ & $\begin{array}{l}\text { ICP-AES; } \\
\text { NIOSH } 7300\end{array}$ & $1 \mu \mathrm{g} / \mathrm{sample}$ & $\begin{array}{l}105 \% \text { at } \\
2.5 \mu \mathrm{g} \\
97 \% \text { at } \\
1 \mathrm{mg}\end{array}$ & NIOSH 1994b \\
\hline $\begin{array}{l}\text { Air, airborne } \\
\text { particulates }\end{array}$ & $\begin{array}{l}\text { Collection on glass or quartz } \\
\text { fiber filter; microwave or hot } \\
\text { acid digestion Method } 10-3.1\end{array}$ & $\begin{array}{l}\text { AAS, graphite } \\
\text { furnace; } \\
\text { Method IO-3.2 }\end{array}$ & $0.10 \mathrm{ng} / \mathrm{m}^{3}$ & No data & EPA 1999 \\
\hline $\begin{array}{l}\text { Air, airborne } \\
\text { particulates }\end{array}$ & $\begin{array}{l}\text { Collection on Teflon (fine PM) } \\
\text { and Nucleopore (coarse PM) } \\
\text { membrane filter }\end{array}$ & $\begin{array}{l}\text { XRF; } \\
\text { Method IO-3.3 }\end{array}$ & $\begin{array}{l}0.18 \mathrm{ng} / \mathrm{m}^{3} \text { (fine } \\
\mathrm{PM}) ; 1.89 \mathrm{ng} / \mathrm{m}^{3} \\
\text { (coarse PM) }\end{array}$ & No data & EPA 1999 \\
\hline $\begin{array}{l}\text { Air, airborne } \\
\text { particulates }\end{array}$ & $\begin{array}{l}\text { Collection on glass or quartz } \\
\text { fiber filter; microwave or hot } \\
\text { acid digestion Method IO-3.1 }\end{array}$ & $\begin{array}{l}\text { ICP-AES; } \\
\text { Method IO-3.4 }\end{array}$ & $3.1 \mathrm{ng} / \mathrm{m}^{3}$ & $96.4 \%$ & EPA 1999 \\
\hline $\begin{array}{l}\text { Air, airborne } \\
\text { particulates }\end{array}$ & $\begin{array}{l}\text { Collection on glass or quartz } \\
\text { fiber filter; microwave or hot } \\
\text { acid digestion Method IO-3.1 }\end{array}$ & $\begin{array}{l}\text { ICP-MS; } \\
\text { Method IO-3.5 }\end{array}$ & $0.02 \mathrm{ng} / \mathrm{m}^{3}$ & $\begin{array}{l}101.7 \% \text { at } \\
20 \mu \mathrm{g} / \mathrm{L} ; \\
102.3 \% \text { at } \\
100 \mu \mathrm{g} / \mathrm{L}\end{array}$ & EPA 1999 \\
\hline $\begin{array}{l}\text { Air, airborne } \\
\text { particulates }\end{array}$ & $\begin{array}{l}\text { Collection on PCTE or Teflon } \\
\text { filters, or Kapton impaction } \\
\text { surface }\end{array}$ & $\begin{array}{l}\text { PIXE; } \\
\text { Method IO-3.6 }\end{array}$ & $2.37 \mathrm{ng} / \mathrm{m}^{3}$ & No data & EPA 1999 \\
\hline $\begin{array}{l}\text { Air, airborne } \\
\mathrm{Ni}(\mathrm{CO})_{4}\end{array}$ & $\begin{array}{l}\text { Collection on low-Ni charcoal } \\
\text { sorbent tube; ultrasonic } \\
\text { digestion with nitric acid }\end{array}$ & $\begin{array}{l}\text { Graphite } \\
\text { furnace AAS; } \\
\text { NIOSH } 6007\end{array}$ & $0.01 \mu \mathrm{g} / \mathrm{sample}$ & $\begin{array}{l}93 \% \text { at } \\
5 \text { to } \\
121 \mu \mathrm{g} / \mathrm{m}^{3}\end{array}$ & NIOSH 1994b \\
\hline Water & $\begin{array}{l}\text { Acid digestion in mixture of } \\
\text { nitric, sulfuric, and perchloric } \\
\text { acids }\end{array}$ & $\begin{array}{l}\text { Electro- } \\
\text { thermal AAS; } \\
\text { Method } 11\end{array}$ & $\begin{array}{l}0.2 \mu \mathrm{g} \mathrm{Ni} / \mathrm{L} \\
\text { fluids }\end{array}$ & $\begin{array}{l}98 \% \text { at } \\
5 \mu \mathrm{g} \mathrm{Ni} / \mathrm{L} \\
97 \% \text { at } \\
8 \mu \mathrm{g} \mathrm{Ni} / \mathrm{L}\end{array}$ & IARC 1986 \\
\hline Water & $\begin{array}{l}\text { Preconcentrated on activated } \\
\text { charcoal; eluted with } 20 \% \\
\text { nitric acid }\end{array}$ & ICP-OES & $82 \mathrm{ng} / \mathrm{L}$ & $\begin{array}{l}96.0 \% \text { at } \\
2.0 \mu \mathrm{g} / \mathrm{L}\end{array}$ & $\begin{array}{l}\text { Yunes et al. } \\
2003\end{array}$ \\
\hline $\begin{array}{l}\text { Drinking, } \\
\text { domestic, } \\
\text { surface } \\
\text { water; } \\
\text { industrial } \\
\text { waste water }\end{array}$ & Filter and acidify sample & $\begin{array}{l}\text { ICP-AES; } \\
\text { Method D1976 }\end{array}$ & $15 \mu \mathrm{g} / \mathrm{L}$ & $92 \%$ & ASTM 2000 \\
\hline $\begin{array}{l}\text { Drinking } \\
\text { water, } \\
\text { surface } \\
\text { water, } \\
\text { groundwat }\end{array}$ & Filter and acidify sample & $\begin{array}{l}\text { ICP-MS; } \\
\text { Method D5673 }\end{array}$ & $4 \mu \mathrm{g} / \mathrm{L}$ & $104 \%$ & ASTM 2000 \\
\hline
\end{tabular}




\section{Table 7-2. Analytical Methods for Determining Nickel in Environmental Samples}

\begin{tabular}{|c|c|c|c|c|c|}
\hline $\begin{array}{l}\text { Sample } \\
\text { matrix }\end{array}$ & Preparation method & $\begin{array}{l}\text { Analytical } \\
\text { method }\end{array}$ & $\begin{array}{l}\text { Sample } \\
\text { detection limit }\end{array}$ & $\begin{array}{l}\text { Percent } \\
\text { recovery }\end{array}$ & Reference \\
\hline \multirow[t]{3}{*}{$\begin{array}{l}\text { Water, } \\
\text { waste water }\end{array}$} & Acid digestion & $\begin{array}{l}\text { AAS, direct } \\
\text { aspiration; } \\
\text { Method } 249.1\end{array}$ & $0.04 \mathrm{mg} / \mathrm{L}$ & $\begin{array}{l}100 \% \text { at } \\
0.20 \mathrm{mg} \\
\mathrm{Ni} / \mathrm{L} ; 97 \% \\
\text { at } 1.0 \mathrm{mg} \\
\mathrm{Ni} / \mathrm{L} ; 93 \% \\
\text { at } 5.0 \mathrm{mg} \\
\mathrm{Ni} / \mathrm{L}\end{array}$ & EPA 1983 \\
\hline & $\begin{array}{l}\text { Acid digestion; sample } \\
\text { solutions should contain } \\
0.5 \% \mathrm{HNO}_{3}\end{array}$ & $\begin{array}{l}\text { AAS, direct } \\
\text { aspiration; } \\
\text { Method } 249.2\end{array}$ & $1 \mu \mathrm{g} / \mathrm{L}$ & $100 \%$ & EPA 1983 \\
\hline & $\begin{array}{l}\text { Filter and acidify sample } \\
\text { (dissolved } \mathrm{Ni} \text { ); digest in nitric } \\
\text { acid (total recoverable } \mathrm{Ni} \text { ) }\end{array}$ & $\begin{array}{l}\text { ICP-AES; } \\
\text { Method } 200.7\end{array}$ & $5 \mu \mathrm{g} / \mathrm{L}$ & $\begin{array}{l}\text { Accuracy: } \\
6.7 \% \text { at } \\
30 \mu \mathrm{g} / \mathrm{L} ; \\
8.3 \% \text { at } \\
60 \mathrm{~g} / \mathrm{L} ; \\
2.0 \% \text { at } \\
120 \mathrm{~g} / \mathrm{L}\end{array}$ & $\begin{array}{l}\text { EPA 1983, } \\
\text { 1994; Martin et } \\
\text { al. } 1992\end{array}$ \\
\hline $\begin{array}{l}\text { Water, } \\
\text { waste water }\end{array}$ & $\begin{array}{l}\text { Filter and acidify sample } \\
\text { (dissolved Ni); digest in nitric } \\
\text { acid (total recoverable Ni) }\end{array}$ & $\begin{array}{l}\text { ICP-MS; } \\
\text { Method } 200.8\end{array}$ & $0.5 \mu \mathrm{g} / \mathrm{L}$ & $\begin{array}{l}100.1 \% \text { at } \\
100 \mu \mathrm{g} / \mathrm{L}\end{array}$ & EPA 1994 \\
\hline $\begin{array}{l}\text { Water, } \\
\text { waste water }\end{array}$ & Acid digestion & $\begin{array}{l}\text { AAS, graphite } \\
\text { furnace; } \\
\text { Method } 7521\end{array}$ & $1 \mu \mathrm{g} / \mathrm{L}$ & No data & EPA 2002 \\
\hline $\begin{array}{l}\text { Water, } \\
\text { waste water }\end{array}$ & $\begin{array}{l}\text { Digestion with nitric and } \\
\text { hydrochloric acids }\end{array}$ & $\begin{array}{l}\text { ICP-AES; } \\
\text { Method 6010C }\end{array}$ & $10 \mu \mathrm{g} / \mathrm{L}$ & $\begin{array}{l}98 \% \text { at } \\
250 \mu \mathrm{g} / \mathrm{L} ; \\
92 \% \text { at } \\
60 \mu \mathrm{g} / \mathrm{L} ; \\
93 \% \text { at } \\
30 \mu \mathrm{g} / \mathrm{L}\end{array}$ & EPA 2002 \\
\hline $\begin{array}{l}\text { Marine } \\
\text { water }\end{array}$ & $\begin{array}{l}\text { Acidified with nitric acid, } \\
\text { undissolved material removed }\end{array}$ & $\begin{array}{l}\text { STPGFAA; } \\
\text { Method } 200.12\end{array}$ & $1.8 \mu \mathrm{g} / \mathrm{L}$ & $\begin{array}{l}92 \% \text { at } \\
15 \mu \mathrm{g} / \mathrm{L} ; \\
93 \% \text { at } \\
37.5 \mu \mathrm{g} / \mathrm{L}\end{array}$ & EPA 1997b \\
\hline Snow & $\begin{array}{l}\text { Samples acidified with nitric } \\
\text { acid }\end{array}$ & ICP-MS & $0.7 \mathrm{pg} / \mathrm{L}$ & $95 \%$ & $\begin{array}{l}\text { Barbante et al. } \\
2002\end{array}$ \\
\hline $\begin{array}{l}\text { Soil, } \\
\text { sediment, } \\
\text { sludge, solid } \\
\text { waste }\end{array}$ & $\begin{array}{l}\text { Digestion with nitric and } \\
\text { hydrochloric acids; } \\
\text { Method } 3050\end{array}$ & $\begin{array}{l}\text { ICP-AES; } \\
\text { Method 6010B }\end{array}$ & $10 \mu \mathrm{g} / \mathrm{L}$ & $\begin{array}{l}98 \% \text { at } \\
250 \mu \mathrm{g} / \mathrm{L} \\
93 \% \text { at } \\
50 \mu \mathrm{g} / \mathrm{L}\end{array}$ & $\begin{array}{l}\text { EPA 1986b; } \\
\text { EPA } 2002\end{array}$ \\
\hline $\begin{array}{l}\text { Soil, } \\
\text { sediment, } \\
\text { sludge, solid } \\
\text { waste }\end{array}$ & $\begin{array}{l}\text { Digestion with nitric and } \\
\text { hydrochloric acids; } \\
\text { Method } 3050\end{array}$ & $\begin{array}{l}\text { AAS, direct } \\
\text { aspiration; } \\
\text { Method } 7520\end{array}$ & $0.04 \mathrm{mg} / \mathrm{L}$ & $\begin{array}{l}100 \% \text { at } \\
0.2 \mathrm{mg} / \mathrm{L} ; \\
97 \% \text { at } \\
1.0 \mathrm{mg} / \mathrm{L} ; \\
93 \% \text { at } \\
5.0 \mathrm{mg} / \mathrm{L}\end{array}$ & EPA 1986b \\
\hline
\end{tabular}




\section{Table 7-2. Analytical Methods for Determining Nickel in Environmental Samples}

\begin{tabular}{|c|c|c|c|c|c|}
\hline $\begin{array}{l}\text { Sample } \\
\text { matrix }\end{array}$ & Preparation method & $\begin{array}{l}\text { Analytical } \\
\text { method }\end{array}$ & $\begin{array}{l}\text { Sample } \\
\text { detection limit }\end{array}$ & $\begin{array}{l}\text { Percent } \\
\text { recovery }\end{array}$ & Reference \\
\hline $\begin{array}{l}\text { Soil (total } \\
\text { nickel) }\end{array}$ & $\begin{array}{l}\text { Digest with nitric acid; oxidize } \\
\text { with hydrogen peroxide at } \\
450{ }^{\circ} \mathrm{C} \text { to destroy organic } \\
\text { matter; digest with sulfuric and } \\
\text { hydrofluoric acids, followed by } \\
\text { digestion with nitric, sulfuric, } \\
\text { and perchloric acids }\end{array}$ & AAS & $0.1 \mu \mathrm{g} \mathrm{Ni} / \mathrm{g}$ soil & No data & $\begin{array}{l}\text { Baker and } \\
\text { Amacher } 1982\end{array}$ \\
\hline $\begin{array}{l}\text { Soil (DPTA } \\
\text { extractable) }\end{array}$ & $\begin{array}{l}\text { Shake soil with } 0.005 M \text { DPTA } \\
\text { extraction solution for } 2 \text { hours }\end{array}$ & AAS & No data & No data & $\begin{array}{l}\text { Baker and } \\
\text { Amacher } 1982\end{array}$ \\
\hline $\begin{array}{l}\text { Soil (acid } \\
\text { extractable) }\end{array}$ & $\begin{array}{l}\text { Shake soil with } 0.1 \mathrm{~N} \text { hydro- } \\
\text { chloric acid for } 5 \text { minutes; } \\
\text { complete } 3 \text { times }\end{array}$ & AAS & No data & No data & $\begin{array}{l}\text { Baker and } \\
\text { Amacher } 1982\end{array}$ \\
\hline \multirow[t]{2}{*}{$\begin{array}{l}\text { Soil and } \\
\text { sediment }\end{array}$} & $\begin{array}{l}\text { Sample is heated to } 110^{\circ} \mathrm{C} \text { in } \\
\text { a mixture of hydrochloric, } \\
\text { nitric, perchloric, and hydro- } \\
\text { fluoric acids and evaporated } \\
\text { to dryness, and then treated } \\
\text { with aqua regia }\end{array}$ & ICP-AES & $3 \mathrm{ppm}$ & $92-114 \%$ & USGS 2002 \\
\hline & $\begin{array}{l}\text { Sample is heated to } 110^{\circ} \mathrm{C} \text { in } \\
\text { a mixture of hydrochloric, } \\
\text { nitric, perchloric, and hydro- } \\
\text { fluoric acids and evaporated } \\
\text { to dryness, and then treated } \\
\text { with aqua regia }\end{array}$ & ICP-MS & 0.16 ppm & $91-104 \%$ & USGS 2002 \\
\hline Food & $\begin{array}{l}\text { Wet oxidation with sulfuric } \\
\text { acid, complexation with } \\
\text { ammonium tetramethylenedi- } \\
\text { thiocarbamate followed by } \\
\text { extraction with methyl butyl } \\
\text { ketone }^{\mathrm{a}}\end{array}$ & $\begin{array}{l}\text { AAS; } \\
\text { Method } 17\end{array}$ & $20 \mu \mathrm{g} / \mathrm{kg}$ & No data & IARC 1986 \\
\hline $\begin{array}{l}\text { Edible } \\
\text { tissues }\end{array}$ & $\begin{array}{l}\text { Samples were homogenized, } \\
\text { mixed with magnesium nitrate } \\
\text { solution }(6.67 \%) \text {, lyophilized, } \\
\text { dry ashed twice, and } \\
\text { dissolved in hydrochloric acid }\end{array}$ & AAS & 0.15 ppm & $101 \%$ & $\begin{array}{l}\text { Coleman et al. } \\
1992\end{array}$ \\
\hline
\end{tabular}




\section{Table 7-2. Analytical Methods for Determining Nickel in Environmental Samples}

\begin{tabular}{llllll}
\hline $\begin{array}{l}\text { Sample } \\
\text { matrix }\end{array}$ & Preparation method & $\begin{array}{l}\text { Analytical } \\
\text { method }\end{array}$ & $\begin{array}{l}\text { Sample } \\
\text { detection limit }\end{array}$ & $\begin{array}{l}\text { Percent } \\
\text { recovery }\end{array}$ & Reference \\
\hline Food & $\begin{array}{l}\text { Samples were homogenized } \\
\text { then solubilized using } \\
\text { atmospheric pressure } \\
\text { microwave digestion in nitric } \\
\text { acid }\end{array}$ & & $7.0 \mathrm{ng} / \mathrm{g}$ & $52-96 \%{ }^{\mathrm{b}}$ & Melnyk et al. \\
& & & & 2003 \\
& & & & \\
\hline
\end{tabular}

${ }^{\mathrm{a}}$ The digestion procedure is not satisfactory for fats and oils. For these substances, sulfuric acid and $50 \%$ hydrogen peroxide should be used.

${ }^{b}$ Percent recoveries of nickel in food samples spiked at 2 times the limit of detection (LOD) of nickel were given as: rice cereal, 94\%; fatty food, 95\%; beverage, 93\%; duplicate diet 1, 52\%; and duplicate diet 2, 90\%. In food samples spiked with nickel at 5 times the LOD, the percent recoveries were given as: fatty food, 96\%; beverage, 94\%; duplicate diet 1, 81\%; and duplicate diet 2, 81\%.

AAS = atomic absorption spectrometry; DPTA = diethylenetriamine pentaacetic acid; $\mathrm{HNO}_{3}=$ nitric acid; ICPAES = inductively coupled plasma-atomic emission spectroscopy; ICP-OES = inductively coupled plasma-optical emission spectrometry; $\mathrm{Ni}=$ nickel; $\mathrm{Ni}(\mathrm{CO})_{4}=$ nickel carbonyl; $\mathrm{NIOSH}=$ National Institute for Occupational Safety and Health; PCTE = polycarbonate track etched; PIXE = proton induced $\mathrm{x}$-ray emission spectroscopy; $\mathrm{PM}=$ particulate matter; STPGFAA = stabilized temperature graphite furnace atomic absorption; $\mathrm{XRF}=\mathrm{x}-\mathrm{ray}$ fluorescence 
Contamination and loss are the main concerns when determining trace metals (Christensen 1995). Nickel-containing knives and needles should be avoided when collecting specimens. A study that compared the effects of using different dissecting tools on trace metal analysis did not report significant differences in the nickel content of fish or mussel samples dissected with stainless steel, lexan, titanium, or Teflon-coated instruments (Iyengar 1986). Contamination can result from impurities in reagents or laboratory apparatus and laboratory dust. Losses may also occur when the analyte adsorbs onto container walls. When collecting air samples on filters, one should be aware that filter material can contain high and variable trace metal concentrations. Glass fiber filters may contain $<80 \mathrm{ng} / \mathrm{cm}^{2}$ of nickel. Silver membrane, cellulose, and polystyrene filters may contain $\approx 100 \mathrm{ng} / \mathrm{cm}^{2}$ of nickel (Schroeder et al. 1987). Trace metals in blanks of different filter types and in different filters of the same type may vary from 5 to 20\% (Brzezinska-Paudyn et al. 1986).

Some investigators have characterized the forms of nickel in an environmental sample by using successively stronger solvents. Each fraction solubilized is subsequently analyzed for nickel by atomic absorption or other procedures. In air, where the speciation of nickel is less complex, a method of sequential selective leaching has been developed to determine the amount of nickel in four phase categories of a dust sample, namely, soluble nickel, sulfidic nickel, metallic nickel, and refractory nickel oxides (Zatka et al. 1992). Soluble nickel salts, mostly nickel sulfates, are leached at pH 4; sulfidic nickel is next solubilized with a peroxide-citrate solution; and metallic nickel is oxidized with bromine. The residue consists of refractory nickel oxides. Wong and $\mathrm{Wu}$ (1991) used an adsorptive stripping voltammetry method to determine different forms of nickel in air at a nickel manufacturing facility. The method distinguished between metallic nickel ions and nickel oxides. The results showed that speciation of nickel from several samples taken at the same location were highly variable. Although it is important to characterize the nickel contained in an environmental sample, methods that determine nickel speciation are difficult and not in widespread use.

Analytical methods and detection limits for standard methods of determining nickel in environmental media are reported in Table 7-2. If the determination of dissolved nickel is required, samples should be filtered with a $0.45-\mu \mathrm{m}$ membrane filter.

\subsection{ADEQUACY OF THE DATABASE}

Section 104(i)(5) of CERCLA, as amended, directs the Administrator of ATSDR (in consultation with the Administrator of EPA and agencies and programs of the Public Health Service) to assess whether 
adequate information on the health effects of nickel is available. Where adequate information is not available, ATSDR, in conjunction with the National Toxicology Program (NTP), is required to assure the initiation of a program of research designed to determine the health effects (and techniques for developing methods to determine such health effects) of nickel.

The following categories of possible data needs have been identified by a joint team of scientists from ATSDR, NTP, and EPA. They are defined as substance-specific informational needs that if met would reduce the uncertainties of human health assessment. This definition should not be interpreted to mean that all data needs discussed in this section must be filled. In the future, the identified data needs will be evaluated and prioritized, and a substance-specific research agenda will be proposed.

\subsubsection{Identification of Data Needs}

\section{Methods for Determining Biomarkers of Exposure and Effect.}

Exposure. Nickel concentrations in hair, nails, blood, or urine are elevated in exposed individuals. A correlation has been established between nickel levels in urine, plasma, and feces in occupationally exposed workers and nickel levels in air (Angerer and Lehnert 1990; Bernacki et al. 1978; Hassler et al. 1983). If the identity of the nickel compounds to which workers are exposed is known, nickel levels in urine and plasma can be used as a biomarker for nickel exposure (Sunderman 1993). Available analytical methods can determine the nickel levels in these media in both unexposed and occupationally exposed persons. Also, reference values for nickel measured in urine and blood in individuals exposed to low levels of nickel are needed to establish norms for the general population (Christensen 1995).

Methods for determining exposure of individuals through the assessment of plasma or urine levels of nickel are adequate, but further method development is needed to determine nickel speciation in biological media. Also, development of assays that make use of biological markers, such as changes in gene expression in blood cells or protein levels in serum, as measured with gene or protein arrays would be useful not only in providing an alternative method for assessing nickel exposure in occupational and public populations, but also in providing information on biological effects to nickel exposures.

Effect. There are no unique biomarkers of effect for nickel. 


\section{Methods for Determining Parent Compounds and Degradation Products in Environmental}

Media. Methods for determining total nickel in environmental media are well developed and adequate. Standardized methods are available from several sources including EPA (EPA 1983, 1986b, 1999, 2002). Most analytical methods measure total nickel content. Sequential extraction techniques are sometimes used to determine the nature of nickel in particles, e.g., they are exchangeable, adsorbed, easily reducible, or organically bound (Adamo et al. 1995; Lottermoser 2002; Rudd et al. 1988; Rybicka 1989). There is a need for more development in this area and the adoption of standard methods for determining nickel species or forms of nickel in various media.

\subsubsection{Ongoing Studies}

Information on ongoing research studies involving sample collection and the characterization and quantification of nickel was derived from a search of Federal Research in Progress (FEDRIP 2004) and are summarized in Table 7-3. 


\section{Table 7-3. Ongoing Studies Involving Sample Collection and the Characterization and Quantification of Nickel ${ }^{\mathrm{a}}$}

\begin{tabular}{llll}
\hline Investigator & Affiliation & Research description & Sponsor \\
\hline Barker, A & $\begin{array}{l}\text { University of } \\
\text { Massachusets }\end{array}$ & $\begin{array}{l}\text { Validate commonly used analytical } \\
\text { methods for measuring trace elements in a } \\
\text { variety of soils, water, and plants }\end{array}$ & Hatch \\
Odom, JW & Auburn University & $\begin{array}{l}\text { Develop analytical techniques for } \\
\text { determining total and extractable heavy } \\
\text { metals in Alabama soils and plant materials } \\
\text { and assess the normal occurrence of } \\
\text { metals in select soil profiles }\end{array}$ & Hatch \\
& &
\end{tabular}

${ }^{\mathrm{a}}$ FEDRIP 2004 



\section{REGULATIONS AND ADVISORIES}

ATSDR has derived an intermediate-duration inhalation minimal risk level (MRL) of $0.0002 \mathrm{mg} \mathrm{Ni} / \mathrm{m}^{3}$ for nickel. This MRL is based on a NOAEL of $0.06 \mathrm{mg} \mathrm{Ni} / \mathrm{m}^{3}$ and a LOAEL of $0.11 \mathrm{mg} \mathrm{Ni} / \mathrm{m}^{3}$ for chronic active lung inflammation in rats exposed to nickel sulfate 6 hours/day, 5 days/week for 13 weeks (NTP 1996c). The MRL was derived by dividing the NOAEL ${ }_{\mathrm{HEC}}$ of $0.0052 \mathrm{mg} \mathrm{Ni} / \mathrm{m}^{3}$ by an uncertainty factor of 30 (3 for animal to human extrapolation with dosimetric adjustments and 10 for human variability).

ATSDR has derived a chronic-duration inhalation MRL of $9 \times 10^{-5} \mathrm{mg} \mathrm{Ni} / \mathrm{m}^{3}$ for nickel. This MRL is based on a NOAEL of $0.03 \mathrm{mg} \mathrm{Ni} / \mathrm{m}^{3}$ and a LOAEL of $0.06 \mathrm{mg} \mathrm{Ni} / \mathrm{m}^{3}$ for chronic active lung inflammation and bronchialization in rats exposed to nickel sulfate 6 hours/day, 5 days/week for 2 years (NTP 1996c). The MRL was derived by dividing the NOAEL ${ }_{\mathrm{HEC}}$ of $0.0027 \mathrm{mg} \mathrm{Ni} / \mathrm{m}^{3}$ by an uncertainty factor of 30 (3 for animal to human extrapolation with dosimetric adjustments and 10 for human variability).

EPA (IRIS 2005) derived an oral reference dose (RfD) of $0.02 \mathrm{mg} / \mathrm{kg} /$ day for nickel soluble salts. The RfD was based on a NOAEL of $5 \mathrm{mg} / \mathrm{kg} /$ day and a LOAEL of $50 \mathrm{mg} / \mathrm{kg} /$ day for decreased body weight and organ weight in rats exposed to dietary nickel for 2 years (Ambrose et al. 1976). The NOAEL was divided by an uncertainty factor of 300 (10 for animal to human extrapolation, 10 to protect sensitive individuals, and 3 for inadequacies in the reproductive toxicity studies).

The Department of Health and Human Services (NTP 2002) has determined that metallic nickel may reasonably be anticipated to be a carcinogen and that nickel compounds are known to be human carcinogens. Similarly, IARC classified metallic nickel in group 2B (possibly carcinogenic to humans) and nickel compounds in group 1 (carcinogenic to humans). EPA has classified nickel refinery dust and nickel subsulfide in Group A (human carcinogen) (IRIS 2005). Other nickel compounds have not been classified by the EPA. Based on the occupational data, inhalation unit risk levels of $2.4 \times 10^{-4}\left(\mu \mathrm{g} / \mathrm{m}^{3}\right)^{-1}$ and $4.8 \times 10^{-4}\left(\mu \mathrm{g} / \mathrm{m}^{3}\right)^{-1}$ were derived for nickel refinery dust and nickel subsulfide, respectively (IRIS 2005).

In an attempt to reduce the prevalence of nickel sensitivity, the European Union has passed a directive to restrict the use of nickel beginning in February 1996 (Delescluse and Dinet 1994). The directive forbids the use of nickel in objects introduced into pierced ears and other parts of the human body during 
epithelialization of the wound. It forbids the use of nickel in products placed in direct and prolonged contact with the skin (e.g., earrings, watches, clothing accessories). The use of nickel is also forbidden in accessories that are plated with another metal, except if the plating is strong enough to restrict liberation of nickel to $<0.5 \mu \mathrm{g} / \mathrm{cm}^{2} /$ week during a normal use of 2 years.

International, national, and state guidelines and regulations regarding exposure to nickel and its compounds are summarized in Table 8-1. 


\section{Table 8-1. Regulations and Guidelines Applicable to Nickel and Nickel Compounds}

\begin{tabular}{|c|c|c|c|}
\hline Agency & Description & Information & Reference \\
\hline \multicolumn{4}{|c|}{$\begin{array}{l}\text { INTERNATIONAL } \\
\text { Guidelines: }\end{array}$} \\
\hline IARC & $\begin{array}{l}\text { Carcinogenicity classification } \\
\text { Nickel compounds } \\
\text { Nickel, metallic }\end{array}$ & $\begin{array}{l}\text { Group } 1^{\mathrm{a}} \\
\text { Group 2B }\end{array}$ & IARC 1990 \\
\hline \multirow[t]{2}{*}{ WHO } & $\begin{array}{l}\text { Air quality guideline } \\
\text { Nickel unit risk }\end{array}$ & $3.8 \times 10^{-5}\left(\mu \mathrm{g} / \mathrm{m}^{3}\right)^{-1}$ & WHO 2000 \\
\hline & $\begin{array}{l}\text { Drinking water guideline } \\
\text { Nickel }\end{array}$ & $0.02 \mathrm{mg} / \mathrm{L}$ & WHO 1998 \\
\hline
\end{tabular}

NATIONAL

Regulations and Guidelines:

a. Air:

ACGIH

$\mathrm{NIOSH}$

OSHA

OSHA
TLV (8-hour TWA)

Nickel, elemental (as $\mathrm{Ni}$ )

Nickel, soluble inorganic

compounds

Nickel, insoluble inorganic

compounds

Nickel subsulfide (as Ni)

Nickel carbonyl (as Ni)

REL (10-hour TWA)

Nickel ${ }^{\mathrm{c}}$

IDLH

Nickel carbonyl ${ }^{\mathrm{C}}$

IDLH

PEL (8-hour TWA) for general industry

Nickel, metal and insoluble compounds (as $\mathrm{Ni}$ )

Nickel, soluble compounds (as $\mathrm{Ni}$ )

Nickel carbonyl

PEL (8-hour TWA) for construction industry

Nickel, metal and insoluble

compounds (as $\mathrm{Ni}$ )

Nickel, soluble compounds (as $\mathrm{Ni}$ )

Nickel carbonyl

PEL (8-hour TWA) for shipyard industry

Nickel, metal and insoluble

compounds (as Ni)

Nickel, soluble compounds (as $\mathrm{Ni}$ )

Nickel carbonyl

Highly hazardous chemicals, toxics, and reactives

Nickel carbonyl

Threshold quantity

$$
\begin{aligned}
& 1.5 \mathrm{mg} / \mathrm{m}^{3} \\
& 0.1 \mathrm{mg} / \mathrm{m}^{3} \\
& 0.2 \mathrm{mg} / \mathrm{m}^{3} \\
& 0.1 \mathrm{mg} / \mathrm{m}^{3} \\
& 0.05 \mathrm{ppm}
\end{aligned}
$$

$0.015 \mathrm{mg} / \mathrm{m}^{3}$

$10 \mathrm{mg} / \mathrm{m}^{3}$

$0.001 \mathrm{ppm}$

$2 \mathrm{ppm}$

$1.0 \mathrm{mg} / \mathrm{m}^{3}$

$1.0 \mathrm{mg} / \mathrm{m}^{3}$

$0.007 \mathrm{mg} / \mathrm{m}^{3}$

$1.0 \mathrm{mg} / \mathrm{m}^{3}$

$1.0 \mathrm{mg} / \mathrm{m}^{3}$

$0.007 \mathrm{mg} / \mathrm{m}^{3}$

$1.0 \mathrm{mg} / \mathrm{m}^{3}$

$1.0 \mathrm{mg} / \mathrm{m}^{3}$

$0.007 \mathrm{mg} / \mathrm{m}^{3}$

ACGIH 2003

NIOSH 2003a,

2003b

OSHA 2003a

29 CFR 1910.1000, Table Z-1

OSHA 2003e

29 CFR 1926.55

OSHA 2003d

29 CFR 1915.1000

OSHA 2003b, $2003 f$

29 CFR 1926.64, 29 CFR 1910.119,

150 pounds
Appendix A 
Table 8-1. Regulations and Guidelines Applicable to Nickel and Nickel Compounds

\begin{tabular}{|c|c|c|c|}
\hline Agency & Description & Information & Reference \\
\hline \multicolumn{4}{|c|}{ NATIONAL (cont.) } \\
\hline \multicolumn{4}{|l|}{ b. Water } \\
\hline \multirow[t]{5}{*}{ EPA } & Drinking water health advisories & & EPA 2002a \\
\hline & 1-day (10-kg child) & $1.0 \mathrm{mg} / \mathrm{L}$ & \\
\hline & 10-day (10-kg child) & $1.0 \mathrm{mg} / \mathrm{L}$ & \\
\hline & DWEL $^{g}$ & $0.7 \mathrm{mg} / \mathrm{L}$ & \\
\hline & Lifetime $^{\mathrm{h}}$ & $0.1 \mathrm{mg} / \mathrm{L}$ & \\
\hline \multicolumn{4}{|l|}{ c. Food } \\
\hline \multirow[t]{4}{*}{ FDA } & Bottled drinking water & & FDA 2003a \\
\hline & Nickel & $0.1 \mathrm{mg} / \mathrm{L}$ & 21 CFR 165.110 \\
\hline & $\begin{array}{l}\text { Generally recognized as safe as a } \\
\text { direct human food ingredient with no } \\
\text { limitation other than current good } \\
\text { manufacturing practices }\end{array}$ & Nickel & $\begin{array}{l}\text { FDA } 2003 b \\
21 \text { CFR } 184.1537\end{array}$ \\
\hline & $\begin{array}{l}\text { Indirect food additives; components of } \\
\text { paper and paperboard }\end{array}$ & Nickel & $\begin{array}{l}\text { FDA } 2003 c \\
21 \text { CFR } \\
176.180(b)(2)\end{array}$ \\
\hline \multicolumn{4}{|l|}{ d. Other } \\
\hline ACGIH & $\begin{array}{l}\text { Carcinogenicity classification } \\
\text { Nickel subsulfide }\end{array}$ & $A 1^{i}$ & ACGIH 2003 \\
\hline EPA & $\begin{array}{l}\text { Carcinogenicity classification } \\
\text { Nickel } \\
\text { Nickel refinery dust } \\
\text { Nickel carbonyl } \\
\text { Nickel subsulfide }\end{array}$ & $\begin{array}{l}\text { Not evaluated } \\
A^{j} \\
B 2^{k} \\
A^{j}\end{array}$ & IRIS 2005 \\
\hline \multirow[t]{2}{*}{ EPA } & $\begin{array}{l}\text { RfC } \\
\text { Nickel } \\
\text { Nickel refinery dust } \\
\text { Nickel carbonyl } \\
\text { Nickel subsulfide }\end{array}$ & $\begin{array}{l}\text { No data } \\
\text { No data } \\
\text { No data } \\
\text { No data }\end{array}$ & IRIS 2005 \\
\hline & $\begin{array}{l}\text { RfD } \\
\text { Nickel } \\
\text { Nickel refinery dust } \\
\text { Nickel carbonyl } \\
\text { Nickel subsulfide }\end{array}$ & $\begin{array}{l}0.02 \mathrm{mg} / \mathrm{kg} / \mathrm{day} \\
\text { No data } \\
\text { No data } \\
\text { No data }\end{array}$ & IRIS 2005 \\
\hline \multirow[t]{2}{*}{ NTP } & $\begin{array}{l}\text { Carcinogenicity } \\
\text { Nickel, metallic }\end{array}$ & $\begin{array}{l}\text { Reasonably } \\
\text { anticipated to be a } \\
\text { human carcinogen }\end{array}$ & NTP 2002 \\
\hline & $\begin{array}{l}\text { Carcinogenicity } \\
\text { Nickel compounds }\end{array}$ & $\begin{array}{l}\text { Known human } \\
\text { carcinogens }\end{array}$ & NTP 2002 \\
\hline \multicolumn{4}{|l|}{ STATE } \\
\hline $\begin{array}{l}\text { a. Air } \\
\text { b. Water }\end{array}$ & \multicolumn{2}{|c|}{ b. Water } & \\
\hline Arizona & $\begin{array}{l}\text { Drinking water guideline } \\
\text { Nickel, elemental }\end{array}$ & $150 \mu \mathrm{g} / \mathrm{L}$ & HSDB 2003 \\
\hline
\end{tabular}




\section{Table 8-1. Regulations and Guidelines Applicable to Nickel and Nickel Compounds}

\begin{tabular}{|c|c|c|c|}
\hline Agency & Description & Information & Reference \\
\hline \multicolumn{4}{|l|}{ STATE (cont.) } \\
\hline Massachusetts & $\begin{array}{l}\text { Drinking water guideline } \\
\text { Nickel and nickel compounds }\end{array}$ & $100 \mu \mathrm{g} / \mathrm{L}$ & HSDB 2003 \\
\hline Maine & $\begin{array}{l}\text { Drinking water guideline } \\
\quad \text { Nickel and nickel compounds }\end{array}$ & $150 \mu \mathrm{g} / \mathrm{L}$ & HSDB 2003 \\
\hline Minnesota & $\begin{array}{l}\text { Drinking water guideline } \\
\text { Nickel and nickel compounds }\end{array}$ & $100 \mu \mathrm{g} / \mathrm{L}$ & HSDB 2003 \\
\hline c. Food & No data & & \\
\hline d. Other & No data & & \\
\hline
\end{tabular}

${ }^{\mathrm{a}}$ Group 1: carcinogenic to humans

${ }^{\mathrm{b}}$ Group 2B: possibly carcinogenic to humans

${ }^{\mathrm{c}}$ Carcinogen

${ }^{\mathrm{d}}$ Class D: refers to the retention (clearance half-times of $<10$ days) for all compounds except those given for W.

${ }^{\mathrm{e}}$ The ALIs and DACs for inhalation are given for an aerosol with an activity median aerodynamic diameter (AMAD) of $1 \mu \mathrm{m}$ and for class $\mathrm{D}$ and $\mathrm{W}$ of radioactive material, which refers to their retention (clearance half-times of $<10$ days and 10-100 days, respectively) in the pulmonary region of the lung.

${ }^{\dagger}$ Class W: refers to the retention (clearance half-times of 10-100 days) for sulfides, oxides, hydroxides, halides, nitrates, and stannic phosphate.

${ }^{9}$ DWEL: a lifetime exposure concentration protection of adverse, non-cancer health effects, that assumes all of the exposure to a contaminant is from drinking water.

hLifetime: the concentration of a chemical in drinking water that is not expected to cause any adverse

noncarcinogenic effects for a lifetime of exposure. The Lifetime health advisory is based on exposure of a 70-kg

adult consuming $2 \mathrm{~L}$ water/day.

A1: confirmed human carcinogen

${ }^{\mathrm{j}} \mathrm{A}$ : human carcinogen

kB2: probable human carcinogen

'designated as a hazardous substances pursuant to Section 307(a) of the Clean Water Act.

m designated as a hazardous substances pursuant to Section 3001 of RCRA.

ndesignated as a hazardous substances pursuant to Section 112 of the Clean Air Act.

$\mathrm{ACGIH}=$ American Conference of Governmental Industrial Hygienists; $\mathrm{ALI}=$ annual limits on intake; CFR = Code of Federal Regulations; DAC = derived air concentration; DWEL = drinking water equivalent level;

EPA = Environmental Protection Agency; FDA = Food and Drug Administration; HSDB = Hazardous Substances

Data Bank; IARC = International Agency for Research on Cancer; IDLH = immediately dangerous to life or health;

IRIS = Integrated Risk Information System; LLI = lower large intestine; $\mathrm{NIOSH}=$ National Institute for Occupational

Safety and Health; NTP = National Toxicology Program; OSHA = Occupational Safety and Health Administration;

$\mathrm{PEL}=$ permissible exposure limit; RCRA = Resource Conservation and Recovery Act; $\mathrm{REL}=$ recommended

exposure limit; $\mathrm{RfC}=$ inhalation reference concentration; $\mathrm{RfD}=$ oral reference dose; $T C L P=$ toxicity characteristic

leachate procedure; TLV = threshold limit values; TSD = treatment, storage, and disposal; TWA = time-weighted

average; USNRC = Nuclear Regulatory Commission; WHO = World Health Organization 



\section{REFERENCES}

*ABMS. 1994. Non-ferrous metal data [for 1993]. Secaucus, NJ: American Bureau of Metal Statistics, Inc.

*ABMS. 2002. Nickel. Non-ferrous metals yearbook. Chatham NJ: Cadmus Professional Communications, Science Press Division, 157-162.

Accominotti M, Bost M, Haudrechy P, et al. 1998. Contribution to chromium and nickel enrichment during cooking of foods in stainless steel utensils. Contact Dermatitis 38(6):305-310.

Acevedo F, Serra MA, Ermolli M, et al. 2001. Nickel-induced proteins in human HaCaT keratinocyte: Annexin II and phosphoglycerate kinase. Toxicology 159(1-2):33-41.

ACGIH. 1996. 1996 TLVs and BEIs: Threshold limit values for chemical substances and physical agents and biological exposure indices. Cincinnati, $\mathrm{OH}$ : American Conference of Governmental Industrial Hygienists.

*ACGIH. 2003. Nickel. Threshold limit values for chemical substances and physical agents and biological exposure indices. Nickel. Cincinnati, OH: American Conference of Governmental Industrial Hygienists.

Ada AO, Coban T, Kapucuoglu N, et al. 2001. The responses of rat testicular CYP and GST enzymes to cadmium and nickel [Abstract]. Toxicol Lett 123(Suppl 1):48.

*Adachi A, Okiayu M, Nishikawa A, et al. 1998. Metal levels in rain water from Kobe City in Japan. Bull Environ Contam Toxicol 60:892-897.

*Adamo P, Dudka S, Wilson MJ, et al. 1996. Chemical and mineralogical forms of $\mathrm{Cu}$ and $\mathrm{Ni}$ in contaminated soils from the Sudbury mining and smelting region, Canada. Environ Pollut 91(1):11-19.

*Adams JF, Kissel DE. 1989. Zinc, copper, and nickel availabilities as determined by soil solution and DTPA extraction of a sludge-amended soil. Commun Soil Sci Plant Anal 20:139-158.

*Ademec JB, Kihlgren TE. 1967. Nickel and nickel alloys. In: Standen A, ed. Kirk-Othmer encyclopedia of chemical technology, vol. 13, 2nd ed. New York, NY: Interscience Publishers, 735-753.

*Adinolfi M. 1985. The development of the human blood-CSF-brain barrier. Dev Med Child Neurol 27:532-537.

*Adkins B Jr, Richards JH, Gardner DE. 1979. Enhancement of experimental respiratory infection following nickel inhalation. Environ Res 20:33-42.

*Adlercreutz H. 1995. Phytoestrogens: Epidemiology and a possible role in cancer protection. Environ Health Perspect Suppl 103(7):103-112.

*Cited in text 


\section{REFERENCES}

*Agency for Toxic Substances and Disease Registry. 1989. Agency for Toxic Substances and Disease Registry. Decision guideline for identifying substance-specific data needs related to toxicological profiles. Part V. Fed Regist 54:37619-37633.

*Agency for Toxic Substances and Disease Registry. 2003. Public health assessment for Circle Smelting Corporation Beckmeyer, Clinton County, Illinois. EPA Facility ID: ILD050231976. May 12, 2003. Illinois State Dept. of Public Health/U.S. Department of Health and Human Services, Agency for Toxic Substances and Disease Registry.

Agner T, Johansen JD, Overgaard L, et al. 2002. Combined effects of irritants and allergens. Contact Dermatitis 47(1):21-26.

*Akasya-Hillenbrand E, Ozkaya-Bayazit E. 2002. Patch test results in 542 patients with suspected contact dermatitis in Turkey. Contact Dermatitis 46(1):17-23.

*Alberici TM, Sopper WE, Storm GL, et al. 1989. Trace metals in soil vegetation and voles from mine land treated with sewage sludge. J Environ Qual 18:115-119.

*Alberti-Fidanza A, Burini G, Perriello G, et al. 2003. Trace element intake and status of Italian subjects living in the Gubbio area. Environ Res 91:71-77.

*Altman PL, Dittmer DS. 1974. Biological handbooks: Biology data book. Vol. III. 2nd ed. Bethesda, MD: Federation of the American Society of Experimental Biologists, 1987-2008, 2041.

Alvarez C, Blade C, Cartana J. 1993. $\alpha 2$-Adrenergic blockade prevents hyperglycemia and hepatic glutathione depletion in nickel-injected rats. Toxicol Appl Pharm 121:112-117.

*Amacher DE, Paillet SC. 1980. Induction of trifluorothymidine resistant mutants by metal ions in L5178Y/TK+/- cells. Mutat Res 78:279-288.

*Ambrose AM, Larson PS, Borzelleca JF, et al. 1976. Long term toxicologic assessment of nickel in rats and dogs. J Food Sci Technol 13:181-187.

*American Biogenics Corporation. 1988. Ninety day gavage study in albino rats using nickel. Final report submitted to U.S. Environmental Protection Agency, Office of Solid Waste. Submitted by Research Triangle Institute and American Biogenics Corporation.

*Andersen O. 1983. Effects of coal combustion products and metal compounds on sister chromatid exchange (SCE) in a macrophage cell line. Environ Health Perspect 47:239-253.

*Andersen I, Svenes KB. 1989. Determination of nickel in lung specimens of thirty-nine autopsied nickel workers. Int Arch Occup Environ Health 61:289-295.

*Andersen I, Svenes K. 2003. X-ray diffraction spectrometric analysis of nickel refinery aerosols, process materials and particulates isolated from worker lung tissues. J Environ Monit 5(2):202-205.

*Andersen ME, Krishnan K. 1994. Relating in vitro to in vivo exposures with physiologically based tissue dosimetry and tissue response models. In: Salem H, ed. Animal test alternatives: Refinement, reduction, replacement. New York, NY: Marcel Dekker, Inc., 9-25. 


\section{REFERENCES}

*Andersen A, Berge SR, Engeland A, et al. 1996. Exposure to nickel compounds and smoking in relation to incidence of lung and nasal cancer among nickel refinery workers. Occup Environ Med 53(10):708-713.

*Andersen ME, Clewell HJ III, Gargas ML, et al. 1987. Physiologically based pharmacokinetics and the risk assessment process for methylene chloride. Toxicol Appl Pharmacol 87:185-205.

*Anderson KE, Nielsen GD, Flyvholm M-A, et al. 1983. Nickel in tap water. Contact Dermatitis 9:140143.

*Anderson S. 1995. Nickel: A year of contradiction and transition. Engineer Min J 196(3):63-67.

*Anderson PR, Christensen TH. 1988. Distribution coefficients of Cd, Co, and Zn in soils. J Soil Sci $35: 15-22$.

Andreassi M, DiGioacchino M, Sabbioni E, et al. 1998. Serum and urine nickel in nickel-sensitized women: effects of oral challenge with the metal. Contact Dermatitis 38(1):5-8.

Andrew A, Barchowsky A. 2000. Nickel-induced plasminogen activator inhibitor-1 expression inhibits the fibrinolytic activity of human airway epithelial cells. Toxicol Appl Pharmacol 168(1):50-57.

*Angerer J, Lehnert G. 1990. Occupational chronic exposure to metals. II: Nickel exposure of stainless steel welders--biological monitoring. Int Arch Occup Environ Health 62:7-10.

*Ankley GT, Phipps GL, Leonard EN, et al. 1991. Acid-volatile sulfide as a factor mediating cadmium and nickel bioavailability in contaminated sediments. Environ Toxicol Chem 10:1299-1307.

*Antonsen DH. 1981. Nickel compounds. In: Grayson M, Eckroth D, eds. Kirk-Othmer encyclopedia of chemical technology, vol. 15, 3rd ed. New York, NY: John Wiley and Sons, 801-819.

*Antonsen DH, Springer DB. 1967. Nickel compounds. In: Standen A, ed. Kirk-Othmer encyclopedia of chemical technology, vol. 13, 2nd ed. New York, NY: Interscience Publishers, 753-765.

*Anttila A, Pukkala E, Aitio A, et al. 1998. Update of cancer incidence among workers at a copper/nickel smelter and nickel refinery. Int Arch Occup Environ Health 71(4):245-250.

APHA. 1992. Standard methods for the examination of water and wastewater. 18th ed. Washington, DC: American Public Health Association, American Water Works Association, Water Environment Federation.

Aremu DA, Olawuyi J, Meshitsuka S, et al. 2002. Heavy metal analysis of groundwater from Warri, Nigeria. Int J Environ Health Res 12:261-267.

*Arena VC, Sussman NB, Redmond CK, et al. 1998. Using alternative comparison populations to assess occupation-related mortality risk. Results for the high nickel alloys workers cohort. J Occup Environ Med 40(10):907-916.

Arild V, Elise PE, Ljudmila T, et al. 2004. The prevalence of selected pregnancy outcome risk factors in the life-style and medical history of the delivering population in north-western Russia. Int J Circumpolar Health 63(1):39-60. 
9. REFERENCES

*Arlauskas A, Baker RS, Bonin AM, et al. 1985. Mutagenicity of metal ions in bacteria. Environ Res 36:379-388.

*Arnich N, Lanhers MC, Cunat L, et al. 2000. Nickel absorption and distribution from rat small intestine in situ. Biol Trace Elem Res 74(2):141-151.

*Arrouijal FZ, Marzin D, Hildebrand HF, et al. 1992. Differences in genotoxic activity of $\alpha$-Ni3S2 on human lymphocytes from nickel-hypersensitized and nickel-unsensitized donors. Mutagenesis 7(3):183187.

Artik S, Haarhuis K, Wu X, et al. 2001. Tolerance to nickel: Oral nickel administration induces a high frequency of anergic T cells with persistent suppressor activity. J Immunol 167(12):6794-6803.

*ASTM. 2000. Water (1), Methods D1976 and D5673. In: Allen RF, Baldini NC, Gutman EL, et al., eds. Annual book of ASTM standards. Vol. 11.01. West Conshococken, PA: American Society for Testing and Materials.

Azar C, Holomberg J, Lindgren K. 1996. Socio-ecological indicators for sustainability. Methodological and ideological options. Ecol Econ 18:89-112.

Banfield CC, Basketter DA, Powell SM. 1998. Cutaneous reactivity of the hands in nickel-sensitive patients with hand eczema. Contact Dermatitis 38(6):316-318.

*Baker DE, Amacher MC. 1982. Nickel, copper, zinc, and cadmium. In: Methods of soil analysis, Part 2. Chemical and microbiological properties - Agronomy Monograph No. 9, 2nd ed. Madison: ASASSSA, 3323-3336.

*Baranowska-Dutkiewicz B, Rozanska R, Dutkiewicz T. 1992. Occupational and environmental exposure to nickel in Poland. Pol J Occup Med Env Health 5(4):335-343.

*Barbante C, Boutron C, Moreau A-L, et al. 2002. Seasonal variations in nickel and vanadium in Mont Blanc snow and ice dated from the 1960's and 1990's. J Environ Monit 4:960-966.

Barcan V. 2002. Nature and origin of multicomponent aerial emissions of the copper-nickel smelter complex. Environ Int 28:451-456.

*Barceloux DG. 1999. Nickel. Clin Toxicol 37(2):239-258.

*Barnes DG, Dourson M. 1988. Reference dose (RfD): Description and use in health risk assessment. Regul Toxicol Pharmacol 8:471-486.

*Barrie LA, Hoff RM. 1985. Five years of air chemistry observations in the Canadian Arctic. Atmos Environ 19:1995-2010.

*Barrie LA, Lindberg SE, Chan WH, et al. 1987. On the concentration of trace metals in precipitation. Atmos Environ 21:1133-1135.

*Bavazzano P, Bolognesi R, Cassinelli C, et al. 1994. Skin contamination and low airborne nickel exposure of electroplaters. Sci Total Environ 155:83-86. 
*Becker N. 1999. Cancer mortality among arc welders exposed to fumes containing chromium and nickel: Results of a third follow-up: 1989-1995. J Occup Environ Med 41(4):294-303.

*Bencko V, Wagner V, Wagnerova M, et al. 1983. Immuno-biochemical findings in groups of individuals occupationally and nonoccupationally exposed to emissions containing nickel and cobalt. $\mathbf{J}$ Hyg Epidemiol Microbiol Immunol 27:387-394.

*Bencko V, Wagner V, Wagnerova M, et al. 1986. Human exposure to nickel and cobalt: Biological monitoring and immunobiological response. Environ Res 40:399-410.

*Bennett BG. 1984. Environmental nickel pathways in man. In: Sunderman FW Jr, ed. Nickel in the human environment. Proceedings of a joint symposium. IARC scientific publication no. 53. Lyon, France: International Agency for Research on Cancer, 487-495.

*Benson JM, Barr EB, Bechtold WE, et al. 1994. Fate of inhaled nickel oxide and nickel subsulfide in F344/N rats. Inhal Toxicol 6:167-183.

*Benson JM, Burt DG, Carpenter RL, et al. 1988. Comparative inhalation toxicity of nickel sulfate to F344/N rats and B6C3F1 mice exposed for twelve days. Fundam Appl Toxicol 10:164-178.

*Benson JM, Burt DG, Cheng YS, et al. 1989. Biochemical responses of rat and mouse lung to inhaled nickel compounds. Toxicology 57:255-266.

*Benson JM, Carpenter RL, Hahn FF, et al. 1987. Comparative inhalation toxicity of nickel subsulfide to F344/N rats and B6C3F1 mice exposed for twelve days. Fundam Appl Toxicol 9:251-265.

*Benson JM, Chang I-Y, Cheng YS. 1995a. Particle clearance and histopathology in lungs of F344/N rats and B6C3F1 mice inhaling nickel oxide or nickel sulfate. Fundam Appl Toxicol 28:232-244.

*Benson JM, Cheng Y-S, Eidson AF, et al. 1995b. Pulmonary toxicity of nickel subsulfide in F344/N rats exposed for 1-22 days. Toxicology 103:9-22.

Benson J, March TH, Divine KK, et al. 2003. Mechanisms of nickel induced lung cancer - Nickel subsulfide vs nickel sulfate. Toxicol Sci 72(S-1):299.

*Berge SR, Skyberg K. 2003. Radiographic evidence of pulmonary fibrosis and possible etiologic factors at a nickel refinery in Norway. J Environ Monit 5(4):681-688.

*Berger GS. 1994. Epidemiology of endometriosis. In: Berger GS, ed. Endometriosis: Advanced management and surgical techniques. New York, NY: Springer-Verlag.

*Berghem L, Hansson M, Lundborg M, et al. 1987. Fibronectin concentrations in lung lavage fluid after inhalation exposure to low levels of metals. Environ Res 43:79-85.

*Berman E, Rehnberg B. 1983. Fetotoxic effects of nickel in drinking water in mice. National Technical Information Service. EPA600183007. PB83225383.

*Bernacki EJ, Parsons GE, Sunderman FW Jr. 1978. Investigation of exposure to nickel and lung cancer mortality. Ann Clin Lab Sci 8:190-194. 


\section{REFERENCES}

*Bernacki EJ, Zugowicz E, Sunderman FW Jr. 1980. Fluctuations of nickel concentrations in urine of electroplating workers. Ann Clin Lab Sci 10:33-39.

*Bhattacharyya MH. 1983. Bioavailability of orally administered cadmium and lead to the mother, fetus, and neonate during pregnancy and lactation: An overview. Sci Total Environ 28:327-342.

*Biedermann KA, Landolph JR. 1987. Induction of anchorage independence in human diploid foreskin fibroblasts by carcinogenic metal salts. Cancer Res 47:3815-3823.

*Biego GH, Joyeux M, Hartemann P, et al. 1998. Determination of mineral contents in different kinds of milk and estimation of dietary intake in infants. Food Addit Contam 15(7):775-781.

*Biggart NW, Costa M. 1986. Assessment of the uptake and mutagenicity of nickel chloride in Salmonella tester strains. Mutat Res 175:209-215.

*Biggart NW, Murphy E Jr. 1988. Analysis of metal-induced mutations altering the expression or structure of a retroviral gene in a mammalian cell line. Mutat Res 198:115-130.

*Biggart NW, Gallick GE, Murphy EC Jr. 1987. Nickel-induced heritable alterations in retroviral transforming gene expression. J Virol 61:2378-2388.

*Bingham E, Barkley W, Zerwas M, et al. 1972. Responses of alveolar macrophages to metals. I. Inhalation of lead and nickel. Arch Environ Health 25:406-414.

*Birge WJ, Black JA. 1980. Aquatic toxicology of nickel. In: Nriagu JO, ed. Nickel in the environment. New York, NY: John Wiley and Sons, Inc., 354-355.

*Borg K, Tjalve H. 1989. Uptake of $63 \mathrm{Ni} 2+$ in the central and peripheral nervous system of mice after oral administration: Effects of treatment with halogenated 8-hydroxyquinolines. Toxicology 54:59-68.

*Borska L, Fiala Z, Smejkalova J. 2003. Health risk of occupational exposure in welding processes. I. Genotoxic risk. Acta Medica (Hradec Kralove) 46(1):25-29.

Boscolo P, Andressi M, Sabbioni E, et al. 1999. Systemic effects of ingested nickel on the immune system of nickel sensitised women. Life Sci 64(17):1485-1491.

*Bowlby JN, Gunn JM, Liimatainen VA. 1988. Metals in stocked lake trout Salvelinus-namaycush in lakes near Sudbury Canada. Water Air Soil Pollut 39:217-230.

*Bowman RS, Essington ME, O'Connor GA. 1981. Soil sorption of nickel: Influence of solution composition. Soil Sci Soc Am J 45:860-865.

*Boyer KW, Horowitz W. 1986. Special considerations in trace element analysis of foods and biological materials. In: O'Neill IK, Schuller P, Fishbein L, eds. Some metals: As, Be, Cd, Cr, Ni, Pb, Se, Zn. IARC Scientific Publication No. 71. Lyon, France: International Agency for Research on Cancer, 191220.

*Bradley RW, Morris JR. 1986. Heavy metals in fish from a series of metal-contaminated lakes near Sudbury, Ontario. Water Air Soil Pollut 27:341-354. 
Bragadin G, Taraschi A, Marchini C, et al. 1999. Allergic contact dermatitis from nickel following electromyography. Contact Dermatitis 41(6):353-354.

Brasch J, Geier J. 1997. Patch test results in schoolchildren: Results from the Information Network of Departments of Dermatology (IVDK) and the German Contact Dermatitis Research Group (DKG). Contact Dermatitis 37:286-293.

*Brito G, Diaz C, Galindo L, et al. 1990. Levels of metals in canned meat products: Intermetallic correlations. Bull Environ Contam Toxicol 44:309-316.

Broday L, Cai J, Costa M. 1999. Nickel enhances telomeric silencing in Saccharomyces cerevisiae. Mutat Res 440(2):121-130.

*Bronstein AC, Currance PL. 1988. Emergency care for hazardous material exposure. Washington, DC: The CV Mosby Company, 147-148.

*Brooks RR. 1980. Hyperaccumulation of nickel by terrestrial plants. In: Nriagu JO, ed. Nickel in the environment. New York, NY: John Wiley and Sons, 410-413.

*Brown SS, Nomoto S, Stoeppler M, et al. 1981. IUPAC reference method for analysis of nickel in serum and urine by electrothermal atomic absorption spectrometry. Clin Biochem 14:295-299.

*Bruce BW, McMahon PB. 1996. Shallow ground-water quality beneath a major urban center: Denver, Colorado, USA. J Hydrol 186:129-151.

Bryld LE, Hindsberger C, Kyvik KO, et al. 2003. Risk factors influencing the development of hand eczema in a population-based twin sample. Br J Dermatol 149(6):1214-1220.

*Brzezinska-Paudyn A, VanLoon JC, Balicki MR. 1986. Multielement analysis and mercury speciation in atmospheric samples from the Toronto area. Water Air Soil Pollut 27:45-56.

Budinger L, Neuser N, Totzke U, et al. 2001. Preferential usage of TCR-Vbeta17 by peripheral and cutaneous T cells in nickel-induced contact dermatitis. J Immunol 167(10):6038-6044.

*Burrows D, Creswell S, Merrett JD. 1981. Nickel, hands, and hip prosthesis. Br J Dermatol 105:437444.

*Butte W, Heinzow B. 2002. Pollutants in house dust as indicators of indoor contamination. Rev Environ Contam Toxicol 175:1-46.

*Cahill TA. 1989. Monitoring of atmospheric particles and ozone in Sequoia National Park: 1985-1987. Sacramento, CA: California State Air Resources Board. PB90157512.

Candura SM, Locatelli C, Butera R, et al. 2001. Widespread nickel dermatitis from inhalation. Contact Dermatitis 45(3):174-175.

* Capar SG, Cunningham WC. 2000. Element and radionuclide concentrations in food: FDA Total Diet Study 1991-1996. JOAC Int 83(1):157-177.

* Carvalho SMM, Ziemer PL. 1982. Distribution and clearance of ${ }^{63} \mathrm{NiCl}_{2}$ in the rat: Intratracheal study. Arch Environ Contam Toxicol 11:245-248. 
*Casey CE, Neville MC. 1987. Studies in human lactation 3: Molybdenum and nickel in human milk during the first month of lactation. Am J Clin Nutr 45(5):921-926.

Cavallo D, Ursinini CL, Setini A, et al. 2003. Evaluation of oxidative damage and inhibition of DNA repair in an in vitro study of nickel exposure. Toxicol in Vitro 17(5-6):603-307.

Cavani A, Mei D, Guerra E, et al. 1998. Patients with allergic contact dermatitis to nickel and nonallergic individuals display different nickel-specific $\mathrm{T}$ cell responses. Evidence for the presence of effector CD8+ and regulatory CD4+ T cells. J Invest Dermatol 111(4):621-628.

Cavani A, Nasorri F, Ottaviani C, et al. 2003. Human CD25+ regulatory T cells maintain immune tolerance to nickel in healthy, nonallergic individuals. J Immunol 171(11):5760-5768.

Cavani A, Nasorri F, Prezzi C, et al. 2000. Human CD4+ T lymphocytes with remarkable regulatory functions on dendritic cells and nickel-specific TH1 immune responses. J Invest Dermatol 114(2):295302.

*Cavelier C, Foussereau J, Gille P, et al. 1988. Nickel allergy: Tolerance to metallic surface-plated samples in nickel-sensitive humans and guinea pigs. Contact Dermatitis 19:358-361.

Cederbrant K, Gunnarsson LG, Marcusson JA. 2000. Mercury intolerance and lymphocyte transformation test with nickel sulfate, palladium chloride, mercuric chloride, and gold sodium thiosulfate. Environ Res 84(2):140-144.

CELDS. 1994. Computer environmental legislative data system database. University of Illinois, Urbana Illinois.

Cempel M, Janicka K. 2002. Distribution of nickel, zinc, and copper in rat organs after oral administration of nickel(II) chloride. Biol Trace Elem Res 90:215-226.

*CEPA. 1994. Canadian Environmental Protection Act. Priority substances list assessment report: Nickel and its compounds. Government of Canada, Environment Canada, Health Canada, 1-82.

Cerveny KA Jr., Brodell RT. 2002. Blue jean button dermatitis. Nickel allergy presenting as a periumbilical rash. Postgrad Med 112(5):79-80.

Chakrabarti SK, Bai C. 1999. Role of oxidative stress in nickel chloride-induced cell injury in rat renal cortical slices. Biochem Pharmacol 58(9):1501-1510.

Chakrabarti SK, Bai C, Subramanian KS. 1999. DNA-protein crosslinks induced by nickel compounds in isolated rat renal cortical cells and its antagonism by specific amino acids and magnesium ion. Toxicol Appl Pharmacol 154(3):245-255.

Chakrabarti SK, Bai C, Subramanian KS. 2001. DNS-protein crosslinks induced by nickel compounds in isolated rat lymphocytes: Role of reactive oxygen species and specific amino acids. Toxicol Appl Pharmacol 170(3):153-165.

*Chamberlain PG. 1985. Nickel. Preprint from the Bureau of Mines mineral yearbook. Pittsburgh, PA: Bureau of Mines, U.S. Department of the Interior, 108-109. 


\section{REFERENCES}

*Chan WH, Tang AJS, Chung DHS, et al. 1986. Concentration and deposition of trace metals in Ontario-1982. Water Air Soil Pollut 29:373-389.

*Chashschin VP, Artunina GP, Norseth T. 1994. Congenital defects, abortion and other health effects in nickel refinery workers. Sci Total Environ, 148:287-291.

Chen CY, Huang YL. 1998. Association between oxidative stress and cytokine production in nickeltreated rats. Arch Biochem Biophys 356(2):127-132.

Chen C-Y, Lin T-H. 2001. Effects of nickel chloride on human platelets: Enchancement of lipid peroxidation, inhibition of aggregation and interaction with ascorbic acid. J Toxicol Environ Health A 62:431-438.

Chen CY, Huang YL, Lin TH. 1998. Lipid peroxidation in liver of mice administered with nickel chloride: With special reference to trace elements and antioxidants. Biol Trace Elem Res 61(2):193-205.

Chen CY, Wang YF, Huang WR, et al. 2003a. Nickel induces oxidative stress and genotoxicity in human lymphocytes. Toxicol Appl Pharmacol 189:153-159.

Chen CY, Wang YF, Lin YH, et al. 2003b. Nickel-induced oxidative stress and effect of antioxidants in human lymphocytes. Arch Toxicol 77(3):123-130.

Cheng RYS, Zhao A, Alvord WG, et al. 2003. Gene expression dose-response changes in microarrays after exposure of human peripheral lung epithelial cells to nickel(II). Toxicol Appl Pharmacol 191(1):2239.

*Chernoff N, Kavlock RJ. 1982. An in vivo teratology screen utilizing pregnant mice. J Toxicol Environ Health 10:541-550.

*Chin YE, Snow ET, Christie NT. 1994. The stimulatory effect of nickel chloride on DNA replication in human HeLa cells and Escherichia coli. Carcinogenesis 15(5):1013-1016.

Chiu A, Katz AJ, Beaubier J, et al. 2004. Genetic and cellular mechanisms in chromium and nickel carcinogenesis considering epidemiologic findings. Mol Cell Biochem 255(1-2):181-194.

*Chovil A, Sutherland RB, Halliday M. 1981. Respiratory cancer in a cohort of nickel sinter plant workers. Br J Ind Med 38:327-333.

*Christensen JM. 1995. Human exposure to toxic metals: Factors influencing interpretation of biomonitoring results. Sci Total Environ 166:89-135.

*Christensen OB, Lagesson V. 1981. Nickel concentrations of blood and urine after oral administration. Ann Clin Lab Sci 11:119-125.

*Christensen OB, Moller H. 1975. External and internal exposure to the antigen in the hand eczema of nickel allergy. Contact Dermatitis 1:136-141.

*Christie NT, Tummolo DM, Klein CB, et al. 1992. Role of Ni(II) in mutation. In: Nieboer E, Nriagu JO, eds. Nickel and human health, current perspectives: Advances in environmental science and technology, Vol. 25. New York: John Wiley \& Sons, 305-317. 
*Ciccarelli RB, Wetterhahn KE. 1982. Nickel distribution and DNA lesions induced in rat tissues by the carcinogen nickel carbonate. Cancer Res 42:3544-3549.

*Claiborn CS, Larson T, Sheppard L. 2002. Testing the metals hypothesis in Spokane, Washington. Environ Health Perspect 101(Suppl 4):547-552

*Clary JJ. 1975. Nickel chloride-induced metabolic changes in the rat and guinea pig. Toxicol Appl Pharmacol 31:55-65.

Clemens F, Landolph JR. 2003. Genotoxicity of samples of nickel refinery dust. Toxicol Sci 73(1):114123.

*Clewell HJ III, Andersen ME. 1985. Risk assessment extrapolations and physiological modeling. Toxicol Ind Health 1(4):111-131.

*Colborn T, Clement C. 1992. Chemically induced alterations in sexual and functional development. The wildlife/human connection. In: Advances in modern environmental toxicology. Volume XXI. Princeton, NJ: Princeton Scientific Publishing Co.

*Cole KL, Engstrom DR, Futyma RP, et al. 1990. Past atmospheric deposition of metals in northern Indiana measured in a peat core from Cowles Bog. Environ Sci Technol 24:543-549.

*Cole RH, Frederick RE, Healy RP, et al. 1984. Preliminary findings of the priority pollutant monitoring project of the nationwide urban runoff program. J Water Pollut Control Fed 56:898-908.

*Coleman ME, Elder RS, Basu P, et al. 1992. Trace metals in edible tissues of livestock and poultry. JOAC Int 75(4):615-625.

*Conway K, Costa M. 1989. Nonrandom chromosomal alterations in nickel-transformed Chinese hamster embryo cells. Cancer Res 49:6032-6038.

*Cornell RG. 1984. Mortality patterns around stainless-steel workers. In: Sunderman FW Jr, Aitio A, Berlin A, eds. Nickel in the human environment. IARC scientific publication no. 53. Lyon, France: International Agency for Research on Cancer, 65-71.

*Cornell RG, Landis JR. 1984. Mortality patterns among nickel/chromium alloy foundry workers. In: Sunderman FW, Jr, Aitio A, Berlin A, eds. Nickel in the human environment. IARC scientific publication no. 53. Lyon, France: International Agency for Research on Cancer, 87-93.

*Costa M. 1989. Perspectives on the mechanism of nickel carcinogenesis gained from models of in vitro carcinogenesis. Environ Health Perspect 81:73-76.

*Costa M. 1995. Model for the epigenetic mechanism of action of nongenotoxic carcinogens. Am J Clin Nutr 61(suppl):666S-669S.

*Costa M, Heck JD. 1982. Specific nickel compounds as carcinogens. Trends Pharmacol Sci 3:408-410.

*Costa M, Mollenhauer HH. 1980. Carcinogenic activity of particulate metal compounds is proportional to their cellular uptake. Science 209:515-517. 
*Costa M, Heck JD, Robinson SH. 1982. Selective phagocytosis of crystalline metal sulfide particles and DNA strand breaks as a mechanism for the induction of cellular transformation. Cancer Res 42:2757-2763.

Costa M, Salnikow K, Sutherland JE, et al. 2002. The role of oxidative stress in nickel and chromate genotoxicty. Mol Cell Biochem 234-235(1-2):265-275.

*Costa M, Yan Y, Zhao D, et al. 2003. Molecular mechanisms of nickel carcinogenesis: Gene silencing by nickel delivery to the nucleus and gene activation/inactivation by nickel-induced cell signaling. J Environ Monit 5(2):222-223.

*Costa M, Zhuang Z, Huang X, et al. 1994. Molecular mechanisms of nickel carcinogenesis. Sci Total Environ 148:191-199.

*Cotton FA, Wilkinson G. 1980. Advanced inorganic chemistry. A comprehensive text. New York, NY: John Wiley and Sons, 783-798.

*Covance Laboratories, Inc. 2003. In vivo rat micronucleus assay with nickel sulfate hexahydrate. Study Number 7454-100 submitted to NiPERA, 4. August, 2003. Vienna, Virginia: Covance Laboratories, Inc.

*Cox JE, Doll R, Scott WA, et al. 1981. Mortality of nickel workers: Experience of men working with metallic nickel. Br J Ind Med 38:235-239.

*Cragle DL, Hollis DR, Newport TH, et al. 1984. A retrospective cohort study among workers occupationally exposed to metallic nickel powder at the Oak Ridge Gaseous Diffusion Plant. In: Sunderman FW Jr, Aitio A, Berlin A, eds. Nickel in the human environment. IARC scientific publication no. 53. Lyon, France: International Agency for Research on Cancer, 57-64.

*CRIS. 1994. Current Research Information System. Beltsville, MD: U.S. Department of Agriculture.

*Cronin E, DiMichiel AD, Brown SS. 1980. Oral challenge in nickel-sensitive women with hand eczema. In: Brown SS, Sunderman FW Jr, eds. Nickel toxicology. New York, NY: Academic Press, $149-152$.

Curstedt T, Casarett-Bruce M, Camner P. 1984. Changes in glycerophosphatides and their ether analogs in lung lavage of rabbits exposed to nickel dust. Exp Mol Pathol 41:26-34.

Curstedt T, Hafman M, Robertson B, et al. 1983. Rabbit lung after long-term exposure to low nickel dust combustion. I. Effects on phospholipid concentration and surfactant activity. Environ Res 30:8994.

*Custer CM, Custer TW, Anteau MJ, et al. 2003. Trace elements in lesser scaup (Aythya affinis) from the Mississippi Flyway. Ecotoxicology 13:47-54.

*Cyr F, Mehra MC, Mallet VN. 1987. Leaching of chemical contaminants from a municipal landfill site. Bull Environ Contam Toxicol 38:775-782.

*Czerczak S, Gromiec JP. 2001. Nickel, ruthenium, rhodium, palladium, osmium, and platinum. In: Bingham E, Cohrssen B, Powell CH, eds. Patty's toxicology. 5th ed., Volume 3. New York, NY: John Wiley \& Sons, Inc., 195, 213, 222, 246. 
*Dabeka RW. 1989. Survey of lead, cadmium, cobalt, and nickel in infant formulas and evaporated milks and estimation of dietary intakes of the elements by infants 0-12 months old. Sci Total Environ 89:279-289.

*Daldrup T, Haarhoff K, Szathmary SC. 1983. Toedliche nickel sulfaye-intoxikation. Berichte zur Serichtlichen Medizin 41:141-144.

*Danadevi K, Rozati R, Reddy PP, et al. 2003. Semen quality of Indian welders occupationally exposed to nickel and chromium. Reprod Toxicol 17:451-456.

Danadevi K, Rozati R, Saleha Banu B, et al. 2004a. Genotoxic evaluation of welders occupationally exposed to chromium and nickel using the comet and micronucleus assays. Mutagenesis 19(1):35-41.

Danadevi K, Rozati R, Saleha Banu B, et al. 2004b. In vivo genotoxic effect of nickel chloride in mice leukocytes using comet assay. Food Chem Toxicol 42(5):751-757.

*Danielsen TE, Langard S, Andersen A. 1996. Incidence of cancer among Norwegian boiler welders. Occup Environ Med 53(4):231-234.

Das KK, Dasgupta S. 2002. Effect of nickel sulfate on testicular steriodogenesis in rats during protein restriction. Environ Health Perspect 110(9):923-926.

Dasika UK, Kanter KR, Vincent R. 2003. Nickel allergy to the percutaneous patent foramen ovale occuder and subsequent systemic nickel allergy. J Thorac Cardiovasc Surg 126(6):2112.

*Davidson CI. 1980. Dry deposition of nickel from the atmosphere. In: Nriagu JO, ed. Nickel in the environment. New York, NY: John Wiley and Sons, 137-149.

De Vito MJ, Maier WE, Diliberto JJ, et al. 1993. Comparative ability of various PCBs, PCDFs, and TCDD to induce cytochrome P450 1A1 and 1A2 activity following 4 weeks of treatment. Fundam Appl Toxicol 20:125-130.

DiGioacchino M, Boscolo P, Cavallucci E, et al. 1998. Lymphocyte subset changes in blood and gastrointestinal mucosa after oral nickel challenge in nickel-sensitized women. Contact Dermatitis 43(4):206-211.

*Deknudt GH, Leonard A. 1982. Mutagenicity tests with nickel salts in the male mouse. Toxicology 25:289-292.

*DeLaune RD, Smith CJ. 1985. Release of nutrients and metals following oxidation of freshwater and saline sediment. J Environ Qual 14:164-168.

*Delescluse J, Dinet Y. 1994. Nickel allergy in Europe: The new European legislation. Dermatology 189(suppl 2):56-57.

*Demirjian YA, Westman TR, Joshi AM, et al. 1984. Land treatment of contaminated sludge with wastewater irrigation. J Water Pollut Control Fed 56:370-377.

DeSilva BD, Doherty VR. 2000. Nickel allergy from orthodontic appliances. Contact Dermatitis 42(2):102-103 
*DHHS. 1994. Seventh annual report on carcinogens: Summary 1994. Research Triangle Park, NC: U.S. Department of Health and Human Services, National Institute of Environmental Health Sciences, 262-269.

*Dhir H, Agarwal K, Sharma A, et al. 1991. Modifying role of Phyllanthus emblica and ascorbic acid against nickel clastogenicity in mice. Cancer Lett 59:9-18.

*Dieter MP, Jameson CW, Tucker AN, et al. 1988. Evaluation of tissue disposition, myelopoietic, and immunologic responses in mice after long-term exposure to nickel sulfate in the drinking water. $\mathbf{J}$ Toxicol Environ Health 24:356-372.

*DiPaolo JA, Casto BC. 1979. Quantitative studies of in vitro morphological transformation of Syrian hamster cells by inorganic metal salts. Cancer Res 39:1008-1013.

*DiPietro ES, Phillips DL, Paschal DC, et al. 1989. Determination of trace elements in human hair: Reference intervals for 28 elements in nonoccupationally exposed adults in the US and effects of hair treatments. Biol Trace Elem Res 22:83-100.

Dogra S, Khanna AK, Kaw JL. 1999. Antibody forming cell response to nickel and nickel-coated fly ash in rats. Hum Exp Toxicol 18(5):333-337.

*Dolan DM, Warry ND, Rossmann R, et al. 1986. Lake Huron, 1980: Intensive survey summary report. Windsor, Ontario, Canada: Surveillance Work Group, 133.

*Doll R, Mathews JD, Morgan LG. 1977. Cancers of the lung and nasal sinuses in nickel workers. A reassessment of the period of risk. Br J Ind Med 34:102-105.

*Doll R, Morgan LG, Speiger FE. 1970. Cancers of the lung and nasal sinuses in nickel workers. Br J Cancer 24:624-632.

*Dolovich J, Evans SL, Nieboer E. 1984. Occupational asthma from nickel sensitivity: I. Human serum albumin in the antigenic determinant. Br J Ind Med 41:51-55.

*Donat JR, Lao KA, Bruland KW. 1994. Speciation of dissolved copper and nickel in South San Francisco Bay: A multi-method approach. Anal Chim Acta 284:547-571.

*Donskoy E, Donskoy M, Forouhar F, et al. 1986. Hepatic toxicity of nickel chloride in rats. Ann Clin Lab Sci 16:108-117.

Dorn CR, Warner RD, Ahmed W. 1988. Comparison of NiSO4 and NiCl2 as sensitizers in the guinea pig. Int Arch Allergy Appl Immunol 85:332-336.

*Dostal LA, Hopfer SM, Lin SM, et al. 1989. Effects of nickel chloride on lactating rats and their suckling pups, and the transfer of nickel through rat milk. Toxicol Appl Pharmacol 101:220-231.

*Dotterud LK, Falk ES. 1994. Metal allergy in north Norwegian schoolchildren and its relationship with ear piercing and atopy. Contact Dermatitis 31:308-313.

*Dressler RL, Storm GL, Tzilkowski WM, et al. 1986. Heavy metals in cottontail rabbits on mined lands treated with sewage sludge. J Environ Qual 15:278-281. 
9. REFERENCES

Duarte I, Lazzarini R, Kobata CM. 2003. Contact dermatitis in adolescents. Am J Contact Dermatitis 14(4):200-202.

*Duce RA, Liss PS, Merrill JT, et al. 1991. The atmospheric input of trace species to the world ocean. J Global Biogeochem Cycles 5(3):193-259

*Duke JM. 1980a. Nickel in rocks and ores. In: Nriagu JO, ed. Nickel in the environment. New York, NY: John Wiley and Sons, Inc., 27-50.

*Duke JM. 1980b. Production and uses of nickel. In: Nriagu JO, ed. Nickel in the environment. New York, NY: John Wiley and Sons, Inc., 51-65.

*Dunnick JK, Benson JM, Hobbs CH, et al. 1988. Comparative toxicity of nickel oxide, nickel sulfate, and nickel subsulfide after 12 days of inhalation exposure to F344/N rats and B6C3F1 mice. Toxicology 50:145-156.

*Dunnick JK, Elwell MR, Benson JM, et al. 1989. Lung toxicity after 13-week inhalation exposure to nickel oxide, nickel subsulfide, or nickel sulfate in F344/N rats and B6C3F1 mice. Fund Appl Toxicol 12:584-594.

*Dunnick JK, Elwell MR, Radovsky AE, et al. 1995. Comparative carcinogenic effects of nickel subsulfide, nickel oxide, or nickel sulfate chronic exposure in the lung. Cancer Res 55:5251-5256.

Edwards MJ. 1986. Hyperthermia as a teratogen: A review of experimental studies and their clinical significance. Teratog Carcinog Mutagen 6:563-582.

*Egedahl R, Rice E. 1984. Cancer incidence at a hydrometallurgical nickel refinery. In: Sunderman FW Jr, Aitio A, Berlin A, eds. Nickel in the human environment. IARC scientific publication no. 53. Lyon, France: International Agency for Research on Cancer, 47-55.

*Egedahl RD, Coppock E, Homik R. 1991. Mortality experience at a hydrometallurgical nickel refinery in Fort Saskatchewan, Alberta between 1954 and 1984. J Soc Occup Med 41(1):29-33.

*Egedahl R, Carpenter M, Lundell D. 2001. Mortality experience among employees at a hydrometallurgical nickel refinery and fertiliser complex in Fort Saskatchewan, Alberta (1954-95) Occup Environ Med 58(11):711-715.

Egedahl RD, Fair M, Homik R. 1993. Mortality among employees at a hydrometallurgical nickel refinery and fertilizer complex in Fort Saskatchewan, Alberta (1954-1984). Can J Public Health 84:4044.

*Elias Z, Mur JM, Pierre F, et al. 1989. Chromosome aberrations in peripheral blood lymphocytes of welders and characterization of their exposure by biological samples analysis. J Occup Med 31:477-483.

*Emmett EA, Risby TH, Jiang L, et al. 1988. Allergic contact dermatitis to nickel: Bioavailability from consumer products and provocation threshold. J Am Acad Dermatol 19(2):314-322.

*English JC, Parker RDR, Sharma RP, et al. 1981. Toxicokinetics of nickel in rats after intratracheal administration of a soluble and insoluble form. Am Ind Hyg Assoc J 42:486-492. 
*Enterline PE, Marsh GM. 1982. Mortality among workers in a nickel refinery and alloy plant in West Virginia. J Natl Cancer Inst 68:925-933.

*EPA. 1978. Water-related environmental fate of 129 priority pollutants: Vol. 1. Washington, DC: Office of Water Planning and Standards, U.S. Environmental Protection Agency, 15-1 to 15-8. EPA440479029a.

*EPA. 1980. Ambient water quality criteria document: Nickel. Washington, DC: U.S Environmental Protection Agency. EPA440580060 (Errata 1981).

*EPA. 1981a. Treatability manual: Volume I. Washington, DC: U.S. Environmental Protection Agency, EPA600282001a, I.4.11-1 to I.4.II-4.

*EPA. 1981b. Database for influent heavy metals in publicly owned treatment works. EPA6005281220. Cincinnati, OH: Municipal Environmental Research Laboratory, U.S. Environmental Protection Agency $1-5$.

*EPA. 1983. Methods for chemical analysis of water and waste. Cincinnati, OH: Environmental Monitoring and Support Laboratory, U.S. Environmental Protection Agency. EPA800479020. 249.2-1 to $249.2-2 ; 249.1-1$ to $249.1-2$.

*EPA. 1985a. Estimation of nickel species in ambient air. Research Triangle Park, NC: Pollution Assessment Branch, U.S. Environmental Protection Agency.

*EPA. 1985b. Drinking water criteria document for nickel. Cincinnati, OH: Environmental Criteria and Assessment Office, U.S. Environmental Protection Agency. EPA600x84193. PB86117801.

*EPA. 1986a. Health assessment document for nickel and nickel compounds. Cincinnati, OH: Office of Health and Environmental Assessment, Environmental Criteria and Assessment Office, U.S.

Environmental Protection Agency. EPA600883012FF.

*EPA. 1986b. Test methods for evaluating solid waste. 3rd ed. Washington, DC: U.S. Environmental Protection Agency, 3050-1 to 3050-6; three-6 to three-7; 6010-1 to 6010-17; 7520-1 to 7520-3.

EPA. 1988. Analysis of clean water act effluent guidelines: Pollutants. Summary of the chemicals regulated by industrial point source category. U.S. Environmental Protection Agency. 40 CFR Parts 400475.

*EPA. 1990a. Interim methods for development of inhalation reference doses. Washington, DC: U.S. Environmental Protection Agency, Office of Research and Development. EPA600890066A.

*EPA. 1990b. Guidelines establishing test procedures for the analysis of pollutants. U.S. Environmental Protection Agency. Fed Regist 55(116):24532-24537.

EPA. 1991a. Environmental Protection Agency. 40 CFR Part 302.4. Hazardous substances and reportable quantities.

EPA. 1991b. Environmental Protection Agency. 40 CFR Part 131. Amendments to the water quality standards regulation. Fed Regist 56:58442. 
EPA. 1991c. Drinking water quantification of toxicologic effects for nickel. U.S. Environmental Protection Agency. PB92173426. ECAO-CIN-443.

*EPA. 1994. Methods for the determination of metals in environmental samples, supplement 1. U.S. Environmental Protection Agency. National Exposure Research Laboratory. EPA600R94111.

EPA. 1995a. Removal of legally obsolete or redundant rules. Fed Regist 60(125):33926-33930.

EPA. 1995b. Toxics criteria for those states not complying with Clean Water Act Section 303(c)(2)(B). Fed Regist 60:44123.

EPA. 1996a. Drinking water regulations and health advisories. Washington, DC. U.S. Environmental Protection Agency, Office of Water. EPA822R96001.

EPA. 1996b. List of hazardous substances and reportable quantities. U.S. Environmental Protection Agency. Code of Federal Regulations. 40 CFR 302.

*EPA. 1996c. Method 1640: Determination of trace elements in ambient waters by on-line chelation preconcentration and inductively coupled plasma-mass spectrometry. Washington DC: U.S. Environmental Protection Agency. EPA821R96007.

*EPA. 1996d. Method 1639: Determination of trace elements in ambient waters by stabilized temperature graphite furnace atomic absorption. Washington, DC: U.S. Environmental Protection Agency. EPA821R96005.

*EPA. 1996e. Method 1638: Determination of trace elements in ambient waters by inductively coupled plasma-mass spectrometry. Washington, DC: U.S. Environmental Protection Agency. EPA821R96005.

*EPA. 1997a. Special report on environmental endocrine disruption: An effects assessment and analysis. Washington, DC: U.S. Environmental Protection Agency, Risk Assessment Forum. EPA630R96012.

EPA. 1997b. Method 200.12: Determination of trace elements in marine waters by stabilized temperature graphite furnace atomic absorption. Cincinnati $\mathrm{OH}$ : National Exposure Research Laboratory.

EPA. 1997c. Method 200.10: Determination of trace elements in marine waters by on-line chelation preconcentration and inductively coupled plasma- mass spectrometry. Cincinnati $\mathrm{OH}$ : National Exposure Research Laboratory.

EPA. 1997d. Method 200.13: Determination of trace elements in marine waters by off-line chelation preconcentration with graphite furnace atomic absorption. Cincinnati $\mathrm{OH}$ : National Exposure Research Laboratory, 1-19.

*EPA. 1999. Compendium of methods for the determination of inorganic compounds in ambient air. Chapters 10-3.2, 10-3.3, 10-3.4, 10-3.5, and 10-3.6. Cincinnati OH: U.S. Environmental Protection Agency, Office of Research and Development. EPA625R96010a.

*EPA. 2000. National air pollutant emission trends, 1900-1998. Research Triangle Park, NC: U.S. Environmental Protection Agency, Office of Air Quality Planning and Standards. EPA454R00002. http://www.epa.gov/ttn/atw/nata/index.htm. 
*EPA. 2002a. 2002 Edition of the drinking water standards and health advisories. Washington, DC: U.S. Environmental Protection Agency. EPA822R02038. http://www.epa.gov/waterscience. June 06, 2003.

*EPA. 2002b. Test methods for evaluating solid waste, physical/chemical methods. Washington, DC: U.S. Environmental Protection Agency. SW-846. http://www.epa.gov/epaoswer/hazwaste/test/up4b.htm.

*EPA. 2003a. Chemical accident prevention provisions. List of substances. Washington, DC: U.S. Environmental Protection Agency. 40 CFR 68.130. http://www.epa.gov/epahome/cfr40.htm. June 06, 2003.

*EPA. 2003b. Chemical accident prevention provisions. Table of toxic endpoints. Washington, DC: U.S. Environmental Protection Agency. 40 CFR 68, Appendix A.

http://www.epa.gov/epahome/cfr40.htm. June 06, 2003.

*EPA. 2003c. Criteria for municipal solid waste landfills. List of hazardous inorganic and organic constituents. Washington, DC: U.S. Environmental Protection Agency. 40 CFR 258, Appendix II. http://www.epa.gov/epahome/cfr40.htm. June 06, 2003.

*EPA. 2003d. Designation, reportable quantities, and notification. Designation of hazardous substance. Washington, DC: U.S. Environmental Protection Agency. 40 CFR 302.4. http://www.epa.gov/epahome/cfr40.htm. June 06, 2003.

EPA. 2003e. Designation, reportable quantities, and notification. Notification requirements. Washington, DC: U.S. Environmental Protection Agency. 40 CFR 302.6. http://www.epa.gov/epahome/cfr40.htm. June 06, 2003.

*EPA. 2003f. Effluent guidelines and standards. General provisions. Toxic pollutants. Washington, DC: U.S. Environmental Protection Agency. 40 CFR 401.15. http://www.epa.gov/epahome/cfr40.htm. June 06, 2003.

*EPA. 2003g. Emergency planning and notification. The list of extremely hazardous substances and their threshold planning quantities. Washington, DC: U.S. Environmental Protection Agency. 40 CFR 355, Appendix A. http://www.epa.gov/epahome/cfr40.htm. June 06, 2003.

EPA. 2003h. Identification and listing of hazardous waste. Hazardous constituents. Washington, DC: U.S. Environmental Protection Agency. 40 CFR 261, Appendix VIII.

http://www.epa.gov/epahome/cfr40.htm. June 06, 2003.

*EPA. 2003i. Land disposal restrictions. Universal treatment standards. Washington, DC: U.S. Environmental Protection Agency. 40 CFR 268.48. http://www.epa.gov/epahome/cfr40.htm. June 06, 2003.

*EPA. 2003j. National emission standards for hazardous air pollutants. List of pollutants. Washington, DC: U.S. Environmental Protection Agency. 40 CFR 61.01. http://www.epa.gov/epahome/cfr40.htm. June 06, 2003.

EPA. 2003k. National primary drinking water regulations. Maximum contaminant level goals for inorganic contaminants. Washington, DC: U.S. Environmental Protection Agency. 40 CFR 141.51. http://www.epa.gov/epahome/cfr40.htm. June 06, 2003. 
*EPA. 20031. National primary drinking water regulations. Maximum contaminant levels for inorganic contaminants. Washington, DC: U.S. Environmental Protection Agency. 40 CFR 141.62. http://www.epa.gov/epahome/cfr40.htm. June 06, 2003.

EPA. 2003m. Standards for owners and operators of hazardous waste treatment, storage, and disposal facilities. Ground-water monitoring list. Washington, DC: U.S. Environmental Protection Agency. 40 CFR 264, Appendix IX. http://www.epa.gov/epahome/cfr40.htm. June 06, 2003.

*EPA. 2003n. Standards for the management of specific hazardous wastes and specific types of hazardous waste management facilities. Washington, DC: U.S. Environmental Protection Agency. 40 CFR 266, Appendix V. http://www.epa.gov/epahome/cfr40.htm. June 06, 2003.

*EPA. 2003o. Standards for the management of specific hazardous wastes and specific types of hazardous waste management facilities. Health-based limits for exclusion of waste-derived residues. Washington, DC: U.S. Environmental Protection Agency. 40 CFR 266, Appendix VII. http://www.epa.gov/epahome/cfr40.htm. June 06, 2003.

EPA. 2003p. Standards for the use or disposal of sewage sludge. Pollutant limits. Washington, DC: U.S. Environmental Protection Agency. 40 CFR 503.13. http://www.epa.gov/epahome/cfr40.htm. June 06, 2003.

*EPA. 2003q. Standards for the use or disposal of sewage sludge. Pollutant limits. Washington, DC: U.S. Environmental Protection Agency. 40 CFR 503.43. http://www.epa.gov/epahome/cfr40.htm. June 06, 2003.

*EPA. 2003r. Toxic chemical release reporting: Community right-to-know. Chemicals and chemical categories to which this part applies. Washington, DC: U.S. Environmental Protection Agency. 40 CFR 372.65. http://www.epa.gov/epahome/cfr40.htm. June 06, 2003.

*EPA. 2003s. Water quality guidance for the Great Lakes system. Pollutants of initial focus in the Great Lakes water quality initiative. Washington, DC: U.S. Environmental Protection Agency. 40 CFR 132, Table 6. http://www.epa.gov/epahome/cfr40.htm. June 06, 2003.

*EPA. 2003t. National human exposure assessment survey (NHEXAS) databases. Washington, DC: U.S. Environmental Protection Agency. http://oaspub.epa.gov/eims/eimsapi.search?partner=ordheds\&frm=simple (query keyword = NHEXAS). August 29, 2003.

*EPA. 2003u. 1996 modeled ambient concentration for nickel compounds. Washington, DC: U.S. Environmental Protection Agency. http://www.epa.gov/ttnatw01/nata/pdf/nicke_conc.pdf. August 28, 2003.

Ermolli M, Menne C, Pozzi G, et al. 2001. Nickel, cobalt and chromium-induced cytotoxicity and intracellular accumulation in human hacat keratinocytes. Toxicology 159(1-2):23-31.

*Eun HC, Marks R. 1990. Dose-response relationships for topically applied antigens. Br J Dermatol 122:491-499.

*Evans LJ. 1989. Chemistry of metal retention by soils. Environ Sci Technol 23:1046-1056.

*Evans EG, Evans GF, Ray DB, et al. 1984. Air quality data for metals 1977 through 1979 from the national air surveillance networks. Research Triangle Park, NC: Office of Research and Development, 
Environmental Monitoring Systems Laboratory, U.S. Environmental Protection Agency. EPA600S483053.

*Evans JE, Miller ML, Andringa A, et al. 1995. Behavioral, histological, and neurochemical effects of nickel(II) on the rat olfactory system. Toxicol Appl Pharmacol 130:209-220.

FDA. 1993. Quality standard for foods with no identity standards; bottled water. Food and Drug Administration. Fed Regist 58:41612-41619.

*FDA. 2000. Total diet study statistics on element results. Washington, DC: Food and Drug Administration. Revision 1, 1991-1998. April 25, 2000.

*FDA. 2003a. Beverages. Bottled water. Washington, DC: Food and Drug Administration. 21 CFR 165.110. http://www.access.gpo.gov/cgi-bin/cfrassemble.cgi?title=200321. June 06, 2003.

*FDA. 2003b. Direct food substances generally recognized as safe. Nickel. Washington, DC: Food and Drug Administration. 21 CFR 184.1537. http://www.access.gpo.gov/cgi-

bin/cfrassemble.cgi?title=200321. June 06, 2003.

*FDA. 2003c. Indirect food additives: Paper and paperboard components. Components of paper and paperboard in contact with dry food. Washington, DC: Food and Drug Administration. 21 CFR 176.180(b)(2). http://www.access.gpo.gov/cgi-bin/cfrassemble.cgi?title=200321. June 06, 2003.

*FEDRIP. 2004. Federal Research in Progress. Springfield, VA: National Technical Information Service.

*Feng X, Melander AP, Klaue B. 2000. Contribution of municipal waste incineration to trace metal deposition on the vicinity. Water Air Soil Pollution 119:295-316.

Figa-Talamanca I, Petrelli G. 2000. Reduction in male births among workers exposed to metal fumes. Int J Epidemiol 29(2):381-383.

Finch GL, Fisher GL, Hayes TL. 1987. The pulmonary effects and clearance of intratracheally instilled Ni3S2 and TiO2 in mice. Environ Res 42:83-93.

*Fischer T. 1989. Occupational nickel dermatitis. In: Maibach HI, Menne T, eds. Nickel and the skin: Immunology and toxicology. Boca Raton, FL: CRC Press, Inc., 117-132.

*Fisher GL, Chrisp CE, McNeill DA. 1986. Lifetime effects of intratracheally instilled nickel subsulfide on B6C3F1 mice. Environ Res 40:313-320.

*Fletcher GG, Rossetto FE, Turnbull JD, et al. 1994. Toxicity, uptake, and mutagenicity of particulate and soluble nickel compounds. Environ Health Perspect 102(suppl 3):69-79.

Fleming CJ, Burden AD, Forsyth A. 1999. The genetics of allergic contact hypersensitivity to nickel. Contact Dermatitis 41(5):251-253.

*Flint GN, Packirisamy S. 1995. Systemic nickel: The contribution made by stainless-steel cooking utensils. Contact Dermatitis 32:218-224. 
*Fomon SJ. 1966. Body composition of the infant: Part I: The male "reference infant". In: Falkner F, ed. Human development. Philadelphia, PA: WB Saunders, 239-246.

*Fomon SJ, Haschke F, Ziegler EE, et al. 1982. Body composition of reference children from birth to age 10 years. Am J Clin Nutr 35:1169-1175.

*Forgacs Z, Paksy K, Lazar P, et al. 1998. Effect of Ni2+ on the testosterone production of mouse primary Leydig cell culture. J Toxicol Environ Health A 55:213-224.

*Forgacs Z, Nemethy Z, Revesz C, et al. 2001. Specific amino acids moderate the effects on Ni2+ on the testosterone production of mouse Leydig cells in vitro. J Toxicol Environ Health A 62(5):349-358.

*Foulkes EC, Blanck S. 1984. The selective action of nickel on tubule function in rabbit kidneys. Toxicology 33:245-259.

*Fournier P-G, Govers TR. 2003. Contamination by nickel, copper and zinc during the handling of euro coins. Contact Dermatitis 48:181-188.

*Francis AJ, Dodge CJ. 1990. Anaerobic microbial remobilization of toxic metals coprecipitated with iron oxide. Environ Sci Technol 24:373-378.

*Frankild S, Andersen KE, Nielsen GD. 1995. Effect of sodium lauryl sulfate (SLS) on in vitro percutaneous penetration of water, hydrocortisone and nickel. Contact Dermatitis 32:338-345.

Frasier LH, Chaudhuri I. 2003. Short-term inhalation toxicity benchmark for nickel oxide. Toxicol Sci 72(S-1):142-143.

*Frenkel K, Karkoska J, Cohen B, et al. 1994. Occupational exposures to Cd, Ni, and Cr modulate titers of antioxidized DNA base autoantibodies. Environ Health Perspect 102(suppl 3):221-225.

*Friedland AJ, Johnson AH, Siccama TG. 1986. Zinc, Cu, Ni and Cd in the forest floor in the northeastern United States. Water Air Soil Pollut 29:233-243.

*FSTRAC. 1990. Summary of state and federal drinking water standards and guidelines, 1990. Washington, DC: American Water Works Association.

*Fullerton A, Andersen JR, Hoelgaard A, et al. 1986. Permeation of nickel salts through human skin in vitro. Contact Dermatitis 15:173-177.

*Funakoshi T, Inoue T, Shimada H, et al. 1997. The mechanisms of nickel uptake by rat primary hepatocyte cultures: Role of calcium channels. Toxicology 124(1):21-26.

*Galbreath KC, Crocker CR, Nyberg CM, et al. 2003. Nickel speciation measurements of urban particulate matter: Method evaluation and relevance to risk assessment. J Environ Monit 4(3):56N-61N.

Galloway WB, Lake JL, Phelps DK, et al. 1983. The mussel watch: Intercomparison of trace level constituent determinations. Environ Toxicol Chem 2:395-410.

Gandini C, Locatelli C, Mennoia NV, et al. 2000. Acute occupational poisoning by inhalation of nickel carbonate [Abstract]. Toxicol Lett 116(Suppl. 1):84. 
*Gawkrodger DJ, Cook SW, Fell GS, et al. 1986. Nickel dermatitis: The reaction to oral nickel challenge. Br J Dermatol 115:33-38.

*Gawkrodger DJ, Healy J, Howe AM. 1995. The prevention of nickel contact dermatitis. A review of the use of binding agents and barrier creams. Contact Dermatitis 32:257-265.

Gawkrodger DJ, Lewis FM, Shah M. 2000. Contact sensitivity to nickel and other metals in jewelry reactors. J Am Acad Dermatol 43(1):31-36.

*Gerin M, Fletcher AC, Gray C, et al. 1993. Development and use of a welding process exposure matrix in a historical prospective study of lung cancer risk in European welders. Int J Epidemiol 22(6, Suppl 2):S22-28.

*Gerritse RG, Vriesema R, Dalenberg J, et al. 1982. Effect of sewage sludge on trace element mobility in soils. J Environ Qual 7:359-364.

*Ghezzi I, Baldasseroni A, Sesana G, et al. 1989. Behaviour of urinary nickel in low-level occupational exposure. Med Lav 80:244-250.

*Gibbons RD, Dolan DG, May H, et al. 1999. Statistical comparison of leachate from hazardous, codisposal, and municipal solid waste landfills. Ground Water Monit Remed (fall) :57-72.

*Gilman JPW. 1962. Metal carcinogenesis. II. A study on the carcinogenic activity of cobalt, copper, iron and nickel compounds. Cancer Res 22:159-162.

*Gitlitz PH, Sunderman FW Jr, Goldblatt PJ. 1975. Aminoaciduria and proteinuria in rats after a single intraperitoneal injection of Ni(II). Toxicol Appl Pharmacol 34:430-440.

*Giusti L, Yang Y-L, Hewitt CN, et al. 1991. The solubility and partitioning of atmospherically derived trace metals in artificial and natural waters: A review. Atmos Environ 27A:1567-1578.

*Giwercman A, Carlsen E, Keiding N, et al. 1993. Evidence for increasing incidence of abnormalities of the human testis: A review. Environ Health Perspect Suppl 101(2):65-71.

*Gladney ES, Gordon SE, Zoller WH. 1978. Coal combustion: Source of elements in urban air. J Environ Sci Health A13:481-491.

*Gladney ES, Perrin DR, Robinson RD, et al. 1984. Multitechnique determination of elemental concentrations in NBS urban air particulate SRM 1648 and evaluation of its use for quality assurance. J Radioanal Nucl Chem 83:379-386.

*Godbold JH, Tompkins EA. 1979. A long-term mortality study of workers occupationally exposed to metallic nickel at the Oak Ridge Gaseous Diffusion Plant. J Occup Med 21:799-806.

*Goldberg M, Goldberg P, Leclerc A, et al. 1987. Epidemiology of respiratory cancers related to nickel mining and refining in New Caledonia (1978-1984). Int J Cancer 15:300-304.

*Goldberg M, Goldberg P, Leclerc A, et al. 1994. A 10-year incidence survey of respiratory cancer and a case-control study within a cohort of nickel mining and refining workers in New Caledonia. Cancer Causes Control 5:15-25. 
*Goldfrank LR, Weisman RS, Flomenbaum NE, et al. 1990. Goldfrank's toxicologic emergencies. 4th ed. Norwalk, CT: Appleton and Lange, 656-658.

*Golomb D, Ryan D, Eby N, et al. 1997. Atmospheric deposition of toxics onto Massachusetts Bay. I. Metals. Atmos Environ 31(9):1349-1359.

*Gordon CJ. 1989. Effect of nickel chloride on body temperature and behavioral thermoregulation in the rat. Neurotoxicol Teratol 11:317-320.

*Gordon T, Amdur MO. 1991. Responses of the respiratory system to toxic agents. In: Amdur MO, Doull J, Klaassen CD, eds. Casarett and Doull's toxicology. 4th ed. New York, NY: McGraw-Hill, Inc., 383-406.

*Gordon CJ, Fogelson L, Stead AG. 1989. Temperature regulation following nickel intoxication in the mouse: Effect of ambient temperature. Comp Biochem Physiol 92:73-76.

*Gosselin RE, Smith RP, Hodge HC. 1984. Clinical toxicology of commercial products, 5th ed. Baltimore, MD: Williams \& Wilkins, II, 145.

*Goutet M, Ban M, Binet S. 2000. Effects of nickel sulfate on pulmonary natural immunity in Wistar rats. Toxicology 145(1):15-26.

*Graham JA, Miller FJ, Daniels MJ, et al. 1978. Influence of cadmium, nickel and chromium on primary immunity in mice. Environ Res 16:77-87.

*Grandjean P. 1984. Human exposure to nickel. In: Sunderman FW Jr, ed. Nickel in the human environment. Proceedings of a joint symposium, IARC scientific publication no. 53. Lyon, France: International Agency for Research on Cancer, 469-485.

*Graney JR, Landis MS, Norris GA. 2004. Concentrations and solubility of metals from indoor and personal exposure PM2.5 samples. Atmos Environ 38(2):237-247.

*Gray LE Jr, Kavlock RJ, Ostby J, et al. 1986. An evaluation of figure-eight maze activity and general behavioral development following prenatal exposure to forty chemicals: Effects of cytosine arabinoside, dinocap, nitrofen, and vitamin A. Neurotoxicology 7:449-462.

*Green MHL, Muriel WJ, Bridges BA. 1976. Use of simplified fluctuation test to detect low levels of mutagens. Mutat Res 38:33-42.

*Greenberg RR, Zoller WH, Gordon SE. 1978. Composition and size distribution of particles released in refuse incinerators. Environ Sci Technol 12:566-573.

*Grimsrud TK, Berge SR, Haldorsen T, et al. 2002. Exposure to different forms of nickel and risk of lung cancer. Am J Epidemiol 156(12):1123-1132.

*Grimsrud TK, Berge SR, Martinsen JI, et al. 2003. Lung cancer incidence among Norweigian nickelrefinery workers 1953-2000. J Environ Monit 5(2):190-197.

Gupta S, Ahmad N, Hussein MM, et al. 2000. Involvement of nitric oxide in nickel-induced hyperglycemia in rats. Nitric Oxide 4(2):129-138. 
*Gutenmann WH, Rutzke M, Kuntz HT, et al. 1994. Elements and polychlorinated biphenyls in sewage sludge of large cities in the United States. Chemosphere 28(4):725-728.

*Guzelian PS, Henry CJ, Olin SS, eds. 1992. Similarities and differences between children and adults: Implications for risk assessment. Washington, DC: International Life Sciences Institute Press.

Haack E, Warren LA. 2003. Biofilm hydrous manganese oxyhydroxides and metal dynamics in acid rock drainage. Environ Sci Technol 37(18):4138-4147.

*Haber LT, Erdreicht L, Diamond GL, et al. 2000. Hazard identification and dose response of inhaled nickel-soluble salts. Regul Toxicol Pharmacol 31:210-230.

Haley PJ, Bise DE, Muggenburg BA, et al. 1987. Immunopathologic effects of nickel subsulfide on the primate pulmonary immune system. Toxicol Appl Pharmacol 88:1-12.

*Haley PJ, Shopp GM, Benson JM, et al. 1990. The immunotoxicity of three nickel compounds following 13-week inhalation exposure in the mouse. Fundam Appl Toxicol 15:476-487.

*Halim M, Conte P, Piccolo A. 2003. Potential availability of heavy metals to phytoextraction from contaminated soils induced by exogenous humic substances. Chemosphere 52:265-275.

*Hamilton-Koch W, Snyder RD, Lavelle JM. 1986. Metal-induced DNA damage and repair in human diploid fibroblasts and Chinese hamster ovary cells. Chem Biol Interact 59:17-28.

*Hansen K, Stern RM. 1984. Toxicity and transformation potency of nickel compounds in BHK cells in vitro. In: Sunderman FW Jr, Aitio A, Berlin A, eds. Nickel in the human environment. IARC scientific publication no. 53. Lyon, France: International Agency for Research on Cancer, 193-200.

*Hansen LD, Fisher JL. 1980. Distribution in coal fly ash particles. Environ Sci Technol 14:1111-1117.

*Hansen KS, Lauritsen JM, Skytthe A. 1996. Cancer incidence among mild steel and stainless steel welders and other metal workers. Am J Ind Med 30(4):373-382.

*Hansson HC, Ekholm AKP, Ross HB. 1988. Rainwater analysis: A comparison between protoninduced x-ray emission and graphite furnace atomic absorption spectroscopy. Environ Sci Technol 22:527-531.

*Hargitai L. 1989. The role of humus status of soils in binding toxic elements and compounds. Sci Total Environ 81/82:643-651.

Harkin A, Hynes MJ, Masterson E, et al. 2003. A toxicokinetic study of nickel-induced immunosuppression in rats. Immunopharmacol Immunotoxicol 45(4):655-670.

*Haro RT, Furst A, Falk H. 1968. Studies on the acute toxicity of nickelocene. Proc West Pharmacol Soc 11:39-42.

Harrison SJ, Vale JA, Watts CD. 1993. The estimation of aerial inputs of metals to estuarine waters from point pattern data using an isoplething technique: Severn Estuary, UK. Atmos Environ 27A:2365-2373.

*Hartwig A, Beyersmann D. 1989. Enhancement of UV-induced mutagenesis and sister-chromatid exchanges by nickel ions in V79 cells: Evidence for inhibition of DNA repair. Mutat Res 217:65-73. 
*Hartwig A, Kruger I, Beyersmann D. 1994. Mechanisms in nickel genotoxicity: The significance of interactions with DNA repair. Toxicol Lett 72:353-358.

*Hasanen E, Pohjola V, Hahkala M, et al. 1986. Emissions from power plants fueled by peat, coal, natural gas and oil. Sci Total Environ 54:29-51.

*Hassler E, Lind B, Nilsson B, et al. 1983. Urinary and fecal elimination of nickel in relation to airborne nickel in a battery factory. Ann Clin Lab Sci 13:217-224.

*Hawley GG. 1981. Condensed chemical dictionary. 10th ed. New York, NY: Van Nostrand Reinhold Co., 724-725.

*Hay DJ, Finkelstein A, Klicius R. 1986. The national incinerator testing and evaluation program twostage incinerator combustion tests. Chemosphere 15:9-12.

*HazDat. 2005. Agency for Toxic Substances and Disease Registry (ATSDR), Atlanta, GA. http://www.atsdr.cdc.gov. January 13, 2005.

Healy J, Johnson S, Little MC, et al. 1998. An in vitro study of the use of chelating agents in cleaning nickel-contaminated human skin: an alternative approach to preventing nickel allergic contact dermatitis. Contact Dermatitis 39:171-181.

*Heit M, Klusek C, Baron J. 1984. Evidence of deposition of anthropogenic pollutants in remote Rocky Mountain air. Water Air Soil Pollut 22:403-416.

*Heit M, Schofield C, Driscoll CT, et al. 1989. Trace element concentrations in fish from three Adirondack lakes New York USA with different pH values. Water Air Soil Pollut 44:9-30.

*Helmers E, Schrems O. 1995. Wet deposition of metals to the tropical north and south Atlantic Ocean. Atmos Environ 29(19):2475-2484.

*Hendel RC, Sunderman FW Jr. 1972. Species variations in the proportions of ultrafiltrable and proteinbound serum nickel. Res Commun Chem Pathol Pharmacol 4:141-146.

Henriksson J, Tallkvist J, Tjalve H. 1997. Uptake of nickel into the brain via olfactory neurons in rats. Toxicol Lett 91(2):153-162.

*Herpin U, Siewers U, Markert B, et al. 2004. Second German heavy-metal survey by means of mosses, and comparison of the first and second approach in Germany and other European countries. Environ Sci Pollut Res 11(1):57-66.

Heule F, Tahapary GJ, Bello CR, et al. 1998. Delayed-type hypersensitivity to contact allergens in psoriasis. A clinical evaluation. Contact Dermatitis 38(2):78-82.

Hindsen M, Bruze M, Christensen OB. 1997. The significance of previous allergic contact dermatitis for elicitation of delayed hypersensitivity to nickel. Contact Dermatitis 37(3):101-106.

*Hindsen M, Bruze M, Christensen OB. 2001. Flare-up reactions after oral challenge with nickel in relation to challenge doses and intensity and time of previous patch test reactions. J Am Acad Dermatol 44(4):616-623. 
*Hindsen M, Christensen OB, Moller B. 1994. Nickel levels in serum and urine in five different groups of eczema patients following oral ingestion of nickel. Acta Derm Venereol 74:176-178.

Hirano S, Asami T, Kodama N, et al. 1994a. Correlation between inflammatory cellular responses and chemotactic activity in bronchoalveolar lavage fluid following intratracheal instillation of nickel sulfate in rats. Arch Toxicol 68(7):444-449.

*Hirano S, Shimada T, Osugi J, et al. 1994b. Pulmonary clearance and inflammatory potency of intratracheally instilled or acutely inhaled nickel sulfate in rats. Arch Toxicol 68:548-554.

*Ho VC, Johnston MM. 1986. Nickel dermatitis in infants. Contact Dermatitis 15:270-273.

*Ho W, Furst A. 1973. Nickel excretion by rats following a single treatment. Proc West Pharmacol Soc $16: 245-248$.

*Hoel DG, Davis DL, Miller AB, et al. 1992. Trends in cancer mortality in 15 industrialized countries, 1969-1986. J Natl Cancer Inst 84(5):313-320.

*Hoey MJ. 1966. The effects of metallic salts on the histology and function of the rat testis. J Reprod Fertil 12:461-471.

*Hopfer SM, Sunderman FW Jr. 1978. Manganese inhibition of nickel subsulfide induction of erythrocytosis in rats. Res Commun Chem Pathol Pharmacol 19(2):337-345.

*Hopfer SM, Sunderman FW Jr. 1988. Hypothermia and deranged rhythm of core body temperature in nickel chloride-treated rats. Res Commun Chem Pathol Pharmacol 62:495-505.

*Hopfer SM, Fay WP, Sunderman FW Jr. 1989. Serum nickel concentrations in hemodialysis patients with environmental exposure. Ann Clin Lab Sci 19:161-167.

*Hopfer SM, Sunderman FW Jr, Reid MC, et al. 1984. Increased immunoreactive erythropoietin in serum and kidney extracts of rats with Ni3S2-induced erythrocytosis. Res Commun Chem Pathol Pharmacol 43:299-305.

Horak E, Sunderman FW Jr. 1973. Fecal nickel excretion by healthy adults. Clin Chem 19:429-430.

*Horak E, Sunderman FW Jr. 1975a. Effects of Ni(II) upon plasma glucagon and glucose in rats. Toxicol Appl Pharmacol 33:388-391.

*Horak E, Sunderman FW Jr. 1975b. Effects of Ni(II), other divalent metal ions, and glucagon upon plasma glucose concentrations in normal, adrenalectomized and hypophysectomized rats. Toxicol Appl Pharmacol 32:316-329.

*Horak E, Sunderman FW Jr, Sarkar B. 1976. Comparisons of antidotal efficacy of chelating drugs upon acute toxicity of Ni(II) in rats. Res Commun Chem Pathol Pharmacol 14:153-165.

*Horie A, Tanaka I, Haratake J, et al. 1985. Electron microscopy of pulmonary lesions including carcinoma, induced by inhalation exposure of rats to nickel oxide aerosol. In: Brown SS, Sunderman FW Jr, eds. Progress in nickel toxicology. Proceedings of the 3rd International Congress on Nickel Metabolism and Toxicology. Oxford, UK: Blackwell, 41-44. 
*Horng CJ, Horng PH, Hsu JW, et al. 2003. Simultaneous determination of urinary cadmium, cobalt, lead, and nickel concentrations in steel production workers by differential pulse stripping voltammetry. Arch Environ Health 58(2):104-110.

HSDB. 1996. Hazardous Substances Data Bank. National Library of Medicine, National Toxicology Information Program, Bethesda, MD.

*HSDB. 2003. Environmental standards and regulations. Hazardous Substances Data Bank. National Library of Medicine, National Toxicology Information Program, Bethesda, MD.

*HSDB. 2004. Environmental standards and regulations. Hazardous Substances Data Bank. National Library of Medicine, National Toxicology Information Program, Bethesda, MD.

*Hsie AW, Johnson NP, Couch DB, et al. 1979. Quantitative mammalian cell mutagenesis and a preliminary study of the mutagenic potential of metallic compounds. In: Kharasch N, ed. Trace metals in health and disease. New York, NY: Raven Press, 55-69.

*Hsieh TH, Yu CP, Oberdörster G. 1999a. A dosimetry model of nickel compounds in the rat lung. Inhal Toxicol 11:229-246.

*Hsieh TH, Yu CP, Oberdörster G. 1999b. Modeling of deposition and clearance of inhaled Ni compounds in the human lung. Regul Toxicol Pharmacol 30(1):18-28.

Huber LT, Redirect L, Diamond GL, et al. 2000. Hazard identification and dose response of inhaled nickel-soluble salts. Regul Toxicol Pharmacol 31:210-230.

*Hueper WC. 1958. Experimental studies in metal carcinogenesis. IX. Pulmonary lesions in guinea pigs and rats exposed to prolonged inhalation of powdered metallic nickel. Arch Pathol 65:600-607.

*IARC. 1986. Environmental carcinogens: Selected methods of analysis. Volume 8. Some metals: As, $\mathrm{Be}, \mathrm{Cd}, \mathrm{Cr}, \mathrm{Ni}, \mathrm{Pb}, \mathrm{Se}, \mathrm{Zn}$. IARC Scientific Publication No. 71. Lyon, France: International Agency for Research on Cancer, World Health Organization. Methods 11 and 17.

*IARC. 1990. IARC monographs on the evaluation of carcinogenic risks to humans. Volume 49: Chromium, nickel and welding. Lyon, France: International Agency for Research on Cancer, World Health Organization, 257-445.

IARC. 1994. IARC monographs on the evaluation of carcinogenic risks to humans. Lists of IARC evaluations. Lyon, France: International Agency for Research on Cancer, World Health Organization.

*Ilback N-G, Fohlman J, Friman GA. 1992. A common viral infection can change nickel target organ distribution. Toxicol Appl Pharmacol 114(1):166-170.

*Ilback N-G, Fohlman J, Friman G. 1994. Changed distribution and immune effects of nickel augment viral-induced inflammatory heart lesions in mice. Toxicology 91:203-219.

*International Committee on Nickel Carcinogenesis in Man. 1990. Report of the International Committee on Nickel Carcinogenesis in Man. Scand J Work Environ Health 16(1):1-82. 
*IOM. 2002. Arsenic, boron, nickel, silicon and vanadium. In: Dietary reference intakes for vitamin A, vitamin $\mathrm{K}$, arsenic, boron, chromium, copper, iodine, iron, manganese, molybdenum, nickel, silicon, vanadium and zinc. Institute of Medicine. Washington, DC: National Academy Press.

*IRIS. 1996. Integrated Risk Information System. Cincinnati, OH: Office of Health and Environmental Assessment, Environmental Criteria and Assessment Office, U.S. Environmental Protection Agency.

*IRIS. 2005. Nickel. Washington, DC: Integrated Risk Information System. http://www.epa.gov/iris/. January 13, 2005.

*Iscan M, Coban T, Eke BC, et al. 1995. Differential responses of hepatic monooxygenases and glutathione $S$-transferases of mice to a combination of cadmium and nickel. Comp Biochem Physiol 111C(1):61-68.

Ishihara Y, Kyono H, Serita F, et al. 2000. Inflammatory responses and mucus secretion in rats with acute bronchiolitis induced by nickel chloride. Inhal Toxicol 14:417-430.

*Ishimatsu S, Kawamoto T, Matsuno K, et al. 1995. Distribution of various nickel compounds in rat organs after oral administration. Biol Trace Elem Res 49(1):43-52.

*Iyengar G. 1986. Sampling, storage and pretreatment of biological material. In: O'Neill IK, Schuller P, Fishbein L, eds. Some metals: As, Be, Cd, Cr, Ni, Pb, Se, Zn. IARC Scientific Publication No. 71. Lyon, France: International Agency for Research on Cancer, 141-158.

*Iyengar G. 1989. Elemental analysis of biological systems. Vol. I: Biomedical, environmental, compositional, and methodological aspects of trace elements. Boca Raton, FL: CRC Press, 173-174.

*Jakobsson K, Mikoczy Z, Skerfving S. 1997. Deaths and tumours among workers grinding stainless steel: A follow up. Occup Environ Med 54(11):825-829.

*Jasim S, Tjalve H. 1986a. Effects of sodium pyridimethione on the uptake and distribution of nickel, cadmium and zinc in pregnant and non-pregnant mice. Toxicology 38:327-350.

*Jasim S, Tjalve H. 1986b. Effect of zinc pyridimethione on the tissue disposition of nickel and cadmium in mice. Acta Pharmacol Toxicol 59:204-208.

*Jenkins DW. 1980. Nickel accumulation in aquatic biota. In: Nriagu JO, ed. Nickel in the environment. New York, NY: John Wiley and Sons, 283-337.

Jensen CS, Lisby S, Larsen JK, et al. 2004. Characterization of lymphocyte subpopulations and ctyokine profiles in peripheral blood of nickel-sensitive individuals with systemic contact dermatitis after oral nickel exposure. Contact Dermatitis 50(1):31-38.

*Jensen CS, Menne T, Lisby S, et al. 2003. Experimental systemic contact dermatitis from nickel: A dose-response study. Contact Dermatitis 49(3):124-132.

Johansen JD, Menne T, Christophersen J, et al. 2000. Changes in the pattern of sensitization to common contact allergens in Denmark between 1985-86 and 1997-98, with a special view to the effect of preventive strategies. Br J Dermatol 142(3):490-495. 
*Johanson CE. 1980. Permeability and vascularity of the developing brain: Cerebellum vs cerebral cortex. Brain Res 190:3-16.

*Johansson A, Camner P. 1986. Adverse effects of metals on the alveolar part of the lung. Scan Electron Microsc 2:631-637.

*Johansson A, Camner P, Jarstrand C, et al. 1980. Morphology and function of alveolar macrophages after long-term nickel exposure. Environ Res 23:170-180.

*Johansson A, Camner P, Robertson B. 1981. Effects of long-term nickel dust exposure on rabbit alveolar epithelium. Environ Res 25:391-402.

*Johansson A, Curstedt T, Jarstrand C, et al. 1988b. Effects on the rabbit lung of combined exposure to nickel and trivalent chromium. J Aerosol Sci 19:1075-1078.

*Johansson A, Curstedt T, Robertson B, et al. 1983. Rabbit lung after inhalation of soluble nickel. II. Effects of lung tissue and phospholipids. Environ Res 31:399-412.

*Johansson A, Curstedt T, Robertson B, et al. 1989. Lung lesions after experimental combined exposure to nickel and trivalent chromium. Environ Res 50:103-119.

*Johansson A, Lundborg M, Skog S, et al. 1987. Lysozyme activity in ultrastructurally defined fractions of alveolar macrophages after inhalation exposure to nickel. Br J Ind Med 44:47-52.

*Johansson A, Wiernik A, Lundborg M, et al. 1988a. Alveolar macrophages in rabbits after combined exposure to nickel and trivalent chromium. Environ Res 46:120-132.

*Johnson DL, Davis BL, Dzubay TG, et al. 1984. Chemical and physical analyses of Houston aerosol for interlaboratory comparison of source apportionment procedures. Atmos Environ 18:1539-1553.

*Jordan WP, King SE. 1979. Nickel feeding in nickel-sensitive patients with hand eczema. J Am Acad Dermatol 1:506-508.

Juhlin L, Johansson GO, Bennich H, et al. 1969. Immunoglobulin E in dermatoses. Arch Dermatol 100:12-16.

*Kaaber K, Veinen NK, Tjell JC. 1978. Low nickel diet in the treatment of patients with chronic nickel dermatitis. Br J Dermatol 98:197-201.

Kaasalainen M, Yli-Halla M. 2003. Use of sequential extraction to assess metal partitioning in soils. Environ Pollut 126(2):225-233.

*Kadota I, Kurita M. 1955. Hyperglycemia and islet cell damage caused by nickelous chloride. Metab Clin Exp 4:337-342.

Kaldor J, Peto J, Easton D, et al. 1986. Models for respiratory cancer in nickel refinery workers. J Natl Cancer Inst 77:841-848.

*Käkelä R, Käkelä A, Hyvarinen H. 1999. Effects of nickel chloride on reproduction of the rat and possible antagonistic role of selenium. Comp Biochem Physiol C 123(1):27-33. 
Kalliomaki PL, Hyvarinen KH, Aitio A, et al. 1986. Kinetics of the metal components of intratracheally instilled stainless steel welding fume suspensions in rats. Br J Ind Med 43:112-119.

*Kamboj VP, Kar AB. 1964. Anti-testicular effect of metallic and rare earth salts. J Reprod Fertil 7:2123.

*Kanematsu M, Hara M, Kada T. 1980. Rec-assay and mutagenicity studies on metal compounds. Mutat Res 77:109-116.

Kanerva L, Jolanki R, Estlander T, et al. 2000. Incidence rates of occupational allergic contact dermatitis caused by metals. Am J Contact Dermatitis 11(3):155-160.

Kang J, Zhang Y, Chen J, et al. 2003. Nickel-induced histone hypoacetylation: The role of reactive oxygen species. Toxicol Sci 74:279-286.

*Kapsenberg ML, Van der Pouw-Kraan T, Stiekeme FE. 1988. Direct and indirect nickel-specific stimulation of $\mathrm{T}$ lymphocytes from patients with allergic contact dermatitis to nickel. Eur J Immunol 18:977-982.

Karaczyn AA, Bal W, North SL, et al. 2003. The octapeptidic end of the C-terminal tail of histone H2A is cleaved off in cells exposed to carcinogenic nickel(II). Chem Res Toxicol 16(12):1555-1559.

*Kargacin B, Klein CB, Costa M. 1993. Mutagenic responses of nickel oxides and nickel sulfides in Chinese hamster v79 cell lines at the xanthine-guanine phosphoribosyl transferase locus. Mutat Res 300:63-72.

*Karjalainen S, Kerttula R, Pukkala E. 1992. Cancer risk among workers at a copper/nickel smelter and nickel refinery in Finland. Int Arch Occup Environ Health 63:547-551.

*Kaschl A, Römheld V, Chen Y. 2002. The influence of soluble organic matter from municipal solid waste compost on trace metal leaching in calcareous soils. Sci Total Environ 291:45-57.

*Kashulin NA, Ratkin NE, Dauvalter VA, et al. 2001. Impact of airborne pollution on the drainage area of subarctic lakes and fish. Chemosphere 42:51-59.

Kasprzak KS, Bal W, Karaczyn AA. 2003a. The role of chromatin damage in nickel-induced carcinogenesis. A review of recent developments. J Environ Monit 5(2):183-187.

*Kasprzak KS, Gabryel P, Jarczewska K. 1983. Carcinogenicity of nickel(II) hydroxides and nickel(II) sulfate in Wistar rats and its relation to the in vitro dissolution rates. Carcinogenesis 4:275-279.

Kasprzak KS, Marchow L, Breborowicz J. 1973. Pathological reactions in rat lungs following intratracheal injection of nickel subsulfide and 3,4-benzopyrene. Res Commun Chem Pathol Pharmacol 6:237-245.

*Kasprzak KS, Sunderman FW Jr, Salnikow K. 2003b. Nickel carcinogenesis. Mutat Res 533(1-2):6797.

*Kasprzak KS, Waalkes MP, Poirier LA. 1986. Effects of magnesium acetate on the toxicity of nickelous acetate in rats. Toxicology 42:57-68. 


\section{REFERENCES}

Kaur P, Dani HM. 2003. Carcinogenicity of nickel is the result of its binding to RNA and not to DNA. J Environ Pathol Toxicol Oncol 22(1):29-39.

Kawanishi S, Inoue S, Oikawa S, et al. 2001. Oxidative DNA damage in cultured cells and rat lungs by carcinogenic nickel compounds. Free Radic Biol Med 31(1):108-116.

Kawanishi S, Oikawa S, Inoue S, et al. 2002. Distinct mechanisms of oxidative DNA damage induced by carcinogenic nickel subsulfide and nickel oxides. Environ Health Perspect 110(Suppl 5):789-791.

*Keczkes K, Basheer AM, Wyatt EH. 1982. The persistence of allergic contact sensitivity: A 10-year follow-up in 100 patients. Br J Dermatol 107:461-465.

*Kempton S, Sterrit RM, Lester JN. 1987. Heavy metal removal in primary sedimentation. I. The influence of metal solubility. Sci Total Environ 63:231-247.

*Kenney MA, McCoy H. 1992. A review of biointeractions of Ni and Mg. I. Enzyme, endocrine, transport, and skeletal systems. Magnes Res 5:215-222.

*Khandelwal S, Tandon SK. 1984. Effect of cadmium pretreatment on nickel toxicity. In: Sunderman FW Jr, Aitio A, Berlin A, eds. Nickel in the human environment. IARC scientific publication no. 53. Lyon France: International Agency for Research on Cancer, 293-300.

*Kilburn KH, Warshaw R, Boylen CT, et al. 1990. Cross-shift and chronic effects of stainless-steel welding related to internal dosimetry of chromium and nickel. Am J Ind Med 17:607-615.

*King LD. 1988. Retention of metals by several soils of the southeastern United States. J Environ Qual 17:239-246.

*Kinney PL, Chillrud SN, Ramstrom S, et al. 2002. Exposure to multiple air toxics in New York City. Environ Health Perspect 110 (Suppl 4):539-546.

*Kirk WS. 1988a. Nickel. Preprint from the Bureau of Mines mineral yearbook. Pittsburgh, PA: Bureau of Mines, U.S. Department of the Interior, 1-12.

*Kirk WS. 1988b. Nickel. In: Mineral commodity summaries 1988. Pittsburgh, PA: Bureau of Mines, U.S. Department of the Interior, 1-12.

*Klein CB, Kargacin B, Su L. 1994. Metal mutagenic Chinese hamster cell lines. Environ Health Perspect 102(Suppl 3):63-7.

*Kodama Y, Ishimatsu S, Matsuno K, et al. 1985a. Pulmonary deposition and clearance of a nickel oxide aerosol by inhalation. Biol Trace Elem Res 7:1-8.

* Kodama Y, Tanaka Z, Matsuno K, et al. 1985b. Pulmonary deposition and clearance of inhaled nickel oxide aerosol. In: Brown SS, Sunderman FW Jr, eds. Progress in nickel toxicology. Proceedings of the 3rd International Congress on Nickel Metabolism and Toxicology. Oxford, UK: Blackwell, 81-84.

*Kollmeier H, Seemann JW, Muller KM, et al. 1987. Increased chromium and nickel content in lung tissue and bronchial carcinoma. Am J Ind Med 11:659-669. 
*Komori M, Nishio K, Kitada M, et al. 1990. Fetus-specific expression of a form of cytochrome P-450 in human livers. Biochemistry 29:4430-4433.

*Koptsik S, Koptsik G, Livantsova S, et al. 2003. Heavy metals in soils near the nickel smelter: Chemistry, spatial variation, and impacts on plant diversity. J Environ Monit 5(3):441-450.

*Koutrakis P, Briggs SLK, Leaderer BP. 1992. Source apportionment of indoor aerosols in Suffolk and Onondaga Counties, New York. Environ Sci Technol 26:521-527.

*Kowalczyk GS, Gordon GE, Rheingrover SW. 1982. Identification of atmospheric particulate sources in Washington, D.C. using chemical element balances. Environ Sci Technol 16:79-90.

Kreyberg L. 1978. Lung cancer in workers in a nickel refinery. Br J Ind Med 35:109-116.

*Krishnan K, Andersen ME. 1994. Physiologically based pharmacokinetic modeling in toxicology. In: Hayes W, ed. Principles and methods of toxicology. 3rd ed. New York, NY: Raven Press, Ltd., 149188.

*Krishnan K, Andersen ME, Clewell HJ III, et al. 1994. Physiologically based pharmacokinetic modeling of chemical mixtures. In: Yang, RSA, ed. Toxicology of chemical mixtures. New York, NY: Academic Press, 399-437.

Kuck PH. 1997. Nickel. In: U.S. geological survey minerals yearbook. http://minerals.usgs.gov/minerals/pubs/commodity/nickel/. June 8, 1997.

*Kuck PH. 2001. Nickel. In: U.S. geological survey minerals yearbook. http://minerals.usgs.gov/minerals/pubs/commodity/nickel/. January 24, 2001.

*Kuck PH. 2002. Nickel. In: U.S. geological survey minerals yearbook. http://minerals.usgs.gov/minerals/pubs/commodity/nickel/. December 30, 2004.

*Kuligowski J, Halperin KM. 1992. Stainless steel cookware as a significant source of nickel, chromium, and iron. Arch Environ Contam Toxicol 23:211-215.

*LaBauve JM, Kotuby-Amacher J, Gambrell RP. 1988. The effect of soil properties and a synthetic municipal landfill leachate on the retention of $\mathrm{Ca}, \mathrm{Ni}, \mathrm{Pb}$, and $\mathrm{Zn}$ in soil and sediment materials. J Water Pollut Control Fed 60:379.

*LaBella FS, Dular R, Lemon P, et al. 1973. Prolactin secretion is specifically inhibited by nickel. Nature 245:330-332.

Laila AMA, Azza FA, El-Sehely M, et al. 2003. Effect of chelating agent (DMSA) and vitamin C on nickel induced testicular toxicity in rats: Toxicological, biochemical and histological study. Toxicology 191(1):52.

*Landing WM, Perry JJ Jr, Guentzel JL. 1995. Relationships between the atmospheric deposition of trace elements, major ions, and mercury in Florida: The FAMS Project (1992-1993). Water Air Soil Pollut 80:343-352. 
*Landsberger S, Jervis RE, Kajrys G, et al. 1983. Characterization of trace elemental pollutants in urban snow using proton induced $\mathrm{x}$-ray emission and instrumental neutron activation analysis. Int $\mathrm{J}$ Environ Anal Chem 16:95-130.

*Larramendy ML, Popescu NC, DiPaolo JA. 1981. Induction by inorganic metal salts of sister chromatid exchanges and chromosome aberrations in human and Syrian hamster cell strands. Environ Mutagen 3:597-606.

*Larsen PF, Zdanowicz V, Johnson AC. 1983. Trace metal distribution in the surficial sediments of Penobscot Bay, Maine. Bull Environ Contam Toxicol 31:566-573.

*Larsson-Stymne B, Widstrom L. 1985. Ear piercing--a cause of nickel allergy in schoolgirls? Contact Dermatitis 13:289-293.

*Laschelles K, Nicholls D. 1991. Nickel compounds. In: Elvers B, Hawkins S, Schulz G, eds. Ullmann's encyclopedia of industrial chemistry. Volume A17. 5th edition. Weinheim, Germany: VCH Verlagsgesellschaft, 235.

*Lechner JF, Tokiwa T, McClendon IA, et al. 1984. Effects of nickel sulfate on growth and differentiation of normal human bronchial epithelial cells. Carcinogenesis 5:1697-1703.

*Lee AY, Lee YS. 1990. A case of allergic contact dermatitis due to nickel in underground water. Contact Dermatitis 22:141-143.

*Lee RE Jr, Crish HL, Riley AE, et al. 1975. Concentration and size of trace metal emissions from a power plant, a store plant and a cotton gin. Environ Sci Technol 9:643-647.

*Lee RE Jr, Goranson SS, Enrione RE, et al. 1972. National air surveillance cascade impactor network. II. Size distribution measurements of trace metal components. Environ Sci Technol 6(12):1025-1030.

*Lee Y-W, Klein CB, Kargacin B, et al. 1995. Carcinogenic nickel silences gene expression by chromatin condensation and DNA methylation: A new model for epigenetic carcinogens. Mol Cell Biol $15: 2547-2557$.

*Lee-Chen SF, Yu CT, Wu DR, et al. 1994. Differential effects of luminol, nickel and arsenite on the rejoining of ultraviolet light and alkylation-induced DNA breaks. Environ Mol Mutagen 23:116-120.

*Leeder JS, Kearns GL. 1997. Pharmacokinetics in pediatrics: Implications for practice. Pediatr Clin North Am 44(1):55-77.

*Leikauf GD. 2002. Hazardous air pollutants and asthma. Environ Health Perspect 110(Suppl 4):505526.

*Leung H-W. 1993. Physiologically-based pharmacokinetic modeling. In: Ballantyne B, Marrs T, Turner P, eds. General and applied toxicology. New York, NY: Stockton Press, I:153-164.

Levin C, Maibach HI. 2000. An overview of the efficacy of topical corticosteroids in experimental human nickel contact dermatitis. Contact Dermatitis 43:317-321. 
*Li J, Davidson G, Huang Y, et al. 2004. Nickel compounds act through phosphatidylinositol-3kinase/Akt-dependent, p70(S6k)-independent pathway to induce hypoxia inducible factor transactivation and Cap43 expression in mouse epidermal Cl41 cells. Cancer Res 64(1):94-101.

Li W, Zhao Y, Gantz DL, et al. 2003. Nickel (Ni2+) enhancement of microtubule assembly in vitro is dependent on GTP function. Toxicol Appl Pharmacol 193:202-208.

*Ligeti L, Rubanyi G, Kaller A, et al. 1980. Effect of nickel ions on hemodynamics, cardiac performance and coronary blood flow in anesthetized dogs. In: Anke M, Schneider HJ, Bruckner C, eds. 3rd Trace Element Symposium: Nickel. Jena, East Germany: Freidrick-Schiller-Universitaet, 117-122.

*Lioy PJ, Daisey JM, Morandi MT, et al. 1987. The airborne toxic element and organic substances (ATEOS) study design. In: Lioy PJ, Daisey JM, eds. Toxic air pollutants: A comprehensive study of non-criteria air pollutants. Chelsea, MI: Lewis Publishers, Inc., 3-42.

Lindermann M, Bohmer J, Zabel M, et al. 2003. ELISpot: A new tool for the detection of nickel sensitization. Clin Exp Allergy 33(7):992-998.

Linneberg A, Nielsen NH, Menne T, et al. 2003. Smoking might be a risk factor for contact allergy. J Allergy Clin Immunol 111(5):980-984.

Lisby S, Hansen LH, Skov L, et al. 1999. Nickel-induced activation of T cells in individuals with negative patch test to nickel sulphate. Arch Dermatol Res 291(5):247-252.

*Livingston, AL. 1978. Forage plant estrogens. J Toxicol Environ Health 4:301-324.

*Lloyd GK. 1980. Dermal absorption and conjugation of nickel in relation to the induction of allergic contact dermatitis: Preliminary results. In: Brown SS, Sunderman FW Jr, eds. Nickel toxicology. London, UK: Academic Press, 145-148.

*Lottermoser B. 2002. Exposure assessment of naturally metal enriched topsoils, Port MacQuarie, Australia. Environ Geochem Health 24:183-190.

*Lu CC, Matsumoto N, Iijimi S. 1979. Teratogenic effects of nickel chloride on embryonic mice and its transfer to embryonic mice. Teratology 19:137-142.

*Lucassen ECHET, Smolders AJP, Roelofs JGM. 2002. Potential sensitivity of mires to drought, acidification and mobilization of heavy metals: The sediment $\mathrm{S} / \mathrm{Ca}+\mathrm{Mg}$ ) ratio as diagnostic tool. Environ Pollut 120:635-646.

Lukin A, Dauvalter V, Kashulin N, et al. 2003. Assessment of copper-nickel industry impact on a subarctic lake ecosystem. Sci Total Environ 306:73-83.

*Lumb G, Sunderman FW. 1988. The mechanism of malignant tumor induction by nickel subsulfide. Ann Clin Lab Sci 18:353-366.

*Lytle TF, Lytle JS. 1990. Heavy metals in the eastern oyster, Crassostrea virginica, of the Mississippi sound. Bull Environ Contam Toxicol 44:142-148.

*Magari SR, Schwartz J, Williams PL, et al. 2002. The association of particulate air metal concentrations with heart rate variability. Environ Health Perspect 110(9):875-880. 
*Magnus K, Andersen A, Hogetveit AC. 1982. Cancer of respiratory organs among workers at a nickel refinery in Norway. Int J Cancer 30:681-685.

*Manceau A, Tamura N, Celestre RS, et al. 2003. Molecular-scale speciation of Zn and Ni in soil ferromanganese nodules from Loess soils of the Mississippi Basin. Environ Sci Technol 37:75-80.

*Mann H, Fyfe WS, Kerrich R, et al. 1989. Retardation of toxic heavy metal dispersion from nickelcopper mine tailings, Sudbury district, Ontario: Role of acidophilic microorganisms. I. Biological pathway of metal retardation. Biorecovery 1:155-172.

*Marcus JM, Thompson AM. 1986. Heavy metals in oyster tissue around three coastal marinas. Bull Environ Contam Toxicol 36:587-594.

*Mart L, Nurnberg HW, Dryssen D. 1984. Trace metal levels in the eastern Arctic Ocean. Sci Total Environ 39:1-14.

*Martin TD, Brockhoff CA, Creed JT, et al. 1992. Determination of metals and trace elements in water and wastes by inductively coupled plasma-atomic emission spectrometry. In: Smoley KC, ed. Methods for the determination of metals in environmental samples. Boca Raton, FL: CRC Press, 33-78.

*Martino M, Turner A, Millward GE. 2003. Influence of organic complexation on the adsorption kinetics of nickel in river waters. Environ Sci Technol 37(11):2383-2388.

*Marzin DR, Phi HV. 1985. Study of the mutagenicity of metal derivatives with Salmonella typhimurium TA102. Mutat Res 155:49-51.

*Mas A, Peligero MJ, Arola L, et al. 1986. Distribution and kinetics of injected nickel in the pregnant rat. Clin Exp Pharmacol Physiol 13:91-96.

*Mastromatteo E. 1986. Yant memorial lecture: Nickel. Am Ind Hyg Assoc J 47:589-601.

*Mathur AK, Gupta BN. 1994. Dermal toxicity of nickel and chromium in guinea pigs. Vet Hum Toxicol 36(2):131-132.

Mathur AK, Gupta BN. 1998. Dermal toxicity of linear alkylbenzene sulfonate, chromium, and nickel in guinea pigs. J Toxicol Cutaneous Ocul Toxicol 17(4):191-196.

*Mathur AK, Agarwal C, Singh A, et al. 1988. Effect of sodium lauryl sulphate and nickel alone and in combination on the skin of guinea pigs. Toxicol Lett 42:249-256.

*Mathur AK, Datta KK, Tandon SK, et al. 1977. Effect of nickel sulphate on male rats. Bull Environ Contam Toxicol 17:241-247.

*Mathur AK, Dikshith TSS, Lal MM, et al. 1978. Distribution of nickel and cytogenetic changes in poisoned rats. Toxicology 10:105-113.

*Mathur AK, Gupta BN, Singh S, et al. 1992. Cutaneous toxicity of sodium lauryl sulphate, nickel, and their combination in guinea pigs: Biochemical and histopathological observations. Bull Environ Contam Toxicol 49:871-878. 
9. REFERENCES

*May TW, Wiedmeyer RH, Gober J, et al. 2001. Influence of mining-related activities on concentrations of metals in water and sediment from streams of the Black Hills, South Dakota. Arch Environ Contam Toxicol 40:1-9.

*Mayer C, Klein RG, Wesch H, et al. 1998. Nickel subsulfide is genotoxic in vitro but shows no mutagenic potential in respiratory tract tissues of BigBlue(TM) rats and Muta(TM) Mouse mice in vivo after inhalation. Mutat Res 420(1-3):85-98.

* Mayr U, Butsch A, Schneider S. 1992. Validation of two in vitro test systems for estrogenic activities with zearalenone, phytoestrogens and cereal extracts. Toxicology 74:135-149.

M'Bemba Meka P, Chakrabarti SK. 2001. Effects of different nickel compounds on the transport of para-aminohippurate ion by rat renal cortical slices. Toxicol Lett 122(3):235-244.

*McConnell LH, Fink JN, Schlueter DP, et al. 1973. Asthma caused by nickel sensitivity. Ann Intern Med 78:888-890.

*McCoy H, Kenney MA. 1992. A review of biointeractions of Ni and Mg. II. Immune system and oncology. Magnes Res 5(3):223-232.

McDowell SA, Gammon K, Bachurski CJ, et al. 2003. Differential gene expression in the initiation and progression of nickel-induced acute lung injury. Am J Respir Cell Mol Biol 28(2):188-198.

*McGeer JC, Brix KV, Skeaff JM, et al. 2003. Inverse relationship between bioconcentration factor and exposure concentration for metals: implications for hazard assessment of metals in the aquatic environment. Environ Toxicol Chem 22(5):1017-1037.

*McGregor DB, Brown A, Cattanch P, et al. 1988. Responses of the L5178Y TK+/TK- mouse lymphoma cell forward mutation assay. III. 72 coded chemicals. Environ Mol Mutagen 12:85-154.

McNeely MD, Nechay MW, Sunderman FW Jr. 1972. Measurements of nickel in serum and urine as indices of environmental exposure to nickel. Clin Chem 18:992-995.

*McNeill DA, Chrisp CE, Fisher GL. 1990. Tumorigenicity of nickel subsulfide in strain A/J mice. Drug Chem Toxicol 13:71-86.

Meding B. 2000. Differences between the sexes with regard to work-related skin disease. Contact Dermatitis 43:65-71.

Meding B. 2003. Epidemiology of nickel allergy. J Environ Monit 5(2):188-189.

*Medinsky MA, Benson JM, Hobbs CH. 1987. Lung clearance and disposition of 63Ni in F344/N rats after intratracheal instillation of nickel sulfate solutions. Environ Res 43:168-178.

*Meijer C, Bredberg M, Fischer T, et al. 1995. Ear piercing, and nickel and cobalt sensitization, in 520 young Swedish men doing compulsory military service. Contact Dermatitis 32:147-149.

*Melnyk LJ, Morgan JN, Fernando R, et al. 2003. Determination of metals in composite diet samples by inductively coupled plasma-mass spectrometry. J AOAC Int 86(2):439-447. 
*Menne T. 1994. Quantitative aspects of nickel dermatitis: Sensitization and eliciting threshold concentrations. Sci Total Environ 148:275-281.

*Menne T, Calvin G. 1993. Concentration threshold of non-occluded nickel exposure in nickel-sensitive individuals and controls with and without surfactant. Contact Dermatitis 29:180-184.

*Menne T, Holm NV. 1983. Nickel allergy in a female twin population. Int J Dermatol 22:22-28.

*Menne T, Maibach HI. 1987. Systemic contact allergy reactions. Sem Dermatol 6:108-118.

*Menne T, Maibach HI. 1989. Nickel allergic contact dermatitis: A review. J Am Coll Toxicol 8:12711273.

Menne T, Thorboe A. 1976. Nickel dermatitis--nickel excretion. Contact Dermatitis 2:353-354.

*Menne T, Brandrup F, Thestrup-Pedersen K, et al. 1987. Patch test reactivity to nickel alloys. Contact Dermatitis 16:255-259.

*Menne T, Christophersen J, Green A. 1989. Epidemiology of nickel dermatitis. In: Maibach HI, Menne T, eds. Nickel and the skin: Immunology and toxicology. Boca Raton, FL: CRC Press, Inc., 109-115.

*Meranger JC, Subramanian KS, Chalifoux C. 1981. Survey for cadmium, cobalt, chromium, copper, nickel, lead, calcium, and magnesium in Canadian drinking water supplies. J AOAC 64:44-53.

Merritt K, Crowe TD, Brown SA. 1989. Elimination of nickel, cobalt, and chromium following repeated injections of high dose metal salts. J Biomed Mater Res 23:845-862.

Merzenich H, Hartwig A, Ahrens W, et al. 2001. Biomonitoring on carcinogenic metals and oxidative DNA damage in a cross-sectional study. Cancer Epidemiol Biomarkers Prev 10(5):515-522.

Migally N, Murthy RC, Doye A, et al. 1982. Changes in pulmonary alveolar macrophages in rats exposed to oxides of zinc and nickel. J Submicrosc Cytol 14:621-626.

Minoia C, Sabbioni E, Ronchi A, et al. 1994. Trace element reference values in tissues from inhabitants of the European Community. IV. Influence of dietary factors. Sci Total Environ 141:181-195.

*Misra M, Athar M, Hasan SK, et al. 1988. Alleviation of nickel-induced biochemical alterations by chelating agents. Fundam Appl Toxicol 11:285-292.

*Miura T, Patierno SR, Sakuramoto T. 1989. Morphological and neoplastic transformation of $\mathrm{C} 3 \mathrm{H} / 10 \mathrm{t} 1 / 2 \mathrm{Cl} 8$ mouse embryo cells by insoluble carcinogenic nickel compounds. Environ Mol Mutagen 14:65-78.

*Miyaki M, Akamatsu N, Ono T, et al. 1979. Mutagenicity of metal cations in cultured cells from Chinese hamster. Mutat Res 68:259-263.

*Moller H. 1984. Attempts to induce contact allergy to nickel in the mouse. Contact Dermatitis 10:6568. 


\section{REFERENCES}

*Moon J, Davison AJ, Smith TJ, et al. 1988. Correlation clusters in the accumulation of metals in human scalp hair: Effects of age, community of residence and abundances of metals in air and water supplies. Sci Total Environ 72:87-112.

*Morgan LG, Rouge PJC. 1984. Biological monitoring in nickel refinery workers. In: Sunderman FW Jr, ed. Proceedings of a joint symposium. Lyon, France: International Agency for Research on Cancer, 507-520.

*Morimoto Y, Nambu Z, Tanaka I, et al. 1995. Effects of nickel oxide on the production of tumor necrosis factor by alveolar macrophages of rats. Biol Trace Elem Res 48(3):287-296.

*Morita T, Asano N, Awogi T, et al. 1997. Evaluation of the rodent micronucleus assay in the screening of IARC carcinogens (groups 1,2A, and 2B). The summary report of the 6th collaborative study by CSGMT/JEMS · MMS. Mutat Res 389:3-122.

Morris DL. 1998. Intradermal testing and sublingual desensitization for nickel. Cutis 61(3):129-132.

*Morselli PL, Franco-Morselli R, Bossi L. 1980. Clinical pharmacokinetics in newborns and infants: Age-related differences and therapeutic implications. Clin Pharmacokin 5:485-527.

*Moschandreas DJ, Karuchit S, Berry MR, et al. 2002. Exposure apportionment: Ranking food items by their contribution to dietary exposure. J Expo Anal Environ Epidemiol 12:233-243.

*Moulin JJ, Clavel T, Roy D, et al. 2000. Risk of lung cancer in workers producing stainless steel and metallic alloys. Int Arch Occup Environ Health 73(3):171-80.

*Moulin JJ, Portefaix P, Wild P, et al. 1990. Mortality study among workers producing ferroalloys and stainless steel in France. Br J Ind Med 47(8):537-543.

*Moulin JJ, Wild P, Mantout B, et al. 1993. Mortality from lung cancer and cardiovascular disease among stainless-steel producing workers. Cancer Causes Control 4:75-81.

* Mouvet C, Bourg ACM. 1983. Speciation (including adsorbed species) of copper, lead, nickel, and zinc in the Meuse River. Water Res 17:641-649.

*Mozzanica N, Rizzolo L, Veneroni G, et al. 1990. HLA-A, B, C and DR antigens in nickel contact sensitivity. Br J Dermatol 122:309-314.

Mudroch A, Arafat N, Davies S. 1984. Changes in chemical speciation of soluble metals in Quebec lakes. Environ Tech Lett 5:237-244.

*Muir DCF, Jadon N, Julian JA, et al. 1994. Cancer of the respiratory tract in nickel sinter plant workers: Effect of removal from sinter plant exposure. Occup Environ Med 51(1):19-22.

*Muir DCF, Julian J, Jadon N, et al. 1993. Prevalence of small opacities in chest radiographs of nickel sinter plant workers. Br J Ind Med 50:428-431.

*Müller-Fassbender M, Elsenhans B, McKie AT, et al. 2003. Different behaviour of $63 \mathrm{Ni}$ and $59 \mathrm{Fe}$ during absorption in iron-deficient and iron-adequate jejunal rat segments ex vivo. Toxicology 185(12):141-153. 
*Mumma RO, Raupach DC, Waldman JP, et al. 1984. National survey of elements and other constituents in municipal sewage sludge. Arch Environ Contam Toxicol 13:75-83.

*Mustafa S, Haq I. 1988. Adsorption of copper, cobalt and nickel on amorphous iron hydroxide from aqueous electrolyte solution. Environ Technol Lett 9:1379-1386.

*Myron DR, Zimmerman TJ, Shuler TR, et al. 1978. Intake of nickel and vanadium by humans. A survey of selected diets. Am J Clin Nutr 31:527-531.

*NAS. 1975. Nickel. National Academy of Sciences. Washington, DC: National Academy Press, 4, 5, 17.

NAS. 1989. Recommended dietary allowance. National Academy of Sciences. Washington DC: National Academy Press.

*NAS. 2002. Dietary reference intakes for vitamin A, vitamin K, arsenic, boron, chromium, copper, iodine, iron, manganese, molybdenum, nickel, silicon, vanadium, and zinc. Washington, DC: National Academy of Sciences. http://books.nap.edu/books/0309072794/html/521.html. January 13, 2005.

NAS/NRC. 1989. Biologic markers in reproductive toxicology. National Academy of Sciences/National Research Council. Washington, DC: National Academy Press, 15-35.

NATICH. 1996. Acceptable ambient air concentration guidelines/standards; concentrations, units, and averaging times report. National Air Toxics Information Clearinghouse.

Nestle FO, Speidel H, Speidel MO. 2002. Metallurgy: High nickel release from 1- and 2-Euro coins. Nature 419(6903): 132.

*Nethercott JR, Holness DL. 1990. Cutaneous nickel sensitivity in Toronto, Canada. J Am Acad Dermatol 22:756-761.

*Newhook R, Hirtle H, Byrne K, et al. 2003. Releases from copper smelters and refineries and zinc plants in Canada: human health exposure and risk characterization. Sci Total Environ 301:25-41.

Nguyen VD, Merks AGA, Valenta P. 1990. Atmospheric deposition of acid, heavy metals, dissolved organic carbon and nutrients in the Dutch Delta Area in 1980-1986. Sci Total Environ 99:71-91.

*Nielsen FH. 1980. Effect of form of iron on the interaction between nickel and iron in rats: Growth and blood parameters. J Nutr 110:965-973.

*Nielsen FH. 1982. Possible future implications of nickel, arsenic, silicon, vanadium, and other ultratrace elements in human nutrition. In: Clinical and biochemical nutritional aspects of trace elements. New York, NY: Alan R. Liss, Inc., 379-404.

*Nielsen FH. 1990. Other trace elements. In: Brown ML, ed. Present knowledge in nutrition. 6th edition, Washington, DC: International Life Sciences Institute Press, 294-307.

*Nielsen FH, Ollerich DA. 1974. Nickel: A new essential trace element. FASEB J 33:1767-1772.

*Nielsen FH, Sandstead HH. 1974. Are nickel, vanadium, silicon, fluorine, and tin essential for man? Ann J Clin Nutr 27:515-520. 
*Nielsen GD, Flyvholm M. 1984. Risks of high nickel intake with diet. In: Sunderman FW Jr, ed. Nickel in the human environment. Proceedings of a joint symposium. IARC scientific publication no. 53. Lyon, France: International Agency for Research on Cancer, 333-338.

*Nielsen FH, Hunt CD, Uthus EO. 1980. Interactions between essential trace and ultratrace elements. Ann NY Acad Sci 355:152-162.

*Nielsen FH, Myron DR, Givand SH, et al. 1975. Nickel deficiency in rats. J Nutr 105:1620-1630.

*Nielsen FH, Shuler TR, McLeod TG, et al. 1984. Nickel influences iron metabolism through physiologic, pharmacologic and toxicologic mechanisms in the rat. J Nutr 114:1280-1288.

*Nielsen GD, Jepson LV, Jorgensen PJ, et al. 1990. Nickel-sensitive patients with vesicular hand eczema: Oral challenge with a diet naturally high in nickel. Br J Dermatol 122:299-308.

*Nielsen NH, Linneberg A, Menne T, et al. 2002. Incidence of allergic contact sensitization in Danish adults between 1990 and 1998; the Copenhagen Allergy Study, Denmark. Br J Dermatol 147(3):487-492.

Nielsen NH, Menne T, Kristiansen J, et al. 1999. Effects of repeated skin exposure to low nickel concentrations: A model for allergic contact dermatitis to nickel on the hands. Br J Dermatol 141(4):676-682.

*Nightingale HI. 1987. Water quality beneath urban runoff water management basins. Water Res Bull 23:197-205.

NIOSH. 1989. National Occupational Exposure Survey (NOES). Cincinnati, OH: U.S. Department of Health and Human Services, Public Health Service, Centers for Disease Control, National Institute for Occupational Safety and Health. March 29, 1989.

*NIOSH. 1990. National Occupational Exposure Survey (NOES). Cincinnati, OH: U.S. Department of Health and Human Services, Public Health Service, Centers for Disease Control, National Institute for Occupational Safety and Health. July 1, 1990.

NIOSH. 1994a. Documentation for immediately dangerous to life or health concentrations (IDLHs). Cincinnati, OH: U.S. Department of Health and Human Services, Public Health Service, Centers for Disease Control, National Institute for Occupational Safety and Health.

*NIOSH. 1994b. NIOSH manual of analytical methods. 4th ed. Methods 6007, 6300, 8005, 8310. Cincinnati, OH: U.S. Department of Health and Human Services, Public Health Service, Centers for Disease Control.

NIOSH. 1994c. NIOSH pocket guide to chemical hazards. Cincinnati, OH: U.S. Department of Health and Human Services, National Institute for Occupational Safety and Health. NIOSH Publications.

*NIOSH. 2003a. NIOSH pocket guide to chemical hazards. Nickel carbonyl. Washington, DC: National Institute for Occupational Safety and Health. http://www.cdc.gov/niosh/npg/npg.html. June 06, 2003.

*NIOSH. 2003b. NIOSH pocket guide to chemical hazards. Nickel metal and other compounds. Washington, DC: National Institute for Occupational Safety and Health. http://www.cdc.gov/niosh/npg/npg.html. June 06, 2003. 
*NOAA. 1987. A summary of selected data on chemical contaminants in tissues collected during 1984, 1985, and 1986. NOAA Tech. Memorandum NOS OMA38. Rockville, MD: National Oceanic Atmospheric Administration.

*Norgaard O. 1955. Investigation with radioactive Ni-57 into the resorption of nickel through the skin in normal and in nickel-hypersensitive persons. Acta Derm Venereol 35:111-117.

* Norgaard O. 1957. Investigations with radioactive nickel, cobalt and sodium on the resorption through the skin in rabbits, guinea pigs and man. Acta Derm Venereol 37:440-445.

*North American Contact Dermatitis Group. 1973. Epidemiology of contact dermatitis in North America: 1972. Arch Dermatol 108:537-549.

*Novey HS, Habib M, Wells ID. 1983. Asthma and IgE antibodies induced by chromium and nickel salts. J Allergy Clin Immunol 72:407-412.

*NRC. 1993. National Research Council. Pesticides in the diets of infants and children. Washington, DC: National Academy Press.

Nriagu JO. 1989. A global assessment of natural sources of atmospheric trace metals. Nature 338:47-49.

*Nriagu JO, Coker RD. 1980. Trace metals in humic and fulvic acids from Lake Ontario sediments. Environ Sci Technol 14:443-446.

*Nriagu JO, Pacyna JM. 1988. Quantitative assessment of worldwide contamination of air, water and soils by trace metals. Nature 333:134-139.

*Nriagu JO, Lawson G, Wong HKT, et al. 1996. Dissolved trace metals in Lakes Superior, Erie, and Ontario. Environ Sci Technol 30:178-187.

NTD. 1995. The export connection. National Trade Data Base.

*NTP. 1996a. NTP technical report on the toxicology and carcinogenesis studies of nickel oxide (CAS No. 1313-99-1) in F344/N rats and B6C3F1 mice (inhalation studies). Research Triangle Park, NC: U.S. Department of Health and Human Services, Public Health Service, National Institutes of Health. NTPTRS No. 451.

*NTP. 1996b. NTP technical report on the toxicology and carcinogenesis studies of nickel subsulfide (CAS No. 12035-72-2) in F344/N rats and B6C3F1 mice (inhalation studies). Research Triangle Park, NC: U.S. Department of Health and Human Services, Public Health Service, National Institutes of Health. NTP-TRS No. 453.

*NTP. 1996c. NTP technical report on the toxicology and carcinogenesis studies of nickel sulfate hexahydrate (CAS No. 10101-97-0) in F344/N rats and B6C3F1 mice (inhalation studies). Research Triangle Park, NC: U.S. Department of Health and Human Services, Public Health Service, National Institutes of Health. NTP-TRS No. 454.

*NTP. 2002. Report on carcinogens. Bethesda, MD: U.S. Department of Health and Human Services, Public Health Service, National Toxicology Program. http://ehp.niehs.nih.gov/roc/toc10/html. June 06, 2003. 
9. REFERENCES

*Nygren O. 2002. New approaches for assessment of occupational exposure to metals using on-site measurements. J Environ Monit 4:623-627.

*Obone E, Chakrabarti SK, Bai C, et al. 1999. Toxicity and bioaccumulation of nickel sulfate in Sprague-Dawley rats following 13 weeks of subchronic exposure. J Toxicol Environ Health A 57:379401.

*Odland JO, Nieboer E, Romanova N, et al. 2003. Intercommunity and temporal variation of eleven essential and five toxic elements in human placentas from deliveries in thirteen arctic and sub-arctic areas of Russia and Norway. J Environ Monit 5(1):166-174.

* Ogawa HI, Shibahara T, Iwata H, et al. 1994. Genotoxic activities in vivo of cobaltous chloride and other metal chlorides as assayed in the Drosophila wing spot test. Mutat Res 320:133-140.

*Ohanian EV. 1986. Health effects of corrosion products in drinking water. Trace Subst Environ Health 20:122-138.

*Ohno H, Hanaoka F, Yamada M. 1982. Inducibility of sister chromatid exchanges by heavy metal ions. Mutat Res 104:141-145.

Ohshima S. 2003. Induction of genetic instability and chromosomal instability by nickel sulfate in V79 Chinese hamster cells. Mutagenesis 18(2):133-137.

*Ojajarvi IA, Partanen TJ, Ahblom A, et al. 2000. Occupational exposures and pancreatic cancer: A meta-analysis. Occup Environ Med 57(5):316-324.

*Oliveira JP, Bastos de Siqueira MEP, Da Silva CS. 2000. Urinary nickel as bioindicator of workers' Ni exposure in a galvanizing plant in Brazil. Int Arch Occup Environ Health 73(1):65-68.

Oller AR. 2002. Respiratory carcinogenicity assessment of soluble nickel compounds. Environ Health Perspect Suppl 110(5):841-844.

*Oller AR, Costa M, Oberdorster G. 1997. Carcinogenicity assessment of selected nickel compounds. Toxicol Appl Pharmacol 143:152-166.

*Olsen I, Jonsen J. 1979. Whole-body autoradiography of 63Ni in mice throughout gestation. Toxicology 12:165-172.

*O'Rourke MK, Van De Water PK, Jin S, et al. 1999. Evaluations of primary metals from NHEXAS Arizona: distributions and preliminary exposures. J Expo Anal Environ Epidemiol 9:435-445.

OSHA. 1989. U.S. Department of Labor. Occupational Safety and Health Administration. Air contaminants: Final rule. 29 CFR 1910, 2946.

OSHA. 1993. U.S. Department of Labor. Occupational Safety and Health Administration. Air contaminants: Final rule. 29 CFR 1910.

*OSHA. 2003a. Occupational safety and health standards. Limits for air contaminants. Washington, DC: Occupational Safety and Health Administration. 29 CFR 1910.1000, Table Z-1. http://www.osha.gov/comp-links.html. June 06, 2003. 
*OSHA. 2003b. Occupational safety and health standards. List of highly hazardous chemicals, toxics, and reactives. Washington, DC: Occupational Safety and Health Administration. 29 CFR 1910.119, Appendix A. http://www.osha.gov/comp-links.html. June 06, 2003.

OSHA. 2003c. Occupational safety and health standards. National Research Council recommendations concerning chemical hygiene in laboratories. Washington, DC: Occupational Safety and Health Administration. 29 CFR 1910.1450, Appendix A. http://www.osha.gov/comp-links.html. June 06, 2003.

*OSHA. 2003d. Occupational safety and health standards for shipyard employment. Air contaminants. Washington, DC: Occupational Safety and Health Administration. 29 CFR 1915.1000. http://www.osha.gov/comp-links.html. June 06, 2003.

*OSHA. 2003e. Safety and health regulations for construction. Gases, vapors, fumes, dusts, and mists. Washington, DC: Occupational Safety and Health Administration. 29 CFR 1926.55, Appendix A. http://www.osha.gov/comp-links.html. June 06, 2003.

*OSHA. 2003f. Safety and health regulations for construction. List of highly hazardous chemicals, toxics, and reactives. Washington, DC: Occupational Safety and Health Administration. 29 CFR 1926.64, Appendix 5. http://www.osha.gov/comp-links.html. June 06, 2003.

Oshima S. 2001. Induction of aneuploidy by nickel sulfate in V79 Chinese hamster cells. Mutat Res 492(1-2):39-50.

*Oskarsson A, Tjalve H. 1979. An autoradiographic study on the distribution of $63 \mathrm{NiCl} 2$ in mice. Ann Clin Lab Sci 9:47-59.

*Ottolenghi AD, Haseman JK, Payne WW, et al. 1974. Inhalation studies of nickel sulfide in pulmonary carcinogenesis of rats. JNCI 54:1165-1172.

*Owen GM, Brozek J. 1966. Influence of age, sex and nutrition on body composition during childhood and adolescence. In: Falkner F, ed. Human development. Philadelphia, PA: WB Saunders, 222-238.

*Pacyna JM. 1984. Estimation of the atmospheric emissions of trace elements from anthropogenic sources in Europe. Atmos Environ 18:41-50.

*Pacyna JM, Ottar B. 1985. Transport and chemical composition of the summer aerosol in the Norwegian arctic. Atmos Environ 19:2109-2120.

*Page GW. 1981. Comparison of groundwater and surface water for patterns and levels of contamination by toxic substances. Environ Sci Technol 15:1475-1481.

*Pandey R, Srivastava SP. 2000. Spermatotoxic effects of nickel in mice. Bull Environ Contam Toxicol 64(2):161-167.

*Pandey R, Kumar R, Singh SP, et al. 1999. Male reproductive effect of nickel sulphate in mice. BioMetals 12(4):339-346.

*Pang D, Burges DC, Sorhan T. 1996. Mortality studies of nickel platers with special reference to cancers of the stomach and lung. Occup Environ Med 53(10):714-717. 
*Patierno SR, Costa M. 1985. DNA-protein cross-links induced by nickel compounds in intact cultured mammalian cells. Chem Biol Interact 55:75-91.

*Patierno SR, Costa M. 1987. Effects of nickel(II) on nuclear protein binding to DNA in intact mammalian cells. Cancer Biochem Biophys 9:113-126.

*Patriarca M, Lyon TD, Fell GS. 1997. Nickel metabolism in humans investigated with an oral stable isotope. Am J Clin Nutr 66(3):616-621.

*Pattenden NJ, Branson JR, Fisher EMR. 1982. Trace element measurement in wet and dry deposition and airborne particulate at an urban site. Deposition Atmos Pollut Proc Colloq, 173-184.

*Pedersen E, Hogetveit AC, Andersen A. 1973. Cancer of respiratory organs among workers at a nickel refinery in Norway. Int J Cancer 12:32-41.

Pellizzari ED, Smith DJ, Clayton CA, et al. 2001. An assessment of the data quality for NHEXAS- Part I: exposure to metals and volatile organic chemicals in Region 5. J Expo Anal Environ Epidemiol 11:140-154.

*Pennington JAT, Jones JW. 1987. Molybdenum, nickel, cobalt, vanadium, and strontium in total diets. J Am Diet Assoc 87:1644-1650.

*Peralta-Videa JR, Gardea-Torresdey E, Gomez E, et al. 2002. Effect of mixed cadmium, copper, nickel and zinc at different pHs upon alfalfa growth and heavy metal uptake. Environ Pollut 119:291-301.

Pereira MC, Pereira ML, Sousa JP. 1998. Evaluation of nickel toxicity on liver, spleen, and kidney of mice after administration of high-dose metal ion. J Biomed Mater Res 40(1):40-47.

*Peto R, Cuckle H, Doll R, et al. 1984. Respiratory cancer mortality of Welsh nickel refinery workers. In: Sunderman FW Jr, Aitio A, Berlin A, eds. Nickel in the human environment. IARC scientific publication no. 53. Lyon, France: International Agency for Research on Cancer, 37-46.

*Pikalek P, Necasek J. 1983. The mutagenic activity of nickel in Cornebacterium sp. Folia Microbiol 26:17-21.

*Pitt R, Field R, Lalor M, et al. 1995. Urban stormwater toxic pollutants: Assessment, sources, and treatability. Water Environ Res 67(3):260-275.

*Polednak AP. 1981. Mortality among welders, including a group exposed to nickel oxides. Arch Environ Health 36:235-242.

*Pool-Zobel BL, Lotzmann N, Knoll M, et al. 1994. Detection of genotoxic effects in human gastric and nasal mucosa cells isolated from biopsy samples. Environ and Mol Mutagen 24:23-45.

Pott F, Ziem U, Reiffer FJ, et al. 1987. Carcinogenicity studies on fibres, metal compounds and some other dusts in rats. Exp Pathol 32:129-152.

*Poulton DJ. 1987. Trace contaminant status of Hamilton Harbor. J Great Lakes Res 13:193-202.

*Poulton DJ, Simpson KJ, Barton DR, et al. 1988. Trace metals and benthic invertebrates in sediments of nearshore Lake Ontario and Hamilton Harbor. J Great Lakes Res 14:52-65. 
Prows DR, Leikauf G. 2001. Quantitative trait analysis of nickel-induced acute lung injury in mice. Am J Respir Cell Mol Biol 24(6):740-746.

Prows DR, McDowell SA, Aronow BJ, et al. 2003. Genetic susceptibility to nickel-induced acute lung injury. Chemosphere 51(10):1139-1148.

*Prystowsky SD, Allen AM, Smith RW, et al. 1979. Allergic contact hypersensitivity to nickel, neomycin, ethylenediamine and benzocaine. Arch Dermatol 115:959-962.

Pulido MD, Parrish AR. 2003. Metal-induced apoptosis: Mechanisms. Mutat Res 533:227-241.

Purello D'Ambrosio F, Bagnato F, Guarneri B, et al. 1998. The role of nickel in foods exacerbating nickel contact dermatitis. Allergy 53:143-145.

Qu W, Kasprzak KS, Kadiiska M, et al. 2001. Mechanisms of arsenic-induced cross-tolerance to nickel cytotoxicity, genotoxicity, and apotosis in rat liver epithelial cells. Toxicol Sci 63(2):189-195.

*QueHee SS, Finelli VN, Fricke FL, et al. 1982. Metal content of stack emissions, coal and fly ash from some eastern and western power plants in the USA as obtained by ICP-AES. Int J Environ Anal Chem 13:1-18.

*Radike M, Warshawsky D, Caruso J, et al. 2002. Distribution and accumulation of a mixture of arsenic, cadmium, chromium, nickel and vanadium in mouse small intestine, kidneys, pancreas, and femur following oral administration in water or feed. J Toxicol Environ Health A 65(23):2029-2052.

*Rai D, Zachara JM. 1984. Chemical attenuation rates, coefficients, and constants in leachate migration. Volume 1: A critical Review. Palo Alto, CA: Electric Power Research Institute.

*Raithel HJ, Schaller KH, Akslen LA, et al. 1989. Analyses of chromium and nickel in human pulmonary tissue. Investigations in lung cancer patients and a control population under special consideration of medical expertise aspects. Int Arch Occup Environ Health 61:507-512.

*Raithel HJ, Schaller KH, Reith A, et al. 1988. Investigations on the quantitative determination of nickel and chromium in human lung tissue industrial medical toxicological and occupational medical expertise aspects. Int Arch Occup Environ Health 60:55-66.

*Rasmuson A. 1985. Mutagenic effects of some water-soluble metal compounds in a somatic eye-color test system in Drosophila melanogaster. Mutat Res 157:157-162.

*Redmond CK. 1984. Site-specific cancer mortality among workers involved in the production of high nickel alloys: In: Sunderman FW Jr, Aitio A, Berlin A, eds. Nickel in the human environment. IARC scientific publication no. 53. Lyon, France: International Agency for Research on Cancer, 73-86.

Reimann C, DeCaritat P, Halleraker JH, et al. 1997. Rainwater composition in eight arctic catchments in Northern Europe (Finland, Norway and Russia). Atmos Environ 31(2):159-170.

*Reith AK, Reichborn-Kjennerud S, Aubele M, et al. 1994. Biological monitoring of chemical exposure in nickel workers by imagining cytometry (ICM) of nasal smears. Anal Cell Path 6:9-21.

*Rendall REG, Phillips JI, Renton KA. 1994. Death following exposure to fine particulate nickel from a metal arc process. Ann Occup Hyg 38(6):921-930. 


\section{REFERENCES}

*Rezuke WN, Knight JA, Sunderman FW Jr. 1987. Reference values for nickel concentrations in human tissues and bile. Am J Ind Med 11:419-426.

*Rice KE. 1999. Trace-element concentrations in streambed sediment across the conterminous United States. Environ Sci Technol 33:2499-2504.

*Richter RO, Theis TL. 1980. Nickel. In: Nriagu JO, ed. Nickel in the environment. New York, NY: John Wiley and Sons, Inc., 189-202.

*Ries MW, Kampmann C, Rupprecht H-J, et al. 2003. Nickel release after implantation of the Amplatzer occluder. Am Heart J 145(4):737-741.

Riley MR, Boesewetter DE, Kim AM, et al. 2003. Effects of metals $\mathrm{Cu}, \mathrm{Fe}, \mathrm{Ni}, \mathrm{V}$, and $\mathrm{Zn}$ on rat lung epithelial cells. Toxicology 190(3):171-184.

*Roberts RS, Julian JA, Muir DCF, et al. 1989a. A study of mortality in workers engaged in the mining, smelting, and refining of nickel. II: Mortality from cancer of the respiratory tract and kidney. Toxicol Ind Health 5(6):975-993.

*Roberts RS, Julian JA, Sweezey D, et al. 1989b. A study of mortality in workers engaged in the mining, smelting, and refining of nickel. I: Methodology and mortality by major cause groups. Toxicol Ind Health 5(6):957-974.

Robertson GL, Lebowitz MD, O'Rourke MK, et al. 1999. The National Human Exposure Assessment Survey (NHEXAS) study in Arizona - introduction and preliminary results. J Expo Anal Environ Epidemiol 9:427-434.

*Robinson SH, Costa M. 1982. The induction of DNA strand breakage by nickel compounds in cultured Chinese hamster ovary cells. Cancer Lett 15:35-40.

*Rodriguez-Arnaiz R, Ramos P. 1986. Mutagenicity of nickel sulphate in Drosophila melanogaster. Mutat Res 170:115-117.

Roelofs-Haarhuis K, Wu X, Nowak M, et al. 2003. Infectious nickel tolerance: A reciprocal interplay of tolerogenic APCs and T suppressor cells that is driven by immunization. J Immunol 171(6):2863-2872.

*Rope SK, Arthur WJ, Craig TH, et al. 1988. Nutrient and trace elements in soil and desert vegetation of southern Idaho. Environ Monit Assess 10:1-24.

*Rossmann R. 1988. Estimation of trace metal storage in Lake St. Clair post-settlement sediments using composite samples. J Great Lakes Res 14:66-75.

Roul S, Ducombe G, Taleb A. 1999. Usefulness of the European standard series for patch testing in children A 3-year single-centre study of 337 patients. Contact Dermatitis 40(5):232-235.

*RTECS. 2004. Registry of Toxic Effects of Chemical Substances. Washington, DC: U.S. Department of Health and Human Services, Public Health Service, Centers for Disease Control, National Institute for Occupational Safety and Health. June 2004. 
*RTI. 1985. Final report (draft): Dose-range finding study of nickel chloride administered to CD rats in the drinking water. Research Triangle Park, NC: Office of Solid Waste Management, U.S. Environmental Protection Agency.

*RTI. 1986. Two-generation reproduction and fertility study of nickel chloride administered to CD rats in the drinking water: 90-Day exposure of CD rats to nickel chloride administered in the drinking water. Final study report (I of III). Research Triangle Park, NC: Office of Solid Waste Management, U.S. Environmental Protection Agency.

*RTI. 1988a. Two-generation reproduction and fertility study of nickel chloride administered to CD rats in the drinking water: Fertility and reproductive performance of the Po generation. Final study report (II of III). Research Triangle Park, NC: Office of Solid Waste Management, U.S. Environmental Protection Agency.

*RTI. 1988b. Two-generation reproduction and fertility study of nickel chloride administered to CD rats in the drinking water: Fertility and reproductive performance of the F1 generation. Final study report (III of III). Research Triangle Park, NC: Office of Solid Waste Management, U.S. Environmental Protection Agency.

*Rubanyi G, Ligeti L, Koller A, et al. 1984. Possible role of nickel ions in the pathogenesis of ischemic coronary vasoconstriction in the dog heart. J Mol Cell Cardiol 16:533-546.

*Rubin ES. 1999. Toxic releases from power plants. Environ Sci Technol 33:3062-3067.

*Rudd T, Lake DL, Mehrotra I, et al. 1988. Characterization of metal forms in sewage sludge by chemical extraction and progressive acidification. Sci Total Environ 74:149-175.

Rudzki E, Rebandel P, Karas Z. 1997. Patch testing with lower concentrations of chromate and nickel. Contact Dermatitis 37(1):46.

*Rybicka EH. 1989. Metals and their chemical and mineralogical forms in industrial pollutants of the atmosphere. Environ Technol Lett 10:921-928.

*Rydh CJ, Svärd B. 2003. Impact on global metal flows arising from the use of portable rechargeable batteries. Sci Total Environ 302:167-184.

*Sabbioni E, Goetz L, Bignoli G. 1984. Health and environmental implications of trace metals released from coal-fired power plants: An assessment study of the situation in the European community. Sci Total Environ 40:141-154.

*Sadiq M, Enfield CG. 1984a. Solid phase formation and solution chemistry of nickel in soils. 1. Theoretical. Soil Sci 138:262-270.

*Sadiq M, Enfield CG. 1984b. Solid phase formation and solution chemistry of nickel in soils. 2. Experimental. Soil Sci 138:335-340.

Salnikow K, Blasgosklonny MV, Ryan H, et al. 2000a. Carcinogenic nickel induces genes involved with hypoxic stress. Cancer Res 60(1):38-41.

Salnikow K, Davidson T, Kluz T, et al. 2003a. Genechip analysis of signaling pathways effected by nickel. J Environ Monit 5(2):206-209. 
Salnikow K, Davidson T, Zhang Q, et al. 2003b. The involvement of hypoxia-induced transcription factor-1-dependent pathway in nickel carcinogenesis. Cancer Res 63(13):3524-3540.

Salnikow K, Li X, Lippmann M. 2004. Effect of nickel and iron co-exposure on human lung cells. Toxicol Appl Pharmacol 196(2):258-265.

*Salnikow K, Su W, Blagosklonny MV, et al. 2000b. Carcinogenic metals induce hypoxia-inducible factor-stimulated transcription by reactive oxygen sepcies-independent mechanism. Cancer Res 60(13):3375-3378.

*Saltzman BE, Cholak J, Schafer JL, et al. 1985. Concentration of six metals in the air of eight cities. Environ Sci Technol 19:328-333.

*Sanford WE, Nieboer E. 1992. Renal toxicity of nickel in humans. In: Nieboer E, Nriagu JO, eds. Nickel and human health: Current perspectives: Proceedings of the Fourth International Conference on Nickel Metabolism and Toxicology; September 1988, Helsinki, Finland. New York: John Wiley \& Sons, Inc., 123-134.

*Santucci B, Manna F, Cannistraci C, et al. 1994. Serum and urine concentrations in nickel-sensitive patients after prolonged oral administration. Contact Dermatitis 30:97-101.

*San̆udo-Wilhelmy SA, Gill GA. 1999. Impact of the Clean Water Act on the levels of toxic metals in urban estuaries: The Hudson River Estuary revisited. Environ Sci Technol 33(20):3477-3481.

Saplakoglu U, Iscan M, Iscan M. 1997. DNA single-strand breakage in rat lung, liver and kidney after single and combined treatments of nickel and cadmium. Mutat Res 394(1-3):133-140.

Saricaoglu H, Tunali S, Bulbul E, et al. 1998. Prevention of nickel-induced allergic contact reactions with pentoxifylline. Contact Dermatitis 39(5):244-247.

Saripalli YV, Gadzia JE, Belsito DV. 2003. Tacrolimus ointment $0.1 \%$ in the treatment of nickelinduced allergic contact dermatitis. J Am Acad Dermatol 49(3):477-482.

*Sarkar B. 1984. Nickel metabolism. In: Sunderman FW Jr, Aitio A, Berlin A, eds. Nickel in the human environment. IARC scientific publication no. 53. Lyon, France: International Agency for Research on Cancer, 367-384.

*Saxholm HJK, Reith A, Brogger A. 1981. Oncogenic transformation and cell lysis in C3H/10T1/2 cells and increased sister chromatid exchange in human lymphocytes by nickel subsulfide. Cancer Res 41:4136-4139.

*Schmidt JA, Andren AW. 1980. The atmospheric chemistry of nickel. In: Nriagu JO, ed. Nickel in the environment. New York, NY: John Wiley and Sons, Inc., 93-135.

Schnuch A, Geier J, Lessmann H, et al. 2003. [Decrease in nickel sensitization in young patientssuccessful intervention through nickel exposure regulation? Results of IVDK, 1992-2001] Hautarzt 54:626-632. (German)

*Schroeder HA, Mitchener M. 1971. Toxic effects of tract elements on the reproduction of mice and rats. Arch Environ Health 23:102. 
*Schroeder HA, Mitchener M. 1975. Life-term effects of mercury, methyl mercury and nine other trace metals on mice. J Nutr 105:452-458.

*Schroeder HA, Balassa JJ, Vintin WH Jr. 1964. Chromium, lead, cadmium, nickel and titanium in mice: Effect on mortality, tumors and tissue levels. J Nutr 83:239-250.

*Schroeder HA, Mitchener M, Nason AP. 1974. Life-term effects of nickel in rats: Survival, tumors, interactions with trace elements and tissue levels. J Nutr 104:239-243.

*Schroeder WH, Dobson M, Kane DM. 1987. Toxic trace elements associated with airborne particulate matter: A review. Air Pollut Control Assoc 11:1267-1287.

Schubert HJ. 2000. Airborne nickel dermatitis. Contact Dermatitis 42:118-119.

Scott LK, Grier LR, Arnold TC, et al. 2002. Respiratory failure from inhalational nickel carbonyl exposure treated with continuous high-volume hemofiltration and disulfiram. Inhal Toxicol 14(11):11031109.

*Scudlark JR, Conko KM, Church TM. 1994. Atmospheric wet deposition of trace elements to Chesapeake Bay: CBAD Study year 1 results. Atmos Environ 28(8):1487-1498.

*Sedlak DL, Phinney JT, Bedsworth WW. 1997. Strongly complexed Cu and Ni in wastewater effluents and surface runoff. Environ Sci Technol 31:3010-3016.

Seidenari S, Belletti B, Mantovani L, et al. 1996. Nickel sulfate 5-20\% aq does not evoke irritation on the skin of non-nickel-sensitive subjects. Contact Dermatitis 35(4):260-261.

*Seidenberg JM, Anderson DG, Becker RA. 1986. Validation of an in vivo developmental toxicity screen in the mouse. Teratog Carcinog Mutagen 6:361-374.

*Seilkop SK. 2001. Occupational exposures and pancreatic cancer: A meta-analysis. Occup Environ Med 58(1):63-64.

*Seilkop SK, Oller AR. 2003. Respiratory cancer risks associated with low-level nickel exposure: An integrated assessment based on animal, epidemiological, and mechanistic data. Regul Toxicol Pharmacol 37:173-190.

Sen P, Costa M. 1986a. Incidence and localization of sister chromatid exchanges induced by nickel and chromium compounds. Carcinogenesis 7:1527-1533.

*Sen P, Costa M. 1986b. Pathway of nickel uptake influences its interaction with heterochromatic DNA. Toxicol Appl Pharmacol 84:278-285.

*Sen P, Conway K, Costa M. 1987. Comparison of the localization of chromosome damage induced by calcium chromate and nickel compounds. Cancer Res 47:2142-2147.

Seoane AI, Dulout FN. 2001. Genotoxic ability of cadmium, chromium and nickel salts studied by kinetochore staining in the cytokinesis-blocked micronucleus assay. Mutat Res 490(2):99-106.

*Setchell BP, Waites GMH. 1975. The blood-testis barrier. In: Creep RO, Astwood EB, Geiger SR, eds. Handbook of physiology: Endocrinology V. Washington, DC: American Physiological Society. 
*Shacklette HT, Boerngen JG. 1984. Element concentration in soils and other surficial materials of the conterminous United States. U.S. Geological Survey professional paper 1270. Alexandria, VA: U.S. Geological Survey.

*Shannon HS, Julian JA, Muir DCF, et al. 1984a. A mortality study of Falcon Bridge workers. In: Sunderman FW Jr, Aitio A, Berlin A, eds. Nickel in the environment. IARC scientific publications no. 53. Lyon, France: International Agency for Research on Cancer, 117-124.

*Shannon HS, Julian JA, Roberts, RS. 1984b. A mortality study of 11,500 nickel workers. J Natl Camcer Inst 73(6):1251-1258.

*Shannon HS, Walsh C, Jadon N, et al. 1991. Mortality of 11,500 nickel workers -- extended follow up and relationship to environmental conditions. Toxicol Ind Health 7:277-294.

Shah M, Lewis FM, Gawkrodger DJ. 1998. Nickel as an occupational allergen. A survey of 368 nickelsensitive subjects. Arch Dermatol 134(10):1231-1236.

Shiao YH, Lee SH, Kasprzak KS. 1998. Cell cycle arrest, apotosis and p53 expression in nickel(II) acetate-treated Chinese hamster ovary cells. Carcinogenesis 19(7):1203-1207.

*Shiller AM, Boyle EA. 1987. Variability of dissolved trace metals in the Mississippi River (USA). Geochim Cosmochim Acta 51:3273-3278.

Shimada H, Funakoshi T, Inoue T, et al. 2000. The effects of sulfhydryl blockers and metal ions on nickel accumulation by rat primary hepatocyte cultures. Toxicol Lett 118(1-2):87-92.

Shiraishi K, Yamagami Y, Kameoka K, et al. 1988. Mineral contents in model diet samples for different age groups. J Nutr Sci Vitaminol (Tokyo) 34(1):55-65.

* Shirakawa T, Kusaka Y, Fujimura N, et al. 1990. Hard metal asthma - cross immunological and respiratory activity between cobalt and nickel. Thorax 45:267-271.

Shum KW, Meyer JD, Chen Y, et al. 2003. Occupational contact dermatitis to nickel: Experience of the British dermatologists (EPIDERM) and occupational physicians (OPRA) surveillance schemes. Occup Environ Med 60(12):954-957.

*Siller GM, Seymour GJ. 1994. Kinetics and specificity of nickel hypersensitivity in the murine model. Australas J Dermatol 35:77-81.

Silvennoinen-Kassinen S, Ilonen J, Tiilikainen A, et al. 1979. No significant association between HLA and nickel contact sensitivity. Tissue Antigens 14(5):459-461.

*Simonato L, Fletcher AC, Andersen A, et al. 1991. A historical prospective study of European stainless steel, mild steel, and shipyard welders. Br J Ind Med 48(3):145-154.

*Simonetti V, Manzini BM, Seidenari S. 1998. Patch testing with nickel sulfate: comparison between 2 nickel sulfate preparations and 2 different test sites on the back. Contact Dermatitis 39(4):187-191.

*Singh I. 1984. Induction of gene conversion and reverse mutation by manganese sulphate and nickel sulphate in Saccharomyces cerevisiae. Mutat Res 137:47-49. 


\section{REFERENCES}

*Sjovall P, Christensen OB, Moller H. 1987. Oral hyposensitization in nickel allergy. J Am Acad Dermatol 17(1):774-778.

*Smart GA, Sherlock JC. 1987. Nickel in foods and the diet. Food Addit Contam 4:61-71.

*Smart GA, Sherlock C, Norman JA. 1987. Dietary intakes of lead and other metals: A study of young children from an urban population in the UK. Food Addit Contam 5(1):85-93.

*Smialowicz RJ, Rogers RR, Riddle MM, et al. 1985. Immunologic effects of nickel. II. Suppression of natural killer cell activity. Environ Res 36:56-66.

*Smialowicz RJ, Rogers RR, Riddle MM, et al. 1986. Immunological studies in mice following in utero exposure to $\mathrm{NiCl} 2$. Toxicology 38:293-303.

*Smialowicz RJ, Rogers RR, Rowe DG, et al. 1987. The effects of nickel on immune function in the rat. Toxicology 44:271-281.

*Smith SR. 1994. Effect of soil pH on availability to crops of metals in sewage sludge-treated soils. I. Nickel, copper and zinc uptake and toxicity to ryegrass. Environ Pollut 85(3):321-327.

Smith JC, Hackley B. 1968. Distribution and excretion of nickel-63 administered intravenously to rats. J Nutr 95:541-546.

Smith GM, Williams FLR, Lloyd OLL. 1987. Respiratory cancer and air pollution from iron foundries in a Scottish town: An epidemiological and environmental study. Br J Ind Med 44:795-802.

*Smith MK, George EL, Stober JA. 1993. Perinatal toxicity associated with nickel chloride exposure. Environ Res 61:200-211.

*Sobti RC, Gill RK. 1989. Incidence of micronuclei and abnormalities in the head of spermatozoa caused by the salts of a heavy metal nickel. Cytologia 54:249-254.

*Solomons NW, Viteri F, Shuler TR, et al. 1982. Bioavailability of nickel in man: Effects of food and chemically defined dietary constituents on the absorption of inorganic nickel. J Nutr 112:39-50.

Sommer S, Wilkinson SM. 2001. Allergic contact dermatitis caused by a nickel-containing headband. Contact Dermatitis 44(3):178.

*Sorahan T. 2004. Mortality of workers at a plant manufacturing nickel alloys, 1958-2000. Occup Med 54(1):28-34.

Sorahan T, Esmen NA. 2004. Lung cancer mortality in UK nickel-cadmium battery workers, 1947-2000. Occup Environ Med 61(2):108-116.

*Sörme L, Lagerkvist R. 2002. Sources of heavy metals in urban wastewater in Stockholm. Sci Total Environ 298:131-145.

Sowder AG, Bertsch PM, Morris PJ. 2003. Partitioning and availability of uranium and nickel in contaminated riparian sediments. J Environ Qual 32(3):885-898. 


\section{REFERENCES}

*Spiegelberg T, Koerdel W, Hochrainer D. 1984. Effect of NiO inhalation on alveolar macrophages and the humoral immune system of rats. Ecotoxicol Environ Safety 8:516-525.

*Springborn Laboratories. 2000a. A one-generation reproduction range-finding study in rats with nickel sulfate hexahydrate. Spencerville, OH: Springborn Laboratories, Inc. SLI Study No. 3472.3.

*Springborn Laboratories. 2000b. An oral (gavage) two-generation reproduction toxicity study in Sprague-Dawley rats with nickel sulfate hexahydrate. Final Report. Volume 1 of 3. Spencerville, OH: Springborn Laboratories, Inc. SLI Study No. 3472.4.

*Springborn Laboratories. 2002. A range-finding 90-day oral (gavage) toxicity study in Fischer 344 rats with nickel sulfate hexahydrate. Spencerville, OH: Springborn Laboratories, Inc. SLI Study No. 3472.6.

*Spruit D, Bongaarts PJM. 1977. Nickel content of plasma, urine and hair in contact dermatitis. Dermatologica 154:291-300.

*Srivastava RC, Hussein MM, Srivastava SK, et al. 1995. Effect of pre-exposure to cadmium and silver on nickel induced toxic manifestations in mice: possible role of ceruloplasmin and metallothionein. Bull Environ Contam Toxicol 54:751-759.

*Stedman DH, Hikade DA. 1980. Nickel toxicology. In: Brown SS, Sunderman FW Jr, eds. Proceedings of the international conference on nickel toxicology, September, Swansea, Wales. London, UK: Academic Press, 183-186.

*Stephenson T, Lawson PS, Rudd T, et al. 1987. Mechanism of metal removal in activated sludge. J Environ Eng 113:1074-1087.

*Stoeppler M. 1980. Analysis of nickel in biological material and natural waters. In: Nriagu JO, ed. Nickel in the environment. New York, NY: John Wiley and Sons, Inc., 661-821.

*Stoeppler M. 1984. Analytical chemistry of nickel. In: Sunderman FW Jr, Aitio A, Berlin A, eds. Nickel in the human environment. IARC scientific publication no. 53. Lyon, France: International Agency for Research on Cancer, 459-485.

*Stoessel RP, Michaelis W. 1986. Wet and dry deposition of heavy metals. In: Proceedings of the 2nd International Conference on Environmental Contamination, Amsterdam, Netherlands: CEP Consultants, Ltd., 85-88.

*Stutz DR, Janusz SJ. 1988. Hazardous materials injuries--a handbook for pre-hospital care. 2nd ed. Beltsville, MD: Bradford Communications Corporation, 218-219.

*Suedel BC, Boraczek JA, Peddicord RK, et al. 1994. Trophic transfer and biomagnification potential of contaminants in aquatic ecosystems. Rev Environ Contam Toxicol 136:21-89.

*Sumino K, Hayakawa K, Shibata T, et al. 1975. Heavy metals in normal Japanese tissues: Amounts of 15 heavy metals in 30 subjects. Arch Environ Health 30:487-494.

Summer B, Sander CA, Przybilla B, et al. 2001. Molecular analysis of T-cell clonality with concomitant specific T-cell proliferation in vitro in nickel-allergic individuals. Allergy 56(8):767-770. 
*Sunderman FW Jr. 1986. Sources of exposure and biological effects of nickel. In: O'Neill IK, Schuller P, Fishbein L, eds. Environmental carcinogens selected methods of analysis. Volume 8: Some metals: $\mathrm{As}, \mathrm{Be}, \mathrm{Cd}, \mathrm{Cr}, \mathrm{Ni}, \mathrm{Pb}, \mathrm{Se}, \mathrm{Zn}$. IARC scientific publication no. 71. Lyon, France: International Agency for Research on Cancer, 79-92.

*Sunderman FW Jr. 1989a. Carcinogenicity of metal alloys in orthopedic prostheses: Clinical and experimental studies. Fundam Appl Toxicol 13:205-216.

*Sunderman FW Jr. 1989b. Mechanisms of nickel carcinogenesis. Scand J Work Environ Health 15:112.

*Sunderman FW Jr. 1993. Biological monitoring of nickel in humans. Scand J Work Environ Health 19(Suppl 1):34-38.

*Sunderman FW Jr, Barber AM. 1988. Finger-loops, oncogenes, and metals. Ann Clin Lab Sci 18:267288.

*Sunderman FW Jr, Horak E. 1981. Biochemical indices of nephrotoxicity, exemplified by studies of nickel nephropathy. In: Brown SS, Davies DS, eds. Organ-directed toxicity: Chemical indices and mechanisms. London, UK: Pergamon Press, 52-64.

*Sunderman FW Jr, Maenza RM. 1976. Comparisons of carcinogenicities of nickel compounds in rats. Res Commun Chem Pathol Pharmacol 14:319-330.

*Sunderman FW Jr, McCully KS. 1983. Effects of manganese compounds on carcinogenicity of nickel subsulfide in rats. Carcinogenesis 4:461-465.

* Sunderman FW Jr, Oskarsson A. 1991. Nickel. In: Merian E, ed. Metals and their compounds in the environment. New York, NY: VCH Verlagsgesellschaft, 1101-1126.

*Sunderman FW Jr, Aitio A, Morgan LG, et al. 1986. Biological monitoring of nickel. Toxicol Ind Health 2:17-78.

*Sunderman FW Jr, Dingle B, Hopfer SM, et al. 1988. Acute nickel toxicity in electroplating workers who accidentally ingested a solution of nickel sulfate and nickel chloride. Am J Ind Med 14:257-266.

*Sunderman FW Jr, Hopfer SM, Knight JA, et al. 1987. Physicochemical characteristics and biological effects of nickel oxides. Carcinogenesis 8:305-313.

*Sunderman FW Jr, Hopfer SM, Sweeney KR, et al. 1989b. Nickel absorption and kinetics in human volunteers. Proc Soc Exp Biol Med 191:5-11.

*Sunderman FW Jr, Hopfer SM, Swift T, et al. 1989c. Cobalt, chromium, and nickel concentrations in body fluids of patients with porous-coated knee or hip prostheses. J Orthop Res 7:307-315.

*Sunderman FW Jr, Kasprzak K, Horak E, et al. 1976. Effects of triethylenetetramine upon the metabolism and toxicity of 63NiCl2 in rats. Toxicol Appl Pharmacol 38:177-188.

*Sunderman FW Jr, Morgan LG, Andersen A, et al. 1989a. Histopathology of sinonasal and lung cancers in nickel refinery workers. Ann Clin Lab Sci 19:44-50. 
*Sunderman FW Jr, Shen SK, Mitchell JM, et al. 1978. Embryotoxicity and fetal toxicity of nickel in rats. Toxicol Appl Pharmacol 43:381-390.

*Svenes KB, Andersen I. 1998. Distribution of nickel in lungs from former nickel workers. Int Arch Occup Environ Health 71(6):424-428.

*Sweeney MD, Naidu AS. 1989. Heavy metal in sediments of the inner shelf of the Beaufort Sea, northern arctic Alaska. Mar Poll Bull 20:140-143.

*Sweet CW, Vermette SJ, Landsberger S. 1993. Sources of toxic trace elements in urban air in Illinois. Environ Sci Technol 27:2502-2510.

*Szakmary E, Movai V, Naray M, et al. 1995. Haemodynamic effect of nickel chloride in pregnant rats. Acta Physiologica Hungarica 83(1):3-12.

Szepietowski JC, McKenzie RC, Keohane SG, et al. 1997. Atopic and non-atopic individuals react to nickel challenge in a similar way. A study of the cytokine profile in nickel-induced contact dermatitis. Br J Dermatol 137(2):195-200.

*Takagi Y, Matsuda S, Imai S, et al. 1986. Trace elements in human hair: An international comparison. Bull Environ Contam Toxicol 36:793-800.

*Takagi Y, Matsuda S, Imai S, et al. 1988. Survey of trace elements in human nails: An international comparison. Bull Environ Contam Toxicol 41:690-695.

Takahashi S, Oishi M, Takeda E, et al. 1999. Physiochemical characteristics and toxicity of nickel oxide particles calcined at different temperatures. Biol Trace Elem Res 69(2):161-174.

*Takenaka S, Hochrainer D, Oldiges H. 1985. Alveolar proteinosis induced in rats by long-term inhalation of nickel oxide. In: Brown SS, Sunderman FW Jr, eds. Progress in nickel toxicology. Proceedings of the 3rd International Congress on Nickel Metabolism and Toxicology, Oxford, UK: Blackwell, 89-92.

*Tallkvist J, Tjalve H. 1994. Nickel absorption from perfused rat jejunal and ileal segments. Pharmacol Toxicol 75:233-243.

*Tallkvist J, Tjälve H. 1997. Effect of dietary iron-deficiency on the disposition of nickel in rats. Toxicol Lett 92:131-138.

Tallkvist J, Tjalve H. 1998. Transport of nickel across monolayers of human intestinal Caco-2 cells. Toxicol Appl Pharmacol 151(1):117-122.

*Tanaka I, Horie A, Haratake J, et al. 1988. Lung burden of green nickel oxide aerosol and histopathological findings in rats after continuous inhalation. Biol Trace Elem Res 16:19-26.

*Tanaka I, Ishimatsu S, Matsuno K, et al. 1985. Biological half time of deposited nickel oxide aerosol in rat lung by inhalation. Biol Trace Element Res 8:203-210.

*Taylor GJ, Crowder AA. 1983. Accumulation of atmospherically deposited metals in wetland boils of Sudbury, Ontario. Water Air Soil Pollut 19:29-42. 
*Tedeschi RE, Sunderman FW. 1957. Nickel poisoning. V. The metabolism of nickel under normal conditions and after exposure to nickel carbonyl. Arch Ind Health 16:486-488.

*Templeton DM, Sunderman FW Jr, Herber RFM. 1994. Tentative reference values for nickel concentrations in human serum, plasma, blood, and urine: Evaluation according to the TRACY protocol. Sci Tot Environ 148:243-251.

*Thomas KW, Pellizzari ED, Berry MR. 1999. Population-based intakes and tap water concentrations for selected elements in the EPA Region V National Human Exposure Assessment Survey (NHEXAS). J Expo Anal Environ Epidemiol 9:402-413.

Thomas P, Barnstorf S, Summer B, et al. 2003. Immuno-allergological properties of aluminum oxide (Al2O3) ceramics and nickel sulfate in humans. Biomaterials 24(6):959-966.

Thomas P, Rueff F, Przybilla B. 2000. Cheilitis due to nickel contact allergy in a trumpet player. Contact Dermatitis 42(6):351-352.

*Tien JK, Howson TE. 1981. Nickel and nickel alloys. In: Grayson M, Eckroth D, eds. Kirk-Othmer encyclopedia of chemical technology, Vol. 15, 3rd ed. New York, NY: John Wiley and Sons, Inc., 787801.

*Tipton IH, Cook MJ. 1963. Trace elements in human tissue. Part II. Adult subjects from the United States. Health Phys 9:103-145.

*Todorovska N, Karadjova I, Stafilov T. 2002. ETAAS determination of nickel in serum and urine. Anal Bioanal Chem 373(4-5):310-313.

*Tola S, Kilpio J, Virtamo M. 1979. Urinary and plasma concentrations of nickel as indicators of exposure to nickel in an electroplating shop. J Occup Med 21:184-188.

*Torjussen W. 1985. Occupational nasal cancer caused by nickel and nickel compounds. Rhinology 23:101-105.

*Torjussen W, Andersen I. 1979. Nickel concentrations in nasal mucosa, plasma and urine in active and retired nickel workers. Ann Clin Lab Sci 9:289-298.

Torjussen W, Zachariasen H, Andersen I. 2003. Cigarette smoking and nickel exposure. J Environ Monit 5(2):198-201.

Toya T, Serita F, Sawatari K, et al. 1997. Lung lesions induced by intratracheal instillation of nickel fumes and nickel oxide powder in rats. Ind Health 35(1):69-77.

*TRI02. 2004. TRI explorer: Providing access to EPA's toxics release inventory data. Washington, DC: Office of Information Analysis and Access, Offices of Environmental Information, U.S.

Environmental Protection Agency. Toxic Release Inventory. http://www.epa.gov/triexplorer/. December 30, 2004.

*Turk JL, Parker D. 1977. Sensitization with Cr, Ni, and Zr salts and allergic type granuloma formation in the guinea pig. J Invest Dermatol 68:341-345. 
9. REFERENCES

Turkall RM, Skowronski GA, Suh DH, et al. 2003. Effect of a chemical mixture on dermal penetration of arsenic and nickel in male pig in vitro. J Toxicol Environ Health A 66(7):647-655.

*Tyler LD, McBride MB. 1982. Mobility and extractability of cadmium, copper, nickel, and zinc in organic and mineral soil columns. Soil Sci 134:198-205.

*United States Treasury. 2004. Fact sheets: Currency \& coins. Manufacturing process for U.S. coins. http://www.treas.gov/education/fact-sheets/currency/manufacturing.shtml. December 30, 2004.

*U.S. Department of Commerce. 1987. Statistical abstracts of the United States. 108th ed. Washington, DC: Bureau of the Census, U.S. Department of Commerce, 670-681.

*U.S. NRC. 2003. Standards for protection against radiation. Annual limits on intake (ALIs) and derived air concentrations (DACs) of radionuclides for occupational exposure; effluent concentrations, concentrations for release to sewerage. Washington, DC: U.S. Nuclear Regulatory Commission. 10 CFR 20, Appendix B. http://www.nrc.gov/reading-rm/doc-collections/cfr/. June 06, 2003.

USC. 2003. Hazardous air pollutants. Washington, DC: United States Code. 42 USC 7412 http://www4.law.cornell.edu/uscode/. June 06, 2003.

USGS. 1999. Selected elements and organic chemicals in bed sediment and fish tissue of the Tualatin River Basin, Oregon, 1992-1996. U.S. Geological Survey. Water-resources investigation report 99-4107.

*USGS. 2000a. Concentrations of selected trace elements in fish tissue and streambed sediment in the Clark Fork-Pend Oreille and Spokane River Basins, Washington, Idaho, and Montana, 1998. US Geological Survey.

*USGS. 2000b. Trace elements and organochlorine compounds in bed sediment and fish tissue at selected sites in New Jersey streams- sources and effects. U.S. Geological Survey. Water-resources investigation report 99-4235.

*USGS. 2002. Analytical methods for chemical analysis of geologic and other materials. Denver, CO: U.S. Geological Survey. Open-File Report 02-223.

*USGS. 2003. Nickel. U.S. Geological Survey, Mineral Commodity Summaries, January 2004. http://minerals.usgs.gov/minerals/pubs/commodity/nickel/nickemcs04.pdf. January 13, 2005.

*Uter W, Pfahlberg A, Gefeller O, et al. 2003. Risk factors for contact allergy to nickel - results of a multifactorial analysis. Contact Dermatitis 48(1):33-38.

Vago II, Gyori Z, Loch J. 1996. Comparison of chromium and nickel uptake of plants grown in different soils. Anal Bioanal Chem 354(5-6):714-717.

*Valentine R, Fisher GL. 1984. Pulmonary clearance of intratracheally administered ${ }^{63} \mathrm{Ni}_{3} \mathrm{~S}_{2}$ in strain A/J mice. Environ Res 34:328-334.

*van den Berg CM, Achterberg EP. 1994. Automated in-line sampling and analysis of trace elements in surface waters. Trends Anal Chem 13(9):348-352.

Vander Pluym CJ, Coe JY. 2001. Does nickel toxicity occur in children after implantation of Amplatzer (R) Occluders. Pediatr Res 49(4):40A. 
*van Geen A, Rosener P, Boyle E. 1988. Entrainment of trace-metal-enriched Atlantic-shelf water in the inflow to the Mediterranean Sea. Nature 331:423-426.

*van Hoogstraten IMW, von Blomberg ME, Boden D, et al. 1994. Effects of oral exposure to nickel or chromium on cutaneous sensitization. Curr Probl Dermatol 20:237-241.

*Van Winkle MR, Scheff PA. 2001. Volatile organic compounds, polycyclic aromatic hydrocarbons and elements in the air of ten urban homes. Indoor Air 11:49-64.

*Veien NK, Menne T. 1990. Nickel contact allergy and a nickel-restricted diet. Semin Dermatol 9(3):197-205.

*Veien NK, Hattel T, Justesen O, et al. 1982. Contact dermatitis in children. Contact Dermatitis 8:373375.

*Veien NK, Hattel T, Justesen O, et al. 1987. Oral challenge with nickel and cobalt in patients with positive patch tests to nickel and/or cobalt. Acta Derm Venereol 67:321-325.

*Versieck J. 1985. Trace elements in human body fluids and tissues. CRC Crit Rev Clin Lab Sci 22(2):97-184.

*Vieira I, Sonnier M, Cresteil T. 1996. Developmental expression of CYP2E1 in the human liver: Hypermethylation control of gene expression during the neonatal period. Eur J Biochem 238:476-483.

Vincent JH, Werner MA. 2003. Critical evaluation of historical occupational aerosol exposure records: Applications to nickel and lead. Ann Occup Hyg 47(1):49-59.

Vollmer J, Fritz M, Dormy A, et al. 1997. Dominance of the BV17 element in nickel-specific human T cell receptors related to severity of contact sensitivity. Eur J Immunol 27(8):1865-1874.

*Vong RJ, Baker BM, Brechtel FJ, et al. 1997. Ionic and trace element composition of cloud water collected on the Olympic Peninsula of Washington state. Atmos Environ 31(13):1991-2001.

Voutsa D, Samara C. 2002. Labile and bioaccessible fractions of heavy metals in the airborne particulate matter from urban and industrial areas. Atmos Environ 36:3583-3590.

*Vyskocil A, Senft A, Viau C, et al. 1994a. Biochemical renal changes in workers exposed to soluble nickel compounds. Hum Exp Toxicol 13:257-261.

*Vyskocil A, Viau C, Cizkova M. 1994b. Chronic nephrotoxicity of soluble nickel in rats. Hum Exp Toxicol 13:689-693.

*Waalkes MP, Kasprzak KS, Ohshima M, et al. 1985. Protective effects of zinc acetate toward the toxicity of nickelous acetate in rats. Toxicology 34:29-41.

*Wahlberg JE. 1976. Sensitization and testing of guinea pigs with nickel sulfate. Dermatologica 152321.

Wahlberg JE, Liden C. 2000. Cross-reactivity patterns of cobalt and nickel studied with repeated open applications (ROATs) to the skin of guinea pigs. Am J Contact Dermatitis 11(1):42-48. 
*Waksvik H, Boysen M. 1982. Cytogenic analysis of lymphocytes from workers in a nickel refinery. Mutat Res 103:185-190.

*Wall LM, Calnan CD. 1980. Occupational nickel dermatitis in the electroforming industry. Contact Dermatitis 6:414-420.

Wallengren J, Larsson B. 2001. Nitric oxide participates in prick test and irritant patch test reactions in human skin. Arch Dermatol Res 293:121-125.

Wang SJ, Paek DM, Kim RH, et al. 2002. Variation of systolic blood pressure in rats exposed to cadmium and nickel. Environ Res 88(2):116-119.

*Wantke F, Hemmer W, Jarisch R, et al. 1996. Patch test reactions in children, adults and the elderly. A comparative study in patients with suspected allergic contact dermatitis. Contact Dermatitis 34(5):316319

*Warner JS. 1984. Occupation exposure to airborne nickel in producing and using primary nickel products. In: Sunderman FW Jr, Aitio A, Berlin A, eds. Nickel in the human environment. IARC scientific publication no. 53. Lyon, France: International Agency for Research on Cancer, 419-437.

Wataha JC, Lockwood PE, Marek M, et al. 1999. Ability of Ni-containing biomedical alloys to activate monocytes and endothelial cells in vitro. J Biomed Mater Res 45(3):251-257.

*Watanabe C, Weiss B, Cox C, et al. 1990. Modification by nickel of instrumental thermoregulatory behavior in rats. Fundam Appl Toxicol 14:578-588.

*Webber MD, Shamess A. 1987. Heavy metal concentrations in Halton Region soils: An assessment for future municipal sludge utilization. J Soil Sci 67:893-903.

*Wehner AP. 1986. 2. Health and environmental effects of aerosols: Biological effects and fate of inhaled man-made and natural aerosols in animal models. J Aerosol Sci 17:305-315.

*Wehner AP, Craig DK. 1972. Toxicology of inhaled NiO and CoO in Syrian golden hamsters. Am Ind Hyg Assoc J 33:147-155.

*Wehner AP, Busch RH, Olson RJ, et al. 1975. Chronic inhalation of nickel oxide and cigarette smoke by hamsters. Am Ind Hyg Assoc J 36:801-809.

*Wehner AP, Stuart BO, Sanders CL. 1979. Inhalation studies with Syrian golden hamsters. Prog Exp Tumor Res 24:177-198.

*Weischer CH, Kordel W, Hochrainer D. 1980. Effects of $\mathrm{NiCl} 2$ and $\mathrm{NiO}$ in Wistar rats after oral uptake and inhalation exposure, respectively. Zent Bakteriol Mikrobiol Hyg (B) 171:336-351.

Weng L, Lexmond TM, Wolthoorn A, et al. 2003. Phytotoxicity and bioavailability of nickel: Chemical speciation and bioaccumulation. Environ Toxicol Chem 22(9):2180-2187.

*Weng LP, Wolthoorn A, Lexmond TM, et al. 2004. Understanding the effects of soil characteristics on phytotoxicity and bioavailability of nickel using speciation models. Environ Sci Technol 38(1):156-162. 
9. REFERENCES

Werfel U, Langen V, Eickhoff I, et al. 1998. Elevated DNA single-strand breakage frequencies in lymphocytes of welders exposed to chromium and nickel. Carcinogenesis 19(3):413-418.

*Werner MA, Thomassen Y, Hefland S, et al. 1999. Correlation of urinary nickel excretion with observed 'total' and inhalable aerosol exposures of nickel refinery workers. J Environ Monit 1:557-562.

*West JR, Smith HW, Chasis H. 1948. Glomerular filtration rate, effective renal blood flow, and maximal tubular excretory capacity in infancy. J Pediatr 32:10-18.

*Whanger PD. 1973. Effects of dietary nickel on enzyme activities and mineral content in rats. Toxicol Appl Pharmacol 25:323-331.

*WHO. 1991. Environmental health criteria 108: Nickel. International programme on chemical safety. World Health Organization. http:Iwww.inchem.org/documents/ehc/ehc/ehc108.htm. January 17, 2005.

*WHO. 1998. Guidelines for drinking water quality. Nickel. Geneva, Switzerland: World Health Organization. http://www.who.int/en/. June 06, 2003.

*WHO. 2000. Air quality guidelines. Geneva, Switzerland: World Health Organization. http://www.who.int/en/. June 06, 2003.

*Widdowson EM, Dickerson JWT. 1964. Chemical composition of the body. In: Comar CL, Bronner F, eds. Mineral metabolism: An advanced treatise. Volume II: The elements Part A. New York: Academic Press.

*Wiersema JM, Wright L, Rogers B, et al. 1984. Human exposure to potentially toxic elements through ambient air in Texas. Proc APCA Annu Meet 1:1-15.

*Wilkinson DS, Wilkinson JD. 1989. Nickel allergy and hand eczema. In: Maibach HI, Menne T, eds. Nickel and the skin: Immunology and toxicology. Boca Raton, FL: CRC Press, Inc., 133-163

*Windholz M. 1983. The Merck index. 10th ed. Rahway, NJ: Merck and Co., 932-933, 1171.

*Windom HL, Schropp SJ, Calder FD, et al. 1989. Natural trace metal concentrations in estuarine and coastal marine sediments of the southeastern United States. Environ Sci Technol 23:314-320.

*Winger PV, Schultz DP, Johnson WW. 1990. Environmental contaminant concentrations in biota from the lower Savannah River, Georgia and South Carolina. Arch Environ Contam Toxicol 19:101-117.

*Wong PK. 1988. Mutagenicity of heavy metals. Bull Environ Contam Toxicol 40:597-603.

*Wong JL, Wu T-G. 1991. Speciation of airborne nickel in occupational exposure. Environ Sci Technol 25(2):306-309.

*Wood JM. 1987. Biological processes in the cycling of elements between soil or sediments and the aqueous environment. Hydrobiologia 149:31-42.

Wozniak K, Blasiak J. 2002. Free radicals-mediated induction of oxidized DNA bases and DNA-protein cross-links by nickel chloride. Mutat Res 514(1-2):233-243. 
*Wulf HC. 1980. Sister chromatid exchanges in human lymphocytes exposed to nickel and lead. Dan Med Bull 27:40-42.

Yadav JS, Yadav AS, Sharma T. 2001. Chromosome damage in nickel-chrome electroplaters. J Hum Ecol 12(3):185-189.

*Yeats PA. 1988. The distribution of trace metals in ocean waters. Sci Total Environ 72:131-149.

*Young TC, DePinto JV, Seger ES. 1982. Transport and fate of heavy metals in Onondaga Lake, New York, USA. Bull Environ Contam Toxicol 29:554-560.

*Yu CP, Hsieh TH, Oller AR, et al. 2001. Evaluation of the human nickel retention model with workplace data. Regul Toxicol Pharmacol 33:165-172.

*Yunes N, Moyano S, Cerutti S, et al. 2003. On-line preconcentration and determination of nickel in natural water samples by flow injection-inductively coupled plasma optical emission spectrometry (FIICP-OES) Talanta 59(5):943-949.

*Zaroogian GE, Johnson M. 1984. Nickel uptake and loss in the bivalves Crassostrea virginica and Mytilus edulis. Arch Environ Contam Toxicol 13:411-418.

*Zatka VJ, Warner JS, Maskery D. 1992. Chemical speciation of nickel in airborne dusts: Analytical method and results of an interlaboratory test program. Environ Sci Technol 26:138-144.

Zhai H, Chang Y-C, Singh M, et al. 1999. In vivo nickel allergic contact dermatitis: Human model for topical therapeutics. Contact Dermatitis 40:203-208.

Zhai H, Chew A, Bashir SJ, et al. 2003. Provocative use test of nickel coins in nickel-sensitized subjects and controls. Br J Dermatol 149(2):311-317.

*Zhang Q, Kusaka Y, Donaldson K. 2000. Comparative injurious and proinflammatory effects of three ultrafine metals in macrophages from young and old rats. Inhal Toxicol 12:267-273.

Zhang Q, Kusaka Y, Sato K, et al. 2003. Comparative toxicity of standard nickel and ultrafine nickel in lung after intratracheal instillation. J Occup Health 45(1):23-30.

Zhang Z, Suo Z, Holm R, et al. 1987. Diagnostic implications of p53 protein reactivity in nasal mucosa of nickel workers. Anal Quant Cytol Histol 19(4):345-350.

*Ziegler EE, Edwards BB, Jensen RL, et al. 1978. Absorption and retention of lead by infants. Pediatr Res 12:29-34.

*Zissu D, Cavelier C, De Ceaurriz J. 1987. Experimental sensitization of guinea pigs to nickel and patch testing with metal samples. Food Chem Toxicol 25:83-85.

*Zoroddu MA, Schinocca L, Kowalik-Jankowska T, et al. 2002. Molecular mechanisms in nickel carcinogenesis: Modeling Ni (II) binding site in histone H4. Environ Health Perspect Suppl 110(5):719723. 



\section{GLOSSARY}

Absorption-The taking up of liquids by solids, or of gases by solids or liquids.

Acute Exposure-Exposure to a chemical for a duration of 14 days or less, as specified in the Toxicological Profiles.

Activity Median Aerodynamic Diameter (AMAD)—The median of the distribution of radiolabelled particles with varying activities and aerodynamic diameters. The aerodynamic diameter takes into account both the density of the particle and the aerodynamic drag.

Adsorption-The adhesion in an extremely thin layer of molecules (as of gases, solutes, or liquids) to the surfaces of solid bodies or liquids with which they are in contact.

Adsorption Coefficient $\left(\mathbf{K}_{\mathbf{o c}}\right)$ - The ratio of the amount of a chemical adsorbed per unit weight of organic carbon in the soil or sediment to the concentration of the chemical in solution at equilibrium.

Adsorption Ratio (Kd) - The amount of a chemical adsorbed by sediment or soil (i.e., the solid phase) divided by the amount of chemical in the solution phase, which is in equilibrium with the solid phase, at a fixed solid/solution ratio. It is generally expressed in micrograms of chemical sorbed per gram of soil or sediment.

Benchmark Dose (BMD)-Usually defined as the lower confidence limit on the dose that produces a specified magnitude of changes in a specified adverse response. For example, a BMD10 would be the dose at the $95 \%$ lower confidence limit on a $10 \%$ response, and the benchmark response (BMR) would be $10 \%$. The BMD is determined by modeling the dose response curve in the region of the dose response relationship where biologically observable data are feasible.

Benchmark Dose Model-A statistical dose-response model applied to either experimental toxicological or epidemiological data to calculate a BMD.

Bioconcentration Factor (BCF) - The quotient of the concentration of a chemical in aquatic organisms at a specific time or during a discrete time period of exposure divided by the concentration in the surrounding water at the same time or during the same period.

Biomarkers-Broadly defined as indicators signaling events in biologic systems or samples. They have been classified as markers of exposure, markers of effect, and markers of susceptibility.

Cancer Effect Level (CEL) - The lowest dose of chemical in a study, or group of studies, that produces significant increases in the incidence of cancer (or tumors) between the exposed population and its appropriate control.

Carcinogen-A chemical capable of inducing cancer.

Case-Control Study - A type of epidemiological study that examines the relationship between a particular outcome (disease or condition) and a variety of potential causative agents (such as toxic chemicals). In a case-controlled study, a group of people with a specified and well-defined outcome is identified and compared to a similar group of people without outcome. 
Case Report-Describes a single individual with a particular disease or exposure. These may suggest some potential topics for scientific research, but are not actual research studies.

Case Series-Describes the experience of a small number of individuals with the same disease or exposure. These may suggest potential topics for scientific research, but are not actual research studies.

Ceiling Value-A concentration of a substance that should not be exceeded, even instantaneously.

Chronic Exposure-Exposure to a chemical for 365 days or more, as specified in the Toxicological Profiles.

Cohort Study - A type of epidemiological study of a specific group or groups of people who have had a common insult (e.g., exposure to an agent suspected of causing disease or a common disease) and are followed forward from exposure to outcome. At least one exposed group is compared to one unexposed group.

Cross-sectional Study-A type of epidemiological study of a group or groups of people that examines the relationship between exposure and outcome to a chemical or to chemicals at one point in time.

Data Needs-Substance-specific informational needs that if met would reduce the uncertainties of human health assessment.

Developmental Toxicity-The occurrence of adverse effects on the developing organism that may result from exposure to a chemical prior to conception (either parent), during prenatal development, or postnatally to the time of sexual maturation. Adverse developmental effects may be detected at any point in the life span of the organism.

Dose-Response Relationship-The quantitative relationship between the amount of exposure to a toxicant and the incidence of the adverse effects.

Embryotoxicity and Fetotoxicity-Any toxic effect on the conceptus as a result of prenatal exposure to a chemical; the distinguishing feature between the two terms is the stage of development during which the insult occurs. The terms, as used here, include malformations and variations, altered growth, and in utero death.

Environmental Protection Agency (EPA) Health Advisory-An estimate of acceptable drinking water levels for a chemical substance based on health effects information. A health advisory is not a legally enforceable federal standard, but serves as technical guidance to assist federal, state, and local officials.

Epidemiology—Refers to the investigation of factors that determine the frequency and distribution of disease or other health-related conditions within a defined human population during a specified period.

Genotoxicity-A specific adverse effect on the genome of living cells that, upon the duplication of affected cells, can be expressed as a mutagenic, clastogenic, or carcinogenic event because of specific alteration of the molecular structure of the genome.

Half-life-A measure of rate for the time required to eliminate one half of a quantity of a chemical from the body or environmental media. 
Immediately Dangerous to Life or Health (IDLH) - The maximum environmental concentration of a contaminant from which one could escape within 30 minutes without any escape-impairing symptoms or irreversible health effects.

Immunologic Toxicity-The occurrence of adverse effects on the immune system that may result from exposure to environmental agents such as chemicals.

Immunological Effects-Functional changes in the immune response.

Incidence-The ratio of individuals in a population who develop a specified condition to the total number of individuals in that population who could have developed that condition in a specified time period.

Intermediate Exposure-Exposure to a chemical for a duration of 15-364 days, as specified in the Toxicological Profiles.

In Vitro-Isolated from the living organism and artificially maintained, as in a test tube.

In Vivo-Occurring within the living organism.

Lethal Concentration $(\mathbf{L o})\left(\mathbf{L C}_{\mathbf{L o}}\right)$ - The lowest concentration of a chemical in air that has been reported to have caused death in humans or animals.

Lethal Concentration(50) $\left(\mathbf{L C}_{50}\right)$ - A calculated concentration of a chemical in air to which exposure for a specific length of time is expected to cause death in $50 \%$ of a defined experimental animal population.

Lethal Dose $\mathbf{e}_{(\mathbf{L o})}\left(\mathbf{L D}_{\mathbf{L o}}\right)$ - The lowest dose of a chemical introduced by a route other than inhalation that has been reported to have caused death in humans or animals.

Lethal Dose $\mathbf{( 5 0}_{\mathbf{5 0}}\left(\mathbf{L D}_{\mathbf{5 0}}\right)$ - The dose of a chemical that has been calculated to cause death in $50 \%$ of a defined experimental animal population.

Lethal Time $\left(\mathbf{5 0}_{\mathbf{0}}\left(\mathbf{L T}_{\mathbf{5 0}}\right)\right.$ - A calculated period of time within which a specific concentration of a chemical is expected to cause death in $50 \%$ of a defined experimental animal population.

Lowest-Observed-Adverse-Effect Level (LOAEL) — The lowest exposure level of chemical in a study, or group of studies, that produces statistically or biologically significant increases in frequency or severity of adverse effects between the exposed population and its appropriate control.

Lymphoreticular Effects-Represent morphological effects involving lymphatic tissues such as the lymph nodes, spleen, and thymus.

Malformations-Permanent structural changes that may adversely affect survival, development, or function.

Mass Median Aerodynamic Diameter (MMAD)—The median of the distribution of particles with varying mass concentrations and aerodynamic diameters. The aerodynamic diameter takes into account both the density of the particle and the aerodynamic drag. 
Minimal Risk Level (MRL) — An estimate of daily human exposure to a hazardous substance that is likely to be without an appreciable risk of adverse noncancer health effects over a specified route and duration of exposure.

Modifying Factor (MF) - A value (greater than zero) that is applied to the derivation of a Minimal Risk Level (MRL) to reflect additional concerns about the database that are not covered by the uncertainty factors. The default value for a MF is 1 .

Morbidity-State of being diseased; morbidity rate is the incidence or prevalence of disease in a specific population.

Mortality-Death; mortality rate is a measure of the number of deaths in a population during a specified interval of time.

Mutagen-A substance that causes mutations. A mutation is a change in the DNA sequence of a cell's DNA. Mutations can lead to birth defects, miscarriages, or cancer.

Necropsy-The gross examination of the organs and tissues of a dead body to determine the cause of death or pathological conditions.

Neurotoxicity-The occurrence of adverse effects on the nervous system following exposure to a chemical.

No-Observed-Adverse-Effect Level (NOAEL) - The exposure level of a chemical at which there were no statistically or biologically significant increases in frequency or severity of adverse effects seen between the exposed population and its appropriate control. Effects may be produced at this dose, but they are not considered to be adverse.

Octanol-Water Partition Coefficient $\left(\mathbf{K}_{\mathbf{o w}}\right)$ - The equilibrium ratio of the concentrations of a chemical in $n$-octanol and water, in dilute solution.

Odds Ratio (OR) - A means of measuring the association between an exposure (such as toxic substances and a disease or condition) that represents the best estimate of relative risk (risk as a ratio of the incidence among subjects exposed to a particular risk factor divided by the incidence among subjects who were not exposed to the risk factor). An OR of greater than 1 is considered to indicate greater risk of disease in the exposed group compared to the unexposed group.

Organophosphate or Organophosphorus Compound-A phosphorus-containing organic compound and especially a pesticide that acts by inhibiting cholinesterase.

Permissible Exposure Limit (PEL) —An Occupational Safety and Health Administration (OSHA) allowable exposure level in workplace air averaged over an 8-hour shift of a 40-hour workweek.

Pesticide-General classification of chemicals specifically developed and produced for use in the control of agricultural and public health pests.

Pharmacokinetics - The dynamic behavior of a material in the body, used to predict the fate (disposition) of an exogenous substance in an organism. Utilizing computational techniques, it provides the means of studying the absorption, distribution, metabolism, and excretion of chemicals by the body. 
Pharmacokinetic Model-A set of equations that can be used to describe the time course of a parent chemical or metabolite in an animal system. There are two types of pharmacokinetic models: data-based and physiologically-based. A data-based model divides the animal system into a series of compartments, which, in general, do not represent real, identifiable anatomic regions of the body, whereas the physiologically-based model compartments represent real anatomic regions of the body.

Physiologically Based Pharmacodynamic (PBPD) Model—A type of physiologically based doseresponse model that quantitatively describes the relationship between target tissue dose and toxic end points. These models advance the importance of physiologically based models in that they clearly describe the biological effect (response) produced by the system following exposure to an exogenous substance.

Physiologically Based Pharmacokinetic (PBPK) Model-Comprised of a series of compartments representing organs or tissue groups with realistic weights and blood flows. These models require a variety of physiological information: tissue volumes, blood flow rates to tissues, cardiac output, alveolar ventilation rates, and possibly membrane permeabilities. The models also utilize biochemical information, such as air/blood partition coefficients, and metabolic parameters. PBPK models are also called biologically based tissue dosimetry models.

Prevalence-The number of cases of a disease or condition in a population at one point in time.

Prospective Study - A type of cohort study in which the pertinent observations are made on events occurring after the start of the study. A group is followed over time.

q1*-The upper-bound estimate of the low-dose slope of the dose-response curve as determined by the multistage procedure. The $\mathrm{q}_{1} *$ can be used to calculate an estimate of carcinogenic potency, the incremental excess cancer risk per unit of exposure (usually $\mu \mathrm{g} / \mathrm{L}$ for water, $\mathrm{mg} / \mathrm{kg} / \mathrm{day}$ for food, and $\mu \mathrm{g} / \mathrm{m}^{3}$ for air).

Recommended Exposure Limit (REL) —A National Institute for Occupational Safety and Health (NIOSH) time-weighted average (TWA) concentration for up to a 10-hour workday during a 40-hour workweek.

Reference Concentration (RfC)—An estimate (with uncertainty spanning perhaps an order of magnitude) of a continuous inhalation exposure to the human population (including sensitive subgroups) that is likely to be without an appreciable risk of deleterious noncancer health effects during a lifetime. The inhalation reference concentration is for continuous inhalation exposures and is appropriately expressed in units of $\mathrm{mg} / \mathrm{m}^{3}$ or $\mathrm{ppm}$.

Reference Dose (RfD)—An estimate (with uncertainty spanning perhaps an order of magnitude) of the daily exposure of the human population to a potential hazard that is likely to be without risk of deleterious effects during a lifetime. The RfD is operationally derived from the no-observed-adverse-effect level (NOAEL, from animal and human studies) by a consistent application of uncertainty factors that reflect various types of data used to estimate RfDs and an additional modifying factor, which is based on a professional judgment of the entire database on the chemical. The RfDs are not applicable to nonthreshold effects such as cancer.

Reportable Quantity (RQ) - The quantity of a hazardous substance that is considered reportable under the Comprehensive Environmental Response, Compensation, and Liability Act (CERCLA). Reportable quantities are (1) 1 pound or greater or (2) for selected substances, an amount established by regulation 
either under CERCLA or under Section 311 of the Clean Water Act. Quantities are measured over a 24-hour period.

Reproductive Toxicity-The occurrence of adverse effects on the reproductive system that may result from exposure to a chemical. The toxicity may be directed to the reproductive organs and/or the related endocrine system. The manifestation of such toxicity may be noted as alterations in sexual behavior, fertility, pregnancy outcomes, or modifications in other functions that are dependent on the integrity of this system.

Retrospective Study-A type of cohort study based on a group of persons known to have been exposed at some time in the past. Data are collected from routinely recorded events, up to the time the study is undertaken. Retrospective studies are limited to causal factors that can be ascertained from existing records and/or examining survivors of the cohort.

Risk-The possibility or chance that some adverse effect will result from a given exposure to a chemical.

Risk Factor-An aspect of personal behavior or lifestyle, an environmental exposure, or an inborn or inherited characteristic that is associated with an increased occurrence of disease or other health-related event or condition.

Risk Ratio-The ratio of the risk among persons with specific risk factors compared to the risk among persons without risk factors. A risk ratio greater than 1 indicates greater risk of disease in the exposed group compared to the unexposed group.

Short-Term Exposure Limit (STEL) - The American Conference of Governmental Industrial Hygienists (ACGIH) maximum concentration to which workers can be exposed for up to 15 minutes continually. No more than four excursions are allowed per day, and there must be at least 60 minutes between exposure periods. The daily Threshold Limit Value-Time Weighted Average (TLV-TWA) may not be exceeded.

Standardized Mortality Ratio (SMR) - A ratio of the observed number of deaths and the expected number of deaths in a specific standard population.

Target Organ Toxicity-This term covers a broad range of adverse effects on target organs or physiological systems (e.g., renal, cardiovascular) extending from those arising through a single limited exposure to those assumed over a lifetime of exposure to a chemical.

Teratogen-A chemical that causes structural defects that affect the development of an organism.

Threshold Limit Value (TLV)—An American Conference of Governmental Industrial Hygienists (ACGIH) concentration of a substance to which most workers can be exposed without adverse effect. The TLV may be expressed as a Time Weighted Average (TWA), as a Short-Term Exposure Limit (STEL), or as a ceiling limit (CL).

Time-Weighted Average (TWA)_An allowable exposure concentration averaged over a normal 8-hour workday or 40-hour workweek.

Toxic Dose(50) (TD50) - A calculated dose of a chemical, introduced by a route other than inhalation, which is expected to cause a specific toxic effect in $50 \%$ of a defined experimental animal population.

Toxicokinetic-The absorption, distribution, and elimination of toxic compounds in the living organism. 
Uncertainty Factor (UF) - A factor used in operationally deriving the Minimal Risk Level (MRL) or Reference Dose (RfD) or Reference Concentration (RfC) from experimental data. UFs are intended to account for (1) the variation in sensitivity among the members of the human population, (2) the uncertainty in extrapolating animal data to the case of human, (3) the uncertainty in extrapolating from data obtained in a study that is of less than lifetime exposure, and (4) the uncertainty in using lowestobserved-adverse-effect level (LOAEL) data rather than no-observed-adverse-effect level (NOAEL) data. A default for each individual UF is 10; if complete certainty in data exists, a value of 1 can be used; however, a reduced UF of 3 may be used on a case-by-case basis, 3 being the approximate logarithmic average of 10 and 1 .

Xenobiotic-Any chemical that is foreign to the biological system. 



\section{APPENDIX A. ATSDR MINIMAL RISK LEVELS AND WORKSHEETS}

The Comprehensive Environmental Response, Compensation, and Liability Act (CERCLA) [42 U.S.C. 9601 et seq.], as amended by the Superfund Amendments and Reauthorization Act (SARA) [Pub. L. 99499], requires that the Agency for Toxic Substances and Disease Registry (ATSDR) develop jointly with the U.S. Environmental Protection Agency (EPA), in order of priority, a list of hazardous substances most commonly found at facilities on the CERCLA National Priorities List (NPL); prepare toxicological profiles for each substance included on the priority list of hazardous substances; and assure the initiation of a research program to fill identified data needs associated with the substances.

The toxicological profiles include an examination, summary, and interpretation of available toxicological information and epidemiologic evaluations of a hazardous substance. During the development of toxicological profiles, Minimal Risk Levels (MRLs) are derived when reliable and sufficient data exist to identify the target organ(s) of effect or the most sensitive health effect(s) for a specific duration for a given route of exposure. An MRL is an estimate of the daily human exposure to a hazardous substance that is likely to be without appreciable risk of adverse noncancer health effects over a specified duration of exposure. MRLs are based on noncancer health effects only and are not based on a consideration of cancer effects. These substance-specific estimates, which are intended to serve as screening levels, are used by ATSDR health assessors to identify contaminants and potential health effects that may be of concern at hazardous waste sites. It is important to note that MRLs are not intended to define clean-up or action levels.

MRLs are derived for hazardous substances using the no-observed-adverse-effect level/uncertainty factor approach. They are below levels that might cause adverse health effects in the people most sensitive to such chemical-induced effects. MRLs are derived for acute (1-14 days), intermediate (15-364 days), and chronic (365 days and longer) durations and for the oral and inhalation routes of exposure. Currently, MRLs for the dermal route of exposure are not derived because ATSDR has not yet identified a method suitable for this route of exposure. MRLs are generally based on the most sensitive chemical-induced end point considered to be of relevance to humans. Serious health effects (such as irreparable damage to the liver or kidneys, or birth defects) are not used as a basis for establishing MRLs. Exposure to a level above the MRL does not mean that adverse health effects will occur. 
MRLs are intended only to serve as a screening tool to help public health professionals decide where to look more closely. They may also be viewed as a mechanism to identify those hazardous waste sites that are not expected to cause adverse health effects. Most MRLs contain a degree of uncertainty because of the lack of precise toxicological information on the people who might be most sensitive (e.g., infants, elderly, nutritionally or immunologically compromised) to the effects of hazardous substances. ATSDR uses a conservative (i.e., protective) approach to address this uncertainty consistent with the public health principle of prevention. Although human data are preferred, MRLs often must be based on animal studies because relevant human studies are lacking. In the absence of evidence to the contrary, ATSDR assumes that humans are more sensitive to the effects of hazardous substance than animals and that certain persons may be particularly sensitive. Thus, the resulting MRL may be as much as 100 -fold below levels that have been shown to be nontoxic in laboratory animals.

Proposed MRLs undergo a rigorous review process: Health Effects/MRL Workgroup reviews within the Division of Toxicology, expert panel peer reviews, and agency-wide MRL Workgroup reviews, with participation from other federal agencies and comments from the public. They are subject to change as new information becomes available concomitant with updating the toxicological profiles. Thus, MRLs in the most recent toxicological profiles supersede previously published levels. For additional information regarding MRLs, please contact the Division of Toxicology, Agency for Toxic Substances and Disease Registry, 1600 Clifton Road NE, Mailstop F-32, Atlanta, Georgia 30333. 


\section{MINIMAL RISK LEVEL (MRL) WORKSHEET}

$\begin{array}{ll}\text { Chemical Name: } & \text { Nickel } \\ \text { CAS Number: } & \text { 7440-02-0 } \\ \text { Date: } & \text { May 13, 2005 } \\ \text { Profile Status: } & \text { Final Post-Public Comment Draft } \\ \text { Route: } & \text { [x] Inhalation [ ] Oral } \\ \text { Duration: } & \text { [ ] Acute [x] Intermediate [ ] Chronic } \\ \text { Graph Key: } & 38 \\ \text { Species: } & \text { F344 Rats }\end{array}$

Minimal Risk Level: 0.0002 [ ] mg/kg/day $[\mathrm{x}] \mathrm{mg} / \mathrm{m}^{3}$

Reference: NTP 1996c. Toxicology and carcinogenesis of nickel sulfate hexahydrate (CAS No. 1010197-0) in F344/N rats and B6C3F1 mice (inhalation studies). U.S. Department of Health and Human Services, Public Health Service, National Institutes of Health, National Toxicology Program, Research Triangle Park, NC.

Experimental design: Groups of 10 male and 10 female F344/N rats were exposed to $0.12,0.25,0.5,1.0$, or $2.0 \mathrm{mg} / \mathrm{m}^{3}$ nickel sulfate hexahydrate $\left(0.03,0.06,0.11,0.22\right.$, or $0.44 \mathrm{mg} \mathrm{Ni} / \mathrm{m}^{3}$, as calculated by study authors) for 6 hours/day, 5 days/week for 13 weeks. The mass median aerodynamic diameter (MMAD) (and sigma g) values reported in Table K1 of the paper were 2.31 (2.1), 2.11 (2.7), 3.08 (2.9), 1.81 (2.2), and 2.01 (2.0) for the $0.03,0.06,0.11,0.22$, and $0.44 \mathrm{mg} \mathrm{Ni} / \mathrm{m}^{3}$ concentrations, respectively. End points examined included body weight gain, clinical observations, hematology, and organ weights, and microscopic examinations of the following organs were completed: adrenal gland, bone, brain, clitoral gland, epididymis, oviduct, esophagus, heart, large intestine, small intestine, kidneys, larynx, liver, lung, lymph nodes, mammary gland, nose, ovary, pancreas, parathyroid gland, pituitary, preputial gland, prostate, salivary gland, seminal vesicle, skin, spleen, stomach, testis, thymus, thyroid gland, trachea, bladder, and uterus.

Effects noted in study and corresponding doses: No exposure related deaths, alterations in body weight gain, or clinical signs were observed. A number of hematological alterations were observed in female rats-increased hematocrit, hemoglobin, and erythrocyte concentrations at $0.22 \mathrm{mg} \mathrm{Ni} / \mathrm{m}^{3}$ and higher; increased reticulocytes at $0.03 \mathrm{mg} \mathrm{Ni} / \mathrm{m}^{3}$ and higher; increased leukocyte levels at $0.11 \mathrm{mg} \mathrm{Ni} / \mathrm{m}^{3}$ and higher; increased segmented neutrophils at $0.06 \mathrm{mg} \mathrm{Ni} / \mathrm{m}^{3}$ and higher; and increased lymphocytes at $0.22 \mathrm{mg} \mathrm{Ni} / \mathrm{m}^{3}$ and higher - the study authors noted that these alterations are consistent with chronic inflammation, hyperplasia of lymph nodes, and mild dehydration. Significant alterations in lung weights were observed at $0.06 \mathrm{mg} \mathrm{Ni} / \mathrm{m}^{3}$ and higher. Lung lesions consisted of minimal alveolar macrophage hyperplasia at $0.03-0.11 \mathrm{mg} \mathrm{Ni} / \mathrm{m}^{3}$, mild to moderate macrophage hyperplasia at 0.22 and $0.44 \mathrm{mg} \mathrm{Ni} / \mathrm{m}^{3}$, interstitial infiltrates at $0.22 \mathrm{mg} \mathrm{Ni} / \mathrm{m}^{3}$ and higher in males and $0.11 \mathrm{mg} \mathrm{Ni} / \mathrm{m}^{3}$ and higher in females, and chronic active inflammation characterized by slight thickening of alveolar septae due to an increase in monnuclear inflammatory cells, and few neutrophils and fibroblasts in the intersitium. Hyperplasia of bronchial and mediastinal lymph nodes was observed at $0.22 \mathrm{mg} \mathrm{Ni} / \mathrm{m}^{3}$ and higher and atrophy of the olfactory epithelium was observed at 0.22 and $0.44 \mathrm{mg} \mathrm{Ni} / \mathrm{m}^{3}$.

The minimal alveolar macrophage hyperplasia observed at $0.03-0.11 \mathrm{mg} \mathrm{Ni} / \mathrm{m}^{3}$ was not considered an adverse health effect because the slight changes in the number of macrophages were considered to be part of the normal physiologic response to inhaled particles and it is not believed to compromise the lung's ability to clear foreign matter. 
Dose and end point used for MRL derivation:

\section{[x] NOAEL [ ] LOAEL}

The NOAEL of $0.06 \mathrm{mg} / \mathrm{m}^{3}$ for chronic active inflammation in rats is the basis of the intermediateduration inhalation MRL for nickel.

Uncertainty Factors used in MRL derivation:

[ ] 10 for use of a LOAEL

[x] 3 for extrapolation from animals to humans with dosimetric adjustment

[x] 10 for human variability

Was a conversion used from $\mathrm{ppm}$ in food or water to a $\mathrm{mg} /$ body weight dose? No.

If an inhalation study in animals, list the conversion factors used in determining human equivalent dose: The exposure concentration was adjusted for intermittent exposure (6 hours/24 hours, 5 days/7 days). A regional deposited dose ratio (RDDR) of 0.474 for the pulmonary region was used to extrapolate from particle deposition in rats to deposition in humans. The RDDR was calculated using EPA's software for calculating RDDRs. The following parameters were used: particle size (MMAD) of $2.11 \mu \mathrm{m}$ and geometric standard deviation (sigma g) of 2.7; default human body weight $(70 \mathrm{~kg})$, minute volume $(13 \mathrm{~L})$ and pulmonary surface area $\left(54 \mathrm{~m}^{2}\right)$; default female F344 rat body weight $(0.124 \mathrm{~kg})$, minute volume $(101.3 \mathrm{~mL})$, and pulmonary surface area $\left(0.34 \mathrm{~m}^{2}\right)$.

$\mathrm{NOAEL}_{\mathrm{ADJ}}=0.06 \mathrm{mg} \mathrm{Ni} / \mathrm{m}^{3} \times 6$ hours $/ 24$ hours $\times 5$ days $/ 7$ days $=0.011 \mathrm{mg} \mathrm{Ni} / \mathrm{m}^{3}$ NOAEL $_{\text {HEC }}=$ NOAEL $_{\text {ADJ }} \times$ RDDR $=0.011 \mathrm{mg} \mathrm{Ni} / \mathrm{m}^{3} \times 0.474=0.0052 \mathrm{mg} \mathrm{Ni} / \mathrm{m}^{3}$

Other additional studies or pertinent information which lend support to this MRL: The identification of the lung as the most sensitive target of nickel toxicity is supported by a number of acute-, intermediate-, and chronic-duration studies of nickel sulfate, nickel subsulfide, and nickel oxide in rats and mice (Benson et al. 1995a, 1995b; Horie et al. 1985; NTP 1996a, 1996b, 1996c; Ottolenghi et al. 1990; Tanaka et al. 1988). In these studies, respiratory effects, in particular chronic lung inflammation, was observed at the lowest LOAEL values. Three other inhalation studies have examined the toxicity of nickel sulfate. Benson et al. (1995a) observed mild alveolitis in rats exposed to $0.11 \mathrm{mg} \mathrm{Ni} / \mathrm{m}^{3} 6$ hours/day, 5 days/week for 6 months; 4 months after exposure termination, alveolitis was still present in the nickel-exposed rats. Minimal alveolar macrophage hyperplasia was observed at $0.03 \mathrm{mg} \mathrm{Ni} / \mathrm{m}^{3}$; this was not observed 4 months after exposure termination. In mice exposed to nickel sulfate ( 6 hours/day, 5 days/week for 13 weeks), chronic lung inflammation and fibrosis were observed at $0.44 \mathrm{mg} \mathrm{Ni} / \mathrm{m}^{3}$; minimal alveolar hyperplasia was observed at $0.11 \mathrm{mg} \mathrm{Ni} / \mathrm{m}^{3}$ and higher (NTP 1996c). Similarly, Benson et al. (1995a) reported minimal alveolar macrophage hyperplasia and interstitial pneumonia in mice exposed to $0.22 \mathrm{mg}$ $\mathrm{Ni} / \mathrm{m}^{3}$.

Similar studies in which rats and mice were exposed to nickel subsulfide (NTP 1996b) or nickel oxide (1996a) confirm that the lungs are the principal target of nickel toxicity following inhalation exposure. Comparison of the NOAEL and LOAEL values identified in the NTP studies of nickel sulfate (NTP 1996c), nickel subsulfide (NTP 1996b), and nickel oxide (NTP 1996a) demonstrate that nickel sulfate is more toxic than nickel subsulfide and nickel oxide. In rats, the NOAEL and LOAEL values for chronic lung inflammation were 0.06 and $0.11 \mathrm{mg} \mathrm{Ni} / \mathrm{m}^{3}$ for nickel sulfate (NTP 1996c), 0.11 and $0.22 \mathrm{mg} \mathrm{Ni} / \mathrm{m}^{3}$ for nickel subsulfide (NTP 1996b), and 2.0 and $3.9 \mathrm{mg} \mathrm{Ni} / \mathrm{m}^{3}$ for nickel oxide (NTP 1996a). Atrophy of the nasal olfactory epithelium was observed at 0.22 and $0.44 \mathrm{mg} \mathrm{Ni} / \mathrm{m}^{3}$ as nickel sulfate (NTP 1996c) and nickel subsulfide (NTP 1996b), respectively. Similar effects were observed in mice. For nickel sulfate 
and nickel subsulfide, the LOAEL values in mice were higher than the LOAELs identified in rats; the LOAEL for chronic inflammation following exposure to nickel oxide was the same in rats and mice.

No intermediate-duration human inhalation exposure studies were identified; a number of chronic exposure studies have examined the potential of nickel and nickel compounds to induce respiratory effects in workers. Most of these studies are cohort mortality studies that did not find significant increases in the number of deaths from nonmalignant respiratory system disease (Arena et al. 1998; Cox et al. 1981; Cragle et al. 1984; Egedahl et al. 2001; Enterline and Marsh 1982; Redmond 1984; Roberts et al. 1989b; Shannon et al. 1984b, 1991). A few studies have examined workers for possible nonlethal respiratory effects. Two studies examined chest x-rays of workers: one found an increased risk of moderate pulmonary fibrosis (Berge and Skyberg 2003) and the other did not find any significant alterations (Muir et al. 1993). Although most of occupational exposure studies did not report exposure levels, workers were typically exposed to nickel levels that far exceed levels found in ambient air.

Agency Contact (Chemical Managers): Mike Fay, Sharon Wilbur, and Henry Abadin 


\section{MINIMAL RISK LEVEL (MRL) WORKSHEET}

$\begin{array}{ll}\text { Chemical Name: } & \text { Nickel } \\ \text { CAS Number: } & \text { 7440-02-0 } \\ \text { Date: } & \text { May 13, 2005 } \\ \text { Profile Status: } & \text { Final Post-Public Comment Draft } \\ \text { Route: } & \text { [x] Inhalation [ ] Oral } \\ \text { Duration: } & \text { [ ] Acute [ ] Intermediate [x] Chronic } \\ \text { Graph Key: } & 77 \\ \text { Species: } & \text { F344 Rats }\end{array}$

Minimal Risk Level: $9 \times 10^{-5} \quad\left[\right.$ ] mg/kg/day $[\mathrm{x}] \mathrm{mg} / \mathrm{m}^{3}$

Reference: NTP 1996c. Toxicology and carcinogenesis of nickel sulfate hexahydrate (CAS No. 1010197-0) in F344/N rats and B6C3F1 mice (inhalation studies). Research Triangle Park, NC: U.S. Department of Health and Human Services, Public Health Service, National Institutes of Health, National Toxicology Program.

Experimental design: Groups of male and female F344 rats were exposed to 0.12, 0.25, or $0.5 \mathrm{mg} / \mathrm{m}^{3}$ nickel sulfate hexahydrate $\left(0,0.03,0.06\right.$, or $0.11 \mathrm{mg} \mathrm{Ni} / \mathrm{m}^{3}$ as calculated by study authors) 6 hours/day, 5 days/week for 2 years. The mean mass median aerodynamic diameter (MMAD) and sigma $\mathrm{g}$ values (reported in Table K2 of the paper) were 2.50 (sigma g of 2.38), 2.24 (2.21), and 2.25 (2.08) for the $0.03,0.06$, and $0.11 \mathrm{mg} \mathrm{Ni} / \mathrm{m}^{3}$ concentrations, respectively. End points examined included body weight gain, clinical observations, hematology, and organ weights. Microscopic examinations of the following organs were completed: adrenal gland, bone, brain, clitoral gland, epididymis, oviduct, esophagus, heart, large intestine, small intestine, kidneys, larynx, liver, lung, lymph nodes, mammary gland, nose, ovary, pancreas, parathyroid gland, pituitary, preputial gland, prostate, salivary gland, seminal vesicle, skin, spleen, stomach, testis, thymus, thyroid gland, trachea, bladder, and uterus.

Effects noted in study and corresponding doses: No significant alterations in survival, body weight, or the occurrence of clinical signs were observed. The only treatment-related changes noted were in the respiratory tract. Lung lesions consisted of chronic active inflammation, hyperplasia of alveolar macrophages, alveolar proteinosis, and fibrosis at 0.06 and $0.11 \mathrm{mg} \mathrm{Ni} / \mathrm{m}^{3}$. The combined incidences of chronic active inflammation in the male and female rats were 28/106, 24/106, 91/106, and 98/107 in the $0,0.03,0.06$, and $0.11 \mathrm{mg} \mathrm{Ni} / \mathrm{m}^{3}$ groups, respectively. The chronic inflammation consisted of multifocal, minimal to mild accumulation of macrophages, neutrophils, and cellular debris within the alveolar spaces. No significant alterations in the malignant tumors were observed in the lungs. Significant increases in the incidence of lymphoid hyperplasia of the bronchial lymph nodes and atrophy of the olfactory epithelium were observed at $0.11 \mathrm{mg} \mathrm{Ni} / \mathrm{m}^{3}$.

Dose and end point used for MRL derivation:

\section{[x] NOAEL [ ] LOAEL}

The NOAEL of $0.03 \mathrm{mg} / \mathrm{m}^{3}$ for chronic active inflammation and lung fibrosis in rats is the basis of the chronic inhalation MRL for nickel. 
Uncertainty Factors used in MRL derivation:

[ ] 10 for use of a LOAEL

[x] 3 for extrapolation from animals to humans with dosimetric adjustment

[x] 10 for human variability

Was a conversion used from $\mathrm{ppm}$ in food or water to a mg/body weight dose? No.

If an inhalation study in animals, list the conversion factors used in determining human equivalent dose: The exposure concentration was adjusted for intermittent exposure (6 hours/24 hours, 5 days/7 days). A regional deposited dose ratio (RDDR) of 0.506 for the pulmonary region was used to extrapolate from a deposited dose in rats to a deposited dose in humans. The RDDR was calculated using EPA's software for calculating RDDRs. The following parameters were used: particle size (MMAD) of $2.5 \mu \mathrm{m}$ and geometric standard deviation (sigma g) of 2.38 ; default human body weight $(70 \mathrm{~kg}$ ), minute volume $(13 \mathrm{~L})$ and pulmonary surface area $\left(54 \mathrm{~m}^{2}\right)$; default female F344 rat body weight $(0.229 \mathrm{~kg})$, minute volume $(167.3 \mathrm{~mL})$, and pulmonary surface area $\left(0.34 \mathrm{~m}^{2}\right)$.

NOAEL $_{A D J}=0.03 \mathrm{mg} \mathrm{Ni} / \mathrm{m}^{3} \times 6$ hours $/ 24$ hours $\times 5$ days $/ 7$ days $=0.0054 \mathrm{mg} \mathrm{Ni} / \mathrm{m}^{3}$

NOAEL $_{\text {HEC }}=$ NOAEL $_{\text {ADJ }} \times$ RDDR $=0.0054 \mathrm{mg} \mathrm{Ni} / \mathrm{m}^{3} \times 0.506=0.0027 \mathrm{mg} \mathrm{Ni} / \mathrm{m}^{3}$

Other additional studies or pertinent information which lend support to this MRL: The identification of the lung as the most sensitive target of nickel toxicity is supported by a number of acute-, intermediate-, and chronic-duration studies of nickel sulfate, nickel subsulfide, and nickel oxide in rats and mice (Benson et al. 1995a, 1995b; Horie et al. 1985; NTP 1996a, 1996b, 1996c; Ottolenghi et al. 1990; Tanaka et al. 1988). In these studies, respiratory effects, in particular chronic lung inflammation, was observed at the lowest LOAEL values. One other inhalation study has examined the toxicity of nickel sulfate. Chronic active lung inflammation was observed in mice exposed to 0.11 or $0.22 \mathrm{mg} \mathrm{Ni} / \mathrm{m}^{3} 6$ hours/day, 5 days/week for 2 years (NTP 1996c); no respiratory tract effects were observed at $0.06 \mathrm{mg} \mathrm{Ni} / \mathrm{m}^{3}$. Chronic-duration studies (all studies involved 6 hour/day, 5 day/week exposures) with different nickel compounds have also found inflammatory lung effects at in rats exposed to $0.11 \mathrm{mg} \mathrm{Ni} / \mathrm{m}^{3}$ as nickel subsulfide for 2 years (NTP 1996b), rats exposed to $0.7 \mathrm{mg} \mathrm{Ni} / \mathrm{m}^{3}$ as nickel subsulfide for 78 weeks (Ottolenghi et al. 1990), mice exposed to $0.44 \mathrm{mg} \mathrm{Ni} / \mathrm{m}^{3}$ as nickel subsulfide for 2 years (NTP 1996b), rats exposed to $0.2 \mathrm{mg} \mathrm{Ni} / \mathrm{m}^{3}$ as nickel oxide for 2 years (NTP 1996a), and mice exposed to $1 \mathrm{mg} \mathrm{Ni} / \mathrm{m}^{3}$ as nickel oxide for 2 years (NTP 1996a).

The potential of nickel to induce nonmalignant respiratory tract effects has been examined in a number of cohort mortality studies. In general, these studies did not find significant increases in the risk of dying from nonmalignant respiratory system disease (Arena et al. 1998; Cox et al. 1981; Cragle et al. 1984; Egedahl et al. 2001; Enterline and Marsh 1982; Redmond 1984; Roberts et al. 1989b; Shannon et al. 1984b, 1991). Mixed results have been found in the few studies examining nonlethal respiratory tract effects. Two studies examined chest x-rays of nickel workers: one found an increased risk of moderate pulmonary fibrosis (Berge and Skyberg 2003) and the other did not find any significant alterations (Muir et al. 1993). Although most of occupational exposure studies did not report exposure levels, workers were typically exposed to nickel levels that far exceed levels found in ambient air.

Agency Contact (Chemical Managers): Mike Fay, Sharon Wilbur, and Henry Abadin 



\section{APPENDIX B. USER'S GUIDE}

\section{Chapter 1}

\section{Public Health Statement}

This chapter of the profile is a health effects summary written in non-technical language. Its intended audience is the general public, especially people living in the vicinity of a hazardous waste site or chemical release. If the Public Health Statement were removed from the rest of the document, it would still communicate to the lay public essential information about the chemical.

The major headings in the Public Health Statement are useful to find specific topics of concern. The topics are written in a question and answer format. The answer to each question includes a sentence that will direct the reader to chapters in the profile that will provide more information on the given topic.

\section{Chapter 2}

\section{Relevance to Public Health}

This chapter provides a health effects summary based on evaluations of existing toxicologic, epidemiologic, and toxicokinetic information. This summary is designed to present interpretive, weightof-evidence discussions for human health end points by addressing the following questions:

1. What effects are known to occur in humans?

2. What effects observed in animals are likely to be of concern to humans?

3. What exposure conditions are likely to be of concern to humans, especially around hazardous waste sites?

The chapter covers end points in the same order that they appear within the Discussion of Health Effects by Route of Exposure section, by route (inhalation, oral, and dermal) and within route by effect. Human data are presented first, then animal data. Both are organized by duration (acute, intermediate, chronic). In vitro data and data from parenteral routes (intramuscular, intravenous, subcutaneous, etc.) are also considered in this chapter.

The carcinogenic potential of the profiled substance is qualitatively evaluated, when appropriate, using existing toxicokinetic, genotoxic, and carcinogenic data. ATSDR does not currently assess cancer potency or perform cancer risk assessments. Minimal Risk Levels (MRLs) for noncancer end points (if derived) and the end points from which they were derived are indicated and discussed.

Limitations to existing scientific literature that prevent a satisfactory evaluation of the relevance to public health are identified in the Chapter 3 Data Needs section.

\section{Interpretation of Minimal Risk Levels}

Where sufficient toxicologic information is available, ATSDR has derived MRLs for inhalation and oral routes of entry at each duration of exposure (acute, intermediate, and chronic). These MRLs are not 
meant to support regulatory action, but to acquaint health professionals with exposure levels at which adverse health effects are not expected to occur in humans.

MRLs should help physicians and public health officials determine the safety of a community living near a chemical emission, given the concentration of a contaminant in air or the estimated daily dose in water. MRLs are based largely on toxicological studies in animals and on reports of human occupational exposure.

MRL users should be familiar with the toxicologic information on which the number is based. Chapter 2, "Relevance to Public Health," contains basic information known about the substance. Other sections such as Chapter 3 Section 3.9, "Interactions with Other Substances," and Section 3.10, "Populations that are Unusually Susceptible" provide important supplemental information.

MRL users should also understand the MRL derivation methodology. MRLs are derived using a modified version of the risk assessment methodology that the Environmental Protection Agency (EPA) provides (Barnes and Dourson 1988) to determine reference doses (RfDs) for lifetime exposure.

To derive an MRL, ATSDR generally selects the most sensitive end point which, in its best judgement, represents the most sensitive human health effect for a given exposure route and duration. ATSDR cannot make this judgement or derive an MRL unless information (quantitative or qualitative) is available for all potential systemic, neurological, and developmental effects. If this information and reliable quantitative data on the chosen end point are available, ATSDR derives an MRL using the most sensitive species (when information from multiple species is available) with the highest no-observed-adverse-effect level (NOAEL) that does not exceed any adverse effect levels. When a NOAEL is not available, a lowest-observed-adverse-effect level (LOAEL) can be used to derive an MRL, and an uncertainty factor (UF) of 10 must be employed. Additional uncertainty factors of 10 must be used both for human variability to protect sensitive subpopulations (people who are most susceptible to the health effects caused by the substance) and for interspecies variability (extrapolation from animals to humans). In deriving an MRL, these individual uncertainty factors are multiplied together. The product is then divided into the inhalation concentration or oral dosage selected from the study. Uncertainty factors used in developing a substance-specific MRL are provided in the footnotes of the levels of significant exposure (LSE) tables.

\section{Chapter 3}

\section{Health Effects}

\section{Tables and Figures for Levels of Significant Exposure (LSE)}

Tables and figures are used to summarize health effects and illustrate graphically levels of exposure associated with those effects. These levels cover health effects observed at increasing dose concentrations and durations, differences in response by species, MRLs to humans for noncancer end points, and EPA's estimated range associated with an upper- bound individual lifetime cancer risk of 1 in 10,000 to 1 in 10,000,000. Use the LSE tables and figures for a quick review of the health effects and to locate data for a specific exposure scenario. The LSE tables and figures should always be used in conjunction with the text. All entries in these tables and figures represent studies that provide reliable, quantitative estimates of NOAELs, LOAELs, or Cancer Effect Levels (CELs).

The legends presented below demonstrate the application of these tables and figures. Representative examples of LSE Table 3-1 and Figure 3-1 are shown. The numbers in the left column of the legends correspond to the numbers in the example table and figure. 


\section{LEGEND}

\section{See Sample LSE Table 3-1 (page B-6)}

(1) Route of Exposure. One of the first considerations when reviewing the toxicity of a substance using these tables and figures should be the relevant and appropriate route of exposure. Typically when sufficient data exist, three LSE tables and two LSE figures are presented in the document. The three LSE tables present data on the three principal routes of exposure, i.e., inhalation, oral, and dermal (LSE Tables 3-1, 3-2, and 3-3, respectively). LSE figures are limited to the inhalation (LSE Figure 3-1) and oral (LSE Figure 3-2) routes. Not all substances will have data on each route of exposure and will not, therefore, have all five of the tables and figures.

(2) Exposure Period. Three exposure periods - acute (less than 15 days), intermediate (15364 days), and chronic (365 days or more) - are presented within each relevant route of exposure. In this example, an inhalation study of intermediate exposure duration is reported. For quick reference to health effects occurring from a known length of exposure, locate the applicable exposure period within the LSE table and figure.

(3) Health Effect. The major categories of health effects included in LSE tables and figures are death, systemic, immunological, neurological, developmental, reproductive, and cancer. NOAELs and LOAELs can be reported in the tables and figures for all effects but cancer. Systemic effects are further defined in the "System" column of the LSE table (see key number 18).

(4) Key to Figure. Each key number in the LSE table links study information to one or more data points using the same key number in the corresponding LSE figure. In this example, the study represented by key number 18 has been used to derive a NOAEL and a Less Serious LOAEL (also see the two "18r" data points in sample Figure 3-1).

(5) Species. The test species, whether animal or human, are identified in this column. Chapter 2, "Relevance to Public Health," covers the relevance of animal data to human toxicity and Section 3.4, "Toxicokinetics," contains any available information on comparative toxicokinetics. Although NOAELs and LOAELs are species specific, the levels are extrapolated to equivalent human doses to derive an MRL.

(6) Exposure Frequency/Duration. The duration of the study and the weekly and daily exposure regimens are provided in this column. This permits comparison of NOAELs and LOAELs from different studies. In this case (key number 18), rats were exposed to "Chemical x" via inhalation for 6 hours/day, 5 days/week, for 13 weeks. For a more complete review of the dosing regimen, refer to the appropriate sections of the text or the original reference paper (i.e., Nitschke et al. 1981).

(7) System. This column further defines the systemic effects. These systems include respiratory, cardiovascular, gastrointestinal, hematological, musculoskeletal, hepatic, renal, and dermal/ocular. "Other" refers to any systemic effect (e.g., a decrease in body weight) not covered in these systems. In the example of key number 18, one systemic effect (respiratory) was investigated.

(8) NOAEL. A NOAEL is the highest exposure level at which no harmful effects were seen in the organ system studied. Key number 18 reports a NOAEL of 3 ppm for the respiratory system, which was used to derive an intermediate exposure, inhalation MRL of $0.005 \mathrm{ppm}$ (see footnote "b"). 
(9) LOAEL. A LOAEL is the lowest dose used in the study that caused a harmful health effect. LOAELs have been classified into "Less Serious" and "Serious" effects. These distinctions help readers identify the levels of exposure at which adverse health effects first appear and the gradation of effects with increasing dose. A brief description of the specific end point used to quantify the adverse effect accompanies the LOAEL. The respiratory effect reported in key number 18 (hyperplasia) is a Less Serious LOAEL of $10 \mathrm{ppm}$. MRLs are not derived from Serious LOAELs.

(10) Reference. The complete reference citation is given in Chapter 9 of the profile.

(11) CEL. A CEL is the lowest exposure level associated with the onset of carcinogenesis in experimental or epidemiologic studies. CELs are always considered serious effects. The LSE tables and figures do not contain NOAELs for cancer, but the text may report doses not causing measurable cancer increases.

(12) Footnotes. Explanations of abbreviations or reference notes for data in the LSE tables are found in the footnotes. Footnote "b" indicates that the NOAEL of 3 ppm in key number 18 was used to derive an MRL of $0.005 \mathrm{ppm}$.

\section{LEGEND}

\section{See Sample Figure 3-1 (page B-7)}

LSE figures graphically illustrate the data presented in the corresponding LSE tables. Figures help the reader quickly compare health effects according to exposure concentrations for particular exposure periods.

(13) Exposure Period. The same exposure periods appear as in the LSE table. In this example, health effects observed within the acute and intermediate exposure periods are illustrated.

(14) Health Effect. These are the categories of health effects for which reliable quantitative data exists. The same health effects appear in the LSE table.

(15) Levels of Exposure. Concentrations or doses for each health effect in the LSE tables are graphically displayed in the LSE figures. Exposure concentration or dose is measured on the log scale "y" axis. Inhalation exposure is reported in $\mathrm{mg} / \mathrm{m}^{3}$ or $\mathrm{ppm}$ and oral exposure is reported in $\mathrm{mg} / \mathrm{kg} / \mathrm{day}$.

(16) NOAEL. In this example, the open circle designated $18 \mathrm{r}$ identifies a NOAEL critical end point in the rat upon which an intermediate inhalation exposure MRL is based. The key number 18 corresponds to the entry in the LSE table. The dashed descending arrow indicates the extrapolation from the exposure level of $3 \mathrm{ppm}$ (see entry 18 in the table) to the MRL of $0.005 \mathrm{ppm}$ (see footnote "b" in the LSE table).

(17) CEL. Key number $38 \mathrm{~m}$ is one of three studies for which CELs were derived. The diamond symbol refers to a CEL for the test species-mouse. The number 38 corresponds to the entry in the LSE table. 
(18) Estimated Upper-Bound Human Cancer Risk Levels. This is the range associated with the upperbound for lifetime cancer risk of 1 in 10,000 to 1 in 10,000,000. These risk levels are derived from the EPA's Human Health Assessment Group's upper-bound estimates of the slope of the cancer dose response curve at low dose levels $\left(\mathrm{q}_{1}{ }^{*}\right)$.

(19) Key to LSE Figure. The Key explains the abbreviations and symbols used in the figure. 


\section{SAMPLE}

$1 \rightarrow \quad$ Table 3-1. Levels of Significant Exposure to [Chemical x] - Inhalation

\begin{tabular}{|c|c|c|c|c|c|c|c|c|c|}
\hline & & \multirow[b]{2}{*}{$\begin{array}{l}\text { Key to } \\
\text { figure }^{a}\end{array}$} & \multirow{2}{*}{\multicolumn{2}{|c|}{$\begin{array}{c}\text { Exposure } \\
\text { frequency/ } \\
\text { Species duration }\end{array}$}} & \multirow[b]{2}{*}{ System } & \multirow[b]{2}{*}{$\begin{array}{l}\text { NOAEL } \\
(\mathrm{ppm})\end{array}$} & \multicolumn{2}{|l|}{ LOAEL (effect) } & \multirow[b]{2}{*}{ Reference } \\
\hline & & & & & & & $\begin{array}{l}\text { Less serious } \\
(\mathrm{ppm})\end{array}$ & Serious (ppm) & \\
\hline \multirow[t]{2}{*}{2} & $\rightarrow$ & \multicolumn{8}{|c|}{ INTERMEDIATE EXPOSURE } \\
\hline & & & 5 & 6 & 7 & 8 & 9 & & 10 \\
\hline 3 & $\rightarrow$ & Systemic & $\downarrow$ & $\downarrow$ & $\downarrow$ & $\downarrow$ & $\downarrow$ & & $\downarrow$ \\
\hline \multirow[t]{7}{*}{4} & $\rightarrow$ & 18 & Rat & $\begin{array}{l}13 \mathrm{wk} \\
5 \mathrm{~d} / \mathrm{wk} \\
6 \mathrm{hr} / \mathrm{d}\end{array}$ & Resp & $3^{b}$ & 10 (hyperplasia) & & Nitschke et al. 1981 \\
\hline & & CHRONIC & XPOSUR & & & & & & \\
\hline & & Cancer & & & & & 11 & & \\
\hline & & & & & & & $\downarrow$ & & \\
\hline & & 38 & Rat & $\begin{array}{l}18 \mathrm{mo} \\
5 \mathrm{~d} / \mathrm{wk} \\
7 \mathrm{hr} / \mathrm{d}\end{array}$ & & & 20 & $\begin{array}{l}\text { (CEL, multiple } \\
\text { organs) }\end{array}$ & Wong et al. 1982 \\
\hline & & 39 & Rat & $\begin{array}{l}89-104 \mathrm{wk} \\
5 \mathrm{~d} / \mathrm{wk} \\
6 \mathrm{hr} / \mathrm{d}\end{array}$ & & & 10 & $\begin{array}{l}\text { (CEL, lung tumors, } \\
\text { nasal tumors) }\end{array}$ & NTP 1982 \\
\hline & & 40 & Mouse & $\begin{array}{l}79-103 \mathrm{wk} \\
5 \mathrm{~d} / \mathrm{wk} \\
6 \mathrm{hr} / \mathrm{d}\end{array}$ & & & 10 & $\begin{array}{l}\text { (CEL, lung tumors, } \\
\text { hemangiosarcomas) }\end{array}$ & NTP 1982 \\
\hline
\end{tabular}




\section{SAMPLE}

Figure 3-1. Levels of Significant Exposure to [Chemical X] - Inhalation 13

\section{Acute ( $\leq 14$ days)}

Intermediate (15-364 days)
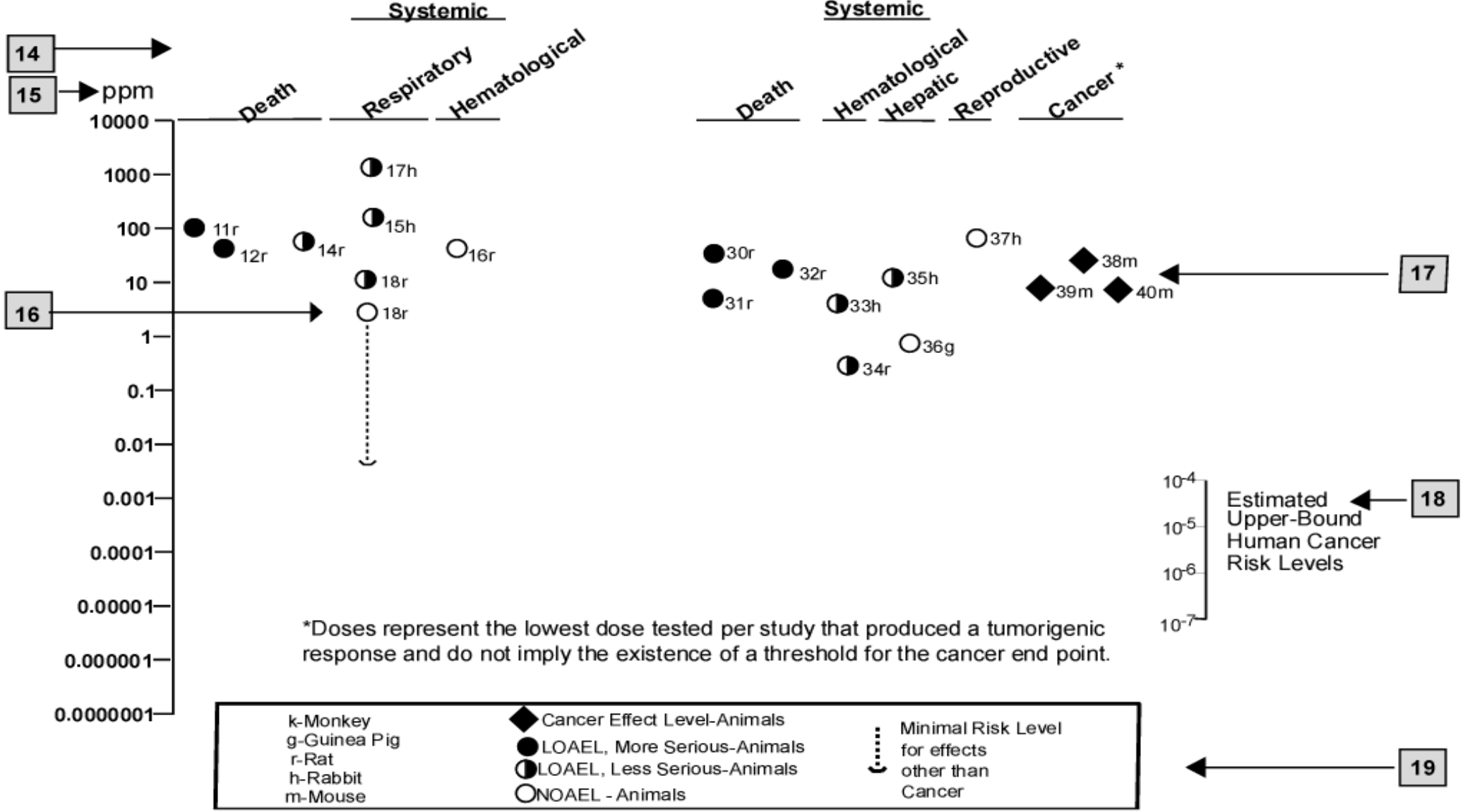



\section{APPENDIX C. ACRONYMS, ABBREVIATIONS, AND SYMBOLS}

\begin{tabular}{|c|c|}
\hline ACGIH & American Conference of Governmental Industrial Hygienists \\
\hline ACOEM & American College of Occupational and Environmental Medicine \\
\hline ADI & acceptable daily intake \\
\hline ADME & absorption, distribution, metabolism, and excretion \\
\hline AED & atomic emission detection \\
\hline AFID & alkali flame ionization detector \\
\hline AFOSH & Air Force Office of Safety and Health \\
\hline ALT & alanine aminotransferase \\
\hline AML & acute myeloid leukemia \\
\hline $\mathrm{AOAC}$ & Association of Official Analytical Chemists \\
\hline AOEC & Association of Occupational and Environmental Clinics \\
\hline AP & alkaline phosphatase \\
\hline APHA & American Public Health Association \\
\hline AST & aspartate aminotransferase \\
\hline atm & atmosphere \\
\hline ATSDR & Agency for Toxic Substances and Disease Registry \\
\hline AWQC & Ambient Water Quality Criteria \\
\hline BAT & best available technology \\
\hline $\mathrm{BCF}$ & bioconcentration factor \\
\hline BEI & Biological Exposure Index \\
\hline BMD & benchmark dose \\
\hline BMR & benchmark response \\
\hline $\mathrm{BSC}$ & Board of Scientific Counselors \\
\hline BUN & Blood urea nitrogen \\
\hline $\mathrm{C}$ & centigrade \\
\hline CAA & Clean Air Act \\
\hline CAG & Cancer Assessment Group of the U.S. Environmental Protection Agency \\
\hline CAS & Chemical Abstract Services \\
\hline $\mathrm{CDC}$ & Centers for Disease Control and Prevention \\
\hline CEL & cancer effect level \\
\hline CELDS & Computer-Environmental Legislative Data System \\
\hline CERCLA & Comprehensive Environmental Response, Compensation, and Liability Act \\
\hline CFR & Code of Federal Regulations \\
\hline $\mathrm{Ci}$ & curie \\
\hline CI & confidence interval \\
\hline $\mathrm{CL}$ & ceiling limit value \\
\hline CLP & Contract Laboratory Program \\
\hline $\mathrm{cm}$ & centimeter \\
\hline CML & chronic myeloid leukemia \\
\hline CPSC & Consumer Products Safety Commission \\
\hline CWA & Clean Water Act \\
\hline DHEW & Department of Health, Education, and Welfare \\
\hline DHHS & Department of Health and Human Services \\
\hline DNA & deoxyribonucleic acid \\
\hline DOD & Department of Defense \\
\hline DOE & Department of Energy \\
\hline DOL & Department of Labor \\
\hline DOT & Department of Transportation \\
\hline
\end{tabular}




\begin{tabular}{|c|c|}
\hline $\begin{array}{l}\text { DOT/UN/ } \\
\text { NA/IMCO }\end{array}$ & $\begin{array}{l}\text { Department of Transportation/United Nations/ } \\
\text { North America/International Maritime Dangerous Goods Code }\end{array}$ \\
\hline DWEL & drinking water exposure level \\
\hline ECD & electron capture detection \\
\hline ECG/EKG & electrocardiogram \\
\hline EEG & electroencephalogram \\
\hline EEGL & Emergency Exposure Guidance Level \\
\hline EPA & Environmental Protection Agency \\
\hline $\mathrm{F}$ & Fahrenheit \\
\hline $\mathrm{F}_{1}$ & first-filial generation \\
\hline FAO & Food and Agricultural Organization of the United Nations \\
\hline FDA & Food and Drug Administration \\
\hline FEMA & Federal Emergency Management Agency \\
\hline FIFRA & Federal Insecticide, Fungicide, and Rodenticide Act \\
\hline FPD & flame photometric detection \\
\hline $\mathrm{fpm}$ & feet per minute \\
\hline FR & Federal Register \\
\hline FSH & follicle stimulating hormone \\
\hline $\mathrm{g}$ & gram \\
\hline GC & gas chromatography \\
\hline $\operatorname{gd}$ & gestational day \\
\hline GLC & gas liquid chromatography \\
\hline GPC & gel permeation chromatography \\
\hline HPLC & high-performance liquid chromatography \\
\hline HRGC & high resolution gas chromatography \\
\hline HSDB & Hazardous Substance Data Bank \\
\hline IARC & International Agency for Research on Cancer \\
\hline IDLH & immediately dangerous to life and health \\
\hline ILO & International Labor Organization \\
\hline IRIS & Integrated Risk Information System \\
\hline $\mathrm{Kd}$ & adsorption ratio \\
\hline $\mathrm{kg}$ & kilogram \\
\hline $\mathrm{kkg}$ & metric ton \\
\hline $\mathrm{K}_{\mathrm{oc}}$ & organic carbon partition coefficient \\
\hline $\mathrm{K}_{\mathrm{ow}}$ & octanol-water partition coefficient \\
\hline $\mathrm{L}$ & liter \\
\hline $\mathrm{LC}$ & liquid chromatography \\
\hline $\mathrm{LC}_{50}$ & lethal concentration, $50 \%$ kill \\
\hline $\mathrm{LC}_{\mathrm{Lo}}$ & lethal concentration, low \\
\hline $\mathrm{LD}_{50}$ & lethal dose, $50 \%$ kill \\
\hline $\mathrm{LD}_{\mathrm{Lo}}$ & lethal dose, low \\
\hline LDH & lactic dehydrogenase \\
\hline LH & luteinizing hormone \\
\hline LOAEL & lowest-observed-adverse-effect level \\
\hline LSE & Levels of Significant Exposure \\
\hline $\mathrm{LT}_{50}$ & lethal time, $50 \%$ kill \\
\hline $\mathrm{m}$ & meter \\
\hline MA & trans,trans-muconic acid \\
\hline MAL & maximum allowable level \\
\hline $\mathrm{mCi}$ & millicurie \\
\hline MCL & maximum contaminant level \\
\hline
\end{tabular}




\begin{tabular}{|c|c|}
\hline MCLG & maximum contaminant level goal \\
\hline MF & modifying factor \\
\hline MFO & mixed function oxidase \\
\hline $\mathrm{mg}$ & milligram \\
\hline $\mathrm{mL}$ & milliliter \\
\hline $\mathrm{mm}$ & millimeter \\
\hline MMAD & Mass median aerodynamic diameter \\
\hline $\mathrm{mmHg}$ & millimeters of mercury \\
\hline mmol & millimole \\
\hline mppcf & millions of particles per cubic foot \\
\hline MRL & Minimal Risk Level \\
\hline MS & mass spectrometry \\
\hline NAAQS & National Ambient Air Quality Standard \\
\hline NAG & $\mathrm{N}$-Acetyl- $\beta$-D-glucosaminidase \\
\hline NAS & National Academy of Science \\
\hline NATICH & National Air Toxics Information Clearinghouse \\
\hline NATO & North Atlantic Treaty Organization \\
\hline NCE & normochromatic erythrocytes \\
\hline $\mathrm{NCEH}$ & National Center for Environmental Health \\
\hline NCI & National Cancer Institute \\
\hline ND & not detected \\
\hline $\begin{array}{l}\text { NFPA } \\
\text { ng }\end{array}$ & $\begin{array}{l}\text { National Fire Protection Association } \\
\text { nanogram }\end{array}$ \\
\hline NHANES & National Health and Nutrition Examination Survey \\
\hline NIEHS & National Institute of Environmental Health Sciences \\
\hline $\mathrm{NIOSH}$ & National Institute for Occupational Safety and Health \\
\hline NIOSHTIC & NIOSH's Computerized Information Retrieval System \\
\hline NLM & National Library of Medicine \\
\hline $\mathrm{nm}$ & nanometer \\
\hline nmol & nanomole \\
\hline NOAEL & no-observed-adverse-effect level \\
\hline NOES & National Occupational Exposure Survey \\
\hline NOHS & National Occupational Hazard Survey \\
\hline NPD & nitrogen phosphorus detection \\
\hline NPDES & National Pollutant Discharge Elimination System \\
\hline NPL & National Priorities List \\
\hline NR & not reported \\
\hline NRC & National Research Council \\
\hline NS & not specified \\
\hline NSPS & New Source Performance Standards \\
\hline NTIS & National Technical Information Service \\
\hline NTP & National Toxicology Program \\
\hline ODW & Office of Drinking Water, EPA \\
\hline OERR & Office of Emergency and Remedial Response, EPA \\
\hline $\mathrm{OHM} / \mathrm{TADS}$ & Oil and Hazardous Materials/Technical Assistance Data System \\
\hline OPP & Office of Pesticide Programs, EPA \\
\hline OPPT & Office of Pollution Prevention and Toxics, EPA \\
\hline OPPTS & Office of Prevention, Pesticides and Toxic Substances, EPA \\
\hline OR & odds ratio \\
\hline OSHA & Occupational Safety and Health Administration \\
\hline OSW & Office of Solid Waste, EPA \\
\hline
\end{tabular}




\begin{tabular}{|c|c|}
\hline OTS & Office of Toxic Substances \\
\hline OW & Office of Water \\
\hline OWRS & Office of Water Regulations and Standards, EPA \\
\hline PAH & polycyclic aromatic hydrocarbon \\
\hline PBPD & physiologically based pharmacodynamic \\
\hline PBPK & physiologically based pharmacokinetic \\
\hline PCE & polychromatic erythrocytes \\
\hline PEL & permissible exposure limit \\
\hline pg & picogram \\
\hline PHS & Public Health Service \\
\hline PID & photo ionization detector \\
\hline pmol & picomole \\
\hline PMR & proportionate mortality ratio \\
\hline ppb & parts per billion \\
\hline ppm & parts per million \\
\hline ppt & parts per trillion \\
\hline PSNS & pretreatment standards for new sources \\
\hline $\mathrm{RBC}$ & red blood cell \\
\hline REL & recommended exposure level/limit \\
\hline $\mathrm{RfC}$ & reference concentration \\
\hline RfD & reference dose \\
\hline RNA & ribonucleic acid \\
\hline RQ & reportable quantity \\
\hline RTECS & Registry of Toxic Effects of Chemical Substances \\
\hline SARA & Superfund Amendments and Reauthorization Act \\
\hline SCE & sister chromatid exchange \\
\hline SGOT & serum glutamic oxaloacetic transaminase \\
\hline SGPT & serum glutamic pyruvic transaminase \\
\hline SIC & standard industrial classification \\
\hline SIM & selected ion monitoring \\
\hline SMCL & secondary maximum contaminant level \\
\hline SMR & standardized mortality ratio \\
\hline SNARL & suggested no adverse response level \\
\hline SPEGL & Short-Term Public Emergency Guidance Level \\
\hline STEL & short term exposure limit \\
\hline STORET & Storage and Retrieval \\
\hline $\mathrm{TD}_{50}$ & toxic dose, $50 \%$ specific toxic effect \\
\hline TLV & threshold limit value \\
\hline TOC & total organic carbon \\
\hline TPQ & threshold planning quantity \\
\hline TRI & Toxics Release Inventory \\
\hline TSCA & Toxic Substances Control Act \\
\hline TWA & time-weighted average \\
\hline UF & uncertainty factor \\
\hline U.S. & United States \\
\hline USDA & United States Department of Agriculture \\
\hline USGS & United States Geological Survey \\
\hline VOC & volatile organic compound \\
\hline WBC & white blood cell \\
\hline WHO & World Health Organization \\
\hline
\end{tabular}




$\begin{array}{ll}> & \text { greater than } \\ \geq & \text { greater than or equal to } \\ = & \text { equal to } \\ < & \text { less than } \\ \leq & \text { less than or equal to } \\ \% & \text { percent } \\ \alpha & \text { alpha } \\ \beta & \text { beta } \\ \gamma & \text { gamma } \\ \delta & \text { delta } \\ \mu \mathrm{m} & \text { micrometer } \\ \mu \mathrm{g} & \text { microgram } \\ \mathrm{q}_{1}{ }^{*} & \text { cancer slope factor } \\ - & \text { negative } \\ + & \text { positive } \\ (+) & \text { weakly positive result } \\ (-) & \text { weakly negative result }\end{array}$





\section{APPENDIX D. INDEX}

absorbed dose 161,181

active transport. 153

adenocarcinomas 80,86 adrenal gland $72,112,134,138$

adrenals 138

adsorbed $212,215,220,223,224,225,229,258,276$

adsorption $213,220,223,224,225,226,231,258,266$

aerobic 212,230

ambient air anaerobic $11,20,22,25,205,211,231,238,243$

bioaccumulation 213,230

bioavailability 228

bioconcentration factor 258

biomarker 227

biomarkers $161,164,275$

body weight effects $18,73,115$

breast milk

$$
18,73,115
$$

cancer $5,6,7,14,16,26,27,79,80,81,86,87,123,130,138,157,159,175,283$

carcinogen $7,16,87,279,282,283$ carcinogenic $7,13,16,17,25,26,79,80,81,86,87,155,157,175,279,283$ carcinogenicity $15,81,153,155,156,169,175,179,183$ carcinoma 87,88 carcinomas 80,86 cardiovascular $69,78,123$ cardiovascular effects 69,109 chromosomal aberrations 155,176

clearance $19,139,143,149,150,151,153,157,163,168,181,283$ contact dermatitis $13,15,25,26,27,28,65,86,90,91,123,170$ death $72,113,127$ dermal effects $130,132,133,134,155,156,161,164,169,176,180$

DNA elimination half-time $72,112,126,154,157,158$ endocrine $72,112,126,158$ endocrine effects 113 erythema $8,120,158,160,182$

fetus

fractional absorption 148 gastrointestinal effects $69,109,110$ general population $4,11,12,13,14,80,113,127,138,160,161,162,178,179$ $205,211,243,244,246,256,263,275$

genotoxic $133,155,176$ genotoxicity $130,156,176$ groundwater $2,11,211,212,219,223,226,227,230,235,253,256,268,270$

half-life $25,135,141,161,163,220$ hematological effects $70,110,123$ hepatic effects $70,111,126$ 
hydroxyl radical . 213

immune system $7,12,73,75$

immunological $25,73,74,76,116,117,172,177,178$

immunological effects $117,172,178$

$\mathrm{K}_{\text {ow }}$ $191,192,193$

$\mathrm{LD}_{50}$ $90,91,173$

lung cancer $13,16,80,86,87$

lymphatic .76

lymphoreticular.

$74,76,174$

metabolic effects 74,115

milk $3,8,91,134,136,142,154,155,160,167,177,242,250,253$

mucociliary $143,163,167$ musculoskeletal effects 70,110 neonatal. $23,160,174,179$ neurobehavioral 158,179

neurochemical 77

neurodevelopmental. 120 ocular effects 28,123 pharmacodynamic 144 pharmacokinetic placenta $115,144,145,146,159,165$ pulmonary fibrosis $8,79,134,140,160$ rate constant $15,20,22,65$ renal effects 147,148 retention $71,111,112$ salivation . sarcoma 130,132 solubility spermatogonia thyroid toxicokinetic $139,140,223,224,226,262,283$ tumors $15,25,68,134,137,143,169,213,226,261$ 
